Portland State University

PDXScholar

$1-1-2010$

\title{
Library Characterization and Static Timing Analysis of Single-Track Circuits in GasP
}

Swetha Mettala Gilla

Portland State University

Follow this and additional works at: https://pdxscholar.library.pdx.edu/open_access_etds

Let us know how access to this document benefits you.

\section{Recommended Citation}

Mettala Gilla, Swetha, "Library Characterization and Static Timing Analysis of Single-Track Circuits in GasP" (2010). Dissertations and Theses. Paper 273.

https://doi.org/10.15760/etd.273

This Thesis is brought to you for free and open access. It has been accepted for inclusion in Dissertations and Theses by an authorized administrator of PDXScholar. Please contact us if we can make this document more accessible: pdxscholar@pdx.edu. 


\title{
Library Characterization and Static Timing Analysis of Single-Track Circuits in GasP
}

\section{by}

\section{Swetha Mettala Gilla}

\begin{abstract}
A thesis submitted in partial fulfillment of the requirements for the degree of
\end{abstract}

\author{
Master of Science \\ in \\ Electrical and Computer Engineering
}

Thesis Committee:

Xiaoyu Song, Chair

Douglas V. Hall

Christof Teuscher

Marly Roncken

Portland State University

(C) 2010 


\section{Abstract}

Library characterization and 'Static Timing Analysis' (STA) are widely used in the design of modern CMOS integrated circuits to confirm that critical timing constraints are met. While many commercial tools are available to do timing validation using library characterization and static timing analysis, their operation depends on calculations relative to a global synchronous clock. This thesis applies timing validation to circuits from which the global synchronous clock is absent, making application of commercial tools difficult. Previous work at the University of Southern California (USC) showed how to overcome the incompatibility of commercial STA tools for asynchronous circuits. This thesis shows how to overcome the incompatibility of library characterization for asynchronous circuits, and ties the results to the STA solution of USC.

The particular family of circuits considered in this thesis is called GasP. GasP circuits are light in area and power, and have demonstrated operation at about twice the throughput one would expect from conventional clocked circuits. This makes GasP an ideal candidate for modern network-on-chip and system-on-chip architectures. In part, GasP circuits achieve their performance advantages by using a 'single-track' signaling protocol. Two GasP modules communicate with each other over a single wire. One module drives the wire up and a second module at the other end of the wire drives the wire down. This conflicts with the common assumption that wires are driven only from one end. As a result, special circuitry is needed to characterize a GasP library module. This thesis shows how to break a GasP module and its timing constraints into manageable pieces and how to simulate and collect the data relevant for characterization and static timing analysis. When combined with software tools for identifying the critical timing constraints, these thesis results will provide confidence in the correct operation of GasP. 


\section{Acknowledgements}

This work was partly sponsored under DARPA grant number HR0011-10-1-0069 for "Fleet Studies". In addition to DARPA, I would like to thank all the people who have helped and inspired me during my thesis work.

I especially want to thank my advisor, Drs. Marly Roncken, for her guidance during my research and study at Portland State University (PSU). Her perpetual energy and enthusiasm in research were a great motivation to me. She was always accessible and willing to help me with my research. She is the greatest personality I ever met in my 24 years life time. I admire her personality after Swami Vivekananda.

I was delighted to interact with Dr. Ivan Sutherland. It was a great opportunity to participate in his seminars on GasP circuits, and an even greater opportunity to get the chance to work with him on a timing flow for these circuits. His insight in asynchronous circuit design is amazing. He explains anything that looks like "eschew obfuscation" into something as obvious as "keep it simple". ${ }^{0.1}$ To me, he defines what a world-class researcher and teacher are about.

The GasP seminar class and my research with Marly and Ivan at the Asynchronous Research Center (ARC) were both a turning point in my life and a wonderful experience. The chance to publish and present my research work at the ASYNC 2010 conference

\footnotetext{
${ }^{0.1}$ The text "eschew obfuscation" is on one of his sweatshirts.
} 
in Grenoble, France, broadened my view of the world, and enabled me to meet other researchers and students working in the domain of asynchronous circuits.

I am much obliged to Professor Xiaoyu Song, for agreeing to be my thesis advisor. One simply could not wish for a more friendly and understanding advisor. I feel blessed by his encouragement to pursue this thesis topic, and to help me plan it. Professor Douglas V. Hall, and Professor Christof Teuscher deserve special thanks as my thesis committee members. I sincerely appreciate their participation and technical feedback.

I want to thank Prasad Joshi, Mallika Prakash, and Professor Peter Beerel from the University of Southern California (USC), for making their static timing analysis flow available, and for sending me their publications and any scripts they could officially provide. The USC flow became the basis for my thesis. I am particularly grateful to Prasad for being so patient in replying to my string of emails with questions, even during the busy time before his wedding.

I would also like to thank my first manager at PSU, Lisa Weldon, for her support in providing me with a student job during my initial struggling days at PSU when finances were tight. My one year stint at HAL India opened my mind and motivated me to attend graduate school. I would like to thank the friends from my college years in India, in particular my badminton friends "6STARS", and my close friend in Portland: Laddu. My deepest gratitude goes to my family for their unflagging love and support. Without them, this thesis would simply not exist. I am indebted to my mother, Indira Bai, for her care and love. She is simply perfect. I have no suitable words to describe her everlasting love to me. I remember her support when I encountered difficulties, and I am daily reminded of her delicious dishes now that I do my own cooking. I thank my father, Gopal Reddy, for believing in me and for supporting my study and career decisions. 


\section{Contents}

Abstract

Acknowledgements ii

List of Figures $\quad$ xi

1 INTRODUCTION 1

1.1 GasP Circuits ....................... 2

1.2 Timing Validation Flow . . . . . . . . . . . . . . 4

1.3 Reading Guide . . . . . . . . . . . . . . . . 6

2 6-4 GASP AND ITS RELATIVE TIMING CONSTRAINTS 10

3 LIBRARY CHARACTERIZATION PART-A: LOOK UP TABLES (LUTs) 14

3.1 Introduction . . . . . . . . . . . . . . . . . . 14

3.2 Step 1: Use Electric to Access the GasP Design . . . . . . . . . . . . 18

3.3 Step 2: Create a Simulation Environment for the Design . . . . . . . . 20

3.4 Step 3: Generate Input Files for SPICE . . . . . . . . . . . . . . . . . . 21 
3.4.1 Generate a SPICE Netlist . . . . . . . . . . . . . . 21

3.4.2 Generate SPICE Simulation Files: HEADER and TRAILER . . 21

3.5 Step 4: Modify the SPICE Input Files . . . . . . . . . . . . . . . 23

3.5.1 Create Sweep Parameters in the SPICE Netlist . . . . . . . . 23

3.5.2 Add Sweep and Measurement Statements to the TRAILER File 25

3.6 Step 5: Generate a SPICE Output File with Look Up Tables . . . . . . . 26

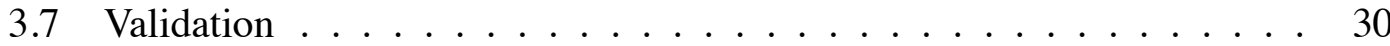

3.8 Conclusion and Future Work for Chapter $3 \ldots \ldots 33$

4 LIBRARY CHARACTERIZATION PART-B: FROM GASP TO LUTs 34

4.1 Introduction . . . . . . . . . . . . . . . . . 34

4.2 Quick Reminder: What's a Look Up Table and How's It Used? . . . . . 37

4.3 STEP 1: Partitioning Complex Paths into Simple Paths . . . . . . . . 37

4.4 STEP 2: Defining an Accurate Simulation Set-Up for Simple Paths . . . 47

4.4.1 Circuit Partitioning for RT1 to RT4 . . . . . . . . . . . . 47

4.4.2 Circuit Enhancements for Min-Max and Repetitive Simulations 55

4.4.2.1 Simulating Minimum Gate Delays . . . . . . . . 56

4.4.2.2 Autonomously Re-Initializing Un-Driven Wires . . . 59

4.4.2.3 Summary of Circuit Enhancements . . . . . . . . 64

4.4.3 Back to PART-A to Generate Look Up Tables . . . . . . . . . . 65

4.4.3.1 Setup for Figure 4.8 and LUTblue:PRED ${ }_{i}-\mathrm{FIRE}_{i}-\quad$. 66 
4.4.3.2 Setup for Figure 4.9 and LUTblue:SUCC $i+\mathrm{FIRE}_{i}-\quad$. 68

4.4.3.3 Setup for Figure 4.7(b) and LUTblue:FIRE $i-$ Dout $_{i}+\quad 69$

4.4.3.4 Setup for Figure 4.10, LUTred/blue:FIRE $i+$ SUCC $_{i}+71$

4.4.3.5 Setup for Figure 4.11, LUTblue/green:FIRE + PRED $_{i}-76$

4.5 General Simulation Setup Covering All Possible Sweep Cases . . . . . 81

4.5.1 Covering All Possible External Input Drives . . . . . . . . . . . 81

4.5.2 Covering Zero Exernal Output Loads . . . . . . . . . . . . 83

4.6 Conclusion and Future Work for Chapter $4 \ldots \ldots$. . . . . . 85

\section{STATIC TIMING ANALYSIS OF 6-4 GASP USING LOOK UP TABLES 88}

5.1 Introduction . . . . . . . . . . . . . . . . . 89

5.2 STEP 1: From SPICED to PrimeTime Look Up Tables . . . . . . . . . 91

5.2.1 From Input Driver Size to Input Transition Slope . . . . . . . . 93

5.2.2 From Output Load Size to Output Load Capacitance . . . . . . 94

5.2.3 A Quick Sanity Check of the PrimeTime Look Up Tables . . . . 103

5.3 STEP 2: From 6-4 GasP Module to PrimeTime Black Box . . . . . . . 105

5.4 STEP 3: Create a Library File with SPICED Look Up Tables . . . . . . 106

5.4.1 STEP 3.1: Rename Look Up Tables to Match Black Box Names 107

5.4.2 STEP 3.2: Create a Library File with SPICED Look Up Tables . 110

5.5 STEP 4: Create Commands for Relative Timing Validation . . . . . . . 112

5.6 STEP 5: Run and Inspect Timing Reports . . . . . . . . . . . . . 113 
5.7 Conclusion and Future Work for Chapter 5 . . . . . . . . . 116

6 SUMMARY AND DISCUSSION OF TIMING RESULTS 118

7 COMPARISON TO RELATED WORK 123

8 CONCLUSION AND FUTURE WORK 126

$\begin{array}{ll}\text { References } & 127\end{array}$

Appendices

A SPICE FILES FOR SPICE SWEEPS 132

A.1 SPICE Netlist Generated by Electric . . . . . . . . . . . . . . . 132

A.2 SPICE Simulation Set-Up File: HEADER . . . . . . . . . . . . 136

A.3 SPICE Simulation Set-Up File: TRAILER . . . . . . . . . . . . . 136

A.4 Modified SPICE Netlist, Ready for Sweep . . . . . . . . . . . . . . . . 137

A.5 Modified SPICE TRAILER File, Ready for Sweep ～. . . . . . . . . . 140

A.6 SPICE Output File . . . . . . . . . . . . . . . . 141

B SPICE FILES FOR SWEEPS IN THE DESIGN SPACE 142

B.1 SPICE Netlist for Reference Design . . . . . . . . . . . . . . . 142

B.2 SPICE TRAILER File for Reference Design . . . . . . . . . . . . . 151

B.3 SPICE Output File for Reference Design . . . . . . . . . . . . . 153 
C SPICE FILES FOR FIGURE 4.8 AND LUTblue:PRED ${ }_{i}-$ FIRE $_{i}-$

C.1 SPICE Netlist . . . . . . . . . . . . . . . . . . . 154

C.2 SPICE Simulation Setup File: HEADER . . . . . . . . . . . . . . . . 160

C.3 SPICE Simulation Setup File: TRAILER _ . . . . . . . . . . . . 161

C.4 SPICE Output File . . . . . . . . . . . . . . . 163

D SPICE FILES FOR FIGURE 4.9 AND LUTblue:SUCC $i+$ FIRE $_{i}-\quad 165$

D.1 SPICE Netlist . . . . . . . . . . . . . . . 165

D.2 SPICE Simulation Setup File: HEADER . . . . . . . . . . . . . . 171

D.3 SPICE Simulation Setup File: TRAILER ～. . . . . . . . . . . 172

D.4 SPICE Output File . . . . . . . . . . . . . . . . 174

E SPICE FILES FOR FIGURE 4.7(b) AND LUTblue:FIRE L $_{i}-$ Dout $_{i}+\quad 176$

E.1 SPICE Netlist . . . . . . . . . . . . . . 176

E.2 SPICE Simulation Setup File: HEADER . . . . . . . . . . . . . . . 180

E.3 SPICE Simulation Setup File: TRAILER . . . . . . . . . . . . 181

E.4 SPICE Output File . . . . . . . . . . . . . . . . . . . 182

F SPICE FILES FOR FIGURE 4.10 AND LUTred/blue:FIRE ${ }_{i}+$ SUCC $_{i}+\quad 183$

F.1 SPICE Netlist . . . . . . . . . . . . . . . . 183

F.2 SPICE Simulation Setup File: HEADER . . . . . . . . . . . . . . . 189

F.3 SPICE Simulation Setup File: TRAILER _ . . . . . . . . . . . . 190

F.4 SPICE Output File . . . . . . . . . . . . . . . . . . 192 
G SPICE FILES FOR FIGURE 4.11 AND LUTblue/green:FIRE ${ }_{i}+$ PRED $_{i}-194$

G.1 SPICE Netlist . . . . . . . . . . . . . . . . . . . . . . 194

G.2 SPICE Simulation Setup File: HEADER . . . . . . . . . . . . . . . 199

G.3 SPICE Simulation Setup File: TRAILER _ . . . . . . . . . . 200

G.4 SPICE Output File . . . . . . . . . . . . . . . . 202

H SPICE FILES FOR FIGURE 4.22 WITH ZERO EXTERNAL LOADS 204

H.1 SPICE Netlist . . . . . . . . . . . . . . . . . . 204

H.2 SPICE Simulation Setup File: HEADER . . . . . . . . . . . . . . . . 214

H.3 SPICE Simulation Setup File: TRAILER _ . . . . . . . . . . 215

H.4 SPICE Output File . . . . . . . . . . . . . . . . . . 217

I TRANSLATING LOAD SIZE INTO LOAD CAPACITANCE 219

I.1 SPICE Netlist . . . . . . . . . . . . . . . . . 219

I.2 SPICE Simulation File: HEADER . . . . . . . . . . . . . . 223

I.3 SPICE Simulation File: TRAILER . . . . . . . . . . . . . . . 224

I.4 SPICE Output File . . . . . . . . . . . . . . . 226

J PRIMETIME NETLIST FOR THE TWO-STAGE GASP FIFO 228

K PRIMETIME LIBRARY WITH SPICED LOOK UP TABLES 229

L PRIMETIME COMMANDS FOR VALIDATING RT1 TO RT4 234 
M.1 Timing Report for RT1 or RT(L2) $)^{\operatorname{TrfF}-\mathrm{RstB}} \ldots \ldots$. . . . . . . . . . . . . 237

M.2 Timing Report for RT2 or RT(L2) $)^{\operatorname{TrfF}-\mathrm{RstF}} \ldots \ldots \ldots$. . . . . . . . 238

M.3 Timing Report for RT3 or RT(L2) $)^{\operatorname{TrfB}-\mathrm{RstF}} \ldots \ldots$. . . . . . . . . . . . 239

M.4 Timing Report for RT4 or RT(L2) $)^{\operatorname{TrfB}-\mathrm{RstB}} \quad \ldots \ldots$. . . . . . . . . . 240

N BONUS MATERIAL: DISTANCE CONSTRAINT GRAPH 241

O BONUS MATERIAL: SHORT-TO-MEDIUM-TO-LONG WIRE STUDY 253

$P \quad$ BONUS MATERIAL: THESIS POWERPOINT PRESENTATION 265 


\section{List of Figures}

1.1 High-level view of a linear pipeline in GasP with a bundled datapath . . 4

1.2 Timing validation flow for GasP circuits . . . . . . . . . . 5

2.1 Two-stage FIFO with two 6-4 GasP modules . . . . . . . . . . . . . . . 11

2.2 Relative timing constraints on single-track wire L2 of Figure $2.1 \ldots 13$

3.1 Half-way GasP circuit . . . . . . . . . . . . . . . . . . 19

3.2 Simulation environment for the Half-way GasP circuit in Figure 3.1 . 19

3.3 Initial SPICE netlist with wrong null values for sweep size parameters . $\quad 22$

3.4 SPICE netlist with corrected sweep size parameters . . . . . . . . . . 24

3.5 SPICE simulation file fragment with sweep and measurement statements 27

3.6 SPICE output file fragment with look up table data . . . . . . . . . 29

3.7 Design sweep of the the Half-way GasP circuit in Figure $3.1 \quad$. . . . . 31

3.8 Comparison of Design sweep versus SPICE sweep results . . . . . . . . 32

4.1 Wide-range look up table with delay and output slopes . . . . . . 38

4.2 Wide-range landscape graphs with delay and output slope information . 38

4.3 Partitioning of the complex paths in relative timing constraint RT1 . . 42 
4.4 Partitioning of the complex paths in relative timing constraint RT2 . . 43

4.5 Partitioning of the complex paths in relative timing constraint RT3 . . 44

4.6 Partitioning of the complex paths in relative timing constraint RT4 . . 45

4.7 6-4 GasP circuit partitioning for RT1 to RT4 . . . . . . . . . . . . 51

4.8 Circuit enhancement for LUTblue:PRED $i-\mathrm{FIRE}_{i}-$ for minimum delay 57

4.9 Circuit enhancement for LUTblue:SUCC $i+\mathrm{FIRE}_{i}-$ for minimum delay 58

4.10 Circuit enhancement LUTred/blue:FIRE $i+$ SUCC $_{i}+$ to self-reset output $\quad 61$

4.11 Circuit enhancement LUTblue/green:FIRE + $+\mathrm{PRED}_{i}-$ to self-reset output 63

4.12 Simulation setup for Figure 4.8 and LUTblue:PRED ${ }_{i}-\mathrm{FIRE}_{i}-\ldots$. . . 67

4.13 Simulation setup for Figure 4.9 and LUTblue:SUCC ${ }_{i}+\mathrm{FIRE}_{i}-\ldots 69$

4.14 Simulation setup for Figure 4.7(b)and LUTblue:FIRE ${ }_{i}-$ Dout $_{i}+\ldots$. . 70

4.15 Simulation setup for Figure 4.10 and LUTred/blue:FIRE $+\mathrm{SUCC}_{i}+\ldots 72$

4.16 VCCS validation-1 for Figure 4.15 and LUTred/blue:FIRE ${ }_{i}+\mathrm{SUCC}_{i}+.74$

4.17 VCCS validation-2 for Figure 4.15 and LUTred/blue:FIRE ${ }_{i}+\mathrm{SUCC}_{i}+\ldots 75$

4.18 Simulation setup for Figure 4.11 and LUTblue/green:FIRE ${ }_{i}+\mathrm{PRED}_{i}-\quad$. 77

4.19 VCCS validation-1 for Figure 4.18 and LUTblue/green:FIRE + PRED $_{i}-\quad 79$

4.20 VCCS validation-2 for Figure 4.18 and LUTblue/green:FIRE + PRED $_{i}-\quad 80$

4.21 General simulation setup for look up table sweeps . . . . . . . . . . 82

4.22 Simulation setup for zero external ouput load . . . . . . . . . . . 84

5.1 Simulation setup for translating load size into load capacitance . . . . . 95 
5.2 Graph showing the translation of load size into load capacitance . . . . 97

5.3 SPICE to PrimeTime look up table for $\mathrm{LUTblue}_{\mathrm{PRED}}-\mathrm{FIRE}_{i}-\ldots 9$

5.4 SPICE to PrimeTime look up table for $\mathrm{LUTblue}_{\mathrm{SUCC}}+\mathrm{FIRE}_{i}$ - . . . 99

5.5 SPICE to PrimeTime look up table for LUTblue:FIRE ${ }_{i}-$ Dout $_{i}+\ldots 100$

5.6 SPICE to PrimeTime look up table for LUTred/blue:FIRE ${ }_{i}+\mathrm{SUCC}_{i}+\quad . \quad 101$

5.7 SPICE to PrimeTime look up table for LUTblue/green:FIRE ${ }_{i}+\mathrm{PRED}_{i}-102$

5.8 Landscape graphs with delay and output slope information . . . . . . 103

5.9 Landscape graphs with delay and output slope information - continued 104

5.10 Split pin architecture of a basic 6-4 GasP module with its black box icon 105

5.11 Split pin architecture of the two-stage GasP FIFO . . . . . . . . . 106

5.12 PrimeTime version of the relative timing constraints from Figure 2.2 . 108

5.13 Renaming of SPICE look up table names into PrimeTime names . . . . 110

6.1 Rising and falling delays at the near and far end of single-track wire L2 120

6.2 Distance Constraint Graph for 6-4 GasP in 90nm CMOS . . . . . . . . 122 


\section{Chapter 1}

\section{INTRODUCTION}

Concurrency and energy efficiency have taken their place as first class criteria in the design of modern circuits and systems along with, and at times even at the cost of, speed in terms of throughput and latency. The design of modern concurrent systems centers around a communication infrastructure that connects a number of functional blocks. Examples are IBM Research's Cell systems [8], Intel's research milestones for many-core and teraflop systems [9, 10], and Sun's (now: Oracle's) UltraSPARC T1 microprocessor, codenamed 'Niagara' [11]. Each block tends to have its own clock or timing regime, fit to best match its operations. From a timing point of view, the blocks operate asynchronously. Even if blocks use the same clock frequency, their operations are likely to be skewed or out of phase with respect to each other.

The Asynchronous Research Center (ARC) at Portland State University is building a concurrent system architecture called 'Fleet' [31,13] centered around an asynchronous communication network, built using GasP modules. Fleet anticipates the use of a variety of computation blocks of low to modest complexity, called 'ships', such as lowpower adders and multipliers, high-speed adders and multipliers, dividers, various shift and sort functions, I/O functions, and memory blocks. The ships can be synchronous 
or asynchronous, including GasP. They can be integrated in a single chip, or spread over different chips or FPGAs. This makes it possible to import asynchronous ships designed by companies like Achronix Semiconductor Corporation [5] for asynchronous FPGA's, Fulcrum Microsystems [7] for high-density ethernet switches, and Tiempo [14] for ultra-low-power functions. Alternatively, Fleet can take ships built using different asynchronous design styles from other academic and research institutes. For an overview of asynchronous design styles and a list of institutes that study and develop asynchronous circuits, see the yearly international symposium on asynchronous circuits and systems, which is approaching its 17-th anniversary [12].

\subsection{GasP Circuits}

The target circuits for this thesis are GasP circuits. These circuits use handshake signaling over a single-track wire and single-rail data encoding. This results in $(\mathrm{N}+1)$ wires to communicate $\mathrm{N}$ data bits. The combination of single-track handshaking and single-rail data results in light-weight circuits - light in area and light in power. GasP circuits also happen to be about twice as fast as one would expect of a globally clocked circuit implemented in the same technology. Add the flexibility and elasticity of asynchronous design to high speed, low power, and low area, and you get an excellent circuit family for on-chip communication - which is why Fleet uses a GasP network on chip.

In addition to the data wires, GasP circuits use a single wire for timing. The single-track handshake is a bi-directional communication on that wire. A logical high voltage level on the wire indicates a request, and a logical low voltage level on the wire indicates an acknowledge signal. The wire has two drivers - one at each end, one for the request and one for the acknowledge. This leads naturally to a two-phase return-to-zero handshake. 
The wire is shared, so each end drives only briefly. The brief drive avoids a drive fight between the two ends. In addition, each driver responds only to changes at its own end. It's this local response that enables the communication to complete in two phases. To make this work, we need some faith: faith in our own engineering and in the design tools that we use. The faith in a single-track handshake depends on two assumptions:

Assumption 1: The brief drive is long enough to traverse the wire.

Assumption 2: The voltage level at the near end of the wire reflects the far-end level.

Data in GasP are exchanged using a bundled-data, also known as single-rail, protocol. Data bits are represented using one wire per bit, just like in conventional synchronous circuits. The single-track request signal indicates that the sender module has new data for the receiver module, i.e. it acts as a 'data valid' signal for the data transfer. The single-track acknowledge signal indicates that the receiver module has finished consuming the data, i.e. it acts as a 'completion' signal for the data transfer. Figure 1.1 gives a high-level overview of a linear pipeline in GasP. The GasP modules are connected with single-track wires. Each GasP module Mi generates a local pulse signal FIRE F $_{i}$ that acts as a local clock or enable signal for the group of latches above it, making the group temporarily transparent. To make this work, we need - again - some faith. The faith in a bundled datapath operation can be expressed in terms of conventional setup and hold times between the data inputs and outputs and the clock enable signals at both ends of the datapath. The data bundling constraints in GasP and their static timing analysis have been solved successfully by Prasad Joshi in his Master of Science thesis [2].

In the rest of my thesis, I will assume that timing constraints related to datapaths are no longer an issue, and I will henceforth ignore the datapath portion of a GasP design. 


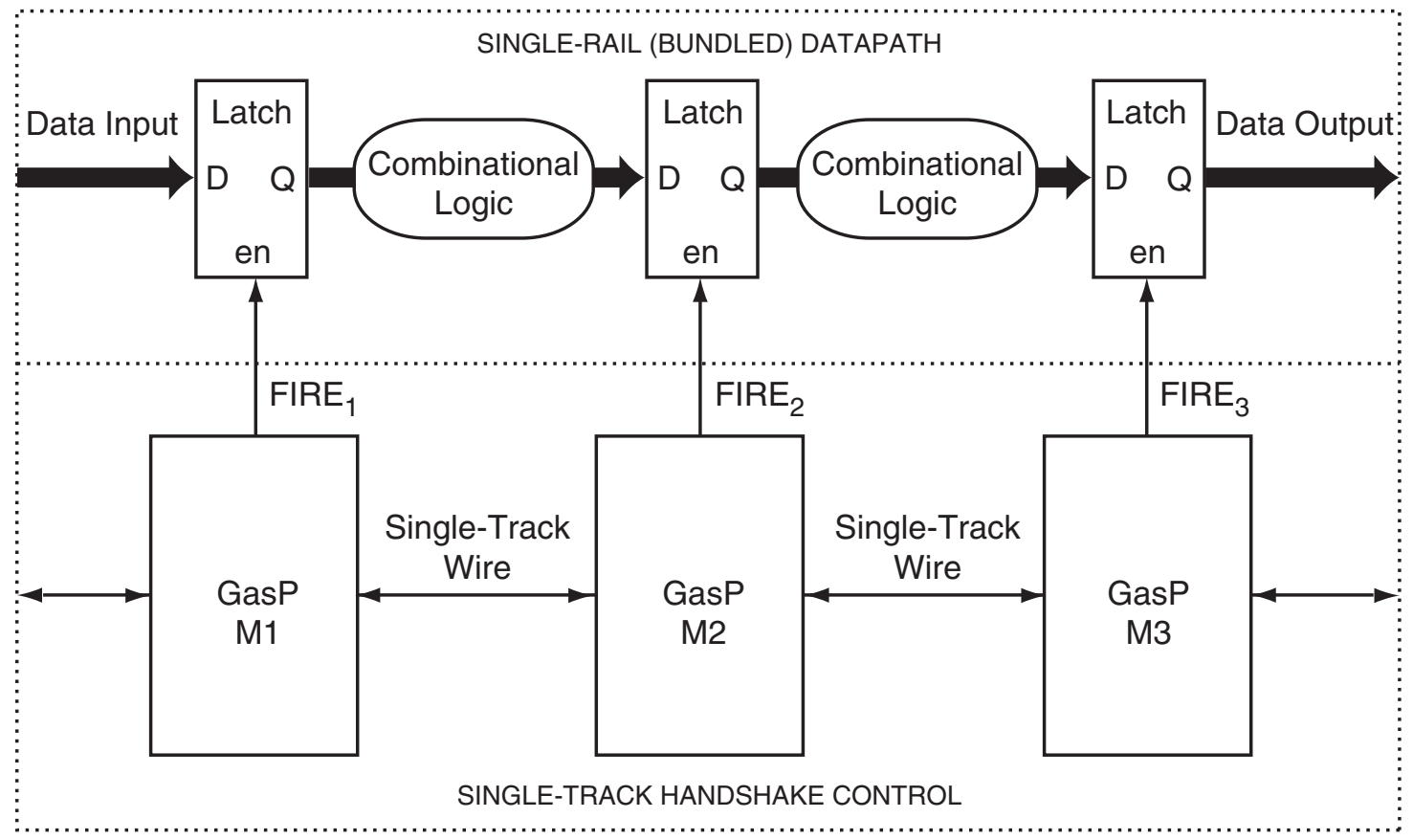

Figure 1.1 High-level view of a linear pipeline in GasP with a bundled datapath. The GasP control modules are connected with bi-directional single-track wires. Their implementations are omitted here, but follow in Chapter 2, Figure 2.1, page 11. Each GasP module $\mathrm{M} i$ generates a local pulse signal $\mathrm{FIRE}_{i}$ that acts as a local clock or enable signal for the group of latches above it, making the group temporarily transparent.

The message to take away is that GasP designs are light-weight because GasP designers use faith where they can and build in measurement where they must. This take-away also explains where my thesis comes in: I developed a timing validation flow that translates faith into measurement. As a result, a GasP designer can rely on faith to make her design light-weight, and use my validation flow to check how well that faith holds up.

\subsection{Timing Validation Flow}

Figure 1.2 gives an overview of the timing validation flow I offer. The complete flow consists of three consecutive parts: (1) generation of the critical timing constraints, and (2) library characterization, and (3) static timing analysis (STA). 


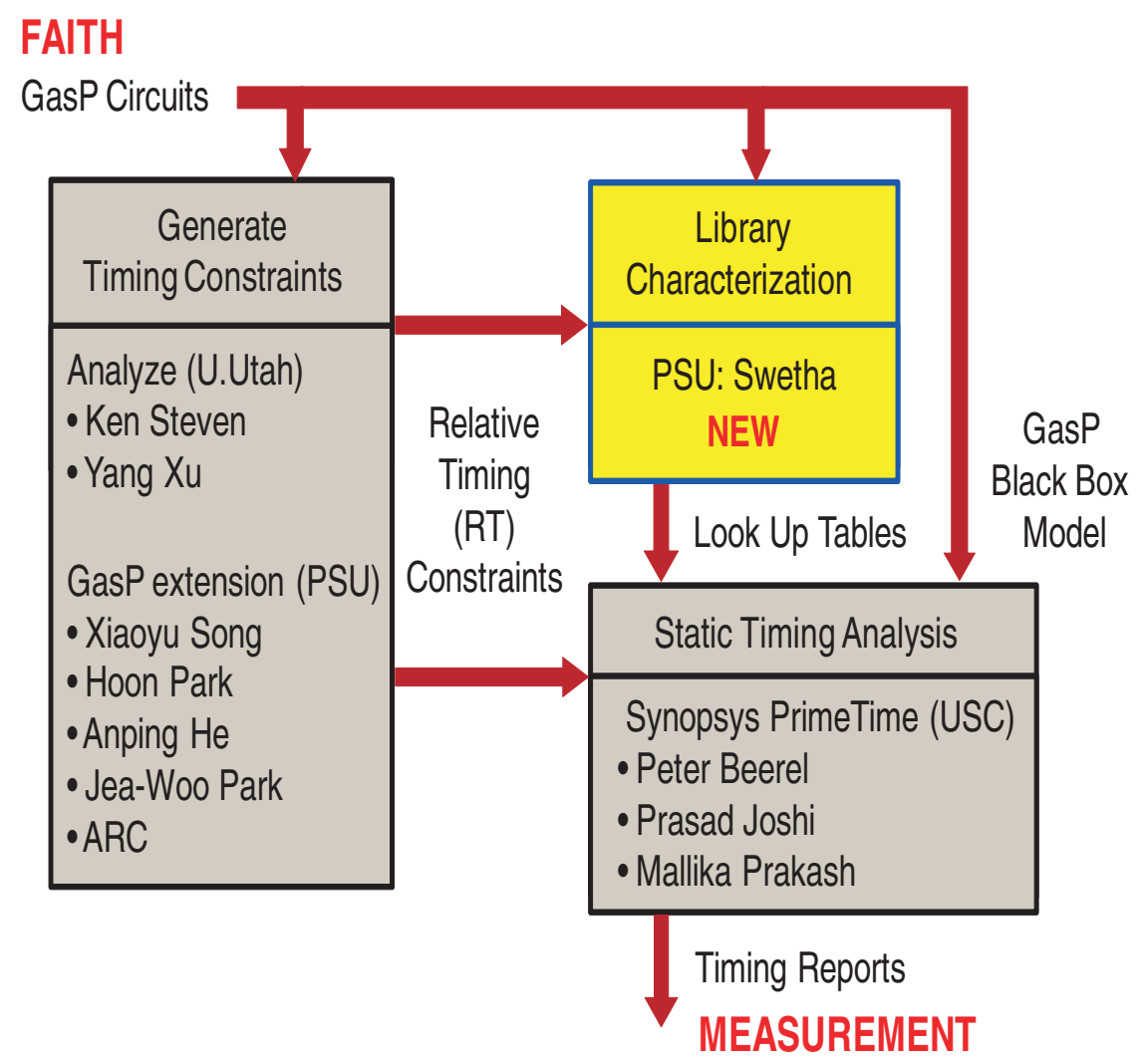

Figure 1.2 Timing validation flow for GasP circuits. The complete flow consists of three parts: (1) generation of the critical timing constraints, (2) library characterization, and (3) static timing analysis (STA). Together the three parts take a GasP circuit, built using faith, and translate it into timing reports that measure how well the faith holds up.

Ideally, the first step takes a GasP circuit and generates its critical timing constraints. There is a tool for this step, developed at the University of Utah by Professor Ken Stevens and his students $[26,37]$. The tool is called 'Analyze'. It is being extended for GasP circuits here at Portland State University by Professor Xiaoyu Song and his students, in collaboration with the Asynchronous Research Center. The tool uses formal verification techniques. The timing constraints are called 'Relative Timing Constraints' . In my thesis, I assume that the first part is done, i.e. I start with a GasP circuit and a given set of relative timing constraints. 
The second step in the flow, and the first step addressed in my thesis is called 'Library Characterization'. This is the key part of my thesis, and it's new work. In this step, I take a GasP circuit and its relative timing constraints. I partition these into manageable pieces that I simulate for various operating conditions. And I generate a collection of so-called 'Look Up Tables' (LUTs) that store the timing information of the paths under those conditions.

I feed these look up tables and a so-called 'Black Box' model of the GasP circuit into static timing analysis. The black box shows only the circuit input and output pins, and hides the combinational loops and single-track details of GasP. This third step is not new. The black box model and the analysis commands for the relative timing constraints were developed at the University of Southern California by Professor Peter Beerel and his students. The tool itself is a commercial tool by Synopsys called 'PrimeTime' [35]. All I had to do was to get it running at Portland State University and to import my timing constraints and look up tables.

The result of static timing analysis is a collection of timing reports, one for each relative timing constraint, showing how much slack there is to make or break the constraint. So, in total, these three flow parts take a GasP circuit, built using faith, and translate it into timing reports that measure how well the faith holds up. In summary: the timing validation flow outlined in Figure 1.2 translates faith into measurement. ${ }^{1.1}$

\subsection{Reading Guide}

I have now told you why I built a timing validation flow for GasP, and which parts I borrowed and which parts are new. The rest of my thesis is organized as follows:

\footnotetext{
${ }^{1.1}$ Of course, the real measurement is the silicon, but that is a very expensive and time-consuming measurement. The measurement I am talking about is a simulated measurement.
} 
- Chapter 2 gives the design details on GasP, and identifies the four key relative timing constraints for single-track communication. In this thesis, I work with TSMC 90nm CMOS implementations of 6-4 GasP, but that's just a choice. This choice has zero impact on the generality and applicability of my flow to other CMOS implementations or other members of the GasP family, such as 4-2 GasP or 3-7 GasP. More GasP details can be found in Appendix O or [30, 29, 18, 13].

- Chapter 3-4 are about library characterization, and cover he biggest part of my thesis. Here, I show how timing constraints are translated into look up tables. This requires three steps. In the first step, I partition the timing constraints into manageable pieces. In the second step, I build a simulation environment for each partitioned timing constraint, using the schematic design environment provided by Electric [24] and the transistor-level simulation environment provided by SPICE [34]. In the third step, I run the SPICE simulator to generate the look up tables. Chapter 3 explains the basic simulation environment in Electric and the SPICE commands for the third step. Chapter 4 takes a 6-4 GasP module and the four key relative timing constraints identified in Chapter 2, and goes into the details of each step.

- Chapter 5 shows how static timing analysis uses the timing information in the look up tables to validate the four relative timing constraints. Here, I explain the STA part of the flow and show how to import the look up tables generated in Chapter 4.

- Chapter 6 analyzes the timing results produced by the STA part of the flow. Because the proof of the pudding is in the eating, I did not just propose and develop a timing validation flow, but I also used it. I used it to validate the four timing constraints that are key to single-track handshake communication in 6-4 GasP. 
Moreover, I show that the results in the timing reports are compatible with the results of my ASYNC 2010 publication in [18] and Appendix O.

- Chapter 7 compares my approach to the approach taken by USC. I deliberately postpone this comparison to the end of my thesis, because this enables me to give a more in-depth explanation than would be possible with an up-front comparison where the reader has not yet had a chance to read any of my work.

- Chapter 8 concludes this thesis and indicates what next steps are needed. As far as future work goes, what this flow needs most is a wire delay model. The wire delay model should distinguish the effective capacitance seen at the near end of the wire from the capacitance and delay seen at the far end. It may be that an Elmore delay model suffices $[23,36]$. Last but not least: this flow needs automation so it can become an integral part of the GasP design environment.

Chapters 2-7 are also available as internal reports $[16,17,19]$ from the Asynchronous Research Center at Portland State University, where I worked as a Research Assistant. The reports are co-authored by Marly Roncken and Ivan Sutherland. For that reason, Chapters 2-7 use as author identity 'we' instead of ' $I$ '. The author identity changes to 'I' again when I conclude this thesis in Chapter 8.

After working with flows that require various tools and translations from file formats in one tool to file formats in another tool, I have come to realize that documentation often lacks the clarity and teaching capability that it should provide. I have therefore set an extra goal for my thesis. Not only do I want to show that I generated a timing validation flow for single-track circuits in GasP, but I also want to explain this flow in a way that is useful for professors as well as for their students. I have set up this thesis so 
that others can easily use the flow, execute it, and validate its results. The many files in Appendices A-M in this thesis are there to support this extra goal.

In addition to Appendices A-M with files, I have added three more Appendices N-P with extra bonus material to complete the picture and provide a quick tour of this thesis:

- Appendix N contains the ARC report on the Distance Constraint Graph. I defined this graph notion when studying 6-4 GasP with short wires. It has turned out to be a useful abstraction for thinking about wire lengths and module distances versus critical timing paths (or relative timing constraints as we call them at the ARC).

- Appendix O contains the paper that I presented and published at ASYNC 2010. It contains the short-to-medium-to-long wire studies for 6-4 GasP. The above ARC report and the ASYNC 2010 paper shaped the topic of this thesis. Both are cited repeatedly in this thesis to motivate their inclusion. IEEE and my co-authors graciously allowed me to include them as appendix to this thesis.

- Appendix P contains the handout of the powerpoint presentation that I presented during my thesis defense, on 29 October 2010. Reading the powerpoint slides, which I printed as a 4-slides-per-page handout may be the quickest way to get a tour of this thesis. I hope you will enjoy the tour! 


\section{Chapter 2}

\section{6-4 GASP AND ITS RELATIVE TIMING CONSTRAINTS}

Chapter 2 introduces the basics of GasP circuits. We use a 6-4 GasP FIFO module as reference design. Figure 2.1 shows a two-stage FIFO with two 6-4 GasP FIFO modules, M1 and M2. Modules M1 and M2 are connected by a bi-directional wire L2 in the middle. Module M1 drives wire L2 high via P-transistor E and module M2 drives wire L2 low via N-transistor X. Wire L2 is called a 'single-track wire', but in GasP we also call it a 'state wire' because it holds state. It is high when it has valid data in which case we call its state 'Full' and it is low when it has a bubble, in which case we call its state 'Empty'. GasP module M1 fires when its $\mathrm{PRED}_{1}$ signal is high, meaning full, and its $\mathrm{SUCC}_{1}$ signal is low, meaning empty.

When M1 fires, it forwards the full state on its left to state wire L2 and module M2 on its right, using the six gates on the red arrow in Figure 2.1: A, B, C, D, E and F. When M2 fires it drains state wire L2, using the 4 gates on the green arrow: A, B, C and X. The name for 6-4 GasP comes from the six gates in the path going forward from one module to the next, and the four gates in the reverse path.

In addition, a 6-4 GasP module has two self-resetting loops of five gates each, as in-

dicated by the blue arrows in Figure 2.1. We distinguish the successor loop through 


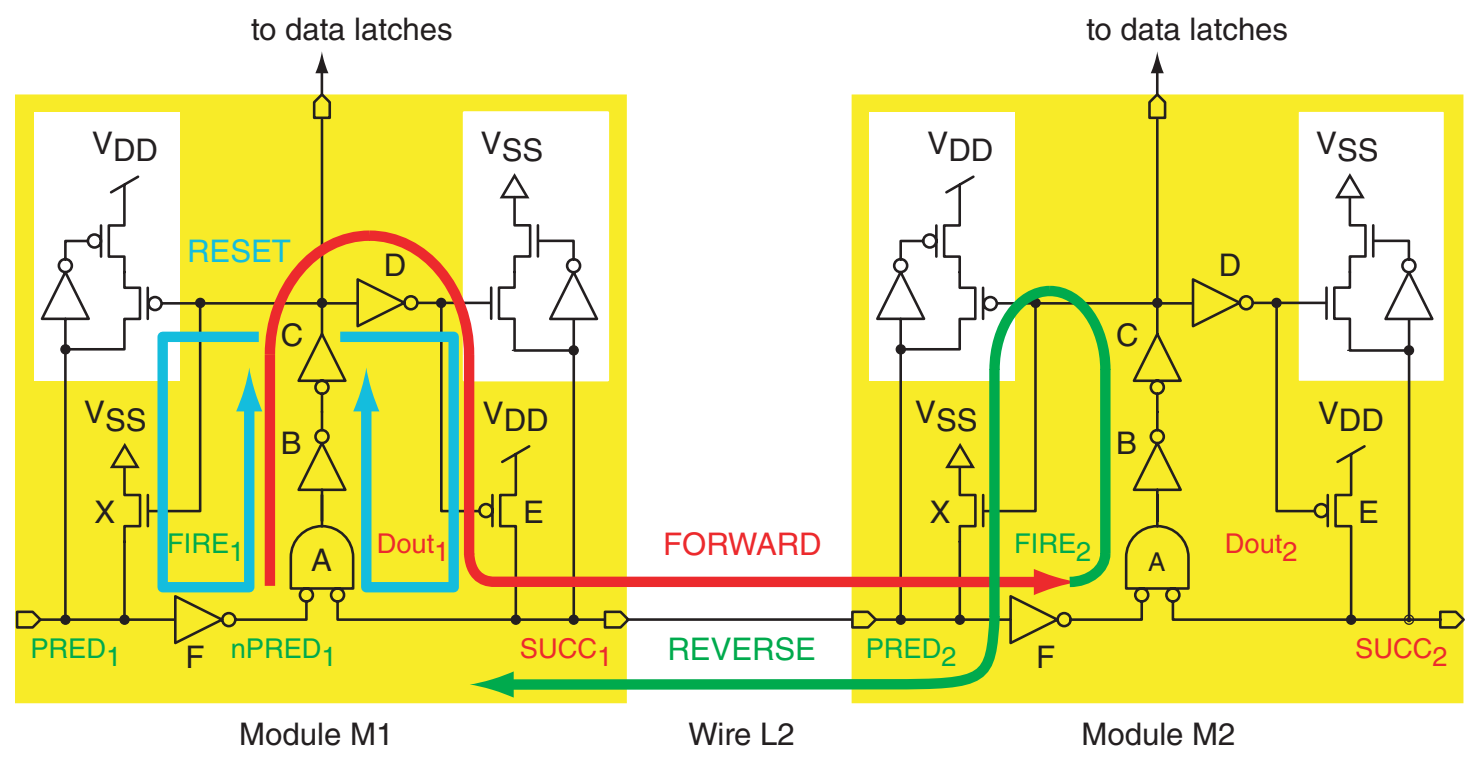

Figure 2.1 Two-stage FIFO with two 6-4 GasP modules, M1 and M2, connected by a single-track wire L2 which is bi-directional. In GasP, we often refer to single-track wire L2 as 'state wire'. The bundled data paths are not shown here, nor are the state wires connected to the left of M1 and the right of M2. For a high-level view with datapaths, see Figure 1.1 on page 4.

the path with gates $\mathrm{A}, \mathrm{B}, \mathrm{C}, \mathrm{D}$, and $\mathrm{E}$ from the predecessor loop through the path with gates A, B, C, X, and F. When state wire L2 is not driven its state is held by the halfkeepers at the two ends of the wire, shown in the white insets of Figure 2.1. Note that the NMOS half-keeper is connected to the $\mathrm{SUCC}_{1}$ signal at the successor side and to the successor loop. Similarly, the PMOS half-keeper is connected to the PRED 1 signal at the predecessor side and to the predecessor loop. Further details on 6-4 GasP can be found in Appendix $\mathrm{O}$ and in $[30,29,18,13]$.

The two self-resetting loops turn off the drive signal to the state wire and we must avoid letting them turn the drive off prematurely. Correct operation of 6-4 GasP requires that state wire L2 is (1) driven long enough in the forward direction to raise both wire ends, $\mathrm{SUCC}_{1}$ and $\mathrm{PRED}_{2}$, and (2) driven long enough in the reverse direction to lower both wire ends. 
To validate that both (1) and (2) hold, we compare the forward and the reverse drive delays for L2 to the self-resetting loop delays and require the former to beat the latter. Thus, state wire L2 in Figure 2.1 produces the following four relative timing constraints:

- RT1 or RT(L2) ${ }^{\operatorname{TrfF}-\mathrm{RstB}}$ : The forward transfer delay to drive both ends of L2 high through DE in module M1 is shorter than the delay of the backward self-resetting loop through XFABCDE in M1 that stops the drive. Figure 2.2(a) illustrates this graphically: the red path must be shorter than the blue path.

- RT2 or RT(L2) ${ }^{\text {TrfF-RstF }}$ : The forward transfer delay to drive both ends of L2 high through DE in module M1 is shorter than the delay of the forward self-resetting loop through DEABCDE in M1 that stops the drive. Figure 2.2(b) illustrates this graphically: the red path must be shorter than the blue path.

- RT3 or RT(L2) ${ }^{\text {TrfB-RstF }}$ : The reverse transfer delay to drive both ends of L2 low through $\mathrm{X}$ in module M2 is shorter than the delay of the forward self-resetting loop through DEABCX in M2 that stops the drive. Figure 2.2(c) illustrates this graphically: the green path must be shorter than the blue path.

- RT4 or RT(L2) ${ }^{\text {TrfB-RstB }}$ : The backward transfer delay to drive both L2 ends low through $\mathrm{X}$ in module M2 is shorter than the delay of the backward self-resetting loop through XFABCX in M2 that stops the drive. Figure 2.2(d) illustrates this graphically: the green path must be shorter than the blue path.

From these relative timing constraints, and their graphical representations in Figure 2.2, it follows that the red, green and blue paths in Figures 2.1-2.2 are the circuit paths whose delays we must calculate. Note that the blue paths contain (combinational) loops. This classifies them as complex paths, and as such as paths too difficult to handle by commercial library characterization and static timing analysis tools. In Chapters 4 and 5 we explain how this can be solved. 


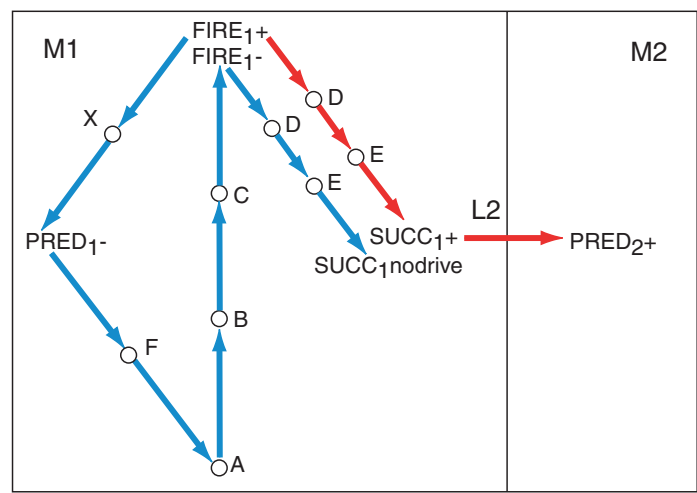

(a) RT(L2) TrfF-RstB : red path < blue path

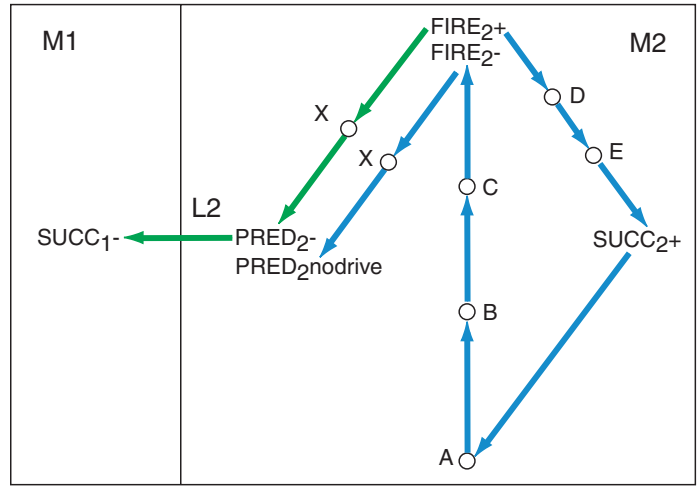

(c) RT(L2) TrfB-RstF : green path < blue path

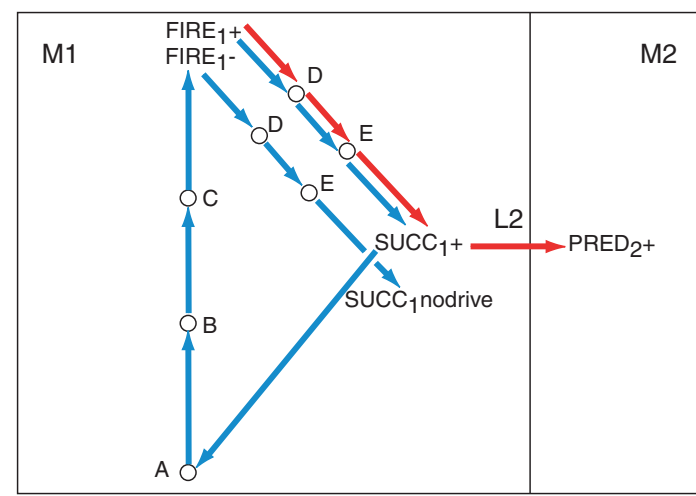

(b) RT(L2) TrfF-RstF : red path < blue path

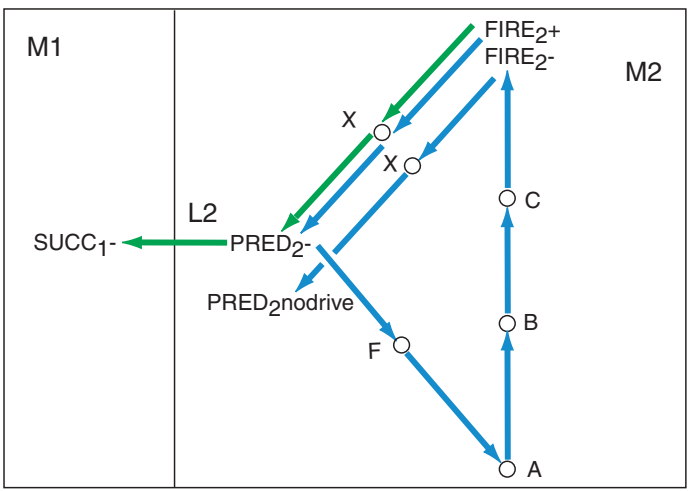

(d) RT(L2) TrfB-RstB : green path < blue path

Figure 2.2 Graphical representation of the four relative timing constraints on state wire L2 in Figure 2.1. Graph (a) is a graphical representation of relative timing constraint RT1 or RT(L2) ${ }^{\text {TrfF-RstB }}$. It requires that the delay for the red-arrow path must be less than the delay for the blue-arrow path. The red-arrow path in graph (a) corresponds to the forward red arrow in Figure 2.1, followed from FIRE 1 to $\mathrm{PRED}_{2}$. The blue-arrow path in (a) correspond to the backward blue reset arrow in Figure 2.1, followed from FIRE $_{1}$ to FIRE 1 and on to the point where M1 stops its forward drive to L2. We use circles for gates and arcs for signal transitions. A ' + ' symbol indicates a rising transition, and a '-' symbol indicates a falling transition. Likewise, graph (b) is a graphical representation of RT2 or RT(L2) TrfF-RstF , graph (c) of RT3 or RT(L2) ${ }^{\text {TrfB-RstF }}$, and (d) of RT4 or RT(L2) ${ }^{\text {TrfB-RstB }}$. The green-arrow paths in graph (c) and graph (d) correspond to the reverse green arrow in Figure 2.1, followed from FIRE $\mathrm{F}_{2}$ to $\mathrm{SUCC}_{1}$. The delay for the green-arrow path in graph (c) must be less than the delay for the blue-arrow path in graph (c), and likewise for graph (d). 


\section{Chapter 3}

\section{LIBRARY CHARACTERIZATION PART-A: LOOK UP TABLES (LUTs)}

Chapter 3 is also available as internal report ARC2010-smg01 [16]. It describes the results of our investigations into creating look up tables that accurately characterize the timing information of a 6-4 GasP module. These tables allow us to bypass tool issues related to loops and other design elements used in asynchronous circuits that standard tools for synchronous circuit designs are not accustomed to. In addition to avoiding tool issues, the use of look up tables can also speed up the static timing analysis (STA) of a large GasP design. We present our results in two parts. PART-A, described here, takes a simple circuit without loops, and shows the basic flow aspects from the schematics entry level of the design in Electric to the SPICE simulation set-up and the SPICE output files with the look up tables. PART-B, described in Chapter 4 shows how to partition complex circuits with loops and multiple inputs and outputs into designs simple enough for the characterization flow in PART-A.

\subsection{Introduction}

A look up table is basically an array with a search key and a corresponding result. look up tables are often used to reduce the run time of a computation. In the old days people used printed look up tables like logarithmic or trigonometry tables to speed up hand 
calculations. Look up tables are used in one of three ways: (1) Exact match or (2) Interpolation, or (3) Extrapolation. When the search key exists, i.e. when it exactly matches a look up table entry, we can simply read out the corresponding result value. When the search key does not match any of the look up table entries, we estimate the result value for a search key that is inside respectively outside the entry range, by using interpolation respectively extrapolation techniques on the result values of the look up table entries that are closest in value to the search key. For our usage, linear interpolation and extrapolation techniques suffice.

We use look up tables (LUTs) for static timing analysis (STA) of GasP circuits. The look up tables that we generate store the path delays for a GasP circuit, under various operating conditions. Our look up tables use a two-dimensional search key (wid1 $\times$ wid2) and store three types of delay:

1. (wid1 $\times$ wid2) $\rightarrow$ slope (in[1])

2. $($ wid1 $\times$ wid2) $\rightarrow$ delay (in[1] to out[1])

3. $($ wid $1 \times$ wid2 $) \rightarrow$ slope $($ out $[1])$

where:

- wid1 is the size of the external gate driving path input in[1].

- wid2 is the size of the external gate driven by path output out[1].

- slope(in[1]) is the input transition slope, a.k.a. input slew time, as denoted by the time difference $\left(\operatorname{tin}_{2}-t i n_{1}\right)$ from time $t i n_{1}$ when a rising input transition on in[1] passes the $20 \%$ supply voltage point to time $\operatorname{tin}_{2}$ when it passes the $80 \%$ voltage point - and vice versa for a falling input transition.

- delay(in[1] to out[1]) is the input-to-output transition delay time (tout-tin) from time tin when the input transition on in[1] passes the $50 \%$ supply voltage point to time tout when the output transition on out[1] passes the $50 \%$ supply point. 
- slope(out[1]) is the output transition slope, a.k.a. output slew, as for instance denoted by the time difference $\left(\right.$ tout $_{2}-$ tout $\left._{1}\right)$ from time tout $t_{1}$ when a falling output transition on out[1] passes the $80 \%$ supply voltage point to time tout $_{2}$ when it passes the $20 \%$ point - and vice versa for a rising output transition.

For those of you who are familiar with standard static timing analysis (STA) tools like PrimeTime of Synopsys: note that the search keys in our look up tables are different. We use sizing information instead of input slopes and output loads. By doing this we can use the theory of Logical Effort [32] in both the design and the characterization of our circuits. We feel that this makes the characterization process less artificial and more realistic. To accommodate standard STA tools, we measure the input slopes for a given drive size and we have methods to translate a driven output size into an output load which we'll explain in Chapter 5. In the present Chapter 3, we show how to generate such look up tables for simple paths in a GasP circuit, i.e. paths with one input and one output, and without feedback. Complex paths are the topic of Chapter 4.

There are basically two ways to sweep the search key values for (wid1 $\times$ wid2) and generate the resulting delay and slope information for a simple path in a GasP circuit. One approach is to design all sweeps in parallel at the schematic entry level and create one composite SPICE run. This works provided the size of the simulation result file is small enough to be read back into Electric's schematic entry and layout environment [24] for validation and debug. We had some trouble with this in our long wire studies for [18]. We will investigate another approach where we design exactly one parameterized sweep schematics in Electric, which we will simulate multiple times, each time with a new set of parameter values, using the .DATA sweep command in SPICE [34]. 
Our look up table generation procedure distinguishes the following five steps:

Step 1: Use schematics and layout tool Electric to access the GasP design.

Step 2: In Electric, create a realistic simulation environment for the GasP design.

Step 3: From Electric, generate input files for SPICE:

- Generate a SPICE netlist for the GasP design and its environment.

- Generate SPICE simulation set-up files.

Step 4: Modify the SPICE input files:

- In the netlist, create sweep parameters for wid1 and wid2.

- In the simulation set-up files, add sweep statements for wid1 and wid2, and measurement statements for in[1] and out[1].

Step 5: Run SPICE and capture the look up table results in an output file.

The outline for the rest of Chapter 3 is as follows. Sections 3.2 to 3.6 each discuss one step in our 5-step look up table generation procedure. We use the 6-4 GasP circuit of Figure 3.1 on page 19 as our baseline example. Section 3.7 compares the results obtained by applying the 5-step procedure to this circuit to the results obtained from the alternative approach: i.e. from a reference design where all sweeps are embedded in parallel at the schematics entry level and where the results are obtained in one SPICE run. The reference design follows in Figure 3.7, page 31. Section 3.8 concludes Chapter 3. All the SPICE input and output files for the baseline example and the reference design can be found in Appendix A and B.

\section{Note:}

We postpone the comparison between our library characterization work and prior work at USC by Mallika Prakash [21,22] and Prasad Joshi $[2,3]$ to Chapter 7. 


\subsection{Step 1: Use Electric to Access the GasP Design}

Figure 3.1 shows the schematic diagram of the 6-4 Half-way GasP circuit that we use as our design example throughout Chapter 3. The circuit name is Half-way GasP because it represents about half of a 6-4 GasP module in Figure 2.1. The Half-way GasP circuit has one input pin, PRED, and one output pin, FIRE. Input PRED drives an inverter called F which subsequently drives a NOR gate called A. The other input to the NOR gate is grounded. Output FIRE is the output of the NOR gate, delayed by two inversions through gates B and C. Half-way GasP is a fictional circuit which uses gate size 10 for all its gates rather than the step-up of 3 sizing strategy of Logical Effort designs [32].

The operation of the Half-way GasP circuit is as follows. When PRED goes low, the corresponding input to NOR gate A goes high, which results in FIRE going low. The nominal delay between PRED going low and FIRE going low is about 4 gate delays. The delay decreases if the output load driven by FIRE decreases or the input drive to PRED increases. The delay increases if the output load driven by FIRE increases or the input drive to PRED decreases. Likewise, when PRED goes high, FIRE will go high after a delay of about 4 gate delays, with a decrease or increase in delay as before. We will characterize the falling path delay from PRED going low to FIRE going low.

Circuit and path are represented by a black box whose icon is shown in the upper right corner of Figure 3.1. Only the information that we need from the black box is visible:

- Input PRED and output FIRE, as these connect to the simulation environment.

- Size of initial gate in the path, i.e. 10 (for F), and of final gate, i.e. 10 (for C), as these determine the sweep sizes for the input driver and output load inverters in the simulation set-up discussed in the next section.

- The path we're characterizing: PRED- to FIRE-. 


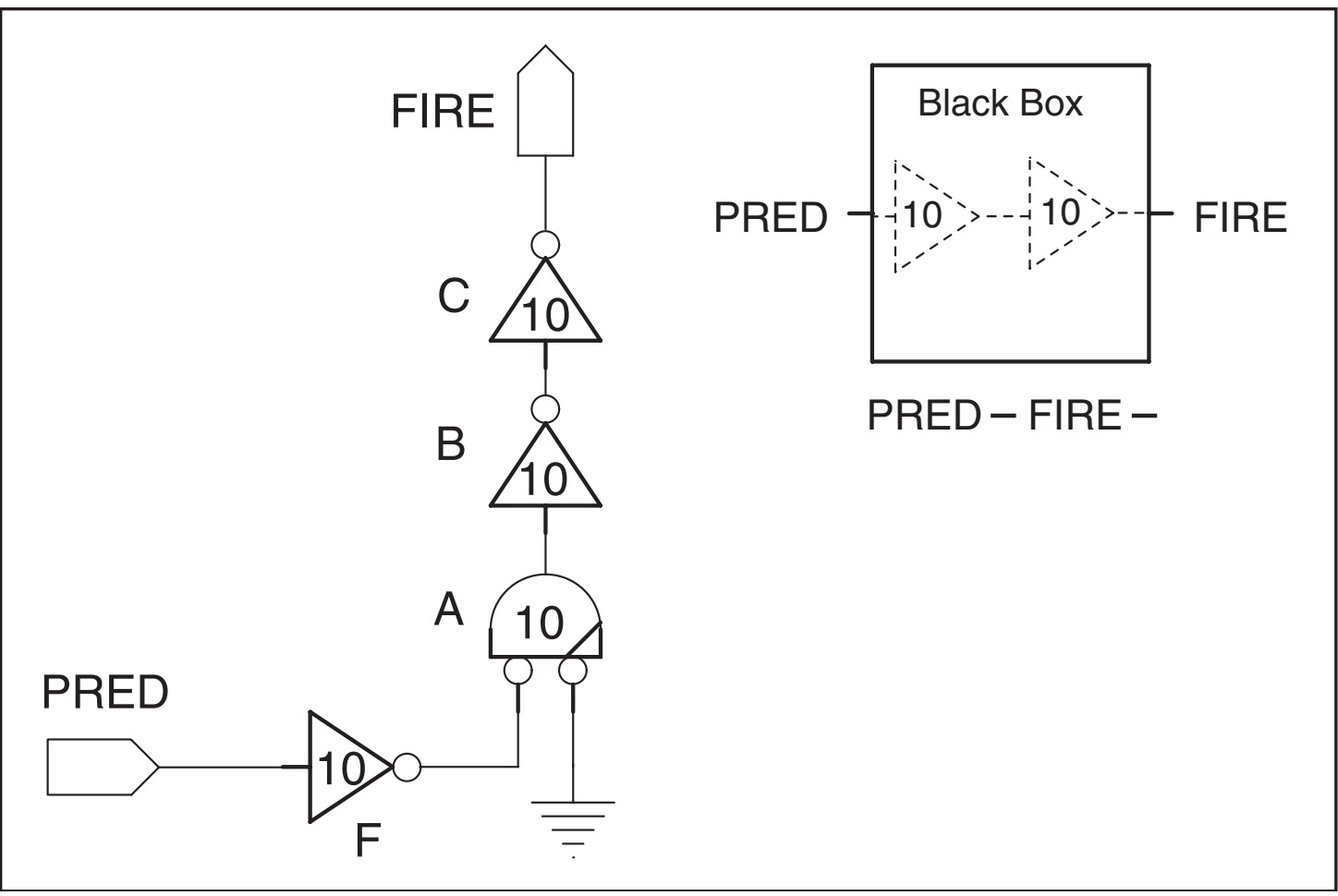

Figure 3.1 Half-way GasP circuit. The icon in the upper right corner of the picture shows the black box representation of the circuit, which has just enough information to characterize the path from PRED falling to FIRE falling.

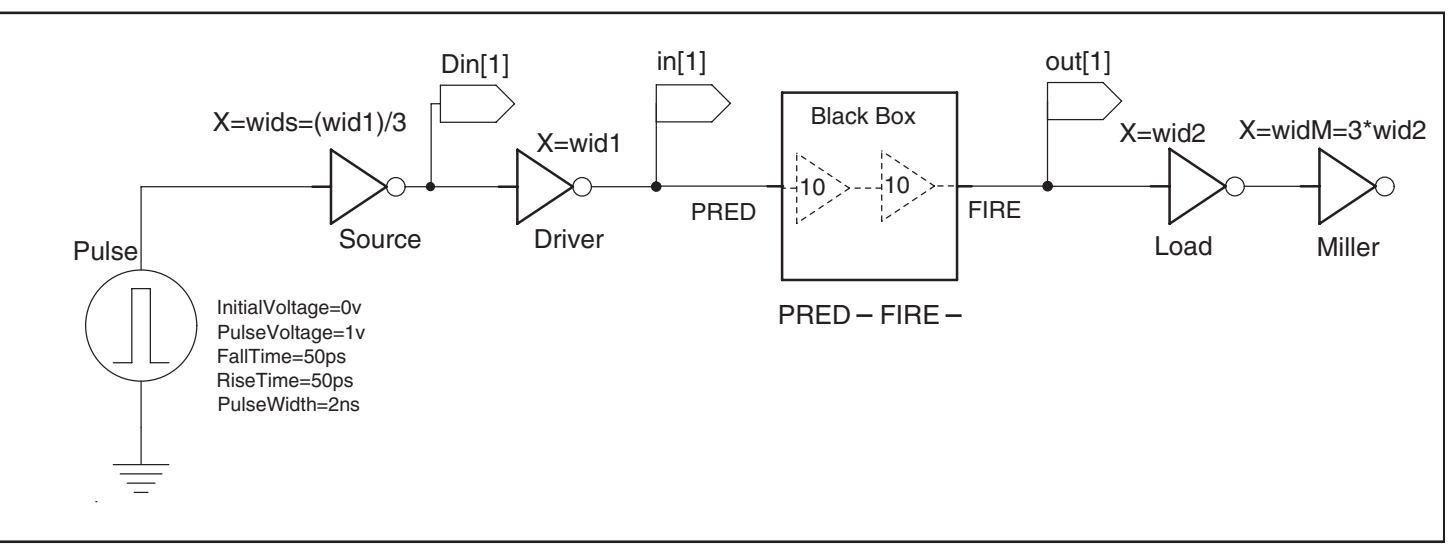

Figure 3.2 Schematics of the simulation environment for the Half-way GasP circuit in Figure 3.1, with sweep parameters wid1, wid2 and observation nodes in[1], out[1]. 


\subsection{Step 2: Create a Simulation Environment for the Design}

Figure 3.2 shows the schematics of the simulation environment using the black box icon for the Half-way GasP circuit in Figure 3.1. We use this simulation environment to generate different input slopes for PRED and different output loads for FIRE. We do this by generating different driver sizes wid 1 for the inverter that drives PRED, and by using different load sizes wid2 for the inverter that is driven by FIRE. Labels Din[1], in[1] and out[1] are the observation points for measuring delay and slope information.

We added a pulse generator to start the different simulation measurements - one per pulse. The generator creates a pulse with a 2 ns width and a full-swing slope of 50ps. ${ }^{3.1}$

Note that we use a series connection of two inverters between the pulse generator and black box input PRED: a so-called source inverter followed by the already mentioned driver inverter. Note also the step-down of 3 from the size wid 1 of the driver inverter to the size wids of the source inverter upstream. A step-down of 3 upstream matches the Logical Effort design strategy [32], and ensures a realistic input slope for the driver input, independent of the slope generated by the pulse generator. We also use a series connection of two inverters for the simulation environment at black box output FIRE: a so-called Miller inverter following the already mentioned load inverter. Note the step-up of 3 downstream from the size wid2 of the load inverter to the size widM of the Miller inverter. The step-up of 3 downstream matches the Logical Effort design strategy, and ensures that the load inverter produces a realistic output load, i.e. it produces just enough source-to-gate 'Miller Effect' for the load inverter as a real environment designed with Logical Effort would produce for the given load size.

\footnotetext{
${ }^{3.1}$ A slope of 50ps works fine for this fictitious example. However, for realistic simulation set-ups, we recommend to use a slope or slew time more typical of a standard inverter operation. For the $90 \mathrm{~nm}$ CMOS process that we currently use, a typical slope for a full signal swing would correspond to $20 \mathrm{ps}$.
} 


\subsection{Step 3: Generate Input Files for SPICE}

The next two Sections 3.4.1 and 3.4.2 cover the following items:

- Generate a SPICE netlist for the GasP design and its environment.

- Generate a SPICE simulation set-up file.

\subsubsection{Generate a SPICE Netlist}

The SPICE netlist generated from Electric for the simulation environment in Figure 3.2 is listed in Appendix A.1. The file extension is '.spi'. Unfortunately, we have thus far been unable to force Electric and SPICE to retain the parameter names for the sizes of the driver and load inverters, wid1 and wid2. Instead of a sweep parameter, we get a 'null' value in the SPICE formulas embedded in the inverter and transistor schematics, as is visible from the highlighted yellow-colored text in Figure 3.3. Fortunately, this is not a show-stopper, and so we will proceed to work with the resulting netlist with 'null' values. In Section 3.5.1 we will substitute the 'null' formulas with the correct parameterized formulas containing wid1 and wid2. However, we will add the challenge of finding a more direct solution for generating an Electric-to-SPICE netlist with sweep parameters to our list of future research items - see Section 3.8.

\subsubsection{Generate SPICE Simulation Files: HEADER and TRAILER}

The two basic SPICE simulation files, the HEADER and the TRAILER, generated from Electric for the simulation environment in Figure 3.2 are listed in Appendix A.2 and Appendix A.3. The file extenstion is '.hsp'. The header file has additional content with process information. We excluded process information for reasons of confidentiality. 


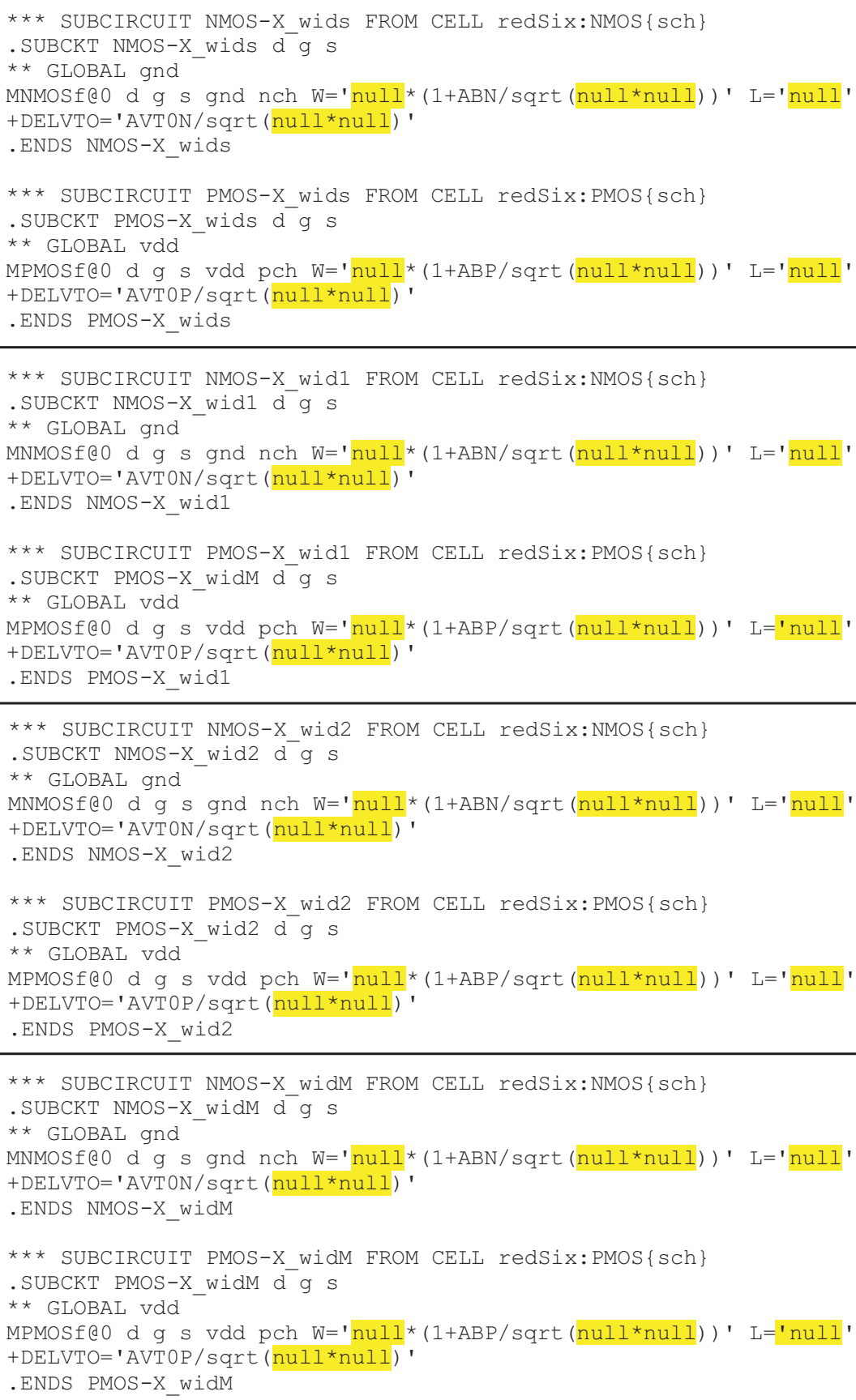

Figure 3.3 SPICE netlist fragments for the source, driver, load and Miller inverters in Figure 3.2. The width and length calculations of the PMOS and NMOS transistors in these parameterized inverter netlists wrongly get 'null' values inserted where the size parameters wid 1 and wid 2 are used. This incorrect insertion is clearly visible in the highlighted yellow text. 


\subsection{Step 4: Modify the SPICE Input Files}

The next two Sections 3.5.1 and 3.5.2 cover the following items:

- In the netlist, create sweep parameters for wid1 and wid2.

- In the simulation set-up files, add sweep and measurement statements.

\subsubsection{Create Sweep Parameters in the SPICE Netlist}

In Section 3.4.1, Figure 3.3, we observed that the parameterized sizes wid1 and wid2 for the driver and load inverters are wrongly expanded into formulas with 'null' in the SPICE netlist parts for the source, driver, load and Miller inverters. We will fix this by substituting the correct formulas. We can derive the correct formulas from the original SPICE text in Electric, or simply by looking at the results for the other inverting gates. Figure 3.4 shows the updated netlist parts with the correct formulas. This substitution process can be easily automated, using a Perl script.

Note that the formulas have size parameters wid1 and wid2. This will enable us to do simulation sweeps and generate look up tables for different values of wid1 and wid2. For completeness and continuity in the Appendices part of this thesis, we have repeated these corrected SPICE netlist portions in Appendix A.4. 


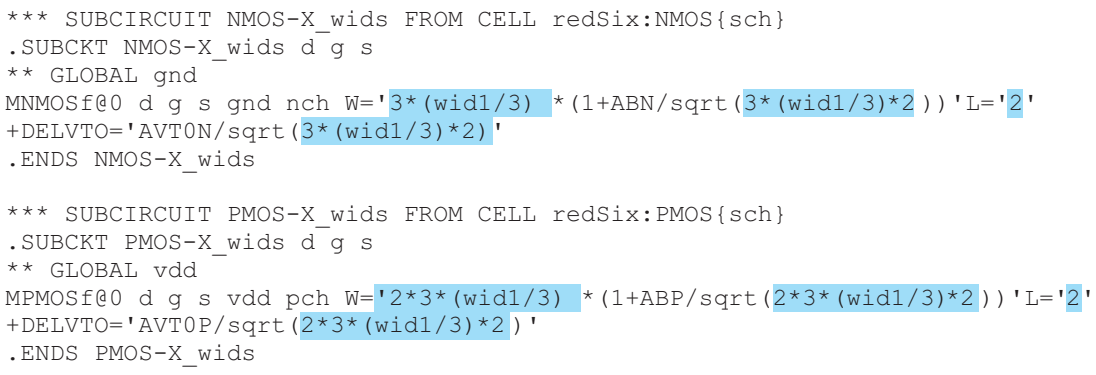

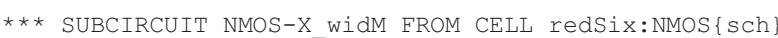
. SUBCKT NMOS-X widM d $g$ s ** GLOBAL gnd

MNMOSf@O d g $s$ gnd nch $\mathrm{W}={ }^{\prime} 3 * 3 * \mathrm{wid} 2 *(1+\mathrm{ABN} / \operatorname{sqrt}(3 * 3 * \mathrm{wid} 2 * 2))^{\prime} \mathrm{L}=\mathbf{\prime}^{\prime} \mathbf{\prime}^{\prime}$ + DELVTO $=$ 'AVTON/sqrt $(3 * 3 * \text { wid } 2 * 2)^{\prime}$

.ENDS NMOS-X_widM

*** SUBCIRCUIT PMOS-X widM FROM CELL redSix: PMOS $\{\mathrm{sch}\}$ . SUBCKT PMOS-X widM d $g$ s

** GLOBAL vdd

MPMOSf@O d g $s$ vdd pch $W=' 2 * 3 * 3 * w i d 2 *(1+A B P / \operatorname{sqrt}(2 * 3 * 3 * w i d 2 * 2))$ ' $L=' 2$ ' $+\mathrm{DELVTO}=$ 'AVTOP/sqrt $(2 * 3 * 3 *$ wid $2 * 2)$ '

.ENDS PMOS-X_widM

Figure 3.4 Corrected SPICE netlist for the source, driver, load and Miller inverters in Figure 3.2. The corrections are highlighted in blue. 


\subsubsection{Add Sweep and Measurement Statements to the TRAILER File}

This is the step where we add the sweep commands for simulating the now correctly parameterized netlist for a specific range of driver and load sizes (wid $1 \times$ wid2) and where we specify what we want to measure at the observation nodes in[1] and out[1] to generate the slope and delay information discussed on page 15:

1. $($ wid $1 \times$ wid2) $\rightarrow$ slope $($ in[1] $)$

2. $($ wid $1 \times$ wid2 $) \rightarrow$ delay $($ in[1] to out[1] $)$

3. $($ wid1 $\times$ wid2 $) \rightarrow$ slope $($ out $[1])$

As sweep command, we use the .DATA sweep command explained in HSPICE reference manual [34], Chapter 2: HSPICE and HSPICE RF Netlist Commands, pages 60-66. Our .DATA command uses a row-column matrix input format, where each column contains the values of a single sweep variable and each row contains the full set of sweep values needed for a single sweep. For instance,

.DATA mysweep wid1 wid2

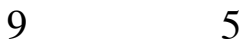

125

\section{ENDDATA}

specifies that the first sweep uses value 9 for driver size wid1 and 5 for load size wid2, the second sweep uses the value 12 for wid 1 and 5 for wid2, etcetera.

The type of analysis done for our DATA sweep 'mysweep' is specified separately. We use a transient analysis similar to the one specified in [34], Chapter 2, page 62. Each of our sweeps starts by default at $0 \mathrm{ps}$, uses time increments of $1 \mathrm{ps}$, and stops at 100ns:

.TRAN 1ps 100ns SWEEP DATA=mysweep 
The measurements done per sweep are specified in a separate .MEASURE statement. The type of .MEASURE statements that we need relate to rise, fall and delay measurements, and are specified in the HSPICE reference manual in [34], Chapter 2, on pages $162-165$. We measure rise times from $20 \%$ to $80 \%$ of the full signal swing from GROUND level (Gnd, VSS) to SUPPLY voltage level (VDD), and we measure fall times from $80 \%$ to $20 \%$. We measure input-to-output delays from $50 \%$ voltage swing at the input to $50 \%$ voltage swing at the output. The syntax for all three measurements is very similar. Here is an example of the syntax for measuring a falling transition on in[1]:

$$
\begin{aligned}
& \text {.MEASURE tfall_i_1 } \\
& +\operatorname{trig} \mathrm{v}(\mathrm{in}[1]) \mathrm{val}={ }^{\prime} 0.8 * \text { SUPPLY’ fall=3 } \\
& +\operatorname{targ} \mathrm{v}(\mathrm{in}[1]) \mathrm{val}={ }^{\prime} 0.2 * \text { SUPPLY' fall=3 }
\end{aligned}
$$

The above example specifies that the measurement is triggered as soon as the voltage level for the third falling transition (fall=3) on in[1] reaches $80 \%$ of the SUPPLY voltage (val='0.8*SUPPLY'). It specifies that the measurement ends when the third falling transition on in[1] reaches the target voltage level of $20 \%$ the SUPPLY voltage.

Figure 3.5 gives the combined text with the .DATA sweep and the transient analysis, and the slope measurement on input in[1]. Note that there is no need to use capitals for the commands, as we did above. The complete text with all the sweep, analysis and measurement statements for the parameterized netlist of Figure 3.2 follows in Appendix A.5.

\subsection{Step 5: Generate a SPICE Output File with Look Up Tables}

We are now ready to run the two SPICE input files modified in Step 4 (Section 3.5) and to generate an output file with the look up table information that we want. The SPICE output file has extension '.mt0'. The full printout follows in Appendix A.6. 


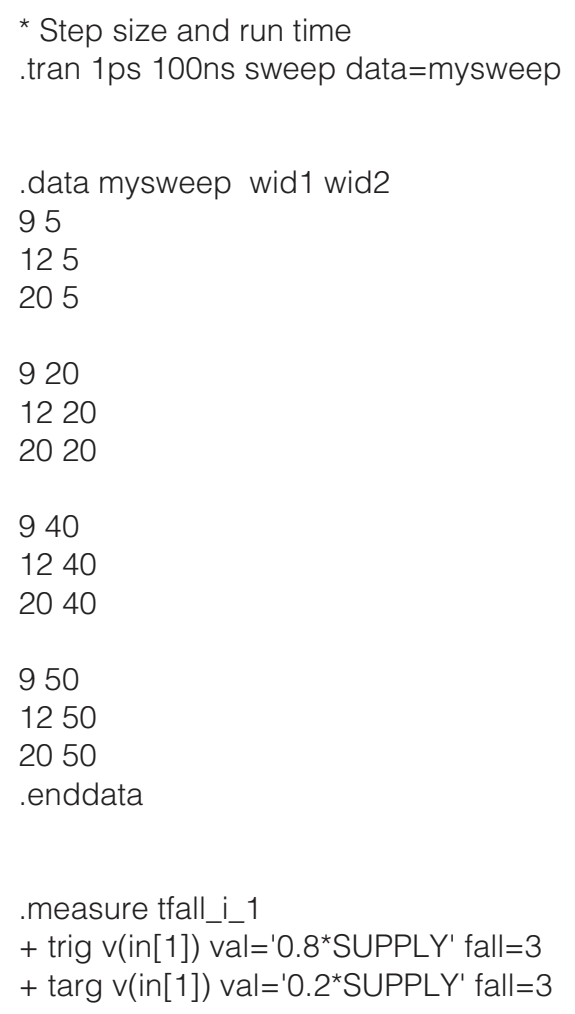

Figure 3.5 SPICE simulation file fragment, showing the .DATA sweep command and the transient analysis that we use for our library characterizations. We show just one measurement statement, measuring the slope of a falling transition on input in[1]. 
Figure 3.6 contains an excerpt, that we'll use to explain the formatting of the output file:

Column 1: The index in the first column gives the sweep iteration number. Note that the numbers use an excessive amount of significant digits. We kept these, because these are the exact results as produced by SPICE. We propose to do any necessary post-processing afterwards, using for instance Microsoft Excel.

Column 2: The second column contains a sequence of three values for each sweep: wid1, tfall_o_1 and alter\#. The last value is of no importance. The value for wid1 is the width of the driver inverter for the current sweep, and the value for tfall_o_1 is the slope or transition time of the falling output transition on out[1]. The significant numbers in the output file are probably realistic only down to a tenth of a picosecond. We can read from Figure 3.6 that the first sweep finds wid1=9 and tfall_o_1=6.9ps.

Column 3: The third column contains a sequence of two values for each sweep: wid2 and tf_delay. Here, wid2 is the width of the load inverter for the current sweep, and tf_delay the input-to-output or propagation delay for a falling transition on in[1] to the corresponding falling transition on out[1]. We can read from Figure 3.6 that the first sweep finds wid2 $=5$ and tf_delay $=48.1 \mathrm{ps}$

Column 4: The fourth column contains again a sequence of two values for each sweep: tfall_i_1 and temper. We will ignore the latter sweep variable for temperature here and focus on tfall_i_1 which is the slope or transition time of the falling input transition on in[1]. We can read from Figure 3.6 that the first sweep finds tfall_i_1=14ps.

So, all with all, columns 1 to 4 together give us the look up table results that we want, phrased as: (wid1 $\times$ wid2) $\rightarrow$ (tfall_i_1,tf_delay,tfall_o_1). 


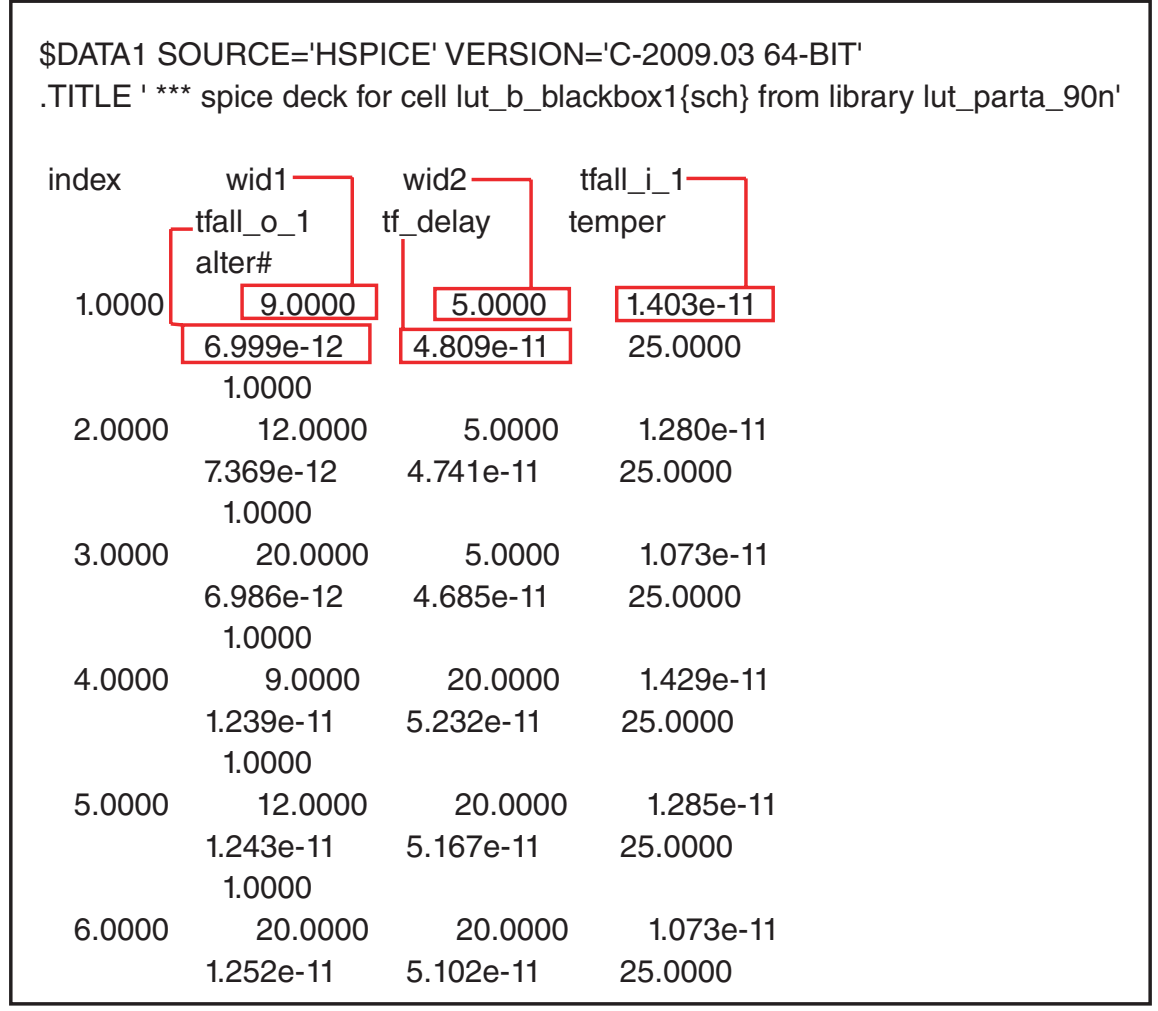

Figure 3.6 Fragment of the SPICE output file with the look up table data, showing $($ wid1 $\times$ wid2) $\rightarrow$ (tfall_i_1, tf_delay, tfall_o_1). 


\subsection{Validation}

In the introduction Section 3.1, we indicated that there are two ways to sweep the search key values for $($ wid1 $\times$ wid2) and to generate the resulting delay and slope information for a simple path in a GasP circuit. 'Way one' is to do the sweep work at design time in Electric, and 'way two' is to do the sweep work in SPICE. We followed 'way two' because the alternative produces much larger SPICE output file sizes. In this section, we compare our results against the results obtained with 'way one'.

Figure 3.7 shows four out of the total of twelve sweeps that we ran, covering all possible driver sizes and all possible load sizes, but only four combinations of driver and load sizes. The four sweeps appear as four parallel designs, all designed in Electric. SPICE will run all four designs in parallel. It does this without needing a .DATA sweep command. However, we do need to specify analysis and measurement statements. So, the original five steps that were needed for 'way two' can be simplified to:

Step 1: (as before) Use Electric to access the GasP design of Figure 3.1.

Step 2: In Electric, create Figure 3.7 with parallel design sweep environments.

Step 3: (as before) From Electric, generate input files for SPICE:

- Generate a SPICE netlist for the GasP design and its environment.

- Generate a SPICE simulation set-up file.

Step 4: Modify only the SPICE simulation set-up file:

- Add transient analysis and measurement statements.

Step 5: Run SPICE and capture the look up table results in an output file.

The corresponding SPICE input and output files follow in Appendix B. 


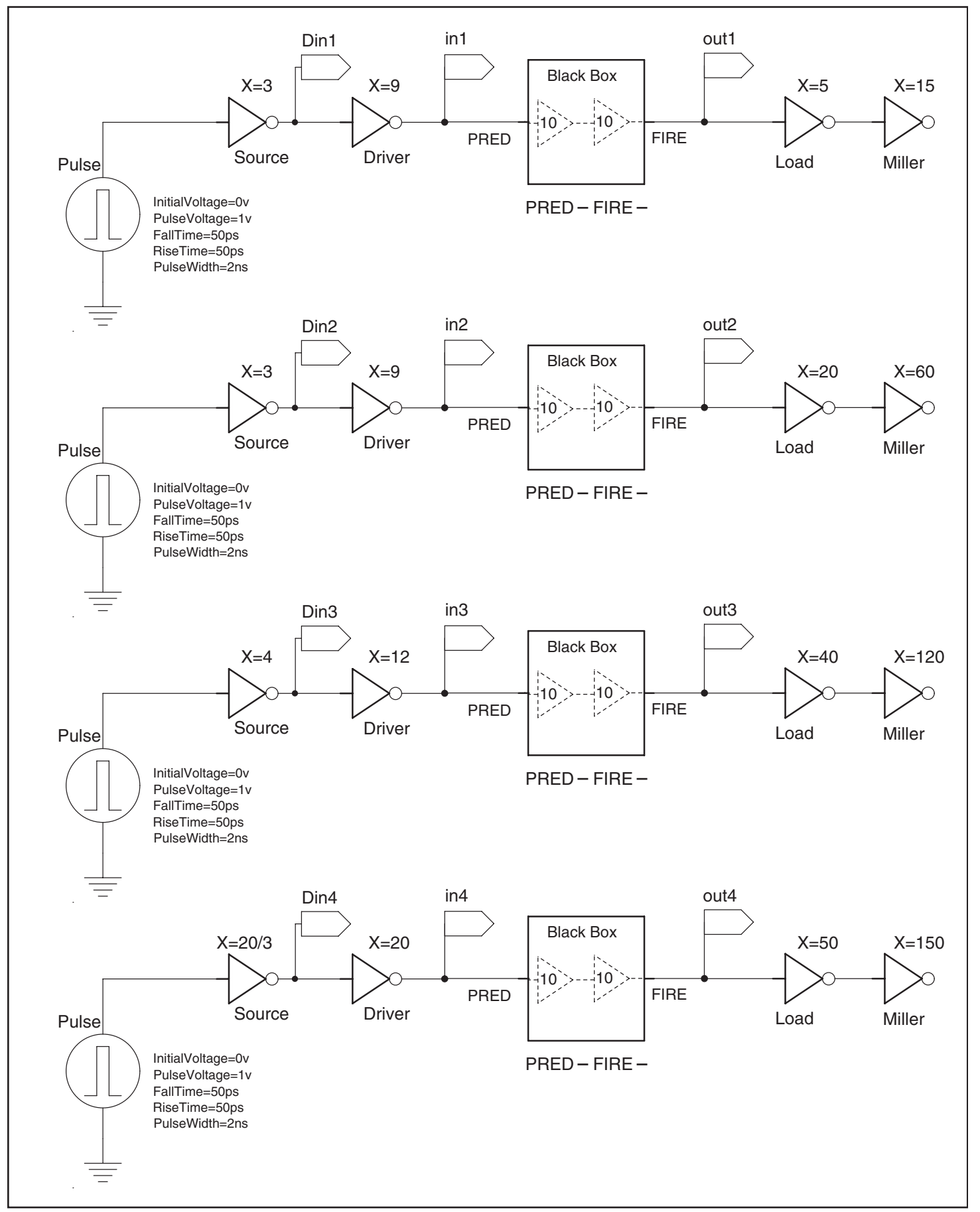

Figure 3.7 Array schematics for sweeping four combinations of driver and source widths in parallel, using four different instances of the simulation set-up of Figure 3.2. Note that the observation signals are now named Din1 to Din4, in 1 to in 4 , out1 to out 4. 


\begin{tabular}{|c|c|c|c|c|c|c|}
\hline & \multicolumn{3}{|c|}{ Driver wid1 $=9$ Load wid2 $=5$} & \multicolumn{3}{|c|}{ Driver wid1 $=9$ Load wid2=20 } \\
\hline & $\begin{array}{c}\text { "Way One" } \\
\text { Design Sweep }\end{array}$ & \begin{tabular}{|c|} 
"Way Two" \\
SPICE Sweep
\end{tabular} & $\%$ Difference & $\begin{array}{c}\text { "Way One" } \\
\text { Design Sweep }\end{array}$ & $\begin{array}{c}\text { "Way Two" } \\
\text { SPICE Sweep }\end{array}$ & $\%$ Difference \\
\hline Input Fall time in ps & $1.38 \mathrm{E}-11$ & $1.40 \mathrm{E}-11$ & $1 \%$ & $1.38 \mathrm{E}-11$ & $1.43 \mathrm{E}-11$ & $3 \%$ \\
\hline Output Fall time in ps & $6.86 \mathrm{E}-12$ & $7.00 \mathrm{E}-12$ & $1.9 \%$ & $1.23 \mathrm{E}-11$ & $1.24 \mathrm{E}-11$ & $1 \%$ \\
\hline \multirow[t]{3}{*}{ Transition delay falling in ps } & $4.79 \mathrm{E}-11$ & $4.81 \mathrm{E}-11$ & $0 \%$ & $5.22 \mathrm{E}-11$ & $5.23 E-11$ & $0 \%$ \\
\hline & \multicolumn{3}{|c|}{ Driver wid1=12 Load wid $2=40$} & \multicolumn{3}{|c|}{ Driver wid1 $=20$ Load wid2 $=50$} \\
\hline & $\begin{array}{c}\text { "Way One" } \\
\text { Design Sweep }\end{array}$ & \begin{tabular}{|c|} 
"Way Two" \\
SPICE Sweep
\end{tabular} & $\%$ Difference & \begin{tabular}{|c|} 
"Way One" \\
Design Sweep
\end{tabular} & \begin{tabular}{|c|} 
"Way Two" \\
SPICE Sweep
\end{tabular} & $\%$ Difference \\
\hline Input Fall time in ps & $1.25 \mathrm{E}-11$ & $1.27 \mathrm{E}-11$ & $2 \%$ & $1.11 \mathrm{E}-11$ & $1.08 \mathrm{E}-11$ & $-3 \%$ \\
\hline Output Fall time in ps & $1.98 \mathrm{E}-11$ & $2.03 E-11$ & $2 \%$ & $2.41 \mathrm{E}-11$ & $2.44 \mathrm{E}-11$ & $1 \%$ \\
\hline Transition delay falling in ps & $5.68 \mathrm{E}-11$ & $5.69 \mathrm{E}-11$ & $0 \%$ & $5.87 \mathrm{E}-11$ & $5.87 \mathrm{E}-11$ & $0 \%$ \\
\hline
\end{tabular}

Figure 3.8 Comparison of the simulation results between 'way one' and 'way two'. 'Way one' is a sweep performed at the design level, using parallel instances of four different simulation environments as illustrated in Figure 3.7. 'Way two' is the approach that we presented in Sections 3.2 to 3.6, where we perform the sweep as part of the SPICE simulation, using .DATA sweep commands.

The table in Figure 3.8 shows a comparison of the results of the four sweeps for 'way one' versus the earlier SPICE sweeps which we have dubbed 'way two'. As before, all measured simulation delays are shown in seconds, with a precision in the order of one tenth of a picosecond. In addition to measured times, we also show the relative difference between 'way one' and 'way two' measurement results, calculated as:

(('way two' or our result) ('way one' or array schematics result)) / ('way two' result)

The results are very similar. The largest differences of $-3 \%$ and $3 \%$ are for input fall times. The good news is that the differences disappear over the length of the path: the differences between the input-to-output path delays are a negligibly $0 \%$.

This is good news because, in the end, it is the path delays that matter. The rise and fall times at input and output nodes are used only to connect the simulation results from one path to the next path in the overall circuit operation, and to compute the combined path delay. The end goal of our look up table results is to allow static timing analysis tools like PrimeTime to compute path delays for relative timing validation of GasP. Relative timing validation amounts to validating that one path has less delay than another path. 
Given this background knowledge and the results in Figure 3.8, we may conclude that a difference of $3 \%$ for the input slope of a path is not going to matter. In summary: the results obtained via 'way one' are similar to those obtained by 'way two'.

\subsection{Conclusion and Future Work for Chapter 3}

We created a characterization flow for generating look up tables with timing information for a simple input-to-output transition in a simplified 6-4 GasP circuit. This chapter is dubbed 'PART-A' because it has a follow-up part. PART-B, in the next chapter, shows how to characterize a complete 6-4 GasP module. In PART-B, we will partition the behavior of a 6-4 GasP FIFO circuit into simple input-to-output transitions and simplified circuits that can be fed back into the library characterization flow developed here in PART-A. Given that "the proof of the pudding is in the eating", we will feed the look up tables that we generate in PART-B to a standard static timing analysis tool to validate the relative timing constrains of the 6-4 GasP FIFO module. This happens in Chapter 5. As far as future work goes, the characterization flow in PART-A can benefit from:

1. Flow automation:

- We need templates for Steps 1-2, to automate the creation of simulation environments like Figure 3.2 and 3.7, for different GasP circuits in Electric.

- We need scripts to automate the SPICE simulation part in Steps 3-5.

2. A direct solution for creating sweep variables widl and wid2 for the driver and load inverters in the simulation environment at the schematic level of Electric:

- Currently, Electric produces 'null' values for the sweep parameters wid1 and wid2 in Figure 3.2. This is visible from the SPICE netlist that we generate in Step 3. We correct this in Step 4, but it would be much better to avoid the need for such a correction. 


\section{Chapter 4}

\section{LIBRARY CHARACTERIZATION PART-B: FROM GASP TO LUTs}

Chapter 4 is also available as internal report ARC2010-smg02 [17]. It is the second of a two-part solution to create look up tables that accurately characterize the timing information of a 6-4 GasP circuit. PART-A in the previous Chapter 3 takes a simple circuit without timing loops, and shows the basic flow aspects from the schematics entry level of the design in Electric to the SPICE simulation set-up and the SPICE output files with the look up tables. PART-B, described here, shows how to partition complex circuits with timing loops and multiple inputs and outputs into designs for which the timing is simple enough to be handled by the characterization flow in PART-A.

\subsection{Introduction}

In PART-A, presented in Chapter 3 and available as report ARC2010-smg01 [16], we showed how one can use SPICE to generate look up tables with timing information of a 6-4 GasP circuit. We generated look up tables for input-to-output propagation times and slew times, a.k.a. slopes or rise and fall times, under various input slopes and output loads. We used a 6-4 GasP sub-circuit, called Half-way GasP, to illustrate the approach. PART-A focused on simple input-to-output paths. PART-B, presented here, extends PART-A, by explaining how to deal with complex paths. We illustrate this extension on the full-fledged 6-4 GasP FIFO design of Figure 2.1 on page 11. 
For complex paths with loops or multiple input and output ports we use a two-step procedure. In the first step, called STEP 1 below, we break the path into a sequence of input-to-output paths, each simple enough to fit in the solution approach of PART-A. In the second step, called STEP 2 below, we show how one should simulate the simple paths to guarantee that the sum of the look up table entries for the propagation times through the simple paths is a sufficiently accurate approximation of the propagation time through the complex path. STEP 2 introduces a unique problem that stems from the single-track handshake protocol in 6-4 GasP: any simple path ending at a single-track output signal is left un-driven at the end of an input pulse. This prevents us from running multiple simulation cycles per sweep - and we typically run a few cycles before we start measuring to get beyond initialization issues. We solve this problem by adding a VCCS device to re-initialize the ouput signal without extra load or delay to the signal. The solution follows in Section 4.4.2.2.

We deploy the look up tables generated with this combined PART-A and PART-B approach to analyze and validate so-called 'Relative Timing' constraints in GasP using standard static timing analysis (STA) tools. Relative timing constraints describe the order in which two signals must arrive at a point of convergence [27, 28]. Specifically: relative timing constraints require that the maximum path delay for the fast signal is below the minimum path delay for the slow signal. This implies that our look up tables must characterize maximum and minimum path delays. This implication simplifies the characterization process because we can ignore all of the intermediary delay scenarios. We use this fact in the naming convention of paths, to express whether the path describes a fast or slow signal. We also use it to incorporate 'The Charlie Effect' [1] into our simulation setup. We use 'The Charlie Effect'only to obtain a maximum or minimum delay 
setup, where needed. For the timing constraints that we characterize and analyze in this thesis, it sufficed to simulate a minimal falling gate delay for gate A in Figure 2.1. We do this by adding a VCVS device. The device enables us to synchronize input events for gates with multiple input signals, without adding extra load or delay. The synchronized (rising) inputs to gate A create a minimal (falling) gate delay for A, which is exactly what we need for the relative timing constraints evaluated in this thesis. The solution follows in Section 4.4.2.1.

The organization of the remainder of Chapter 4 is as follows. Section 4.2 is a quick reminder of what a look up table is and how it is used. Section 4.3 explains STEP 1 of our two-step procedure: how to partition complex circuit paths with loops and multiple input and output ports into simple paths without loops and with one input and one output. Section 4.4 explains STEP 2 of our two-step procedure: how to simulate simple input-to-output paths with sufficient accuracy so that the generated look up tables are a good black box representation for maximum and minimum input-to-output propagation delays and input and output slopes. Section 4.5 generalizes the simulation setup and solves outstanding issues that we encountered in PART-B while using the simulation setup presented in PART-A. Section 4.6 concludes this Chapter 4. Appendices $\mathrm{C}$ to $\mathrm{H}$ contain the SPICE simulation files generated in STEP 2.

\section{Note:}

We postpone the comparison between our library characterization work and prior work at USC by Mallika Prakash [21, 22] and Prasad Joshi [2, 3] to Chapter 7. 


\subsection{Quick Reminder: What's a Look Up Table and How's It Used?}

As a quick preview or reminder of what a look up table is and how it is used in static timing analysis, Figure 4.1 shows a two-dimenstional look up table for a simple path in a 6-4 GasP circuit. The path starts with a rising transition on signal $\mathrm{SUCC}_{i}$ and ends with a falling transition on signal $\mathrm{FIRE}_{i}$. We simulated this path using the general simulation setup of Figure 4.21, page 82. We simulated it for a wide range of input slopes, using drive sizes, and output loads, using load sizes. It is the most detailed look up table that you will find in this thesis. Figure 4.2 plots the landscapes for the input-to-output delay and the output slopes reported in the look up table of Figure 4.1. As you can see, the landscape graphs are very amenable to linear approximation techniques, which is how static timing analysis uses a look up table.

Because emphasis in Chapters 3-4 is about creating a 'flow' for look up tables, the other tables in this thesis are smaller, to simplify the presentation.

\subsection{STEP 1: Partitioning Complex Paths into Simple Paths}

In Chapter 2, we learned about the basic 6-4 GasP module and its four foremost critical relative timing constraints, RT1 to RT4. From Figure 2.1 on page 11 we know that all four timing constraints deal with loops and have multiple inputs and outputs. Below, we will explain STEP 1 of our two-step look up table generation procedure by showing how to break the complex paths in RT1 to RT4 into a sequence of input-to-output paths, where each path is simple enough so we can use the solution approach of PART-A, presented in [16] and Chapter 3, to generate the look up tables for it. 


\begin{tabular}{|c|c|c|c|c|c|c|c|c|c|c|c|c|}
\hline $\begin{array}{r}\text { Drive size } \\
\text { wid1 } \\
\text { Load } \\
\text { size wid2 }\end{array}$ & \multicolumn{2}{|c|}{36} & \multicolumn{2}{|c|}{18} & \multicolumn{2}{|c|}{9} & \multicolumn{2}{|c|}{6} & \multicolumn{2}{|c|}{5} & \multicolumn{2}{|c|}{4} \\
\hline 0 & 35.0 & 7.8 & 36.0 & 8.0 & 37.6 & 8.1 & 39.3 & 7.7 & 40.6 & 7.7 & 42.2 & 8.0 \\
\hline 40 & 34.0 & 10.0 & 38.1 & 10.3 & 39.5 & 10.0 & 41.2 & 10.1 & 42.5 & 9.8 & 44.0 & 9.9 \\
\hline 240 & 44.7 & 19.2 & 45.8 & 19.2 & 47.5 & 19.4 & 49.0 & 19.1 & 50.2 & 19.1 & 51.7 & 18.9 \\
\hline 320 & 47.5 & 23.0 & 48.8 & 23.0 & 50.0 & 22.6 & 51.7 & 22.8 & 52.8 & 22.8 & 54.4 & 23.2 \\
\hline 400 & 50.1 & 26.8 & 51.0 & 27.1 & 52.7 & 27.2 & 54.0 & 27.2 & 55.5 & 27.1 & 56.8 & 26.5 \\
\hline
\end{tabular}

Figure 4.1 Look up table (driver size wid $1 \times$ load size wid2) $\rightarrow$ (delay, output slope). for a simple path in a 6-4 GasP circuit. The path starts with a rising transition on signal $\mathrm{SUCC}_{i}$ and ends with a falling transition on signal FIRE. We simulated it for a wide range of input slopes (using drive sizes) and output loads (using load sizes). In Section 5.2 we translate (wid $1 \times$ wid2) into (input slope $\times$ output load) coordinates. The translation of a - more limited-range - version of the above look up table follows in Figure 5.4, page 99.
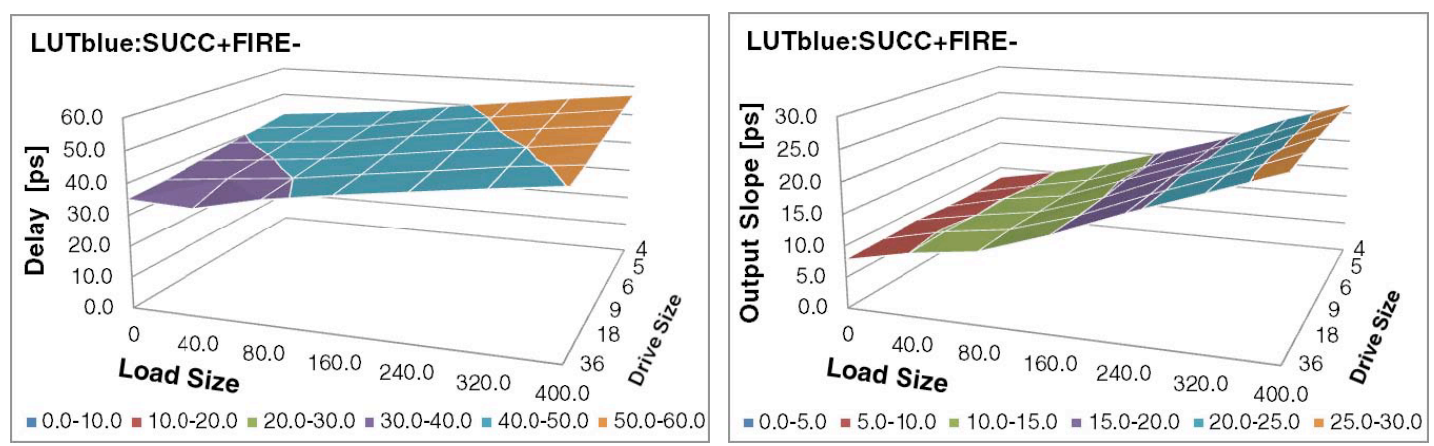

Figure 4.2 Landscape graphs with the delay and output slope information from the look up table in Figure 4.1 above. Delays go from 0ps to 60ps with a color distinction every $10 \mathrm{ps}$, and output slopes from $0 \mathrm{ps}$ to $30 \mathrm{ps}$ with a color distinction every $5 \mathrm{ps}$. We dropped the index ' $i$ ' from the path name, because the path is fully contained within a 6-4 GasP module. Note that these landscapes use (load size $\times$ drive size) rather than (load capacitance $\times$ input slope) coordinates. In Section 5.2 we translate the former coordinate system into the latter. The translations of - more-limited range - landscape versions for the landscapes in this Figure 4.2 follow in Figure 5.9(top), page 104. 
The caveat is that look up tables deal with circuit parameters that can be measured reasonably well in advance. For instance, it takes reasonably low effort to make accurate look up tables that capture the internal path delays in modules M1 and M2 of Figure 2.1. Capturing these internal path delays takes hardly any effort, because each module is a hard macro with pre-defined internal loads, internal wire lengths, and internal drive strengths - custom-designed according to the theory of Logical Effort [32]. But to make an accurate look up table that captures the path delay through state wire L2 between modules M1 and M2 is a tremendous amount of work, because (1) we do not know the exact length and width and spacing of L2 until the system has been placed and routed, and (2) the dependency of the state wire delay to layout wire parameters such as length, width and spacing becomes more complex with longer wires, as we show in [18]. To ensure that timing analysis and relative timing validation for GasP are both accurate and practical, we are going to leave the delay and slope measurements associated with state wire L2 to static timing analysis (STA) with back-annotated wire information from the final layout results. In the partitioning method, to be presented below, we will indicate which of the final paths we are going to leave to STA, and which ones we will characterize using the LUT or look up table approach.

To partition the paths, we first cut them at basic input-output signals, because these are the points where external drives and loads are attached. For our example circuit in Figure 2.1 with its four relative timing constraints in Figure 2.2, the basic input-output signals are $\mathrm{PRED}_{1}, \mathrm{SUCC}_{1}, \mathrm{FIRE}_{1}$ and $\mathrm{PRED}_{2}, \mathrm{SUCC}_{2}, \mathrm{FIRE}_{2}$. We will introduce a few more input-output signals, to control or observe the delay and slope simulation measurements as we go along. The partitioning for the paths that constitute each relative timing constraint follows in Figures 4.3-4.6. 
We will explain our partitioning strategy using the partitioning for RT1 in Figure 4.3. Figure 4.3 partitions the complex paths in RT1 into simple input-to-output paths. The graph at the top is a copy of the graphical representation for RT1 from Figure 2.2(a). It shows a red-arrow path, which we will simply call the red path, and it shows a bluearrow path, which we will simply call the blue path. RT1 requires that the red path delay is less than the blue path delay.

We cut the red path of RT1 at the basic input-output signals FIRE $1, \mathrm{SUCC}_{1}$ and PRED 2 . This yields two simple paths, indicated by the two graphs below the fat black arrow on the right and separated by a fat ' + ' sign, shown in Figure 4.3. For the first path we can reasonably well measure input-to-output delays and slopes and capture these in look up tables. We named this path LUTred:FIRE $1+\mathrm{SUCC}_{1}+$. Note that the name keeps the information about the path color, namely red, and about how the timing is captured, namely by LUT or look up table. The color is important for the simulation set-up: the (maximum) total delay of the red path must be less than the (minimum) total delay of the blue path, and so for red paths we are interested in simulating maximum delays. The second path goes through state wire L2, and so, we are going to leave its delay and slope measurements to STA or static timing analysis with back-annotated wire information from the final layout results. We named this second path STAred:SUCC $1+\mathrm{PRED}_{2}+$. Note that also this name keeps the information about the path color, namely red, and about how the information is to be captured, namely by STA.

We initially cut the blue path of RT1 at the basic input-output signals FIRE $1, \mathrm{PRED}_{1}$ and $\mathrm{SUCC}_{1}$. This yields three paths, indicated by the three graphs below the fat black arrow on the left and separated by a fat '+' sign. For the first two paths we can reasonably well measure input-to-output delays and slopes and capture these in look up tables. 
We named the first path LUTblue:FIRE $1+\mathrm{PRED}_{1}-$ and we named the second path LUTblue:PRED $1-$ FIRE $_{1}-$. Note that, as before, both names keep the information about path color, namely blue, and about how the information is to be captured, namely by LUT or look up table. The total (minimum) delay of the blue path must exceed the total (maximum) delay of the red path, and so for blue paths we are interested in simulating minimum delays. The string 'blue' in the path name helps us remember that this path must be simulated under minimum delay conditions.

The third blue path goes from $\mathrm{FIRE}_{1}-$ to $\mathrm{SUCC}_{1}$ nodrive. The name $\mathrm{SUCC}_{1}$ nodrive expresses that module M1 has stopped driving input-output signal $\mathrm{SUCC}_{1}$. We know of no direct way to measure $\mathrm{SUCC}_{1}$ nodrive, using voltage levels. The best approximation and most practical way for us to measure when $\mathrm{SUCC}_{1}$ nodrive holds is to measure when the voltage level of input signal Dout ${ }_{1}$ to drive transistor E drops below E's threshold level, because that's what stops module M1's drive of $\mathrm{SUCC}_{1}$. To enable such a measurement, we add Dout 1 to the set of input-output signals of module M1, so it becomes available for look up table generation and moreover it remains available for static timing analysis where we tend to treat M1 as a black box with input-output signals and with input-to-output timing information stored in look up tables. Following the path naming convention introduced above, we call this third path: LUTblue:FIRE $1-$ Dout $_{1}+$.

Note how the graph for LUTblue:FIRE $1-$ Dout $_{1}+$ emphasizes the difference between observing Dout ${ }_{1}$ and observing the indirect target $\mathrm{SUCC}_{1}$ nodrive by changing the path line and arrow color from solid blue for the first part of the path to dashed grey for the indirect observation part. 


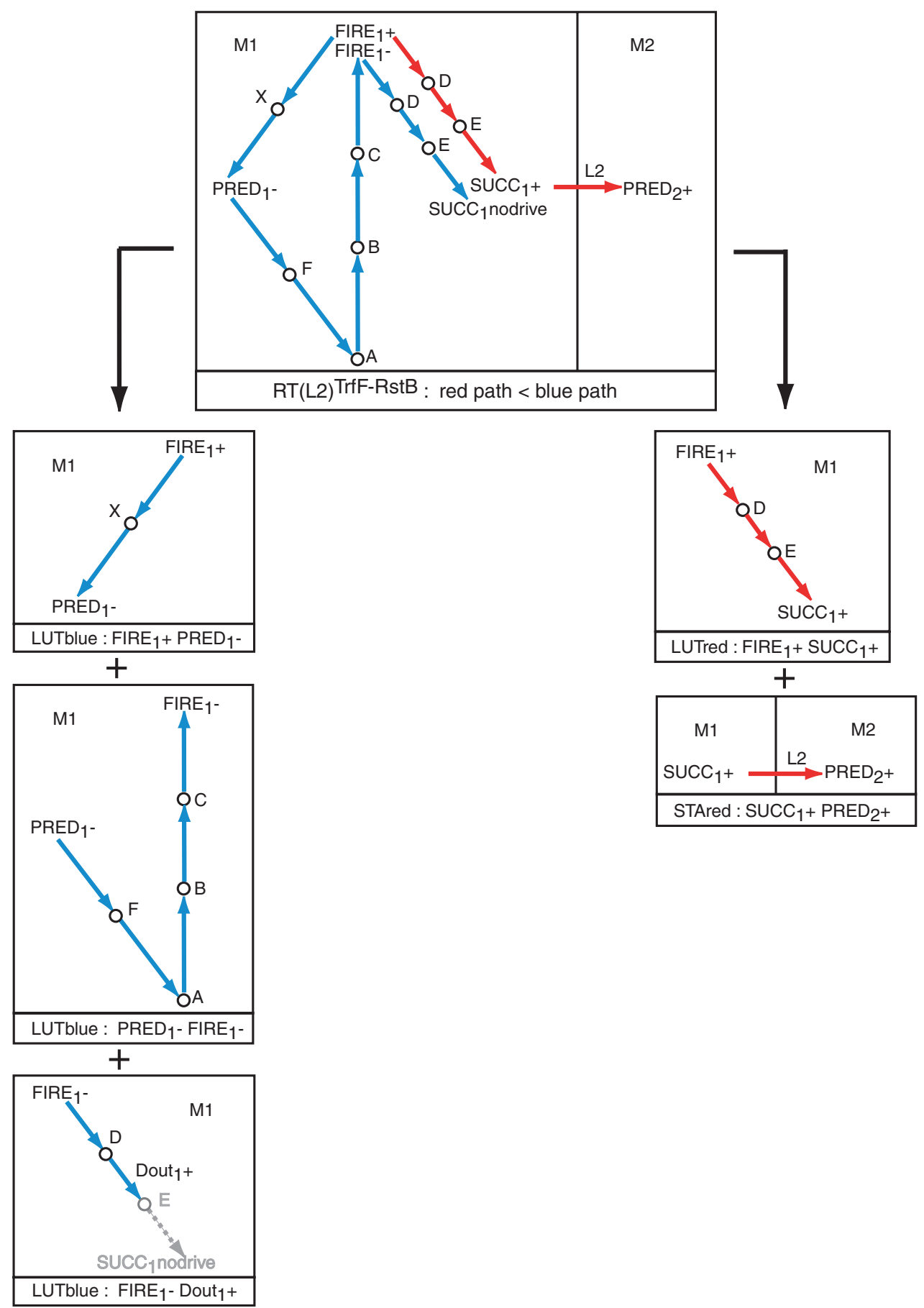

Figure 4.3 Partitioning of the complex paths in relative timing constraint RT1 or RT(L2) ${ }^{\text {TrfF-RstB }}$ of Figure 2.2(a) into simple timing paths for its blue path (left) and for its red path (right). 


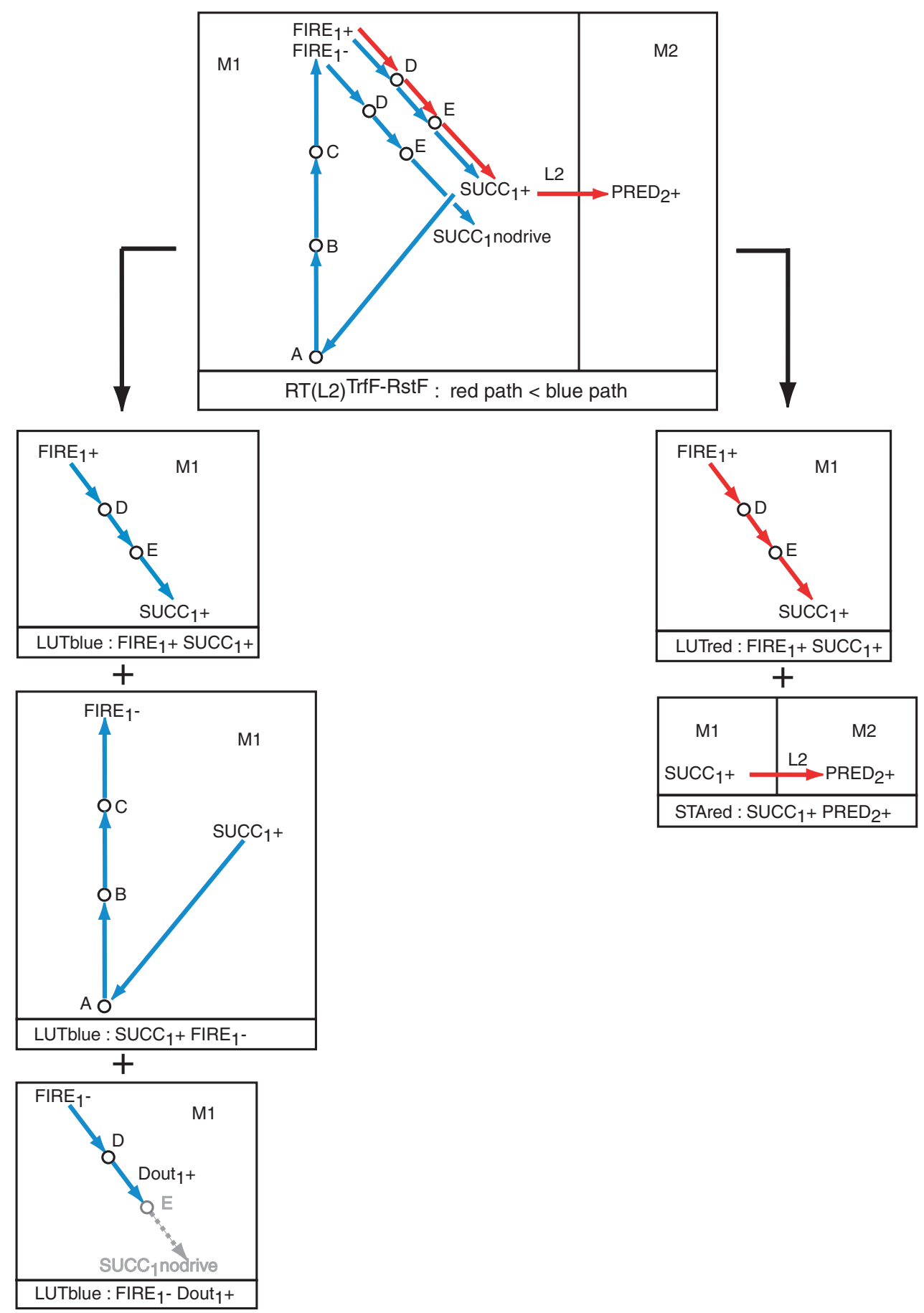

Figure 4.4 Partitioning of the complex paths in relative timing constraint RT2 or RT(L2) ${ }^{\operatorname{TrfF}-\mathrm{RstF}}$ of Figure 2.2(b) into simple timing paths for its blue path (left) and for its red path (right). 


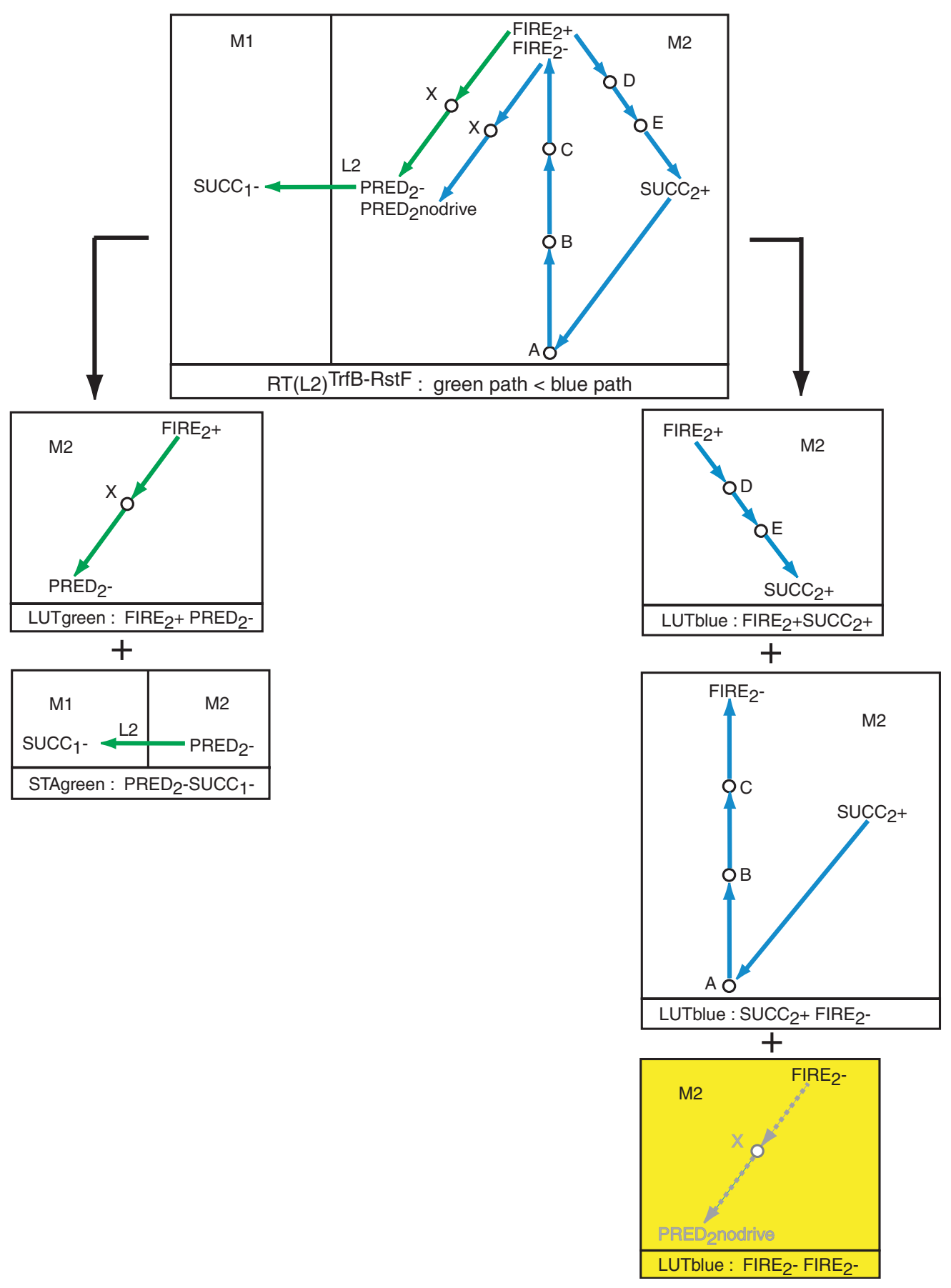

Figure 4.5 Partitioning of the complex paths in relative timing constraint RT3 or RT(L2) ${ }^{\text {TrfB-RstF }}$ of Figure 2.2(c) into simple timing paths for its green path (left) and for its blue path (right). 


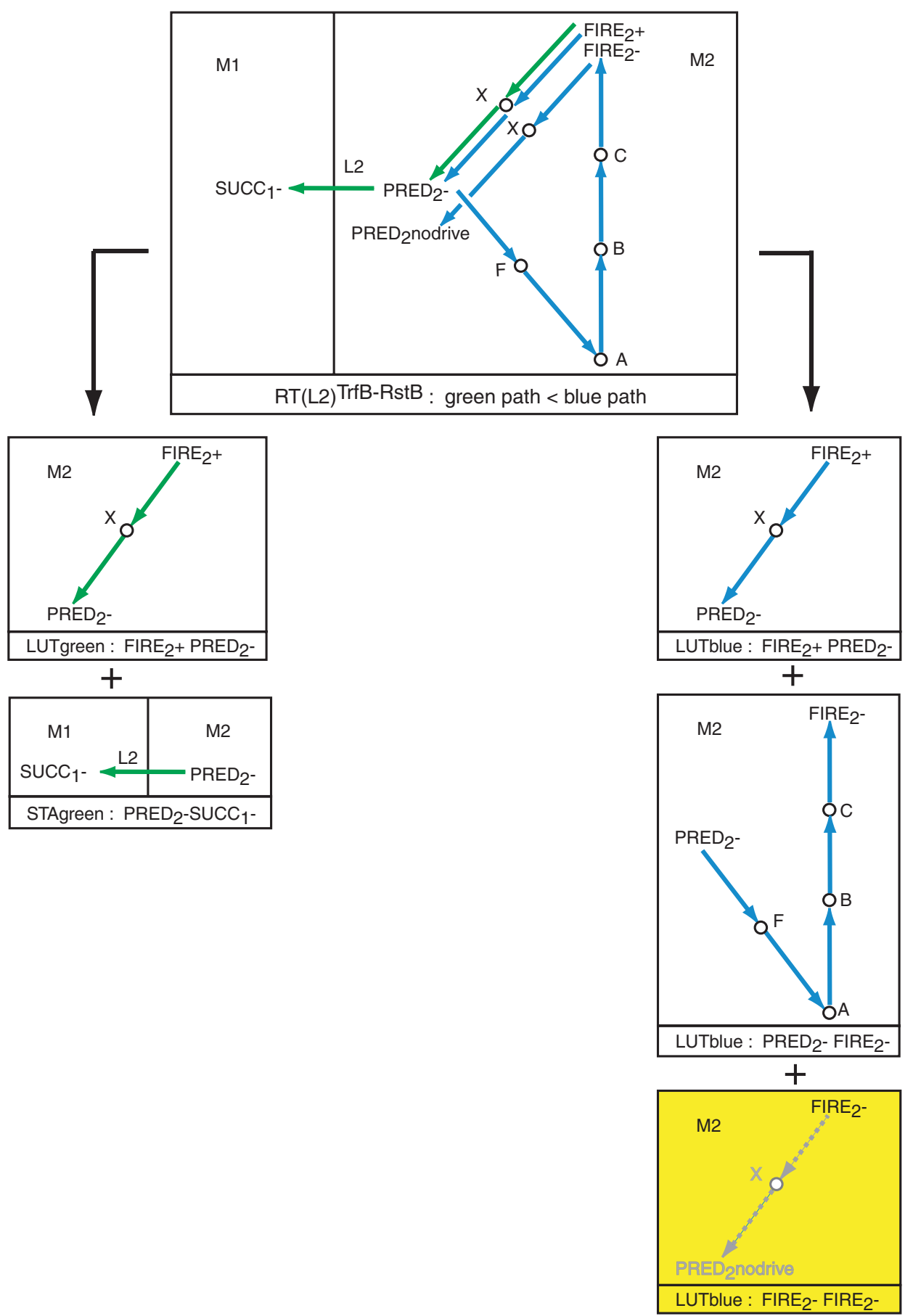

Figure 4.6 Partitioning of the complex paths in relative timing constraint RT4 or RT(L2) ${ }^{\text {TrfB-RstB }}$ of Figure 2.2(d) into simple timing paths for its green path (left) and for its blue path (right). 
This concludes the explanation of the partition for RT1 in Figure 4.3. The partitionings for RT2 to RT4 in Figure 4.4 to 4.6 follow the same approach - specifically:

- Path names for each of the simple paths include information about path color, namely red or green or blue, and about how the information is captured, namely by look up table (LUT) or via static timing analysis (STA). So, by looking at the path name we will know how to set up the path simulations for delays and slopes. Green and red paths will be simulated for maximum delays. Blue paths will be simulated for minimum delays. That way, we can easily validate RT1 to RT4, which are all of one of the two following forms:

$\circ$ the total red path delay is less than the total blue path delay, or

o the total green path delay is less than the total blue path delay.

- Each blue path partition ends in a final path with a nodrive target. The final blue path in Figure 4.4 ends in $\mathrm{SUCC}_{1}$ nodrive. The final blue paths in Figure 4.5 and Figure 4.6 end in $\mathrm{PRED}_{2}$ nodrive. As in Figure 4.3, we approximate these targets by observing the input signal to the drive transistor: instead of $\mathrm{SUCC}_{1}$ nodrive we observe Dout 1 , and instead of $\mathrm{PRED}_{2}$ nodrive we observe FIRE 2 . As before, we emphasize this in the graph by changing the path line and arrow color from solid blue to dashed grey for the indirect observation part.

- The final graphs for the paths with nodrive target $\mathrm{PRED}_{2}$ nodrive in Figure 4.5 and Figure 4.6 have a yellow background to indicate that something special is happening here. Both paths start when signal $\mathrm{FIRE}_{2}$ reaches a pre-determined output voltage level in the previous path and they end when FIRE $_{2}$ drops below the threshold level of drive transistor X. We will not distinguish these two points. In other words: we declare the paths in the yellow-colored graphs of Figure 4.5 and Figure 4.6 empty and will delete these paths from the RT3 and RT4 partitions. 


\subsection{STEP 2: Defining an Accurate Simulation Set-Up for Simple Paths}

In Section 4.4, we will create accurate simulation set-ups for the input-to-output delay and slope calculations of the simple paths for RT1 to RT4. We will do this as follows. Using the complex to simple path partitions for RT1 to RT4 in Figure 4.3 to Figure 4.6 of Section 4.3, we will partition the 6-4 GasP modules M1 and M2 in Figure 2.1 into sub-circuits that allow us to simulate and capture the input-to-output delay and slope information for each particular simple path in RT1 to RT4. We do this in Section 4.4.1

In Section 4.4.2 we will enhance this collection of sub-circuits with extra features so we can (1) simulate minimum and maximum delay scenarios using 'The Charlie Effect', and (2) enable simulation sweeps for simple paths ending in un-driven state wires.

In Section 4.4.3 we revisit the solution approach of PART-A of Chapter 3 and show how we can simulate this collection of enhanced sub-circuits to generate look up tables for each simple path in RT1 to RT4 that has a name starting with 'LUT'. We leave the paths with names starting with 'STA' to static timing analysis. The look up tables will capture the path delays and output slopes under various input slopes and output loads.

Section 4.5 shows how we can generalize the limited simulation setups that we used in Section 4.4.3, and make them work for all possible sweep cases.

\subsubsection{Circuit Partitioning for RT1 to RT4}

In this section, we will partition the two-stage GasP FIFO in Figure 2.1 into sub-circuits that act as the core simulation set-up for the simple paths of RT1-RT4 in Figures 4.3 to 4.6. So, for instance, we partition the circuit in Figure 2.1 to fit the RT1 partition in Figure 4.3. It is not necessary that this sub-circuit partitioning matches the sub-circuit partitioning for RT2 or RT3 or RT4, but we will try to share sub-circuits where we can, because this will reduce the total number of simulation set-ups. 
One way to reduce and simplify simulation set-ups is to work with input slopes, like most of the look up table generation methods do - and to not work with input loads. There is a strong motivation to do it this way:

- If we generate look up tables by simulating input-to-output delays and output slopes for a range of input slopes and output loads, then it does not matter how we obtain the input slopes. In particular: it does not matter which output loads we used in the simulation set-up for a preceding path to generate the output slope that we now use as input slope for the present path. As a result, the simulation set-ups for subsequent paths are independent of each other.

- Were we to use input loads, and generate look up tables by simulating input-tooutput delays and output slopes for a range of input loads and output loads, the dependency between the simulation set-ups of subsequent paths would make it hard or at least more complex to share the simulation set-up between paths with different continuations or with different previous paths.

As an example, consider the simple path $\mathrm{LUTblue}_{\mathrm{PRED}}-\mathrm{FIRE}_{i}-$, which is in the partition of RT1 in Figure 4.3 for $\mathrm{i}=1$, and in the partition of RT4 in Figure 4.6 for $\mathrm{i}=2$. Given that Modules M1 and M2 use the same circuit template, the paths are the same for $i=1$ and $i=2$. But the successor path in RT1 is LUTblue:FIRE ${ }_{i}-$ Dout $_{i}+$ and has the capacitive load of gate D on its input FIRE $_{1}$, while the successor path in RT4 is empty and thus carries no load on its input FIRE ${ }_{2}$. Simulating the joint path LUTblue:PRED ${ }_{i}-\mathrm{FIRE}_{i}-$ with all its output loads, i.e. for X and D and the VDD-connected PMOS half-keeper and the external latches, would give the correct output delay and slope for RT4. But the delays would be skewed for the RT1 continuation into LUTblue:FIRE ${ }_{i}-$ Dout $_{i}+$ because load D is used twice. 
To fix this, we can either simulate the joint path twice, once for RT1 with all the output loads, and once more for RT4 with all the output loads except for D. Or, we can simulate the joint path for a wider range of loads to cover both simulation set-ups in one run. For RT1 we would use the results for the look up table index $\left(C_{\text {in }}, C_{\text {out }}-C_{D}\right)$ and for RT4 we would use the results at index $\left(C_{\text {in }}, C_{\text {out }}\right)$, where $C_{\text {in }}, C_{\text {out }}, C_{D}$ are the input load capacitance at $\mathrm{PRED}_{i}$, the full load capacitance at output $\mathrm{FIRE}_{i}$, and the load capacitance of gate D.

This gets more complex when the number of successor or predecessor paths grows. The more successor and predecessor paths there are to a joint path, the more dependencies we will need to track and the more complex look up table generation and usage will become. For us, this is the key motivation to generate look up tables by using input slopes instead of input loads.

Figure 4.7 shows a complete collection of sub-circuits of Figure 2.1 for covering the relative timing partitions of RT1, RT2, RT3 and RT4 in Figure 4.3 to Figure 4.6. The signals in the sub-circuits of Figure 4.7 are indexed using $(i-1)$ and $i$ and $(i+1)$ instead of 1 for M1 and 2 for M2. The variable indexing enables us to exploit the fact that modules M1 and M2 have identical schematics and use similar single-track wire connections to both their predecessor and successor modules. The index variables also make it easier to identify a shared circuit and simulation set-up for simple relative timing paths that differ only in their index number. 
Figures 4.3-4.6 define the following nine simple relative timing paths:

- LUTblue:PRED ${ }_{i}-$ FIRE $_{i}-$

$\circ$ from RT1 partition, Figure 4.3 ( $i=1$ )

$\circ$ from RT4 partition, Figure $4.6(\mathrm{i}=2)$

- LUTblue:SUCC $i+\mathrm{FIRE}_{i}-$

○ from RT2 partition, Figure 4.4 ( $i=1$ )

$\circ$ from RT3 partition, Figure $4.5(\mathrm{i}=2)$

- LUTred:FIRE ${ }_{i}+\mathrm{SUCC}_{i}+$

$\circ$ from RT1 partition, Figure 4.3 ( $i=1)$

$\circ$ from RT2 partition, Figure $4.4(\mathrm{i}=1)$

- LUTblue:FIRE ${ }_{i}+\mathrm{SUCC}_{i}+$

○ from RT2 partition, Figure $4.4(\mathrm{i}=1)$

○ from RT3 partition, Figure $4.5(\mathrm{i}=2)$

- LUTblue:FIRE ${ }_{i}-$ Dout $_{i}+$

$\circ$ from RT1 partition, Figure 4.3 ( $\mathrm{i}=1$ )

$\circ$ from RT2 partition, Figure $4.4(\mathrm{i}=1)$

- LUTblue:FIRE + PRED $_{i}-$

- from RT1 partition, Figure 4.3 ( $\mathrm{i}=1$ )

$\circ$ from RT4 partition, Figure $4.6(\mathrm{i}=2)$

- LUTgreen:FIRE ${ }_{i}+\mathrm{PRED}_{i}-$

$\circ$ from RT3 partition, Figure 4.5 ( $\mathrm{i}=2$ )

$\circ$ from RT4 partition, Figure $4.6(\mathrm{i}=2)$

- STAgreen:PRED $(i+1)-\mathrm{SUCC}_{i}$

○ from RT3 partition, Figure $4.5(\mathrm{i}=1)$

$\circ$ from RT4 partition, Figure $4.6(\mathrm{i}=1)$

- STAred:SUCC ${ }_{i}+\mathrm{PRED}_{(i+1)}+$

- from RT1 partition, Figure 4.3 (i=1)

○ from RT2 partition, Figure $4.4(\mathrm{i}=1)$ 


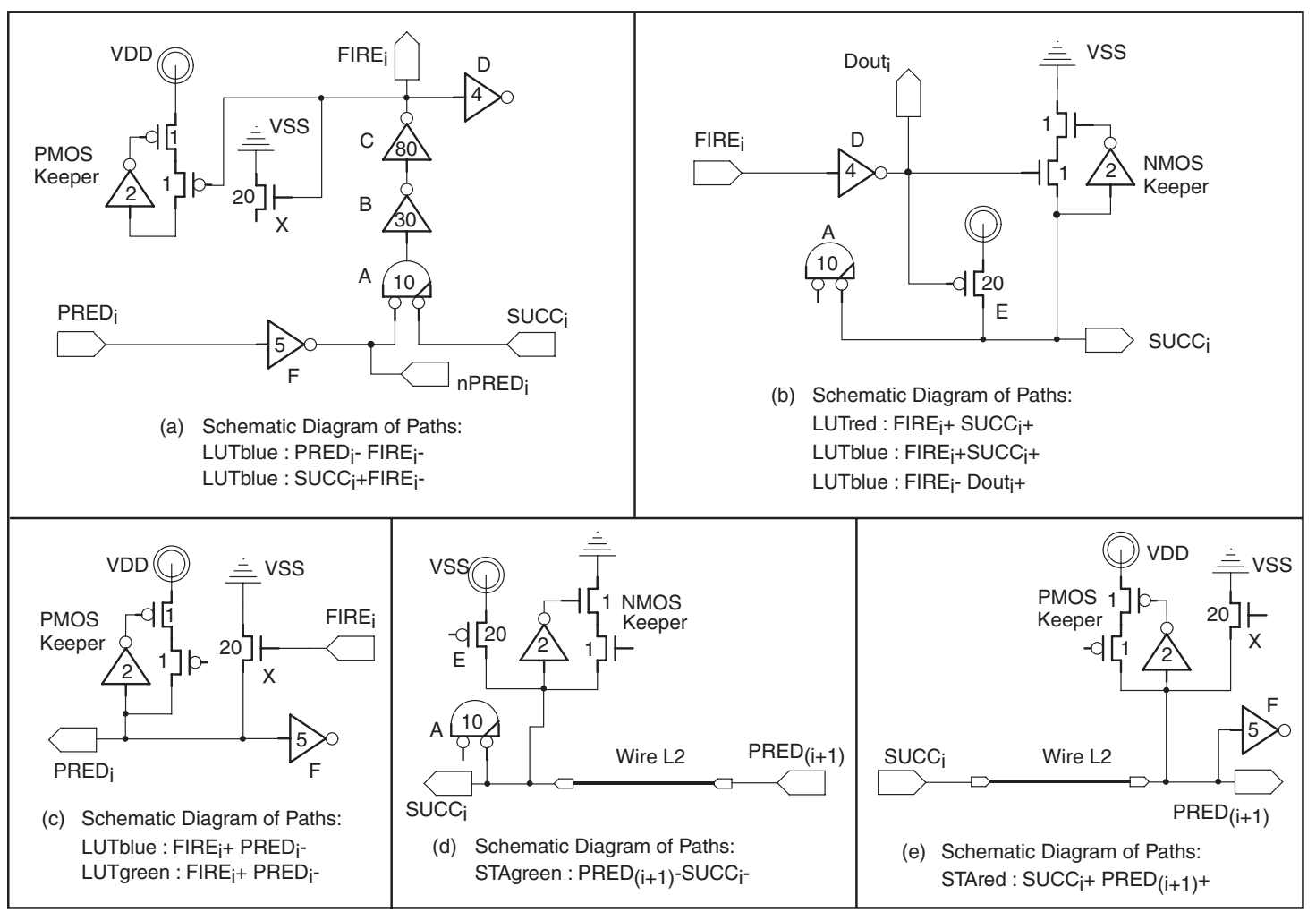

Figure 4.7 Partitioning of the 6-4 GasP Modules M1 and M2 of Figure 2.1 into sub-circuits that match the relative timing partitioning in Figures 4.3-4.6. For each sub-circuit we give a list of the simple relative timing paths that match this sub-circuit. The sub-circuit and its list of simple paths contain sufficient information to finalize the simulation environment for path delay and slope calculations. The simulation results will be captured either by look up tables (LUT) or via static timing analysis (STA). Blue paths will be simulated for minimum path delay calculations. Red and green paths will be simulated for maximum path delay calculations. In Section 4.4.2, Figures 4.8-4.11, we will finalize the above sub-circuits for (a), (b) and (c) to support minimum and maximum delay calculations and serial sweeps.

Note: The careful reader who has read PART-A, Chapter 3, will notice that we did not add Miller inverters following the internal path loads in Figure 4.7(a)-(e) as we should have. Alternatively, we could add second-level internal loads, e.g. we could add gate E as a load to gate D in Figure 4.7 (a) to create a realistic Miller effect for D. 
Each of the above nine paths maps to one of the sub-circuits in Figure 4.7, as follows:

LUTblue:PRED ${ }_{i}-$ FIRE $_{i}-$ : This path goes through gates F, A, B, C. From Figure 2.1 we know that the loads at output signal FIRE $_{i}$ are (1) gates D and X and the PMOS keeper as internal load and (2) the data latches as external load. We explicitly include the total internal load for $\mathrm{FIRE}_{i}$ in the sub-circuit. The external load remains external and becomes an input parameter of the simulation sweep, as discussed in Section 4.4.3. The resulting sub-circuit is shown in Figure 4.7(a). Note that it has three inputs: $\mathrm{PRED}_{i}, \mathrm{nPRED}_{i}$ and $\mathrm{SUCC}_{i}$. In Section 4.4.2, we will re-arrange the input connections and add circuitry to obtain a single input to single output simulation environment for minimum path delay calculations of the two blue paths that map to this sub-circuit.

LUTblue:SUCC $\mathbf{C F}_{i}+\mathbf{F I R E}_{i}-$ : This path goes through gates A, B, C, and maps to the same sub-circuit as previous path LUTblue:PRED $i-$ FIRE $_{i}-$, i.e. Figure 4.7(a).

LUTred:FIRE $i+$ SUCC $_{i}+$ : This path goes through gates D and E. From Figure 2.1 we know that the loads at output signal $\mathrm{SUCC}_{i}$ are (1) gates $\mathrm{A}$ and $\mathrm{X}$ and the NMOS keeper as internal load and (2) state wire L2 to the successor module of $i$ as external load. As we did above for the other paths, we include all internal loads in the sub-circuit and we bring the external load in as input parameter to the simulation sweep. The resulting sub-circuit is shown in Figure 4.7(b). The extra output Dout ${ }_{i}$ of this sub-circuit can be ignored for this path.

LUTblue:FIRE $E_{i}+$ SUCC $_{i}+$ : This path maps to the same sub-circuit as its 'red' version LUTred:FIRE $i+\mathrm{SUCC}_{i}+$, i.e. Figure 4.7(b). The only difference in the simulation set-up would be the selection of PVT (Process, Voltage, Temperature) settings to get minimum path delay calculations for the blue path and maximum ones for the red path. Output Dout ${ }_{i}$ of the circuit can be ignored for this path. 
LUTblue:FIRE $E_{i}-$ Dout $_{i}+$ : This path goes through just gate D. From Figure 2.1 we know that the internal loads at output signal Dout ${ }_{i}$ are gate E and the NMOS keeper, and that there are no external loads connected to Dout ${ }_{i}$. This path can be mapped to the same sub-circuit as the previous two paths, namely Figure 4.7(b). The extra output $\mathrm{SUCC}_{i}$ of this sub-circuit can be ignored for this specific path.

LUTblue:FIRE F $_{i}+$ PRED $_{i}-$ : This path goes through just gate X. From Figure 2.1 we know that the loads at output signal $\mathrm{PRED}_{i}$ are (1) gates $\mathrm{X}$ and $\mathrm{F}$ and the PMOS keeper as internal load and (2) state wire L2 to the predecessor module of $i$ as external load. As we did above for the other paths, we include all internal loads in the sub-circuit and we bring the external load in as input parameter to the simulation sweep. The resulting sub-circuit is shown in Figure 4.7(c).

LUTgreen:FIRE $\mathbf{F}_{i}+\mathbf{P R E D}_{i}-$ : This path maps to the same sub-circuit as its 'blue' version LUTblue:FIRE $i+\mathrm{PRED}_{i}-$ above, i.e. Figure 4.7(c). The only difference in the simulation set-up would be the selection of Process, Voltage, Temperature (PVT) settings to obtain minimum path delay calculations for the blue path and maximum path delay calculations for the green path.

STAgreen:PRED ${ }_{(i+1)}-\mathbf{S U C C}_{i}-$ : This path covers the state wire connection L2 from module $\mathbf{M}(i+1)$ to $\mathbf{M} i$. From Figure 2.1 we know that the loads at output signal $\mathrm{SUCC}_{i}$ are (1) gates A, E and the NMOS keeper as internal load and (2) wire L2 as external load. As we did above for the other paths, we include all internal loads in the sub-circuit and we bring the external load in as input parameter to the simulation sweep. The resulting sub-circuit is shown in Figure 4.7(d).

STAred:SUCC ${ }_{i}+$ PRED $_{(i+1)}+$ : This path covers the state wire connection L2 from module $\mathbf{M}(i)$ to module $\mathbf{M}(i+1)$. From Figure 2.1 we know that the loads at output signal $\operatorname{PRED}_{(i+1)}$ are (1) gates $\mathrm{F}$ and $\mathrm{X}$ and the PMOS keeper as internal 
load and (2) state wire L2 as external load. As we did above for the other paths, we include all internal loads in the sub-circuit and we bring the external load in as input parameter to the simulation sweep. The resulting sub-circuit is Figure 4.7(e).

Note that all but the final two sub-circuits in Figure 4.7(d)-(e) are associated with paths whose timing information goes into look up tables (LUT). The paths associated with the final two sub-circuits will be simulated with methods available during static timing analysis (STA), using layout information, as explained on page 39 in Section 4.3. Note too that the two STA-based paths cross module boundaries: they go from $\mathrm{M}(i+1)$ to M $i$ or vice versa, whereas the LUT-based paths are confined to a single module M $i$. This makes it possible to view and validate LUT-based relative timing constraints on a module by module basis, rather than on the state wire by state wire basis that we followed thus far. The module based view may fit better into standard tool flows than a wire based view. For instance, it would enable us to annotate the relative timing constraints in the schematics description of the module, as is done in the pipeline compilation flow for 'Click' by Handshake Solutions [20].

This is the point where we hand over the STA-based paths to future researchers of timing flows for GasP. The rest of Chapter 4 focuses on LUT-based paths. 


\subsubsection{Circuit Enhancements for Min-Max and Repetitive Simulations}

The sub-circuits and simple relative timing paths in Figure 4.7(a)-(c) are not yet ready for simulation with the look up table generation approach of PART-A of Chapter 3 for the following three reasons:

1. Gate A in Figure 4.7(a) exhibits different input-to-output delays depending on the arrival times of the transitions at its two inputs, $\mathrm{nPRED}_{i}$ and $\mathrm{SUCC}_{i}$. We must minimize the delay for gate A when we are simulating blue paths, and we must maximize the delay for A when we are simulating red and green paths. Given that the relative timing constraints through gate A in this report use strictly blue paths, it suffices to set up the simulation so that the gate delay for A is minimal.

2. Figure 4.7(a) has multiple inputs. The solution presented in PART-A handles only single input to single output look up tables.

3. Figure 4.7(b) cannot be used directly for serial simulations from $\mathrm{FIRE}_{i}$ to $\mathrm{SUCC}_{i}$ because output $\mathrm{SUCC}_{i}$ starts to float when the pulse generator on $\mathrm{FIRE}_{i}$ resets the input to low before starting the next high transition. To obtain valid input to output delay and slope measurement, we must re-initialize the output signal to low before starting the next high transition. Likewise, Figure 4.7(c) cannot be used directly for sweeps that contain multiple simulation cycles from $\mathrm{FIRE}_{i}$ to $\mathrm{PRED}_{i}$.

Section 4.4.2.1 discusses how we can solve the issues indicated in items 1-2 above. Section 4.4.2.2 discusses how we can solve the issues explained in item 3 above. And Section 4.4.2.3 summarizes the new list of sub-circuits and simple relative timing paths that we obtain after solving items 1-3 for the old list of sub-circuits in Figure 4.7(a)-(c). We will pass the new list on to Section 4.4.3 where we generate the look up tables. 


\subsubsection{Simulating Minimum Gate Delays}

Any AND or OR type logic will have series transistors as well as transistors in parallel. The 6-4 GasP gate A in the sub-circuit shown in Figure 4.7(a) has two PMOS transistors in series and two NMOS transistors in parallel. When transistors in series turn on at the same time it takes longer to conduct current than when one of the transistors is already turned on or turned on partially. In contrast, when transistors in parallel turn on at the same time, current is conducted faster than when one of the transistors is already on or partially on. We call this 'The Charlie Effect' [1], after the late Charles Molnar. The result is that the delay of NOR gate $\mathrm{A}$ is maximal when the two input signals $\mathrm{nPRED}_{i}$ and $\mathrm{SUCC}_{i}$ fall at the same time, and the delay is minimal when the two input signals rise at the same time.

For the blue-colored relative timing paths covered by Figure 4.7(a) we are interested in the minimal propagation time for rising input transitions on $\mathrm{nPRED}_{i}$ and $\mathrm{SUCC}_{i}$. This means that we want the rising transitions to coincide. We can force this in SPICE by using a so-called Voltage Controlled Voltage Source (VCVS) on the two signals.

A VCVS is a fictional device - or, as Ivan Sutherland calls it in our ARC report [17]: a 'demon in a box' - that (or according to Ivan "who") can be used in a simulation environment to achieve perfectly synchronized transitions. In this case: it enables us to generate perfectly synchronized rising transitions on gate $\mathrm{A}$ inputs $\mathrm{nPRED}_{i}$ and $\mathrm{SUCC}_{i}$.

The VCVS in Figure 4.8 has two ports that relate to $\mathrm{nPRED}_{i}$, namely in and $\mathrm{GND}_{\text {in }}$, and two ports that relate to $\mathrm{SUCC}_{i}$, namely out and $\mathrm{GND}_{\text {out }}$. It copies the voltage difference between in and $\mathrm{GND}_{\text {in }}$ onto out and $\mathrm{GND}_{\text {out }}$ - and it does this without delay, i.e. instantaneously. 
We can adjust the copy function of a VCVS device in two ways: (1) by scaling the voltage range up or down by a factor called 'Gain', and (2) by cutting off the range at a predefined minimum or maximum voltage, called 'Minimum' and 'Maximum', where Minimum $\leq$ Maximum. This yields the following relation:

$$
\begin{array}{ll}
\left(\text { in }-\mathrm{GND}_{\text {in }}\right) & \\
=\text { Gain } \times\left(\text { out }-\mathrm{GND}_{\text {out }}\right) & \text { if Minimum } \leq \text { Gain } \times\left(\text { out }-\mathrm{GND}_{\text {out }}\right) \leq \text { Maximum } \\
=\text { Minimum } & \text { if Gain } \times\left(\text { out }-\mathrm{GND}_{\text {out }}\right) \leq \text { Minimum } \\
=\text { Maximum } & \text { if Maximum } \leq \text { Gain } \times\left(\text { out }-\mathrm{GND}_{\text {out }}\right)
\end{array}
$$

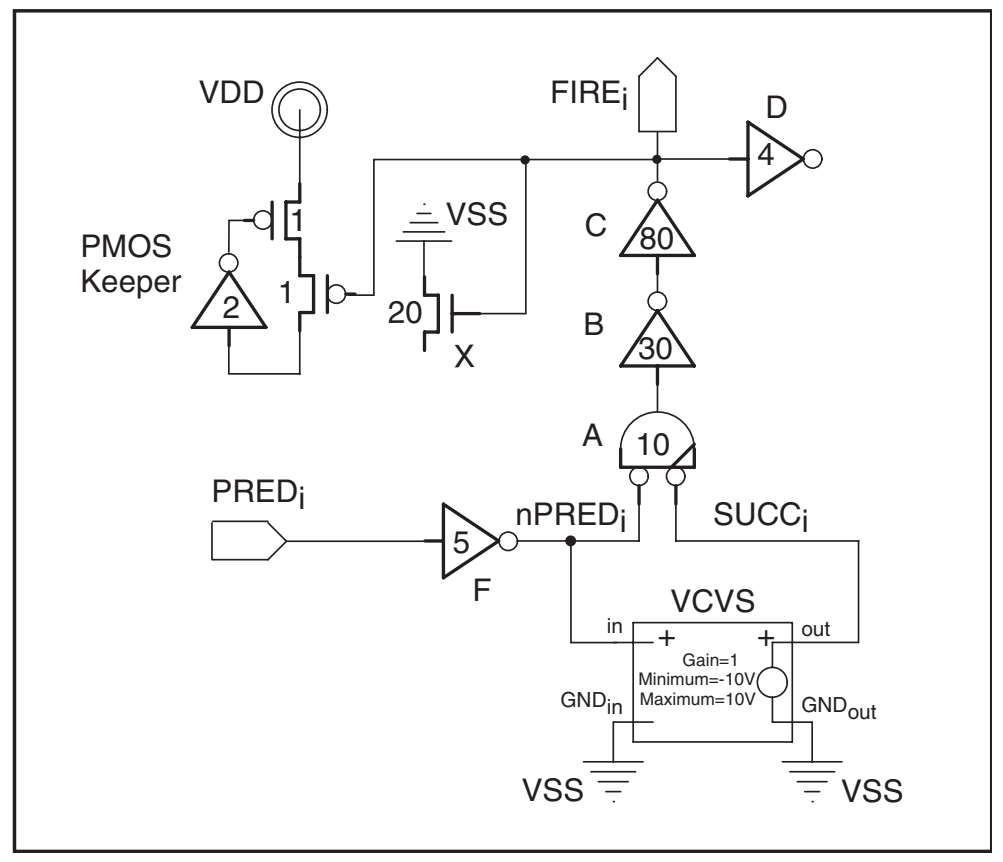

Figure 4.8 Enhancement for Figure 4.7(a) and LUTblue:PRED $-\mathrm{FIRE}_{i}-$. The changes over the sub-circuit in Figure 4.7(a) are (1) an additional Voltage Controlled Voltage Source (VCVS) between the inputs to gate A to minimize the gate delay for a falling output transition on A, and (2) a reduction of the total number of sub-circuit inputs from three $\left(\mathrm{PRED}_{i}, \mathrm{nPRED}_{i}, \mathrm{SUCC}_{i}\right)$ to one $\left(\mathrm{PRED}_{i}\right)$ to make this circuit fit into the LUT generation approach in PART-A, Chapter 3. The VCVS has Gain=1, Minimum $=10 \mathrm{~V}$ and Maximum $=10 \mathrm{~V}$. 


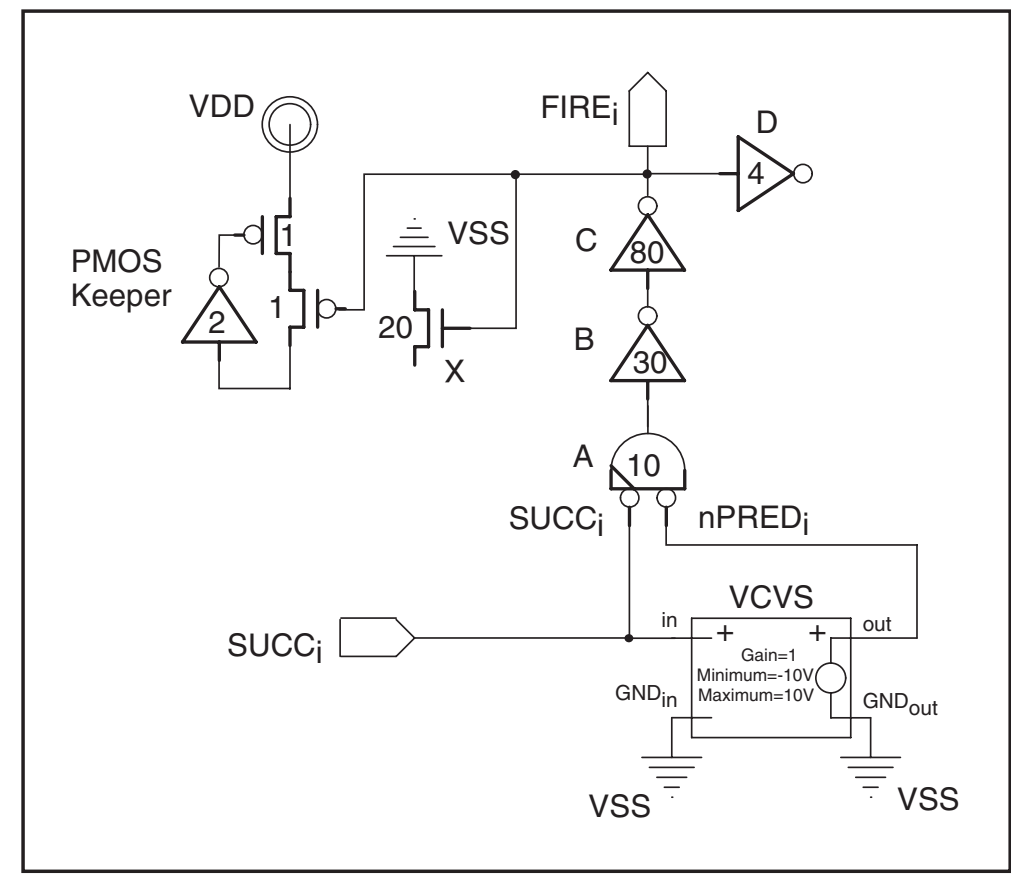

Figure 4.9 Enhancement for Figure 4.7(a) and LUTblue:SUCC $+\mathrm{FIRE}_{i}-$. The changes are similar to those for Figure 4.8, showing (1) an additional Voltage Controlled Voltage Source (VCVS) between the inputs to gate A to minimize the gate delay for a falling output transition on $\mathrm{A}$, and (2) a reduction of the total number of sub-circuit inputs from three $\left(\mathrm{PRED}_{i}, \mathrm{nPRED}_{i}, \mathrm{SUCC}_{i}\right)$ to one $\left(\mathrm{SUCC}_{i}\right)$ to make this circuit fit into the LUT generation approach published in PART-A, Chapter 3. The VCVS has Gain=1, Minimum $=10 \mathrm{~V}$ and Maximum $=10 \mathrm{~V}$.

For relative timing path LUTblue: $\mathrm{PRED}_{i}-\mathrm{FIRE}_{i}-$ we will use a VCVS device with in $=\mathrm{nPRED}_{i}$, out $=\mathrm{SUCC}_{i}, \mathrm{GND}_{\mathrm{in}}=\mathrm{GND}_{\text {out }}=\mathrm{VSS}, \mathrm{Gain}=1, \mathrm{Minimum}=10 \mathrm{~V}$, and Maximum $=10 \mathrm{~V}$. For the other relative timing path LUTblue: $\mathrm{SUCC}_{i}+\mathrm{FIRE}_{i}-$ covered by Figure 4.7(a) we will use a VCVS device with in $=\mathrm{SUCC}_{i}$, out $=\mathrm{nPRED}$, and $\mathrm{GND}_{\mathrm{in}}=\mathrm{GND}_{\text {out }}=\mathrm{VSS}$, Gain $=1$, Minimum $=10 \mathrm{~V}$, and Maximum $=10 \mathrm{~V}$. The correspond ing enhanced sub-circuits follow in Figure 4.8 and Figure 4.9. Note that the resulting circuits have exactly one input and one output, and that they use minimal gate delays for gate A. Thus, they solve the issues brought up in items 1-2 on page 55, at the beginning of Section 4.4.2. 


\subsubsection{Autonomously Re-Initializing Un-Driven Wires}

The GasP family communicates bi-directionally over single-track state wires between modules. A module connected to a state wire can either raise the wire or lower the wire, but it cannot do both. For instance, module M1 in Figure 2.1, page 11, can raise state wire L2 via its local pull-up transistor E, and module M2 can lower L2 via its local pulldown transistor $\mathrm{X}$. In between these active pull-up and pull-down events, that each last for about five gate delays, the state of the wire is maintained by the keepers. For state wire L2 in Figure 2.1, the pull-up and pull-down circuit of its keeper are partitioned over the two modules: L2-pull-up module M1 gets the maintain-low-state or NMOS keeper, and L2-pull-down module M2 gets the maintain-high-state or PMOS keeper. As a result, the sub-circuits in Figure 4.7(b)-(c) miss half of the L2 keeper circuitry. Missing half of the keeper circuitry turns out not to be an issue for the simulation setup. In fact, it will turn out that the sub-circuits have exactly the right half-keeper to make the setup work.

The issue is that Figure 4.7(b) can pull $\mathrm{SUCC}_{i}$ high and keep it low, but it cannot actively lower $\mathrm{SUCC}_{i}$. This is a problem if we want to simulate more than one input-to-output transition: a high input pulse on $\mathrm{FIRE}_{i}$ will initially pull $\mathrm{SUCC}_{i}$ high but then leave $\mathrm{SUCC}_{i}$ un-driven and hence floating as soon as $\mathrm{FIRE}_{i}$ is low. If we fail to reset $\mathrm{SUCC}_{i}$ back to VSS, the next simulation will start with $\mathrm{SUCC}_{i}$ at an unknown voltage level and thereby render the simulated path delay and output slope for LUTred:FIRE $i+\mathrm{SUCC}_{i}+$ and LUTblue:FIRE $i+$ SUCC $_{i}+$ meaningless. ${ }^{4.1}$ This is a problem because we typically run a few simulation cycles before we start measuring the timing, to get past initialization issues. To support multiple simulation cycles, we must reset $\mathrm{SUCC}_{i}$ to its initial state after completion of the current simulation and before the start of the next.

\footnotetext{
${ }^{4.1}$ Even if module M1 had a full L2 keeper, leaving $\mathrm{SUCC}_{i}$ at a stable high voltage at the end of the FIRE $_{i}$ pulse, we would still have to reset $\mathrm{SUCC}_{i}$ to its initialization state before the next simulation cycle.
} 
We can do this with a Voltage Controlled Current Source (VCCS). Like the VCVS that we used earlier, a VCCS is a fictional device or 'demon in a box'. A VCCS device can be used in a simulation environment to re-initialize un-driven wires. The VCCS in Figure 4.10 has two ports, $\mathrm{in}_{1}$ and $\mathrm{in}_{2}$, that compare the voltage levels of $\mathrm{SUCC}_{i}$ and a FIRE -related signal called FIRE_CS $_{i}$. It has two ports, out ${ }_{1}$ and out $_{2}$, that uncharge $\mathrm{SUCC}_{i}$ to re-initialize it to VSS when needed. The difference $\left(\mathrm{in}_{1}-\mathrm{in}_{2}\right)$ in Volts is translated into Amperes for a current flowing from out 1 to out 2 . The translation is without delay, i.e. instantaneously. We can adjust the voltage-to-current translation function of a VCCS device in two ways: (1) by scaling the target range up or down by a factor called 'Gain', and (2) by cutting off the range at a predefined minimum or maximum level, called 'Minimum' and 'Maximum', where Minimum $\leq$ Maximum. This yields the following relation: ${ }^{4.2}$

$$
\begin{array}{ll}
\mathrm{I}_{\text {out1-to-out2 }}[\mathrm{A}] & \\
\sim \text { Gain } \times\left(\mathrm{in}_{1}-\mathrm{in}_{2}\right)[\mathrm{V}] & \text { if Minimum } \leq \text { Gain } \times\left(\mathrm{in}_{1}-\mathrm{in}_{2}\right) \leq \text { Maximum } \\
\sim \text { Minimum } & \text { if Gain } \times\left(\mathrm{in}_{1}-\mathrm{in}_{2}\right) \leq \text { Minimum } \\
\sim \text { Maximum } & \text { if Maximum } \leq \text { Gain } \times\left(\mathrm{in}_{1}-\mathrm{in}_{2}\right)
\end{array}
$$

Signal FIRE_CS ${ }_{i}$ in Figure 4.10 goes from 0 Volts (VSS) to 1.5 Volts (50\% above VDD). It rises before FIRE $_{i}$ rises and it falls after FIRE falls. FIRE_CS $_{i}$ is high indicates

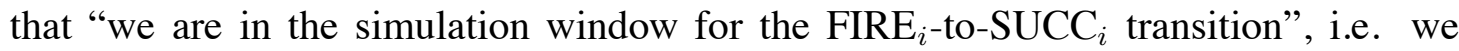
are somewhere in the critical section for the simulation measurement. By widening and raising the pulse for FIRE_CS $i$ relative to $\mathrm{FIRE}_{i}$, we can guarantee that (1) the simulated transition measurement is over when FIRE_CS ${ }_{i}$ goes low, and (2) the VCCS is unaffected by any transition noise on output signal $\mathrm{SUCC}_{i}$ as long as FIRE_CS $_{i}$ is at an ultra-high $1.5 \mathrm{~V}$.

\footnotetext{
${ }^{4.2}$ For convenience of notation, we assume we can mix and match Voltage and Ampere scales.
} 


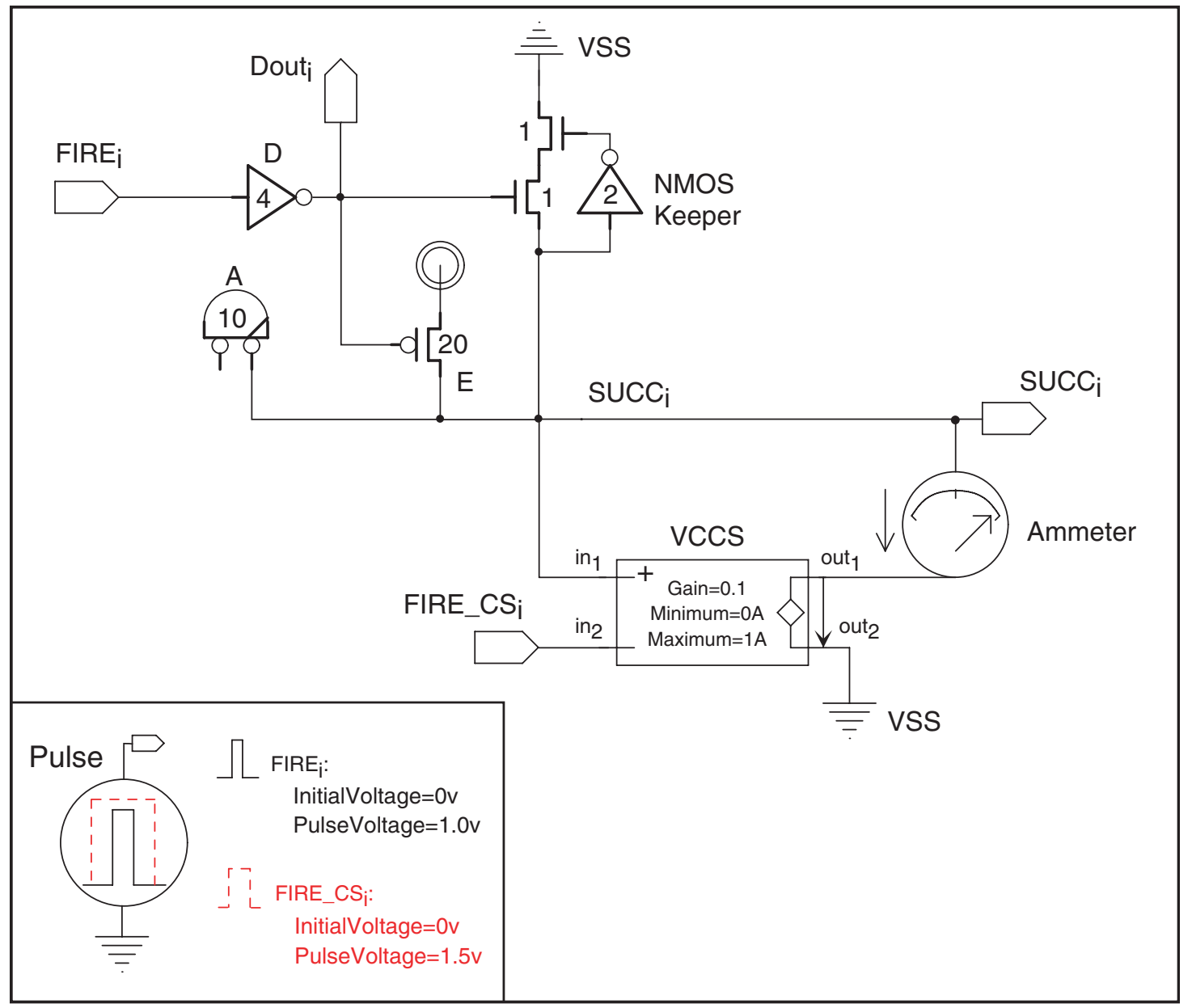

Figure 4.10 Enhancement for the sub-circuit in Figure 4.7(b) and the relative timing paths LUTred:FIRE $i+$ SUCC $_{i}+$ and LUTblue:FIRE $i+$ SUCC $_{i}+$. The enhancement enables the repetitive simulation sweeps needed to create look up tables with timing information for a range of input slopes and output loads. The change over Figure 4.7(b) is an additional Voltage Controlled Current Source (VCCS) on output signal SUCC $i$ to force the signal out of an un-driven high state and to re-initialize it to VSS for the next simulation. The VCCS has Gain=0.1, Minimum=0A and Maximum=1A. Note that we indicated the relation between the pulse generators for VCCS control signal FIRE_CS; and sub-circuit input signal FIRE $i$. We will use this information in Section 4.4.3 to set up the look up table sweeps. 
Moreover, if we make sure that FIRE_CS ${ }_{i}$ is low for long enough to pull $\mathrm{SUCC}_{i}$ low, then the NMOS half-keeper in Figure 4.10 will take over the maintenance of the 0V VSS level on $\mathrm{SUCC}_{i}$. When the keeper kicks in, we no longer need the VCCS device to drive $\mathrm{SUCC}_{i}$ and so we can safely raise FIRE_CS $i$ in preparation for the next simulation.

In summary: Figure 4.10 gives the enhancement for the sub-circuit in Figure 4.7(b) and the two relative timing paths LUTred:FIRE $i+\mathrm{SUCC}_{i}+$ and LUTblue:FIRE $i+\mathrm{SUCC}_{i}+$. It has a VCCS device with in $_{1}=\mathrm{SUCC}_{i}$, in $_{2}=$ FIRE_CS $_{i}$, out $_{1}=\mathrm{SUCC}_{i}$, out $_{2}=\mathrm{VSS}$, Gain=0.1, Minimum=0A, and Maximum=1A. A gain of 0.1 is high enough to make the voltage change on out ${ }_{1}$ 's $\mathrm{SUCC}_{i}$ closely track the downgoing slope of the pulse

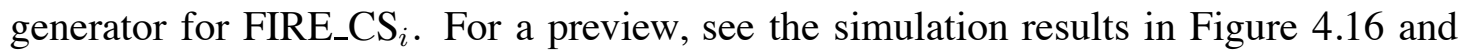
Figure 4.17 on pages $74-75$. This VCCS device comes with the following relational simulation set-up requirements for signals FIRE $_{i}$ and FIRE_CS :

- FIRE_CS ${ }_{i}$ rises to an ultra-high $1.5 \mathrm{~V}$ before $\mathrm{FIRE}_{i}$ starts rising to $1.0 \mathrm{~V}$ VDD.

- FIRE_CS ${ }_{i}$ starts falling to a low VSS level after FIRE $i$ is already low.

- Signal pulse widths and periods must be long enough for $\mathrm{SUCC}_{i}$ to rise and fall.

Figure 4.11 gives a similar enhancement for the sub-circuit in Figure 4.7(c) and the two relative timing paths LUTgreen:FIRE ${ }_{i}+\mathrm{PRED}_{i}-$ and $\mathrm{LUTblue}_{\mathrm{FIRE}}+\mathrm{PRED}_{i}-$. It has a VCCS device with in $_{1}=$ PRED $_{i}$, in $_{2}=$ FIRE_CS $_{i}$, out $_{1}=$ PRED $_{i}$, out $_{2}=$ VSS, Gain=0.1, Minimum=1A, Maximum=0A. Here, the purpose of the VCCS device is to re-initialize the un-driven low $\mathrm{PRED}_{i}$ signal to a high 1.0V VDD level after FIRE ${ }_{i}$ falls. We do this by inverting the FIRE_CS ${ }_{i}$ signal in relation to $\mathrm{FIRE}_{i}$ :

- FIRE_CS ${ }_{i}$ falls to an ultra-low $-0.5 \mathrm{~V}$ before $\mathrm{FIRE}_{i}$ starts rising to VDD.

- FIRE_CS ${ }_{i}$ starts rising to VDD level after FIRE $_{i}$ is already low.

- Signal pulse widths and periods must be long enough for $\operatorname{PRED}_{i}$ to fall and rise. 


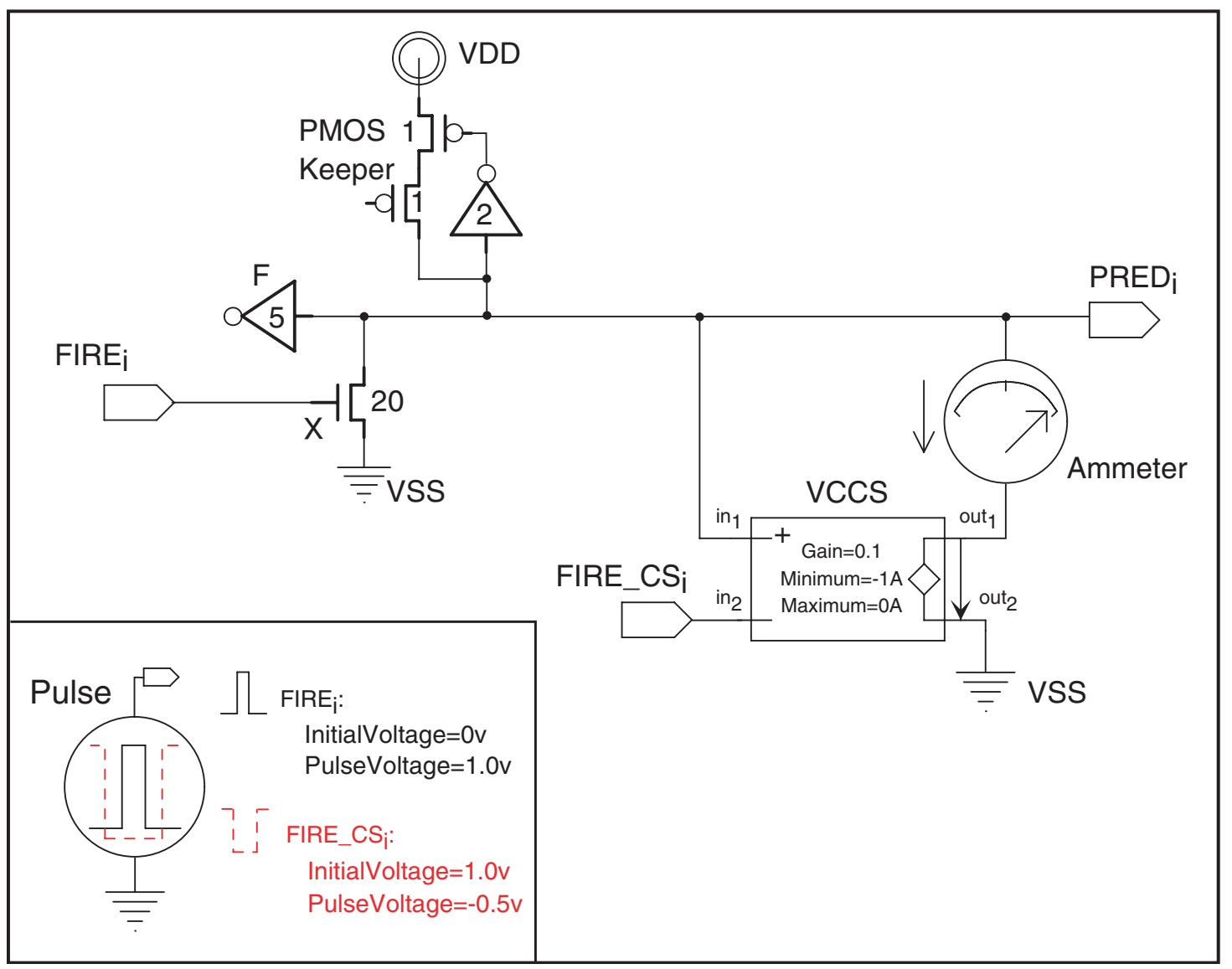

Figure 4.11 Enhancement for the sub-circuit in Figure 4.7(c) and relative timings LUTblue:FIRE $i+$ PRED $_{i}-$ and LUTgreen:FIRE $i+$ PRED $_{i}-$ to enable the repetitive simulation sweeps needed to create look up tables with timing information for a range of input slopes and output loads. The enhancement is similar to Figure 4.10. It uses an additional Voltage Controlled Current Source (VCCS) on output signal PRED $_{i}$ to force the signal out of an undriven low state and to re-initialize it to VDD for the next simulation. The VCCS device has Gain=0.1, Minimum=1A and Maximum=0A. Note that we indicated the relation between the pulse generators for VCCS control signal FIRE CS, and sub-circuit input signal FIRE . We will use this information in Section 4.4.3 to set up the look up table sweeps. 
The VCCS in Figure 4.11 translates a negative voltage difference $\left(\mathrm{PRED}_{i}-\right.$ FIRE_CS $\left._{i}\right)$ into a negative current $\mathrm{I}_{\text {out1-to-out2 }}$ in the range of $1 \mathrm{~A}$ to $0 \mathrm{~A}$. This corresponds to a positive current flow in the range of $0 \mathrm{~A}$ to $1 \mathrm{~A}$ from $\mathrm{out}_{2}$ to out 1 , i.e. to $\mathrm{PRED}_{i}$, which charges $\mathrm{PRED}_{i}$ until the voltage on $\mathrm{PRED}_{i}$ matches that of VDD. Note that this works independent of the actual voltage source for out $_{2}-\mathrm{out}_{2}$ can be VSS or VDD or $-200 \mathrm{~V}$. What matters is the out 2 -to-out 1 direction of the current flow and the gain. A gain of 0.1 is high enough to make the voltage change on out ${ }_{1}$ 's $\mathrm{PRED}_{i}$ closely track the upgoing

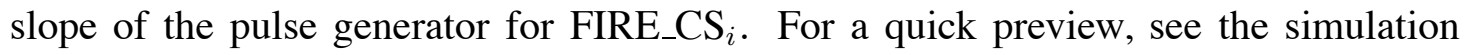
results in Figure 4.19 and Figure 4.20 on pages $79-80$.

Note that both Figure 4.10 and Figure 4.11 have an Ampere meter to measure $\mathrm{I}_{\text {out1-to-out2 }}$. We use the Ampere meter to validate that the VCCS operation does not interfere with the actual simulation measurement. We will come back to this again in Sections 4.4.3.4-4.4.3.5.

\subsubsection{Summary of Circuit Enhancements}

The new list of sub-circuits and simple relative timing paths that we get by solving the issues on page 55 for the old sub-circuits in Figure 4.7(a)-(c) on page 51 is as follows:

1. Figure 4.8, replacing Figure 4.7(a), with LUTblue:PRED $i-$ FIRE $_{i}-$

2. Figure 4.9, replacing Figure 4.7(a), with LUTblue:SUCC $i+\mathrm{FIRE}_{i}-$

3. Figure 4.7(b) with LUTblue:FIRE $i-$ Dout $_{i}+$

4. Figure 4.10, replacing Figure 4.7(b), with LUTred/blue:FIRE $+\mathrm{SUCC}_{i}+$

5. Figure 4.11, replacing Figure 4.7(c), with LUTblue/green:FIRE $+\mathrm{PRED}_{i}-$

We will pass this list on to Section 4.4.3 where we generate the look up tables. 


\subsubsection{Back to PART-A to Generate Look Up Tables}

In this section, we will take the sub-circuits and simple relative timing paths listed in the previous Section 4.4.2.3 and build the final simulation environments to generate look up tables with timing information on input-to-output delay and output slope, for a range of input slopes and output load conditions. To build the look up table simulation environments, we follow the procedure explained in PART-A, Chapter 3, which can be summarized as follows:

- To create realistic input slopes, we use a so-called driver inverter at the input of the sub-circuit. We sweep the input slope by sweeping the size of the driver inverter. To simulate a range of driver sizes, we use a pulse generator with a pulse width and period that are long enough for the path transition to complete and stabilize. We add a so-called source inverter between pulse generator and driver inverter to provide realistic input slopes to the driver inverter. The source inverter is a step-up of 3 smaller than the driver inverter.

- To create realistic output loads, we use a so-called load inverter. We sweep the capacitive output load by sweeping the size of the load inverter. We add a socalled Miller inverter at the output of the load inverter for a realistic Miller effect. The Miller inverter is a step-up of 3 larger than the load inverter.

In our sweeps, we maintain a relationship between driving and driven inverters where the size of the driving inverter is $0.20(1 / 5$ th $)$ to 2 times the size of the gate that it drives. This makes sense from a design perspective. In summary: we sweep the driver inverter from 0.20 to 2 times the size of the sub-circuit gate that it drives, and we sweep the load inverter from $0.5(1 / 2$ nd) to 5 times the size of the sub-circuit gate that drives it. We use wids, wid1, wid2, widM to denote the size of respectively the source inverter, 
the driver inverter, the load inverter, and the Miller inverter. We maintain a step-up of 3 size relationship between the source inverter and the driver inverter. This means that we sweep both inverters simultaneously, using the current wid1 value for the driver inverter and wids $=($ wid1 $) / 3$ for the source inverter. Similarly, we use widM=3 $\times$ wid 2 .

In the following five Sections 4.4.3.1-4.4.3.5, we prepare the final look up table simulation sweep for each of the five sub-circuit-with-simple-paths listed in Section 4.4.2.3.

\subsubsection{Setup for Figure 4.8 and LUTblue:PRED ${ }_{i}-$ FIRE $_{i}-$}

Figure 4.12 shows the simulation setup for the sub-circuit in Figure 4.8 and simple relative timing path LUTblue:PRED $i-\mathrm{FIRE}_{i}-$. The sub-circuit and path are represented by a black box. Only the information that we need from the black box is visible:

- Input $\mathrm{PRED}_{i}$ and output $\mathrm{FIRE}_{i}$, as these connect to the simulation environment.

- Size of initial gate in the path, i.e. 5 (for F), and of final gate, i.e. 80 (for C), as these determine the sweep sizes for the source, driver, load, Miller inverters.

- Path name, LUTblue:PRED $i-\mathrm{FIRE}_{i}-$, because this specifies the transition type (input/output rising/falling, fast/slow).

The sweep values for source, driver, load, Miller inverters are calculated as follows:

- Size wid1 of the driver inverter has a sweep range from $0.2 \times \mathrm{F}$ to $2 \times \mathrm{F}$, where $\mathrm{F}=5$ — the size of the first black box gate in the path. We divided this 1-10 range into five more-or-less linear integer sizes, giving wid1=1, 3, 6, 8, 10 .

- Size wids for the source is a step down of 3 the size of the driver inverter, wid1. For now, we exclude wid1=1 from our sweep, because it would yield a size for wids below the minimum inverter size of 1 . In Section 4.5 , we will give a general solution for obtaining a step down of 3 with buildable inverter sizes, i.e. inverter sizes $\geq 1$. For now, the sweep values for wids are: $1,2,3,3$. 


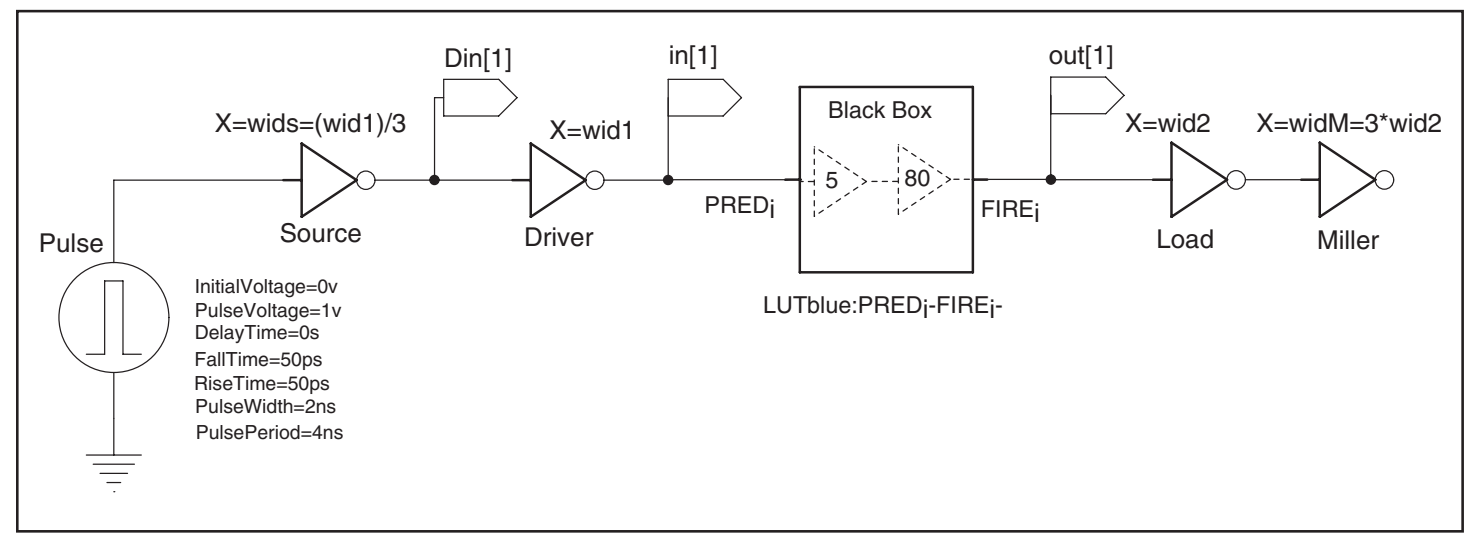

Figure 4.12 Final simulation setup for the sub-circuit in Figure 4.8 and simple path LUTblue:PRED $i-$ FIRE $_{i}-$. The black box represents the sub-circuit. We use a pulse generator followed by a source and a driver inverter at the PRED input of the black box, and we use a load and a Miller inverter at the output. The pulse generator goes from $0 \mathrm{~V}$ (VSS) to $1 \mathrm{~V}$ (VDD) and creates a high pulse of $1 \mathrm{~V}$ with a pulse width of $2 \mathrm{~ns}$ and a period of $4 \mathrm{~ns}$. We sweep the driver and load sizes: wid1 and wid2. We measure propagation and transition delays by observing black box input and output in[1], out[1].

- Size wid2 for the load inverter is swept from $0.5 \times \mathrm{C}$ to $5 \times \mathrm{C}$, where $\mathrm{C}=80-$ the size of the last black box gate in the path. We linearized the 40-400 range into five more-or-less linear integer sizes, giving wid2=40, 130, 220, 310, 400 .

- The size of the Miller inverter varies with the load size: widM=3×wid2.

We use the schematics entry tool Electric to generate (1) a SPICE netlist for Figure 4.12 and (2) a basic SPICE simulation file which contains the settings for the 90nm CMOS process used for this schematics. We modify the SPICE netlist to add the sweep variables for wid1 and wid2, as explained in PART-A, Chapter 3. We adapt the SPICE simulation file by adding the sweep, analysis and measurement statements needed to generate the three look up tables:

- $($ wid1 $\times$ wid2) $\rightarrow$ slope $($ in[1] $)$

- $($ wid1 $\times$ wid2) $\rightarrow$ delay (in[1] to out[1])

- $($ wid $1 \times$ wid2) $\rightarrow$ slope $($ out[1] $)$ 
SPICE netlist, simulation, and result files are shown in Appendix C. We have used typical Process, Voltage and Temperature (PVT) library settings, here. In general, though, we would advise using fast settings to characterize blue paths, because we are interested in minimum delays for blue paths.

\subsubsection{Setup for Figure 4.9 and LUTblue:SUCC + FIRE $_{i}-$}

Figure 4.13 shows the simulation setup for the sub-circuit in Figure 4.9 and simple relative timing path LUTblue:SUCC ${ }_{i}+\mathrm{FIRE}_{i}-$. The sub-circuit and path are represented by a black box. Only the information that we need from the black box is visible:

- Input $\mathrm{SUCC}_{i}$ and output FIRE ${ }_{i}$, as these connect to the simulation environment.

- Size of initial gate ${ }^{4.3}$ in the path, i.e. 10 (for A), and of final gate, i.e. 80 (for C), as these determine the sweep sizes for the source, driver, load, Miller inverters.

- Path name, LUTblue:SUCC $i+\mathrm{FIRE}_{i}-$, because this specifies the transition type (input/output rising/falling, fast/slow).

The sweep values for source, driver, load, Miller inverters are calculated in the same way as we calculated the sweep values for the previous Figure 4.12 in Section 4.4.3.1:

- Driver sweep values for wid1 are: $2(=0.20 \times 10), 6,10,16,20(=2 \times 10)$.

- Source sweep values for wids are a step down of 3 from the size of the driver inverter, wid1. As in Section 4.4.3.1, we exclude wid1=2 from our sweep, because it yields a size for wids below the minimum inverter size of 1. In Section 4.5, we show how this can be solved. For now, the sweep values for wids are: 1, 2, 3, 3 .

- load sweep values for wid2 are: $40(=0.5 \times 80), 130,220,310,400(=5 \times 80)$.

- The size of the Miller inverter varies with the load size: widM=3 $\times$ wid2.

\footnotetext{
${ }^{4.3}$ We simplified this calculation. The real size seen by the driver is $10 \times($ LogicalEffort(A) $\stackrel{\text { def }}{=} 5 / 3) \sim 17$.
} 


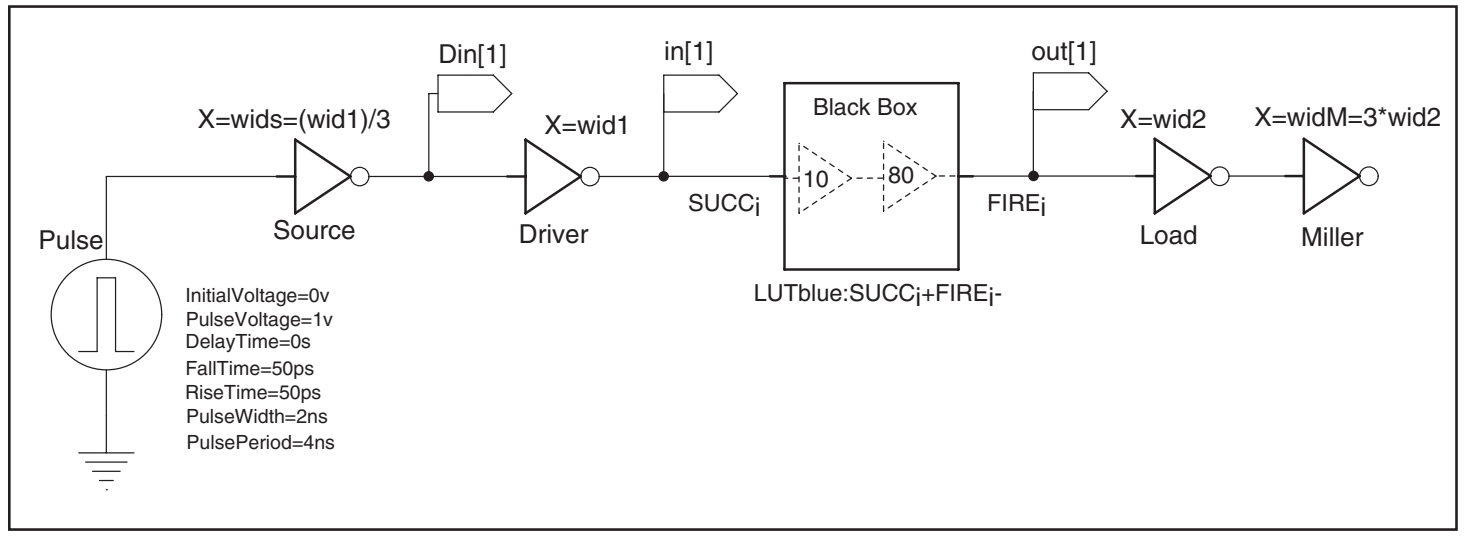

Figure 4.13 Final simulation setup for the sub-circuit in Figure 4.9 and simple path LUTblue:SUCC $i+\mathrm{FIRE}_{i}-$. The black box represents the sub-circuit. We use a pulse generator followed by a source and a driver inverter at the $\mathrm{SUCC}_{i}$ input of the black box, and we use a load and a Miller inverter at the output. The pulse generator goes from $0 \mathrm{~V}$ (VSS) to $1 \mathrm{~V}$ (VDD) and creates a high pulse of $1 \mathrm{~V}$ with a pulse width of $2 \mathrm{~ns}$ and a period of $4 \mathrm{~ns}$. We sweep the driver and load sizes: wid1 and wid2. We measure propagation and transition delays by observing black box input and output in[1], out[1].

The SPICE files for Figure 4.13 are generated like those for the previous Figure 4.12 in Section 4.4.3.1. The final SPICE netlist and simulation setup and results files follow in Appendix D. We have used typical Process, Voltage and Temperature (PVT) library settings, here, though we advise using fast settings to characterize blue-colored paths.

\subsubsection{Setup for Figure 4.7(b) and LUTblue:FIRE ${ }_{i}-$ Dout $_{i}+$}

Figure 4.14 shows the simulation setup for the sub-circuit in Figure 4.7(b) and simple relative timing path LUTblue:FIRE ${ }_{i}-$ Dout $_{i}+$. The sub-circuit and path are represented by a black box. Only the information that we need from the black box is visible:

- Input FIRE $\mathrm{F}_{i}$ and output Dout ${ }_{i}$, as these connect to the simulation environment.

- Size of initial gate in the path, i.e. 4 (for D), because this determines the sweep sizes for the source and driver inverters.

- There is no size indication for the final gate because Dout ${ }_{i}$ is an internal signal. This also explains why there are no load and Miller inverter in Figure 4.14. 


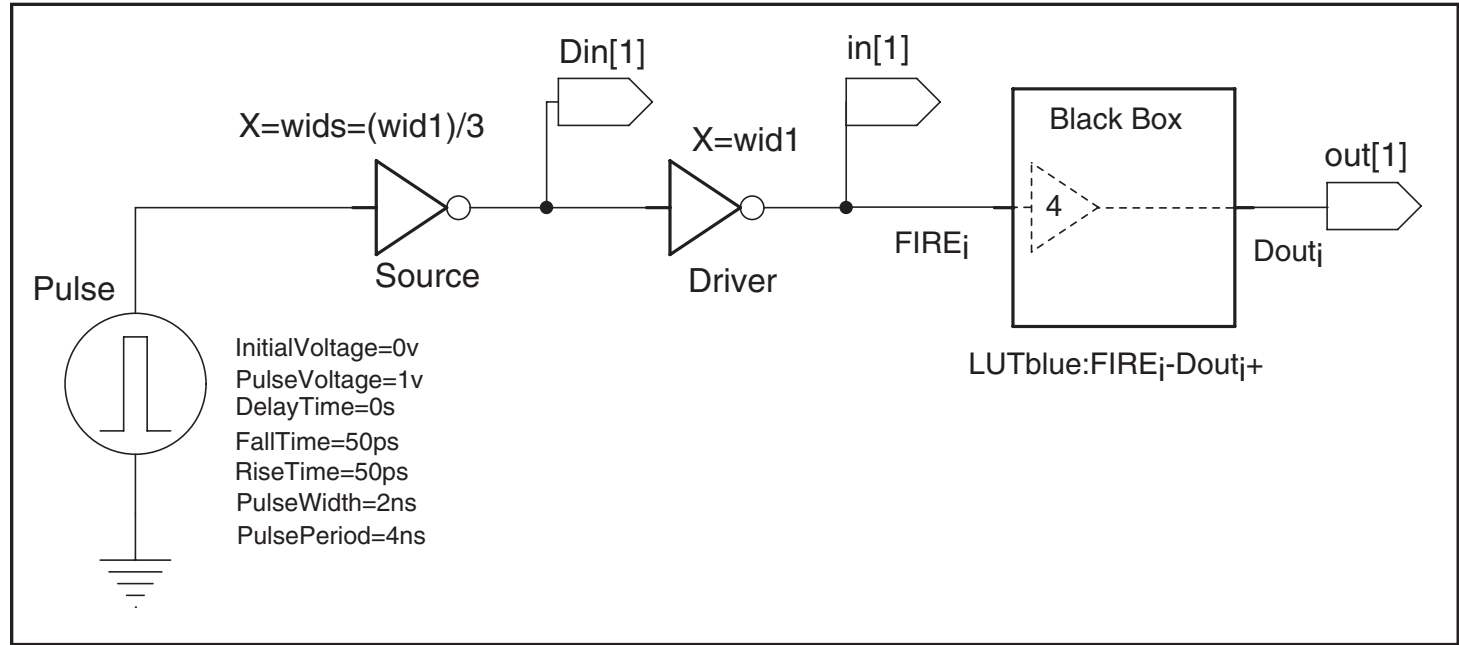

Figure 4.14 Final simulation setup for the sub-circuit in Figure 4.7(b) and simple path LUTblue:FIRE $i-$ Dout $_{i}+$. The black box represents the sub-circuit. We use a pulse generator followed by a source and a driver inverter at the FIRE $i$ input of the black box. The pulse generator goes from $0 \mathrm{~V}$ (VSS) to $1 \mathrm{~V}$ (VDD) and creates a high pulse of $1 \mathrm{~V}$ with a pulse width of $2 \mathrm{~ns}$ and a period of $4 \mathrm{~ns}$. We sweep the driver wid1. We measure propagation and transition delays by observing black box input and output in[1], out[1].

- Path name, LUTblue:FIRE ${ }_{i}-$ Dout $_{i}+$, because this specifies the transition type (input/output rising/falling, fast/slow).

The sweep values for the source and driver inverters are calculated in the same way as we calculated the sweep values for Figure 4.12 in Section 4.4.3.1:

- Driver sweep values for wid1 are: 1 (=0.20×4 rounded up), 3, 4, 6, $8(=2 \times 4)$.

- Source sweep values for wids are a step down of 3 from the size of the driver inverter, wid1. As in Section 4.4.3.1, we exclude wid1=1 from our sweep, because it yields a size for wids below the minimum inverter size of 1. In Section 4.5, we give a general solution to obtain a step down of 3 with buildable inverter sizes $\geq 1$. For now, the sweep values for wids are: 1, 1,2, 3 . 
The SPICE files for Figure 4.14 are generated like those for Figure 4.12 in Section 4.4.3.1 and follow in Appendix E. We have used typical Process, Voltage and Temperature (PVT) library settings. In general, we would advise using fast settings to characterize blue paths, because we are interested in minimum delays for blue paths.

\subsubsection{Setup for Figure 4.10, LUTred/blue:FIRE $_{i}+\mathrm{SUCC}_{i}+$}

Figure 4.15 shows the simulation setup for the sub-circuit in Figure 4.10 and simple relative timing paths LUTred:FIRE $i+\mathrm{SUCC}_{i}+$ and LUTblue:FIRE $i+\mathrm{SUCC}_{i}+$. Subcircuit and paths are represented by a black box. Only the information that we need from the black box is visible:

- Input FIRE $\mathrm{F}_{i}$ and output $\mathrm{SUCC}_{i}$, as these connect to the simulation environment.

- Size of initial gate in the paths, i.e. 4 (for D), and of final gate, i.e. 20 (for E), as these determine the sweep sizes for the source, driver, load, Miller inverters.

- Path names LUTred:FIRE $i+$ SUCC $_{i}+$ and LUTblue:FIRE $i+\mathrm{SUCC}_{i}+$, because these specify the transition type (input/output rising/falling, fast/slow).

- Pulse generator for VCCS input FIRE_CS $i$, because it must be aligned to FIRE . $_{\text {. }}$

- Ampere meter monitoring the VCCS current flow for $\mathrm{I}_{\text {out1-to-out2 }}$ to validate that the VCCS operation does not interfere with the actual simulation measurement.

The sweep values for source, driver, load, Miller inverters are calculated in the same way as we calculated the sweep values for Figure 4.12 in Section 4.4.3.1:

- Driver sweep values for wid1 are: 1 (=0.20×4 rounded up), 3, 4, 6, $8(=2 \times 4)$.

- Source sweep values for wids are a step down of 3 from the size of the driver inverter, wid1. As in Section 4.4.3.1, we exclude wid1=1 from our sweep, because it yields a size for wids below the minimum inverter size of 1. In Section 4.5, we give a general solution to obtain a step down of 3 with buildable inverter sizes $\geq 1$. For now, the sweep values for wids are: $1,1,2,3$. 


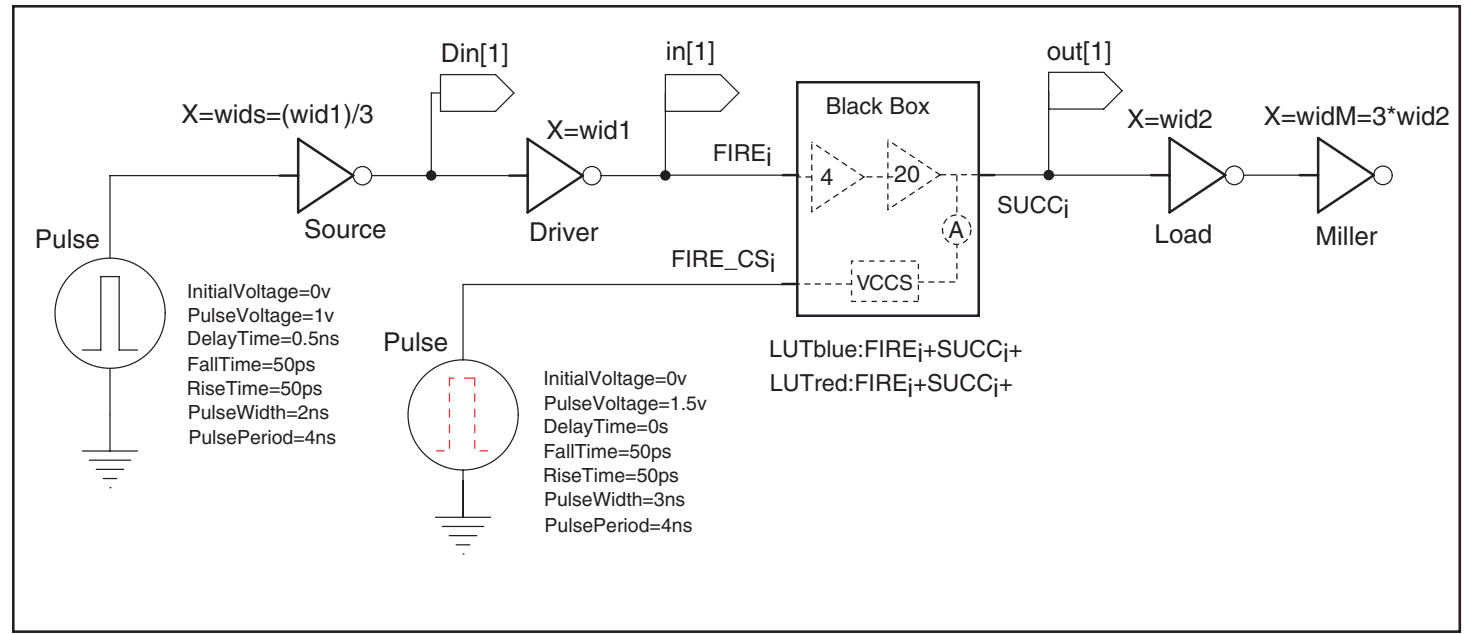

Figure 4.15 Final simulation setup of the sub-circuit in Figure 4.10 and simple paths LUTred:FIRE $i+\mathrm{SUCC}_{i}+$ and LUTblue:FIRE $i+\mathrm{SUCC}_{i}+$. The black box represents the sub-circuit. We use a pulse generator followed by a source and a driver inverter at the $\mathrm{FIRE}_{i}$ input of the black box. The pulse generator goes from 0V (VSS) to 1V (VDD) and creates a high pulse of $1 \mathrm{~V}$ with a pulse width of $2 \mathrm{~ns}$ and a period of $4 \mathrm{~ns}$. The pulse generator for black box signal FIRE_CS ${ }_{i}$ goes from 0V (VSS) to $1.5 \mathrm{~V}$. It has the same period but a wider pulse width of $3 \mathrm{~ns}$. As a result, the FIRE $\mathrm{F}_{i}$ pulse falls completely within the FIRE_CS $i$ pulse window. This guarantees that the VCCS device in the black box works as intended. We can show that this is the case by observing the Ampere meter and validating that it stays at $0 \mathrm{~A}$ for the full duration of the FIRE $i$ pulse. At the output end for $\mathrm{SUCC}_{i}$, we use a load inverter and Miller inverter. We sweep driver and load sizes wid 1 and wid 2 and measure propagation and transition delays by observing black box input and output in[1] and out[1].

- Load sweep values for wid2 are: $10(=0.5 \times 20), 33,56,78,100(=5 \times 20)$.

- The size of the Miller inverter varies with the load size: widM=3 $\times$ wid2.

The SPICE files for Figure 4.15 follow in Appendix F. They are generated similar to the SPICE files for Figure 4.12 in Section 4.4.3.1. We simulated both paths with typical Process, Voltage and Temperature (PVT) settings of the library. But, in general, we would advice simulating the red path with slow PVT settings and the blue path with fast PVT settings, because the relative timing assumptions need maximum delay estimates for red paths and minimum delay estimates for blue paths. 
Figure 4.16 and Figure 4.17 show the two extreme, worst-case operating conditions for the VCCS device in the black box. They validate that the VCCS operates as intended:

- The waveforms in Figure 4.16 were generated using the smallest simulated driver size wid1 $=3$, and the largest load size wid2 $=100$. As a result, the pulse on input signal FIRE $_{i}$ is maximally delayed relative to the FIRE_CS $i$ pulse and so is the measured transition from the rising input signal on $\mathrm{FIRE}_{i}$ to the rising output signal on $\mathrm{SUCC}_{i}$. Note that the transition on $\mathrm{SUCC}_{i}$ falls clearly inside the $\mathrm{FIRE}_{i}$ pulse. Note too that the FIRE $_{i}$ pulse clearly fits inside the FIRE_CS $i$ pulse, with slack to spare. Finally notice that $\mathrm{SUCC}_{i}$ is re-initialized to a clean $0 \mathrm{~V}$ VSS level sufficiently long before the start of the next $\mathrm{FIRE}_{i}$ pulse, and that the Ampere meter measurements indicate that there is no $\mathrm{I}_{\text {out1-to-out2 }}$ current flowing during the FIRE $_{i}$ pulse with the critical timing measurements for LUTred:FIRE ${ }_{i}+\mathrm{SUCC}_{i}+$ respectively LUTblue:FIRE $+\mathrm{SUCC}_{i}+$. In summary: the VCCS device operates correctly and does not interfere with the actual simulation measurement.

- The waveforms in Figure 4.17 were generated using the largest simulated driver size wid1=8, and the largest load size wid2=100. As a result, the pulse on $\mathrm{FIRE}_{i}$ is minimally delayed relative to the pulse on FIRE_CS ${ }_{i}$, creating a minimal time interval for FIRE_CS ${ }_{i}$ to re-initialize SUCC $_{i}$ before the next FIRE $_{i}$ pulse and the next FIRE_CS ${ }_{i}$ pulse. As before in Figure 4.16: the actual measurement for LUTred:FIRE + SUCC + respectively LUTblue:FIRE ${ }_{i}+\mathrm{SUCC}_{i}+$ falls clearly inside the FIRE $_{i}$ pulse, the FIRE $_{i}$ pulse falls clearly inside the FIRE_CS $i$ pulse, $\mathrm{SUCC}_{i}$ is re-initialized cleanly before the next pulse on FIRE_CS ${ }_{i}$ and the next pulse on $\mathrm{FIRE}_{i}$ start, and there is no $\mathrm{I}_{\text {out1-to-out2 }}$ current flowing during the FIRE $_{i}$ pulse. So, also in this scenario, the VCCS device operates correctly and does not interfere with the actual simulation measurement. 


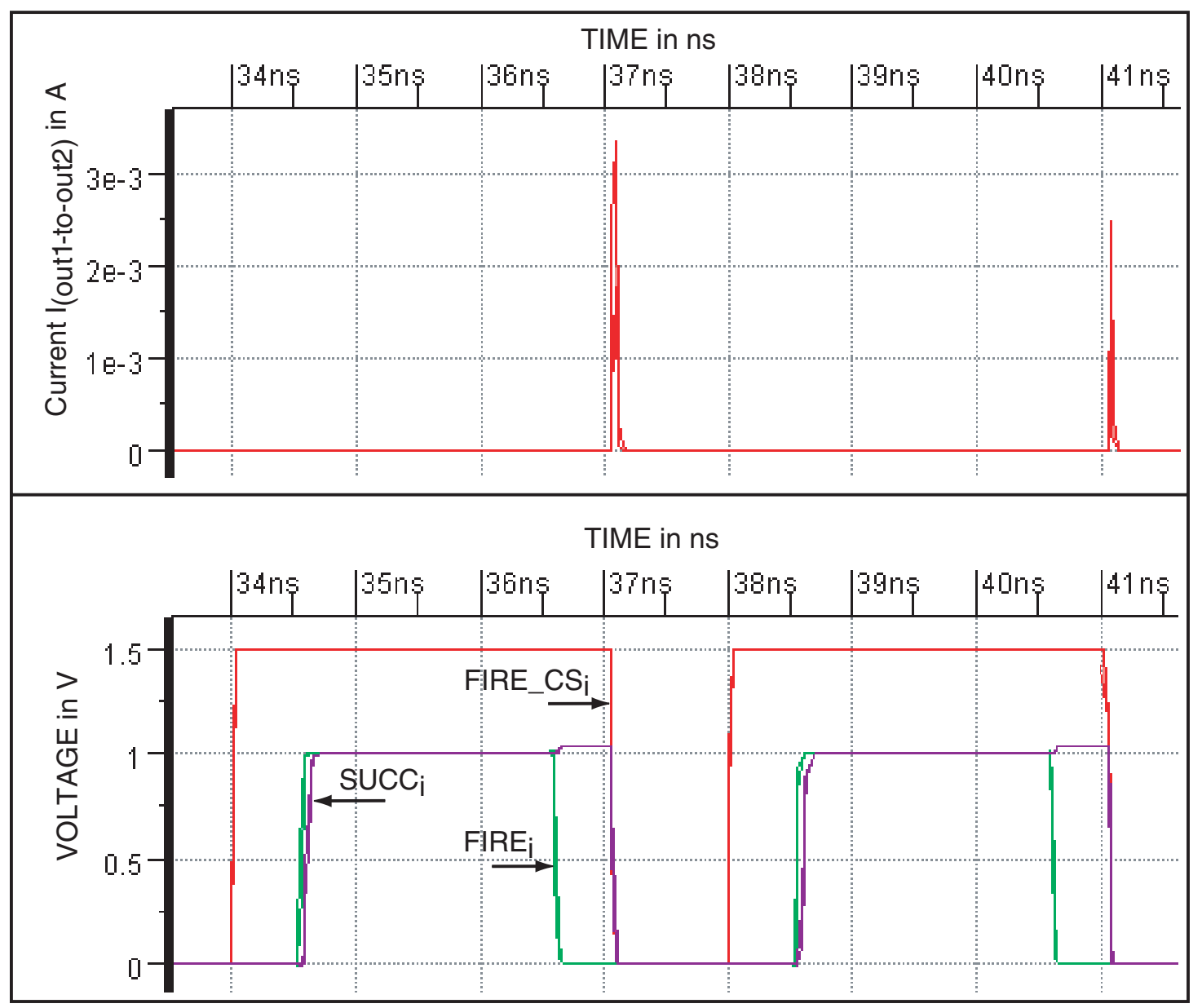

Figure 4.16 This is the first of two validation runs showing that the VCCS used in the black box of Figure 4.15 operates correctly and does not interfere with the actual simulation measurements for the relative timing paths LUTred:FIRE $i+\mathrm{SUCC}_{i}+$ and LUTblue:FIRE $i+\mathrm{SUCC}_{i}+$. We generated this information from SPICE simulations of Figure 4.15, using the smallest driver (wid1=3) and the largest load (wid2=100). The bottom simulation window shows two (red) FIRE_CS $i$ pulses and two (green) FIRE pulses, and their relation in terms of voltage levels and time to each other and to (blue/purple) output signal $\mathrm{SUCC}_{i}$. Both pulse generators produce a high pulse. The top simulation window shows the VCCS current for $\mathrm{I}_{\text {out1-to-out2 }}$ as monitored by the Ampere meter. The properties to validate are: (1) correct alignment of pulse generators, i.e. the (green) FIRE $_{i}$ pulse window lies within the (red) FIRE_CS ${ }_{i}$ pulse window, and (2) correct measurement window, i.e. the rising transition for the (blue/purple) $\mathrm{SUCC}_{i}$ signal lies within the (green) $\mathrm{FIRE}_{i}$-high pulse window, and (3) mutual exclusive VCCS and measurement operations, i.e. $\mathrm{I}_{\text {out } 1-\text { to }- \text { out } 2}=0 \mathrm{~A}$ when $\mathrm{FIRE}_{i}$ is high. Observe that all three properties hold. 


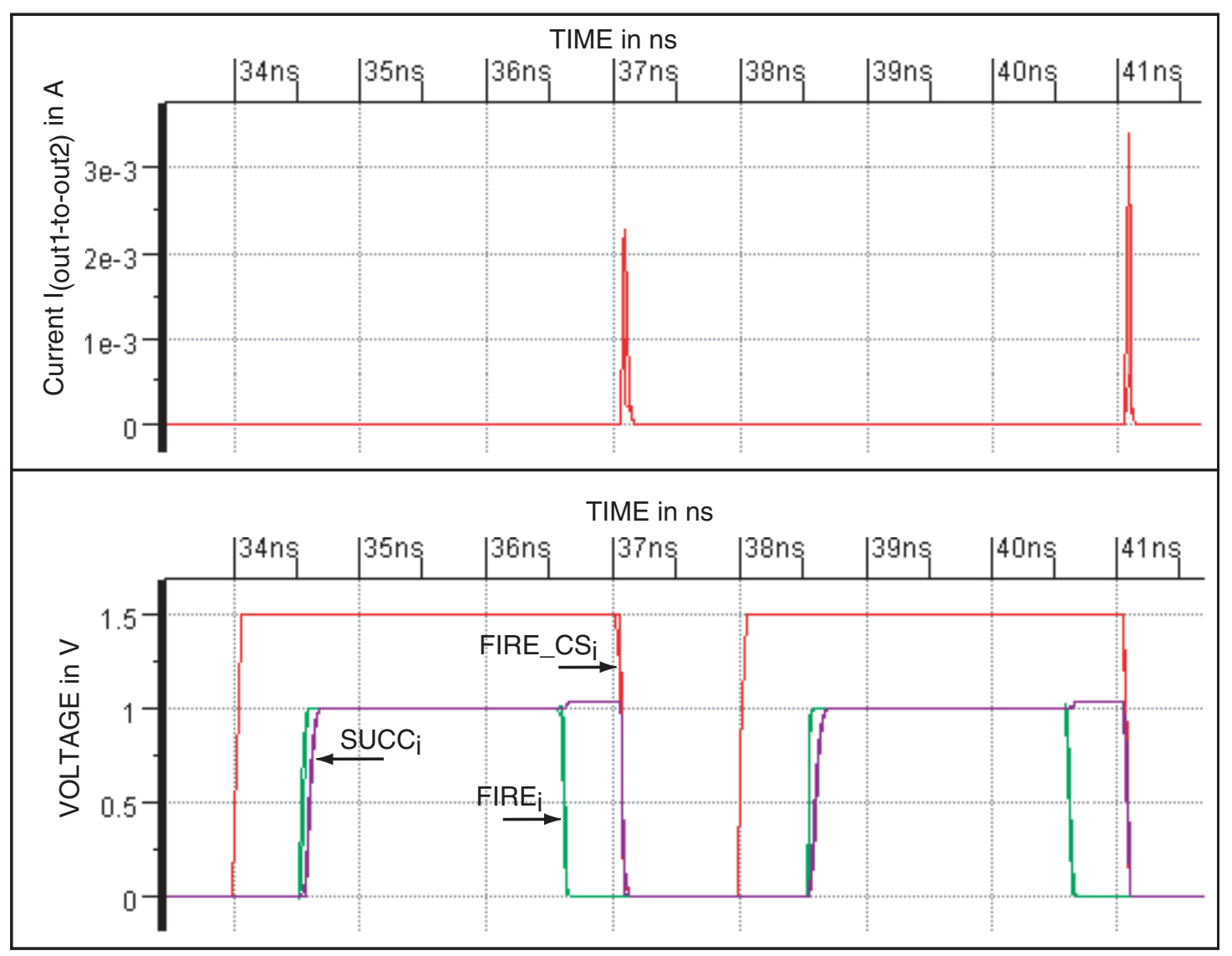

Figure 4.17 Second of the two validation runs showing that the VCCS used in the black box of Figure 4.15 operates correctly and does not interfere with the actual simulation measurements for the relative timing paths LUTred:FIRE $i+\mathrm{SUCC}_{i}+$ and LUTblue:FIRE $i+$ SUCC $_{i}+$. We generated this information from SPICE simulations of Figure 4.15, using the largest driver (wid1=8) and the largest load (wid2=100). The simulation windows follow the same organization as the previous validation run in Figure 4.16. As before, the properties to validate are: (1) correct alignment of pulse generators, i.e. the (green) FIRE $_{i}$ pulse lies within the (red) FIRE_CS $i$ pulse window, (2) correct measurement window, i.e. the rising transition for the (blue/purple) $\mathrm{SUCC}_{i}$ signal lies within the (green) FIRE $_{i}$-high window, and (3) VCCS and measurement operations are mutually exclusive, i.e. $\mathrm{I}_{\text {out } 1-\text { to }- \text { out } 2}=0 \mathrm{~A}$ when $\mathrm{FIRE}_{i}$ is high. As before, all three properties hold. 


\subsubsection{Setup for Figure 4.11, LUTblue/green:FIRE $i+$ PRED $_{i}-$}

Figure 4.18 shows the simulation setup for the sub-circuit in Figure 4.11 and simple relative timing paths LUTblue:FIRE $i+\mathrm{PRED}_{i}-$ and LUTgreen:FIRE ${ }_{i}+\mathrm{PRED}_{i}-$. The sub-circuit and the paths are represented by a black box. Only the information that we need from the black box is visible. Similar to the information listed on page 71 for the black box in Figure 4.15, we need:

- Input FIRE $i$ and output $\operatorname{PRED}_{i}$, as these connect to the simulation environment.

- Size of initial gate ${ }^{4.4}$ in the path, i.e. 20 (for X), and of final gate, i.e. 20 (again X), as these determine the sweep sizes for the source, driver, load, Miller inverters.

- Path names LUTblue:FIRE ${ }_{i}+\mathrm{PRED}_{i}-$ and LUTgreen:FIRE ${ }_{i}+\mathrm{PRED}_{i}-$, because these specify the transition type (input/output rising/falling, fast/slow).

- Pulse generator for VCCS input FIRE_CS ${ }_{i}$, because it must be aligned to $\mathrm{FIRE}_{i}$.

- Ampere meter monitoring the VCCS current flow for $\mathrm{I}_{\text {out1-to-out2 }}$ to validate that the VCCS operation does not interfere with the actual simulation measurement.

The sweep values for the source, driver, load, and Miller inverters are calculated in the same way as we calculated the sweep values for Figure 4.12 in Section 4.4.3.1:

- Driver sweep values for wid1 are: $4(=0.20 \times 20), 13,22,31,40(=2 \times 20)$.

- Source sweep values for wids are a step down of 3 from the size of the driver inverter, wid1. We took the following values for wids: 1, 4, 7, 10,13.

- Load sweep values for wid2 are: $10(=0.5 \times 20), 33,56,78,100(=5 \times 20)$.

- The size of the Miller inverter varies with the load size: widM=3×wid 2 .

\footnotetext{
${ }^{4.4}$ We simplified this calculation. The real size seen by the driver is $20 \times($ LogicalEffort $(X) \stackrel{\text { def }}{=} 1 / 3) \sim 7$.
} 


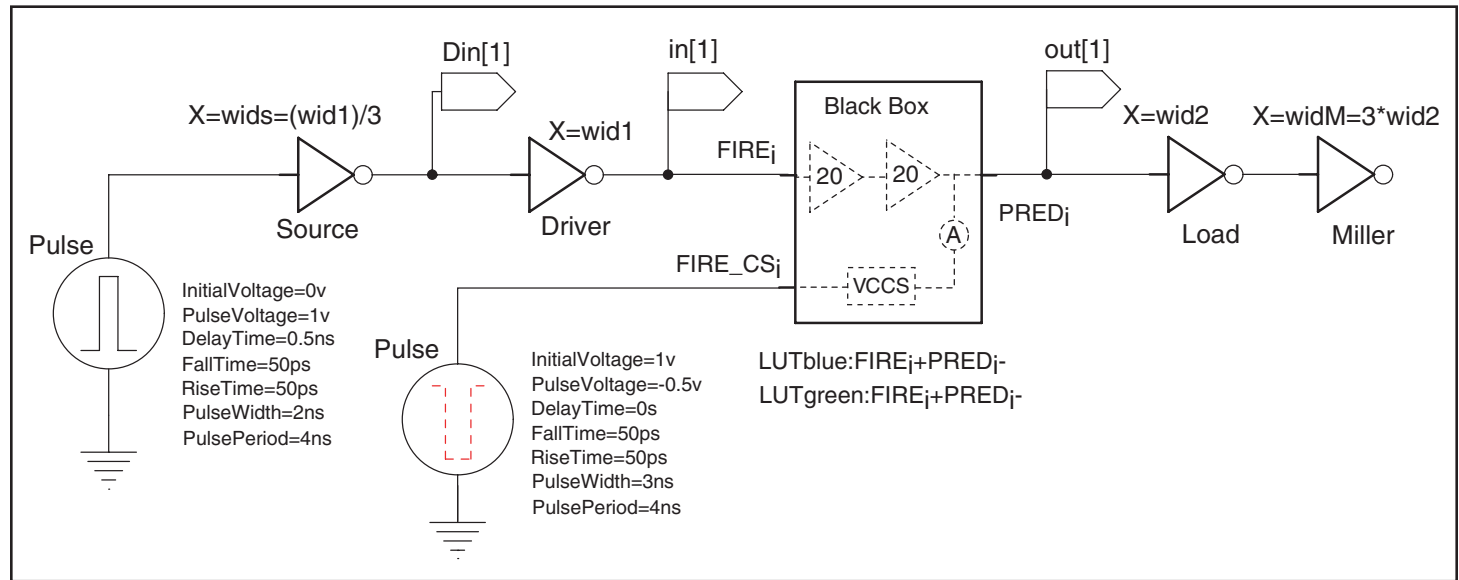

Figure 4.18 Final simulation setup of the sub-circuit in Figure 4.11 and simple paths LUTblue:FIRE $i+$ PRED $_{i}-$ and LUTgreen:FIRE $i+\mathrm{PRED}_{i}-$. The black box represents the sub-circuit. We use a pulse generator followed by a source and a driver inverter at the FIRE $_{i}$ input of the black box. The pulse generator goes from 0V (VSS) to 1V (VDD) and creates a high pulse of $1 \mathrm{~V}$ with a pulse width of $2 \mathrm{~ns}$ and a period of $4 \mathrm{~ns}$. The pulse generator for black box signal FIRE_CS $i$ generates a low pulse and goes from $1 \mathrm{~V}$ (VDD) to $-0.5 \mathrm{~V}$. It has the same period but a wider pulse width of $3 \mathrm{~ns}$. As a result, the FIRE $_{i}$ pulse falls completely within the FIRE_CS $i$ pulse window. This guarantees that the VCCS device in the black box works as intended. We can show that this is the case by observing the Ampere meter and validating that it stays at $0 \mathrm{~A}$ for the full duration of the FIRE $i$ pulse. At the output end for $\mathrm{PRED}_{i}$, we use a load inverter and a Miller inverter. We sweep the driver and load sizes wid1 and wid2 and measure propagation and transition delays by observing the black box input and output in[1] and out[1].

The SPICE files for Figure 4.18 are generated in a way similar to those for Figure 4.12 in Section 4.4.3.1, and follow in Appendix G. We show simulation results under typical Process, Voltage, and Temperature (PVT) setting of the library. However, as a general rule, we would advice simulating the green path with slow PVT settings and the blue path with fast settings because the relative timing assumptions need maximum delay estimates for green and minimum delay estimates for blue paths. 
Figures 4.19-4.20 show the two extreme, worst-case operating conditions for the VCCS in the black box of Figure 4.18. They validate that the VCCS operates as intended:

- The waveforms in Figure 4.19 were generated using the smallest simulated driver size wid1 $=4$, and the largest load size wid2 $=100$. As a result, the pulse on input signal $\mathrm{FIRE}_{i}$ is maximally delayed relative to the FIRE_CS $i$ pulse and so is the measured transition from the rising input signal on $\mathrm{FIRE}_{i}$ to the falling output signal on $\mathrm{PRED}_{i}$. Note that the transition on $\mathrm{PRED}_{i}$ falls clearly inside the $\mathrm{FIRE}_{i}$ pulse. Note too that the $\mathrm{FIRE}_{i}$ pulse clearly fits inside the FIRE_CS ${ }_{i}$ pulse, with slack to spare. Finally notice that $\operatorname{PRED}_{i}$ is re-initialized to a clean $1 \mathrm{~V}$ VDD level sufficiently before the start of the next $\mathrm{FIRE}_{i}$ pulse, and that the Ampere meter measurements indicate that there is no $\mathrm{I}_{\text {out1-to-out2 }}$ current flowing during the FIRE $_{i}$ pulse with the critical timing measurements for LUTblue:FIRE ${ }_{i}+\mathrm{PRED}_{i}-$ respectively LUTgreen:FIRE $+\mathrm{PRED}_{i}-$. In summary: the VCCS device operates correctly and does not interfere with the actual simulation measurement.

- The waveforms in Figure 4.20 were generated using the largest simulated driver size wid1 $=40$, and the largest load size wid2=100. As a result, the pulse on FIRE is minimally delayed relative to the pulse on FIRE_CS ${ }_{i}$, creating a minimal time interval for FIRE_CS ${ }_{i}$ to re-initialize PRED $_{i}$ before the next FIRE $i$ pulse and the next FIRE_CS ${ }_{i}$ pulse. As before in Figure 4.19: the actual measurement for LUTblue:FIRE $i+\mathrm{PRED}_{i}-$ respectively LUTgreen:FIRE $i+\mathrm{PRED}_{i}-$ falls clearly inside the $\mathrm{FIRE}_{i}$ pulse, the FIRE $i$ pulse falls clearly inside the FIRE_CS $i$ pulse, $\mathrm{PRED}_{i}$ is re-initialized cleanly before the next pulse on FIRE_CS ${ }_{i}$ and the next pulse on $\mathrm{FIRE}_{i}$ start, and there is no $\mathrm{I}_{\text {out1-to-out2 }}$ current flowing during the $\mathrm{FIRE}_{i}$ pulse. So, also in this scenario, the VCCS device operates correctly and does not interfere with the actual simulation measurement. 


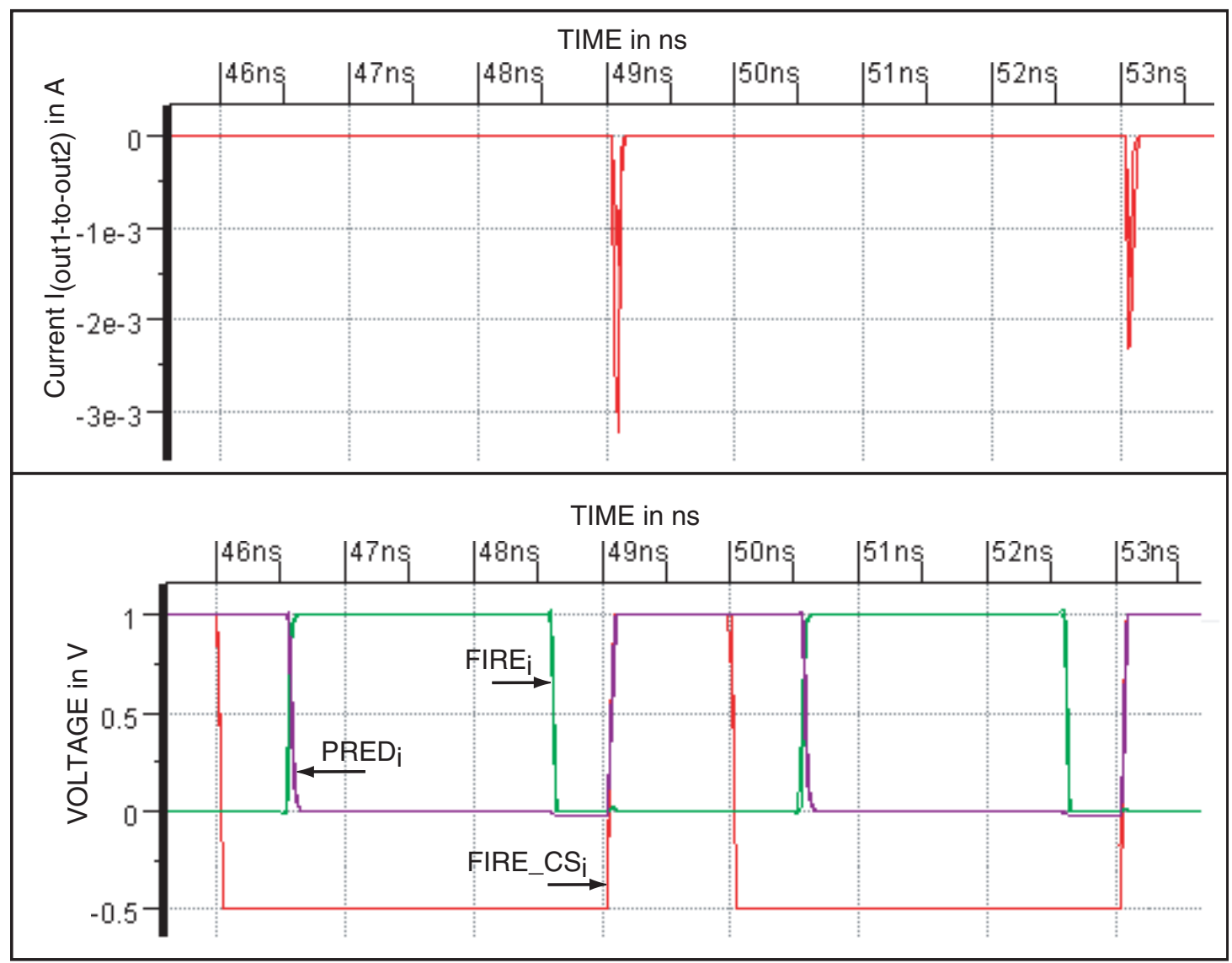

Figure 4.19 First of the two validation runs showing that the VCCS used in the black box of Figure 4.18 operates correctly and does not interfere with the actual simulation measurements for the relative timing paths LUTblue:FIRE $i+\mathrm{PRED}_{i}-$ and LUTgreen:FIRE $i+\mathrm{PRED}_{i}-$. We generated this information from SPICE simulations of Figure 4.18, using the smallest driver (wid1=4) and the largest load (wid2=100). The bottom simulation window shows two (red) FIRE_CS $i$ pulses and two (green) FIRE $_{i}$ pulses, and their relation in terms of voltage levels and time to each other and to (blue/purple) output signal $\operatorname{PRED}_{i}$. The pulse generators produce a low pulse on

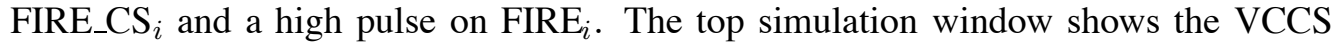
current for $\mathrm{I}_{\text {out1-to-out2 }}$ as monitored by the Ampere meter in Figure 4.18. The properties to validate are: (1) correct alignment of pulse generators, i.e. the FIRE pulse lies within the FIRE_CS $i$ pulse window, (2) correct measurement window, i.e. the rising transition on $\operatorname{PRED}_{i}$ lies within the FIRE $i$ window, and (3) the VCCS and measurement operations are mutually exclusive, i.e. $\mathrm{I}_{\text {out } 1-\text { to }- \text { out } 2}=0 \mathrm{~A}$ when $\mathrm{FIRE}_{i}$ is high. Observe that all three properties hold. 


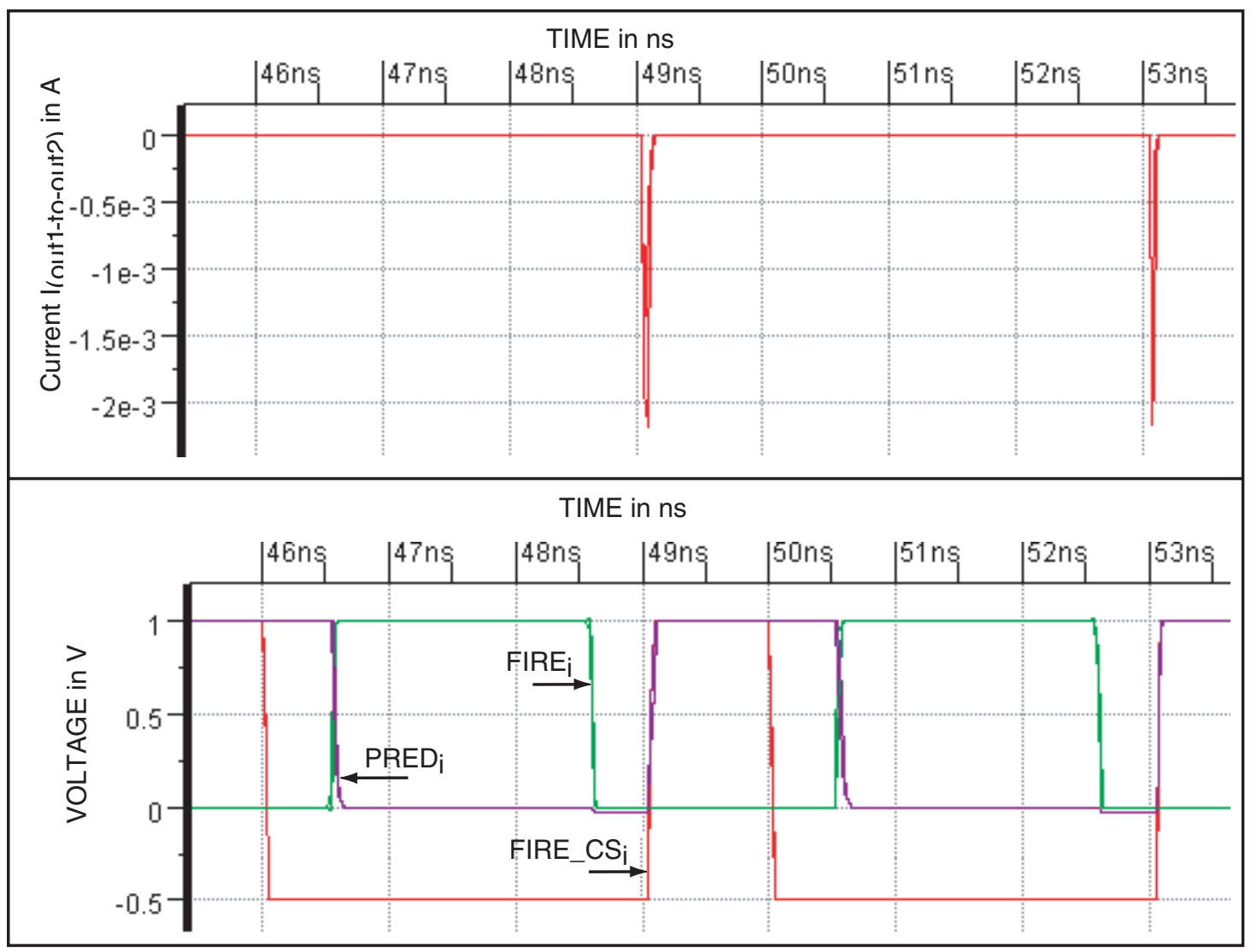

Figure 4.20 Second of the two validation runs showing that the VCCS used in the black box of Figure 4.18 operates correctly and does not interfere with the actual simulation measurements for the relative timing paths LUTblue:FIRE $i+\mathrm{PRED}_{i}-$ and LUTgreen:FIRE $i+$ PRED $_{i}-$. We generated this information from SPICE simulations of Figure 4.18, using the largest driver (wid1=40) and the largest load (wid2=100). The simulation windows follow the same organization as in the previous validation run in Figure 4.19. As before, the properties to validate are as follows: (1) correct alignment of pulse generators, i.e. the (green) FIRE $_{i}$ pulse window lies within the (red) FIRE_CS $i$ pulse window, and (2) correct measurement window, i.e. the rising transition on output signal $\operatorname{PRED}_{i}$ (in blue/purple) lies within the (green) FIRE $i$-high window, and (3) VCCS and measurement operations are mutually exclusive, i.e. $\mathrm{J}_{\text {out } 1-\text { to }- \text { out } 2}=0 \mathrm{~A}$ when FIRE $_{i}$ is high. As before, all three properties hold. 


\subsection{General Simulation Setup Covering All Possible Sweep Cases}

Section 4.5.1 below shows a simulation environment that provides arbitrary external input drives, as weak or as strong as needed. The issue with the simulation environment defined in PART-A, Chapter 3, is that it does not support weak input drives in the order of 0.20 times the size of the path input gate for four out of the five relative timing paths characterized here in PART-B, Chapter 4.

Section 4.5.2 shows how to deal with zero external output loads. We like to include zero external output load simulations to obtain lowerbounds for path propagation delays and output slopes.

\subsubsection{Covering All Possible External Input Drives}

The first four out of five simulation setups, namely those described in Section 4.4.3.1 to Section 4.4.3.4, lack the smallest-size driver. Only the fifth simulation setup described in Section 4.4.3.5, contains the full sweep range. We defined the smallest driver size to be 0.20 times the size of the first gate on the relative timing path that we plan to simulate, rounded off to the closest integer greater or equal to 1 , with 1 being the size of the minimum inverter for the CMOS process of the design - see the introduction of Section 4.4.3 on page 65. The reason for deleting the smallest-size driver from the first four simulation setups is not because the driver size is too small, but because the extra source inverter that we insert between the pulse generator and the driver is too small.

We require a step up of 3 in size between the source and driver, to ensure that the driver receives a realistic input slope, independent of the slope of the pulse generator. The smallest source inverter that we can build in this process has size wids $=1$. The smallest driver size wid1 that this input configuration supports is a step-up of 3 bigger, i.e. wid $1=3 \times$ wids $=3$. To support a sweep value of 0.2 , a driver size wid $1=3$ will support 


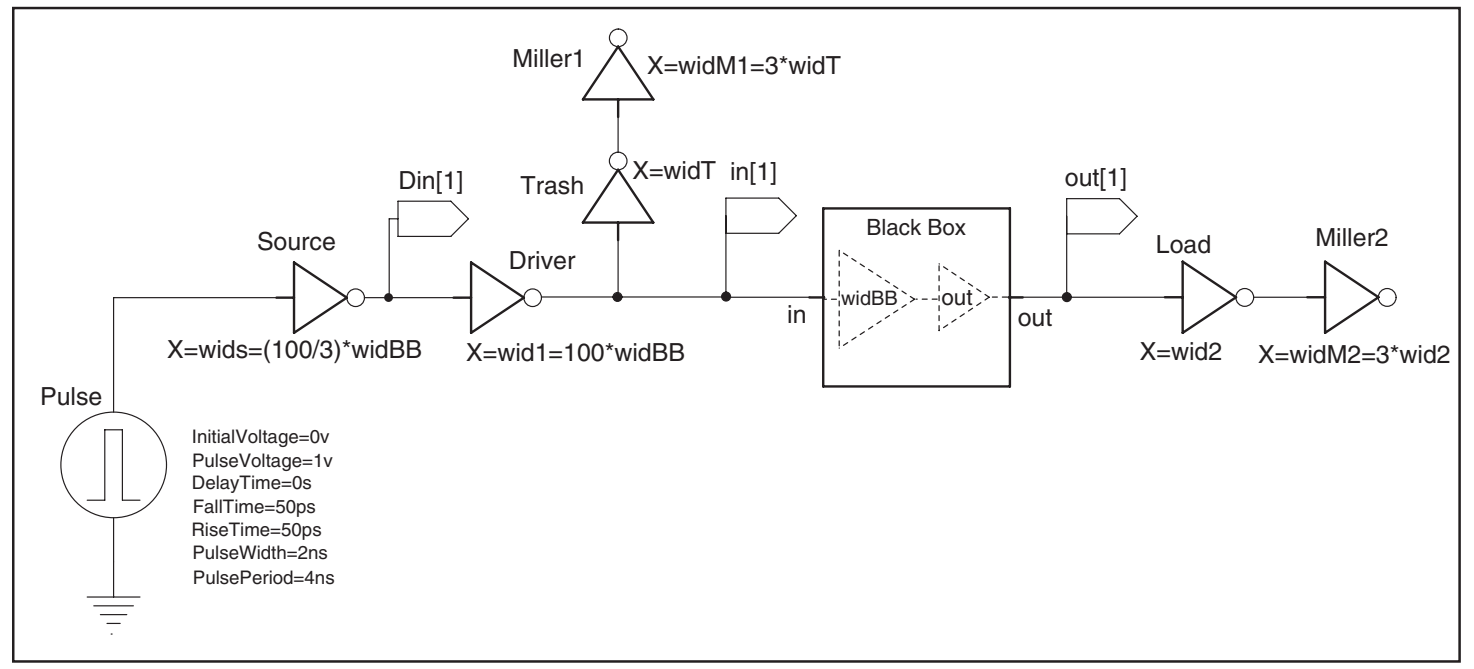

Figure 4.21 General simulation setup for look up table sweeps, ignoring potential VCVS and VCCS add-ons. We sweep widT to set the input slope at in[1], and we sweep wid2 to set the capacitive output load at out[1]. Note that we fixed driver and source sizes to wid $1=100 \times$ widBB and wids $=(100 / 3) \times$ widBB .

a smallest first gate size widBB=wid1/0.2=15 for the first gate of the timed path. The fifth configuration in Figure 4.18, page 77 has size widBB=20 $\geq 15$, and so the fifth configuration can support the complete sweep. But the first four simulation setups all have widBB $\leq 10$, which explains why we cannot support the complete sweep. We can solve this issue, and support complete sweeps for any input gate size widBB $\geq 1$ by using a slightly different input configuration.

Figure 4.21 shows the new simulation setup. The new setup adds two inverters to the original input setup: a so-called Trash inverter and its Miller inverter, called Miller1. Note that we renamed the Miller inverter at the output to Miller2. The Trash inverter and its Miller inverter are inserted as extra load for the driver inverter, in addition to the black box. The result will be that the driver output is divided over the two loads as follows:

- $\operatorname{Val}_{\text {sweep }}=($ widBB + widT $) /$ wid1, where widT is the size of the Trash inverter. 
We can make the sweep almost independent of size widBB, by using very large driver and Trash sizes in relation to the size of the input gate:

- Let wid $1=100 \times$ widBB

- Then a sweep value $\operatorname{Val}_{\text {sweep }}=0.2$ will yield $0.2=($ widBB + widT $) /(100 \times$ widBB $)$ i.e. widT $=(20 \times$ widBB $)-$ widBB $=19 \times$ widBB

- Sweep value $\mathrm{Val}_{\text {sweep }}=2$ will yield widT $=(200 \times$ widBB $)-$ widBB $=199 \times$ widBB

In this new input setup, we keep the driver and source sizes fixed to wid $1=100 \times$ widBB, wids $=(100 / 3) \times$ widBB , and we sweep Trash size widT and size widM1 $=3 \times$ widT of its Miller inverter to sweep the input slope to the black box. The output setup is as before.

\subsubsection{Covering Zero Exernal Output Loads}

In Section 4.5.2, we will show how to deal with zero external output loads. We like to include zero load simulations to obtain lowerbounds for path propagation delays and output slopes. So far, we ran exactly one relative timing path with an external output load of zero. The simulation environment for LUTblue:FIRE ${ }_{i}-$ Dout $_{i}+$ in Figure 4.14 on page 70 has no external output load, because signal Dout ${ }_{i}$ is an internal signal.

The following Figure 4.22 contains a simulation setup for simulating the other four relative timing paths using zero external output loads. We could have done this by using the original simulation setups, and by using the SPICE command file to disconnect the wire connections to the external output loads. Here, in Figure 4.22, we have done it in a way similar to Figure 4.14: we simply deleted the output loads from the original setups

and created new setups. We simulated the resulting setups in parallel. The corresponding SPICE netlist, simulation and output files follow in Appendix H. 


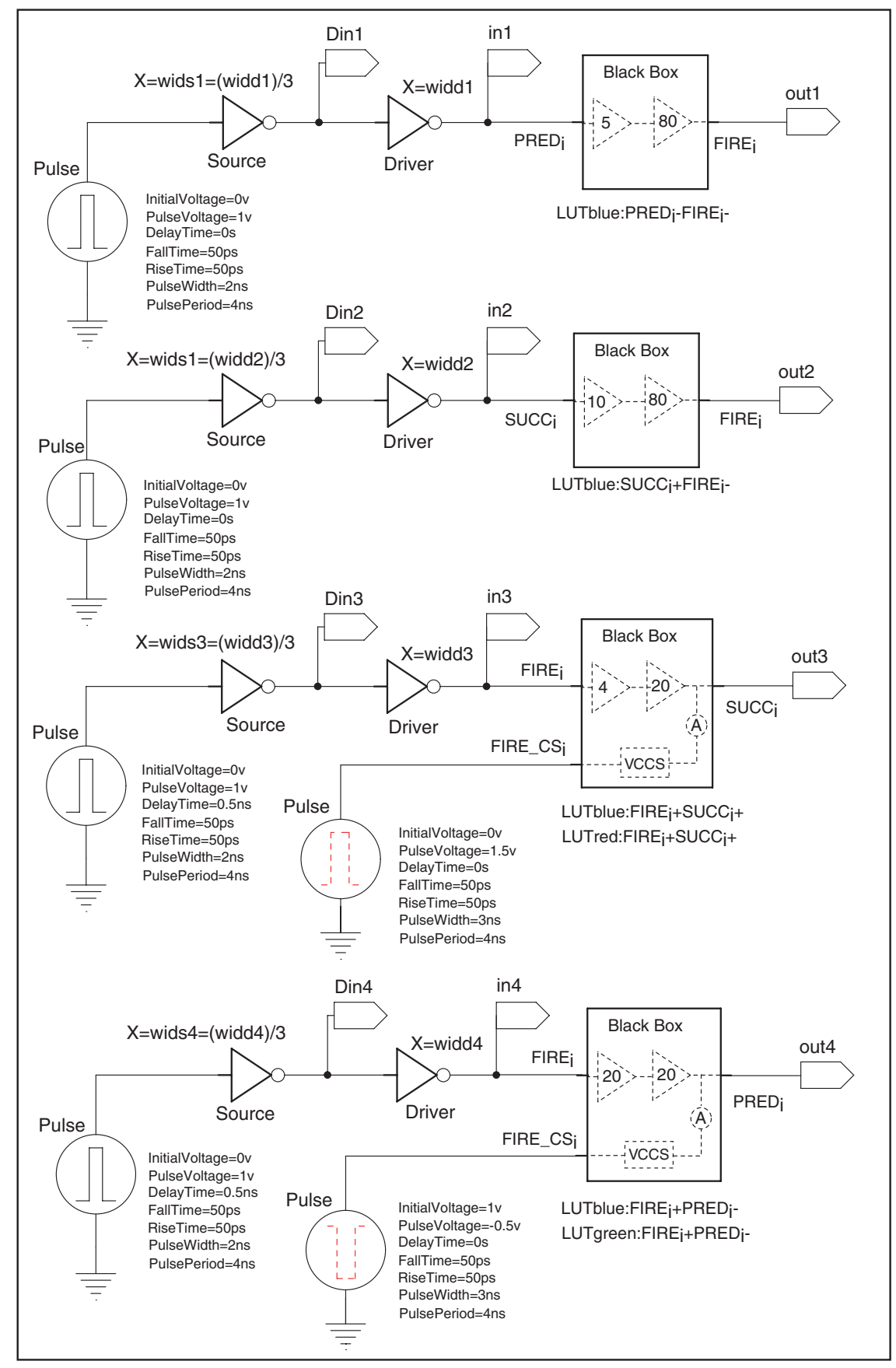

Figure 4.22 Simulation setup with zero external output loads for the original setups in Figure 4.12 (top), Figure 4.13 (second), Figure 4.15 (third), and Figure 4.18 (bottom). We sweep widd1, widd2, widd 3 and widd4 to set the input slope for each path input. We measure the propagation delays and slopes by observing in 1 to in 4 and out 1 to out 4 . 


\subsection{Conclusion and Future Work for Chapter 4}

In Chapter 4, we designed a characterization flow to generate look up tables with timing information for a typical 6-4 GasP circuit. This chapter is dubbed 'PART-B' because it is the second of a two-part study in look up table generation for 6-4 GasP. PART-A in Chapter 3 is the first part. It shows how to characterize a simple 6-4 GasP library module without loops and with a single input and output. PART-B extends the characterization flow in PART-A to general 6-4 GasP circuits, by partitioning the timing behavior of a 6-4 GasP circuit into simple input-to-output transitions and simplified circuits that can be fed back into PART-A. Not only do we show how to do this, but we actually do it: we partition complex relative timing paths and feed the sub-paths back into PART-A. The SPICE input and output files in Appendix $\mathrm{C}-\mathrm{H}$ bear witness to this. Proof of the pudding follows in Chapter 5 where we run the standard STA tool PrimeTime of Synopsys on the look up tables in Appendix $\mathrm{C}-\mathrm{H}$ to validate the four key relative timing constraints in a 6-4 GasP module, listed on page 12.

Here is a summary of the key steps in PART-B:

STEP 1: Partition complex GasP paths by cutting them at module boundaries, i.e. at $\mathrm{PRED}_{i}, \mathrm{FIRE}_{i}, \mathrm{SUCC}_{i}$, and at internal signals that control the single-track wire drive, i.e. at Dout ${ }_{i}$. The names of the sub-path cuttings reflect key properties of the paths. For instance, sub-path name 'LUTblue:PRED ${ }_{i}-\mathrm{FIRE}_{i}-$ ' indicates that (1) the timing information of the path will be captured in a look up table (LUT),

(2) we are interested in simulating this path with minimal delay settings (blue), (3) the path starts with $\operatorname{PRED}_{i}$ falling and ends with a falling transition on $\mathrm{FIRE}_{i}$. 
STEP 2: Set up an accurate simulation environment for each sub-path as follows:

1. From the 6-4 GasP schematics, include all the gates on the sub-path. For all gates on the sub-path, include all the load capacitances on the gate by including the gates and wires connected to the gate output. The resulting circuit is a simplification of the original 6-4 GasP circuit. In particular, the input signal goes to the first gate and nothing else, and the output signal carries all the load that it carries in the 6-4 GasP schematics.

2. Model 'The Charlie Effect' needed for the sub-path, i.e. add a VCVS device to synchronize the input transitions of a multi-input gate, or connect nonleading gate inputs to VSS or VDD to let the leading input transition set the gate delay. Two of the sub-paths characterized in Chapter 4 required a VCVS device to synchronize the inputs of two-input gate A.

3. Re-initialize un-driven wires by adding a VCCS device. Four sub-paths that we characterized in Chapter 4 required a VCCS device for this purpose.

4. Embed the resulting circuit into the simulation setup of PART-A to generate SPICE input files and to create a SPICE output file with the look up tables.

As far as future work on PART-B goes, we have the following suggestions and hints:

1. Create wire models with near-end capacitive load and far-end delay information.

- It may be that an Elmore delay model suffices $[23,36]$.

2. Flow automation, also mentioned in the conclusion of PART-A, becomes even more a necessity given the complexity of PART-A-to-B combined. Specifically:

- The path and circuit partitioning in PART-B can be simplified if we could use pre-defined single-rail datapath designs with a fixed load on $\mathrm{FIRE}_{i}$. 
- There may be other, perhaps easier, ways to partition the circuit. We could for instance keep the full schematics but avoid false feedback paths by splitting signals that act as output as well as input into separate pins, as is done in the static timing analysis approach for 6-4 GasP in [2] and in Chapter 5.

3. Use the general simulation setup described in Section 4.5, Figure 4.21.

- Figure 4.21 guarantees a simulation environment designed according to the theory of Logical Effort, and avoids that the transistor sizes shrink below the minimum size of 1 , as we experienced for 4 out of our 5 simulation setups.

4. Use asymmetric sweeps and add sweep values for the two extreme ends.

- Asymmetric sweeps make it easier to validate that the rows and columns of the look up tables are correctly copied and not accidentally swapped during the transfer process from SPICE output files to PrimeTime input files.

- Sweep values for driver size wid1 and load size wid2 below and above the anticipated sweep range of a GasP design make outlier values more accessible and their effects more predictable. 


\section{Chapter 5}

\section{STATIC TIMING ANALYSIS OF 6-4 GASP USING LOOK UP TABLES}

Chapter 5 is also available as internal report ARC2010-smg04 [19]. It describes how we can use standard static timing analysis (STA) tools to validate the relative timing constraints on which the correctness of 6-4 GasP circuits depends. We chose Synopsys PrimeTime as the de facto STA tool standard. GasP is a non-standard circuit family for STA tools like PrimeTime. The combinational loops in a GasP module and the bi-directionality of the single-track communication channels between two GasP modules make GasP hard to digest for STA tools that are accustomed to clocked circuits where such loops are restricted to state-holding elements like flipflops. To make GasP digestible, we take the following approach:

1. We replace each GasP module by a black box in which each combinational loop is modeled as a sequence of loop-less input-output paths whose timing information is stored in look up tables.

2. We replace each bi-directional single-track wire by two uni-directional wires.

3. We reformulate each relative timing constraint as a data-to-data check from a common point of divergence. We use this common point of divergence as a clock signal. This brings our path1-to-path2 relative timing constraints into the standard STA jargon of clock-to-(path1-versus-path2) constraints. 
This approach was initially developed at the University of Southern California (USC) by Mallika Prakash for asynchronous circuits from USC, Fulcrum and Silistix [21, 22] and it was extended by Prasad Joshi, also USC, to the single-track circuit family that we use in 6-4 GasP $[2,3]$. What we add to it is (1) the use of realistic look up tables for timing the loop-less sub-paths that make up the combinational loops in a 6-4 GasP circuit $[16,17]$, and (2) additional relative timing constraints that our short to medium to long wire studies for 6-4 GasP have identified as necessary timing constraints [18].

\subsection{Introduction}

The term 'static' in static timing analysis alludes to the fact that the timing is analyzed in a data- or input- independent manner, and contrasts to the 'dynamic' or 'simulated' timing analysis that we used in our library characterization approach in Chapters 3-4. Static timing analysis (STA) tends to be much faster than simulated timing analysis. It is used to analyze large complex designs: think 'systems' rather than 'circuit modules'. The speed-up is largely due to its use of simplified delay models and to its limited ability to incorporate signal dependencies. Our simplified delay models are look up tables, which we generate with SPICE, using the library characterization approach described in Chapters 3 and 4. A quick overview of techniques used in static timing analysis and a list of references about the state of the art can be found in [25].

Chapter 5 shows how to run static timing analysis, using Synopsys PrimeTime [35]. The flow that we use comes from the University of Southern California (USC), and was developed by Mallika Prakash and Prasad Joshi two former Master of Science students

in the research group of Professor Peter Beerel. Mallika developed an STA flow for asynchronous circuits from USC, Fulcrum and Silistix [21, 22] and Prasad extended her flow to the single-track circuit family that we use in $6-4$ GasP $[2,3]$. The value that 
we add to the USC flow is the use of SPICED look up tables for 6-4 GasP, and the methodology to generate these Tables.

We use static timing analysis to validate so-called 'Relative Timing' constraints [27, 28] in our circuits. Relative timing constraints describe the order in which two signals must arrive at a point of convergence. The signals typically start from a common point, the point of divergence, and they are all of the form: 'the delay for path A is less than the delay for path B'. In this chapter, we will use static timing analysis to validate four relative timing constraints. These four constraints capture the key communication aspects of single-track handshake signaling between two 6-4 GasP modules. They include the two relative timing constraints for GasP with a lumped-capacitance single-track wire model $[4,15]$. In Chapter 4 we already characterized the timing information needed to validate these four relative timing constraints, and we stored the information in a collection of look up tables. The search key $($ wid1 $\times$ wid2) that we use to index our look up tables is different than the indexing used by PrimeTime. So, our first task is to translate our SPICED look up tables into PrimeTime look up tables. After that, we follow the USC static timing analysis flow for asynchronous circuits.

The resulting flow has five steps:

Step 1: Translate SPICED look up tables into PrimeTime look up tables.

Step 2: Replace each 6-4 GasP module in the (Verilog) design by a black box.

- Our black box model is slightly different than the one Prasad uses in [2].

Step 3: Create a (Liberty) library with the black box timing in look up tables.

- This requires renaming the tables to reflect the new black box pin names.

Step 4: Create a (Tcl) command file to validate the four relative timings.

Step 5: Run PrimeTime and inspect the timing reports. 
The rest of Chapter 5 is organized as follows. Section 5.2 covers the translation of our SPICED look up tables into PrimeTime look up tables, i.e. it covers Step 1 in our fivestep STA flow. Section 5.3 covers the black box model for the 6-4 GasP module, i.e. it covers Step 2 in our five-step STA flow. Sections 5.4-5.6 cover the remaining Steps 3-5. The timing results in Section 5.6 exclude single-track wire delays, because our look up tables do not generate these. So, instead of letting PrimeTime analyze the effects that short, medium, and long wires have on the four relative timing constraints, we will do the analysis ourselves, using the simulation results from our short-to-medium-to-long wire study in Appendix $\mathrm{O}$ and [18]. The analysis can be found in Chapter 6. Section 5.7

concludes Chapter 5. Appendices I-M contain all the files generated in Steps 1-5, ranging from SPICED look up table translations to PrimeTime commands and timing reports.

\section{Note:}

We postpone the comparison between our library characterization work and prior work at USC by Mallika Prakash [21,22] and Prasad Joshi [2, 3] to Chapter 7.

\subsection{STEP 1: From SPICED to PrimeTime Look Up Tables}

The look up tables that we generated in Chapter 4 store the timing information for the relative timing paths RT1 to RT4 of Figure 2.2, using a two-dimensional search key (wid1 $\times$ wid2). We store three types of timing information - see also page 15 :

1. (wid1 $\times$ wid2) $\rightarrow$ slope (in[1])

2. $($ wid $1 \times$ wid2 $) \rightarrow$ delay (in[1] to out[1])

3. $($ wid $1 \times$ wid2) $\rightarrow$ slope $($ out $[1])$

where: 
- wid1 is the size of the external gate driving path input in[1].

- wid2 is the size of the external gate driven by path output out[1].

- slope(in[1]) is the input transition slope, a.k.a. input slew time, as denoted by the time difference $\left(t i n_{2}-t i n_{1}\right)$ from time $\operatorname{tin}_{1}$ when a rising input transition on in[1] passes the $20 \%$ supply voltage point to time $\operatorname{tin}_{2}$ when it passes the $80 \%$ voltage point - and vice versa for a falling input transition.

- delay(in[1] to out[1]) is the input-to-output transition delay time (tout-tin) from time $t$ in when the input transition on in[1] passes the 50\% supply voltage point to time tout when the output transition on out[1] passes the 50\% supply point.

- slope(out[1]) is the output transition slope, a.k.a. output slew, as for instance denoted by the time difference $\left(\right.$ tout $_{2}-$ tout $\left._{1}\right)$ from time tout $_{1}$ when a falling output transition on out[1] passes the $80 \%$ supply voltage point to time tout $_{2}$ when it passes the $20 \%$ point - and vice versa for a rising output transition.

The two-dimensional search key (wid1 $\times$ wid2) in our look up tables is different than the (input slope $\times$ capacitive output load) indexing key used by standard static timing analysis tools like PrimeTime of Synopsys. We use sizing information instead of input slopes and output loads, because it allows us to use the theory of Logical Effort [32] in both the design and the characterization of our circuits. We feel that this brings the characterization process closer to the designer's world, which - in our opinion makes the characterization process less artificial and more realistic. To accommodate standard STA tools, we measure the input slopes for a given drive size and we have methods to translate a driven output size into an output load.

Section 5.2.1 shows how we translate our index key wid1 into an index for input slope. Section 5.2.2 shows how we translate our index key wid2 into an index for output load. Section 5.2.3 analyzes the plausibility of the resulting PrimeTime look up tables. 


\subsubsection{From Input Driver Size to Input Transition Slope}

In Chapter 4 we needed a total of five look up tables to cover the loop-less sub-paths in relative timing constraints RT1 to RT4. We refrained from generating look up tables for the paths crossing single-track wire L2. We left the timing measurements associated with L2 to static timing analysis (STA) with back-annotated wire information from the final layout results. We will come back to this in Chapter 6, where we use the L2 timing results from [18] to bring the timing information for L2 back into the STA validation for RT1 to RT4 of Figure 2.2, page 13. We copied the five look up tables that we generated in Chapter 4 as the top look up tables in Figure 5.3 to Figure 5.7 below.

We will use Figure 5.3 to explain the organization of each table. External driver size wid 1 acts as the column index, and size wid 2 of the external load acts as the row index. The timing information stored at table index (wid1 $\times$ wid2) is as indicated: input slope, input-to-output delay, and output slope - all in picoseconds (ps). The numbers match

those in the SPICE output files in Appendices $\mathrm{C}-\mathrm{G}$, but we display them here with a more realistic precision to only a tenth of a picosecond.

The middle of the three look up tables in Figure 5.3 is a translation of the top table: we replaced column index wid1 by the average input slope produced by driver size wid1. The calculations for the average input slope per wid1 size are shown in the top table. We also calculated the relative difference between the average input slope and the input slope that we measured for driver size wid1 under the various load sizes wid2. To be precise, we calculated:

$\%$ Diff $=100 \times($ average-input-slope - measured-input-slope $) /($ average-input-slope $)$ 
The relative differences for all five look up tables in Figure 5.3 to Figure 5.7 vary from $0 \%$ to less than $25 \%$. This is fine, because it's the path delay that we're interested in, and the input slope turns out to matter a lot less to the total path delay than the output load. We already elaborated on this in Section 3.7 of Chapter 3. We will come back to this again in Section 5.2.3, where we analyze the input-to-output (path) delay landscapes captured by the final translated look up tables.

To summarize: we translated the top look up tables in Figure 5.3-5.7 of the form:

- $($ wid1 $\times$ wid2) $\rightarrow$ slope (in[1]), delay (in[1] to out[1]), slope (out[1])

into the middle look up tables, which use the form:

- $($ slope $($ in[1] $\times$ wid2) $\rightarrow$ delay (in[1] to out[1]), slope (out[1])

This brings our SPICED look up tables one step closer to the look up table format needed in PrimeTime. Follow-up Section 5.2.2 completes the translation.

\subsubsection{From Output Load Size to Output Load Capacitance}

The solid red graph in Figure 5.2 below shows the relationship between the size wid2 of an external load inverter (horizontal axis), and the capacitive load that it represents (vertical axis). It is a linear relationship, as one would expect it to be. The ten dots on the graph show the 90nm CMOS SPICE simulation results for the ten load inverter sizes wid2 that we used in our look up tables. The simulation setup is shown in Figure 5.1. The top setup is similar to the simulation setup that we defined earlier in our library characterization approach in Chapter 3 and hence also similar to the setups that we used in Chapter 4 for generating the look up tables in Figure 5.3 to Figure 5.7. In a nutshell: the setup follows the step-up of 3 Logical Effort design strategy, where each gate drives three times its own load; the source inverter guarantees a decent driver input slope; the Miller inverter is there to give the load inverter a realistic load profile. 


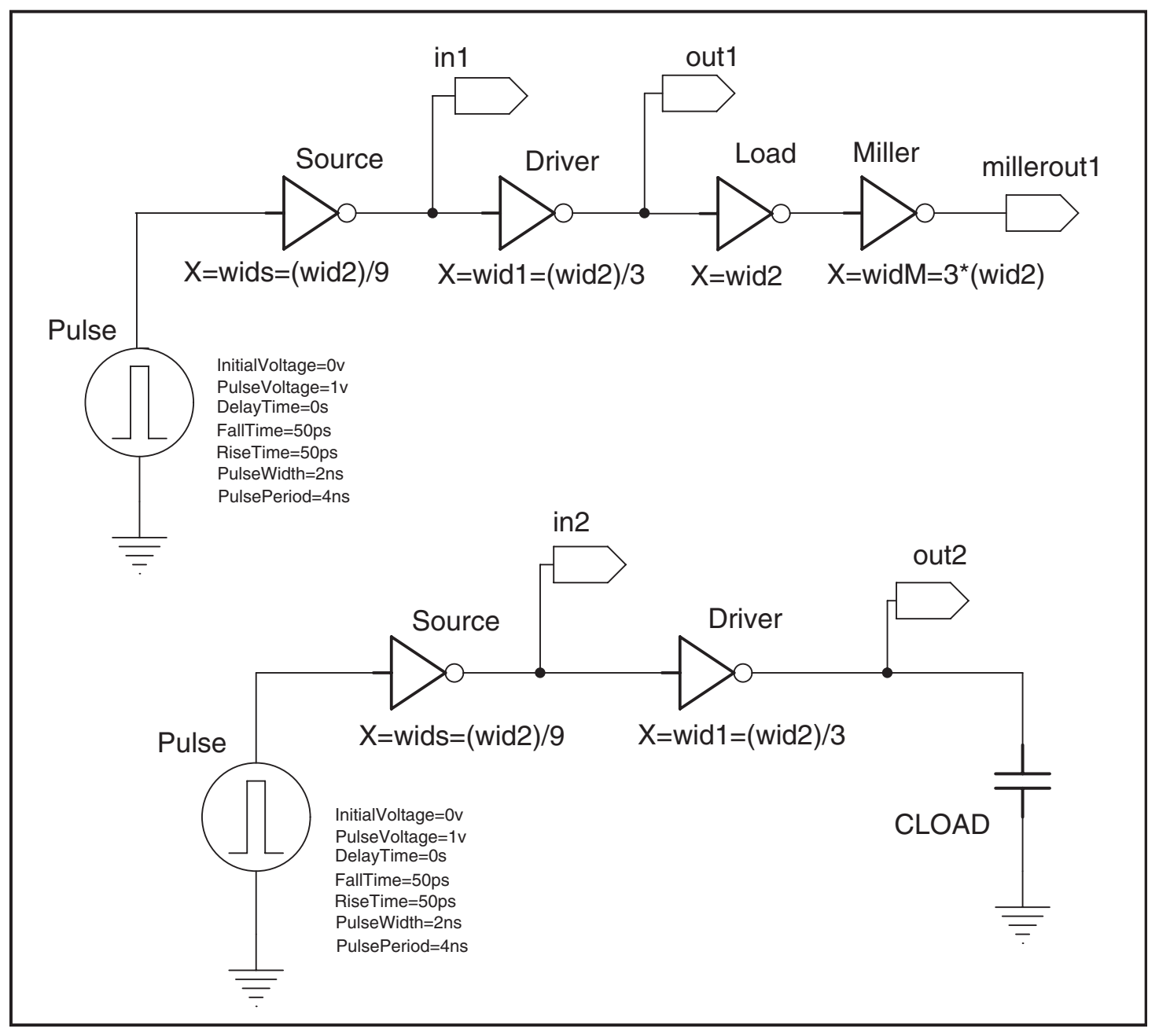

Figure 5.1 Simulation setup for translating load size into load capacitance. The load size is represented by (1) the value of wid 2 in the top configuration and (2) the load circuitry consisting of a wid2-size load inverter followed by a $(3 \times$ wid2)-size Miller inverter. The load capacitance is represented by the value of the load capacitor CLOAD in the bottom configuration. The values for wid 2 and CLOAD go together if the average delay of a rising and falling transition from in 1 to out 1 in the top configuration matches the average delay of a rising and falling transition from in 2 to out 2 in the bottom one. 
We are interested in finding the capacitive load of the wid2 load inverter in the top schematics of Figure 5.1, for a range of sizes wid2. We find the equivalent capacitance through a dual sweep process. First, we select a sweep value for wid2 and we measure the average delay of a rising and falling transition from in 1 to out 1 through the driver inverter. We use this average delay to start a goal-driven sweep for the bottom simulation setup in Figure 5.1, where we have replaced the load circuitry by a load capacitor. We sweep the capacitor value until we measure an average delay from in 2 to out 2 that (closely) matches the average delay from in1 to out1 that we just measured for the top simulation setup.

The SPICE netlist, simulation, and output files can be found in Appendix I. The SPICE output file maps the ten red dots to the following (wid $2 \times$ CLOAD) values:

\begin{tabular}{|l|c|c|c|c|c|c|c|c|c|c|}
\hline Size wid2 & 10 & 33 & 40 & 56 & 78 & 100 & 130 & 220 & 310 & 400 \\
\hline CLOAD [fF] & 4.6 & 15.0 & 18.2 & 25.5 & 35.5 & 45.6 & 59.3 & 101.4 & 142.8 & 185.1 \\
\hline
\end{tabular}

In addition to the red-colored solid-line graph in Figure 5.2, which we annotated with the text 'with Miller inverter', there is a black-colored dotted-line graph, annotated with the text 'no Miller inverter'. The dotted-line graph is the result of a re-simulation of Figure 5.1 with a top configuration that lacks a Miller inverter following the load inverter. Without the Miller inverter, the wid2-size load inverter is perceived to have a higher capacitive load, as one would expect, but the difference is very small for the simulated wid2 range. 


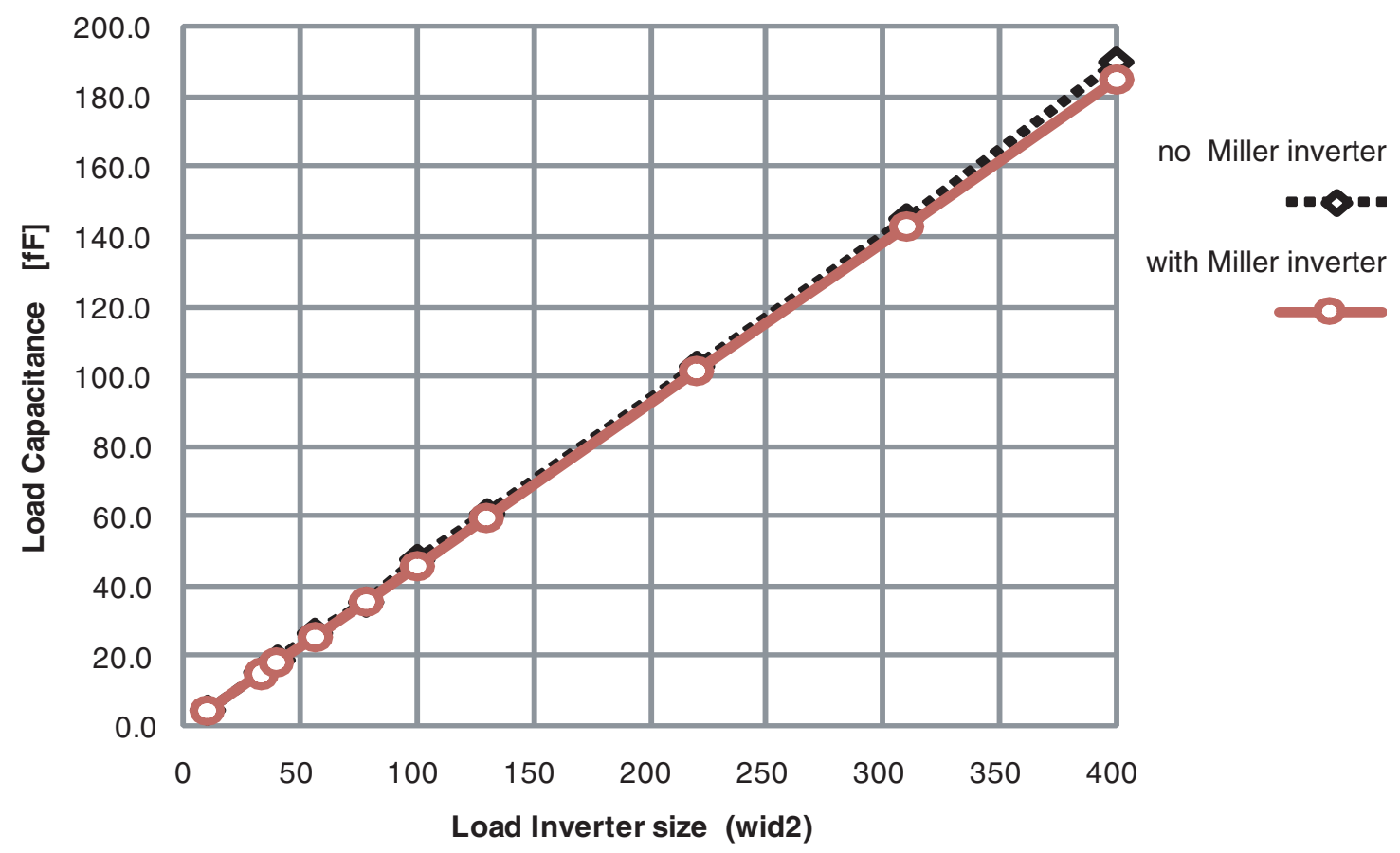

Figure 5.2 Graphical representation of the relationship between the size wid 2 of the load inverter in the top configuration of Figure 5.1 and the load capacitance CLOAD in the bottom configuration of Figure 5.1, as perceived by the driver inverter. The red solid-line graph shows the relationship for Figure 5.1 as is. The black dotted-line graph shows the relationship for Figure 5.1 without the Miller inverter in the top configuration.

We used the red solid-line graph in Figure 5.2 to translate the look up tables in the middle of Figure 5.3-5.7, which are of the form:

- $($ slope $($ in[1] $\times$ wid2) $\rightarrow$ delay (in[1] to out[1]), slope (out[1])

into the bottom look up tables, which use the form:

- $(\operatorname{slope}($ in[1] $\times \operatorname{cload}($ out[1]) $) \rightarrow$ delay (in[1] to out[1]), slope (out[1])

This completes the reformatting of our SPICED look up table for use in PrimeTime. 


\section{LUTblue:PREDi-FIRE;-}

\begin{tabular}{|c|c|c|c|c|c|c|c|c|c|c|c|c|c|c|c|c|}
\hline \multirow[t]{2}{*}{ wid2 I wid1 } & \multicolumn{4}{|c|}{10} & \multicolumn{4}{|c|}{8} & \multicolumn{4}{|c|}{6} & \multicolumn{4}{|c|}{3} \\
\hline & $\begin{array}{l}\text { input } \\
\text { slope } \\
\text { [ps] }\end{array}$ & $\begin{array}{l}\% \text { Diff } \\
\text { to Avg }\end{array}$ & $\begin{array}{l}\text { delay } \\
\text { [ps] }\end{array}$ & $\begin{array}{l}\text { output } \\
\text { slope } \\
\text { [ps] }\end{array}$ & $\begin{array}{c}\text { input } \\
\text { slope } \\
\text { [ps] }\end{array}$ & $\begin{array}{l}\% \text { Diff } \\
\text { to Avg }\end{array}$ & $\begin{array}{l}\text { delay } \\
\text { [ps] }\end{array}$ & $\begin{array}{l}\text { output } \\
\text { slope } \\
\text { [ps] }\end{array}$ & $\begin{array}{l}\text { input } \\
\text { slope } \\
\text { [ps] }\end{array}$ & $\begin{array}{l}\% \text { Diff } \\
\text { to Avg }\end{array}$ & $\begin{array}{l}\text { delay } \\
\text { [ps] }\end{array}$ & $\begin{array}{l}\text { output } \\
\text { slope } \\
\text { [ps] }\end{array}$ & $\begin{array}{c}\text { input } \\
\text { slope } \\
\text { [ps] }\end{array}$ & $\begin{array}{l}\% \text { Diff } \\
\text { to Avg }\end{array}$ & \begin{tabular}{|} 
delay \\
{$[p s]$}
\end{tabular} & $\begin{array}{l}\text { output } \\
\text { slope } \\
\text { [ps] }\end{array}$ \\
\hline 0 & 11.5 & $-0.9 \%$ & 60.3 & 8.0 & 12.4 & $-2.5 \%$ & 60.8 & 8.5 & 13.1 & $0.0 \%$ & 61.1 & 8.3 & 17.9 & $-2.9 \%$ & 63.2 & 8.4 \\
\hline 40 & 11.4 & $0.0 \%$ & 62.1 & 9.9 & 12.2 & $-0.8 \%$ & 62.4 & 9.9 & 13.3 & $-1.2 \%$ & 62.8 & 10.1 & 17.6 & $-1.1 \%$ & 64.7 & 9.6 \\
\hline 130 & 11.3 & $0.9 \%$ & 65.9 & 13.8 & 12.1 & $0.2 \%$ & 66.1 & 13.8 & 13.4 & $-2.1 \%$ & 66.6 & 13.9 & 17.6 & $-0.9 \%$ & 68.3 & 14.0 \\
\hline 220 & 11.1 & $2.6 \%$ & 69.3 & 18.3 & 11.6 & $4.2 \%$ & 69.5 & 18.4 & 13.2 & $-0.4 \%$ & 69.9 & 18.4 & 16.3 & $6.6 \%$ & 71.7 & 18.6 \\
\hline 310 & 11.4 & $0.0 \%$ & 72.3 & 22.7 & 12.2 & $-1.2 \%$ & 72.6 & 22.6 & 12.5 & $5.0 \%$ & 73.1 & 22.3 & 17.6 & $-1.4 \%$ & 74.8 & 22.0 \\
\hline 400 & 11.5 & $-0.9 \%$ & 75.1 & 27.1 & 12.2 & $-0.8 \%$ & 75.4 & 27.3 & 13.1 & $0.2 \%$ & 75.9 & 27.4 & 17.6 & $-1.0 \%$ & 77.7 & 27.3 \\
\hline $\begin{array}{l}\text { Average } \\
\text { input slope }\end{array}$ & 11.4 & $0.0 \%$ & & & 12.1 & $0.0 \%$ & & & 13.1 & $0.0 \%$ & & & 17.4 & $0.0 \%$ & & \\
\hline
\end{tabular}

Replace Driver size wid1 by the Average input slope that it produces

\begin{tabular}{|c|c|c|c|c|c|c|c|c|}
\hline wid2 \input-slope1 [ps] & \multicolumn{2}{|c|}{11.4} & \multicolumn{2}{|c|}{12.1} & \multicolumn{2}{|c|}{13.1} & \multicolumn{2}{|c|}{17.4} \\
\hline & $\begin{array}{c}\text { delay } \\
{[\mathrm{ps}]}\end{array}$ & $\begin{array}{c}\text { output } \\
\text { slope } \\
{[\mathrm{ps}]}\end{array}$ & $\begin{array}{c}\text { delay } \\
{[\mathrm{ps}]}\end{array}$ & $\begin{array}{c}\text { output } \\
\text { slope } \\
\text { [ps] }\end{array}$ & $\begin{array}{c}\text { delay } \\
{[\mathrm{ps}]}\end{array}$ & $\begin{array}{c}\text { output } \\
\text { slope } \\
{[\mathrm{ps}]}\end{array}$ & $\begin{array}{c}\text { delay } \\
{[\mathrm{ps}]}\end{array}$ & $\begin{array}{c}\text { output } \\
\text { slope } \\
{[\mathrm{ps}]}\end{array}$ \\
\hline 0 & 60.3 & 8.0 & 60.8 & 8.5 & 61.1 & 8.3 & 63.2 & 8.4 \\
\hline 40 & 62.1 & 9.9 & 62.4 & 9.9 & 62.8 & 10.1 & 64.7 & 9.6 \\
\hline 130 & 65.9 & 13.8 & 66.1 & 13.8 & 66.6 & 13.9 & 68.3 & 14.0 \\
\hline 220 & 69.3 & 18.3 & 69.5 & 18.4 & 69.9 & 18.4 & 71.7 & 18.6 \\
\hline 310 & 72.3 & 22.7 & 72.6 & 22.6 & 73.1 & 22.3 & 74.8 & 22.0 \\
\hline 400 & 75.1 & 27.1 & 75.4 & 27.3 & 75.9 & 27.4 & 77.7 & 27.3 \\
\hline
\end{tabular}

Replace Load size wid2 by its equivalent Load capacitance

\begin{tabular}{|c|c|c|c|c|c|c|c|c|}
\hline & $\begin{array}{c}\text { delay } \\
\text { output-load2 [fF] I input-slope1 [ps] }\end{array}$ & $\begin{array}{c}\text { output } \\
\text { slope } \\
\text { [ps] }\end{array}$ & $\begin{array}{c}\text { delay } \\
{[\mathrm{ps}]}\end{array}$ & $\begin{array}{c}\text { output } \\
\text { slope } \\
\text { [ps] }\end{array}$ & $\begin{array}{c}\text { delay } \\
{[\mathrm{ps}]}\end{array}$ & $\begin{array}{c}\text { output } \\
\text { slope } \\
\text { [ps] }\end{array}$ & $\begin{array}{c}\text { delay } \\
\text { [ps] }\end{array}$ & $\begin{array}{c}\text { output } \\
\text { slope } \\
\text { [ps] }\end{array}$ \\
\hline 0.0 & 60.3 & 8.0 & 60.8 & 8.5 & 61.1 & 8.3 & 63.2 & 8.4 \\
\hline 18.2 & 62.1 & 9.9 & 62.4 & 9.9 & 62.8 & 10.1 & 64.7 & 9.6 \\
\hline 59.3 & 65.9 & 13.8 & 66.1 & 13.8 & 66.6 & 13.9 & 68.3 & 14.0 \\
\hline 101.4 & 69.3 & 18.3 & 69.5 & 18.4 & 69.9 & 18.4 & 71.7 & 18.6 \\
\hline 142.8 & 72.3 & 22.7 & 72.6 & 22.6 & 73.1 & 22.3 & 74.8 & 22.0 \\
\hline 185.1 & 75.1 & 27.1 & 75.4 & 27.3 & 75.9 & 27.4 & 77.7 & 27.3 \\
\hline
\end{tabular}

Figure 5.3 SPICE to PrimeTime look up table for LUTblue:PRED ${ }_{i}-\mathrm{FIRE}_{i}-$. 


\section{LUTblue:SUCC $\mathbf{j}_{\mathbf{j}} \mathrm{FIRE}_{\mathbf{j}}$}

\begin{tabular}{|c|c|c|c|c|c|c|c|c|c|c|c|c|c|c|c|c|}
\hline \multirow[t]{2}{*}{ wid2 I wid1 } & \multicolumn{4}{|c|}{20} & \multicolumn{4}{|c|}{16} & \multicolumn{4}{|c|}{10} & \multicolumn{4}{|c|}{6} \\
\hline & $\begin{array}{c}\text { input } \\
\text { slope } \\
\text { [ps] }\end{array}$ & $\begin{array}{l}\% \text { Diff } \\
\text { to Avg }\end{array}$ & $\begin{array}{c}\text { delay } \\
{[p s]}\end{array}$ & $\begin{array}{c}\text { output } \\
\text { slope } \\
\text { [ps] }\end{array}$ & $\begin{array}{c}\text { input } \\
\text { slope } \\
\text { [ps] }\end{array}$ & $\begin{array}{l}\% \text { Diff } \\
\text { to Avg }\end{array}$ & $\begin{array}{l}\text { delay } \\
\text { [ps] }\end{array}$ & $\begin{array}{c}\text { output } \\
\text { slope } \\
\text { [ps] }\end{array}$ & \begin{tabular}{|c|} 
input \\
slope \\
[ps]
\end{tabular} & $\begin{array}{l}\% \text { Diff } \\
\text { to Avg }\end{array}$ & $\begin{array}{l}\text { delay } \\
{[p s]}\end{array}$ & $\begin{array}{c}\text { output } \\
\text { slope } \\
\text { [ps] }\end{array}$ & $\begin{array}{c}\text { input } \\
\text { slope } \\
\text { [ps] }\end{array}$ & $\begin{array}{l}\% \text { Diff } \\
\text { to Avg }\end{array}$ & \begin{tabular}{|c|} 
delay \\
{$[\mathrm{ps}]$}
\end{tabular} & $\begin{array}{c}\text { output } \\
\text { slope } \\
\text { [ps] }\end{array}$ \\
\hline 0 & 15.3 & $-7.7 \%$ & 35.8 & 8.8 & 17.7 & $-17.2 \%$ & 36.3 & 8.6 & 15.9 & $2.5 \%$ & 37.4 & 8.5 & 21.7 & $-1.4 \%$ & 39.2 & 8.3 \\
\hline 40 & 14.9 & $-4.9 \%$ & 37.7 & 10.0 & 15.3 & $-1.3 \%$ & 38.0 & 10.1 & 15.8 & $3.1 \%$ & 39.3 & 9.6 & 20.8 & $2.8 \%$ & 40.8 & 10.8 \\
\hline 130 & 15.1 & $-6.3 \%$ & 41.6 & 13.8 & 14.5 & $4.0 \%$ & 41.9 & 14.2 & 15.8 & $3.1 \%$ & 43.0 & 13.9 & 21.6 & $-0.9 \%$ & 44.7 & 13.9 \\
\hline 220 & 13.0 & $8.5 \%$ & 45.0 & 18.0 & 14.1 & $6.6 \%$ & 45.3 & 18.0 & 16.0 & $1.8 \%$ & 46.3 & 18.3 & 21.3 & $0.5 \%$ & 48.1 & 18.2 \\
\hline 310 & 13.2 & $7.0 \%$ & 48.0 & 22.8 & 13.7 & $9.3 \%$ & 48.4 & 22.5 & 16.5 & $-1.2 \%$ & 49.3 & 22.8 & 21.5 & $-0.5 \%$ & 51.2 & 22.7 \\
\hline 400 & 13.9 & $2.1 \%$ & 50.9 & 26.9 & 15.0 & $0.7 \%$ & 51.2 & 27.0 & 17.6 & $-8.0 \%$ & 52.1 & 27.1 & 21.3 & $0.5 \%$ & 54.0 & 27.0 \\
\hline $\begin{array}{l}\text { Average } \\
\text { input slope }\end{array}$ & 14.2 & $0.0 \%$ & & & 15.1 & $0.0 \%$ & & & 16.3 & $0.0 \%$ & & & 21.4 & $0.0 \%$ & & \\
\hline
\end{tabular}

Replace Driver size wid1 by the Average input slope that it produces

\begin{tabular}{|c|c|c|c|c|c|c|c|c|}
\hline wid2 \input-slope1 [ps] & \multicolumn{2}{|c|}{14.2} & \multicolumn{2}{|c|}{15.1} & \multicolumn{2}{|c|}{16.3} & \multicolumn{2}{|c|}{21.4} \\
\hline & $\begin{array}{c}\text { delay } \\
{[\mathrm{ps}]}\end{array}$ & $\begin{array}{c}\text { output } \\
\text { slope } \\
\text { [ps] }\end{array}$ & $\begin{array}{c}\text { delay } \\
\text { [ps] }\end{array}$ & $\begin{array}{c}\text { output } \\
\text { slope } \\
\text { [ps] }\end{array}$ & $\begin{array}{c}\text { delay } \\
{[\mathrm{ps}]}\end{array}$ & $\begin{array}{c}\text { output } \\
\text { slope } \\
{[\mathrm{ps}]}\end{array}$ & $\begin{array}{c}\text { delay } \\
{[\mathrm{ps}]}\end{array}$ & $\begin{array}{c}\text { output } \\
\text { slope } \\
\text { [ps] }\end{array}$ \\
\hline 0 & 35.8 & 8.8 & 36.3 & 8.6 & 37.4 & 8.5 & 39.2 & 8.3 \\
\hline 40 & 37.7 & 10.0 & 38.0 & 10.1 & 39.3 & 9.6 & 40.8 & 10.8 \\
\hline 130 & 41.6 & 13.8 & 41.9 & 14.2 & 43.0 & 13.9 & 44.7 & 13.9 \\
\hline 220 & 45.0 & 18.0 & 45.3 & 18.0 & 46.3 & 18.3 & 48.1 & 18.2 \\
\hline 310 & 48.0 & 22.8 & 48.4 & 22.5 & 49.3 & 22.8 & 51.2 & 22.7 \\
\hline 400 & 50.9 & 26.9 & 51.2 & 27.0 & 52.1 & 27.1 & 54.0 & 27.0 \\
\hline
\end{tabular}

Replace Load size wid2 by its equivalent Load capacitance

\begin{tabular}{|c|c|c|c|c|c|c|c|c|}
\hline output-load2 [fF] \input-slope1 [ps] & \multicolumn{2}{|c|}{14.2} & \multicolumn{2}{|c|}{15.1} & \multicolumn{2}{|c|}{16.3} & \multicolumn{2}{|c|}{21.4} \\
\hline & $\begin{array}{c}\text { delay } \\
{[\mathrm{ps}]}\end{array}$ & $\begin{array}{c}\text { output } \\
\text { slope } \\
\text { [ps] }\end{array}$ & $\begin{array}{c}\text { delay } \\
{[\mathrm{ps}]}\end{array}$ & $\begin{array}{c}\text { output } \\
\text { slope } \\
{[\mathrm{ps}]}\end{array}$ & $\begin{array}{c}\text { delay } \\
{[\mathrm{ps}]}\end{array}$ & $\begin{array}{c}\text { output } \\
\text { slope } \\
{[\mathrm{ps}]}\end{array}$ & $\begin{array}{c}\text { delay } \\
{[\mathrm{ps}]}\end{array}$ & $\begin{array}{c}\text { output } \\
\text { slope } \\
\text { [ps] }\end{array}$ \\
\hline 0.0 & 35.8 & 8.8 & 36.3 & 8.6 & 37.4 & 8.5 & 39.2 & 8.3 \\
\hline 18.2 & 37.7 & 10.0 & 38.0 & 10.1 & 39.3 & 9.6 & 40.8 & 10.8 \\
\hline 59.3 & 41.6 & 13.8 & 41.9 & 14.2 & 43.0 & 13.9 & 44.7 & 13.9 \\
\hline 101.4 & 45.0 & 18.0 & 45.3 & 18.0 & 46.3 & 18.3 & 48.1 & 18.2 \\
\hline 142.8 & 48.0 & 22.8 & 48.4 & 22.5 & 49.3 & 22.8 & 51.2 & 22.7 \\
\hline 185.1 & 50.9 & 26.9 & 51.2 & 27.0 & 52.1 & 27.1 & 54.0 & 27.0 \\
\hline
\end{tabular}

Figure 5.4 SPICE to PrimeTime look up table for LUTblue:SUCC $+\mathrm{FIRE}_{i}-$. 
LUTblue:FIREi-Douti+

\begin{tabular}{|c|c|c|c|c|c|c|c|c|c|c|c|c|c|c|c|c|}
\hline \multirow[t]{2}{*}{ wid2 I wid1 } & \multicolumn{4}{|c|}{8} & \multicolumn{4}{|c|}{6} & \multicolumn{4}{|c|}{4} & \multicolumn{4}{|c|}{3} \\
\hline & slope & $\begin{array}{l}\% \text { Diff } \\
\text { to Avg }\end{array}$ & $\begin{array}{c}\text { delay } \\
\text { [ps] }\end{array}$ & $\begin{array}{l}\text { output } \\
\text { slope } \\
\text { [ps] }\end{array}$ & $\begin{array}{l}\text { input } \\
\text { slope } \\
\text { [ps] }\end{array}$ & $\begin{array}{l}\% \text { Diff } \\
\text { to Avg }\end{array}$ & $\begin{array}{l}\text { delay } \\
\text { [ps] }\end{array}$ & $\begin{array}{l}\text { output } \\
\text { slope } \\
\text { [ps] }\end{array}$ & $\begin{array}{c}\text { input } \\
\text { slope } \\
\text { [ps] }\end{array}$ & $\begin{array}{l}\% \text { Diff } \\
\text { to Avg }\end{array}$ & \begin{tabular}{|c|} 
delay \\
[ps]
\end{tabular} & $\begin{array}{l}\text { output } \\
\text { slope } \\
\text { [ps] }\end{array}$ & $\begin{array}{c}\text { input } \\
\text { slope } \\
\text { [ps] }\end{array}$ & $\begin{array}{l}\% \text { Diff } \\
\text { to Avg }\end{array}$ & $\begin{array}{c}\text { delay } \\
\text { [ps] }\end{array}$ & $\begin{array}{l}\text { output } \\
\text { slope } \\
\text { [ps] }\end{array}$ \\
\hline 0 & 11.6 & $0.0 \%$ & 20.5 & 21.0 & 12.4 & $0.0 \%$ & 20.9 & 21.5 & 14.4 & $0.0 \%$ & 21.7 & 21.5 & 14.7 & $0.0 \%$ & 22.3 & 21.7 \\
\hline $\begin{array}{c}\text { Average } \\
\text { input slope }\end{array}$ & 11.6 & $0.0 \%$ & & & 12.4 & $0.0 \%$ & & & 14.4 & $0.0 \%$ & & & 14.7 & $0.0 \%$ & & \\
\hline
\end{tabular}

Replace Driver size wid1 by the Average input slope that it produces

\begin{tabular}{|c|c|c|c|c|c|c|c|c|}
\hline \multirow[t]{2}{*}{ wid2 \input-slope1 [ps] } & \multicolumn{2}{|c|}{11.6} & \multicolumn{2}{|c|}{12.4} & \multicolumn{2}{|c|}{14.4} & \multicolumn{2}{|c|}{14.7} \\
\hline & $\begin{array}{c}\text { delay } \\
\text { [ps] }\end{array}$ & $\begin{array}{l}\text { output } \\
\text { slope } \\
\text { [ps] }\end{array}$ & $\begin{array}{l}\text { delay } \\
{[\mathrm{ps}]}\end{array}$ & $\begin{array}{l}\text { output } \\
\text { slope } \\
\text { [ps] }\end{array}$ & $\begin{array}{l}\text { delay } \\
\text { [ps] }\end{array}$ & $\begin{array}{l}\text { output } \\
\text { slope } \\
\text { [ps] }\end{array}$ & $\begin{array}{c}\text { delay } \\
\text { [ps] }\end{array}$ & $\begin{array}{l}\text { output } \\
\text { slope } \\
\text { [ps] }\end{array}$ \\
\hline 0 & 20.5 & 21.0 & 20.9 & 21.5 & 21.7 & 21.5 & 22.3 & 21.7 \\
\hline
\end{tabular}

Replace Load size wid2 by its equivalent Load capacitance

\begin{tabular}{|c|c|c|c|c|c|c|c|c|}
\hline output-load2 [fF] \ input-slope1 [ps] & \multicolumn{2}{|c|}{11.6} & \multicolumn{2}{|c|}{12.4} & \multicolumn{2}{|c|}{14.4} & \multicolumn{2}{|c|}{14.7} \\
\hline & $\begin{array}{c}\text { delay } \\
{[\mathrm{ps}]}\end{array}$ & $\begin{array}{c}\text { output } \\
\text { slope } \\
\text { [ps] }\end{array}$ & $\begin{array}{c}\text { delay } \\
{[\mathrm{ps}]}\end{array}$ & $\begin{array}{c}\text { output } \\
\text { slope } \\
\text { [ps] }\end{array}$ & $\begin{array}{c}\text { delay } \\
{[\mathrm{ps}]}\end{array}$ & $\begin{array}{c}\text { output } \\
\text { slope } \\
\text { [ps] }\end{array}$ & $\begin{array}{c}\text { delay } \\
{[\mathrm{ps}]}\end{array}$ & $\begin{array}{c}\text { output } \\
\text { slope } \\
\text { [ps] }\end{array}$ \\
\hline 0.0 & 20.5 & 21.0 & 20.9 & 21.5 & 21.7 & 21.5 & 22.3 & 21.7 \\
\hline
\end{tabular}

Figure 5.5 SPICE to PrimeTime look up table for LUTblue:FIRE $i-$ Dout $_{i}+$. 


\section{LUTred/blue:FIRE $\mathbf{j}_{\mathbf{j}} \mathrm{SUCC}_{\mathbf{j}+}$}

\begin{tabular}{|c|c|c|c|c|c|c|c|c|c|c|c|c|c|c|c|c|}
\hline \multirow[t]{2}{*}{ wid2 \ wid1 } & \multicolumn{4}{|c|}{8} & \multicolumn{4}{|c|}{6} & \multicolumn{4}{|c|}{4} & \multicolumn{4}{|c|}{3} \\
\hline & $\begin{array}{c}\text { input } \\
\text { slope } \\
\text { [ps] }\end{array}$ & $\begin{array}{l}\% \text { Diff } \\
\text { to Avg }\end{array}$ & $\begin{array}{l}\text { delay } \\
\text { [ps] }\end{array}$ & $\begin{array}{l}\text { output } \\
\text { slope } \\
\text { [ps] }\end{array}$ & $\begin{array}{l}\text { input } \\
\text { slope } \\
\text { [ps] }\end{array}$ & $\begin{array}{l}\% \text { Diff } \\
\text { to Avg }\end{array}$ & $\begin{array}{l}\text { delay } \\
\text { [ps] }\end{array}$ & $\begin{array}{l}\text { output } \\
\text { slope } \\
\text { [ps] }\end{array}$ & $\begin{array}{l}\text { input } \\
\text { slope } \\
\text { [ps] }\end{array}$ & $\begin{array}{l}\% \text { Diff } \\
\text { to Avg }\end{array}$ & $\begin{array}{l}\text { delay } \\
\text { [ps] }\end{array}$ & $\begin{array}{l}\text { output } \\
\text { slope } \\
\text { [ps] }\end{array}$ & $\begin{array}{l}\text { input } \\
\text { slope } \\
\text { [ps] }\end{array}$ & $\begin{array}{l}\% \text { Diff } \\
\text { to Avg }\end{array}$ & $\begin{array}{r}\text { delay } \\
\text { [ps] }\end{array}$ & $\begin{array}{l}\text { output } \\
\text { slope } \\
\text { [ps] }\end{array}$ \\
\hline 0 & 10.7 & $10.1 \%$ & 26.3 & 12.2 & 12.2 & $3.2 \%$ & 26.7 & 12.5 & 14.4 & $4.6 \%$ & 27.6 & 12.7 & 16.6 & $3.5 \%$ & 28.5 & 12.9 \\
\hline 10 & 11.7 & $1.7 \%$ & 28.9 & 15.2 & 12.7 & $-0.8 \%$ & 29.0 & 14.9 & 15.4 & $-2.0 \%$ & 30.0 & 15.4 & 17.2 & $0.0 \%$ & 30.9 & 16.0 \\
\hline 33 & 11.7 & $1.7 \%$ & 33.1 & 20.7 & 12.7 & $-0.8 \%$ & 33.7 & 20.8 & 15.2 & $-0.7 \%$ & 34.7 & 21.3 & 17.4 & $-1.2 \%$ & 35.6 & 21.4 \\
\hline 56 & 11.2 & $5.9 \%$ & 37.1 & 27.0 & 12.7 & $-0.8 \%$ & 37.8 & 27.4 & 15.2 & $-0.7 \%$ & 38.8 & 27.6 & 17.5 & $-1.7 \%$ & 39.9 & 27.7 \\
\hline 78 & 11.5 & $3.4 \%$ & 41.0 & 34.1 & 12.6 & $0.0 \%$ & 41.6 & 33.9 & 15.3 & $-1.3 \%$ & 42.4 & 34.0 & 17.1 & $0.6 \%$ & 43.4 & 34.0 \\
\hline 100 & 14.5 & $-21.8 \%$ & 44.3 & 40.5 & 12.7 & $-0.8 \%$ & 45.3 & 40.4 & 14.9 & $1.3 \%$ & 46.4 & 40.4 & 17.4 & $-1.2 \%$ & 47.2 & 41.0 \\
\hline $\begin{array}{l}\text { Average } \\
\text { input slope }\end{array}$ & 11.9 & $0.0 \%$ & & & 12.6 & $0.0 \%$ & & & 15.1 & $0.0 \%$ & & & 17.2 & $0.0 \%$ & & \\
\hline
\end{tabular}

Replace Driver size wid1 by the Average input slope that it produces

\begin{tabular}{|c|c|c|c|c|c|c|c|c|}
\hline wid2 Input-slope1 [ps] & \multicolumn{2}{|c|}{11.9} & \multicolumn{2}{c|}{12.6} & \multicolumn{2}{|c|}{15.1} & \multicolumn{2}{|c|}{17.2} \\
\hline & $\begin{array}{c}\text { delay } \\
\text { [ps] }\end{array}$ & $\begin{array}{c}\text { output } \\
\text { slope } \\
\text { [ps] }\end{array}$ & $\begin{array}{c}\text { delay } \\
{[\mathrm{ps}]}\end{array}$ & $\begin{array}{c}\text { output } \\
\text { slope } \\
\text { [ps] }\end{array}$ & $\begin{array}{c}\text { delay } \\
\text { [ps] }\end{array}$ & $\begin{array}{c}\text { output } \\
\text { slope } \\
\text { [ps] }\end{array}$ & $\begin{array}{c}\text { delay } \\
{[\mathrm{ps}]}\end{array}$ & $\begin{array}{c}\text { output } \\
\text { slope } \\
\text { [ps] }\end{array}$ \\
\hline 0 & 26.3 & 12.2 & 26.7 & 12.5 & 27.6 & 12.7 & 28.5 & 12.9 \\
\hline 10 & 28.9 & 15.2 & 29.0 & 14.9 & 30.0 & 15.4 & 30.9 & 16.0 \\
\hline 33 & 33.1 & 20.7 & 33.7 & 20.8 & 34.7 & 21.3 & 35.6 & 21.4 \\
\hline 56 & 37.1 & 27.0 & 37.8 & 27.4 & 38.8 & 27.6 & 39.9 & 27.7 \\
\hline 78 & 41.0 & 34.1 & 41.6 & 33.9 & 42.4 & 34.0 & 43.4 & 34.0 \\
\hline 100 & 44.3 & 40.5 & 45.3 & 40.4 & 46.4 & 40.4 & 47.2 & 41.0 \\
\hline
\end{tabular}

Replace Load size wid2 by its equivalent Load capacitance

\begin{tabular}{|c|c|c|c|c|c|c|c|c|}
\hline & $\begin{array}{c}|c| \\
\text { output-load2 [fF] Iinput-slope1 [ps] }\end{array}$ & \multicolumn{2}{|c|}{11.9} & \multicolumn{2}{|c|}{12.6} & \multicolumn{2}{|c|}{15.1} & \multicolumn{2}{|c|}{17.2} \\
\hline & $\begin{array}{c}\text { output } \\
\text { slope } \\
{[\mathrm{ps}]}\end{array}$ & $\begin{array}{c}\text { delay } \\
{[\mathrm{ps}]}\end{array}$ & $\begin{array}{c}\text { output } \\
\text { slope } \\
{[\mathrm{ps}]}\end{array}$ & $\begin{array}{c}\text { delay } \\
{[\mathrm{ps}]}\end{array}$ & $\begin{array}{c}\text { output } \\
\text { slope } \\
{[\mathrm{ps}]}\end{array}$ & $\begin{array}{c}\text { delay } \\
{[\mathrm{ps}]}\end{array}$ & $\begin{array}{c}\text { output } \\
\text { slope } \\
{[\mathrm{ps}]}\end{array}$ \\
\hline 0.0 & 26.3 & 12.2 & 26.7 & 12.5 & 27.6 & 12.7 & 28.5 & 12.9 \\
\hline 4.6 & 28.9 & 15.2 & 29.0 & 14.9 & 30.0 & 15.4 & 30.9 & 16.0 \\
\hline 15.0 & 33.1 & 20.7 & 33.7 & 20.8 & 34.7 & 21.3 & 35.6 & 21.4 \\
\hline 25.5 & 37.1 & 27.0 & 37.8 & 27.4 & 38.8 & 27.6 & 39.9 & 27.7 \\
\hline 35.5 & 41.0 & 34.1 & 41.6 & 33.9 & 42.4 & 34.0 & 43.4 & 34.0 \\
\hline 45.6 & 44.3 & 40.5 & 45.3 & 40.4 & 46.4 & 40.4 & 47.2 & 41.0 \\
\hline
\end{tabular}

Figure 5.6 SPICE to PrimeTime look up table for LUTred/blue:FIRE $i+\mathrm{SUCC}_{i}+$. 


\section{LUTblue/green:FIREj+PREDi-}

\begin{tabular}{|c|c|c|c|c|c|c|c|c|c|c|c|c|c|c|c|c|c|c|c|c|}
\hline wid2 \ wid1 & \multicolumn{4}{|c|}{40} & \multicolumn{4}{|c|}{31} & \multicolumn{4}{|c|}{22} & \multicolumn{4}{|c|}{13} & \multicolumn{4}{|c|}{4} \\
\hline 0 & 10 & $3.8 \%$ & 3.7 & 5.2 & 11.6 & $-7.4 \%$ & 3.6 & 5.5 & 12.3 & $-9.8 \%$ & 3.7 & 5.6 & 12.2 & $-2.5 \%$ & 3.4 & 6.0 & 21.7 & $-8.0 \%$ & 3.8 & 8.5 \\
\hline 10 & \begin{tabular}{|l|}
10.8 \\
\end{tabular} & $-3.8 \%$ & 4.6 & 7.5 & \begin{tabular}{|l|}
11.2 \\
\end{tabular} & $-3.7 \%$ & 4.7 & 7.6 & 11.8 & $-5.4 \%$ & 5.0 & 7.8 & 11.8 & $0.8 \%$ & 5.7 & 7.5 & 21.5 & $-7.0 \%$ & 6.8 & 10.5 \\
\hline 78 & 9.7 & $6.7 \%$ & 14.2 & 19.1 & 10.0 & $7.4 \%$ & 14.4 & 19.0 & 10.8 & $3.6 \%$ & 14.4 & 19.6 & 12.0 & $-0.8 \%$ & 15.1 & 20.0 & 19.3 & $4.0 \%$ & 18.0 & 22.7 \\
\hline 100 & 10.3 & $1.0 \%$ & 16.6 & 24.0 & 10.1 & $6.5 \%$ & 17.0 & 24.1 & 10.5 & $6.2 \%$ & 17.3 & 24.0 & 11.2 & $5.9 \%$ & 17.9 & 24.4 & 18.5 & $8.0 \%$ & 20.9 & 27.4 \\
\hline $\begin{array}{c}\text { Average } \\
\text { input slope }\end{array}$ & 10.4 & $0.0 \%$ & & & 10.8 & $0.0 \%$ & & & 11.2 & $0.0 \%$ & & & 11.9 & $0.0 \%$ & & & 20.1 & $0.0 \%$ & & \\
\hline
\end{tabular}

Replace Driver size wid1 by the Average input slope that it produces

\begin{tabular}{|c|c|c|c|c|c|c|c|c|c|c|}
\hline & \multicolumn{2}{|c|}{10.4} & \multicolumn{2}{c|}{10.8} & \multicolumn{2}{c|}{11.2} & \multicolumn{2}{|c|}{11.9} & \multicolumn{2}{c|}{20.1} \\
\hline & $\begin{array}{c}\text { delay } \\
{[\mathrm{ps}]}\end{array}$ & $\begin{array}{c}\text { output } \\
\text { slope } \\
{[\mathrm{ps}]}\end{array}$ & $\begin{array}{c}\text { delay } \\
\text { [ps] }\end{array}$ & $\begin{array}{c}\text { output } \\
\text { slope } \\
{[\mathrm{ps}]}\end{array}$ & $\begin{array}{c}\text { delay } \\
{[\mathrm{ps}]}\end{array}$ & $\begin{array}{c}\text { output } \\
\text { slope } \\
{[\mathrm{ps}]}\end{array}$ & $\begin{array}{c}\text { delay } \\
{[\mathrm{ps}]}\end{array}$ & $\begin{array}{c}\text { output } \\
\text { slope } \\
\text { [ps] }\end{array}$ & $\begin{array}{c}\text { delay } \\
{[\mathrm{ps}]}\end{array}$ & $\begin{array}{c}\text { output } \\
\text { slope } \\
\text { [ps] }\end{array}$ \\
\hline 0 & 3.7 & 5.2 & 3.6 & 5.5 & 3.7 & 5.6 & 3.4 & 6.0 & 3.8 & 8.5 \\
\hline 10 & 4.6 & 7.5 & 4.7 & 7.6 & 5.0 & 7.8 & 5.7 & 7.5 & 6.8 & 10.5 \\
\hline 33 & 8.1 & 10.9 & 8.3 & 11.0 & 9.1 & 10.7 & 9.1 & 11.7 & 11.1 & 14.5 \\
\hline 56 & 11.2 & 15.1 & 11.4 & 15.2 & 11.9 & 15.0 & 12.2 & 15.5 & 14.9 & 18.4 \\
\hline 78 & 14.2 & 19.1 & 14.4 & 19.0 & 14.4 & 19.6 & 15.1 & 20.0 & 18.0 & 22.7 \\
\hline 100 & 16.6 & 24.0 & 17.0 & 24.1 & 17.3 & 24.0 & 17.9 & 24.4 & 20.9 & 27.4 \\
\hline
\end{tabular}

Replace Load size wid2 by its equivalent Load capacitance

\begin{tabular}{|c|c|c|c|c|c|c|c|c|c|c|}
\hline \multirow[t]{2}{*}{ output-load2 [fF] \ input-slope1 [ps] } & \multicolumn{2}{|c|}{10.4} & \multicolumn{2}{|c|}{10.8} & \multicolumn{2}{|c|}{11.2} & \multicolumn{2}{|c|}{11.9} & \multicolumn{2}{|c|}{20.1} \\
\hline & $\begin{array}{l}\text { delay } \\
\text { [ps] }\end{array}$ & $\begin{array}{c}\text { output } \\
\text { slope } \\
\text { [ps] }\end{array}$ & $\begin{array}{l}\text { delay } \\
\text { [ps] }\end{array}$ & $\begin{array}{c}\text { output } \\
\text { slope } \\
\text { [ps] }\end{array}$ & $\begin{array}{l}\text { delay } \\
\text { [ps] }\end{array}$ & $\begin{array}{c}\text { output } \\
\text { slope } \\
\text { [ps] }\end{array}$ & $\begin{array}{l}\text { delay } \\
\text { [ps] }\end{array}$ & $\begin{array}{c}\text { output } \\
\text { slope } \\
{[\mathrm{ps}]}\end{array}$ & $\begin{array}{l}\text { delay } \\
\text { [ps] }\end{array}$ & $\begin{array}{l}\text { output } \\
\text { slope } \\
\text { [ps] }\end{array}$ \\
\hline 0.0 & 3.7 & 5.2 & 3.6 & 5.5 & 3.7 & 5.6 & 3.4 & 6.0 & 3.8 & 8.5 \\
\hline 4.6 & 4.6 & 7.5 & 4.7 & 7.6 & 5.0 & 7.8 & 5.7 & 7.5 & 6.8 & 10.5 \\
\hline 15.0 & 8.1 & 10.9 & 8.3 & 11.0 & 9.1 & 10.7 & 9.1 & 11.7 & 11.1 & 14.5 \\
\hline 25.5 & 11.2 & 15.1 & 11.4 & 15.2 & 11.9 & 15.0 & 12.2 & 15.5 & 14.9 & 18.4 \\
\hline 35.5 & 14.2 & 19.1 & 14.4 & 19.0 & 14.4 & 19.6 & 15.1 & 20.0 & 18.0 & 22.7 \\
\hline 45.6 & 16.6 & 24.0 & 17.0 & 24.1 & \begin{tabular}{|l}
17.3 \\
\end{tabular} & 24.0 & 17.9 & 24.4 & 20.9 & 27.4 \\
\hline
\end{tabular}

Figure 5.7 SPICE to PrimeTime look up table for LUTblue/green:FIRE ${ }_{i}+\mathrm{PRED}_{i}-$. 


\subsubsection{A Quick Sanity Check of the PrimeTime Look Up Tables}

Figure 5.8 and Figure 5.9 below jointly show five pairs of landscape graphs with the delay and output slope information from the five look up tables displayed in Figure 5.3

to Figure 5.7. Both the absolute numbers in picoseconds and the relative changes in the landscape elevations are plausible for the given range of input slopes and output loads and the given paths in the 6-4 GasP circuit.

We normalized the vertical axes to enable apples-to-apples comparisons between the paths: delays go from $0 \mathrm{ps}$ to $80 \mathrm{ps}$, with a color distinction every $10 \mathrm{ps}$, and output slopes go from $0 \mathrm{ps}$ to $45 \mathrm{ps}$, with a color distinction every $5 \mathrm{ps}$.
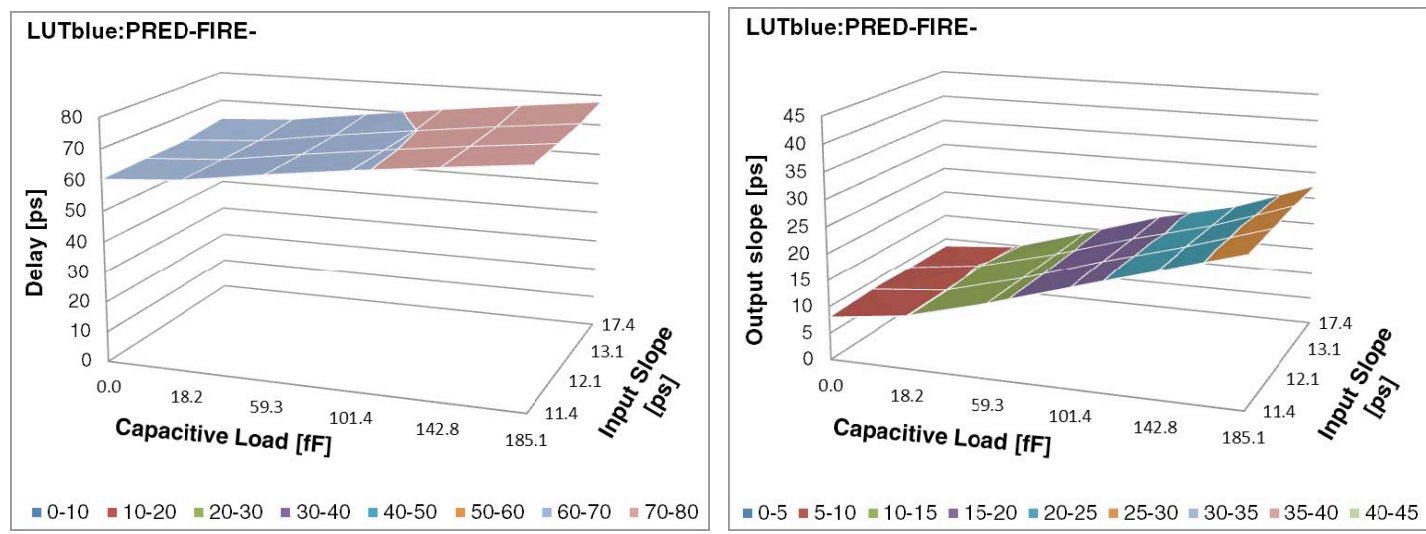

Figure 5.8 (continues in Figure 5.9) Landscape graphs with the delay and output slope information from the five look up tables in Figure 5.3 to Figure 5.7. We normalized the vertical axes to enable apples-to-apples comparisons between the paths: delays go from $0 \mathrm{ps}$ to $80 \mathrm{ps}$ with a color distinction every $10 \mathrm{ps}$, and output slopes from $0 \mathrm{ps}$ to $45 \mathrm{ps}$ with a color distinction every $5 \mathrm{ps}$. We dropped the index ' $i$ ' from the path names, because the paths are all contained within a 6-4 GasP module. For instance, we use LUTblue:PRED-FIRE - instead of the indexed name LUTblue:PRED ${ }_{i}-\mathrm{FIRE}_{i}-$. 

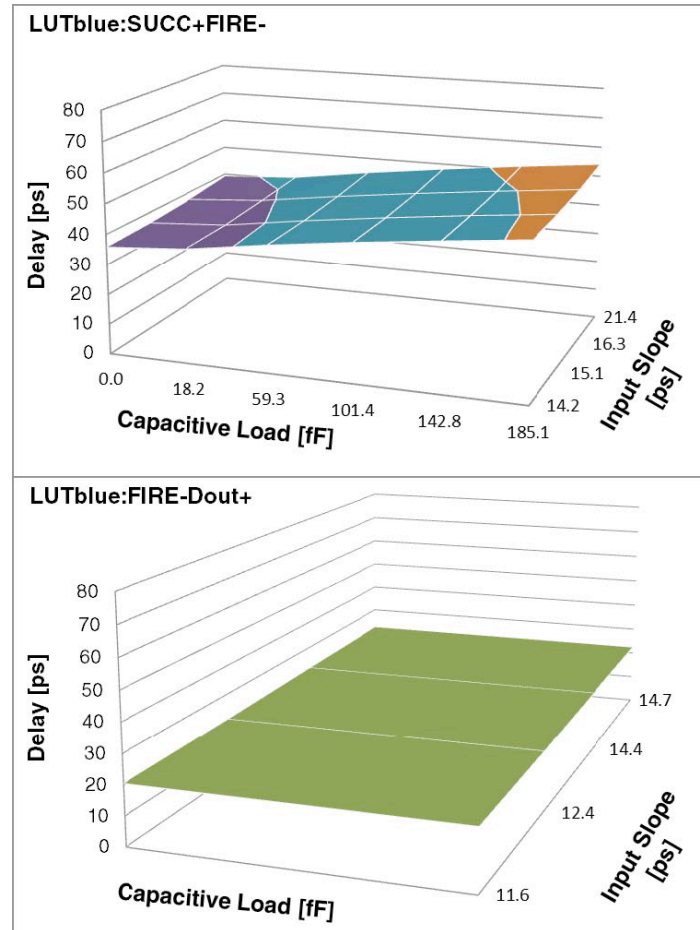

LUTred/blue:FIRE+SUCC+

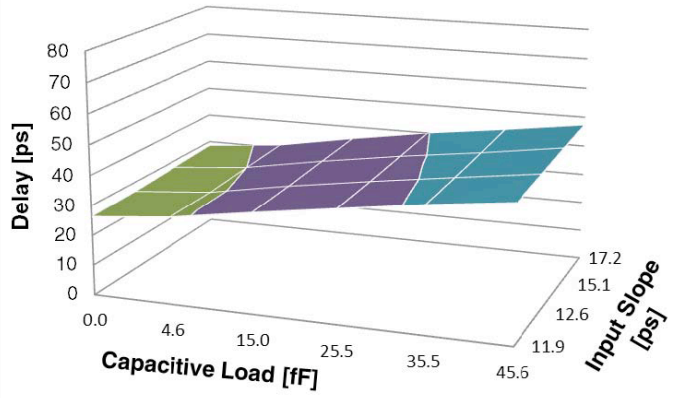

LUTblue/green:FIRE+PRED-

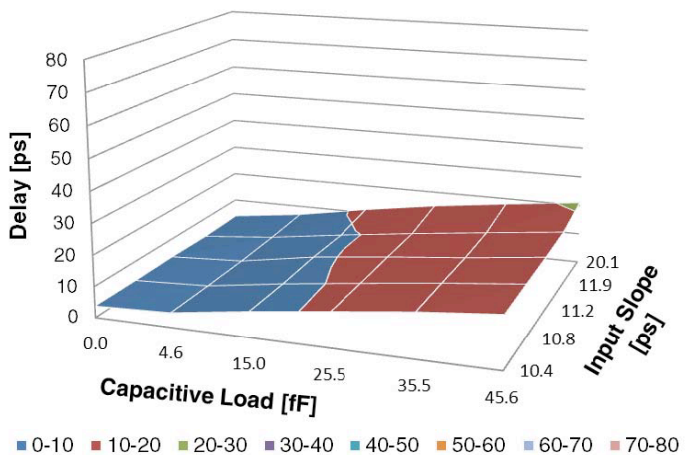

LUTblue:SUCC+FIRE-
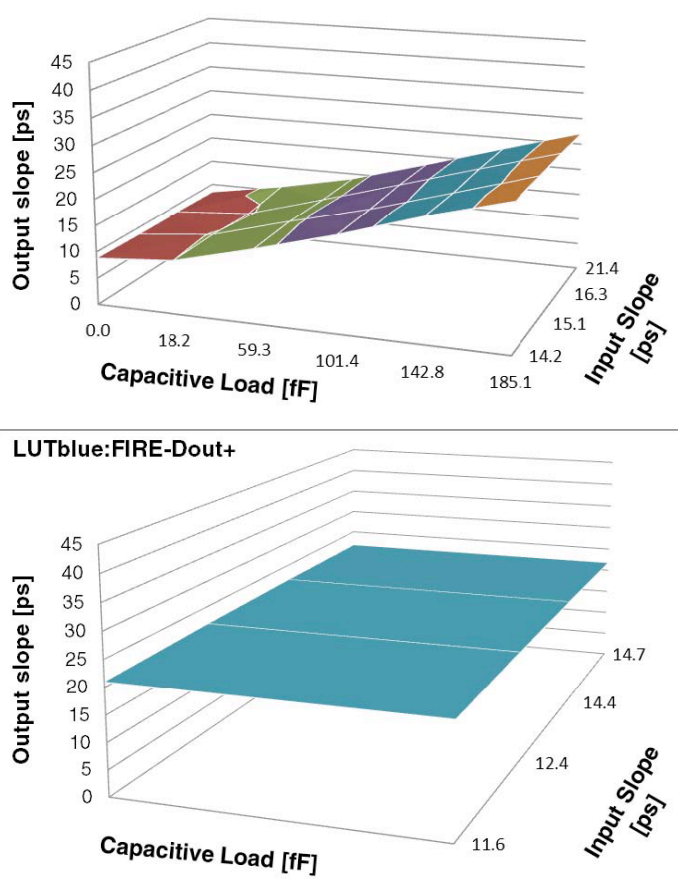

LUTred/blue:FIRE+SUCC+

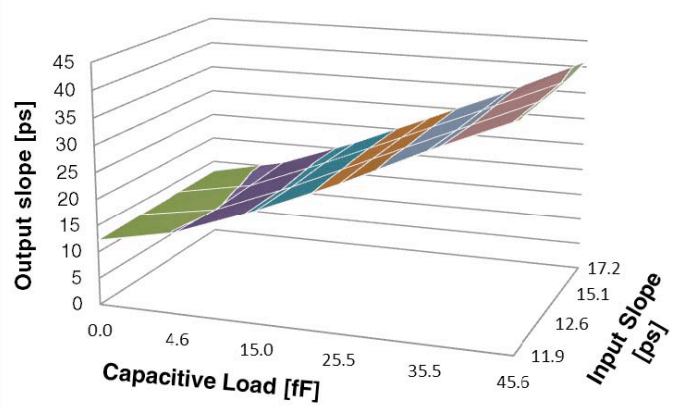

LUTblue/green:FIRE+PRED-

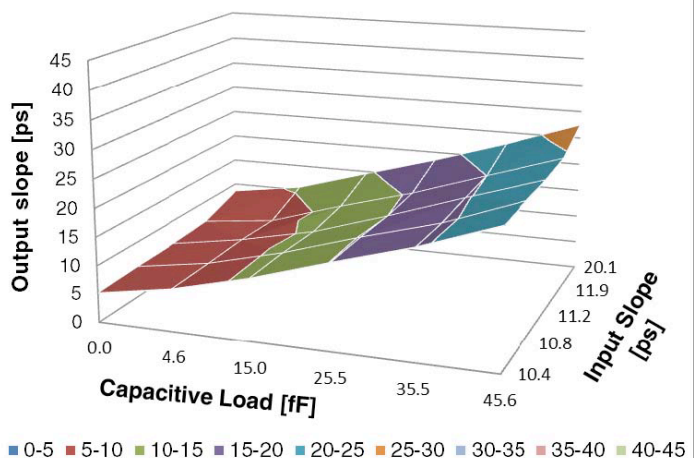

Figure 5.9 Landscape graphs with delay and output slope information - continued. 


\subsection{STEP 2: From 6-4 GasP Module to PrimeTime Black Box}

We now get to the USC part of the static timing analysis flow. In his Master of Science thesis [2, 3], Prasad Joshi presents a 6-4 GasP black box solution approach that solves the issues that PrimeTime has with bi-directional pins and combinational loops. He splits each bi-directional pin into separate input and output pins. He cuts combinational loops by unrolling them and by renaming the loop-back pins as additional pseudo-pins. He calls his black box model a 'Split Pin Architecture'.

Figure 5.10 shows the split pin architecture for GasP modules M1 and M2 in Figure 2.1, with its black box icon in the top-right corner. PrimeTime sees only the black box name, which is 'GasP', and its pins: split pins PRED_OUT and PRED_IN, split pins SUCC_OUT and SUCC_IN, pin FIRE and its pseudo-pin FIRE_PS, and pin Dout. The internal circuitry is invisible to PrimeTime. Prasad's version does not use the pin Dout. We have added Dout to our black box model because our versions of the relative timing constraints for 6-4 GasP are more detailed and more accurate than those used by Prasad.

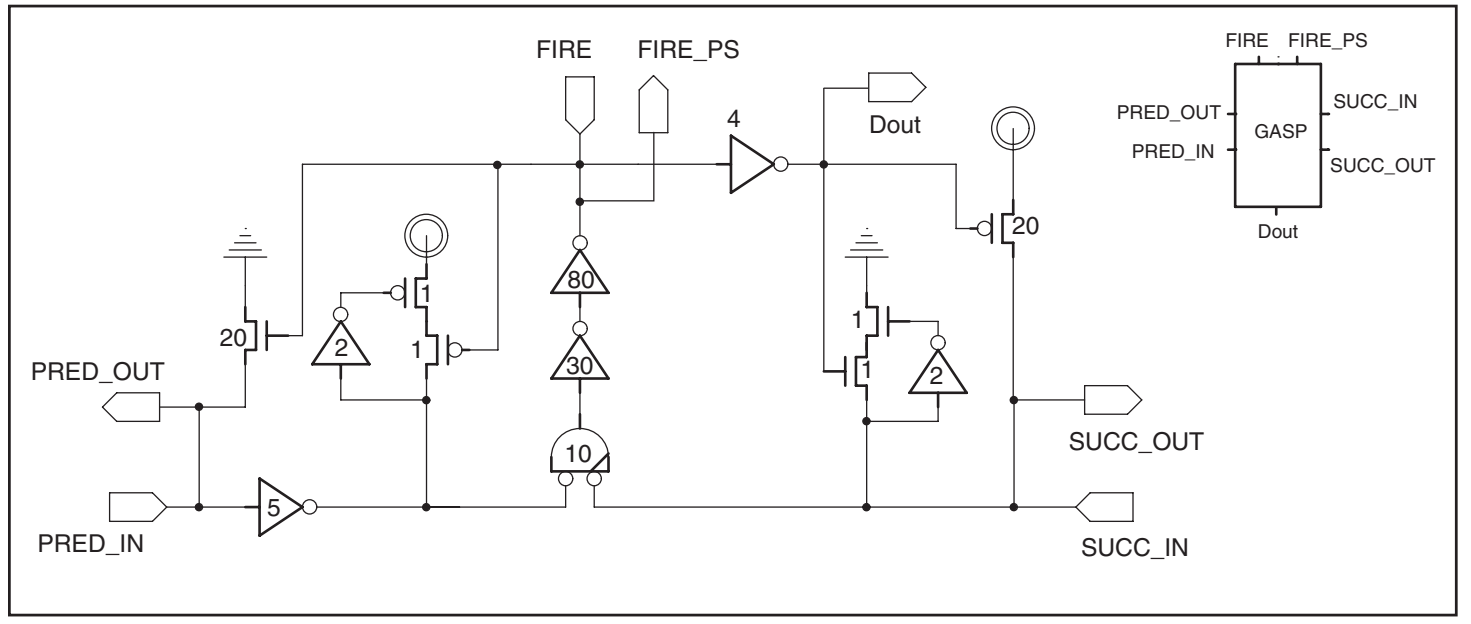

Figure 5.10 Split pin architecture of a basic 6-4 GasP module with its black box icon. 


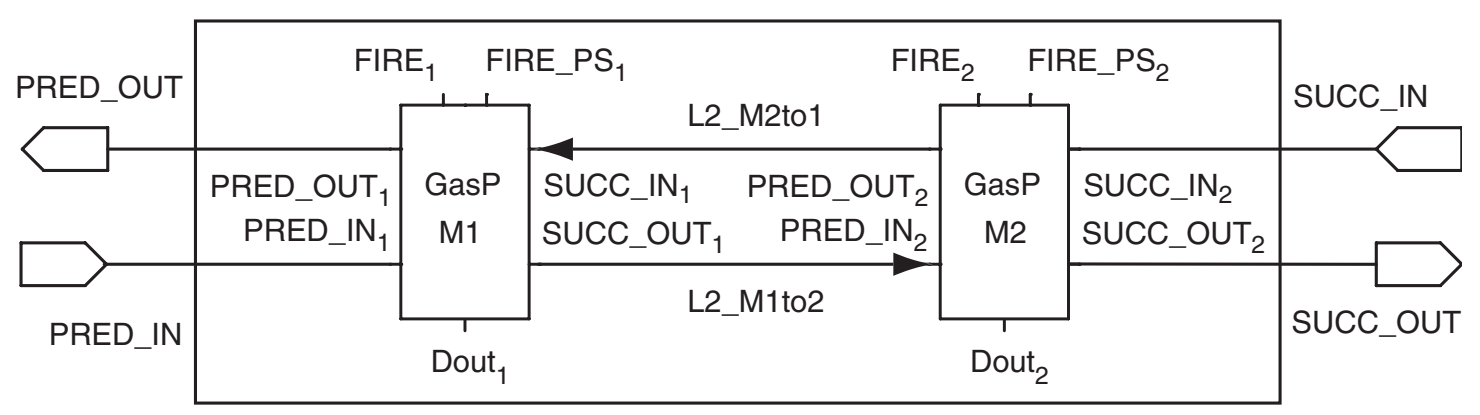

Figure 5.11 PrimeTime view of the two-stage FIFO in Figure 2.1, page 11. The two 6-4 GasP modules M1 and M2 have each been replaced by the black box icon from Figure 5.10. The bi-directional wire for single-track channel L2 has been replaced by two uni-directional wires going in opposite directions.

Figure 5.11 shows what the two-stage FIFO of Figure 2.1 on page 11 looks like when we replace the 6-4 GasP modules M1 and M2 by their black box icons. This is how PrimeTime gets to see Figure 2.1 . We will use Verilog to describe the corresponding netlist configuration in Figure 5.11. The netlist can be found in Appendix J.

\subsection{STEP 3: Create a Library File with SPICED Look Up Tables}

We continue with the USC part of the static timing analysis flow by constructing a PrimeTime library file with the timing information for the 6-4 GasP black box. Most of the groundwork has been done by Prasad Joshi in [2, 3]. Our contribution consists of replacing Prasad's fictitious one-dimensional look up tables with the real, SPICEsimulated, two-dimensional versions in Figures 5.3-5.7. There is one caveat: the look up tables in Figures 5.3-5.7 were generated for the GasP schematics in Figure 2.1 on page 11, and not for the split pin versions in Figures 5.10-5.11. So, our first task is to map the original path name associated with each look up table to the PrimeTime path name for the black box split pin architecture. In Section 5.4.1 we translate the original names for our look up tables into PrimeTime names. In Section 5.4.2, we use the PrimeTime names to create a library file for the two-stage FIFO in Figure 5.11. 


\subsubsection{STEP 3.1: Rename Look Up Tables to Match Black Box Names}

This section discusses our approach in renaming the input and output signal names and relative timing paths in the original two-stage GasP FIFO in Figure 2.1, page 11, to the corresponding names and paths in the new two-stage black box FIFO in Figure 5.11, with the black box as defined in Figure 5.10. Our renaming follows the renaming that Prasad took in his Master of Science thesis [2]. Nevertheless, we would like to explicitly emphasize the renaming part, because it is less trivial than one might think. The crux in making a valid renaming for signal names is to make it valid for what we do with PrimeTime and for what PrimeTime does with these names.

We use PrimeTime to validate the relative timing constraints RT1 to RT4 of Figure 2.2, page 13. This means that we instruct PrimeTime to compare the timing results of the red and the blue paths in RT1 in Figure 2.2(a), and to validate that the delay of the red path is less than that of the blue path. It also means that we instruct PrimeTime to compare the timing results of the green and the blue paths in RT3 in Figure 2.2(c), and to validate that the delay of the green path is less than that of the blue path. We instruct PrimeTime to do similar comparisons for RT2 and RT4 - see Figure 2.2 for details. PrimeTime may use only the input and output signals names that the black box icon allows it to see. It uses these names to construct a path through the circuit, to time this path, and to compare the timing information of this particular path to that of other paths. PrimeTime can connect black box signal names in precisely two ways. It connects the signal names if either the netlist or a look up table connects them.

For a valid renaming, the connectivity that PimeTime sees must match the connectivity in Figure 2.2. One way to achieve this is to redraw Figure 2.2 with black box signal names, and to make sure that the connectivity matches that of the original Figure 2.2. 
The result follows below in Figure 5.12. Note that we cut off the 'nodrive' paths endings at Dout 1 for Figure 5.12(a)-(b) and at FIRE_PS 2 for Figure 5.12(c)-(d). This is consistent with the partitioned relative timing paths in Figures 4.3-4.6, pages 42-45, from which we generated the look up tables in Figures 5.3-5.7.

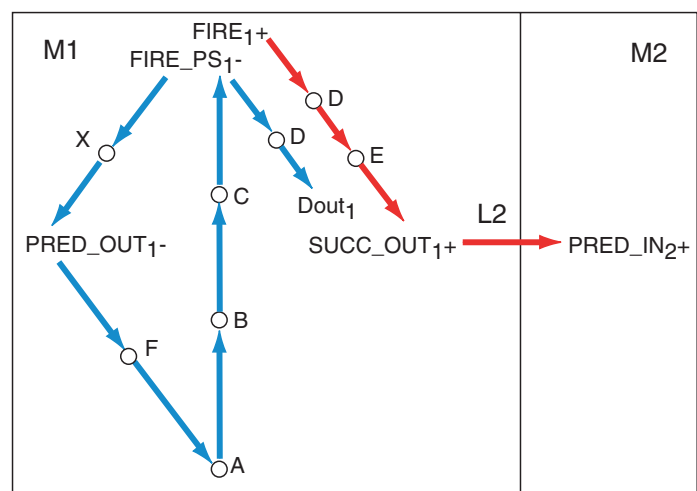

(a) RT1 or RT(L2) TrfF-RstB : red path < blue path

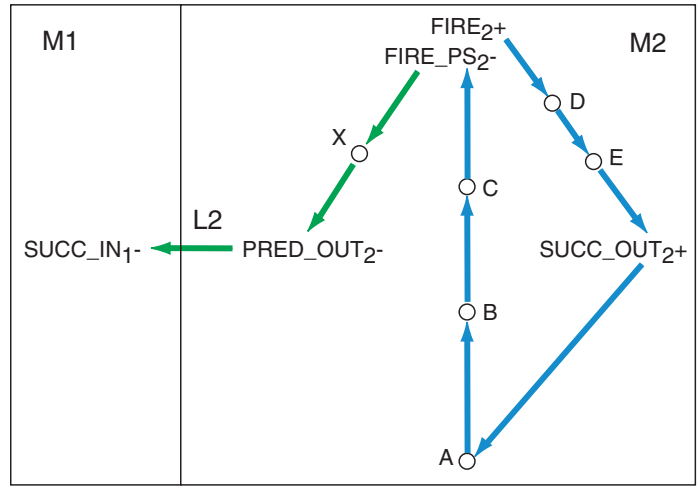

(c) RT3 or RT(L2) TrfB-RstF : green path < blue path

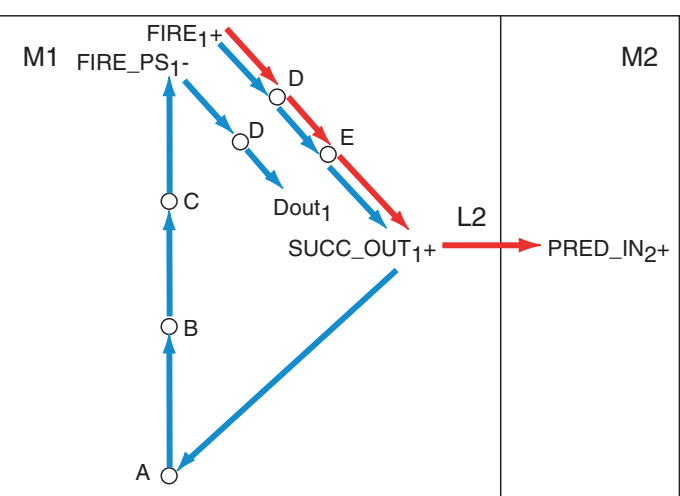

(b) RT2 or RT(L2) TrfF-RstF : red path < blue path

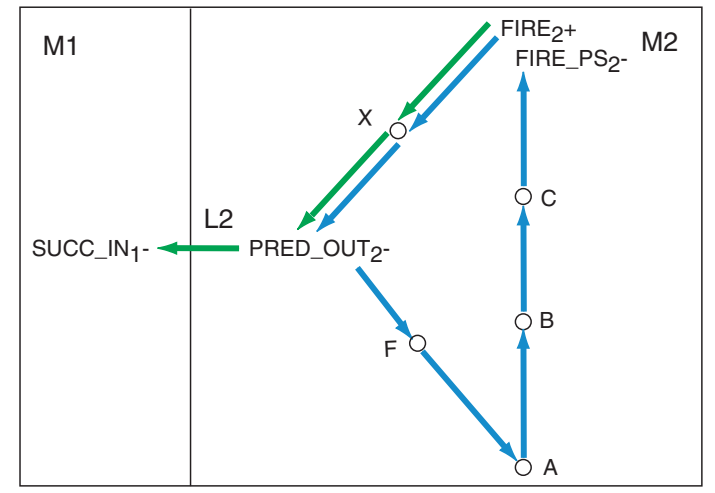

(d) RT4 or RT(L2) TrfB-RstB : green path < blue path

Figure 5.12 PrimeTime version of Figure 2.2, page 13. The pin names for RT1 in (a), RT2 in (b), RT3 in (c), and RT4 in (d) match with the names of the split pin architecture of the 6-4 GasP black box of Figure 5.10. The blue, red and green paths go through the same gates as the original blue, red and green paths in Figure 2.2, with one exception: the 'nodrive' endings now end in Dout ${ }_{1}$ or FIRE_PS ${ }_{2}$. This is consistent with the Dout or $\mathrm{FIRE}_{2}$ path endings for the look up tables in Figure 5.3 to Figure 5.7, pages 98-102. 
At the beginning of this section, we mentioned that the renaming is less trivial than one might think. The reason is that the renaming is not determined by the input or output direction of a pin, which is a typical first guess. Rather it's determined by the connectivity of the blue, red and green relative timing paths. Take for instance the RT2 blue path in Figure 2.2(b). As a first guess, looking at input-to-output path directions and ignoring path continuity, one is tempted to map the individual sub-paths as follows:

\section{Wrong signal name mapping:}

1. FIRE $_{1}+$ to $\mathrm{SUCC}_{1}+\quad \rightarrow \quad$ FIRE $_{1}+$ to SUCC_OUT $_{1}+$

2. SUCC $_{1}+$ to $\mathrm{FIRE}_{1}-\quad \rightarrow \quad \mathrm{SUCC}_{-} \mathrm{IN}_{1}+$ to FIRE_PS $1-$

3. FIRE $_{1}-$ to Dout $_{1}+\left(\right.$ to SUCC $_{1}$ nodrive $) \rightarrow$ FIRE $_{1}-$ to Dout $_{1}+$

This mapping would kill PrimeTime's ability to correctly time the self-resetting loop. Connecting the loop now requires a path extension from SUCC_OUT $1+$ to $\mathrm{SUCC}_{-} \mathrm{IN}_{1}+$ and from FIRE_PS 1 - to FIRE $_{1}-$. The two extensions are non-existent in Figure 5.11. As a result, PrimeTime will not be able to construct RT2's blue path. But even if the extensions would exist, the resulting loop would very likely go through more gates than the original self-resetting loop in Figure 2.2(b). To correctly map the self-resetting loop, we must mimic its connectivity and continuity, as we do in Figure 5.12(b):

\section{Correct signal name mapping:}

1. FIRE $_{1}+$ to $\mathrm{SUCC}_{1}+\quad \rightarrow$ FIRE $_{1}+$ to $\mathrm{SUCC}_{-} \mathrm{OUT}_{1}+$

2. SUCC $_{1}+$ to FIRE $_{1}-\quad \rightarrow \quad$ SUCC_OUT $_{1}+$ to FIRE_PS $1-$

3. FIRE $_{1}-$ to Dout $_{1}+\left(\right.$ to SUCC $_{1}$ nodrive $) \rightarrow$ FIRE_PS $_{1}-$ to Dout $_{1}+$ 
The table in Figure 5.13 shows the new PrimeTime names for the look up tables in Figure 5.3 -5.7, pages 98-102. The names are based on the signal names used in Figure 5.12, and include the (output load $\times$ input slope) table size information to facilitate readability and validation of the table contents. Their syntax is compatible with the Synopsys Liberty library naming conventions [33]. Each new name maintains the timing information embedded in the original name and reflects the size of the table.

\begin{tabular}{|c|c|}
\hline Original name & PrimeTime name \\
\hline LUTblue:PRED ${ }_{i}-$ FIRE $_{i}-$ & :PREDOU' \\
\hline LUTblue:SUCC ${ }_{i}+\mathrm{FIRE}_{i}-$ & LUTblue:SUCCOUTi_r_FIREPSi_f_6x4 \\
\hline LUTblue:FIRE $_{i}-$ Dout $_{i}+$ & LUTblue:FIREPSi_f_ Douti_r_1x4 \\
\hline LUTred/blue:FIRE $_{i}+$ SUCC $_{i}+$ & LUTredblue:FIREi_r_SUCCOUTi_r_6x4 \\
\hline LUTred/blue:FIRE $_{i}+$ PRED $_{i}-$ & LUTbluegreen:FIREi_r_PREDOUTi_f_6 \\
\hline
\end{tabular}

Figure 5.13 Renaming of our SPICED look up tables into PrimeTime look up table names that reflect the black box pin names in Figure 5.10. Notice that we replaced the ' + ' and ' - ' signs for rising and falling transitions by the letters ' $r$ ' and ' $f$ ' respectively.

\subsubsection{STEP 3.2: Create a Library File with SPICED Look Up Tables}

The USC flow for static timing analysis of asynchronous circuits in $[21,22,2,3]$ uses a Synopsys Liberty file format for its PrimeTime library [33]. The library contains the timing information of all the cells used in the PrimeTime netlist. The netlist for the twostage GasP FIFO in Figure 5.11 contains two 6-4 GasP black boxes and the two wire connections between them. The timing information of a 6-4 GasP black box is fully captured in look up tables. The look up tables are split into two parts. The first part simply defines the name of the look up table and the template with the index variables and their range. For instance, look up table LUTblue:PREDOUTi_f_FIREPSi_f_6x4, which corresponds to the look up table in Figure 5.3 on page 98 gets as its first part: 


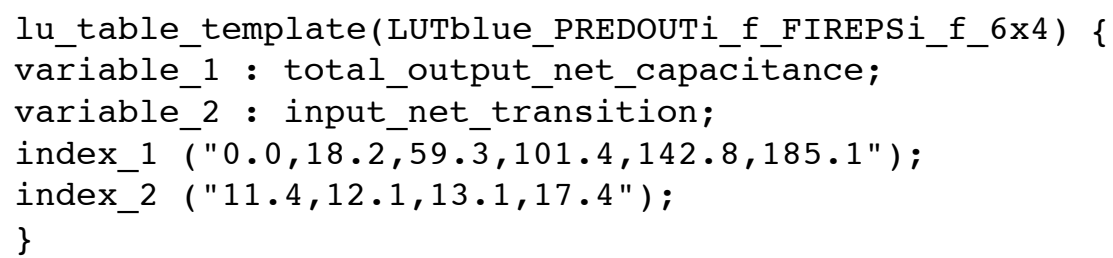

It uses variable_1, total_output_net_capacitance, for the (external) load capacitance on FIRE_PS $_{i}$, and it uses variable_2, input_net_transition, for the falling input slope on PRED_OUT $i$. Just as in the look up table in Figure 5.3, page 98, capacitive load index_1 ranges from 0.0-185.1 fF, and input slope index 2 ranges from 11.4-17.4 ps.

The second part refers to the first part by name, and contains the timing information measured per table entry. This part of the table is moved into the cell definition for the 6-4 GasP black box, where it is stored under the output pin of the path for which the look up table was created. In the case of LUTblue:PREDOUTi_f_FIREPSi_f_6x4, the corresponding output pin is FIRE_PS. The table contents, captured in Figure 5.3, are distributed over two tables: one with the falling input to falling output delays, called cell_fall, and one with the falling output transition time, called fall_transition. The twodimensional table is copied row by row, top row first, each row copied from left to right:

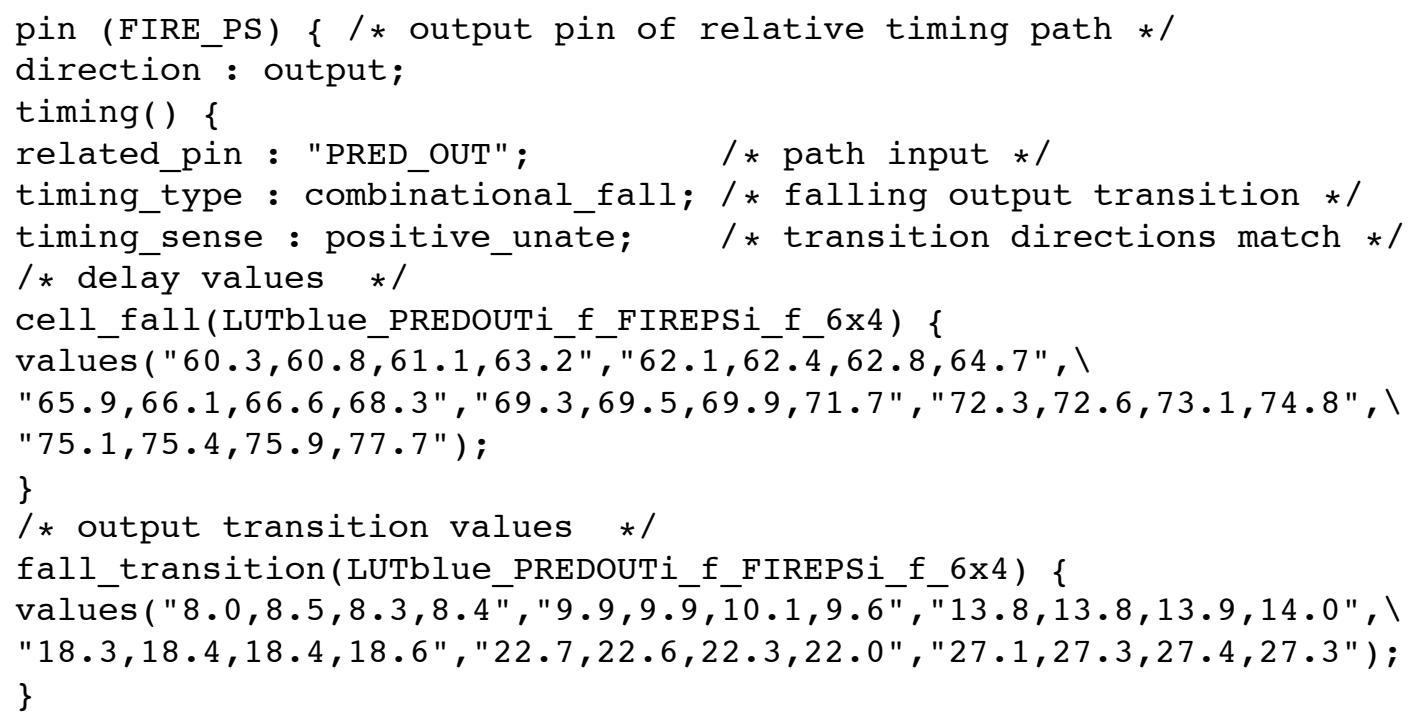

The complete library file and additional explanations follow in Appendix K. 


\subsection{STEP 4: Create Commands for Relative Timing Validation}

We continue with the USC part of the static timing analysis flow by creating the commands for validating the four relative timing constraints, RT1 to RT4 of Figure 5.12, page 108, which are the black box versions of the original relative timings of Figure 2.2, page 13. We follow the command scheme set up by Mallika Prakash [21] and tuned to 6-4 GasP by Prasad Joshi [2]. Our contribution is that we validate two extra relative timing constraints, namely RT2 and RT4. Prasad ignored these two constraints because the initial 6-4 GasP studies used the lumped wire capacitance model to time L2, and RT2 and RT4 are automatically satisfied by this model $[4,15]$. Our latest GasP studies use a distributed RC model to time L2. With a distributed RC wire model, none of the four relative timing constraints RT1 to RT4 are automatically satisfied by either the model or the design [18]. All four need explicit validation via static timing analysis.

Here is the command scheme for validating RT2 of Figure 5.12(b), which requires that 'the forward transfer delay (in red) beats the forward self-resetting loop delay (in blue)' - see Chapter 2 for a textual and graphical explanation:

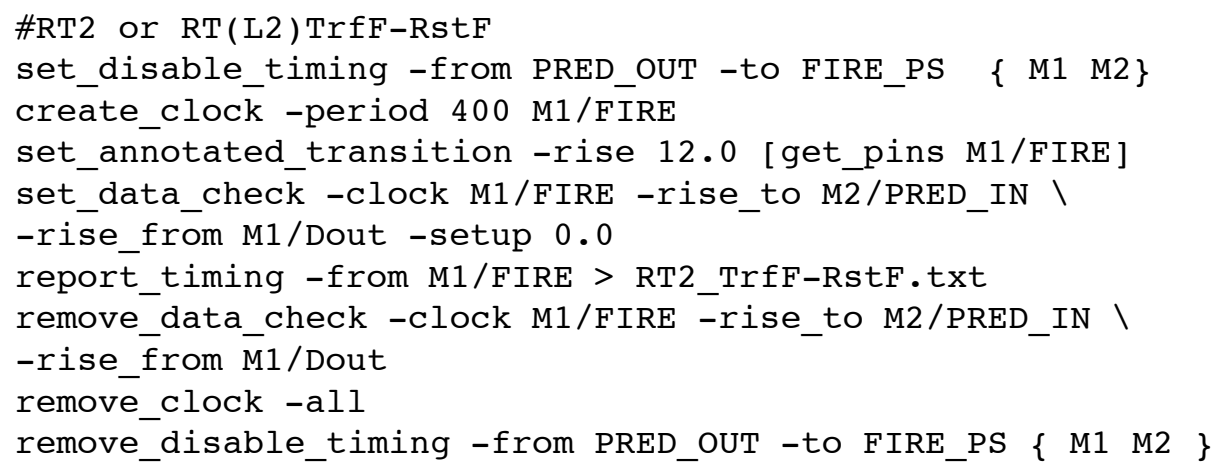

This reads as follows:

1. To avoid interference from the backward self-resetting loop, we disable any timing paths from M $i$ /PRED_OUT to M $i$ /FIRE_PS, $i=1,2$ (disabling M1 suffices).

2. We create a pulse generator with a sufficiently large period on the start signal, 
a.k.a. 'Point of Divergence', for the blue and the red paths in RT2, i.e. on M1/FIRE. We are interested in the rising transition on M1/FIRE, which we define to be a nominal $20 \%-80 \%$ rise time of $12 \mathrm{ps}$.

3. We request PrimeTime to validate that the delay of the forward transfer from M1/FIRE to M2/PRED (the red path), is smaller than the delay of the forward self-resetting loop from M1/FIRE to M1/Dout (the blue path) with a minimum slack of $0.0 \mathrm{ps}$, and to report the timing results to output file 'RT2_TrfF-RstF.txt'.

4. We clean up any side effects, so the next command scheme starts from scratch. The full command file can be found in Appendix L.

\subsection{STEP 5: Run and Inspect Timing Reports}

The PrimeTime run commands follow in the introduction of Appendix M. The output files with the timing reports for the four relative timing constraints RT1 to RT4 follow in Appendices M.1-M.4. We verified that the delay times generated by PrimeTime can be generated using linear interpolation and linear extrapolation on the look up table entries in Figure 5.3-5.7, pages 98-102, using the look up table re-naming in Figure 5.13 on page 110. As an example, we will show how we verified the PrimeTime delays for RT2:

\section{RT2 red and blue path, from M1/FIRE+ to M1/SUCC_OUT+}

- M1/FIRE receives a rising transition with a rise time of $12 \mathrm{ps}$, measured from when the signal voltage passes $20 \%$ VDD to when it reaches $80 \%$ VDD. Our simulation setup uses a default external output load of $0 \mathrm{fF}$ for this path.

- A table entry with an input slope of 12 ps and output load of OfF falls within the look up table range for LUTredblue:FIREi_r_SUCCOUTi_r_6x4, which translates to the look up table name LUTred/blue:FIRE $i+\mathrm{SUCC}_{i}+$ with table entries in Figure 5.6 (bottom), page 101. 
- In Figure 5.6 (bottom), the non-existent entry $(0.0 \mathrm{fF}, 12 \mathrm{ps})$ falls between the existing entry (0.0fF,11.9ps), which has a delay of $26.3 \mathrm{ps}$ and an output slope of $12.2 \mathrm{ps}$, and the existing entry $(0.0 \mathrm{fF}, 12.6 \mathrm{ps})$, which has a delay of $26.7 \mathrm{ps}$ and an output slope of $12.5 \mathrm{ps}$. Linear interpolation thus gives:

$$
\begin{gathered}
\operatorname{delay}(0.0 \mathrm{fF}, 12 \mathrm{ps})=26.3 \mathrm{ps}+((12-11.9) /(12.6-11.9)) \times 0.4 \mathrm{ps}=26.36 \mathrm{ps} \\
\text { output_slope }(0.0 \mathrm{fF}, 12 \mathrm{ps})=12.2+((12-11.9) /(12.6-11.9)) \times 0.3=12.24 \mathrm{ps}
\end{gathered}
$$

- The delay number matches exactly with the delay given by PrimeTime.

\section{RT2 red path, from M1/SUCC_OUT+ to M2/PRED_IN+ via L2}

- This path has no timing information. PrimeTime correctly gives $0.0 p s$ delay.

\section{RT2 blue path, from M1/SUCC_OUT+ to M1/FIRE_PS-}

- The predecessor blue path in item 1 ended with an output slope of $12.24 \mathrm{ps,}$ which becomes the input slope for this path. Our simulation setup uses a default external output load of OfF for this path.

- A table entry with an input slope of 12.24 ps and output load of 0fF falls outside the look up table range for LUTblue:SUCCOUTi_r_FIREi_f_6x4, which translates to the look up table name LUTblue:SUCC $i+\mathrm{FIRE}_{i}-$ with table entries in Figure 5.4 (bottom), page 99.

- In Figure 5.4 (bottom), non-existent entry (0.0fF,12.24ps) falls beyond the existing entry (0.0fF,14.2ps), which has a delay of 35.8ps and an output slope of $8.8 \mathrm{ps}$, and beyond the existing entry $(0.0 \mathrm{fF}, 15.1 \mathrm{ps})$, which has a delay of 36.3ps and an output slope of 8.6ps. Linear extrapolation thus gives:

$$
\begin{aligned}
& \operatorname{delay}(0.0 \mathrm{fF}, 12.24 \mathrm{ps})=35.8 \mathrm{ps}-((14.2-12.24) /(15.1-14.2)) \times 0.5 \mathrm{ps}=34.71 \mathrm{ps} \\
& \text { output_slope }(0.0 \mathrm{fF}, 12.24 \mathrm{ps})=8.8+((14.2-12.24) /(15.1-14.2)) \times 0.2=9.24 \mathrm{ps}
\end{aligned}
$$

- The delay number matches exactly with the delay given by PrimeTime. 


\section{RT2 blue path, from M1/FIRE_PS- to M1/Dout+}

- The predecessor blue path in item 3 ended with an output slope of 9.24ps, which becomes the input slope for this path. Our simulation setup uses a default external output load of OfF for this path.

- A table entry with an input slope of 9.24ps and an output load of OfF falls outside the look up table range for LUTblue:FIREPSi_f_Douti_r_1x4. This table name translates to the look up table name LUTblue:FIRE ${ }_{i}-$ Dout $_{i}+$ with table entries in Figure 5.5 (bottom), page 100.

- In Figure 5.5 (bottom), the non-existent entry (0.0fF, 9.24ps) falls beyond the existing entries (0.0fF, 11.6ps) and (0.0fF, $12.4 \mathrm{ps})$, which have delays $20.5 \mathrm{ps}$ respectively $20.9 \mathrm{ps}$. The output slope is irrelevant in this case, because the RT blue path ends here. Linear extrapolation thus gives:

$$
\operatorname{delay}(0.0 \mathrm{fF}, 9.24 \mathrm{ps})=20.5 \mathrm{ps}-((11.6-9.24) /(12.4-11.6)) * 0.4 \mathrm{ps}=19.32 \mathrm{ps}
$$

- The delay number matches exactly with the delay given by PrimeTime.

\section{RT2 Slack calculation}

- The total delay of the red path in RT2 is the sum of items $1-2=26.36 \mathrm{ps}$.

- The total delay of the blue path is the sum of items 1,3 , and $4=80.39$ ps.

- The slack or (total blue path delay - total red path delay) is thus 54.03ps.

- This is exactly what PrimeTime reports as slack for RT2.

In summary: PrimeTime's delay calculation for RT2 is based on linear interpolation and linear extrapolation of the table entries in our look up tables. This is also true for the PrimeTime delay calculations of the three other relative timing constraints, RT1, RT3, and RT4. 
RT2 has a reported slack of 54.03ps. The reason why this slack is so large is because the L2 wire delay for the red path takes 0ps in the current simulation setup, as can be seen in item 2 in the above validation reasoning for RT2. This implies that the delay for a rising transition from the near end of L2 (M1/SUCC_OUT) to the far end of L2 (M2/PRED_IN) can take up to 54.03ps. A delay beyond 54.03ps invalidates RT2. Invalidating RT2 amounts to M1 stopping its drive before M2 sees the transition. This does not immediately invalidate the circuit operation, as we demonstrate in [18], but it makes the circuit less robust and more noise sensitive. In Chapter 6 we will compare the PrimeTime results to the results of our short-to-medium-to-long wire study in [18].

\subsection{Conclusion and Future Work for Chapter 5}

Chapter 5 is the "proof of the pudding" as we call it in the the conclusion sections of Chapters 3 and 4 . It concludes our quest to create a flow for library characterization and static timing analysis of single-track circuits in GasP.

In Chapter 3, we showed how SPICE can be used to generate the timing information of a simple circuit without loops and without single-track communication wires, and to capture that information in look up tables. Our quest in Chapter 3 was more of a warming-up quest to understand enough of the design environment in Electric and of the simulation environment in SPICE to make things work.

The real quest came in Chapter 4, where we showed how to partition the circuit and the timing constraints that it needs to make the SPICE approach of Chapter 3 work for full-fledged GasP circuits with loops and single-track communication wires. Our timing characterization flow in Chapter 4 is new, as far as we know. 
In Chapter 5, we took the look up tables that we generated in Chapter 4, and fed them to a standard static timing analysis (STA) flow, namely PrimeTime of Synopsys. The STA flow is not new: it was specifically created for asynchronous or self-timed circuits at the University of Southern California (USC), by Mallika Prakash and Prasad Joshi two former Master of Science students in the research group of Professor Peter Beerel. Having access to the USC STA flow for asynchronous circuits was a major help in completing our quest. It limited the final stretch of our quest to (1) importing our look up tables into PrimeTime and (2) validating the timing reports and comparing them to the results of our earlier studies published in [18]. Chapter 5 is primarily about (1) and (2), but it also fills in and explains the connections to the USC flow.

Last but not least: not only did we (1) generate and (2) explain a timing validation flow for single-track circuits in 6-4 GasP but we also (3) used this flow and we (4) validated its results. The many files in Appendices $\mathrm{A}-\mathrm{M}$ in this thesis bear witness to this. For truly: the proof of the pudding is in the eating.

As far as future work goes, we covered most of it in the conclusions of Chapters 3-4. Key to static timing analysis are: flow automation and wire delay models. 


\section{Chapter 6}

\section{SUMMARY AND DISCUSSION OF TIMING RESULTS}

The slack timings computed by PrimeTime and reported in Appendices M.1-M.4 are between 50.29ps and 57.66ps. RT2, for instance, has a reported slack of 54.03ps. The reason why this slack is so large is because the L2 wire delay for the red path takes 0ps in the current simulation setup - as we can see in item 2 for the R2 validation reasoning of the PrimeTime results in Section 5.6. The implication of this is that the delay for a rising transition from the near end of L2 (M1/SUCC_OUT) to the far end of L2 (M2/PRED_IN) can take up to 54.03ps. A delay beyond 54.03ps invalidates RT2. Invalidating RT2 amounts to M1 stopping its drive before M2 sees the transition. This does not immediately invalidate the circuit operation, as we demonstrate in [18], but it makes the circuit less robust and more noise sensitive.

The 54.03ps slack that PrimeTime reports for RT2 is $20-25 \%$ smaller than the slack that we reported in [18]. The reason for this $20-25 \%$ difference is that our PrimeTime simulations use OfF external output loads, whereas the simulations in [18] use realistic output loads. The difference in slack between the red and blue paths (ignoring L2) comes from four gates in the blue path: gates A, B, C, and D in Module M1 of Figure 2.1, page 11. The outputs of gates A, B and D are internal to the circuit, but the output of 
gate $\mathrm{C}$ is external. The Primetime delays for gates A, B and D are correctly simulated, but the delay for gate $\mathrm{C}$ is simulated with $0 \mathrm{fF}$ load. As a result, the slack is off for up to 1 out of 4 gates. All GasP gates are sized to have about the same delay under realistic external load conditions. So, we may expect that the RT2 slack reported by PrimeTime is off by up to $1 / 4$-th or $25 \%$ over the slack reported in [18].

The same is true for the other relative timing constraints: all have a slack $20-25 \%$ smaller than the slack reported in [18].

The slack can be translated in terms of wire length. The interesting question to ask is: "How long can we make L2 until the relative timing constraints break?"

Figure 6.1 shows the simulation results for the forward and the reverse transfer delays over L2 as reported in [18]. It relates wire delay to wire length. More precisely: it relates the near-end delay for a transition over L2 and the far-end delay for that transition to the wire length of L2. The top graph in Figure 6.1 shows these relations for a forward rising transition over L2, and the bottom graph does the same for a falling transition over L2 in the reverse direction. The delay numbers in Figure 6.1 use the process-normalized time metric tau $(\tau)$ rather than the absolute metric of picoseconds (ps). Tau is about 8ps for the 90nm CMOS process that we used in [18]. We use the same process here. A slack of 54.03ps from the near end to the far end of L2 thus corresponds to a slack of about 6.75 $\tau$. In Figure 6.1, the grey graph with near-end delays and the black graph with far-end delays reach a difference of $6.75 \tau$ when the wire length is about $12500 \lambda$ long. 


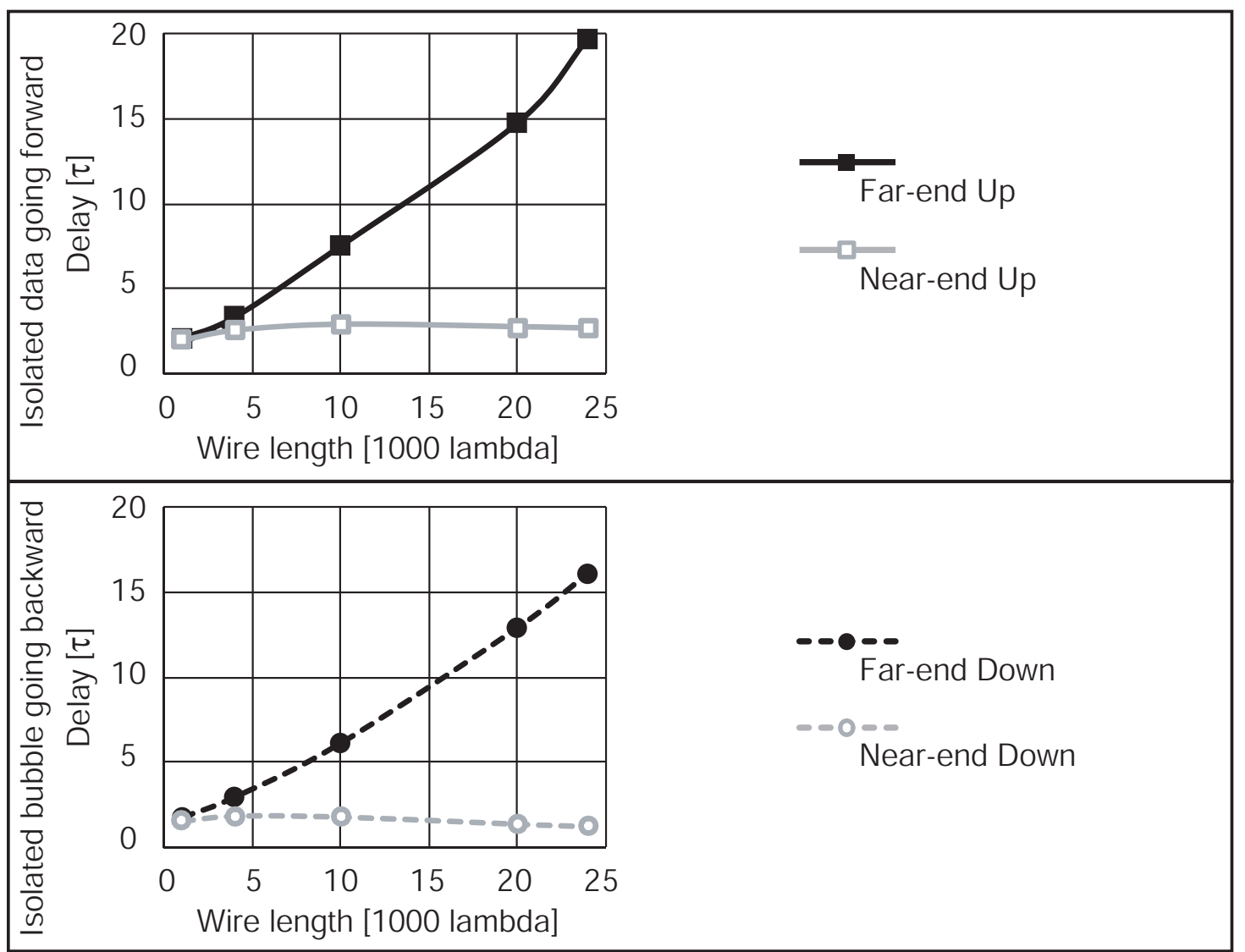

Figure 6.1 Excerpt taken from Figure 4 in [18] showing simulated delays for rising and falling transitions at the near and far end of a given state wire L2 in a ten-stage FIFO ring, for various lengths of that state wire. The FIFO design was done in the same 90nm CMOS process used throughout all the 6-4 GasP studies in $[2,3,4,15,16$, $17,18,19]$ and in this thesis. The top window shows the near- and far-end delays for a forward transfer over L2, with one empty space (bubble) in the ring - the worstcase scenario for a forward transfer. The bottom window shows the near- and far-end delays for a transfer over L2 in the reverse direction, with one data item in the ring, which is the worst-case scenario for the reverse transfer. Wire length and time are measured in lambda $(\lambda)$ and tau $(\tau)$, which for this $90 \mathrm{~nm}$ process translate to $50 \mathrm{~nm}$ and 8 ps respectively. 
With the help of Figure 6.1, we can map all four PrimeTime generated slack numbers for RT1 to RT4 into wire lengths and obtain a maximum allowable wire length for L2 below which RT1 to RT4 hold and beyond which at least one of the constraints fails:

- RT1 has a slack of 50.29ps, i.e. $6.29 \tau$, and holds for an L2 length $\leq 12000 \lambda$.

- RT2 has a slack of 54.03ps, i.e. $6.75 \tau$, and holds for an L2 length $\leq 12500 \lambda$.

- RT3 has a slack of 57.66ps, i.e. 7.21 $\tau$, and holds for an L2 length $\leq 14000 \lambda$.

- RT4 has a slack of 56.46ps, i.e. $7.06 \tau$, and holds for an L2 length $\leq 13500 \lambda$.

So, in summary: the maximum allowable wire length for L2 is $12000 \lambda$.

As mentioned earlier, $12000 \lambda$ is about $25 \%$ shorter than the maximum allowable wire length reported in Figure 6.1, which is $16000 \lambda$. To put these numbers in perspective and to fuel a discussion on how to extend our static timing analysis flow with wire delay models, we end with a so-called 'Distance Constraint Graph'. A Distance Constraint Graph is a graphical representation that Swetha developed in [15] and which shows how relative timing assumptions constrain the distances between 6-4 GasP modules.

Figure 6.2 shows the Distance Constraint Graph for 6-4 GasP that we constructed from the results of our short-to-medium-to-long wire study in [18]. The graph contains four different regions, colored white, light grey, dark grey, and black.

Our current PrimeTime calculations aim for the white region in Figure 6.2. This is the region where the state of single-track wire L2 is either driven high or driven low or maintained by one of the two half-keepers - see Chapter 2 and Figure 2.1, page 11. In the white region of Figure 6.2, all L2 wires have wire lengths ranging from $0 \lambda$ to $16000 \lambda$ (this would be $0 \lambda$ to $12000 \lambda$ in the PrimeTime-equivalent version of Figure 6.2). The white region uses a distributed $\mathrm{RC}$ wire model, similar to the wire model for the given 90nm CMOS manufacturing process that we use in our schematics design environment. 


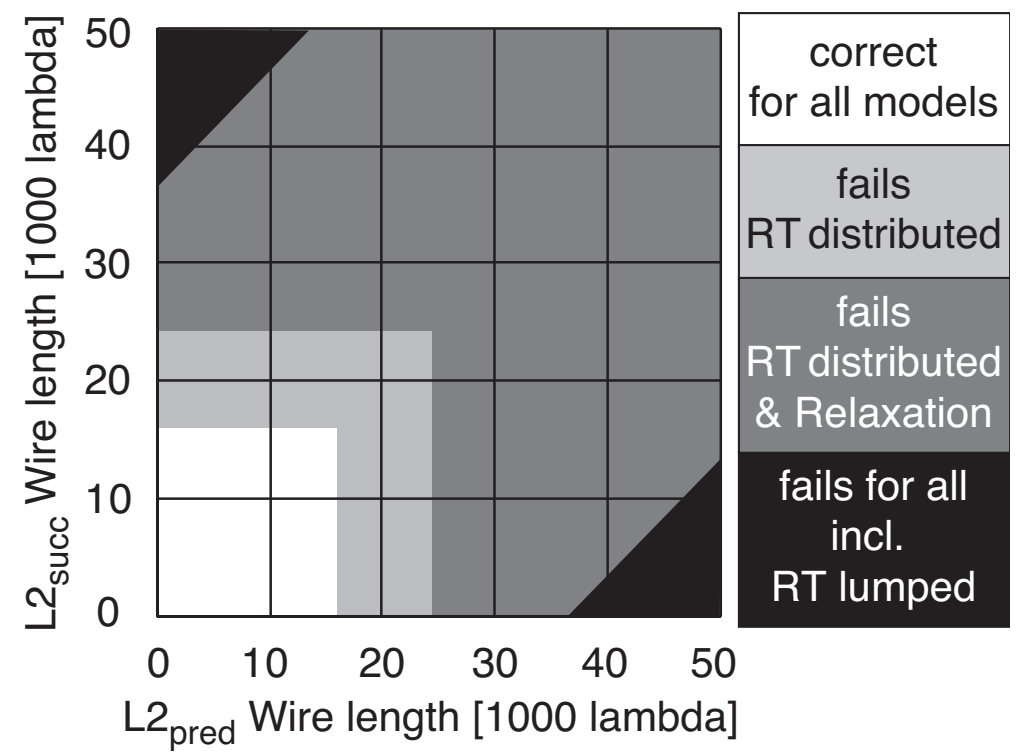

Figure 6.2 Copy of Figure 7 in our short-to-medium-to-long wire study published in [18], showing the Distance Constraint Graph for 6-4 GasP in 90nm CMOS. The graph shows how the distance $\mathrm{L} 2$ pred from a given module to its predecessor, and likewise $\mathrm{L} 2_{\text {succ }}$ from the module to its successor, are constrained. The white area is the domain of the relative timing constraints under a distributed RC wire model; the constraints RT1, RT2, RT3 and RT4 hold for distances up to 16000 lambda. The light-grey area is the domain of charge relaxation; the relative timing constraints no longer hold, but the 6-4 Gasp design still functions correctly. The white, light-grey and dark-grey areas together, i.e. the non-black areas, form the domain of the relative timing constraints under a lumped capacitance wire model. For a lumped capacitance wire model, the constraints are predicted to hold when $\mathrm{L} 2_{\text {pred }}$ and $\mathrm{L} 2_{\text {succ }}$ differ by less than 37000 lambda.

The light-grey region is the surprise in Figure 6.2 and the surprise of our long-wire study. It shows that 6-4 GasP keeps working correctly for L2 wire lengths up to $24000 \lambda$, beyond the validity of relative timing constraints and despite drift periods in L2. The light-grey region works on charge relaxation, as we explain in detail in [18].

To fuel the discussion on how to extend our static timing analysis (STA) flow with wire delay models, here is the question we would like to see answered: "How can STA capture the white and the light-grey regions in Figure 6.2?" 


\section{Chapter 7}

\section{COMPARISON TO RELATED WORK}

Our research builds upon prior research work presented and published by Mallika Prakash [21, 22] and Prasad Joshi [2, 3] - two former Master of Science students in the research group of Professor Peter Beerel at the University of Southern California (USC). Mallika investigated and developed a library characterization and static timing analysis flow for asynchronous circuits with uni-directional communication channels. Her work covers power as well as timing characterization. Prasad extended Mallika's static timing analysis solution to circuits with bi-directional channels. Specifically, he covered the bi-directional communication mechanism for single-track handshaking that we use in 6-4 GasP. Prasad did not extend Mallika's look up table characterization approach to 6-4 GasP - the look up tables in his work are fictional.

The look up table generation approach that we present in Chapters 3-4 differs from Mallika's approach in two major ways:

- Mallika's thesis partly covers single-track circuits. She presents a simulation environment that simulates the asynchronous circuit in its entirety to characterize forward and reverse latencies for various input slopes and output loads. This suffices to characterize the throughput of the circuit, but is insufficient for characterizing 
and validating the circuit's relative timing constraints, i.e. timing assumptions on which the correct operation of the circuit depends. To generate look up tables for validating relative timing constraints in GasP, we must open up the circuit and simulate specific internal paths. The library characterization method that we created and which we describe in Chapters 3-4 does exactly that.

- The asynchronous circuits investigated by Mallika are all two-ported devices: there is a data input side and a data output side. GasP is a three-ported device. Module M1 in Figure 2.1 has a predecessor input, $\mathrm{PRED}_{1}$, a FIRE 1 signal to capture the data, and a successor output, $\mathrm{SUCC}_{1}$. As a result, the approach to look up table generation for Mallika is a straightforward two-dimensional simulation process from (input-drive $\times$ output-load) $\rightarrow$ timing. In contrast, GasP requires:

1. either a table for (input-drive $\times$ load-on-FIRE ${ }_{1} \times$ output-load) $\rightarrow$ timing, which is a three-dimensional look up table,

2. or a partitioning into two-dimensional look up tables,

3. or a pre-defined single-rail datapath design with a fixed load on FIRE $_{1}$, to turn GasP into a two-ported device with two-dimensional look up tables. Lacking a fixed load on $\mathrm{FIRE}_{1}$, we present a solution in the style of item 2 above, because this requires fewer simulations than a solution approach based on item 1 .

An additional difference between the USC flow and our version that is worth mentioning is the way that wires are modeled. In the USC flow, the wires are included with a lumped capacitance model. In contrast, we separate the wire models from our look up table generation process, and make them a plug-and-play object that we can insert later when we run static timing analysis. Our motivation to do so comes from the short-tomedium-to-long wire studies that we did for 6-4 GasP and which we published in [18]. 
The results of these wire studies show that there are two crucial ingredients that make a good sender-receiver wire model:

1. The capacitive load at the near end of the wire, as seen by the sender, as a function of the wire length, width, spacing, and the load capacitance at the wire's far-end:

- For GasP, this information will be used to simulate the relative timing paths related to the blue-colored self-resetting loops in Figures 2.1, 2.2, and 5.12 on pages 11,13 and 108 respectively.

2. Wire resistance and capacitive wire load as seen by the receiver - again as a function of wire length, width, spacing, and the capacitive load at the far-end:

- For GasP, this information will be used to simulate the relative timing paths related to the forward red-colored arrows and reverse green-colored arrows in Figures 2.1 and 2.2 and 5.12 on pages 11, 13 and 108 respectively. 


\section{Chapter 8}

\section{CONCLUSION AND FUTURE WORK}

In this thesis, I have created a timing validation flow for single-track circuits in 6-4 GasP. The flow consists of two parts: library characterization and static timing analysis (STA). The library characterization part is new. The STA part ties into an existing STA flow for asynchronous circuits from the University of Southern California.

I have applied this flow to validate the four relative timing constraints that are key to single-track communication. I have validated the results by comparing them to the timing results obtained in an earlier and independent study. The results - obtained in different ways and for different purposes - are compatible.

The three conclusion sections of Chapter 3, Chapter 4 and Chapter 5, on pages 33, 85 and 116, give an excellent overview of work done and work still outstanding.

Key topics for future work are flow automation and wire delay models. 


\section{References}

[1] Jo Ebergen, Scott Fairbanks, and Ivan Sutherland. Predicting Performance of Micropipelines Using Charlie Diagrams. In Proc. IEEE International Symposium on Advanced Research in Asynchronous Circuits and Systems (ASYNC), pages 238-246, 1998.

[2] Prasad Joshi. Static Timing Analysis of GasP. Master of Science thesis, Electrical Engineering, Faculty of the USC Viterbi School of Engineering, University of Southern California, December 2008.

[3] Prasad Joshi, Peter Beerel, Jonathan Gainsley, Ivan Sutherland, and Marly Roncken. Static Timing Analysis of GasP. User Track poster, Design Automation Conference (DAC), 2009.

[4] Prasad Joshi, Peter Beerel, Marly Roncken, and Ivan Sutherland. Timing Verification of GasP Asynchronous Circuits: Predicted Delay Variations Observed by Experiment. In D. Dams, U. Hannemann, M. Steffen (Eds.): Willem-Paul de Roever Festschrift, LNCS 5930, pages 260-276. Springer-Verlag Berlin Heidelberg, 2010.

[5] Link to Achronix Semiconductor Corporation. http://www.achronix.com. 
[6] Link to FLEET posted at the University of California at Berkeley. http://research.cs.berkeley.edu/class/fleet/docs.

[7] Link to Fulcrum Microsystems. http://www.fulcrummicro.com.

[8] Link to IBM Research Cell Systems. http://www.research.ibm.com/cell.

[9] Link to Intel's Many-Core Computing. http://software.intel.com/enus/blogs/2009/12/02/intel-labs-new-research-milestone-in-many-core-computing.

[10] Link to Intel's Teraflop Research Chip. http://techresearch.intel.com/ProjectDetails.aspx?Id=151.

[11] Link to Sun's (now: Oracle's) UltraSPARC T1. http://en.wikipedia.org/wiki/UltraSPARC_T1.

[12] Link to the 17-th International Symposium on Asynchronous Circuits and Systems (ASYNC 2011). http://asyncsymposium.org/async2011/Home.html.

[13] Link to the Asynchronous Research Center. http://arc.cecs.pdx.edu, with older material posted at http://research.cs.berkeley.edu/class/fleet/docs.

[14] Link to Tiempo. http://www.tiempo-ic.com.

[15] Swetha Mettala Gilla. Distance Constraint Graph: A Graphical Representation for 6-4 GasP showing how Relative Timings constrain the Module Distances. Technical Report, ARC2009-smg01, Asynchronous Research Center, Portland State University, September 2009.

[16] Swetha Mettala Gilla, Marly Roncken, and Ivan Sutherland. Library Characterization of 6-4 GasP: Generating Look Up Tables with SPICE for Static 
Timing Analysis and Relative Timing Validation (PART-A). Technical Report, ARC2010-smg01, Asynchronous Research Center, Portland State University, March 2010.

[17] Swetha Mettala Gilla, Marly Roncken, and Ivan Sutherland. Library Characterization of 6-4 GasP: Generating Look Up Tables with SPICE for Static Timing Analysis and Relative Timing Validation (PART-B). Technical Report, ARC2010-smg02, Asynchronous Research Center, Portland State University, April 2010.

[18] Swetha Mettala Gilla, Marly Roncken, and Ivan Sutherland. Long-Range GasP with Charge Relaxation. In Proc. IEEE International Symposium on Asynchronous Circuits and Systems (ASYNC), pages 185-195, 2010.

[19] Swetha Mettala Gilla, Marly Roncken, and Ivan Sutherland. Static Timing Analysis of 6-4 GasP using PrimeTime and SPICED Look Up Tables. Technical Report, ARC2010-smg04, Asynchronous Research Center, Portland State University, August 2010.

[20] Ad Peeters, Frank te Beest, Mark de Wit, and Willem Mallon. Click Elements: An Implementation Style for Data-Driven Compilation. In Proc. IEEE International Symposium on Asynchronous Circuits and Systems (ASYNC), pages 3-14, 2010.

[21] Mallika Prakash. Library Characterization and Static Timing Analysis of Asynchronous Circuits. Master of Science thesis, Computer Engineering, Faculty of the USC Viterbi School of Engineering, University of Southern California, December 2007. 
[22] Mallika Prakash, Pankaj Golani, Amit Bandlish, Rahul Rithe, and Peter Beerel. Accurate Timing and Power Characterization of Static Single-Track Full-Buffers. SRC Techcon, 2007.

[23] Jan Rabaey, Anantha Chandrakasan, and Borivoye Nikolić. Digital Integrated Circuits: A Design Perspective. Prentice-Hall, 2003.

[24] Steven M. Rubin. Using the ELECTRIC ${ }^{\text {TM }}$ VLSI Design System, Version 8.11. Static Free Software and Sun Microsystems, ISBN 0-9727514-3-2, R.L. Ranch Press, 2010.

[25] Sachin Sapatnekar. Static Timing Analysis. In L. Scheffer, L. Lavagno, G. Martin (Eds.): Electronic Design Automation for Integrated Circuits Handbook, Vol. 2, Ch. 6. CRC Press, Taylor \& Francis Group, 2006.

[26] Ken Stevens. Practical Verification and Synthesis of Low Latency Asynchronous Systems. Doctor of Philosophy thesis, Computer Science, University of Calgary, Alberta, September 1994.

[27] Ken Stevens, Ran Ginosar, and Shai Rotem. Relative Timing. In Proc. IEEE International Symposium on Advanced Research in Asynchronous Circuits and Systems (ASYNC), pages 208-218, 1999.

[28] Ken Stevens, Ran Ginosar, and Shai Rotem. Relative Timing. IEEE Transactions on Very Large Scale Integration (VLSI) Systems, 11(1):129-140, 2003.

[29] Ivan Sutherland. A Six-Four GasP Tutorial. Internal Report ICIES2007-is49, http://research.cs.berkeley.edu/class/fleet/docs, 2007. 
[30] Ivan Sutherland and Scott Fairbanks. GasP: A Minimal FIFO Control. In Proc. IEEE International Symposium on Asynchronous Circuits and Systems (ASYNC), pages 46-53, 2001.

[31] Ivan Sutherland, Adam Megacz, and Igor Benko. FLEET - A One-Instruction Computer. Internal Report ICIES2007-is44, http://research.cs.berkeley.edu/class/fleet/docs, 2007.

[32] Ivan Sutherland, Bob Sproull, and David Harris. Logical Effort: Designing Fast CMOS Circuits. Morgan Kaufmann, San Francisco, 1999.

[33] Synopsys. Liberty User Guides and Reference Manual Suite, Version 2007.12, 2007.

[34] Synopsys. HSPICE ${ }^{\circledR}$ Reference Manual: Commands and Control Options, Version C-2009.09, 2009.

[35] Synopsys. PrimeTime ${ }^{\circledR}$ Fundamentals User Guide, Version D-2010.06, 2010.

[36] Neil Weste and David Harris. CMOS VLSI Design: A Circuits and Systems Perspective. Addison-Wesley, 2005.

[37] Yang Xu and Ken Stevens. Automatic Synthesis of Computation Interference Constraints for Relative Timing Verification. In Proc. IEEE International Conference on Computer Design (ICCD), pages 16-22, 2009. 


\section{Appendix A}

\section{SPICE FILES FOR SPICE SWEEPS}

The files in Appendix A.1 to Appendix A.6 below are generated in Sections 3.2 to 3.7 of Chapter 3. For generation details, see these sections.

\section{A.1 SPICE Netlist Generated by Electric}

We generated the following SPICE netlist from the design schematics in Electric. The file extension is '.spi'. Inverters with size parameters wid1 and wid2 receive erroneous 'null' values. We will correct the erroneous text segments in Appendix A.4.

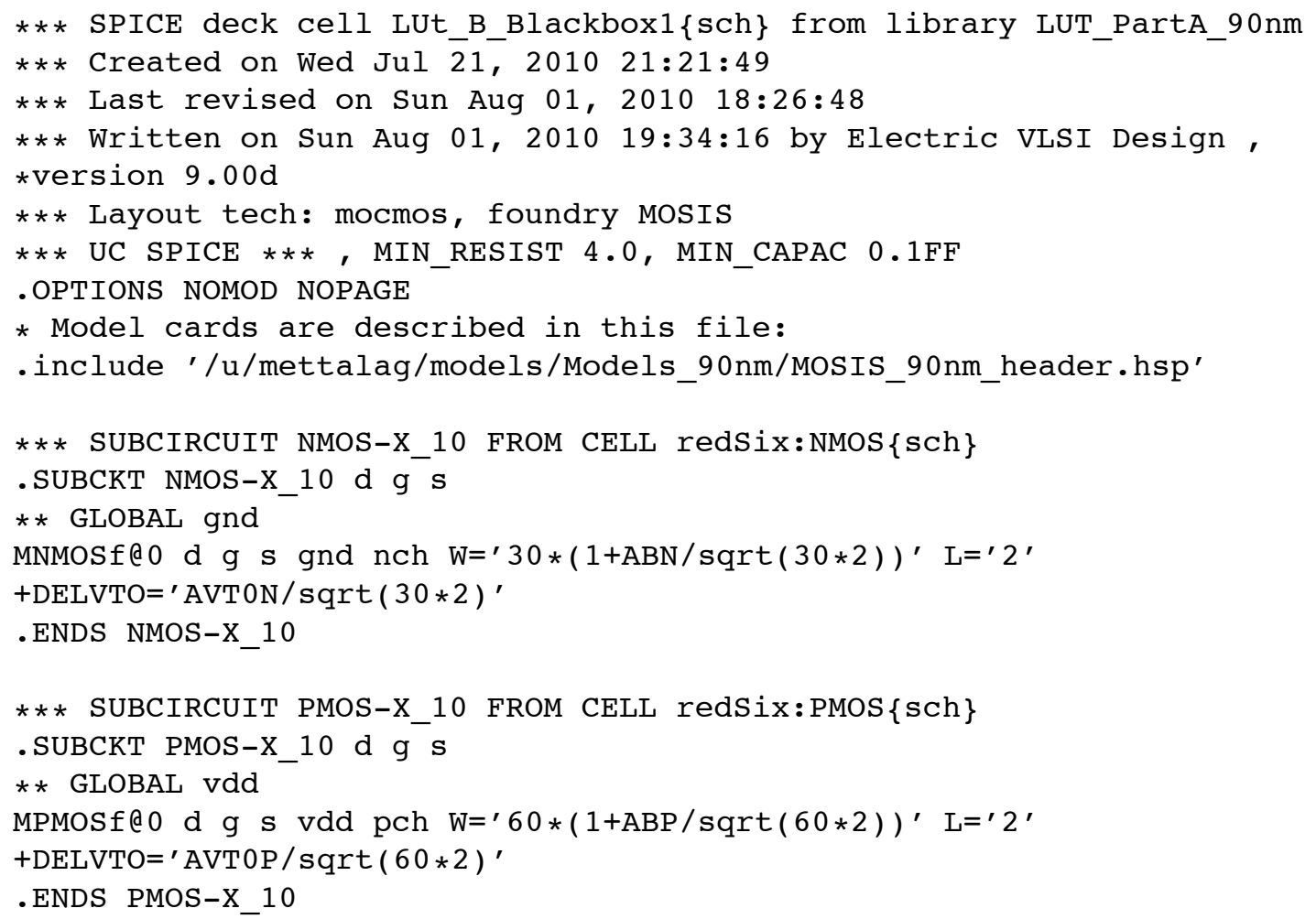




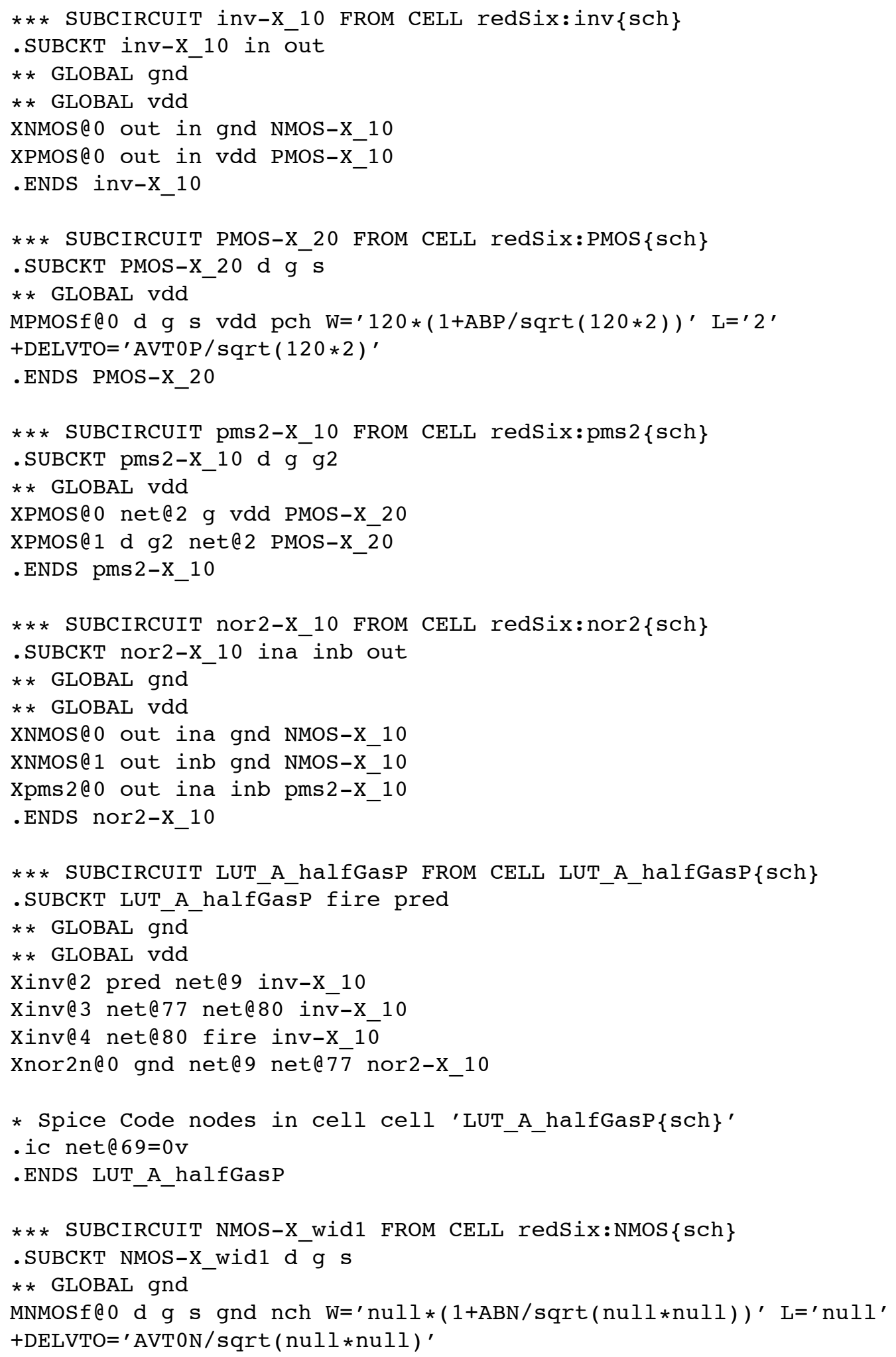




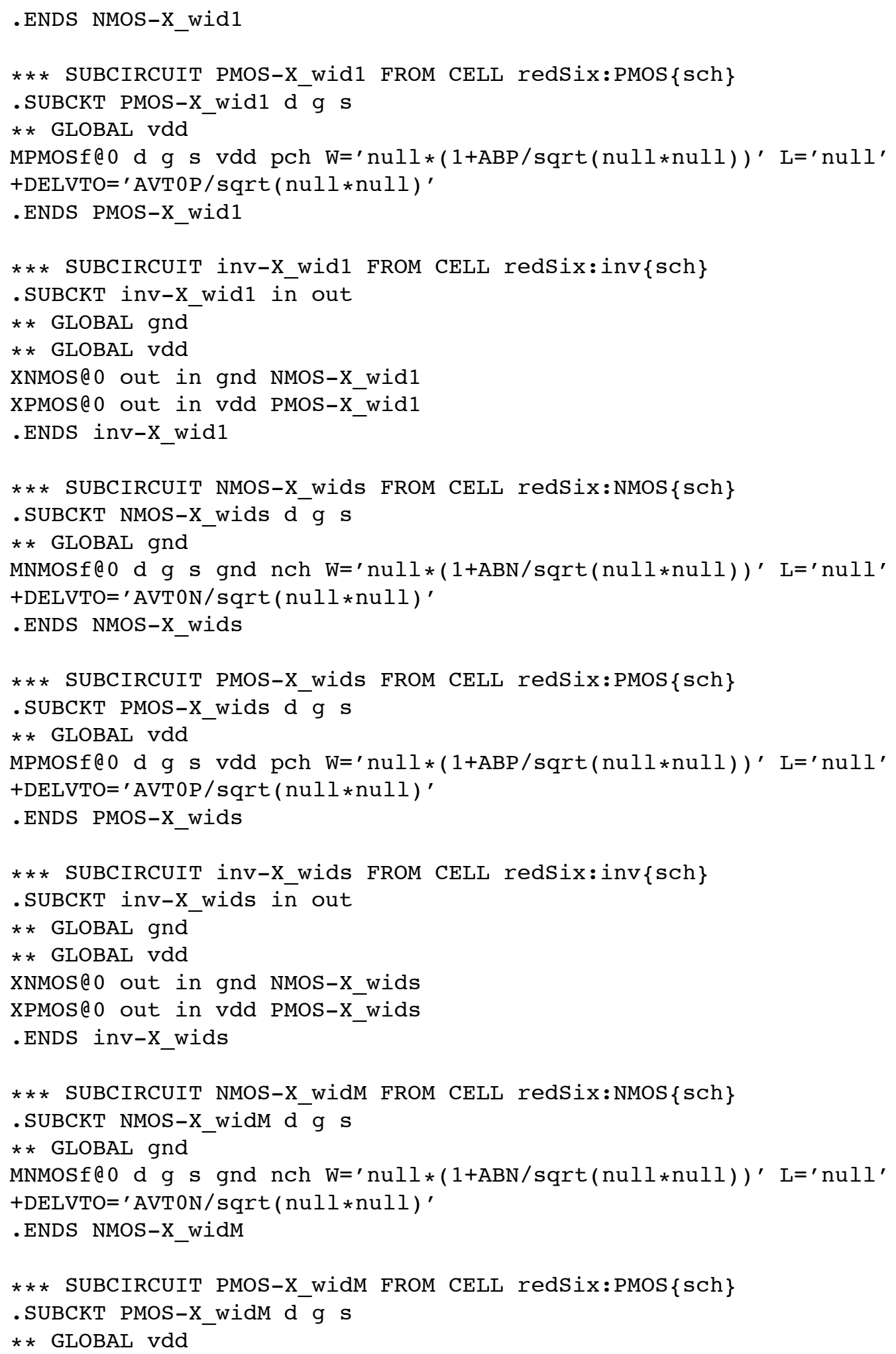


MPMOSf@O d g s vdd pch W='null*(1+ABP/sqrt(null*null))' $L=$ 'null' + DELVTO $={ }^{\prime}$ AVTOP $/$ sqrt $(\text { null } * \text { null })^{\prime}$

.ENDS PMOS-X_widM

*** SUBCIRCUIT inv-X_widM FROM CELL redSix:inv\{sch\}

.SUBCKT inv-X_widM in out

** GLOBAL gnd

** GLOBAL vdd

XNMOS@O out in gnd NMOS-X_widM

XPMOS@O out in vdd PMOS-X_widM

.ENDS inv-X_widM

*** SUBCIRCUIT NMOS-X_wid2 FROM CELL redSix:NMOS $\{\mathrm{sch}\}$

. SUBCKT NMOS-X_wid2 d $g \mathrm{~s}$

** GLOBAL gnd

MNMOSf@O d g s gnd nch $\mathrm{W}=$ 'null $*(1+\mathrm{ABN} / \operatorname{sqrt}(\mathrm{null} * \mathrm{null}))^{\prime} \mathrm{L}=\mathrm{h}^{\mathrm{n}} \mathrm{nul}$ '

+ DELVTO $={ }^{\prime}$ AVTON $/$ sqrt $(\text { null } * \text { null })^{\prime}$

.ENDS NMOS-X_wid2

*** SUBCIRCUIT PMOS-X_wid2 FROM CELL redSix:PMOS $\{$ sch $\}$

.SUBCKT PMOS-X_wid2 d g s

** GLOBAL vdd

MPMOSf@o d g s vdd pch W='null*(1+ABP/sqrt(null*null))' L='null'

+ DELVTO $=$ ' AVTOP/sqrt $($ null $*$ null $) '$

.ENDS PMOS-X_wid2

*** SUBCIRCUIT inv-X_wid2 FROM CELL redSix:inv\{sch\}

.SUBCKT inv-X_wid2 in out

** GLOBAL gnd

** GLOBAL vdd

XNMOS@O out in gnd NMOS-X_wid2

XPMOS@ 0 out in vdd PMOS-X_wid2

.ENDS inv-X_wid2

.global gnd vdd

*** TOP LEVEL CELL: LUt_B_Blackbox $1\{\mathrm{sch}\}$

XLUT_A ha@o out[1] in[1] LUT_A_halfGasP

VPulse@0 net@139 gnd pulse 0 ' $1 \mathrm{v}^{\prime}$ '0ns' '50ps' '50ps' '2ns' '4ns'

Xinv@1 Din[1] in[1] inv-X_wid1

Xinv@2 net@139 Din[1] inv-X_wids

xinv@3 net@64 inv@3_out inv-X_widM

Xinv@ 4 out[1] net@ 64 inv-X_wid2

* Trailer cards are described in this file:

.include '/u/mettalag/models/Models_90nm/MosIs_90nm_trailer.hsp' . END 


\section{A.2 SPICE Simulation Set-Up File: HEADER}

Here is the basic file with default simulation parameters. Its extension is '.hsp'. It contains a pointer to the $90 \mathrm{~nm}$ CMOS process models for our design. Note that we use typical (TT) Process, Voltage, and Temperature (PVT) corners.

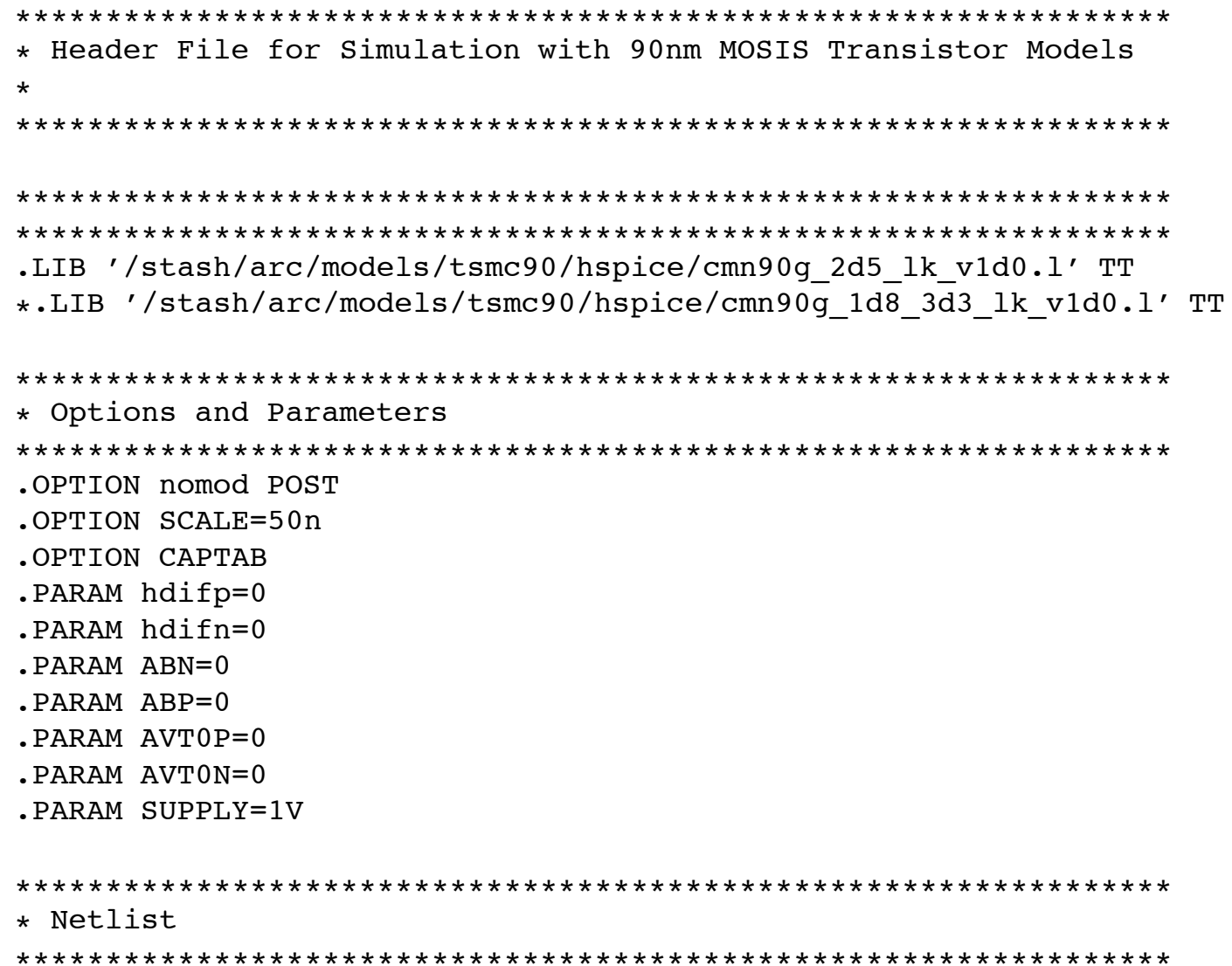

\section{A.3 SPICE Simulation Set-Up File: TRAILER}

Below follows the so-called trailer file with the analysis details of the SPICE simulation. Its extension is '.hsp'. We will modify this file in Appendix A.5, where we add sweep commands and measurement statements.

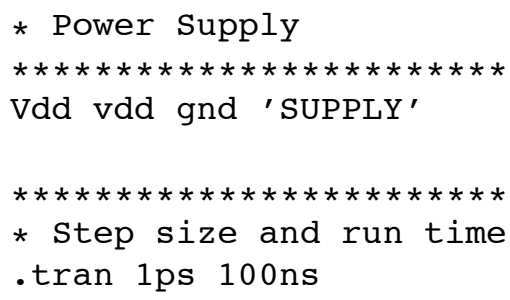




\section{A.4 Modified SPICE Netlist, Ready for Sweep}

In Appendix A.4, we modify the incorrect SPICE netlist from Appendix A.1 by replacing all formulas with 'null' by the correct sweep parameter formulas.

As example, we show how the 'null'-s in the NMOS transistor of the source inverter are replaced by formulas using (wid1)/3, which is the size of the source inverter:

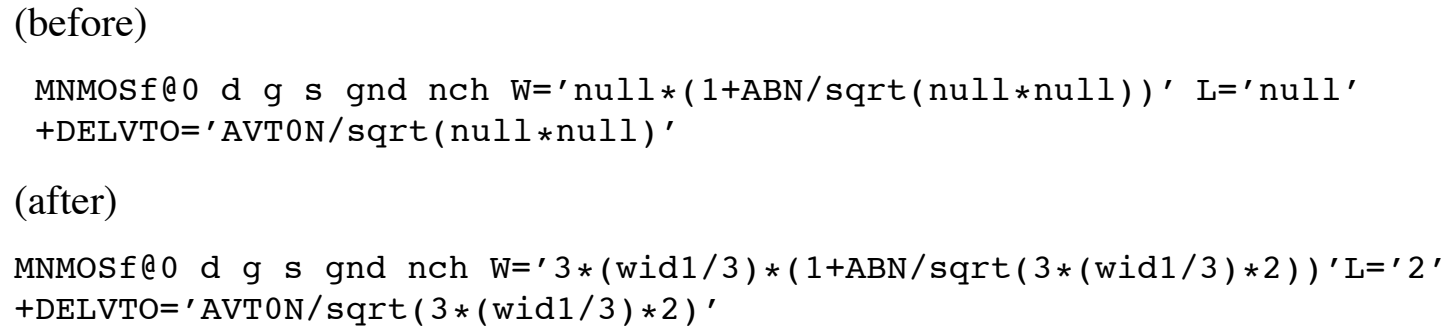

The changes for the PMOS transistor and for transistors in the driver, load and Miller inverters are similar. The modified netlist from the first change onwards is as follows:

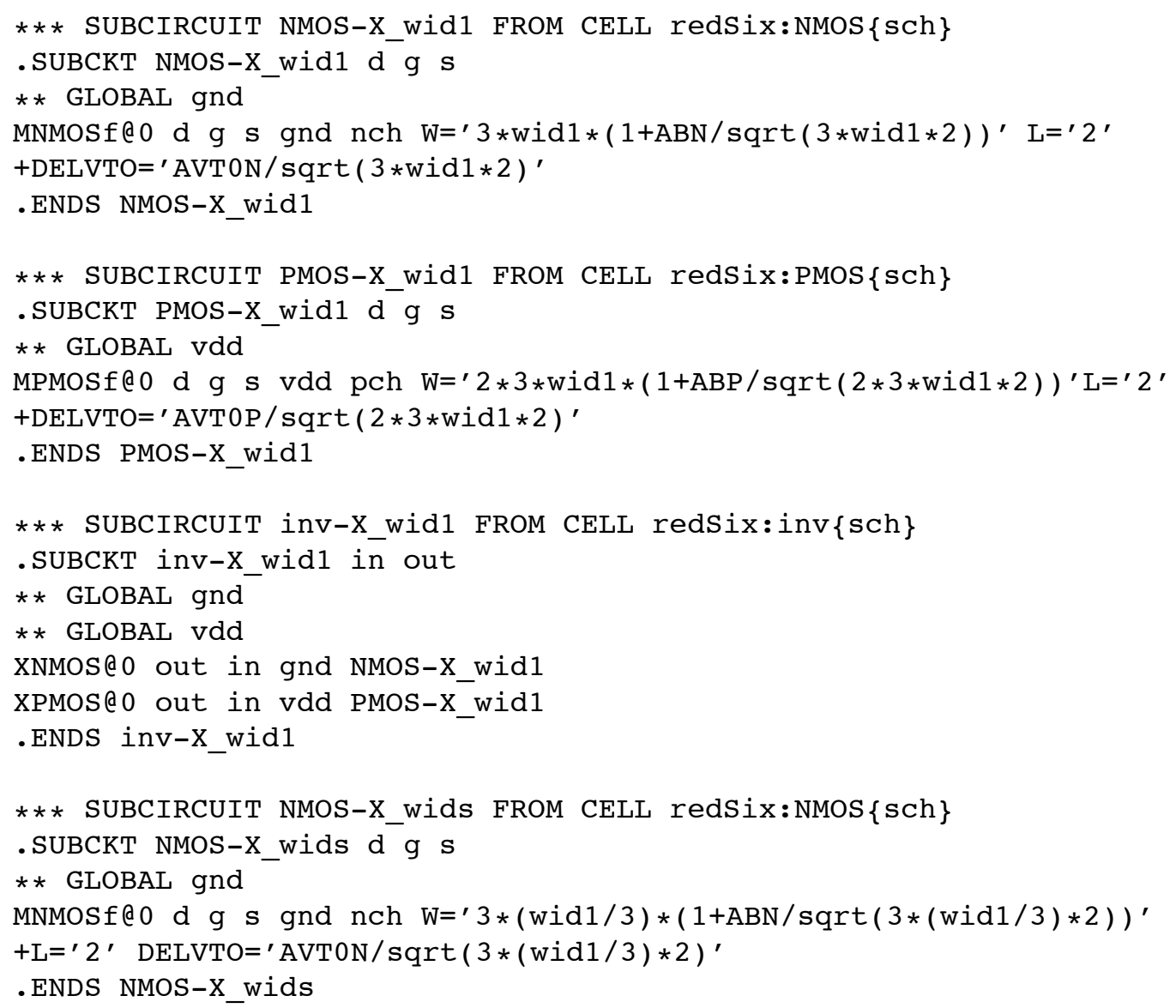




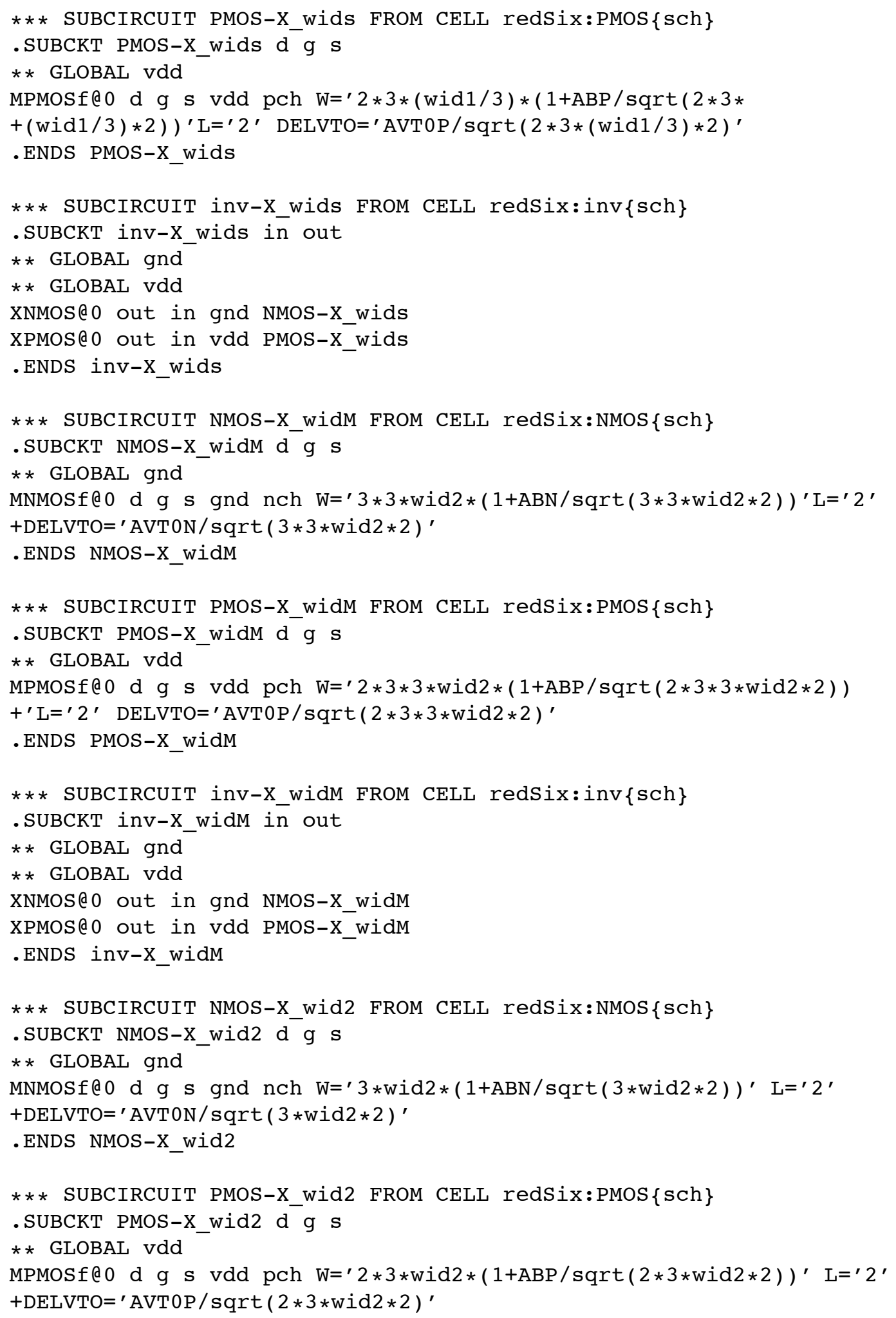


.ENDS PMOS-X_wid2

*** SUBCIRCUIT inv-X_wid2 FROM CELL redSix:inv\{sch $\}$

.SUBCKT inv-X_wid2 in out

* * GLOBAL gnd

** GLOBAL vdd

XNMOS@0 out in gnd NMOS-X_wid2

XPMOS@0 out in vdd PMOS-X_wid2

. ENDS inv-X_wid2

-global gnd vdd

*** TOP LEVEL CELL: LUt B Blackbox $1\{\mathrm{sch}\}$

XLUT A ha@0 out[1] in[1] LUT_A halfGasP

VPulse@o net@139 gnd pulse $0{ }^{-}, \overline{1} \mathrm{v}^{\prime}$ '20ns' '50ps' '50ps' '2ns' '4ns'

Xinve1 Din[1] in[1] inv-X_wid1

Xinv@2 net@139 Din[1] inv-X_wids

Xinve3 net@64 inve3_out inv-X_widM

Xinve 4 out[1] net@ 64 inv-X_wid2

* Trailer cards are described in this file:

. include '/u/mettalag/models/Models_90nm/MOSIS_trailer_partA1.hsp' . END 


\section{A.5 Modified SPICE TRAILER File, Ready for Sweep}

The transient analysis command '.tran' of the trailer file in Appendix A.3 is modified to include the .DATA sweep command for sweeping the driver and load sizes wid1, wid2. For an explanation on the .DATA sweep command below, see Section 3.5.2, page 25. For more details on .DATA sweep commands, see the HSPICE reference manual [34], Chapter 2: HSPICE and HSPICE RF Netlist Commands, pages 60-66.

We also added .MEASUREMENT statements to measure signal transition and path delays. Again, for a quick explanation, see Section 3.5.2. For more details, see [34], Chapter 2, pages 162-165.

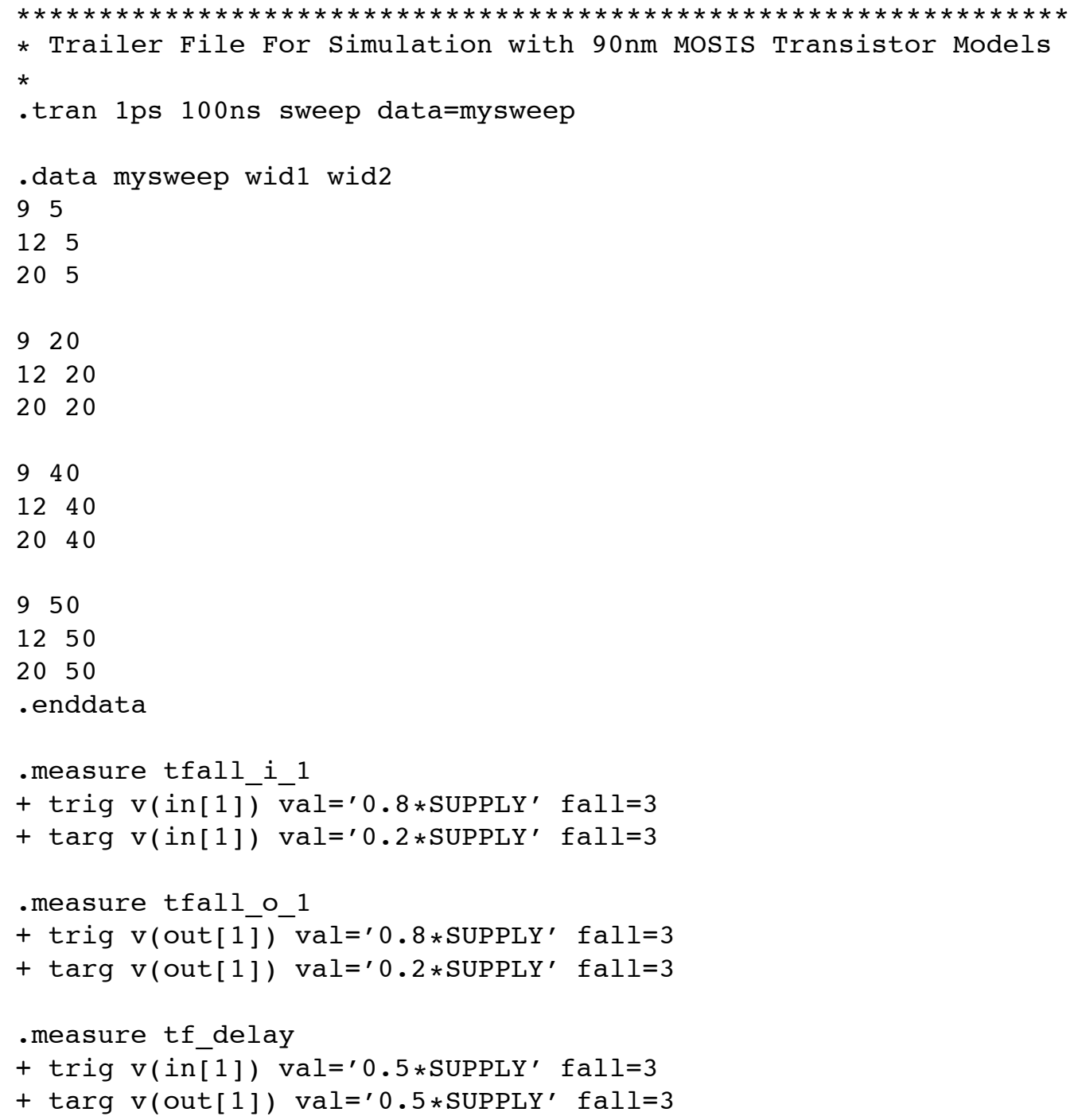




\section{A.6 SPICE Output File}

Below is the SPICE output file with the look up table results. Its file extension is ' $m t 0$ '. For an explanation of the output format, see Section 3.6, page 26. These results belong to the schematics in Figure 3.1-Figure 3.2 and were obtained by doing the simulation sweep in SPICE. In Section 3.7, Figure 3.8, we validate these results against the results obtained for the schematics in Figure 3.7 where the sweep happens in the design space. Specifically: we validated the simulation results for run index $1,4,8,12$.

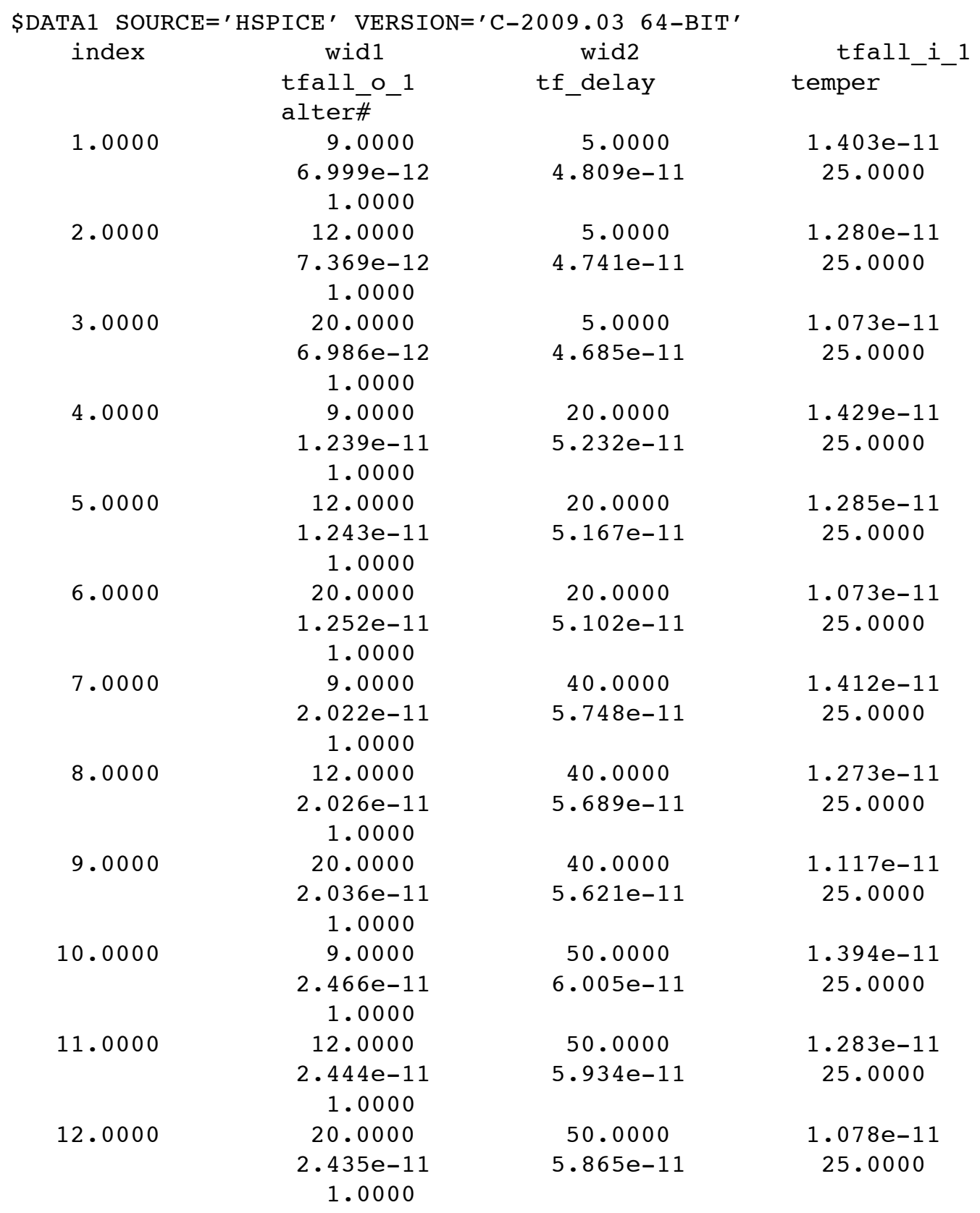




\section{Appendix B}

\section{SPICE FILES FOR SWEEPS IN THE DESIGN SPACE}

Appendix B shows the files that we used for the reference design in Figure 3.7, page 31, where we did the sweep in Electric's design space rather than in SPICE. Appendix B.1 shows the SPICE netlist generated from Electric, Appendix B.2 has the SPICE simulation trailer file, and Appendix B.3 shows the SPICE output file.

\section{B.1 SPICE Netlist for Reference Design}

Here is the netlist for Figure 3.7, page 31, with four simulation environments in parallel:

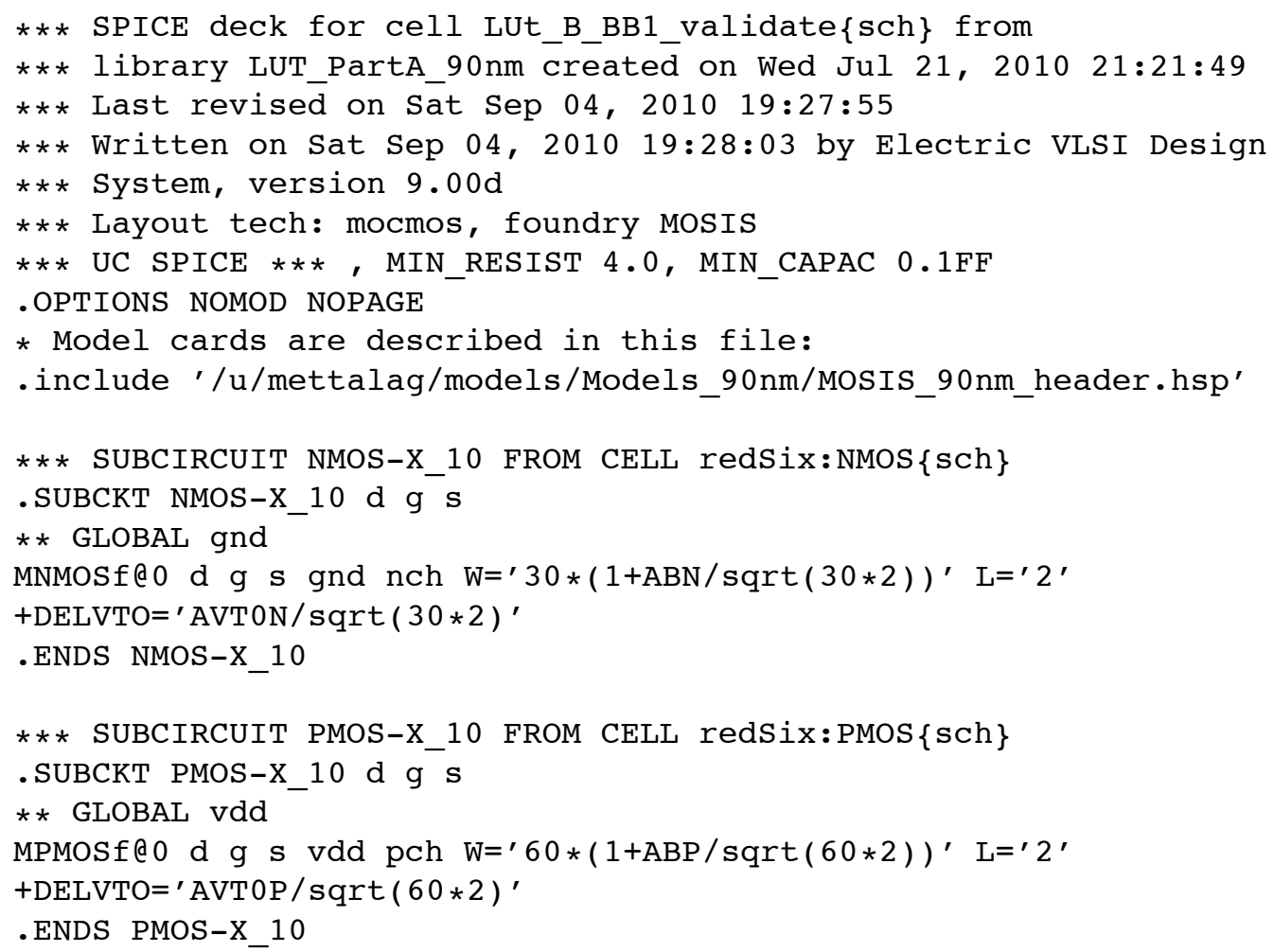




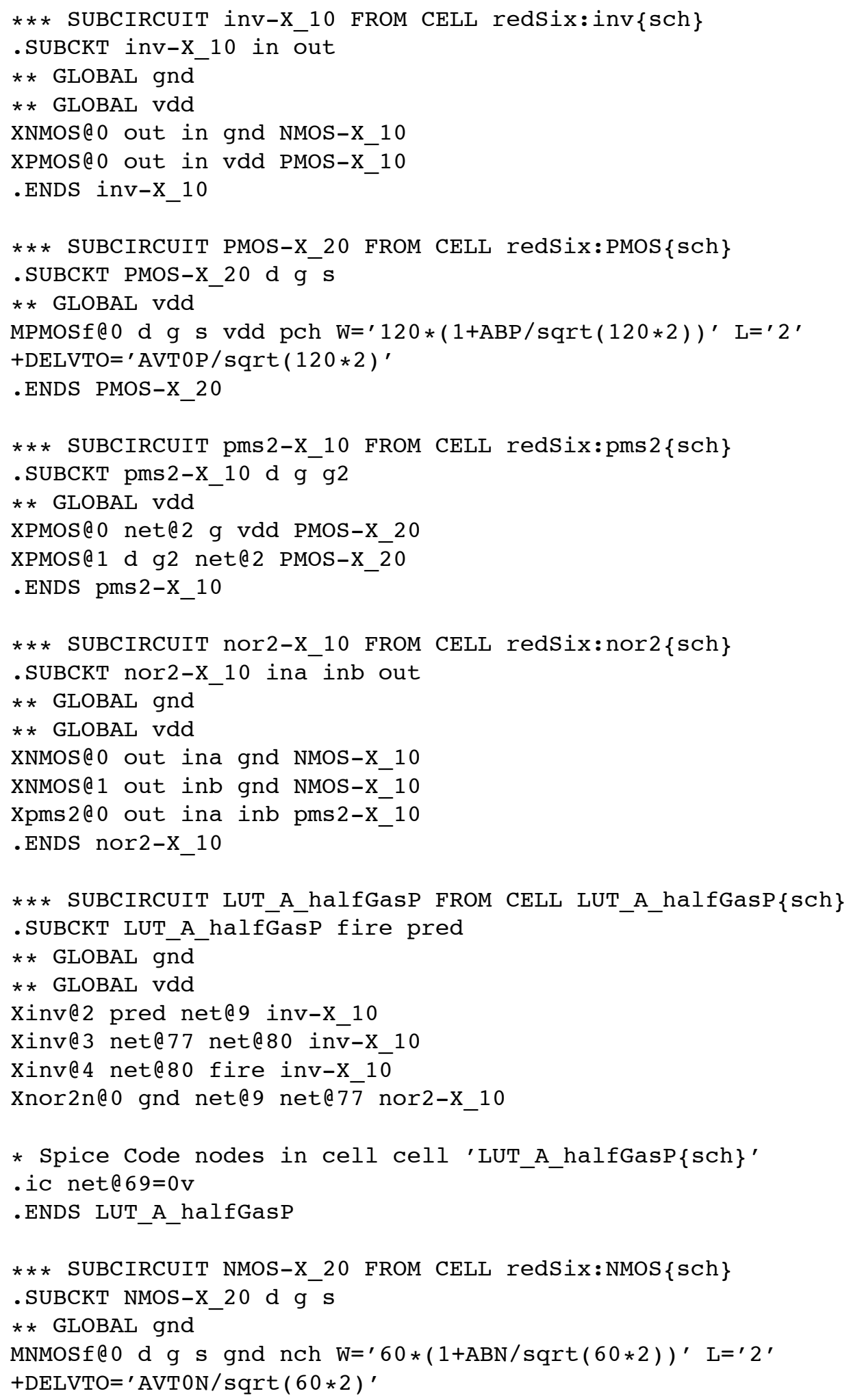


.ENDS NMOS-X_20

*** SUBCIRCUIT inv-X_20 FROM CELL redSix:inv\{sch\}

.SUBCKT inv-X_20 in out

** GLOBAL gnd

** GLOBAL vdd

XNMOS@O out in gnd NMOS-X_20

XPMOS@ 0 out in vdd PMOS-X_20

.ENDS inv-X_20

*** SUBCIRCUIT NMOS-X_6_33 FROM CELL redSix:NMOS $\{\mathrm{sch}\}$

.SUBCKT NMOS-X_6_33 d g s

** GLOBAL gnd

MNMOSf@0 d g s gnd nch $\mathrm{W}=\prime 18.99 *(1+\mathrm{ABN} / \operatorname{sqrt}(18.99 * 2))^{\prime} \mathrm{L}=\mathbf{\prime}^{\prime}$ ' $^{\prime}$

+ DELVTO= 'AVTON $/$ sqrt $(18.99 * 2)^{\prime}$

.ENDS NMOS-X_6_33

*** SUBCIRCUIT PMOS-X_6_33 FROM CELL redSix:PMOS $\{\mathrm{sch}\}$

.SUBCKT PMOS-X_6_33 d g s

** GLOBAL vdd

MPMOSf@0 d g s vdd pch $W=\prime 37.98 *(1+\mathrm{ABP} / \operatorname{sqrt}(37.98 * 2))^{\prime} \mathrm{L}=\mathbf{\prime}^{\prime}$ ' $^{\prime}$

+DELVTO $={ }^{\prime}$ AVTOP $/ \operatorname{sqrt}(37.98 * 2)^{\prime}$

.ENDS PMOS-X_6_33

*** SUBCIRCUIT inv-X_6_33 FROM CELL redSix:inv\{sch\}

.SUBCKT inv-X_6_33 in out

** GLOBAL gnd

** GLOBAL vdd

XNMOS@O out in gnd NMOS-X_6_33

XPMOS@ 0 out in vdd PMOS-X_6_33

.ENDS inv-X_6_33

*** SUBCIRCUIT NMOS-X_150 FROM CELL redSix:NMOS\{sch $\}$

.SUBCKT NMOS-X_150 d $\mathrm{g}$ s

** GLOBAL gnd

MNMOSf@0 d g s gnd nch $\mathrm{W}={ }^{\prime} 450 *(1+\mathrm{ABN} / \operatorname{sqrt}(450 * 2))^{\prime} \mathrm{L}={ }^{\prime} 2^{\prime}$

+ DELVTO $={ }^{\prime}$ AVTON $/ \operatorname{sqrt}(450 * 2)^{\prime}$

.ENDS NMOS-X_150

*** SUBCIRCUIT PMOS-X_150 FROM CELL redSix:PMOS $\{\mathrm{sch}\}$

.SUBCKT PMOS-X_150 d g s

** GLOBAL vdd

MPMOSf@o d g s vdd pch $\mathrm{W}=\prime 900 *(1+\mathrm{ABP} / \operatorname{sqrt}(900 * 2))^{\prime} \mathrm{L}=\mathbf{\prime}^{\prime} \mathbf{2}^{\prime}$

+DELVTO $=$ 'AVTOP/sqrt $(900 * 2)^{\prime}$

.ENDS PMOS-X_150

*** SUBCIRCUIT inv-X_150 FROM CELL redSix:inv\{sch\}

.SUBCKT inv-X_150 in out

* * GLOBAL gnd 


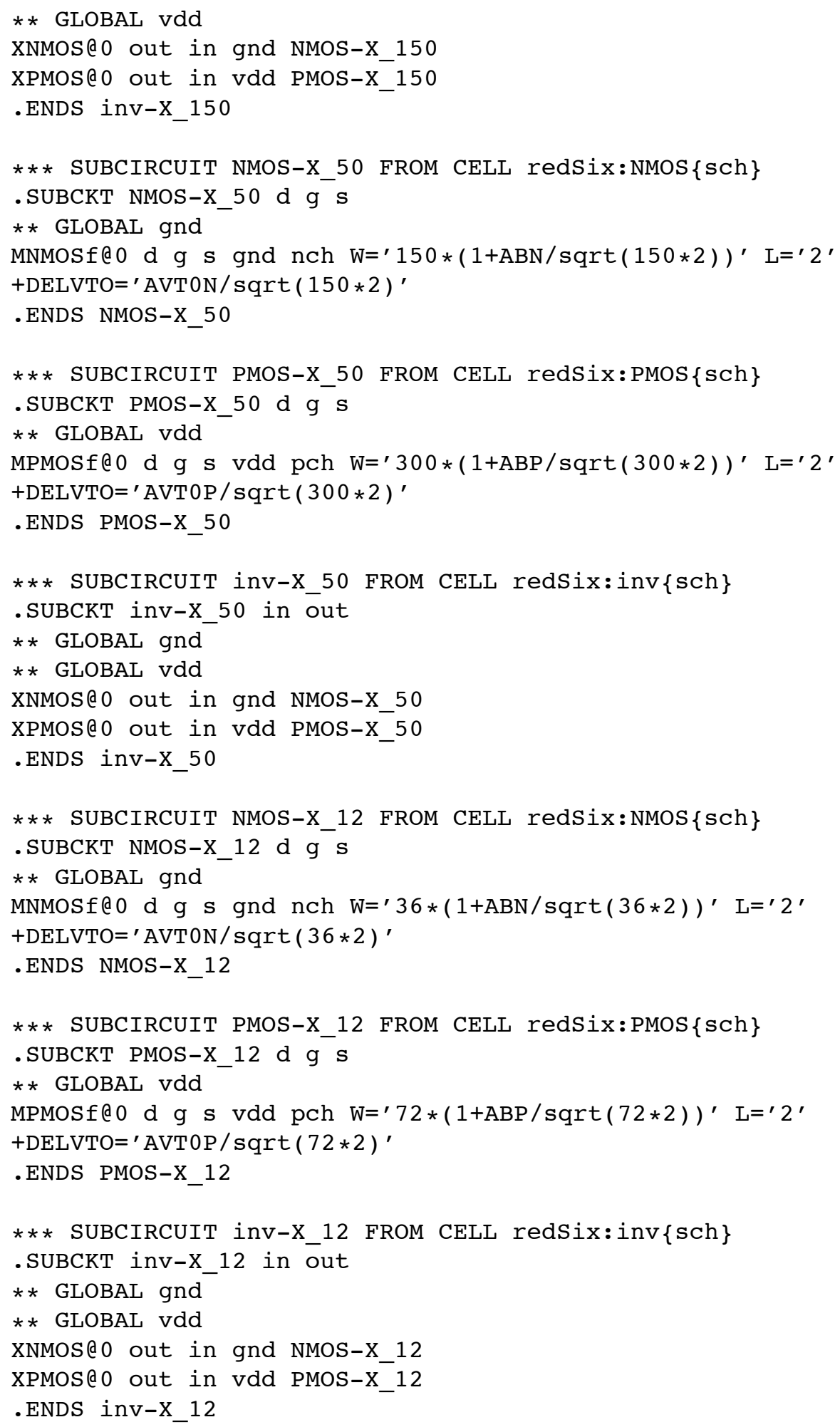




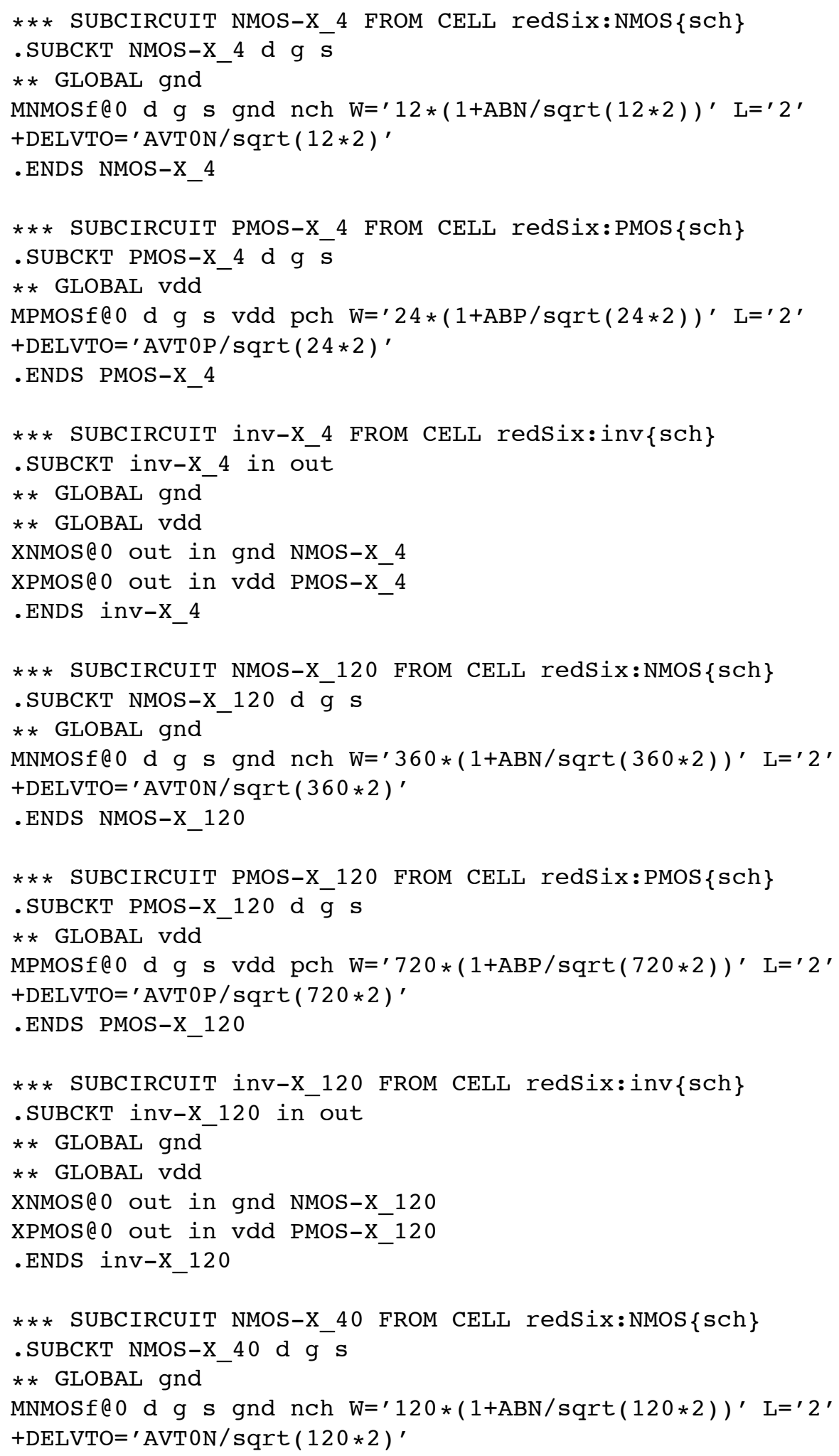




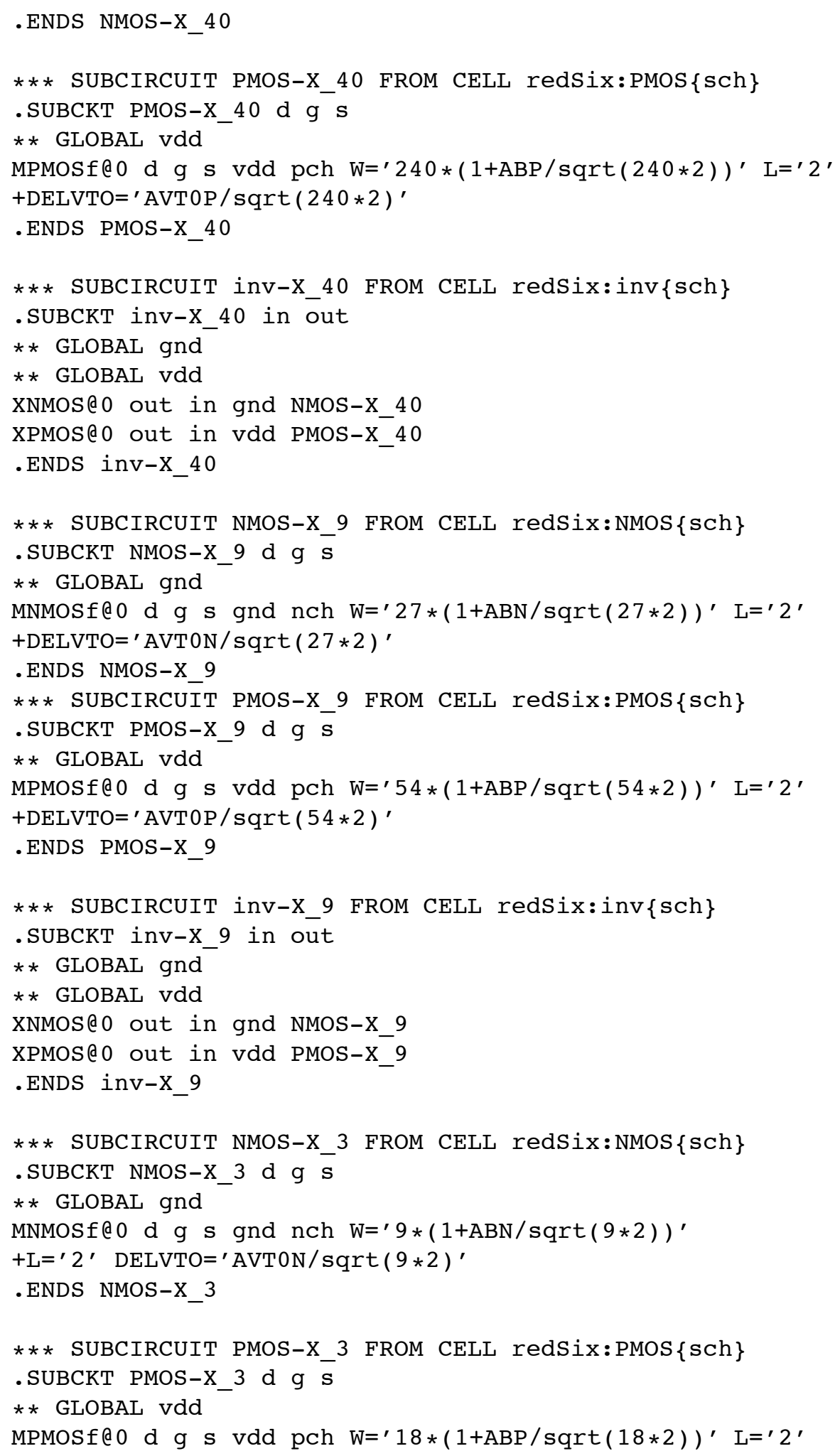




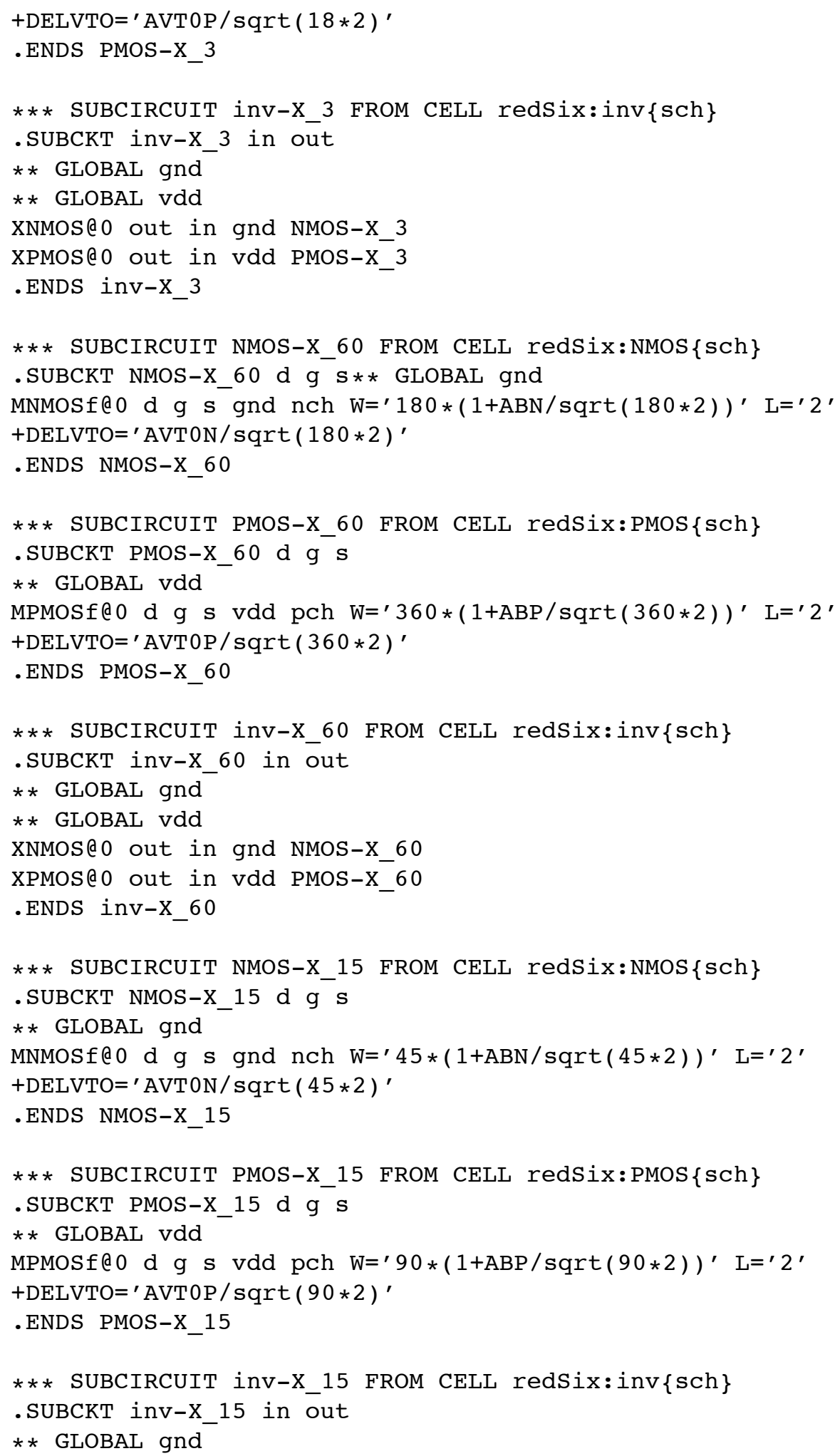




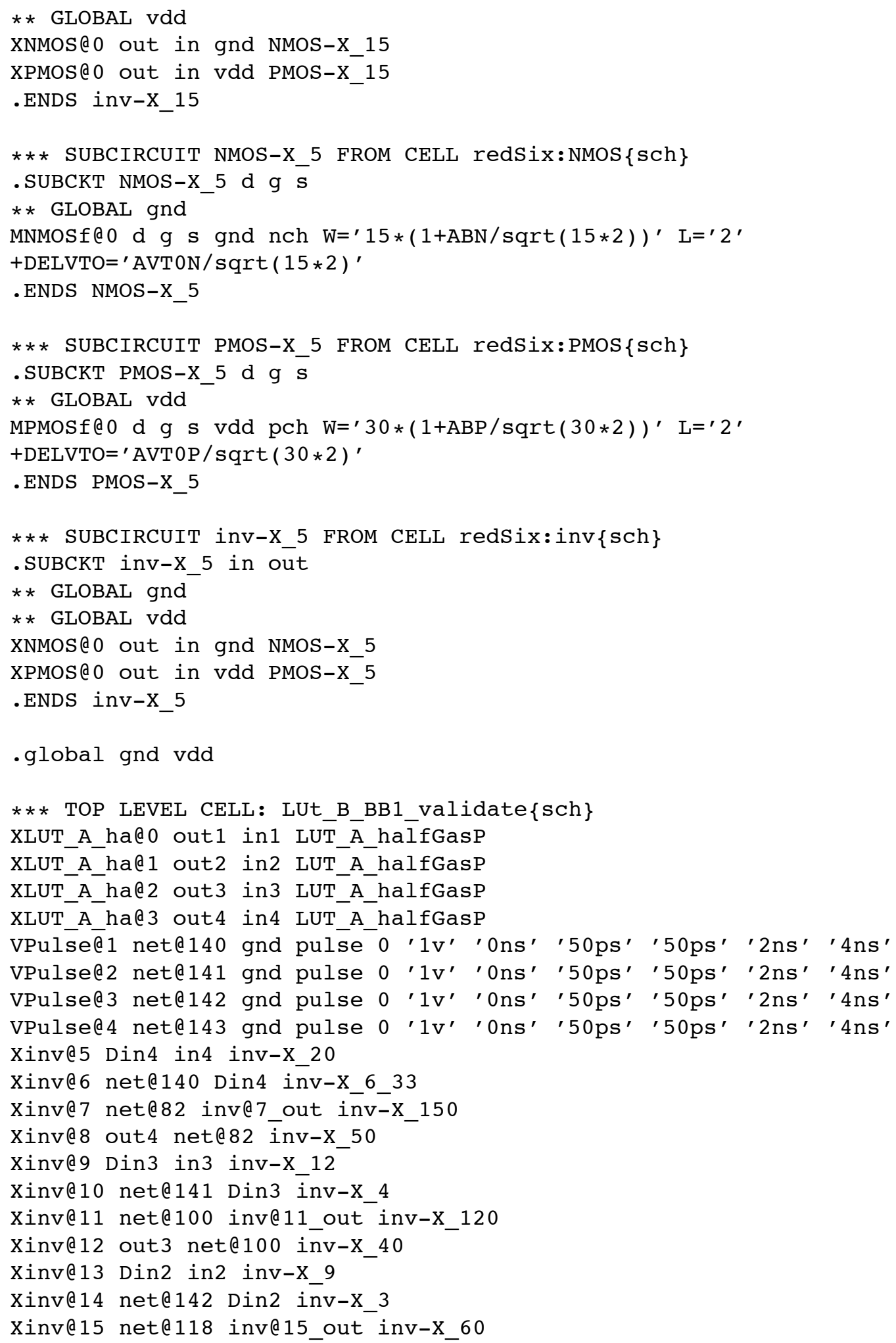


Xinv@16 out2 net@118 inv-X_20

xinv@17 Din1 in 1 inv-x 9

Xinv@18 net@143 Din1 inv-X_3

Xinv@19 net@136 inv@19_out inv-X_15

Xinv@20 out1 net@136 inv-X_5

* Trailer cards are described in this file:

-include '/u/mettalag/models/Models_90nm/MOSIS_trailer_partA2.hsp' . END 


\section{B.2 SPICE TRAILER File for Reference Design}

The trailer file includes measurement statements to obtain the input and output transition slopes and the input-to-output delays for the four parallel simulation environments in Figure 3.7, page 31:

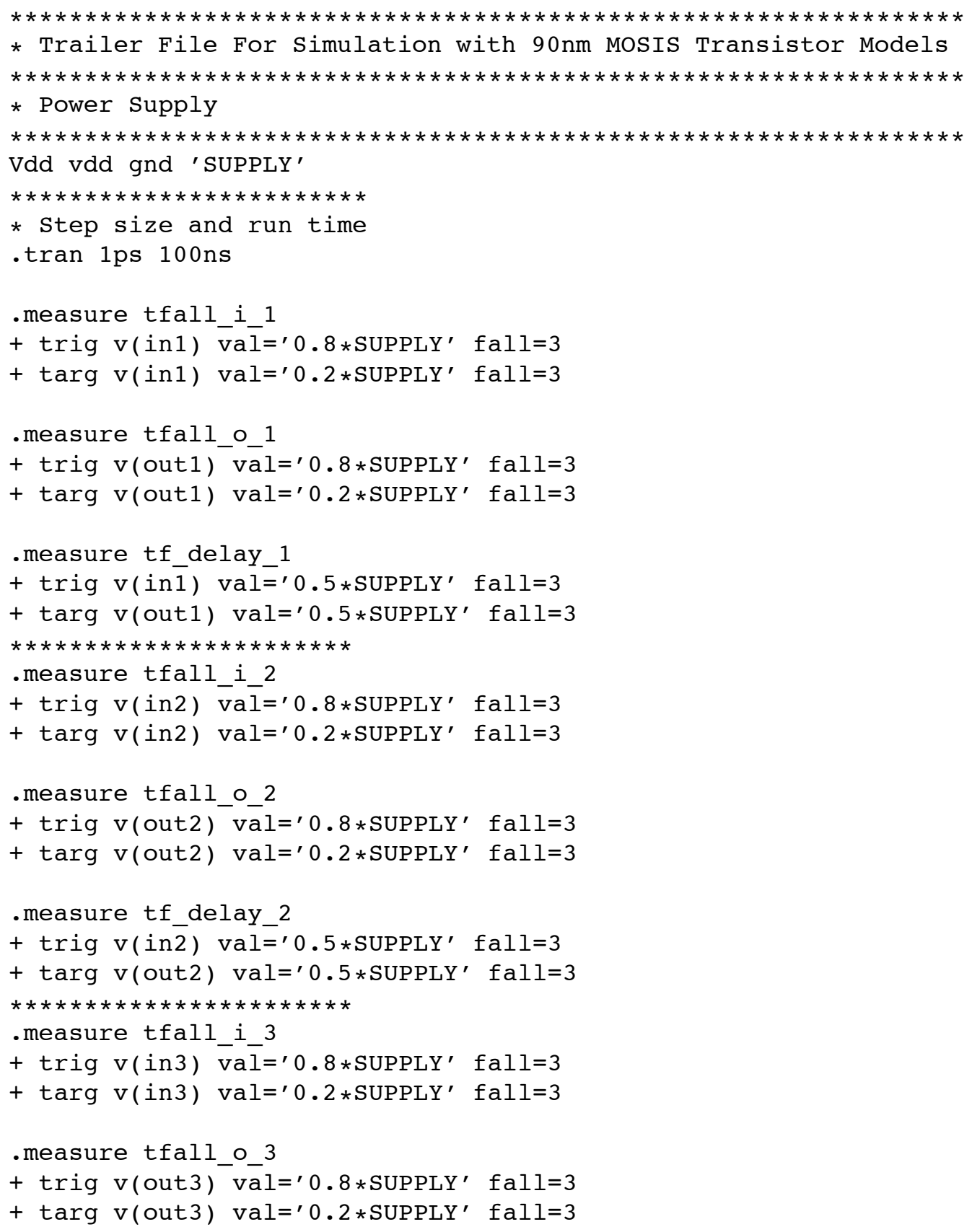




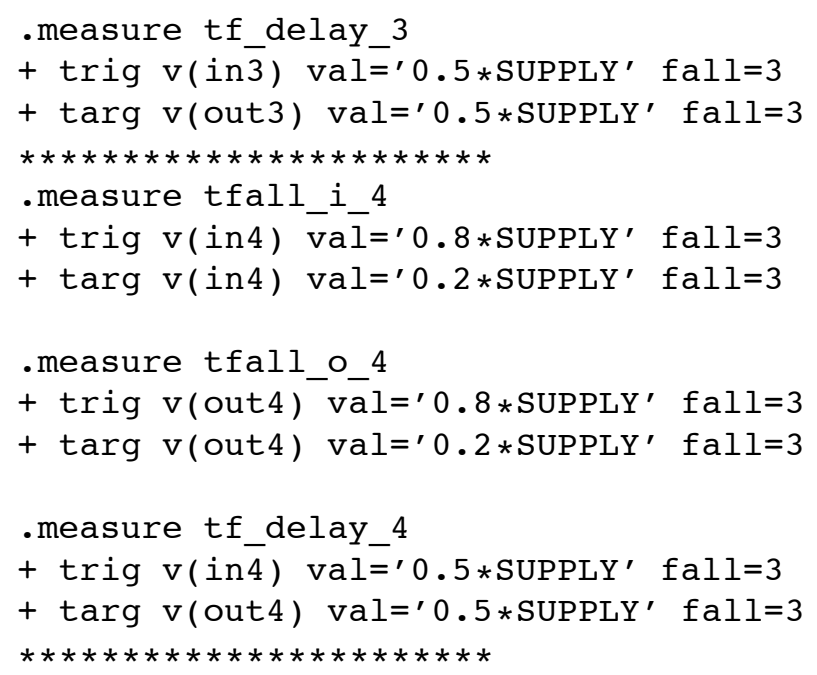




\section{B.3 SPICE Output File for Reference Design}

Here are the results of the reference design experiment in Figure 3.7, page 31, with the slope and delay times for a falling input-to-output transition in each of the four simulation environments:

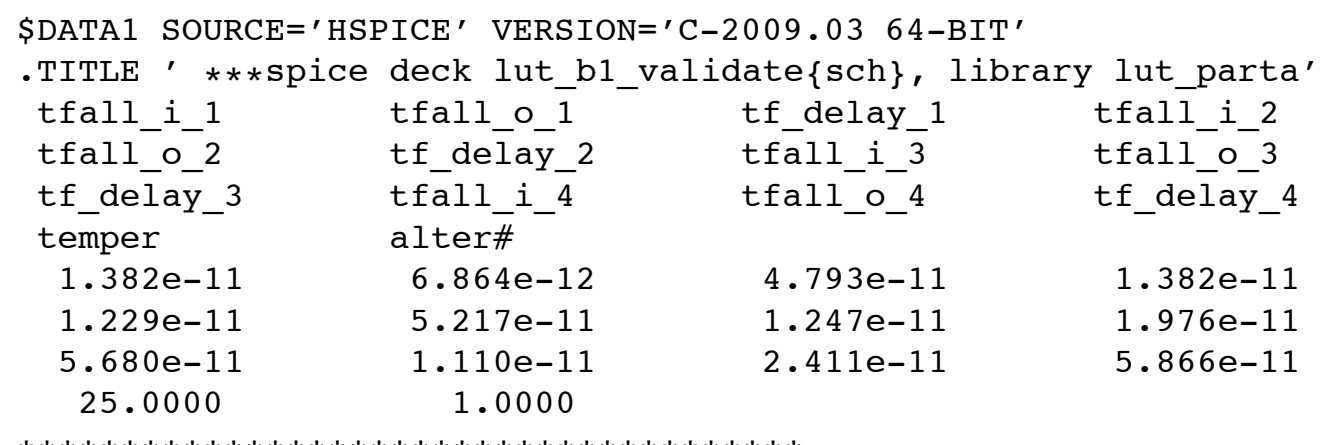




\section{Appendix C}

\section{SPICE FILES FOR FIGURE 4.8 AND LUTblue:PRED ${ }_{i}-$ FIRE $_{i}-$}

\section{C.1 SPICE Netlist}

The SPICE netlist file generated from the design schematics in Electric has the details of all the components and devices used in the schematics of Figure 4.12, page 67, and in the black box schematics of Figure 4.8, page 57. The black box schematics belongs to relative timing path LUTblue:PRED $-\mathrm{FIRE}_{i}-$. The sweep parameters wid1 and wid2 are the sizes of the driver and load inverters. The file extension is '.spi'.

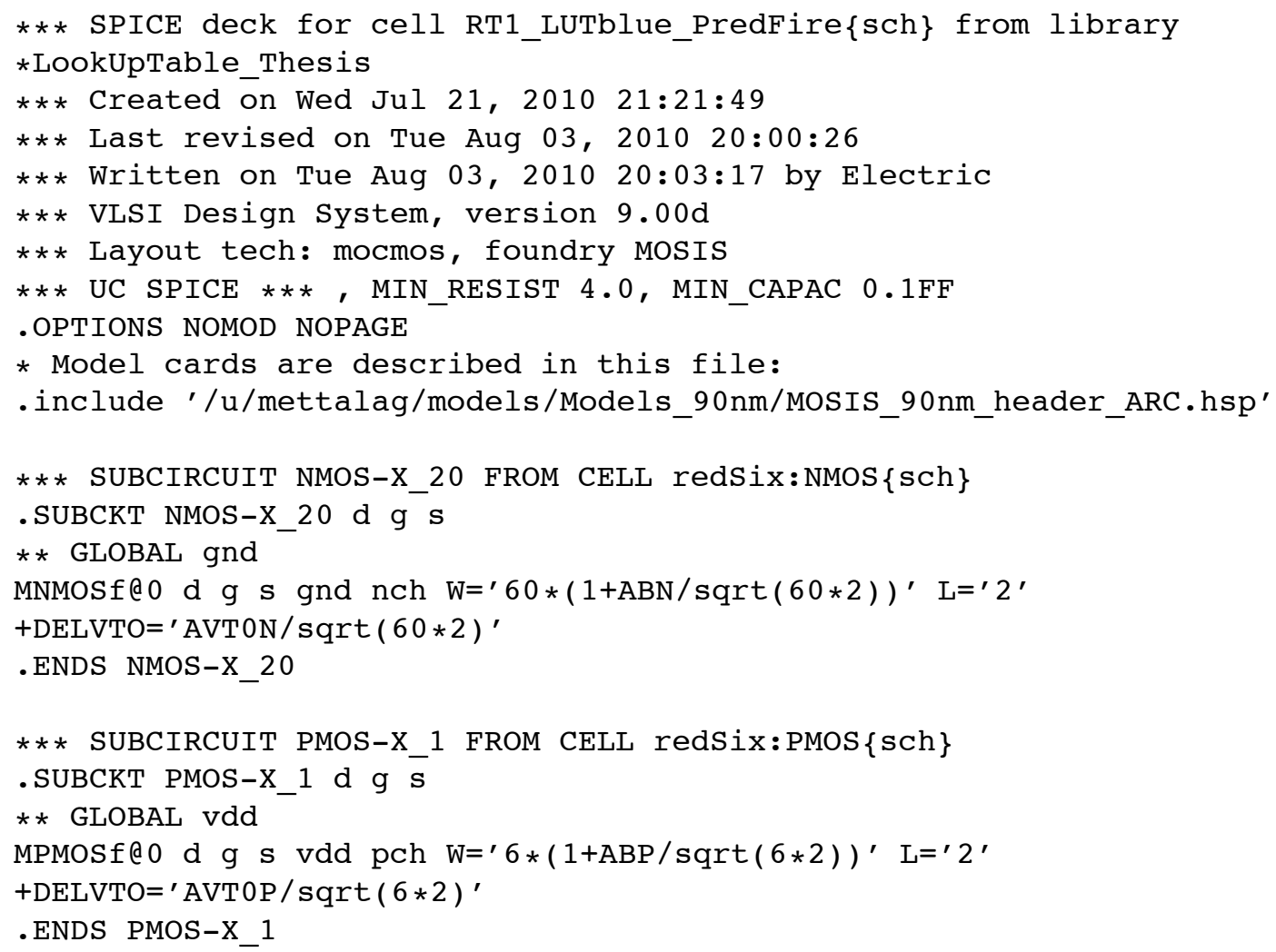




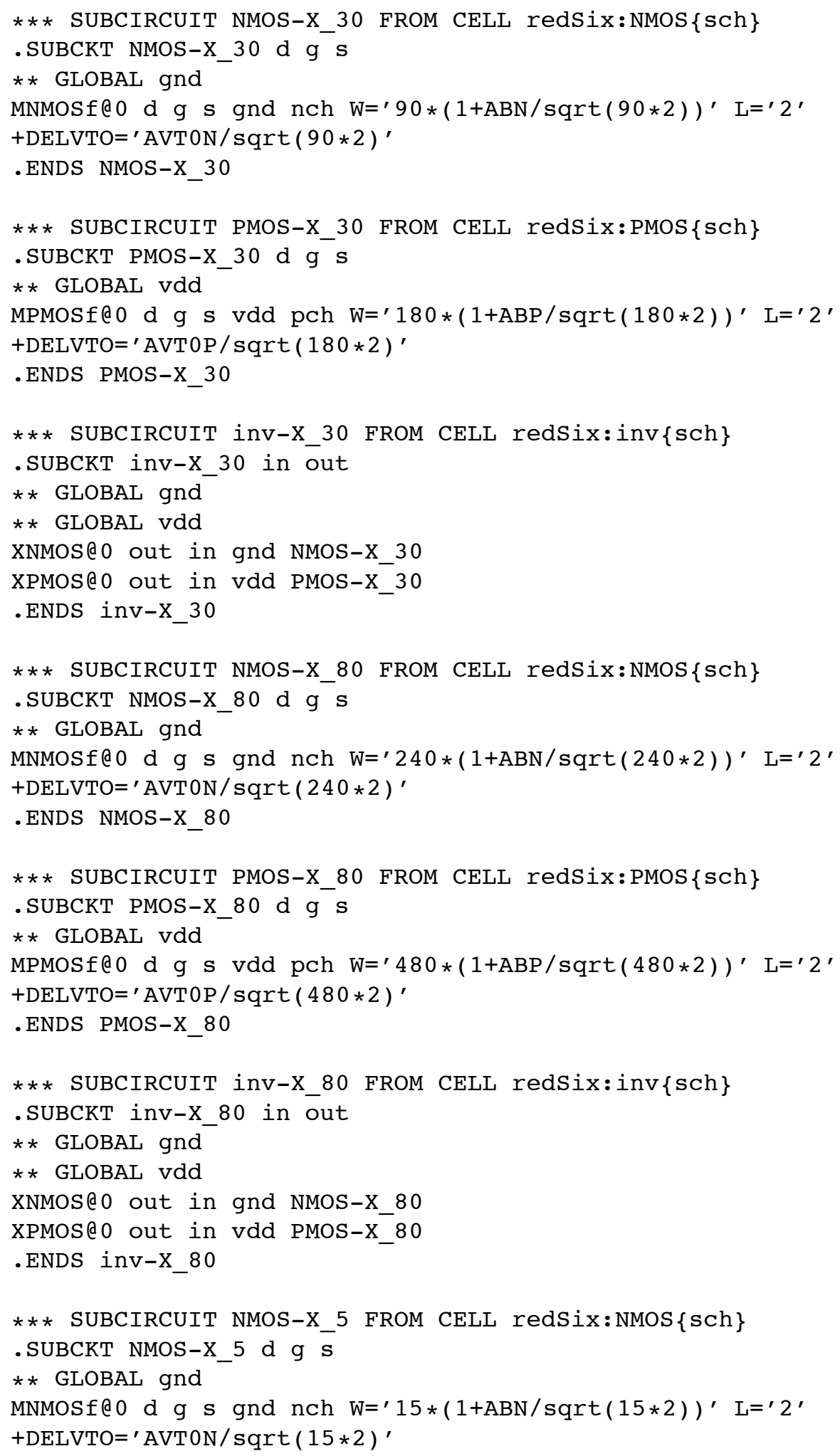




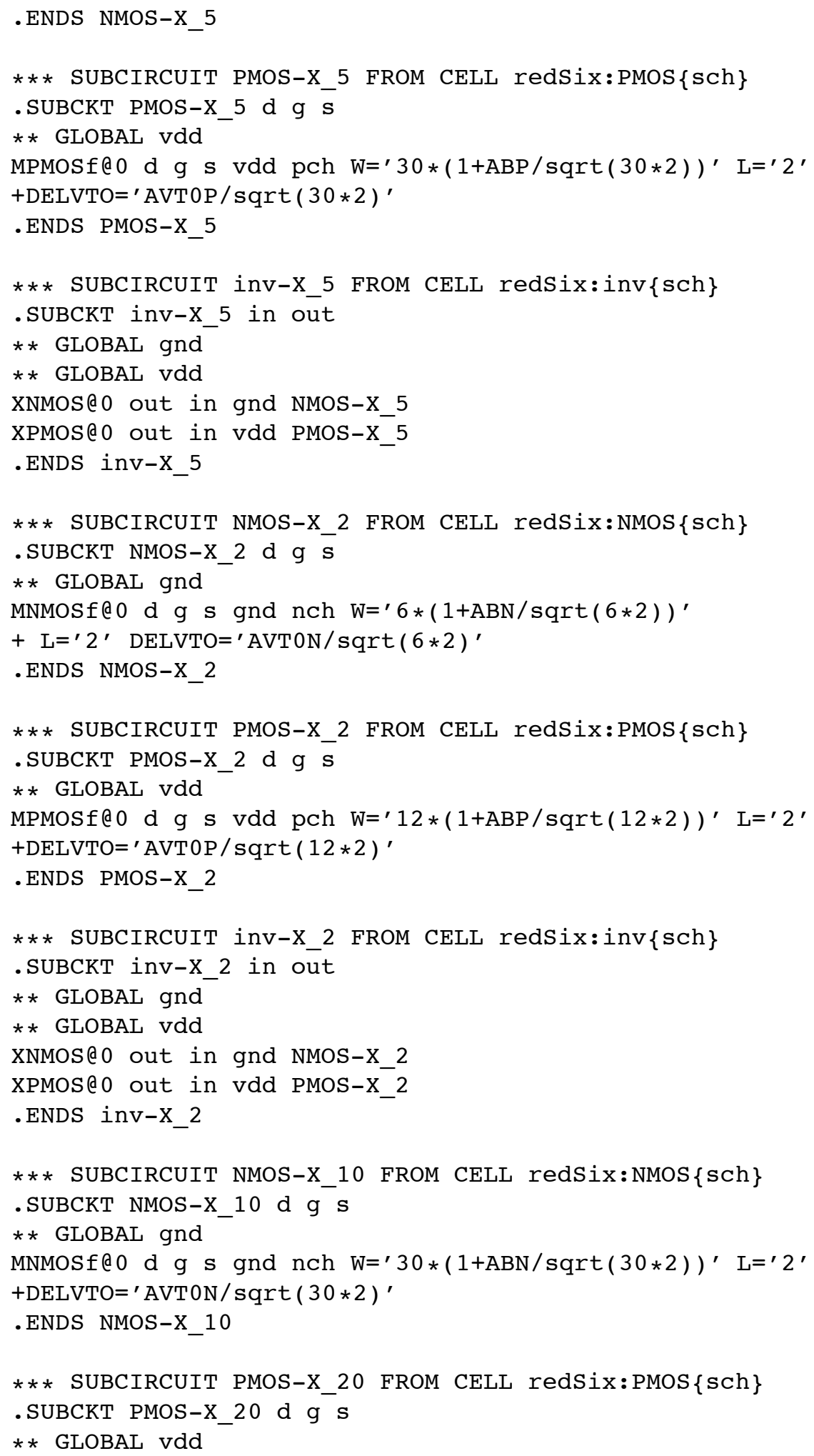


MPMOSf@0 d g s vdd pch $\mathrm{W}=$ ' $120 *(1+\mathrm{ABP} / \operatorname{squt}(120 * 2))^{\prime} \mathrm{L}=\mathbf{\prime}^{\prime}$

$+\mathrm{DELVTO}=$ ' AVTOP/sqrt $(120 * 2)$ '

.ENDS PMOS-X_20

*** SUBCIRCUIT pms2-X_10 FROM CELL redSix:pms 2 sch

.SUBCKT pms2-X_10 d g g2

** GLOBAL vdd

XPMOS@0 net@2 g vdd PMOS-X_20

XPMOS@1 d g2 nete2 PMOS-X_20

- ENDS pms2-X_10

*** SUBCIRCUIT nor2-X_10 FROM CELL redSix:nor $2\{\mathrm{sch}\}$

.SUBCKT nor2-X_10 ina inb out

* * GLOBAL gnd

* * GLOBAL vdd

XNMOS@O out ina gnd NMOS-X_10

XNMOSe1 out inb gnd NMOS-X_10

Xpms 2 @ out ina inb pms $2-\mathrm{X} 10$

. ENDS nor $2-\mathrm{X} \_10$

*** SUBCIRCUIT LUTblue_RT1_path FROM CELL LUTblue_RT1_path\{sch\} .SUBCKT LUTblue_RT1_path fire pred

** GLOBAL gnd

** GLOBAL vdd

XNMOS@O NMOS@O d fire gnd NMOS-X 20

XPMOS@0 net@57 net@ 2 vdd PMOS-X_ $\overline{1}$

XPMOS@1 net@57 fire net@ 4 PMOS $-\bar{X} \_1$

EVCVS@0 net@13 gnd vcvs net@9 gnd 1 max='10V' $\min ='-10 \mathrm{~V}$ '

Xinv@0 net@20 net@19 inv-X_30

Xinv@1 net@19 fire inv-X_8

Xinve2 pred net@ 9 inv-X_ $\overline{5}$

Xinve 4 net@ 4 net@ 2 inv-X_2

Xnor2ne0 nete13 nete 9 nete 20 nor $2-x_{-} 10$

* Spice Code nodes in cell cell 'LUTblue_RT1_path\{sch\}'

-ic $\mathrm{succ}=0 \mathrm{v}$ net@ $68=1 \mathrm{v}$

.ENDS LUTblue_RT1_path

*** SUBCIRCUIT NMOS-X_wid1 FROM CELL redSix:NMOS $\{\mathrm{sch}\}$

.SUBCKT NMOS-X_wid1 d $g$ s

** GLOBAL gnd

MNMOSflo d g $\mathrm{s}$ gnd $\mathrm{nch} \mathrm{W}={ }^{\prime} 3 * \mathrm{Wid} 1 *(1+\mathrm{ABN} / \operatorname{sqrt}(3 * \operatorname{Wid} 1 * 2))^{\prime} \mathrm{L}=$ ' $^{\prime}$

+ DELVTO $=$ ' AVTON/sqrt $(3 *$ wid $1 * 2)$ '

. ENDS NMOS-X_wid1

*** SUBCIRCUIT PMOS-X_wid1 FROM CELL redSix:PMOS $\{\mathrm{sch}\}$

.SUBCKT PMOS-X_wid1 d $g$ s

* * GLOBAL vdd

MPMOSf@0 d g s vdd pch $\mathrm{W}={ }^{\prime} 2 * 3 * \operatorname{Wid} 1 *(1+\mathrm{ABP} / \operatorname{sqrt}(2 * 3 * \operatorname{Wid} 1 * 2))^{\prime}$ 
$+\mathrm{L}={ }^{\prime} 2^{\prime} \mathrm{DELVTO}={ }^{\prime} \mathrm{AVT} 0 \mathrm{P} / \operatorname{sqrt}(2 * 3 * \mathrm{wid} 1 * 2)^{\prime}$

.ENDS PMOS-X_wid1

*** SUBCIRCUIT inv-X_wid1 FROM CELL redSix:inv\{sch .SUBCKT inv-X_wid1 in out

** GLOBAL gnd

** GLOBAL vdd

XNMOS@O out in gnd NMOS-X_wid1

XPMOS@0 out in vdd PMOS-X_wid1

.ENDS inv-X_wid1

*** SUBCIRCUIT NMOS-X_wids FROM CELL redSix:NMOS $\{\mathrm{sch}\}$

.SUBCKT NMOS-X_wids d $g$ s

* * GLOBAL gnd

MNMOSf@0 d g s gnd $\mathrm{nch} \mathrm{W}=\prime 3 *(\mathrm{wid} 1 / 3)$ *

$+(1+\mathrm{ABN} / \operatorname{sqrt}(3 *(\mathrm{wid} 1 / 3) * 2))^{\prime}$

$+\mathrm{L}={ }^{\prime} 2^{\prime} \mathrm{DELVTO}={ }^{\prime} \operatorname{AVT} 0 \mathrm{~N} / \operatorname{sqrt}(3 *(\operatorname{wid} 1 / 3) * 2)^{\prime}$

. ENDS NMOS-X_wids

*** SUBCIRCUIT PMOS-X_wids FROM CELL redSix:PMOS $\{\mathrm{sch}\}$

. SUBCKT PMOS-X_wids d $g$ s

* * GLOBAL vdd

MPMOSf@0 d g s vdd pch $W=\prime 2 * 3 *($ widl $/ 3) *(1+\operatorname{ABP} / \operatorname{sqrt}(2 * 3 *$

$+(\operatorname{wid} 1 / 3) * 2))^{\prime} \mathrm{L}={ }^{\prime} 2^{\prime} \mathrm{DELVTO}={ }^{\prime} \mathrm{AVTOP} / \operatorname{sqrt}(2 * 3 *(\operatorname{wid} 1 / 3) * 2)^{\prime}$

.ENDS PMOS-X_wids

*** SUBCIRCUIT inv-X_wids FROM CELL redSix:inv\{sch

.SUBCKT inv-X_wids in out

* * GLOBAL gnd

** GLOBAL vdd

XNMOS@O out in gnd NMOS-X_wids

XPMOS@O out in vdd PMOS-X_wids

. ENDS inv-X_wids

*** SUBCIRCUIT NMOS-X_widM FROM CELL redSix:NMOS $\{\mathrm{sch}\}$

.SUBCKT NMOS-X_widM d g s

* * GLOBAL gnd

MNMOSf@0 d $\mathrm{g}$ s gnd nch $\mathrm{W}=\prime 3 * 3 * \mathrm{wid} 2 *$

$+(1+\mathrm{ABN} / \operatorname{sqrt}(3 * 3 * \mathrm{wid} 2 * 2))^{\prime}$

$+\mathrm{L}={ }^{\prime} 2$ ' DELVTO='AVTON $/ \operatorname{sqrt}(3 * 3 * \text { wid } 2 * 2)^{\prime}$

.ENDS NMOS-X_widM

*** SUBCIRCUIT PMOS-X_widM FROM CELL redSix:PMOS $\{\mathbf{s c h}\}$

.SUBCKT PMOS-X_widM d $g$ s

* * GLOBAL vdd

MPMOSf@0 d g s vdd pch $\mathrm{W}={ }^{\prime} 2 * 3 * 3 * \mathrm{wid} 2 *$

$+(1+\mathrm{ABP} / \operatorname{sqrt}(2 * 3 * 3 * \mathrm{Wid} 2 * 2))^{\prime} \mathrm{L}={ }^{\prime} 2^{\prime}$

$+\mathrm{DELVTO}={ }^{\prime} \mathrm{AVTOP} / \operatorname{sqrt}(2 * 3 * 3 * \mathrm{Wid} 2 * 2)^{\prime}$

.ENDS PMOS-X_widM 


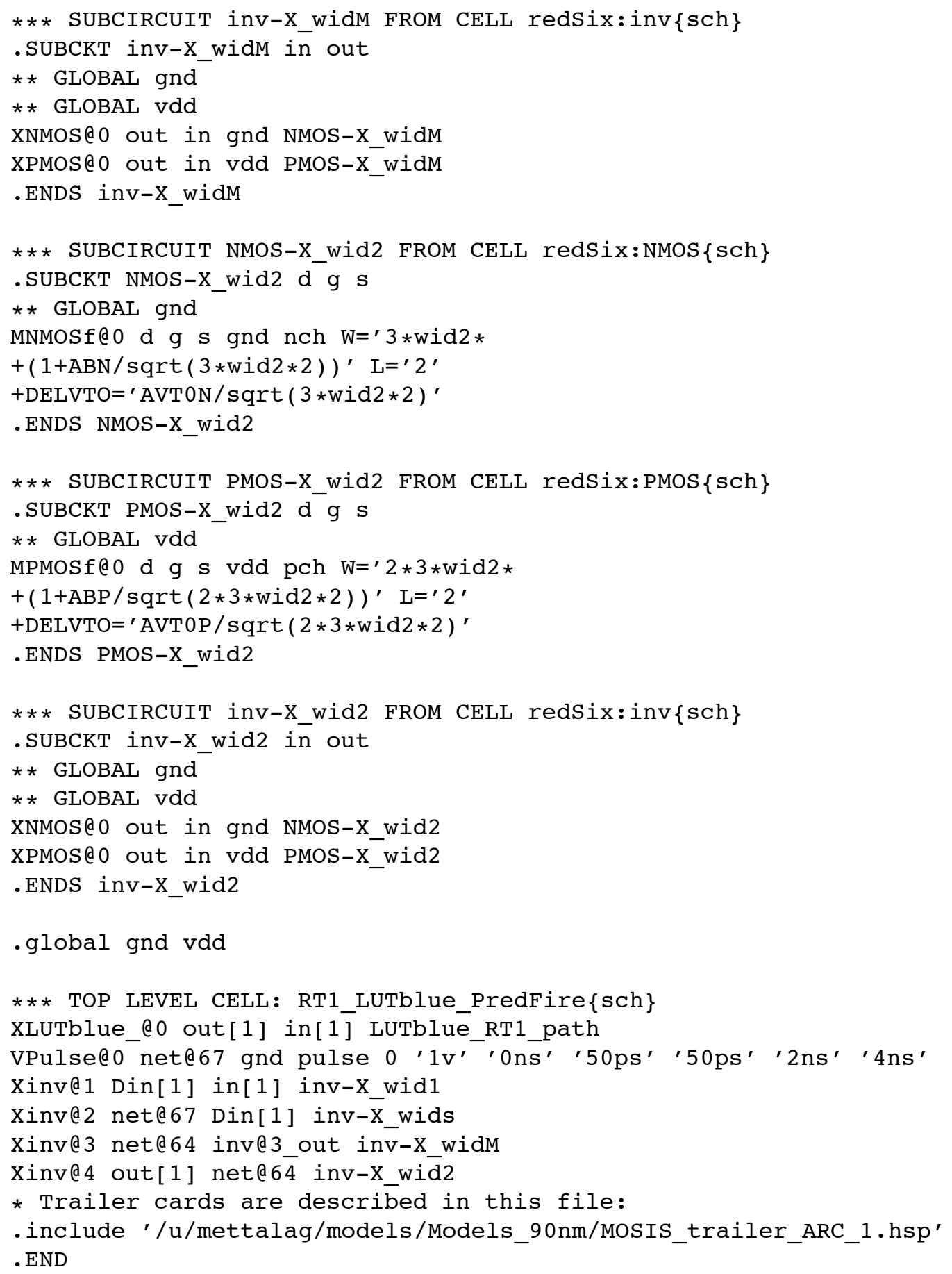




\section{C.2 SPICE Simulation Setup File: HEADER}

Here is the basic file with default simulation parameters for SPICE. Its extension is '.hsp'. It contains a pointer to the 90nm CMOS process models for our GasP designs. We use the library with typical (TT) Process, Voltage and Temperature (PVT) settings.

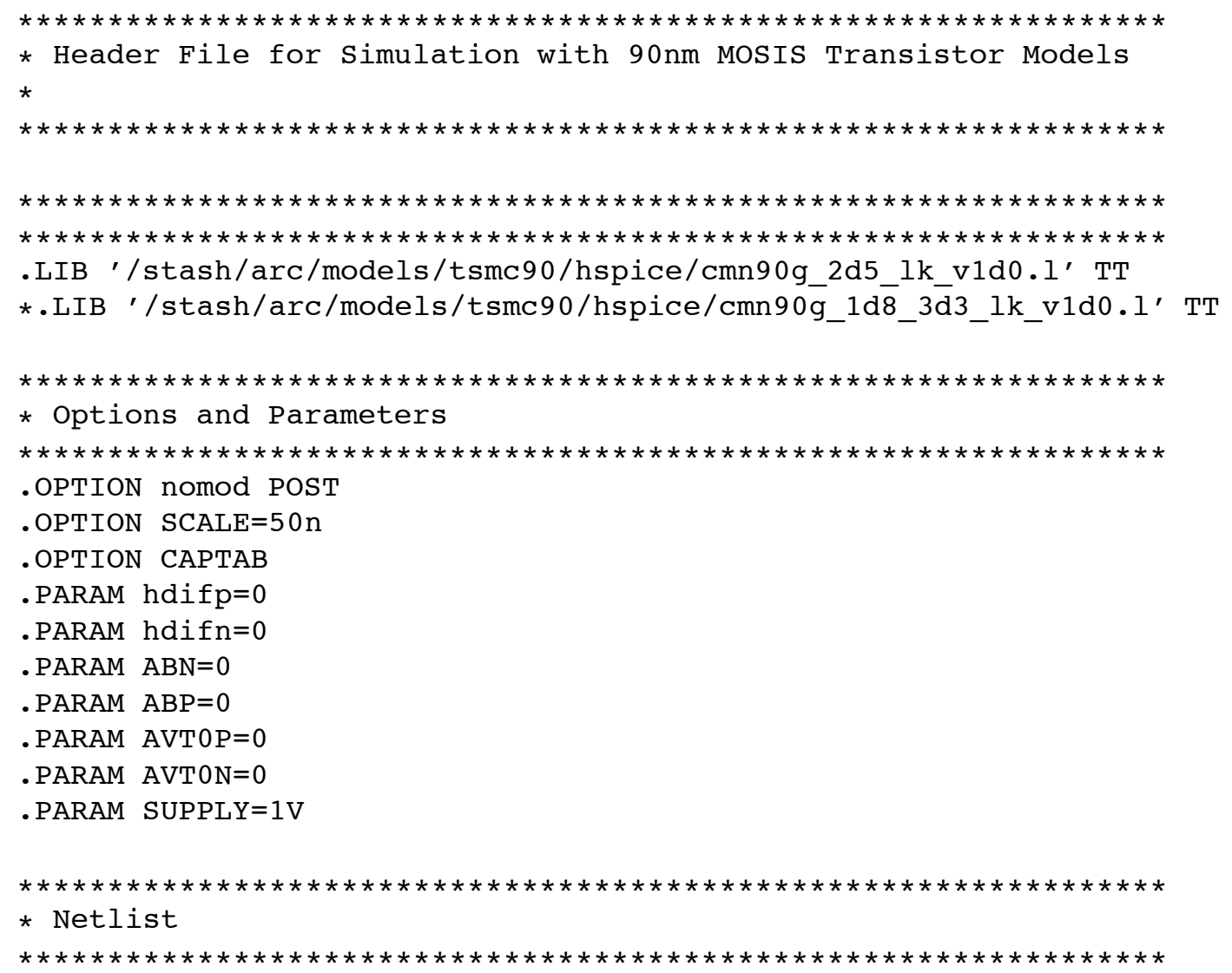




\section{C.3 SPICE Simulation Setup File: TRAILER}

Below follows the SPICE trailer file with the .DATA sweep, transient analysis, and measurement details for the simulation environment in Figure 4.12, page 67, and the black box schematics of Figure 4.8, page 57. We are generating look up tables for relative timing path LUTblue:PRED $i-\mathrm{FIRE}_{i}-$. To do so, we sweep driver size wid1 and load size wid2, and we measure voltage levels on in[1] and out[1].

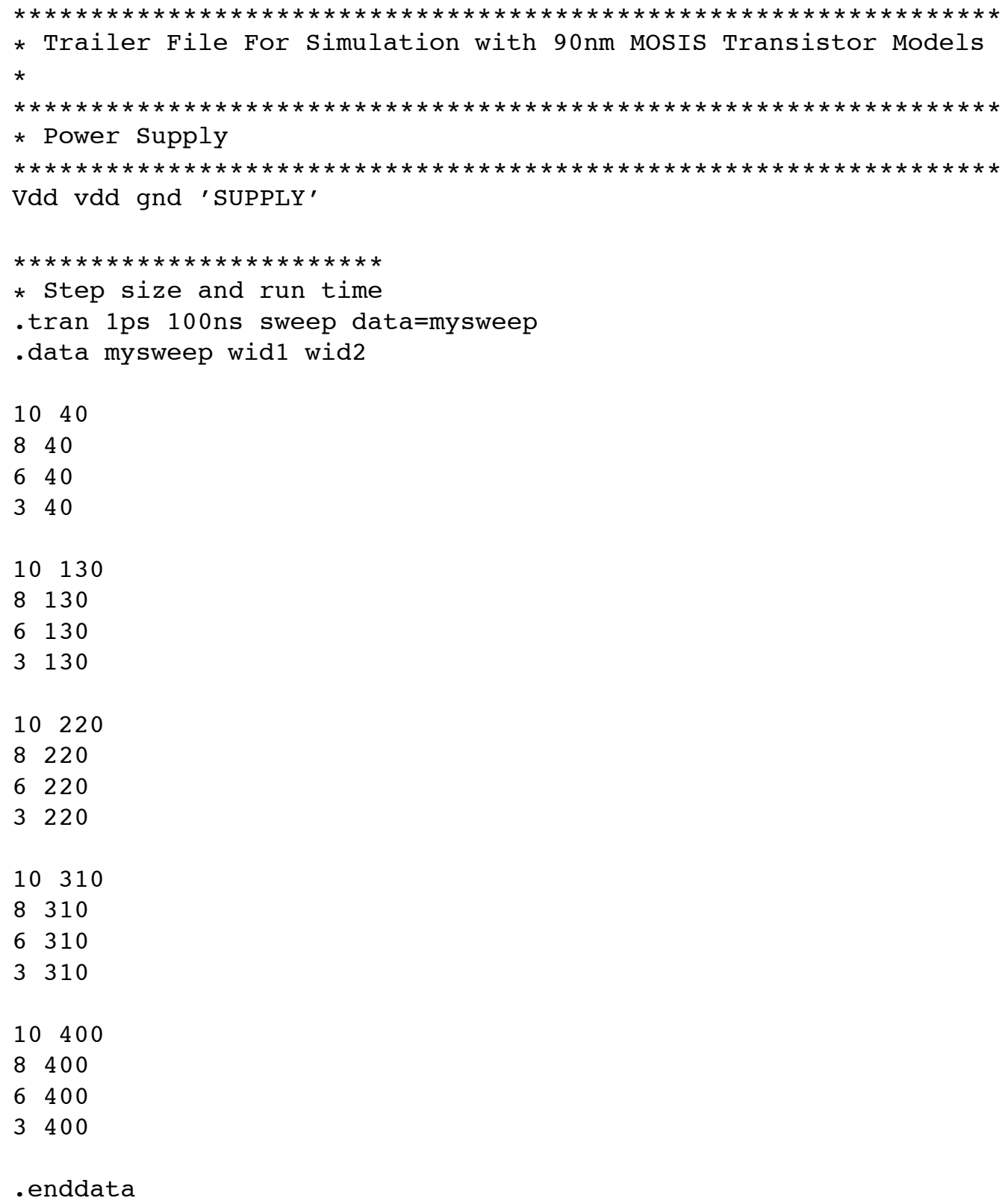




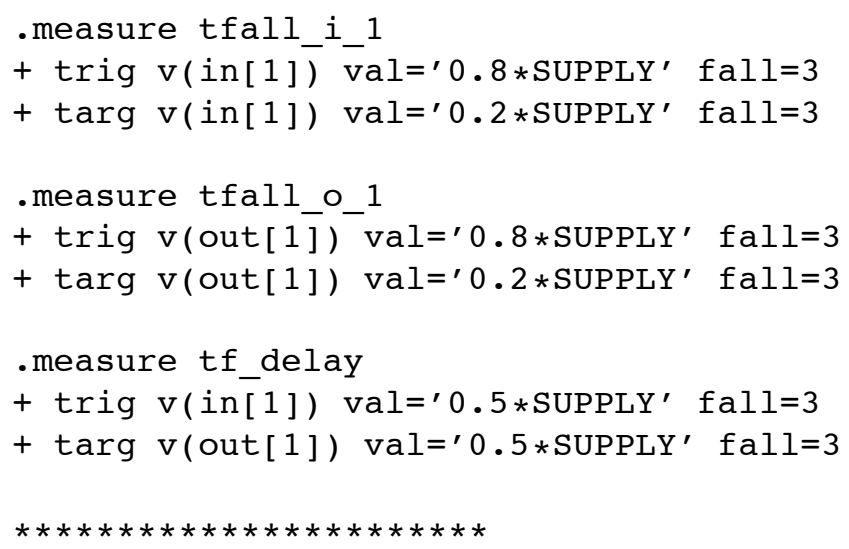




\section{C.4 SPICE Output File}

Below is the SPICE output file with the look up table results for relative timing path LUTblue:PRED $i-$ FIRE $_{i}-$, as measured for the schematic of Figure 4.12, page 67, and the black box schematics of Figure 4.8, page 57. The file extension is '.mt0'.

The file contains the $4 \times 5$ wid $1 \times$ wid 2 sweep index, i.e. index 1 through 20 . Per sweep, it shows the sizes of sweep variables wid1 and wid2, and the measured timing results in seconds. We measure the following timing information:

- tfall_i_1, the transition fall time, a.k.a. slope or slew time, on input in[1]

- tfall_o_1, the transition fall time, a.k.a. slope or slew time, on output out[1]

- tf_delay, the input-to-output delay for the falling transition from in[1] to out[1]

Note: the additional process parameters temper and alter\# can be ignored.

\begin{tabular}{|c|c|c|c|}
\hline $\begin{aligned} \cdot \operatorname{TITLE} & * * * \\
\text { index } & \end{aligned}$ & $\begin{array}{l}\text { spice deck for cell } \\
\text { widl } \\
\text { tfall_o_1 } \\
\text { alter\# }\end{array}$ & $\begin{array}{l}\text { rt1_predfire }\{\mathrm{sch}\} \\
\text { wid2 } \\
\text { tf_delay }\end{array}$ & $\begin{array}{l}\text { from library' } \\
\text { tfall_i_1 } \\
\text { temper }\end{array}$ \\
\hline \multirow[t]{3}{*}{1.0000} & 10.0000 & 40.0000 & $1.144 \mathrm{e}-11$ \\
\hline & $9.980 e-12$ & $6.211 \mathrm{e}-11$ & 25.0000 \\
\hline & 1.0000 & & \\
\hline \multirow[t]{3}{*}{2.0000} & 8.0000 & 40.0000 & $1.215 \mathrm{e}-11$ \\
\hline & $9.877 e-12$ & $6.236 e-11$ & 25.0000 \\
\hline & 1.0000 & & \\
\hline \multirow[t]{3}{*}{3.0000} & 6.0000 & 40.0000 & $1.326 \mathrm{e}-11$ \\
\hline & $1.008 e-11$ & $6.277 e-11$ & 25.0000 \\
\hline & 1.0000 & & \\
\hline \multirow[t]{2}{*}{4.0000} & 3.0000 & 40.0000 & $1.759 \mathrm{e}-11$ \\
\hline & $\begin{array}{c}9.605 e-12 \\
1.0000\end{array}$ & $6.468 \mathrm{e}-11$ & 25.0000 \\
\hline \multirow[t]{2}{*}{5.0000} & $\begin{array}{c}10.0000 \\
1.382 \mathrm{e}-11\end{array}$ & $\begin{array}{l}130.0000 \\
6.590 e-11\end{array}$ & $\begin{array}{c}1.132 \mathrm{e}-11 \\
25.0000\end{array}$ \\
\hline & 1.0000 & & \\
\hline \multirow[t]{2}{*}{6.0000} & 8.0000 & 130.0000 & $1.207 e-11$ \\
\hline & $\begin{array}{c}1.381 \mathrm{e}-11 \\
1.0000\end{array}$ & $6.611 \mathrm{e}-11$ & 25.0000 \\
\hline \multirow[t]{2}{*}{7.0000} & 6.0000 & 130.0000 & $1.337 e-11$ \\
\hline & $\begin{array}{c}1.390 \mathrm{e}-11 \\
1.0000\end{array}$ & $6.655 e-11$ & 25.0000 \\
\hline \multirow[t]{2}{*}{8.0000} & 3.0000 & 130.0000 & $1.756 \mathrm{e}-11$ \\
\hline & $\begin{array}{c}1.404 \mathrm{e}-11 \\
1.0000\end{array}$ & $6.834 \mathrm{e}-11$ & 25.0000 \\
\hline \multirow[t]{2}{*}{9.0000} & 10.0000 & 220.0000 & $1.112 \mathrm{e}-11$ \\
\hline & $\begin{array}{c}1.831 \mathrm{e}-11 \\
1.0000\end{array}$ & $6.931 \mathrm{e}-11$ & 25 . \\
\hline
\end{tabular}




\begin{tabular}{|c|c|c|c|}
\hline \multirow[t]{3}{*}{10.0000} & 8.0000 & 220.0000 & $1.159 \mathrm{e}-11$ \\
\hline & $1.842 e-11$ & $6.954 e-11$ & 25.0000 \\
\hline & 1.0000 & & \\
\hline \multirow[t]{3}{*}{11.0000} & 6.0000 & 220.0000 & $1.315 e-11$ \\
\hline & $1.838 e-11$ & $6.990 e-11$ & 25.0000 \\
\hline & 1.0000 & & \\
\hline \multirow[t]{3}{*}{12.0000} & 3.0000 & 220.0000 & $1.625 e-11$ \\
\hline & $1.856 \mathrm{e}-11$ & $7.169 e-11$ & 25.0000 \\
\hline & 1.0000 & & \\
\hline \multirow[t]{3}{*}{13.0000} & 10.0000 & 310.0000 & $1.140 \mathrm{e}-11$ \\
\hline & $2.265 e-11$ & $7.231 e-11$ & 25.0000 \\
\hline & 1.0000 & & \\
\hline \multirow[t]{3}{*}{14.0000} & 8.0000 & 310.0000 & $1.224 \mathrm{e}-11$ \\
\hline & $2.259 e-11$ & $7.257 e-11$ & 25.0000 \\
\hline & 1.0000 & & \\
\hline \multirow[t]{3}{*}{15.0000} & 6.0000 & 310.0000 & $1.245 e-11$ \\
\hline & $2.234 \mathrm{e}-11$ & $7.308 e-11$ & 25.0000 \\
\hline & 1.0000 & & \\
\hline \multirow[t]{3}{*}{16.0000} & 3.0000 & 310.0000 & $1.756 \mathrm{e}-11$ \\
\hline & $2.200 e-11$ & $7.476 e-11$ & 25.0000 \\
\hline & 1.0000 & & \\
\hline \multirow[t]{3}{*}{17.0000} & 10.0000 & 400.0000 & $1.153 e-11$ \\
\hline & $2.714 \mathrm{e}-11$ & $7.513 e-11$ & 25.0000 \\
\hline & 1.0000 & & \\
\hline \multirow[t]{3}{*}{18.0000} & 8.0000 & 400.0000 & $1.220 \mathrm{e}-11$ \\
\hline & $2.728 e-11$ & $7.539 e-11$ & 25.0000 \\
\hline & 1.0000 & & \\
\hline \multirow[t]{3}{*}{19.0000} & 6.0000 & 400.0000 & $1.308 e-11$ \\
\hline & $2.736 e-11$ & $7.591 e-11$ & 25.0000 \\
\hline & 1.0000 & & \\
\hline \multirow[t]{3}{*}{20.0000} & 3.0000 & 400.0000 & $1.758 e-11$ \\
\hline & $2.734 e-11$ & $7.769 e-11$ & 25.0000 \\
\hline & 1.0000 & & \\
\hline
\end{tabular}




\section{Appendix D}

\section{SPICE FILES FOR FIGURE 4.9 AND LUTblue:SUCC + FIRE $_{i}-$}

\section{D.1 SPICE Netlist}

The SPICE netlist file generated from the design schematics in Electric has the details of all the components and devices used in the schematics of Figure 4.13, page 69, and in the black box schematics of Figure 4.9, page 58. The black box schematics belongs to relative timing path LUTblue:SUCC $i+\mathrm{FIRE}_{i}-$. The sweep parameters wid1 and wid2 are the sizes of the driver and load inverters. The file extension is 'spi'.

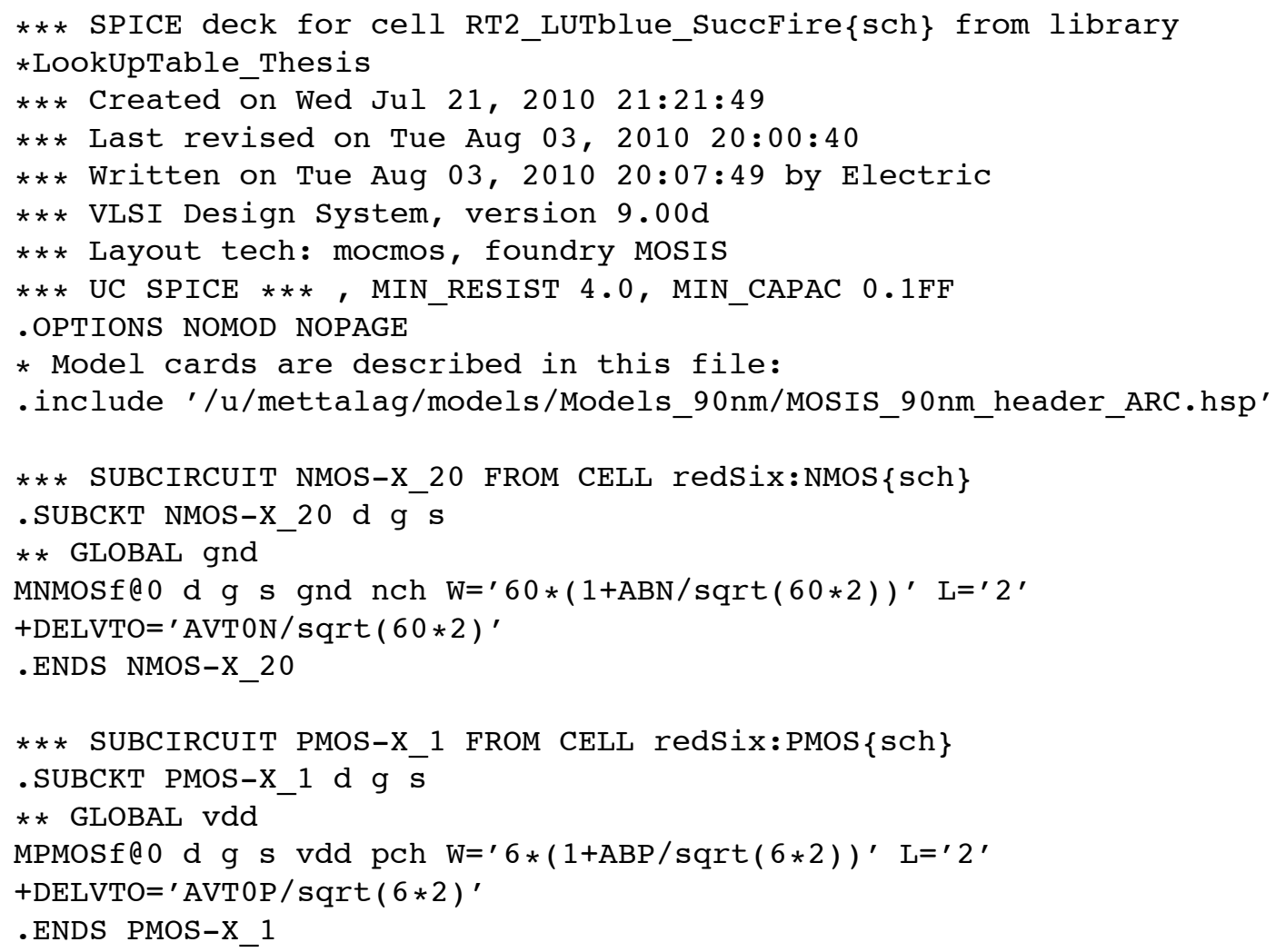




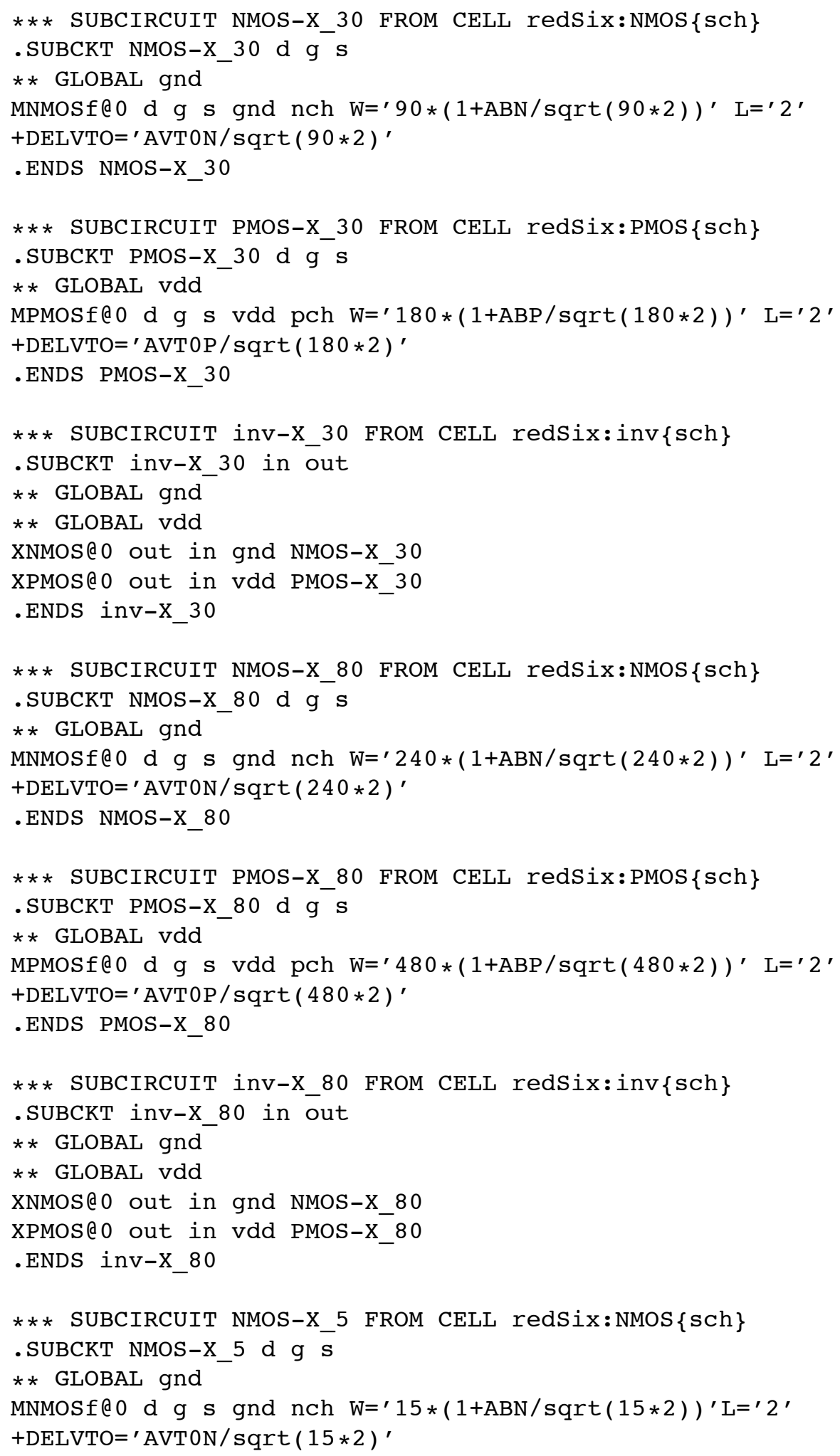




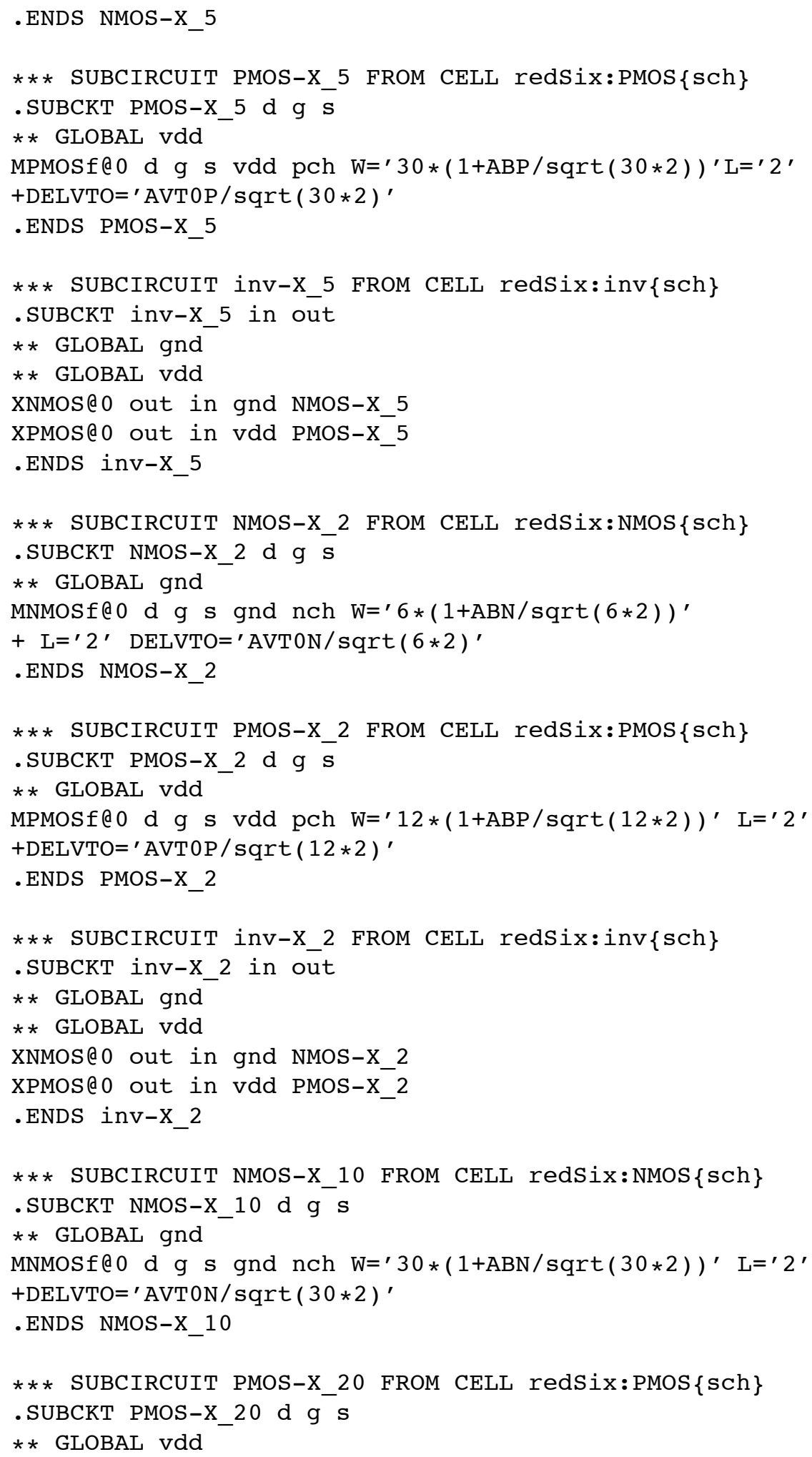


MPMOSf@0 d g s vdd pch $\mathrm{W}=$ ' $120 *(1+\mathrm{ABP} / \operatorname{squt}(120 * 2))^{\prime} \mathrm{L}=\mathbf{\prime}^{\prime}$

$+\mathrm{DELVTO}=$ ' AVTOP/sqrt $(120 * 2)$ '

.ENDS PMOS-X_20

*** SUBCIRCUIT pms2-X_10 FROM CELL redSix:pms 2 sch

. SUBCKT pms $2-\mathrm{X} 10 \mathrm{~d} g \mathrm{~g} 2$

** GLOBAL vdd

XPMOS@0 net@2 g vdd PMOS-X_20

XPMOS@1 d g2 nete2 PMOS-X_20

- ENDS pms2-X_10

*** SUBCIRCUIT nor2-X_10 FROM CELL redSix:nor $2\{\mathrm{sch}\}$

.SUBCKT nor2-X_10 ina inb out

* * GLOBAL gnd

* * GLOBAL vdd

XNMOS@O out ina gnd NMOS-X_10

XNMOSe1 out inb gnd NMOS-X_10

Xpms 2 @ out ina inb pms $2-\mathrm{X} 10$

. ENDS nor $2-\mathrm{X} \_10$

*** SUBCIRCUIT LUTblue_RT2_path FROM CELL LUTblue_RT2_path\{sch\} .SUBCKT LUTblue_RT2_path fire succ

** GLOBAL gnd

* * GLOBAL vdd

XNMOS@O NMOS@O d fire gnd NMOS-X 20

XPMOS@0 net@57 net@2 vdd PMOS-X $\overline{1}$

XPMOS@1 net@57 fire net@ 4 PMOS-X

EVCVS@0 net@9 gnd vcvs succ gnd $\overline{1} \max =' 10 \mathrm{~V}$ ' $\min ={ }^{\prime}-10 \mathrm{~V}$ '

Xinv@0 net@20 net@19 inv-X_30

Xinv@1 net@19 fire inv-X_8

Xinve2 inve2 in nete9 inv-X_5

Xinve 4 net@ 4 net@ 2 inv-X_2

Xnor $2 n @ 0$ succ net@9 net@20 nor $2-x_{-} 10$

* Spice Code nodes in cell cell 'LUTblue_RT2_path\{sch\}'

. ic $\mathrm{succ}=0 \mathrm{v}$ net@ $85=1 \mathrm{v}$

.ENDS LUTblue_RT2_path

*** SUBCIRCUIT NMOS-X_wid1 FROM CELL redSix:NMOS $\{\mathrm{sch}\}$

.SUBCKT NMOS-X_wid1 d $g$ s

** GLOBAL gnd

MNMOSflo d g $\mathrm{s}$ gnd $\mathrm{nch} \mathrm{W}=$ ' $3 * \mathrm{Wid} 1 *(1+\mathrm{ABN} / \operatorname{sqrt}(3 * \operatorname{Wid} 1 * 2))^{\prime} \mathrm{L}=$ ' $^{\prime}$

+ DELVTO $=$ ' AVTON/sqrt $(3 *$ wid $1 * 2)$ '

. ENDS NMOS-X_wid1

*** SUBCIRCUIT PMOS-X_wid1 FROM CELL redSix:PMOS $\{\mathrm{sch}\}$

.SUBCKT PMOS-X_wid1 d $g$ s

* * GLOBAL vdd

MPMOSf@0 d g s vdd pch $\mathrm{W}={ }^{\prime} 2 * 3 * \operatorname{Wid} 1 *(1+\mathrm{ABP} / \operatorname{sqrt}(2 * 3 * \operatorname{Wid} 1 * 2))^{\prime}$ 
$+\mathrm{L}={ }^{\prime} 2^{\prime} \mathrm{DELVTO}={ }^{\prime} \mathrm{AVT} 0 \mathrm{P} / \operatorname{sqrt}(2 * 3 * \mathrm{wid} 1 * 2)^{\prime}$

.ENDS PMOS-X_wid1

*** SUBCIRCUIT inv-X_wid1 FROM CELL redSix:inv\{sch

.SUBCKT inv-X_wid1 in out

** GLOBAL gnd

** GLOBAL vdd

XNMOS@O out in gnd NMOS-X_wid1

XPMOS@0 out in vdd PMOS-X_wid1

.ENDS inv-X_wid1

*** SUBCIRCUIT NMOS-X_wids FROM CELL redSix:NMOS $\{\mathrm{sch}\}$

.SUBCKT NMOS-X_wids d $g$ s

** GLOBAL gnd

MNMOSf@0 d g s gnd nch $\mathrm{W}=\prime 3 *(\operatorname{wid} 1 / 3) *(1+\mathrm{ABN} / \operatorname{sqrt}(3 *(\operatorname{wid} 1 / 3) * 2))^{\prime}$

$+\mathrm{L}={ }^{\prime} 2{ }^{\prime} \mathrm{DELVTO}={ }^{\prime} \operatorname{AVT} 0 \mathrm{~N} / \operatorname{sqrt}(3 *(\mathrm{wid} 1 / 3) * 2)^{\prime}$

.ENDS NMOS-X_wids

*** SUBCIRCUIT PMOS-X_wids FROM CELL redSix:PMOS $\{\mathrm{sch}\}$

.SUBCKT PMOS-X_wids d $g$ s

** GLOBAL vdd

MPMOSf@O d g s vdd pch $\mathrm{W}={ }^{\prime} 2 * 3 *(\mathrm{wid} 1 / 3) *$

$+(1+\mathrm{ABP} / \operatorname{sqrt}(2 * 3 *(\mathrm{wid} 1 / 3) * 2))^{\prime} \mathrm{L}={ }^{\prime} 2^{\prime}$

$+\mathrm{DELVTO}={ }^{\prime} \mathrm{AVTOP} / \operatorname{sqrt}(2 * 3 *(\mathrm{wid} 1 / 3) * 2)^{\prime}$

.ENDS PMOS-X_wids

*** SUBCIRCUIT inv-X_wids FROM CELL redSix:inv\{sch

.SUBCKT inv-X_wids in out

* * GLOBAL gnd

** GLOBAL vdd

XNMOS@O out in gnd NMOS-X_wids

XPMOS@O out in vdd PMOS-X_wids

. ENDS inv-X_wids

*** SUBCIRCUIT NMOS-X_widM FROM CELL redSix:NMOS $\{\mathrm{sch}\}$

.SUBCKT NMOS-X_widM d $g$ s

* * GLOBAL gnd

MNMOSf@0 d g s gnd nch $\mathrm{W}=\prime 3 * 3 * w i d 2 *(1+\mathrm{ABN} / \operatorname{sqrt}(3 * 3 * \mathrm{Wid} 2 * 2))$ '

$+\mathrm{L}={ }^{\prime} 2^{\prime} \mathrm{DELVTO}={ }^{\prime} \mathrm{AVTON} / \operatorname{sqrt}(3 * 3 * \mathrm{wid} 2 * 2)^{\prime}$

.ENDS NMOS-X_widM

*** SUBCIRCUIT PMOS-X_widM FROM CELL redSix:PMOS $\{\mathrm{sch}\}$

.SUBCKT PMOS-X_widM d $g$ s

* * GLOBAL vdd

MPMOSf@0 d g s vdd pch $\mathrm{W}={ }^{\prime} 2 * 3 * 3 * \mathrm{wid} 2 *$

$+(1+\mathrm{ABP} / \operatorname{sqrt}(2 * 3 * 3 * \mathrm{wid} 2 * 2))^{\prime} \mathrm{L}={ }^{\prime} 2^{\prime}$

$+\mathrm{DELVTO}={ }^{\prime} \mathrm{AVTOP} / \operatorname{sqrt}(2 * 3 * 3 * \operatorname{wid} 2 * 2)^{\prime}$

.ENDS PMOS-X_widM 


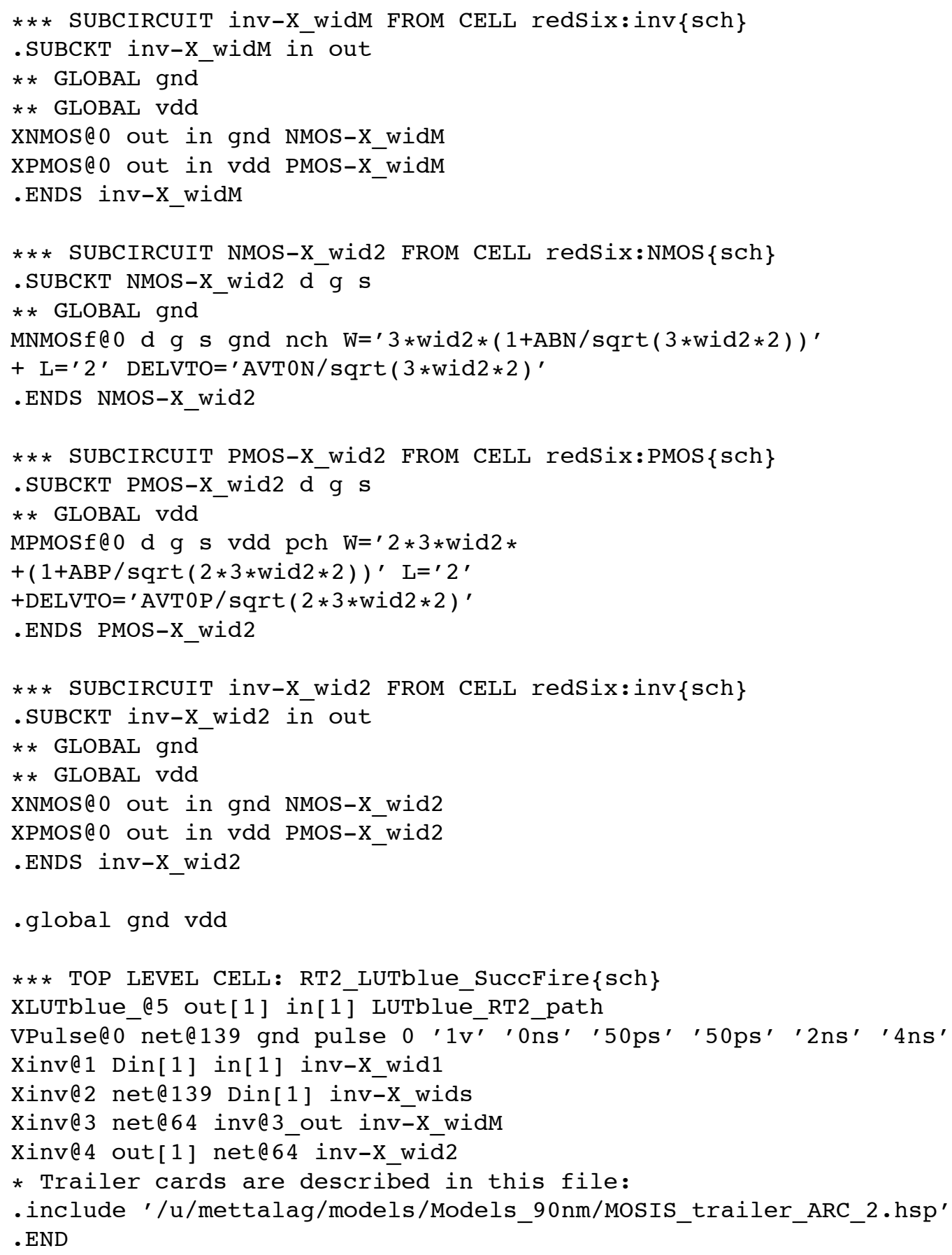




\section{D.2 SPICE Simulation Setup File: HEADER}

Here is the basic file with default simulation parameters for SPICE. Its extension is '.hsp'. It contains a pointer to the 90nm CMOS process models for our GasP designs. We use the library with typical (TT) Process, Voltage and Temperature (PVT) settings.

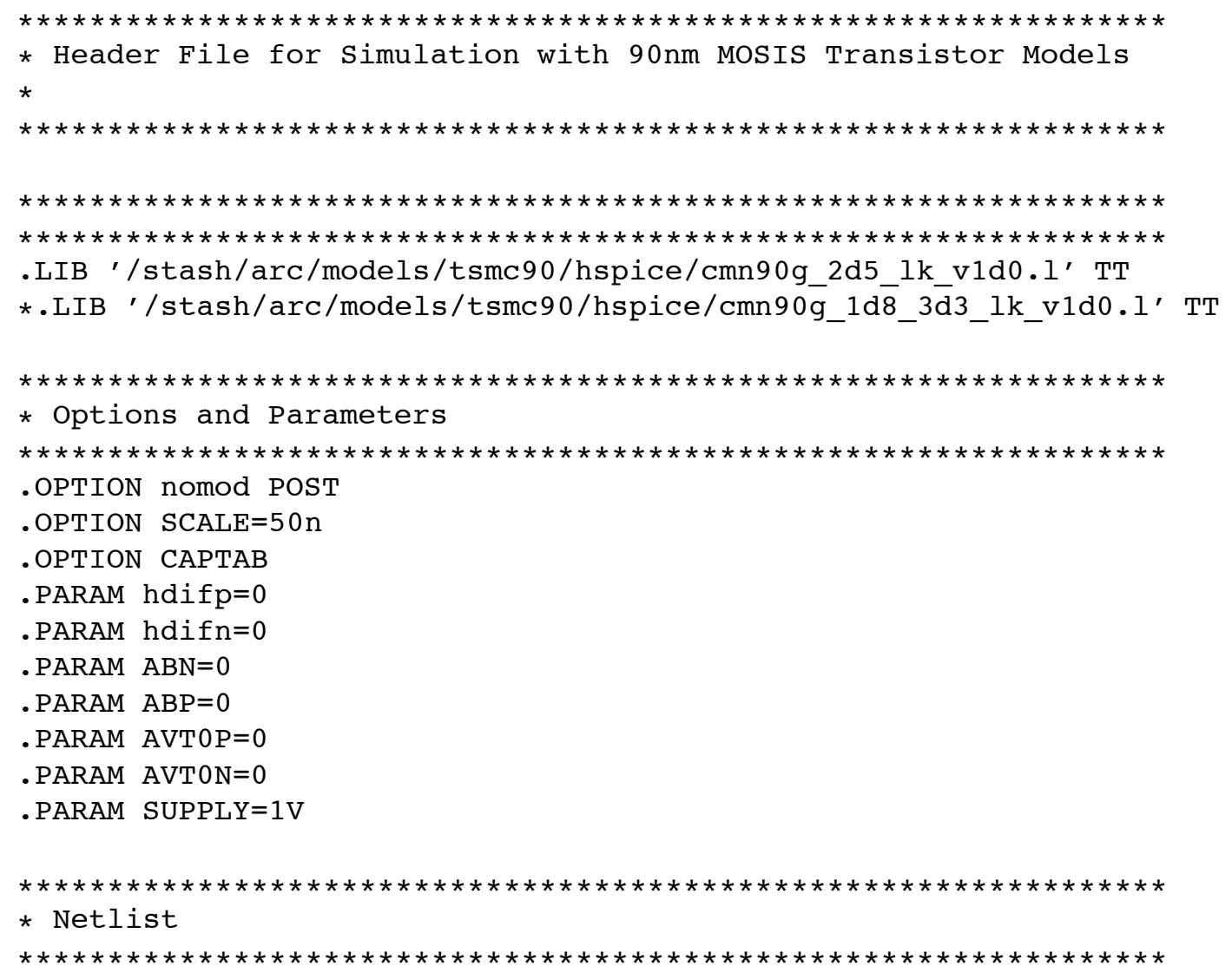




\section{D.3 SPICE Simulation Setup File: TRAILER}

Below follows the SPICE trailer file with the .DATA sweep, transient analysis, and measurement details for the simulation environment in Figure 4.13, page 69, and the black box schematics of Figure 4.9, page 58. We are generating look up tables for relative timing path LUTblue:SUCC ${ }_{i}+\mathrm{FIRE}_{i}-$. To do so, we sweep driver size wid1 and load size wid2, and we measure voltage levels on in[1] and out[1].

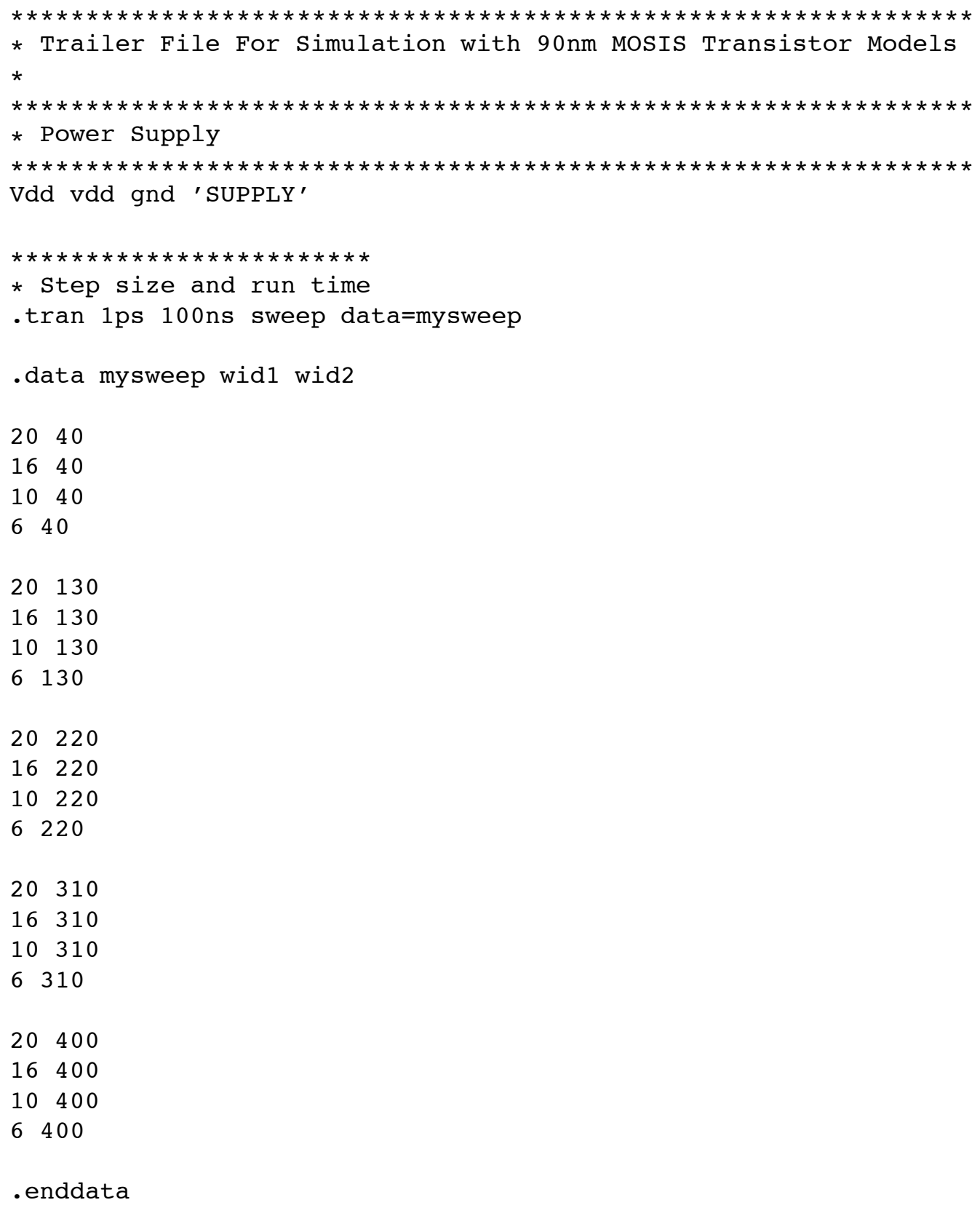




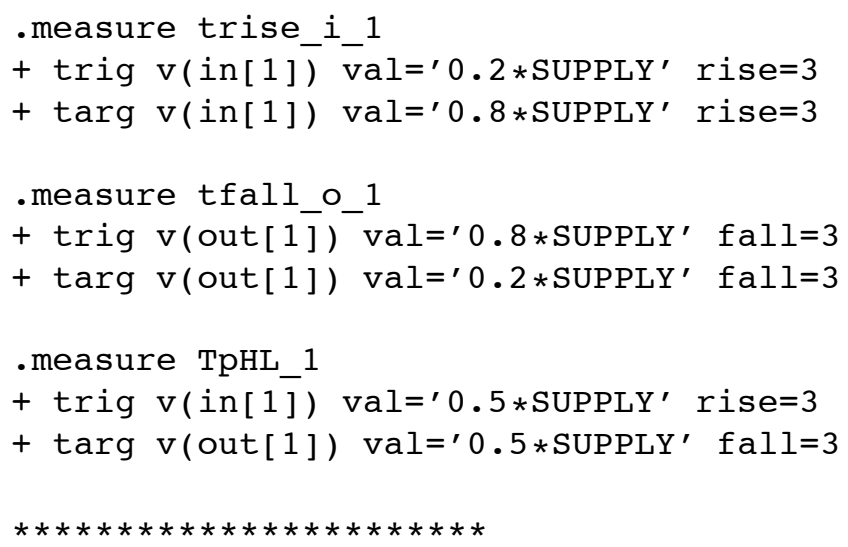




\section{D.4 SPICE Output File}

Below is the SPICE output file with the look up table results for relative timing path LUTblue:SUCC ${ }_{i}+\mathrm{FIRE}_{i}-$, as measured for the schematics of Figure 4.13, page 69, and the black box schematics of Figure 4.9, page 58. The file extension is '. $m t 0$ '.

The file contains the $4 \times 5$ wid $1 \times$ wid 2 sweep index, i.e. index 1 through 20 . Per sweep, it shows the sizes of sweep variables wid 1 and wid 2 , and the measured timing results in seconds. We measure the following timing information:

- trise_i_1, the transition rise time, a.k.a. slope or slew time, on input in[1]

- tfall_o_1, the transition fall time, a.k.a. slope or slew time, on output out[1]

- tphl_1, input-to-output delay for the high to low transition from in[1] to out[1]

Note: the additional process parameters temper and alter\# can be ignored.

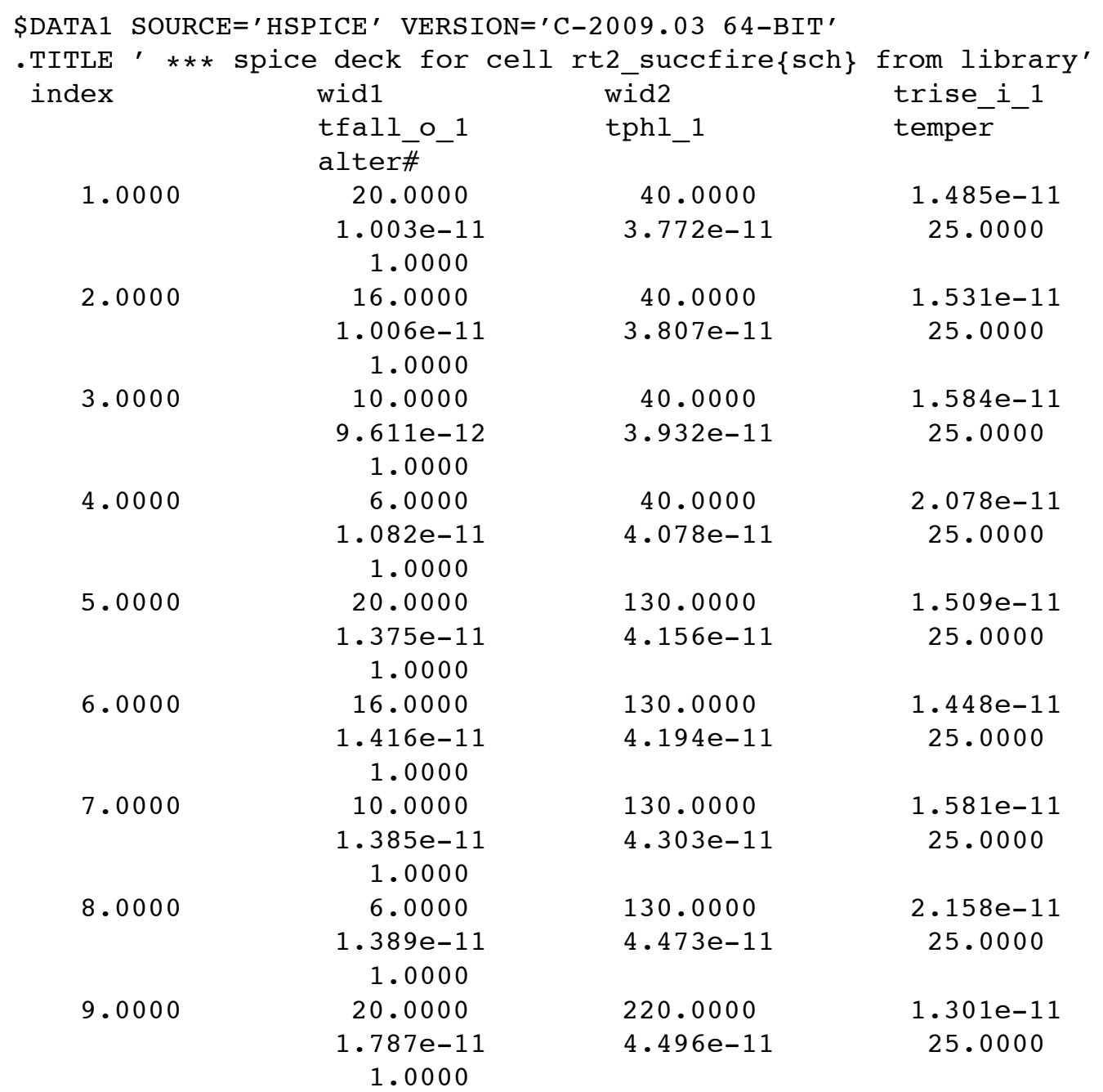




\begin{tabular}{|c|c|c|c|}
\hline \multirow[t]{3}{*}{10.0000} & 16.0000 & 220.0000 & $1.411 \mathrm{e}-11$ \\
\hline & $1.797 e-11$ & $4.531 e-11$ & 25.0000 \\
\hline & 1.0000 & & \\
\hline \multirow[t]{3}{*}{11.0000} & 10.0000 & 220.0000 & $1.596 \mathrm{e}-11$ \\
\hline & $1.827 e-11$ & $4.631 e-11$ & 25.0000 \\
\hline & 1.0000 & & \\
\hline \multirow[t]{3}{*}{12.0000} & 6.0000 & 220.0000 & $2.132 e-11$ \\
\hline & $1.815 e-11$ & $4.808 e-11$ & 25.0000 \\
\hline & 1.0000 & & \\
\hline \multirow[t]{3}{*}{13.0000} & 20.0000 & 310.0000 & $1.321 \mathrm{e}-11$ \\
\hline & $2.284 e-11$ & $4.799 e-11$ & 25.0000 \\
\hline & 1.0000 & & \\
\hline \multirow[t]{3}{*}{14.0000} & 16.0000 & 310.0000 & $1.373 e-11$ \\
\hline & $2.250 e-11$ & $4.836 e-11$ & 25.0000 \\
\hline & 1.0000 & & \\
\hline \multirow[t]{3}{*}{15.0000} & 10.0000 & 310.0000 & $1.652 \mathrm{e}-11$ \\
\hline & $2.280 e-11$ & $4.930 e-11$ & 25.0000 \\
\hline & 1.0000 & & \\
\hline \multirow[t]{3}{*}{16.0000} & 6.0000 & 310.0000 & $2.149 e-11$ \\
\hline & $2.268 e-11$ & $5.115 e-11$ & 25.0000 \\
\hline & 1.0000 & & \\
\hline \multirow[t]{3}{*}{17.0000} & 20.0000 & 400.0000 & $1.390 \mathrm{e}-11$ \\
\hline & $2.688 e-11$ & $5.091 e-11$ & 25.0000 \\
\hline & 1.0000 & & \\
\hline \multirow[t]{3}{*}{18.0000} & 16.0000 & 400.0000 & $1.504 \mathrm{e}-11$ \\
\hline & $2.709 e-11$ & $5.121 e-11$ & 25.0000 \\
\hline & 1.0000 & & \\
\hline \multirow[t]{3}{*}{19.0000} & 10.0000 & 400.0000 & $1.755 e-11$ \\
\hline & $2.714 \mathrm{e}-11$ & $5.212 e-11$ & 25.0000 \\
\hline & 1.0000 & & \\
\hline \multirow[t]{3}{*}{20.0000} & 6.0000 & 400.0000 & $2.129 e-11$ \\
\hline & $2.703 e-11$ & $5.403 e-11$ & 25.0000 \\
\hline & 1.0000 & & \\
\hline
\end{tabular}




\section{Appendix E}

\section{SPICE FILES FOR FIGURE 4.7(b) AND LUTblue:FIRE ${ }_{i}-$ Dout $_{i}+$}

\section{E.1 SPICE Netlist}

The SPICE netlist file generated from the design schematics in Electric has the details of all the components and devices used in the schematics of Figure 4.14, page 70, and in the black box schematics of Figure 4.7(b), page 51. The black box schematics belongs to relative timing path LUTblue:FIRE ${ }_{i}-$ Dout $_{i}+$. Sweep parameters wid1 is the size of the driver inverter. The file extension is '.spi'.

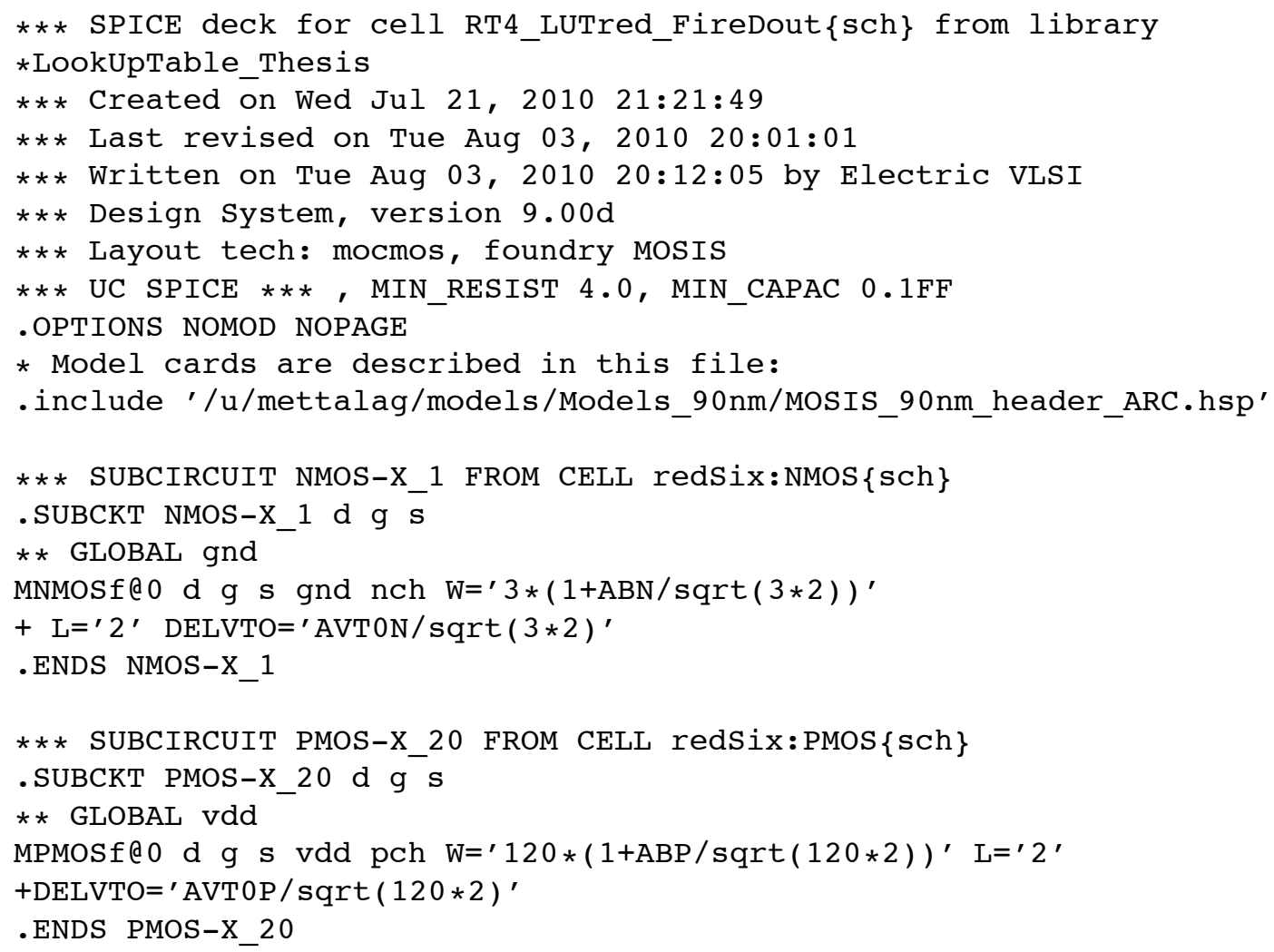




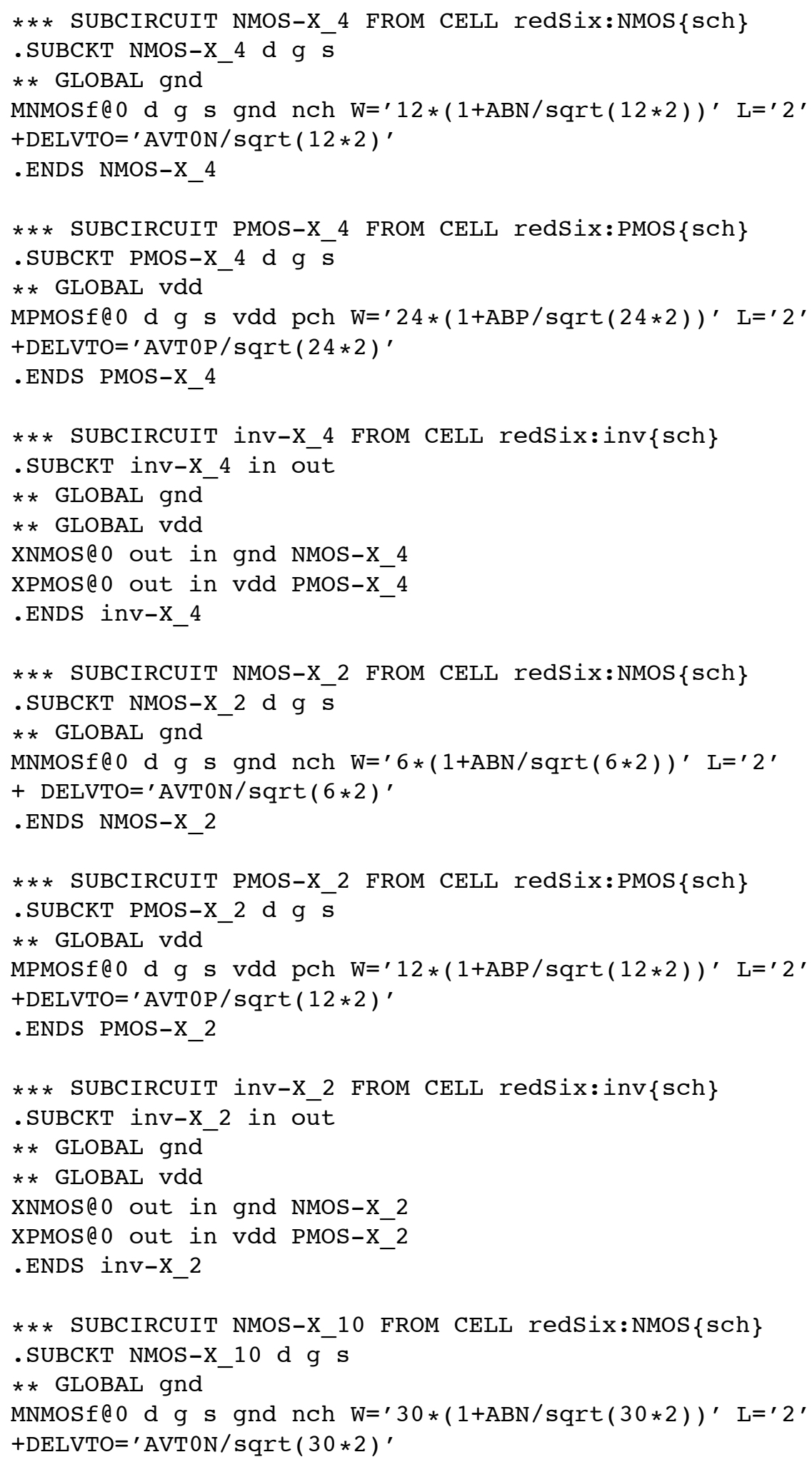


.ENDS NMOS-X_10

*** SUBCIRCUIT pms2-X_10 FROM CELL redSix:pms $2\{\mathrm{sch}\}$

.SUBCKT pms $2-\mathrm{X} \_10 \mathrm{~d} g \mathrm{~g} 2$

* * GLOBAL vdd

XPMOS@O nete2 g vdd PMOS-X_20

XPMOS@ 1 d g2 net@ 2 PMOS-X_20

.ENDS pms2-X_10

*** SUBCIRCUIT nor2-10 FROM CELL redSix:nor2\{sch\}

.SUBCKT nor2-10 ina inb out

* * GLOBAL gnd

** GLOBAL vdd

XNMOS@O out ina gnd NMOS-X 10

XNMOS@ 1 out inb gnd NMOS-X_10

Xpms2@0 out ina inb pms2-X_10

.ENDS nor $2-10$

*** SUBCIRCUIT LUTblue_RT4_path FROM CELL LUTblue_RT4_path\{sch\}

.SUBCKT LUTblue_RT4_path dout fire

** GLOBAL gnd

** GLOBAL vdd

XNMOS@ 1 net@ 64 net@ 1 gnd NMOS-X_1

XNMOS@ 2 net@ 64 dout net@ 3 NMOS $-\bar{x}_{-} 1$

XPMOS@ 2 net@ 3 dout vdd PMOS-X_20

Xinv@0 fire dout inv-X_4

Xinv@1 net@ 3 net@ 1 inv-X_2

Xnor2n@0 net@ 3 nor2n@0_inb nor2n@0_out nor2-10

.ENDS LUTblue_RT4_path

*** SUBCIRCUIT NMOS-X_wid1 FROM CELL redSix:NMOS $\{$ sch $\}$

.SUBCKT NMOS-X_wid1 d g s

** GLOBAL gnd

MNMOSf@o d g s gnd nch $\mathrm{W}={ }^{\prime} 3 * \mathrm{Wid} 1 *(1+\mathrm{ABN} / \operatorname{sqrt}(3 * \mathrm{Wid} 1 * 2))^{\prime}$

$+\mathrm{L}={ }^{\prime} 2^{\prime}$ DELVTO='AVTON $/ \operatorname{sqrt}(3 * \mathrm{Wid} 1 * 2)^{\prime}$

.ENDS NMOS-X_wid1

*** SUBCIRCUIT PMOS-X_wid1 FROM CELL redSix:PMOS $\{\mathrm{sch}\}$

. SUBCKT PMOS-X_wid1 d g s

** GLOBAL vdd

MPMOSf@0 d g s vdd pch $\mathrm{W}=\prime 2 * 3 * \mathrm{Wid} 1 *(1+\mathrm{ABP} / \operatorname{sqrt}(2 * 3 * \mathrm{Wid} 1 * 2))^{\prime}$

$+\mathrm{L}={ }^{\prime} 2^{\prime}$ DELVTO='AVTOP $/ \operatorname{sqrt}(2 * 3 * \mathrm{Wid} 1 * 2)^{\prime}$

.ENDS PMOS-X_wid1

*** SUBCIRCUIT inv-X_wid1 FROM CELL redSix:inv\{sch\}

.SUBCKT inv-X_widl in out

** GLOBAL gnd

** GLOBAL vdd

XNMOS@O out in gnd NMOS-X_wid1 
XPMOS@ 0 out in vdd PMOS-X_wid1

.ENDS inv-X_wid1

*** SUBCIRCUIT NMOS-X_wids FROM CELL redSix:NMOS $\{\mathrm{sch}\}$

.SUBCKT NMOS-X_wids d g $s$

** GLOBAL gnd

MNMOSf@o d g s gnd nch $\mathrm{W}={ }^{\prime} 3 *(\operatorname{wid} 1 / 3) *(1+\mathrm{ABN} / \operatorname{sqrt}(3 *(\operatorname{wid} 1 / 3) * 2))^{\prime}$

$+\mathrm{L}={ }^{\prime} 2^{\prime}$ DELVTO='AVTON $/ \operatorname{sqrt}(3 *(\operatorname{wid} 1 / 3) * 2)^{\prime}$

.ENDS NMOS-X_wids

*** SUBCIRCUIT PMOS-X_wids FROM CELL redSix:PMOS $\{$ sch $\}$

.SUBCKT PMOS-X_wids d g $\mathrm{s}$

** GLOBAL vdd

MPMOSf@O d g s vdd pch $\mathrm{W}=\prime 2 * 3 *(\operatorname{widl} / 3)$ *

$+(1+\mathrm{ABP} / \operatorname{sqrt}(2 * 3 *(\operatorname{wid} 1 / 3) * 2))^{\prime} \mathrm{L}={ }^{\prime} 2^{\prime}$

+ DELVTO $={ }^{\prime}$ AVTOP $/ \operatorname{sqrt}(2 * 3 *($ wid1 $/ 3) * 2)$ '

.ENDS PMOS-X_wids

*** SUBCIRCUIT inv-X_wids FROM CELL redSix:inv\{sch\}

.SUBCKT inv-X_wids in out

** GLOBAL gnd

** GLOBAL vdd

XNMOS@O out in gnd NMOS-X_wids

XPMOS@O out in vdd PMOS-X_wids

.ENDS inv-X_wids

.global gnd vdd

*** TOP LEVEL CELL: RT4_LUTred_FireDout $\{\mathrm{sch}\}$

XLUTblue_@ 7 out[1] in[1] LUTblue_RT4_path

VPulse@o net@139 gnd pulse 0 '1v' '0ns' '50ps' '50ps' '2ns' '4ns'

Xinve1 Din[1] in[1] inv-X_wid1

Xinv@2 net@139 Din[1] inv-X_wids

* Trailer cards are described in this file:

.include '/u/mettalag/models/Models_90nm/MOSIS_trailer_ARC_4.hsp' .END 


\section{E.2 SPICE Simulation Setup File: HEADER}

Here is the basic file with default simulation parameters for SPICE. Its extension is '.hsp'. It contains a pointer to the 90nm CMOS process models for our GasP designs. We use the library with typical (TT) Process, Voltage and Temperature (PVT) settings.

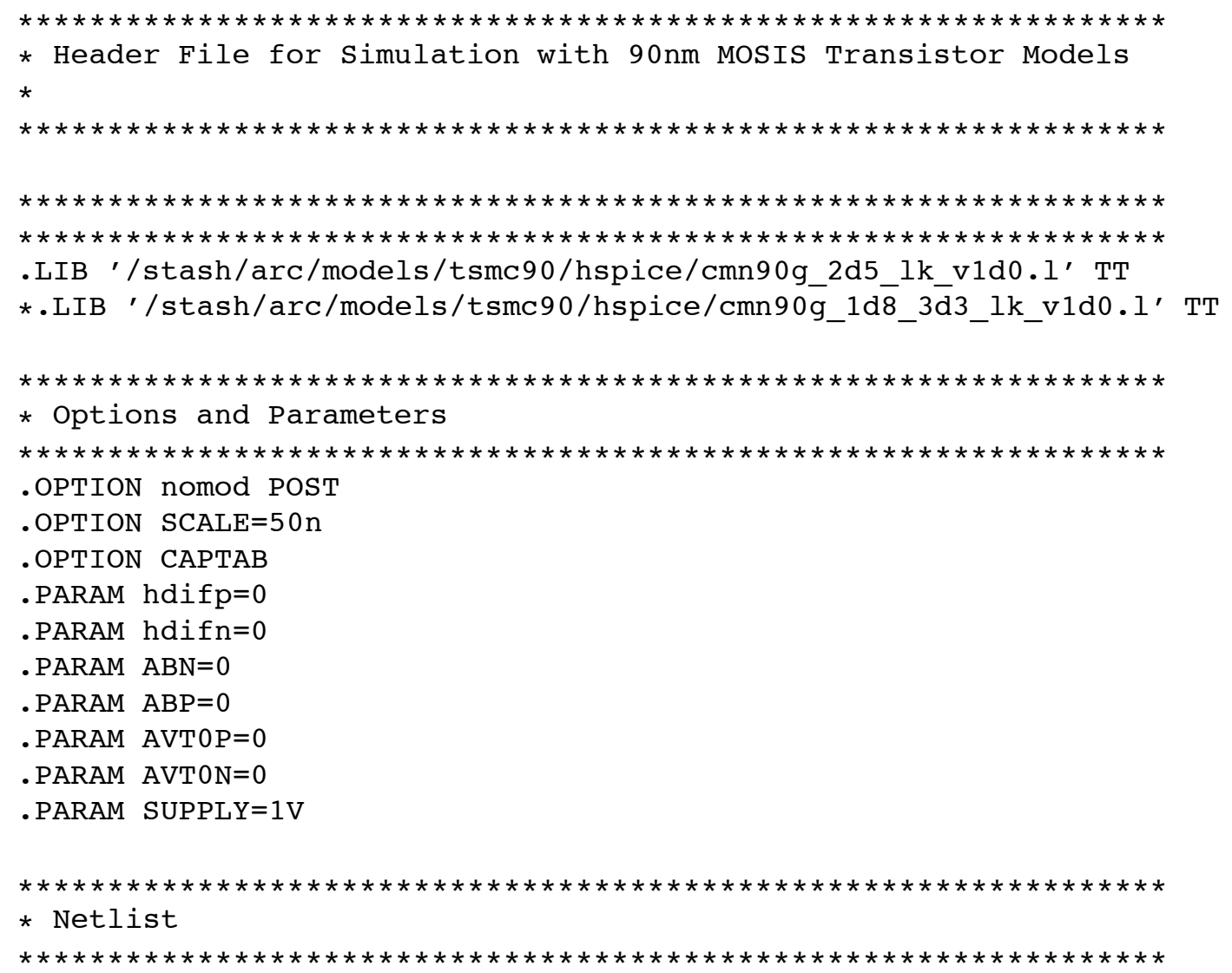




\section{E.3 SPICE Simulation Setup File: TRAILER}

Below follows the SPICE trailer file with the .DATA sweep, transient analysis, and measurement details for the simulation environment in Figure 4.14, page 70, and the black box schematics of Figure 4.7(b), page 51. We are generating look up tables for relative timing path LUTblue:FIRE ${ }_{i}-$ Dout $_{i}+$. To do so, we sweep driver size wid1, and we measure voltage levels on in[1] and out[1].

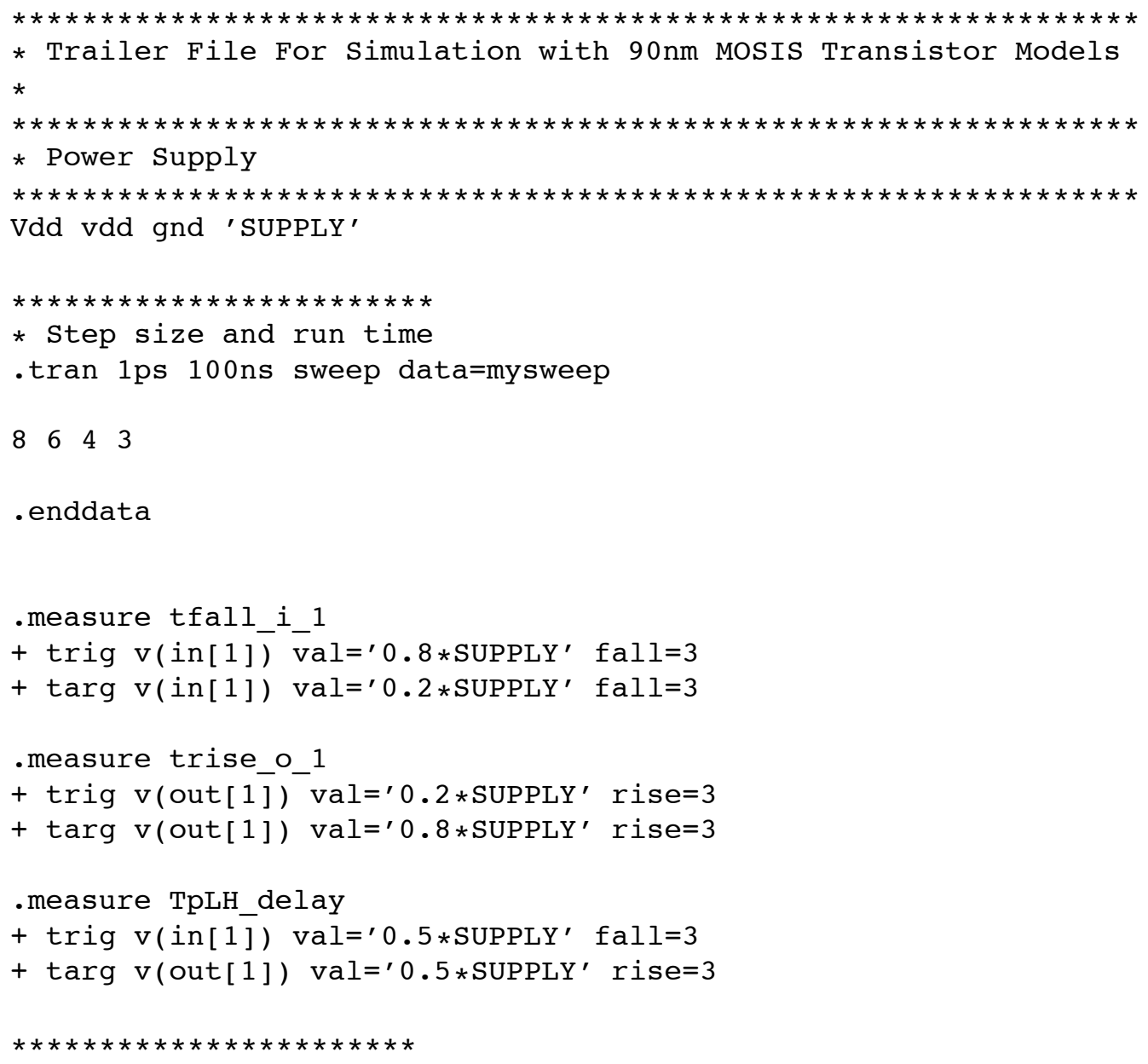




\section{E.4 SPICE Output File}

Below is the SPICE output file with the look up table results for relative timing path LUTblue:FIRE ${ }_{i}-$ Dout $_{i}+$, as measured for the schematic of Figure 4.14, page 70, and the black box schematics of Figure 4.7(b), page 51. The file extension is '.mt0'.

The file contains the $4 \times 1$ wid $1 \times$ wid 2 sweep index, i.e. index 1 through 4 . There is just one wid2 value, because Dout ${ }_{i}$ is an internal signal without external load, i.e. wid2=0 . $^{2}$ Per sweep, the file shows the sizes of sweep variable wid1 and the measured timing results in seconds. We measure the following timing information:

- tfall_i_1, the transition fall time, a.k.a. slope or slew time, on input in[1]

- trise_o_1, the transition rise time, a.k.a. slope or slew time, on output out[1]

- tplh_delay, input-to-output delay for the low to high transition from in[1] to out[1]

Note: the additional process parameters temper and alter\# can be ignored.

\begin{tabular}{|c|c|c|c|}
\hline $\begin{array}{l}\text { TITLE ' } \\
\text { index }\end{array}$ & $\begin{array}{c}\text { spice deck for cell } \\
\text { widl } \\
\text { tplh delay }\end{array}$ & $\begin{array}{l}\text { rt4_firedout }\{\mathrm{sch}\} \\
\text { tfall_i_1 } \\
\text { temper }\end{array}$ & $\begin{array}{l}\text { from library' } \\
\text { trise_o_1 } \\
\text { alter\# }\end{array}$ \\
\hline 1.0000 & $\begin{array}{c}8.0000 \\
2.048 \mathrm{e}-11\end{array}$ & $\begin{array}{c}1.157 \mathrm{e}-11 \\
25.0000\end{array}$ & $\begin{array}{c}2.104 \mathrm{e}-11 \\
1.0000\end{array}$ \\
\hline 2.0000 & $\begin{array}{c}6.0000 \\
2.090 \mathrm{e}-11\end{array}$ & $\begin{array}{c}1.242 e-11 \\
25.0000\end{array}$ & $\begin{array}{c}2.153 e-11 \\
1.0000\end{array}$ \\
\hline 3.0000 & $\begin{array}{c}4.0000 \\
2.168 \mathrm{e}-11\end{array}$ & $\begin{array}{c}1.438 \mathrm{e}-11 \\
25.0000\end{array}$ & $\begin{array}{c}2.153 e-11 \\
1.0000\end{array}$ \\
\hline 4.0000 & $\begin{array}{c}3.0000 \\
2.226 \mathrm{e}-11\end{array}$ & $\begin{array}{c}1.473 e-11 \\
25.0000\end{array}$ & $\begin{array}{c}2.167 e-11 \\
1.0000\end{array}$ \\
\hline
\end{tabular}




\section{Appendix F}

\section{SPICE FILES FOR FIGURE 4.10 AND LUTred/blue:FIRE + SUCC $_{i}+$}

Ideally, the SPICE simulations for Figure 4.15 on page 72 will run the red path for maximum delay conditions by using slow PVT library settings, and they will run the blue path for minimum delay conditions by using fast settings. We show only one simulation (covering both paths) using typical Process, Voltage, Temperature (PVT) settings.

\section{F.1 SPICE Netlist}

The SPICE netlist file generated from the design schematics in Electric has the details of all the components and devices used in the schematics of Figure 4.15, page 72, and in the black box schematics of Figure 4.10, page 61. The black box schematics belongs to relative timing paths LUTred:FIRE $i+\mathrm{SUCC}_{i}+$ and LUTblue:FIRE $i+\mathrm{SUCC}_{i}+$. The sweep parameters wid 1 and wid 2 are the sizes of the driver and load inverters. This configuration has two pulse generators, one for signal $\mathrm{FIRE}_{i}$ to start each measurement, one for signal FIRE_CS $i$ to reset the output signal $\mathrm{SUCC}_{i}$ prior to the next measurement. The file extension is 'spi'.

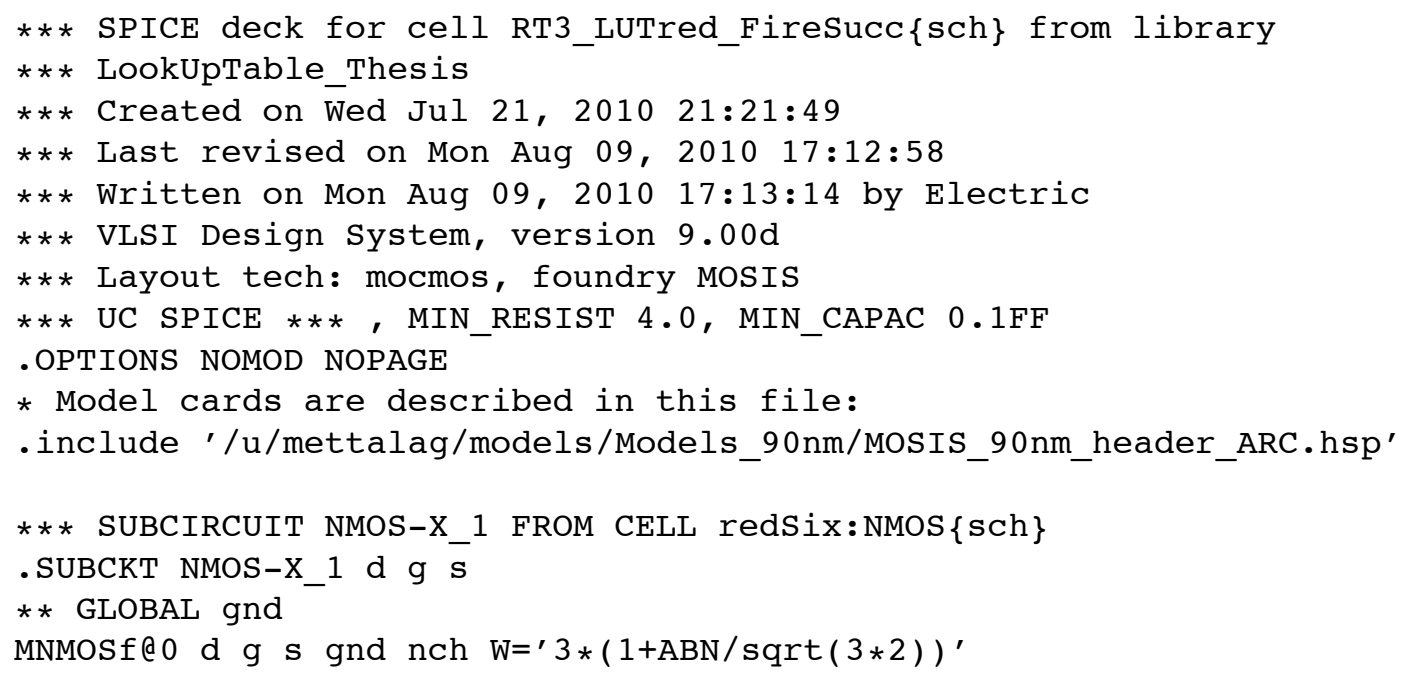




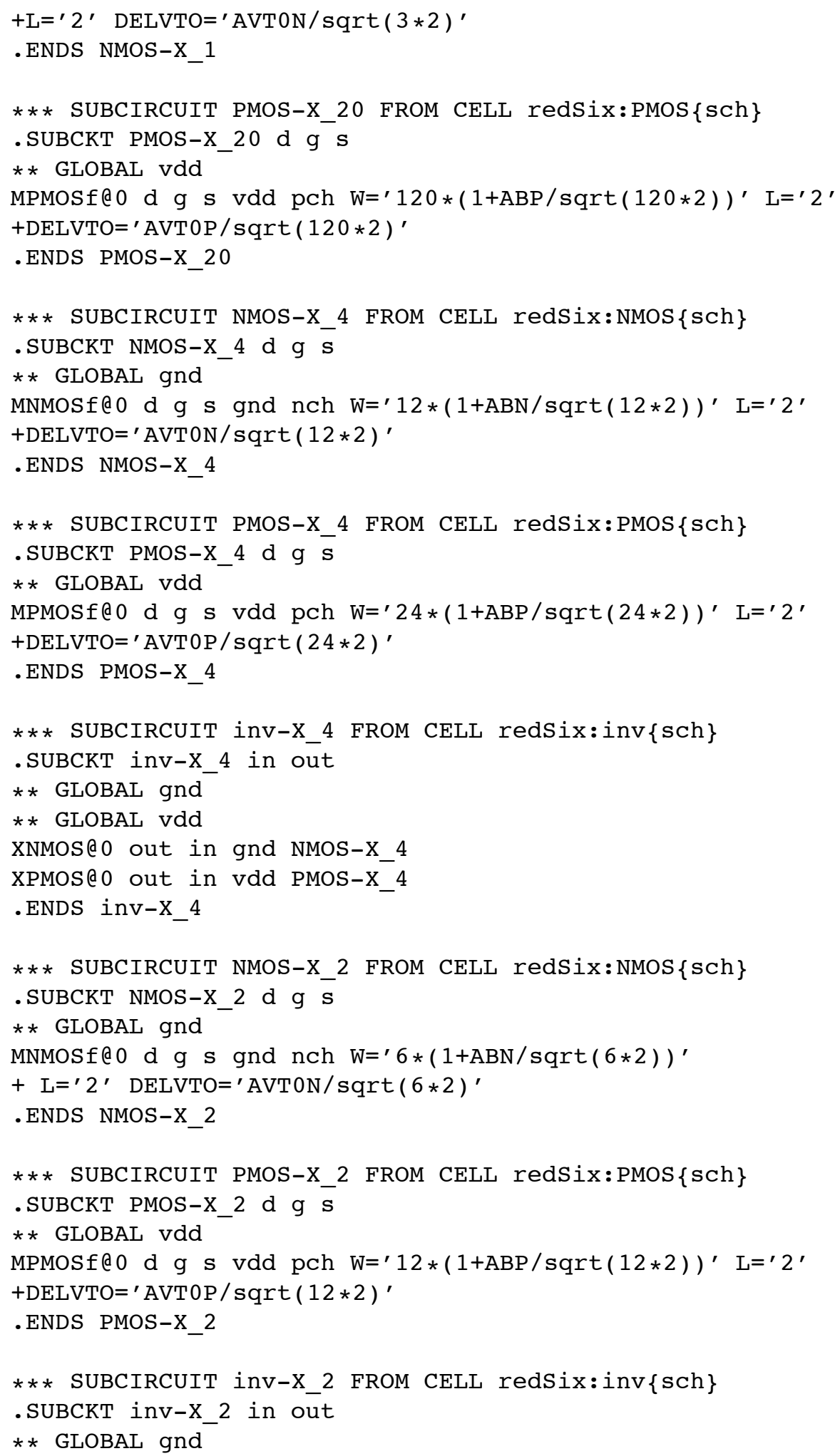




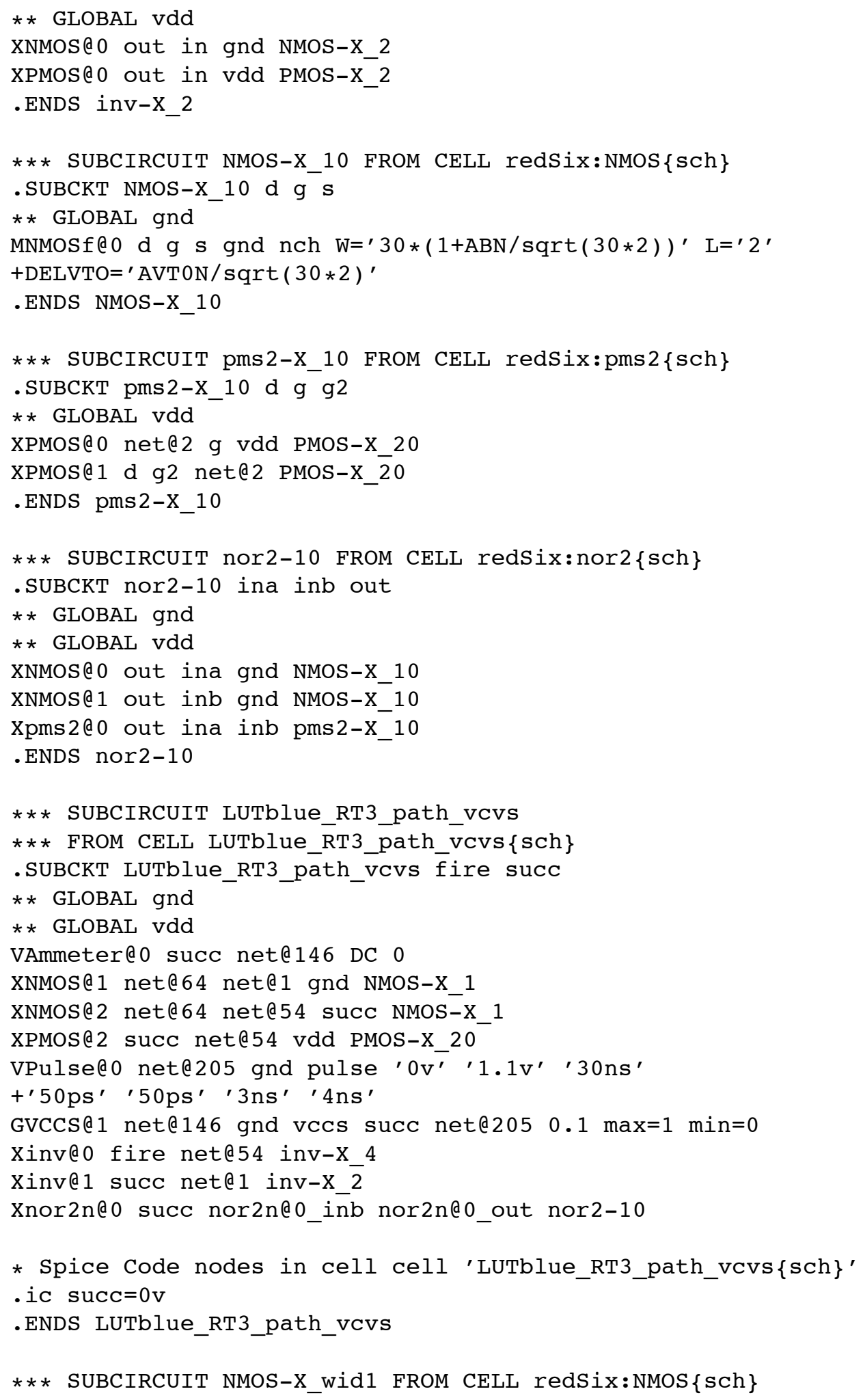




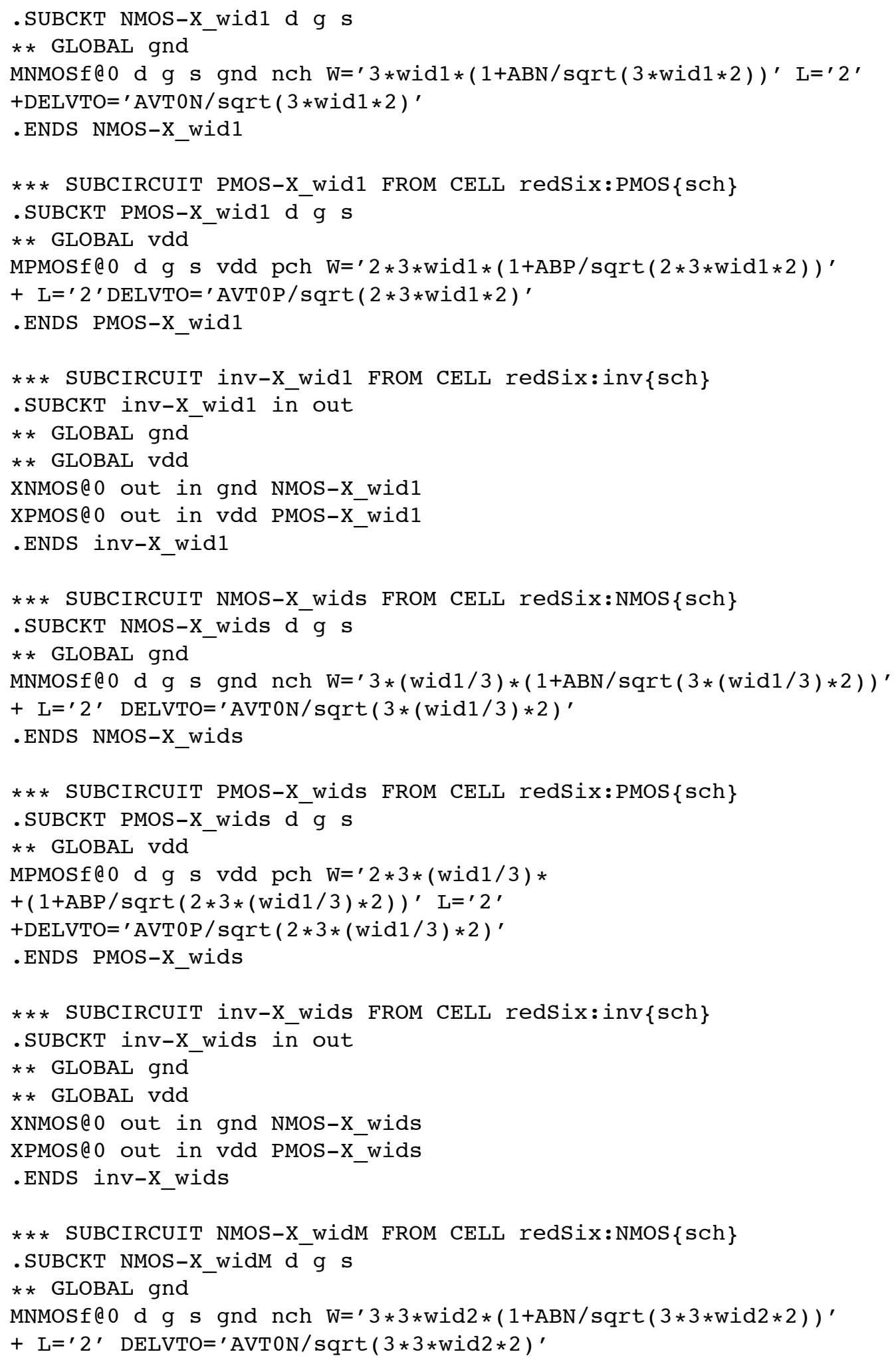


.ENDS NMOS-X_widM

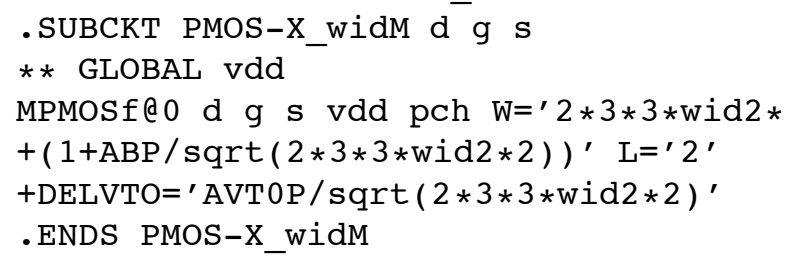


Xinv@3 net@64 inv@3_out inv-X_widM

Xinv@ 4 out[1] net@ $6 \overline{4}$ inv-X wiď 2

* Trailer cards are described in this file:

.include '/u/mettalag/models/Models_90nm/MOSIS_trailer_ARC_3.hsp'

. END 


\section{F.2 SPICE Simulation Setup File: HEADER}

Here is the basic file with default simulation parameters for SPICE. Its extension is '.hsp'. It contains a pointer to the 90nm CMOS process models for our GasP designs. We use the library with typical (TT) Process, Voltage and Temperature (PVT) settings.

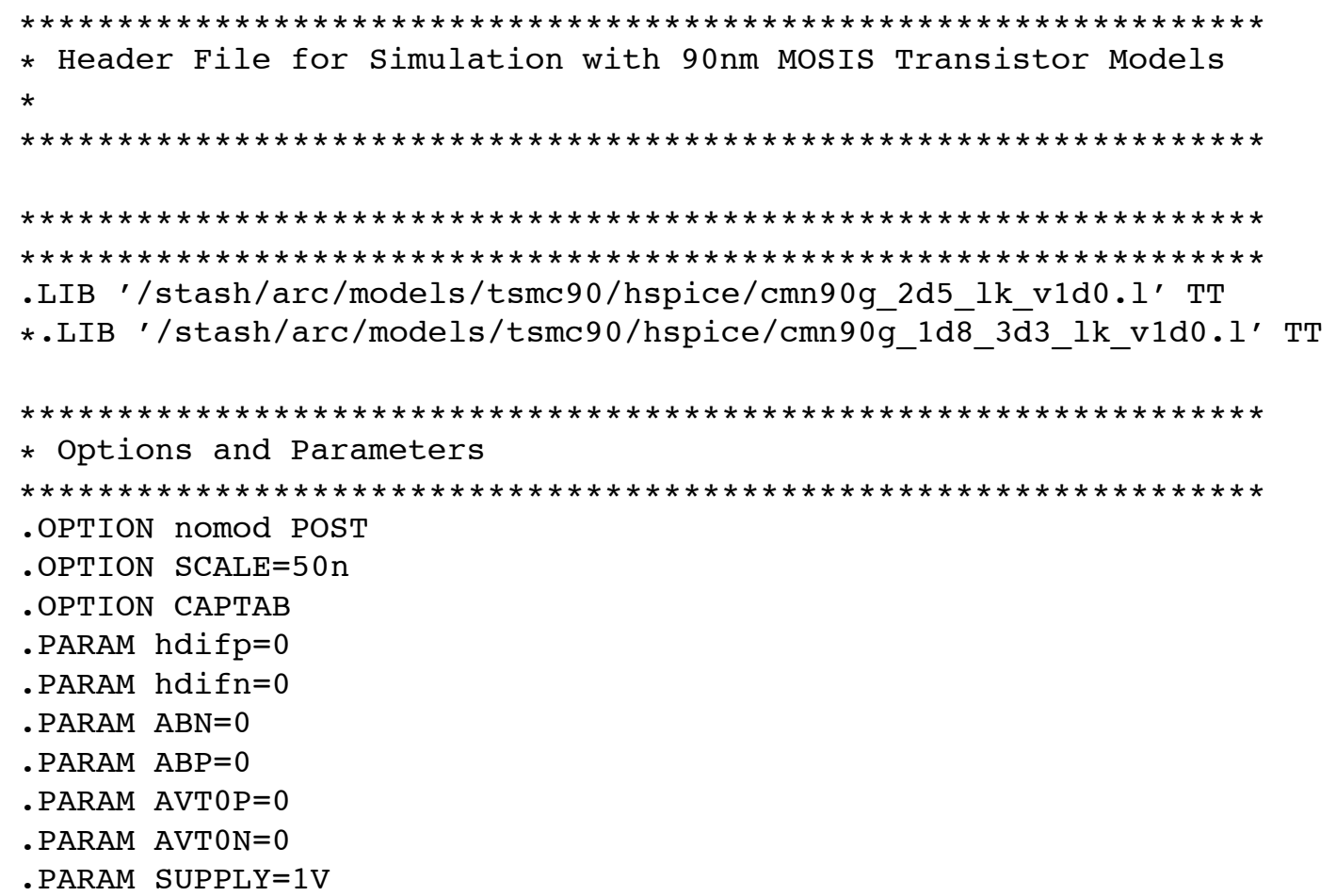




\section{F.3 SPICE Simulation Setup File: TRAILER}

Below follows the SPICE trailer file with the .DATA sweep, transient analysis, and measurement details for the simulation environment in Figure 4.15, page 72, and in the black box schematics of Figure 4.10, page 61. We generate look up tables for relative timing paths LUTred:FIRE $i+$ SUCC $_{i}+$ and LUTblue:FIRE $i+\mathrm{SUCC}_{i}+$. To do so, we sweep driver size wid1 and load size wid2 we measure the voltage on in[1], out[1].

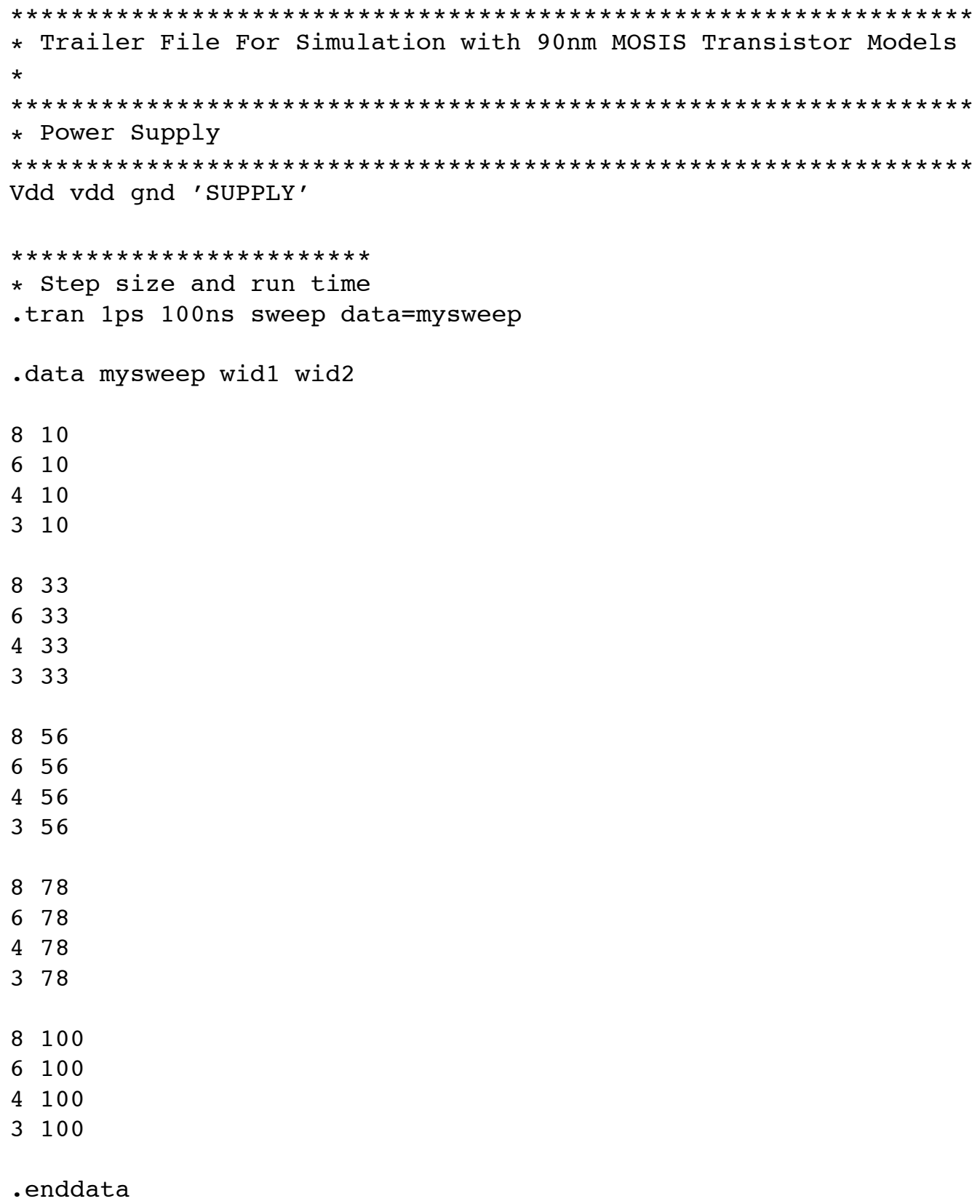




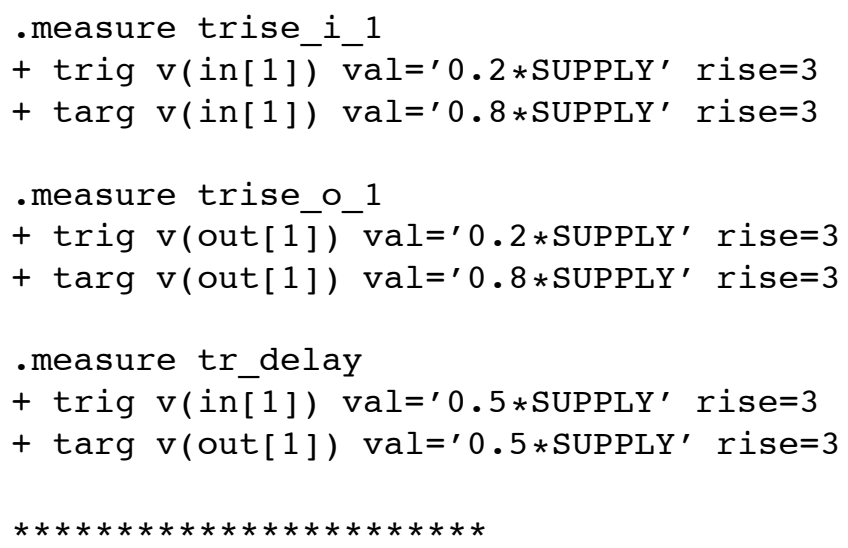




\section{F.4 SPICE Output File}

Below is the SPICE output file with the look up table results for the two relative timing paths LUTred:FIRE ${ }_{i}+\mathrm{SUCC}_{i}+$ and LUTblue:FIRE $i+\mathrm{SUCC}_{i}+$, as measured for the schematic of Figure 4.15, page 72, and the black box schematics of Figure 4.10, page 61 . The file extension is '. $m t 0$ '.

The file contains the $4 \times 5$ wid $1 \times$ wid 2 sweep index, i.e. index 1 through 20 . Per sweep, the file shows the sizes of sweep variables wid1 and wid 2 and the measured timing results in seconds. We measure the following timing information:

- trise_i_1, the transition rise time, a.k.a. slope or slew time, on input in[1]

- trise_o_1, the transition rise time, a.k.a. slope or slew time, on output out[1]

- tr_delay, input-to-output delay for the rising transition from in[1] to out[1]

Note: the additional process parameters temper and alter\# can be ignored.

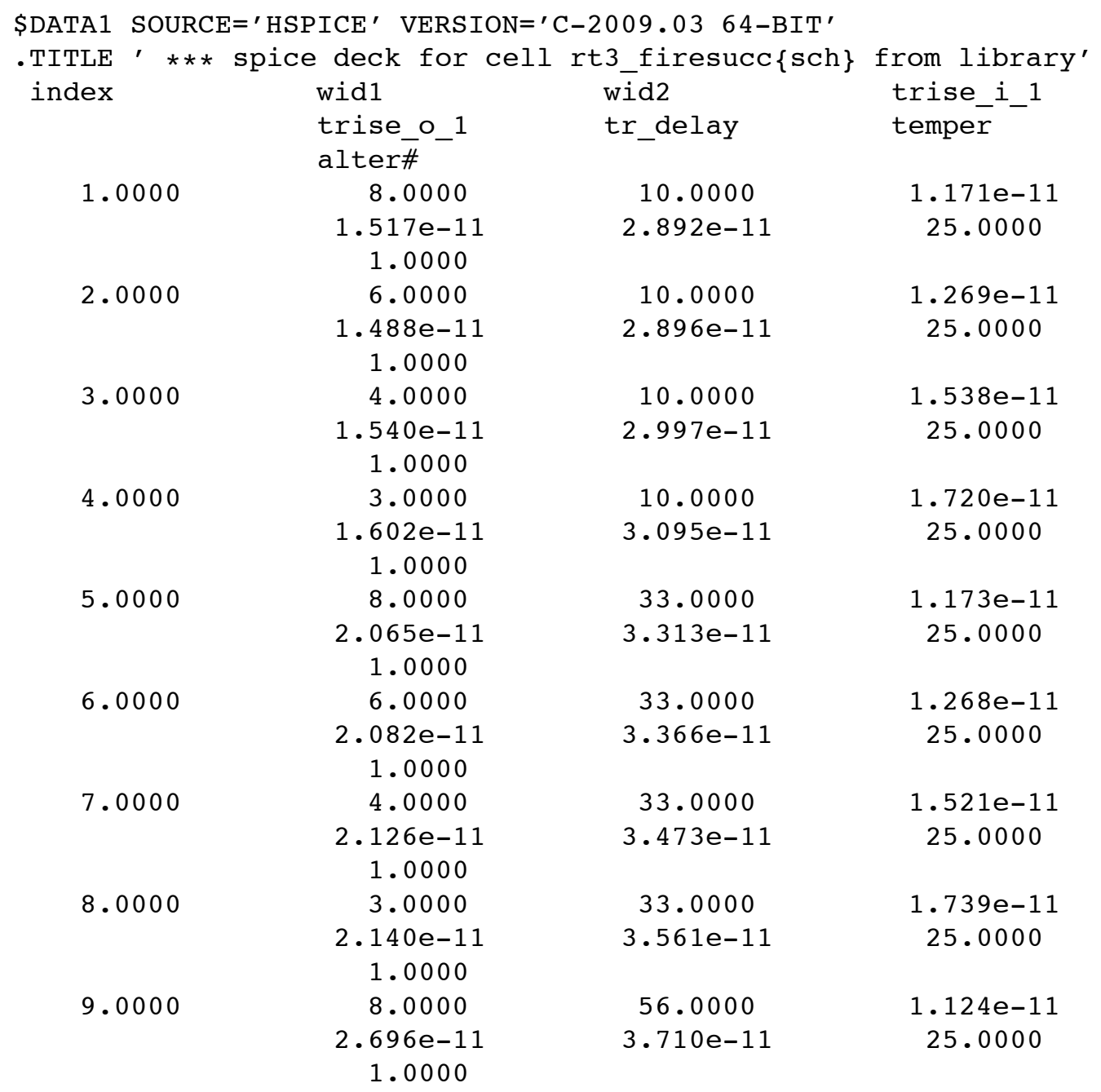




\begin{tabular}{|c|c|c|c|}
\hline \multirow[t]{3}{*}{10.0000} & 6.0000 & 56.0000 & $1.268 e-11$ \\
\hline & $2.742 e-11$ & $3.778 e-11$ & 25.0000 \\
\hline & 1.0000 & & \\
\hline \multirow[t]{3}{*}{11.0000} & 4.0000 & 56.0000 & $1.521 e-11$ \\
\hline & $2.756 e-11$ & $3.877 e-11$ & 25.0000 \\
\hline & 1.0000 & & \\
\hline \multirow[t]{3}{*}{12.0000} & 3.0000 & 56.0000 & $1.745 e-11$ \\
\hline & $2.773 e-11$ & $3.987 e-11$ & 25.0000 \\
\hline & 1.0000 & & \\
\hline \multirow[t]{3}{*}{13.0000} & 8.0000 & 78.0000 & $1.151 e-11$ \\
\hline & $3.414 e-11$ & $4.101 e-11$ & 25.0000 \\
\hline & 1.0000 & & \\
\hline \multirow[t]{3}{*}{14.0000} & 6.0000 & 78.0000 & $1.256 e-11$ \\
\hline & $3.388 e-11$ & $4.158 e-11$ & 25.0000 \\
\hline & 1.0000 & & \\
\hline \multirow[t]{3}{*}{15.0000} & 4.0000 & 78.0000 & $1.528 e-11$ \\
\hline & $3.399 e-11$ & $4.236 e-11$ & 25.0000 \\
\hline & 1.0000 & & \\
\hline \multirow[t]{3}{*}{16.0000} & 3.0000 & 78.0000 & $1.712 \mathrm{e}-11$ \\
\hline & $3.404 e-11$ & $4.338 e-11$ & 25.0000 \\
\hline & 1.0000 & & \\
\hline \multirow[t]{3}{*}{17.0000} & 8.0000 & 100.0000 & $1.454 e-11$ \\
\hline & $4.048 e-11$ & $4.425 e-11$ & 25.0000 \\
\hline & 1.0000 & & \\
\hline \multirow[t]{3}{*}{18.0000} & 6.0000 & 100.0000 & $1.267 e-11$ \\
\hline & $4.038 e-11$ & $4.528 e-11$ & 25.0000 \\
\hline & 1.0000 & & \\
\hline \multirow[t]{3}{*}{19.0000} & 4.0000 & 100.0000 & $1.486 e-11$ \\
\hline & $4.043 e-11$ & $4.636 e-11$ & 25.0000 \\
\hline & 1.0000 & & \\
\hline \multirow[t]{3}{*}{20.0000} & 3.0000 & 100.0000 & $1.740 e-11$ \\
\hline & $4.095 e-11$ & $4.724 e-11$ & 25.0000 \\
\hline & 1.0000 & & \\
\hline
\end{tabular}




\section{Appendix G}

\section{SPICE FILES FOR FIGURE 4.11 AND LUTblue/green:FIRE ${ }_{i}+$ PRED $_{i}-$}

Ideally, the SPICE simulations for Figure 4.18 on page 77 will run the green path for maximum delay conditions by using slow PVT library setting, and they wil run the blue path for minimum delay conditions by using fast settings. We show only one simulation (covering both paths) using typical Process, Voltage, Temperature (PVT) settings.

\section{G.1 SPICE Netlist}

The SPICE netlist file generated from the design schematics in Electric has the details of all the components and devices used in the schematics of Figure 4.18 on page 77 and in the black box schematics of Figure 4.11 on page 63 . The black box belongs to relative timing paths LUTblue:FIRE $+\mathrm{PRED}_{i}-$ and $\mathrm{LUT}_{i}$ reen:FIRE $i+\mathrm{PRED}_{i}-$. The sweep parameters wid1 and wid 2 are the sizes of the driver and load inverters. This configuration has two pulse generators, one for signal $\mathrm{FIRE}_{i}$ to start each measurement, one for signal FIRE_CS ${ }_{i}$ to reset the output signal $\mathrm{PRED}_{i}$ prior to the next measurement. The file extension is 'spi'.

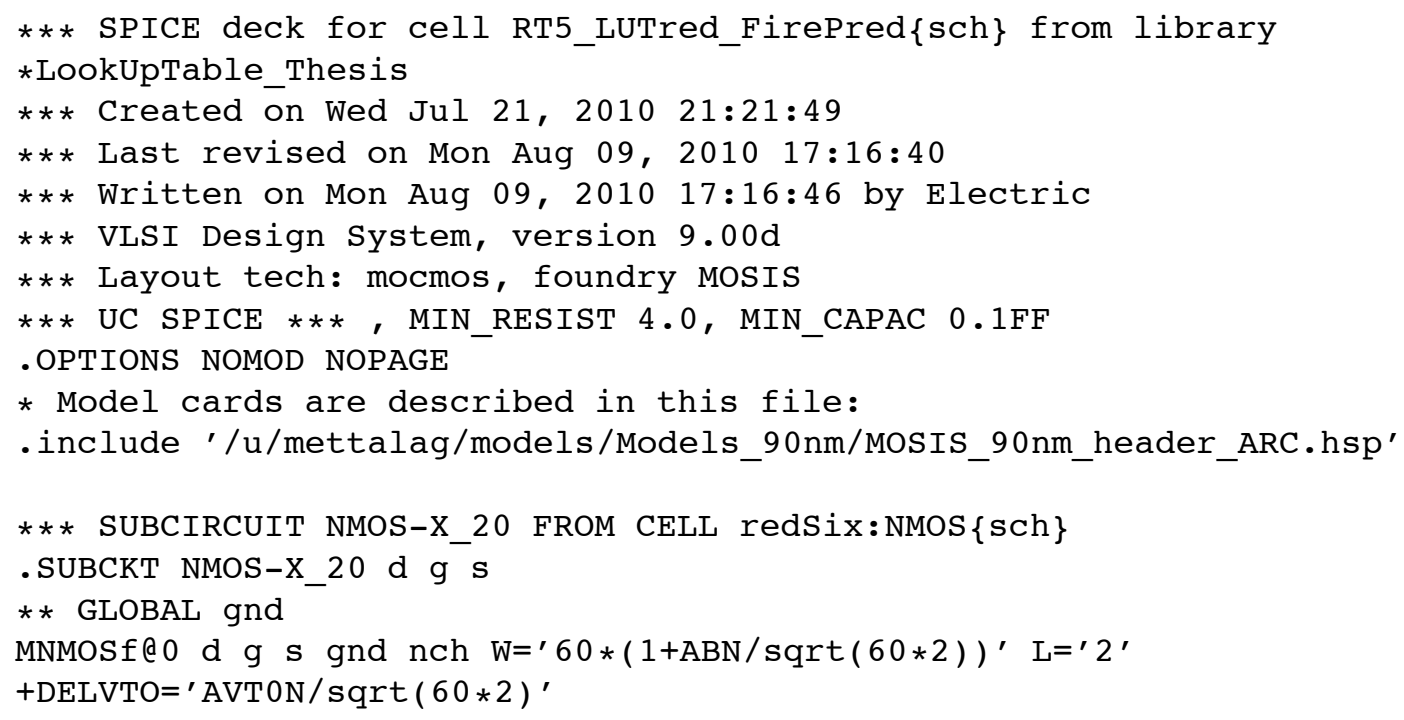


.ENDS NMOS-X_2

*** SUBCIRCUIT PMOS-X_1 FROM CELL redSix:PMOS $\{\mathrm{sch}\}$

.SUBCKT PMOS-X_1 d g $\mathrm{s}$

** GLOBAL vdd

MPMOSf@0 d g s vdd pch $\mathrm{W}={ }^{\prime} 6 *(1+\mathrm{ABP} / \operatorname{sqrt}(6 * 2))^{\prime} \mathrm{L}={ }^{\prime} 2^{\prime}$

+ DELVTO $={ }^{\prime}$ AVTOP $/ \operatorname{sqrt}(6 * 2)^{\prime}$

.ENDS PMOS-X_1

*** SUBCIRCUIT NMOS-X_5 FROM CELL redSix:NMOS $\{\mathrm{sch}\}$

.SUBCKT NMOS-X_5 d g $\mathrm{s}$

** GLOBAL gnd

MNMOSf@0 d g s gnd nch $\mathrm{W}=\prime 15 *(1+\mathrm{ABN} / \operatorname{sqrt}(15 * 2))^{\prime} \mathrm{L}=\mathbf{\prime}^{\prime}$ ' $^{\prime}$

+ DELVTO $=$ ' AVTON $/ \operatorname{sqrt}(15 * 2)$ '

.ENDS NMOS-X_5

*** SUBCIRCUIT PMOS-X_5 FROM CELL redSix:PMOS $\{\mathrm{sch}\}$

.SUBCKT PMOS-X_5 d g $\mathrm{s}$

** GLOBAL vdd

MPMOSf@0 d g s vdd pch $W=\prime 30 *(1+A B P / \operatorname{sqrt}(30 * 2))^{\prime} \mathrm{L}={ }^{\prime} 2^{\prime}$

+ DELVTO $=$ ' AVTOP $/ \operatorname{sqrt}(30 * 2)$ '

.ENDS PMOS-X_5

*** SUBCIRCUIT inv-X_5 FROM CELL redSix:inv\{sch $\}$

.SUBCKT inv-X_5 in out

** GLOBAL gnd

** GLOBAL vdd

XNMOS@O out in gnd NMOS-X_5

XPMOS@ 0 out in vdd PMOS-X 5

.ENDS inv-X_5

*** SUBCIRCUIT NMOS-X_2 FROM CELL redSix:NMOS $\{\mathrm{sch}\}$

.SUBCKT NMOS-X_2 d g $\mathrm{s}$

** GLOBAL gnd

MNMOSf@0 d g s gnd nch $\mathrm{W}=\prime 6 *(1+\mathrm{ABN} / \operatorname{sqrt}(6 * 2))^{\prime}$

$+\mathrm{L}={ }^{\prime} 2^{\prime} \mathrm{DELVTO}={ }^{\prime} \mathrm{AVTON} / \operatorname{sqrt}(6 * 2)^{\prime}$

.ENDS NMOS-X_2

*** SUBCIRCUIT PMOS-X_2 FROM CELL redSix:PMOS $\{\mathrm{sch}\}$

.SUBCKT PMOS-X_2 d g $\bar{s}$

** GLOBAL vdd

MPMOSf@O d g s vdd pch $\mathrm{W}=\prime 12 *(1+\mathrm{ABP} / \operatorname{sqrt}(12 * 2))^{\prime} \mathrm{L}=\mathbf{\prime}^{\prime}$ ' $^{\prime}$

+ DELVTO='AVTOP $/ \operatorname{sqrt}(12 * 2)^{\prime}$

.ENDS PMOS-X_2

*** SUBCIRCUIT inv-X_2 FROM CELL redSix:inv\{sch

.SUBCKT inv-X_2 in out

** GLOBAL gnd

* * GLOBAL vdd 
XNMOS@O out in gnd NMOS-X_2

XPMOS@O out in vdd PMOS-X_2

.ENDS inv-X 2

*** SUBCIRCUIT LUTblue_RT5_path FROM CELL LUTblue_RT5_path\{sch\}

.SUBCKT LUTblue_RT5_path fire pred

** GLOBAL gnd

** GLOBAL vdd

VAmmeter@0 pred net@70 DC 0

XNMOS@0 pred fire gnd NMOS-X_20

XPMOS@ 0 net@ 48 net@ 46 vdd PMOS-X 1

XPMOS@1 net@48 PMOS@1_g pred PMOS-X_1

VPulse@0 net@106 gnd pulse ' $1 \mathrm{v}$ ' '-0.5v' '30ns' '50ps'

+ '50ps' '3ns' '4ns'

GVCCS@0 net@70 gnd vccs pred net@106 $0.1 \mathrm{max}=0 \mathrm{~min}=-1$

Xinve2 pred inv@2_out inv-X_5

Xinv@5 pred net@ $4 \overline{6}$ inv-X_2

* Spice Code nodes in cell cell 'LUTblue_RT5_path\{sch\}'

. ic nete83=1v

.ENDS LUTblue_RT5_path

*** SUBCIRCUIT NMOS-X_wid1 FROM CELL redSix:NMOS $\{$ sch $\}$

.SUBCKT NMOS-X_wid1 d g s

* * GLOBAL gnd

MNMOSf@o d g s gnd nch $\mathrm{W}={ }^{\prime} 3 *$ Wid $1 *(1+\mathrm{ABN} / \operatorname{sqrt}(3 *$ Wid $1 * 2))$ '

$+\mathrm{L}={ }^{\prime} 2^{\prime}$ DELVTO $=$ 'AVT0N $/ \operatorname{sqrt}(3 * \text { Wid } 1 * 2)^{\prime}$

.ENDS NMOS-X_wid1

*** SUBCIRCUIT PMOS-X_wid1 FROM CELL redSix:PMOS $\{$ sch $\}$

.SUBCKT PMOS-X_wid1 d g s

** GLOBAL vdd

MPMOSf@0 d g s vdd pch $\mathrm{W}={ }^{\prime} 2 * 3 * \mathrm{Wid} 1 *(1+\mathrm{ABP} / \operatorname{sqrt}(2 * 3 * \mathrm{Wid} 1 * 2))^{\prime}$

$+\mathrm{L}={ }^{\prime} 2{ }^{\prime} \mathrm{DELVTO}={ }^{\prime} \mathrm{AVT} O \mathrm{P} / \operatorname{sqrt}(2 * 3 * \mathrm{Wid} 1 * 2)^{\prime}$

.ENDS PMOS-X_wid1

*** SUBCIRCUIT inv-X_wid1 FROM CELL redSix:inv\{sch\}

.SUBCKT inv-X_widl in out

** GLOBAL gnd

** GLOBAL vdd

XNMOS@O out in gnd NMOS-X_wid1

XPMOS@O out in vdd PMOS-X_wid1

.ENDS inv-X_wid1

*** SUBCIRCUIT NMOS-X_wids FROM CELL redSix:NMOS $\{$ sch $\}$

.SUBCKT NMOS-X_wids d $g \mathrm{~s}$

** GLOBAL gnd

MNMOSf@0 d g s gnd nch $W=\prime 3 *(\operatorname{wid} 1 / 3) *(1+\operatorname{ABN} / \operatorname{sqrt}(3 *(\operatorname{wid} 1 / 3) * 2))^{\prime}$

$+\mathrm{L}={ }^{\prime} 2^{\prime} \mathrm{DELVTO}={ }^{\prime} \mathrm{AVT} 0 \mathrm{~N} / \operatorname{sqrt}(3 *(\operatorname{wid} 1 / 3) * 2)^{\prime}$ 
.ENDS NMOS-X_wids

*** SUBCIRCUIT PMOS-X_wids FROM CELL redSix:PMOS\{sch $\}$

.SUBCKT PMOS-X_wids d g s

** GLOBAL vdd

MPMOSf@O d g s vdd pch $\mathrm{W}={ }^{\prime} 2 * 3 *(\operatorname{wid} 1 / 3) *$

$+(1+\mathrm{ABP} / \operatorname{sqrt}(2 * 3 *(\text { widl } 1 / 3) * 2))^{\prime} \mathrm{L}=\mathbf{\prime}^{\prime} 2^{\prime}$

+ DELVTO $={ }^{\prime}$ AVT0P $/ \operatorname{sqrt}(2 * 3 *(\text { wid1 } 1 / 3) * 2)^{\prime}$

.ENDS PMOS-X_wids

*** SUBCIRCUIT inv-X_wids FROM CELL redSix:inv\{sch

.SUBCKT inv-X_wids in out

$* *$ GLOBAL gnd

** GLOBAL vdd

XNMOS@O out in gnd NMOS-X_wids

XPMOS@O out in vdd PMOS-X_wids

.ENDS inv-X_wids

*** SUBCIRCUIT NMOS-X_widM FROM CELL redSix:NMOS $\{\mathrm{sch}\}$

.SUBCKT NMOS-X_widM d g s

** GLOBAL gnd

MNMOSf@o d g s gnd nch $\mathrm{W}={ }^{\prime} 3 * 3 * \operatorname{wid} 2 *(1+\mathrm{ABN} / \operatorname{sqrt}(3 * 3 * \operatorname{wid} 2 * 2))^{\prime}$

$+\mathrm{L}={ }^{\prime} 2^{\prime}$ DELVTO='AVTON $/ \operatorname{sqrt}(3 * 3 * \mathrm{wid} 2 * 2)^{\prime}$

.ENDS NMOS-X_widM

*** SUBCIRCUIT PMOS-X_widM FROM CELL redSix:PMOS $\{\mathrm{sch}\}$

.SUBCKT PMOS-X_widM d g $\mathrm{s}$

** GLOBAL vdd

MPMOSf@0 d g s vdd pch $\mathrm{W}={ }^{\prime} 2 * 3 * 3 *$ wid $2 *$

$+(1+\mathrm{ABP} / \operatorname{sqrt}(2 * 3 * 3 * \mathrm{wid} 2 * 2))^{\prime} \mathrm{L}={ }^{\prime} 2^{\prime}$

+ DELVTO $=$ 'AVTOP $/ \operatorname{sqrt}(2 * 3 * 3 * \text { wid } 2 * 2)^{\prime}$

.ENDS PMOS-X_widM

*** SUBCIRCUIT inv-X_widM FROM CELL redSix:inv\{sch\}

. SUBCKT inv-X_widM in out

** GLOBAL gnd

** GLOBAL vdd

XNMOS@O out in gnd NMOS-X_widM

XPMOS@O out in vdd PMOS-X_widM

.ENDS inv-X_widM

*** SUBCIRCUIT NMOS-X_wid2 FROM CELL redSix:NMOS $\{\mathrm{sch}\}$

.SUBCKT NMOS-X_wid2 d g s

** GLOBAL gnd

MNMOSf@o d g s gnd nch $\mathrm{W}={ }^{\prime} 3 * \mathrm{Wid} 2 *(1+\mathrm{ABN} / \operatorname{sqrt}(3 * \mathrm{Wid} 2 * 2))^{\prime}$

$+\mathrm{L}={ }^{\prime} 2^{\prime}$ DELVTO='AVTON $/ \operatorname{sqrt}(3 * \text { Wid2 } 2 * 2)^{\prime}$

.ENDS NMOS-X_wid2

*** SUBCIRCUIT PMOS-X_wid2 FROM CELL redSix:PMOS $\{\mathrm{sch}\}$ 


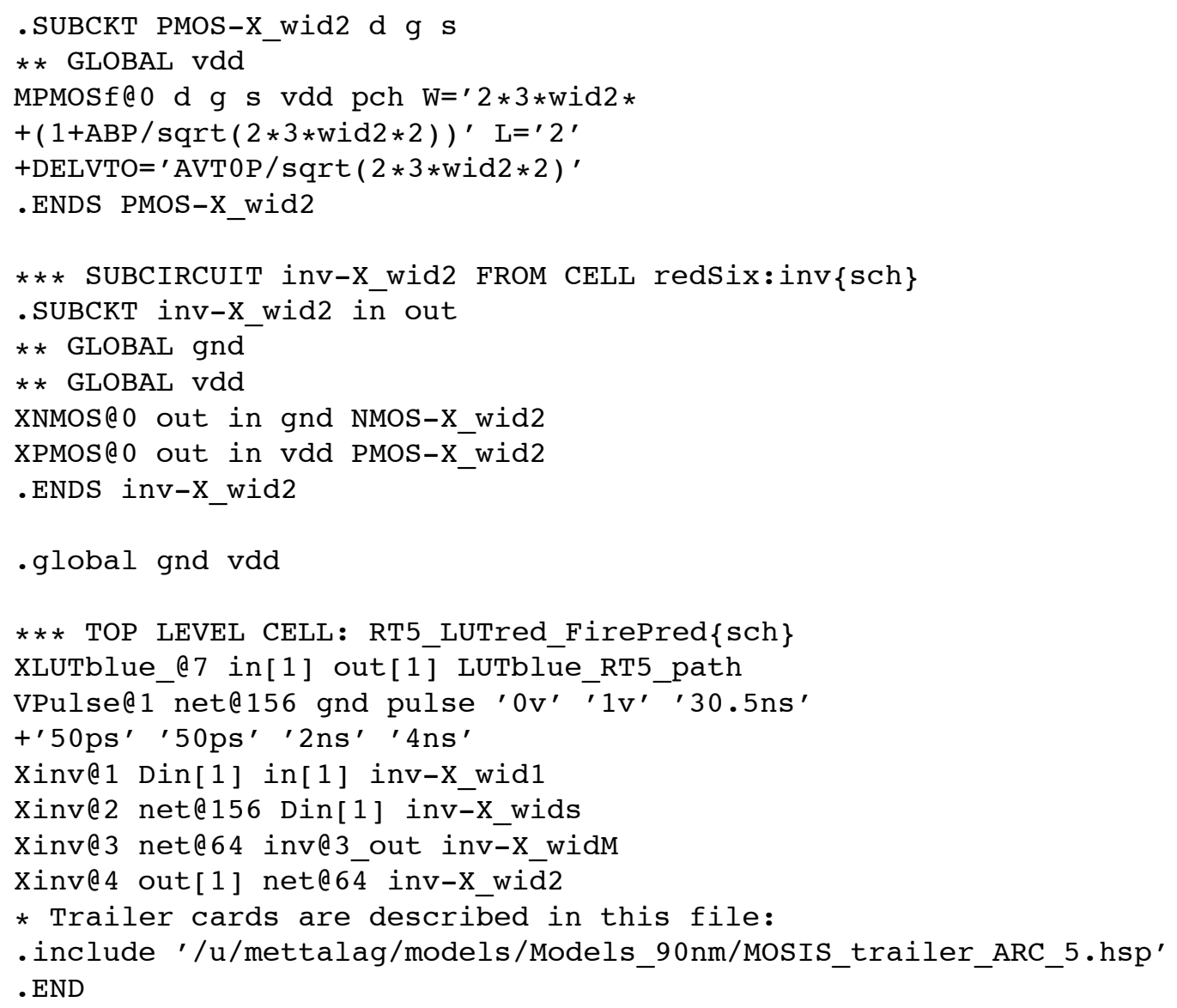




\section{G.2 SPICE Simulation Setup File: HEADER}

Here is the basic file with default simulation parameters for SPICE. Its extension is '.hsp'. It contains a pointer to the 90nm CMOS process models for our GasP designs. We use the library with typical (TT) Process, Voltage and Temperature (PVT) settings.

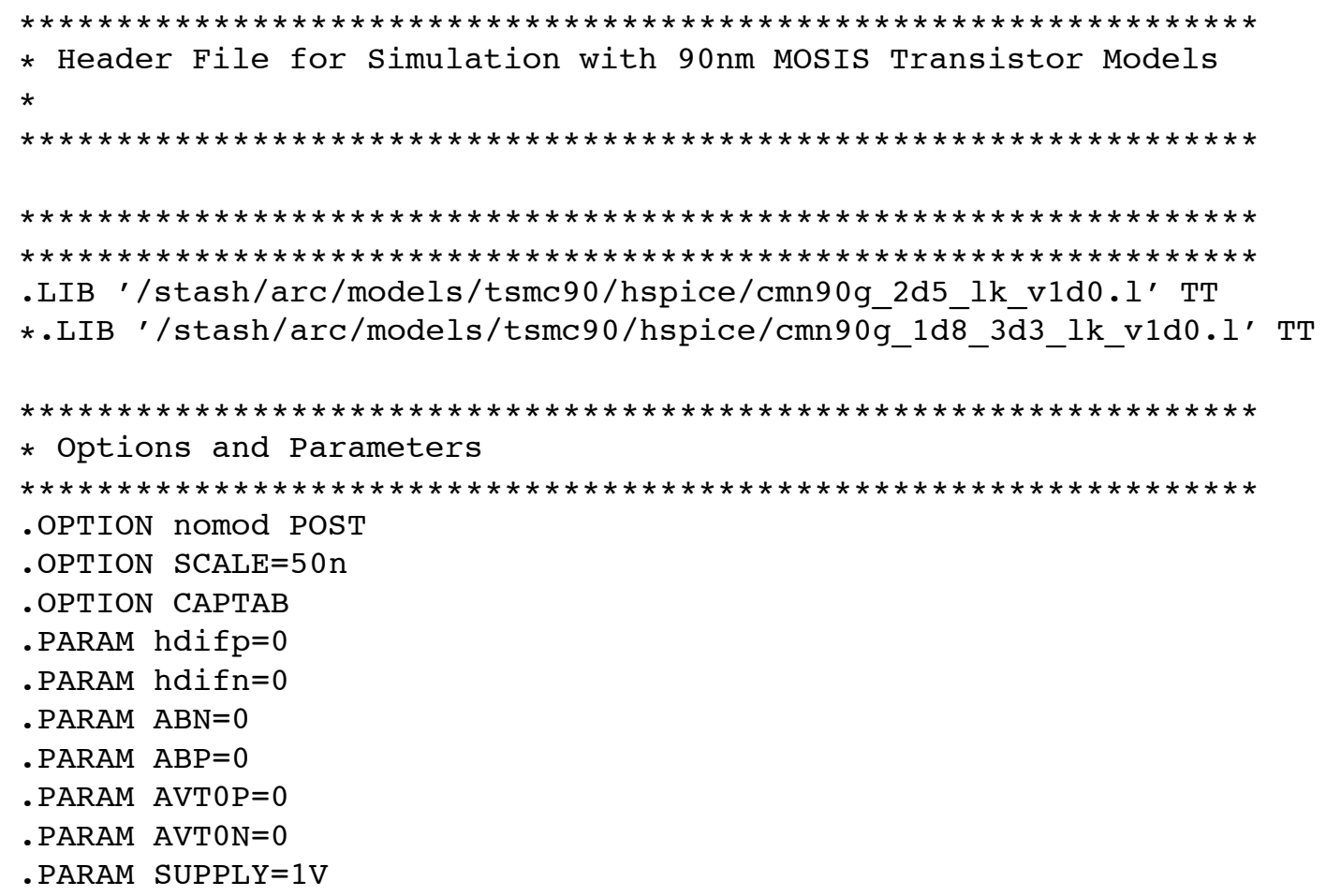




\section{G.3 SPICE Simulation Setup File: TRAILER}

Below follows the SPICE trailer file with the .DATA sweep, transient analysis, and measurement details for the simulation environment in Figure 4.18 on page 77 and for the black box schematics of Figure 4.11 on page 63. We are generating look up tables for relative timing paths LUTblue:FIRE + $+\mathrm{PRED}_{i}-$ and LUTgreen:FIRE $i+\mathrm{PRED}_{i}-$. To do so, we sweep driver size wid1 and load size wid2, and we measure the voltage levels on in[1] and out[1].

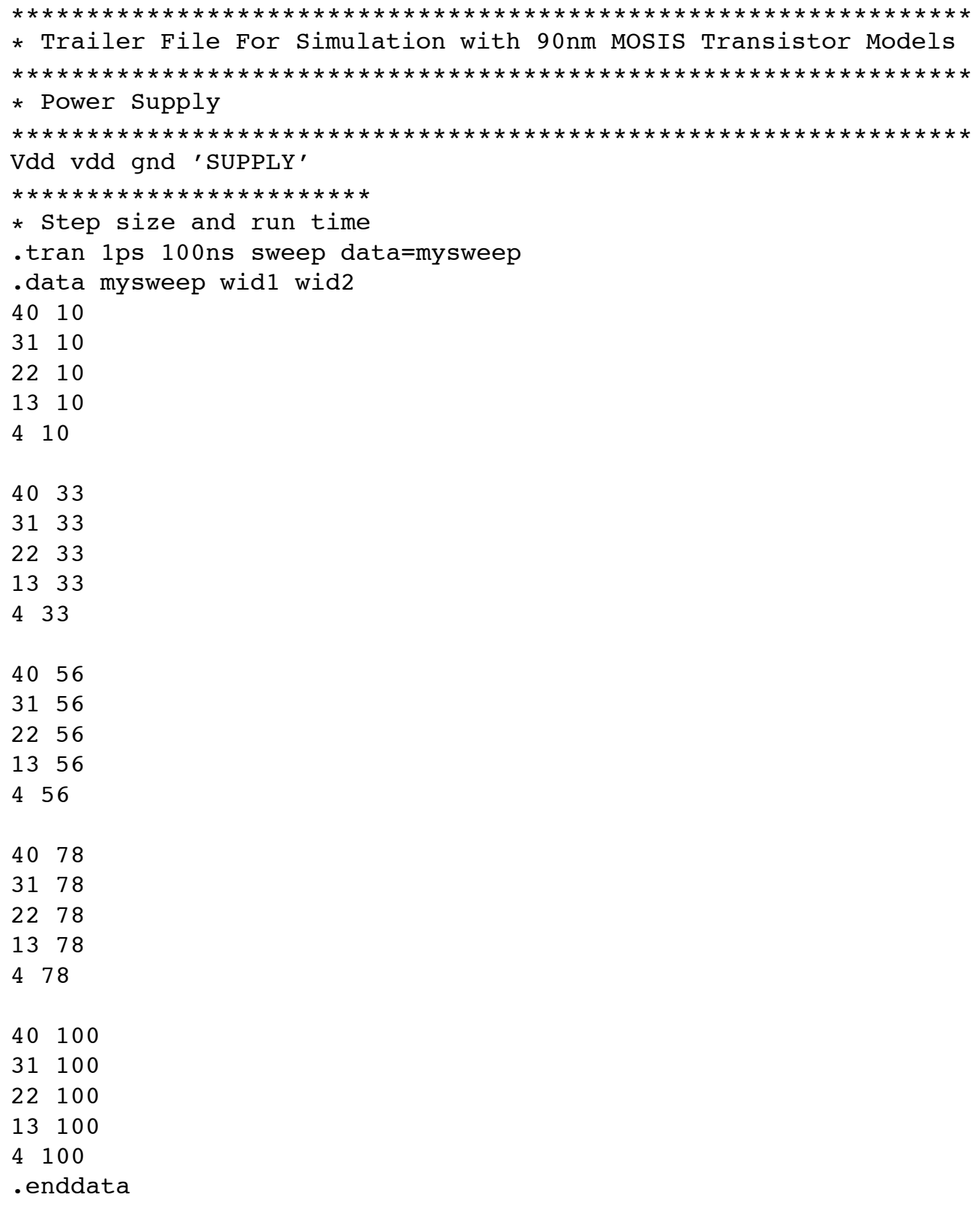




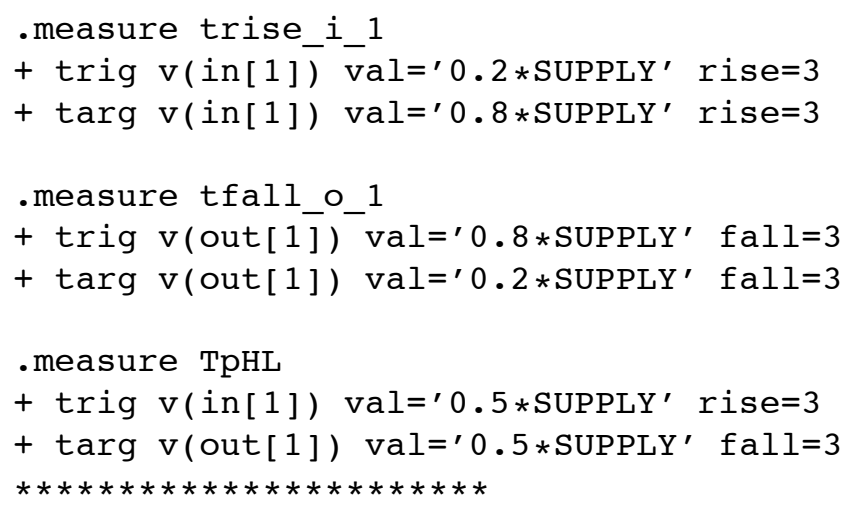




\section{G.4 SPICE Output File}

Below is the SPICE output file with the look up table results for the two relative timing paths LUTblue:FIRE $i+\mathrm{PRED}_{i}-$ and LUTgreen:FIRE $i+\mathrm{PRED}_{i}-$, as measured for the schematic of Figure 4.18 on page 77 and the black box schematics of Figure 4.11 on page 63 . The file extension is ' $m t 0$ '.

The file contains the $5 \times 5$ wid $1 \times$ wid 2 sweep index, i.e. index $1-25$. We have 25 sweeps because Figure 4.18 is the only configuration where the minimum source and driver sizes fit the Logical Effort step-up-of-3 design strategy for the simulation environment. Per sweep, the file shows the sizes of sweep variables wid 1 and wid 2 and the measured timing results in seconds. We measure the following timing information:

- trise_i_1, the transition rise time, a.k.a. slope or slew time, on input in[1]

- tfall_o_1, the transition fall time, a.k.a. slope or slew time, on output out[1]

- tphl, the input-to-output delay for the high to low transition from in[1] to out[1]

Note: the additional process parameters temper and alter\# can be ignored.

\begin{tabular}{|c|c|c|c|}
\hline $\begin{array}{l}. \operatorname{TITLE} \quad * * * \\
\text { index }\end{array}$ & $\begin{array}{l}\text { spice deck for cell } \\
\text { widl } \\
\text { tfall_o_1 } \\
\text { alter\# }\end{array}$ & $\begin{array}{l}\text { rt5_firepred }\{\mathrm{sch}\} \\
\text { wid2 } \\
\text { tph1 }\end{array}$ & $\begin{array}{l}\text { from library' } \\
\text { trise_i_1 } \\
\text { temper }\end{array}$ \\
\hline 1.0000 & 40.0000 & 10.0000 & $1.078 \mathrm{e}-11$ \\
\hline & $\begin{array}{c}7.457 e-12 \\
1.0000\end{array}$ & $4.580 e-12$ & 25.0000 \\
\hline 2.0000 & $\begin{array}{c}31.0000 \\
7.618 \mathrm{e}-12\end{array}$ & $\begin{array}{c}10.0000 \\
4.742 \mathrm{e}-12\end{array}$ & $\begin{array}{c}1.115 \mathrm{e}-11 \\
25.0000\end{array}$ \\
\hline & 1.0000 & & \\
\hline 3.0000 & $\begin{array}{c}22.0000 \\
7.818 \mathrm{e}-12 \\
1.0000\end{array}$ & $\begin{array}{c}10.0000 \\
4.979 e-12\end{array}$ & $\begin{array}{c}1.184 \mathrm{e}-11 \\
25.0000\end{array}$ \\
\hline 4.0000 & $\begin{array}{c}13.0000 \\
7.469 e-12 \\
1.0000\end{array}$ & $\begin{array}{c}10.0000 \\
5.703 e-12\end{array}$ & $\begin{array}{c}1.182 \mathrm{e}-11 \\
25.0000\end{array}$ \\
\hline 5.0000 & $\begin{array}{c}4.0000 \\
1.045 \mathrm{e}-11 \\
1.0000\end{array}$ & $\begin{array}{c}10.0000 \\
6.805 e-12\end{array}$ & $\begin{array}{c}2.147 e-11 \\
25.0000\end{array}$ \\
\hline 6.0000 & $\begin{array}{c}40.0000 \\
1.088 e-11 \\
1.0000\end{array}$ & $\begin{array}{c}33.0000 \\
8.104 \mathrm{e}-12\end{array}$ & $\begin{array}{c}1.106 \mathrm{e}-11 \\
25.0000\end{array}$ \\
\hline 7.0000 & $\begin{array}{c}31.0000 \\
1.104 \mathrm{e}-11 \\
1.0000\end{array}$ & $\begin{array}{c}33.0000 \\
8.261 \mathrm{e}-12\end{array}$ & $\begin{array}{c}1.128 \mathrm{e}-11 \\
25.0000\end{array}$ \\
\hline 8.0000 & $\begin{array}{c}22.0000 \\
1.069 \mathrm{e}-11 \\
\quad 1.0000\end{array}$ & $\begin{array}{c}33.0000 \\
9.067 e-12\end{array}$ & $\begin{array}{c}1.123 e-11 \\
25.0000\end{array}$ \\
\hline 9.0000 & $\begin{array}{c}13.0000 \\
1.171 \mathrm{e}-11\end{array}$ & $\begin{array}{c}33.0000 \\
9.103 e-12\end{array}$ & $\begin{array}{c}1.238 \mathrm{e}-11 \\
25.0000\end{array}$ \\
\hline
\end{tabular}




\begin{tabular}{|c|c|c|c|}
\hline & 1.0000 & & \\
\hline \multirow[t]{3}{*}{10.0000} & 4.0000 & 33.0000 & $2.060 e-11$ \\
\hline & $1.452 \mathrm{e}-11$ & $1.111 e-11$ & 25.0000 \\
\hline & 1.0000 & & \\
\hline \multirow[t]{3}{*}{11.0000} & 40.0000 & 56.0000 & $1.063 e-11$ \\
\hline & $1.514 \mathrm{e}-11$ & $1.117 e-11$ & 25.0000 \\
\hline & 1.0000 & & \\
\hline \multirow[t]{3}{*}{12.0000} & 31.0000 & 56.0000 & $1.078 e-11$ \\
\hline & $1.516 e-11$ & $1.135 \mathrm{e}-11$ & 25.0000 \\
\hline & 1.0000 & & \\
\hline \multirow[t]{3}{*}{13.0000} & 22.0000 & 56.0000 & $1.043 e-11$ \\
\hline & $1.498 e-11$ & $1.187 e-11$ & 25.0000 \\
\hline & 1.0000 & & \\
\hline \multirow[t]{3}{*}{14.0000} & 13.0000 & 56.0000 & $1.201 e-11$ \\
\hline & $1.554 e-11$ & $1.220 e-11$ & 25.0000 \\
\hline & 1.0000 & & \\
\hline \multirow[t]{3}{*}{15.0000} & 4.0000 & 56.0000 & $1.896 e-11$ \\
\hline & $1.837 e-11$ & $1.486 e-11$ & 25.0000 \\
\hline & 1.0000 & & \\
\hline \multirow[t]{3}{*}{16.0000} & 40.0000 & 78.0000 & $9.690 e-12$ \\
\hline & $1.913 e-11$ & $1.422 \mathrm{e}-11$ & 25.0000 \\
\hline & 1.0000 & & \\
\hline \multirow[t]{3}{*}{17.0000} & 31.0000 & 78.0000 & $9.993 e-12$ \\
\hline & $1.904 e-11$ & $1.437 e-11$ & 25.0000 \\
\hline & 1.0000 & & \\
\hline \multirow[t]{3}{*}{18.0000} & 22.0000 & 78.0000 & $1.079 e-11$ \\
\hline & $1.963 e-11$ & $1.442 \mathrm{e}-11$ & 25.0000 \\
\hline & 1.0000 & & \\
\hline \multirow[t]{3}{*}{19.0000} & 13.0000 & 78.0000 & $1.201 e-11$ \\
\hline & $1.998 e-11$ & $1.505 e-11$ & 25.0000 \\
\hline & 1.0000 & & \\
\hline \multirow[t]{3}{*}{20.0000} & 4.0000 & 78.0000 & $1.930 e-11$ \\
\hline & $2.269 e-11$ & $1.801 \mathrm{e}-11$ & 25.0000 \\
\hline & 1.0000 & & \\
\hline \multirow[t]{3}{*}{21.0000} & 40.0000 & 100.0000 & $1.030 e-11$ \\
\hline & $2.398 e-11$ & $1.659 e-11$ & 25.0000 \\
\hline & 1.0000 & & \\
\hline \multirow[t]{3}{*}{22.0000} & 31.0000 & 100.0000 & $1.005 e-11$ \\
\hline & $2.408 e-11$ & $1.700 e-11$ & 25.0000 \\
\hline & 1.0000 & & \\
\hline \multirow[t]{3}{*}{23.0000} & 22.0000 & 100.0000 & $1.053 e-11$ \\
\hline & $2.400 e-11$ & $1.730 e-11$ & 25.0000 \\
\hline & 1.0000 & & \\
\hline \multirow[t]{3}{*}{24.0000} & 13.0000 & 100.0000 & $1.121 \mathrm{e}-11$ \\
\hline & $2.437 e-11$ & $1.791 e-11$ & 25.0000 \\
\hline & 1.0000 & & \\
\hline \multirow{3}{*}{25.0000} & 4.0000 & 100.0000 & $1.852 e-11$ \\
\hline & $2.740 e-11$ & $2.093 e-11$ & 25.0000 \\
\hline & 1.0000 & & \\
\hline
\end{tabular}




\section{Appendix H}

\section{SPICE FILES FOR FIGURE 4.22 WITH ZERO EXTERNAL LOADS}

Appendix H.1 below contains the netlist for Figure 4.22 on page 84. Appendices H.2 and H.3 contain the SPICE HEADER and the SPICE TRAILER simulation files with the sweep, analysis, and measurement statements. Appendix H.4 contains the SPICE output file with the look up tables containing the propagation delays, and input and output slopes under the given input sweep and zero-output-load simulation conditions.

\section{H.1 SPICE Netlist}

The SPICE netlist file generated from the design schematics in Electric has the details of all the components and devices used in the schematics of Figure 4.22 on page 84. Specifically, it contains the details of the sub-circuits for the simple paths LUTblue:PRED ${ }_{i}-\mathrm{FIRE}_{i}-$, LUTblue:SUCC $i+\mathrm{FIRE}_{i}-$, the pair of paths LUTred/blue:FIRE $i+$ SUCC $_{i}+$, and the pair of paths LUTblue/green:FIRE $i+$ PRED $_{i}$. Sweep parameters widd1, widd2, widd3, widd4 are the sizes of the driver inverters of the four sub-circuits. File suffix is '.spi'.

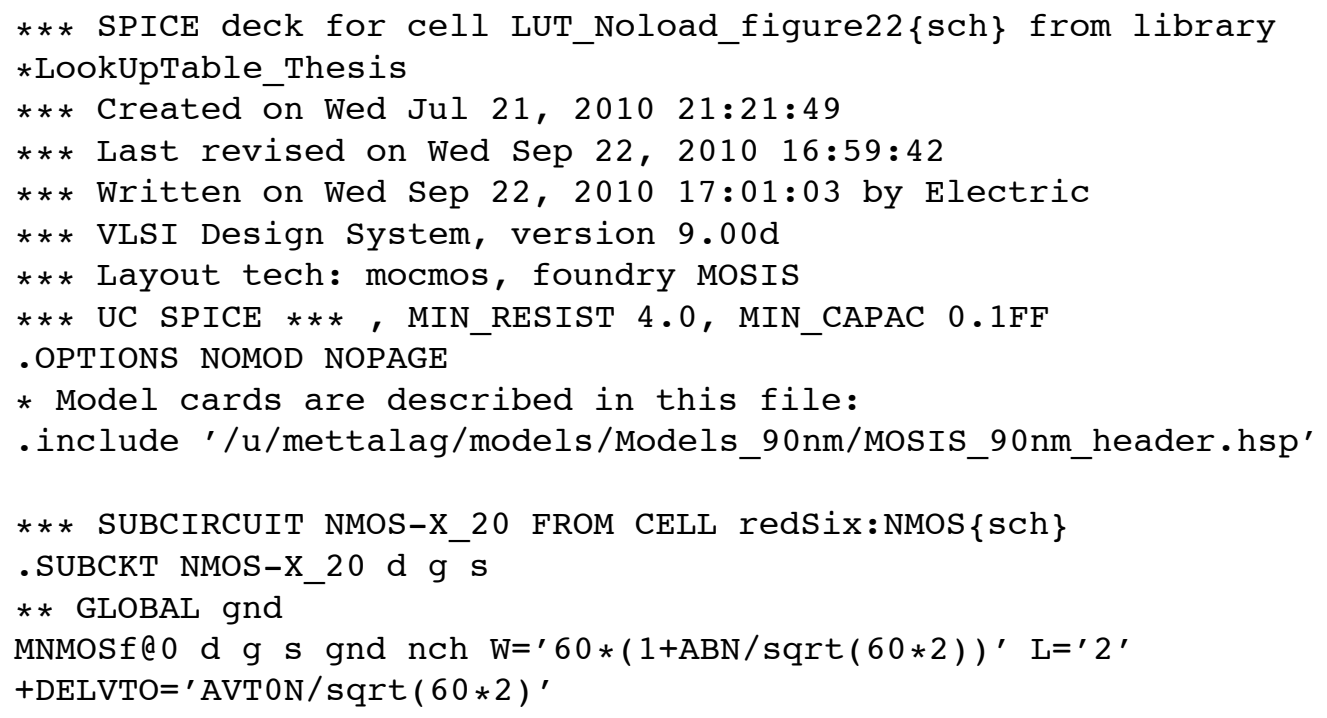


- ENDS NMOS-X_20

*** SUBCIRCUIT PMOS-X_1 FROM CELL redSix:PMOS $\{\mathrm{sch}\}$

.SUBCKT PMOS-X_1 d $g \bar{s}$

* * GLOBAL vdd

MPMOSf@o d g $\mathrm{s}$ vdd pch $\mathrm{W}={ }^{\prime} 6 *(1+\mathrm{ABP} / \operatorname{sqrt}(6 * 2))^{\prime} \mathrm{L}={ }^{\prime} 2^{\prime}$

$+\mathrm{DELVTO}={ }^{\prime} \mathrm{AVTOP} / \operatorname{sqrt}(6 * 2)$ '

.ENDS PMOS-X_1

*** SUBCIRCUIT NMOS-X_30 FROM CELL redSix:NMOS $\{\mathrm{sch}\}$

.SUBCKT NMOS-X_30 d $g$ s

* * GLOBAL gnd

MNMOSf@0 d $g$ s gnd $\mathrm{nch} W=\prime 90 *(1+\mathrm{ABN} / \operatorname{sqrt}(90 * 2))^{\prime} \mathrm{L}={ }^{\prime} \mathbf{2}^{\prime}$

$+\mathrm{DELVTO}={ }^{\prime} \mathrm{AVTON} / \operatorname{sqrt}(90 * 2)$ '

- ENDS NMOS-X_30

*** SUBCIRCUIT PMOS-X_30 FROM CELL redSix:PMOS $\{\mathrm{sch}\}$

.SUBCKT PMOS-X_30 d $\mathrm{g}$ s

** GLOBAL vdd

MPMOSf@0 d g s vdd pch $W=\prime 180 *(1+A B P / \operatorname{sqrt}(180 * 2))^{\prime} L=2^{\prime}$

+DELVTO='AVT0P/sqrt $(180 * 2)$ '

.ENDS PMOS-X_30

*** SUBCIRCUIT inv-X_30 FROM CELL redSix:inv $\{\mathrm{sch}\}$

.SUBCKT inv-X_30 in out

** GLOBAL gnd

* * GLOBAL vdd

XNMOS@O out in gnd NMOS-X_30

XPMOS@0 out in vdd PMOS-X_30

. ENDS inv-X_30

*** SUBCIRCUIT NMOS-X_80 FROM CELL redSix:NMOS $\{\mathrm{sch}\}$

.SUBCKT NMOS-X_80 d $g$ s

** GLOBAL gnd

MNMOSf@0 d g s gnd nch $W=' 240 *(1+\mathrm{ABN} / \operatorname{squt}(240 * 2))^{\prime} \mathrm{L}={ }^{\prime} 2^{\prime}$

$+\mathrm{DELVTO}=$ ' AVTON/sqrt $(240 * 2)$ '

. ENDS NMOS-X_80

*** SUBCIRCUIT PMOS-X_80 FROM CELL redSix:PMOS $\{\mathrm{sch}\}$

.SUBCKT PMOS-X_80 d g $\mathrm{s}$

* * GLOBAL vdd

MPMOSf@0 d g s vdd pch $W=' 480 *(1+\mathrm{ABP} / \operatorname{sqrt}(480 * 2))^{\prime} \mathrm{L}=\mathbf{\prime}^{\prime}$

+ DELVTO= 'AVTOP/sqrt $(480 * 2)$ '

- ENDS PMOS-X_80

*** SUBCIRCUIT inv-X_80 FROM CELL redSix:inv $\{\mathrm{sch}\}$

.SUBCKT inv-X_80 in out

* * GLOBAL gnd

* * GLOBAL vdd 
XNMOS@ 0 out in gnd NMOS-X_80

XPMOS@0 out in vdd PMOS-X_80

.ENDS inv-X_80

*** SUBCIRCUIT NMOS-X_5 FROM CELL redSix:NMOS $\{\mathrm{sch}\}$

.SUBCKT NMOS-X_5 d g $\mathrm{s}$

** GLOBAL gnd

MNMOSf@0 d g $s$ gnd nch $\mathrm{W}=\prime 15 *(1+\mathrm{ABN} / \operatorname{sqrt}(15 * 2))^{\prime} \mathrm{L}=\mathbf{\prime}^{\prime}$ ' $^{\prime}$

+ DELVTO= 'AVTON $/ \operatorname{sqrt}(15 * 2)^{\prime}$

.ENDS NMOS-X_5

*** SUBCIRCUIT PMOS-X_5 FROM CELL redSix:PMOS $\{\mathrm{sch}\}$

.SUBCKT PMOS-X_5 d g $\mathrm{s}$

** GLOBAL vdd

MPMOSf@O d g s vdd pch $\mathrm{W}=\prime 30 *(1+\mathrm{ABP} / \operatorname{sqrt}(30 * 2))^{\prime} \mathrm{L}=\mathbf{\prime}^{\prime}$ ' $^{\prime}$

+ DELVTO $={ }^{\prime}$ AVTOP $/ \operatorname{sqrt}(30 * 2)$ '

.ENDS PMOS-X_5

*** SUBCIRCUIT inv-X_5 FROM CELL redSix:inv\{sch $\}$

.SUBCKT inv-X_5 in out

** GLOBAL gnd

** GLOBAL vdd

XNMOS@ 0 out in gnd NMOS-X_5

XPMOS@O out in vdd PMOS-X_5

.ENDS inv-X_5

*** SUBCIRCUIT NMOS-X_2 FROM CELL redSix:NMOS $\{\mathrm{sch}\}$

.SUBCKT NMOS-X_2 d $\mathrm{g} \mathbf{s}$

** GLOBAL gnd

MNMOSfe0 d g s gnd nch $\mathrm{W}={ }^{\prime} 6 *(1+\mathrm{ABN} / \operatorname{sqrt}(6 * 2))^{\prime}$

$+\mathrm{L}={ }^{\prime} 2^{\prime}$ DELVTO='AVTON $/ \operatorname{sqrt}(6 * 2)^{\prime}$

.ENDS NMOS-X_2

*** SUBCIRCUIT PMOS-X_2 FROM CELL redSix:PMOS $\{\mathrm{sch}\}$ .SUBCKT PMOS-X_2 d g $\mathrm{s}$

** GLOBAL vdd

MPMOSf@O d g s vdd pch $W=\prime 12 *(1+A B P / \operatorname{sqrt}(12 * 2))$ '

$+\mathrm{L}={ }^{\prime} 2^{\prime}$ DELVTO='AVTOP/sqrt $(12 * 2)^{\prime}$

.ENDS PMOS-X_2

*** SUBCIRCUIT inv-X_2 FROM CELL redSix:inv\{sch $\}$

.SUBCKT inv-X_2 in out

** GLOBAL gnd

** GLOBAL vdd

XNMOS@O out in gnd NMOS-X_2

XPMOS@O out in vdd PMOS-X_2

.ENDS inv-X_2

*** SUBCIRCUIT NMOS-X_10 FROM CELL redSix:NMOS $\{\mathrm{sch}\}$ 


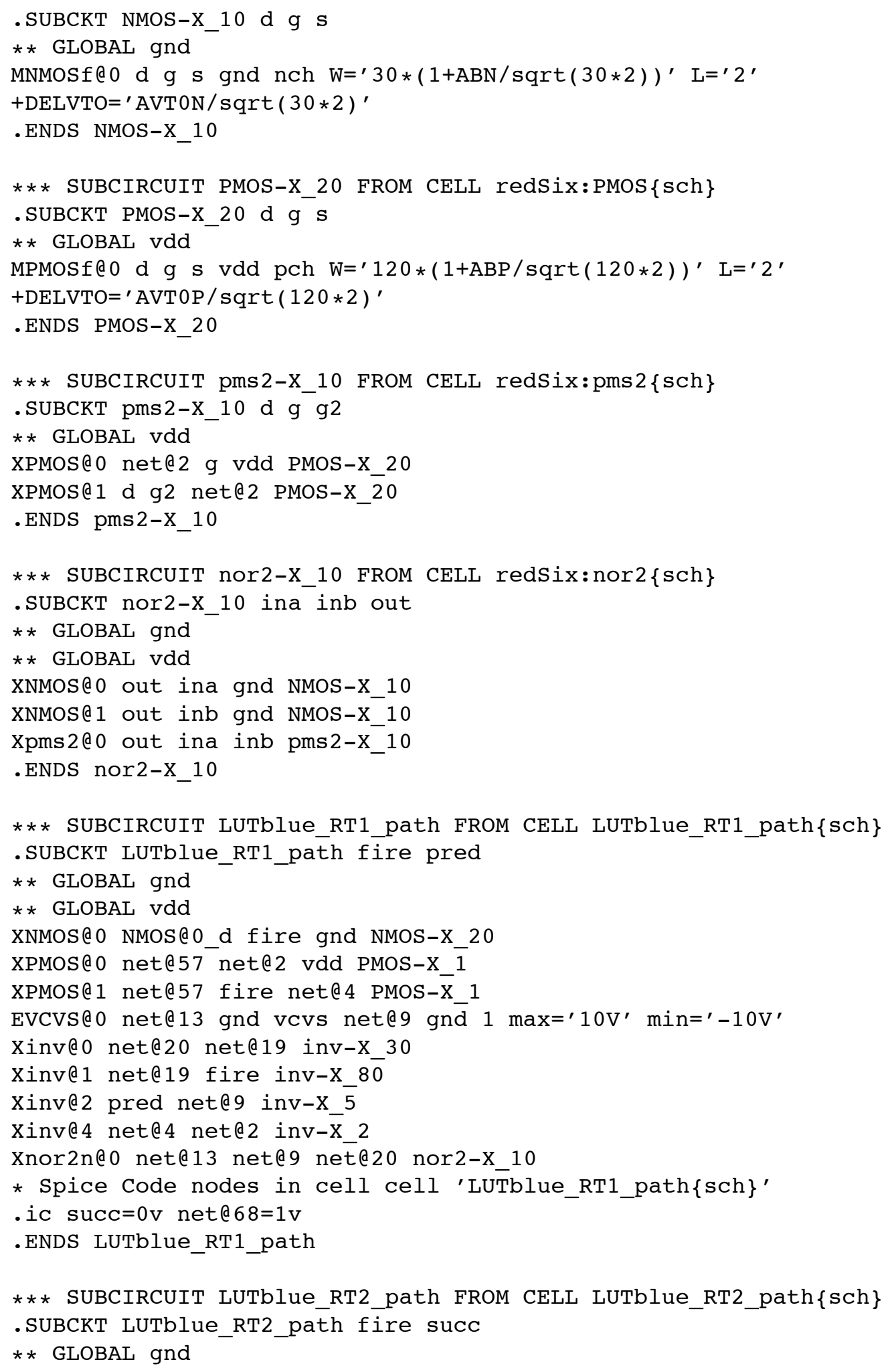




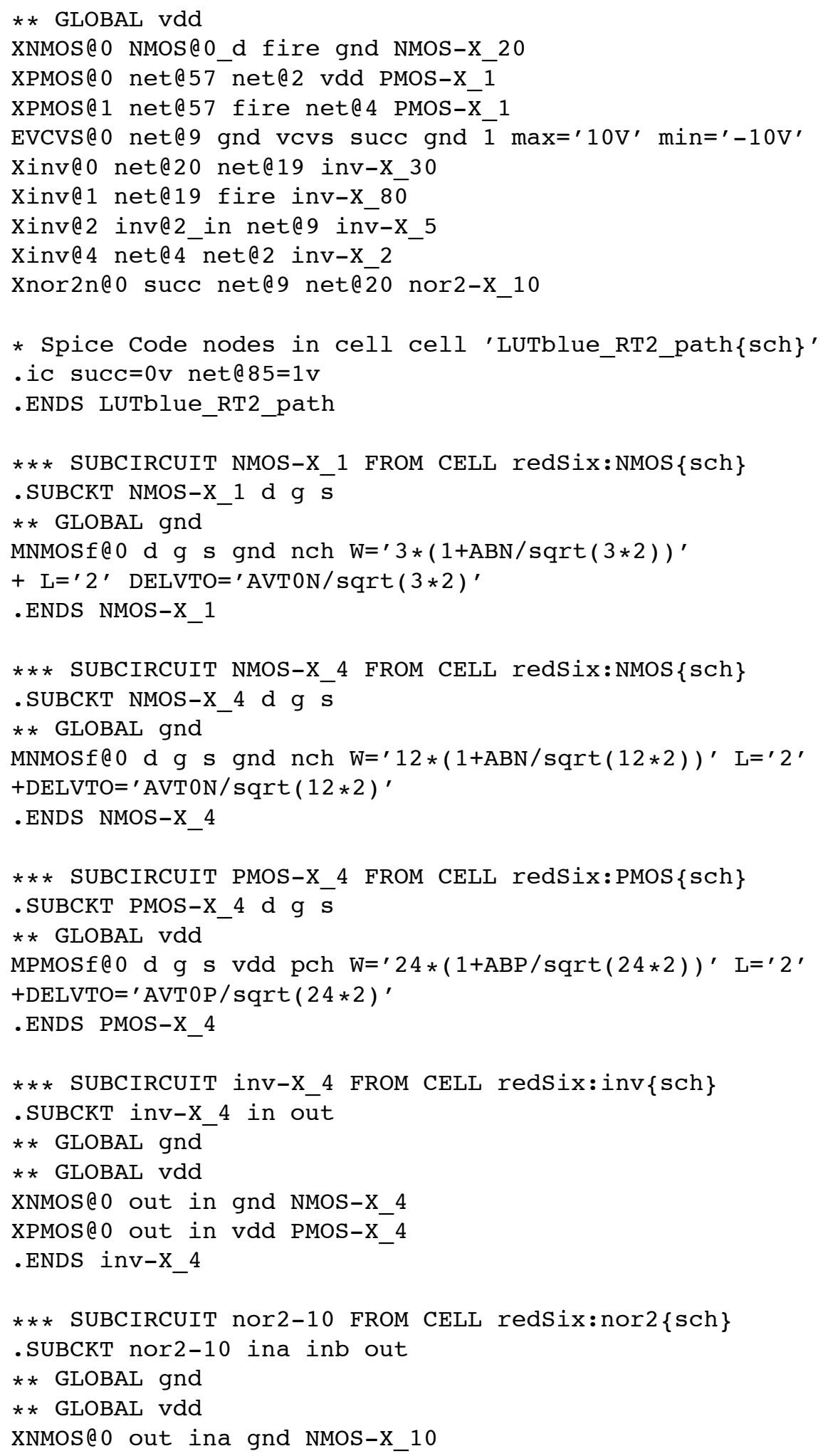


XNMOS@ 1 out inb gnd NMOS-X_10

Xpms2@0 out ina inb pms2-X_10

.ENDS nor2-10

*** SUBCIRCUIT LUTblue_RT3_path FROM CELL LUTblue_RT3_path\{sch\}

.SUBCKT LUTblue_RT3_path fire succ

** GLOBAL gnd

** GLOBAL vdd

VAmmeter@0 succ net@146 DC 0

XNMOS@1 net@64 nete1 gnd NMOS-X_1

XNMOS@2 nete64 net@54 succ NMOS-X_1

XPMOS@2 succ net@54 vdd PMOS-X_20

VPulse@o net@205 gnd pulse ' $0 \mathrm{v}^{\prime}$ ' $1.5 \mathrm{v}$ '

+'30ns' '50ps' '50ps' '3ns' '4ns'

GVCCS@1 net@146 gnd vccs succ net@205 $0.1 \max =1 \min =0$

Xinve0 fire nete54 inv-X_4

Xinv@1 succ net@1 inv-X_z

Xnor2n@0 succ nor2n@0_inb nor2n@0_out nor2-10

* Spice Code nodes in cell cell 'LUTblue_RT3_path\{sch\}'

- ic $\mathrm{succ}=0 \mathrm{v}$

.ENDS LUTblue_RT3_path

*** SUBCIRCUIT LUTblue_RT5_path FROM CELL LUTblue_RT5_path\{sch\} .SUBCKT LUTblue_RT5_path fire pred

** GLOBAL gnd

* * GLOBAL vdd

VAmmeter@0 pred net@70 DC 0

XNMOS@O pred fire gnd NMOS-X_20

XPMOS@0 net@48 net@46 vdd PMOS-X_1

XPMOS@1 net@48 PMOS@1_g pred PMOS-X_1

VPulse@0 net@106 gnd pulse ' $1 \mathrm{v}^{\prime}$ ' $-0.5 \mathrm{v}$ ' '30ns'

+'50ps' '50ps' '3ns' '4ns'

GVCCS@0 net@70 gnd vccs pred net@106 $0.1 \max =0 \mathrm{~min}=-1$

Xinve2 pred inve2_out inv-X_5

Xinve5 pred net@ $4 \overline{6}$ inv-X_2

* Spice Code nodes in cell cell 'LUTblue_RT5_path\{sch\}'

- ic net@83=1v

.ENDS LUTblue_RT5_path

*** SUBCIRCUIT NMOS-X_widd4 FROM CELL redSix:NMOS $\{$ sch $\}$

.SUBCKT NMOS-X_widd4 d g s

* * GLOBAL gnd

MNMOSf@0 d g s gnd nch $\mathrm{W}=' 3 * \mathrm{widd} 4 *(1+\mathrm{ABN} / \operatorname{sqrt}(3 * \mathrm{Widd} 4 * 2))$ '

$+\mathrm{L}={ }^{\prime} 2^{\prime}$ DELVTO='AVTON/sqrt $(3 *$ widd $4 * 2)$ '

. ENDS NMOS-X_widd4

*** SUBCIRCUIT PMOS-X_widd4 FROM CELL redSix:PMOS $\{$ sch $\}$ 


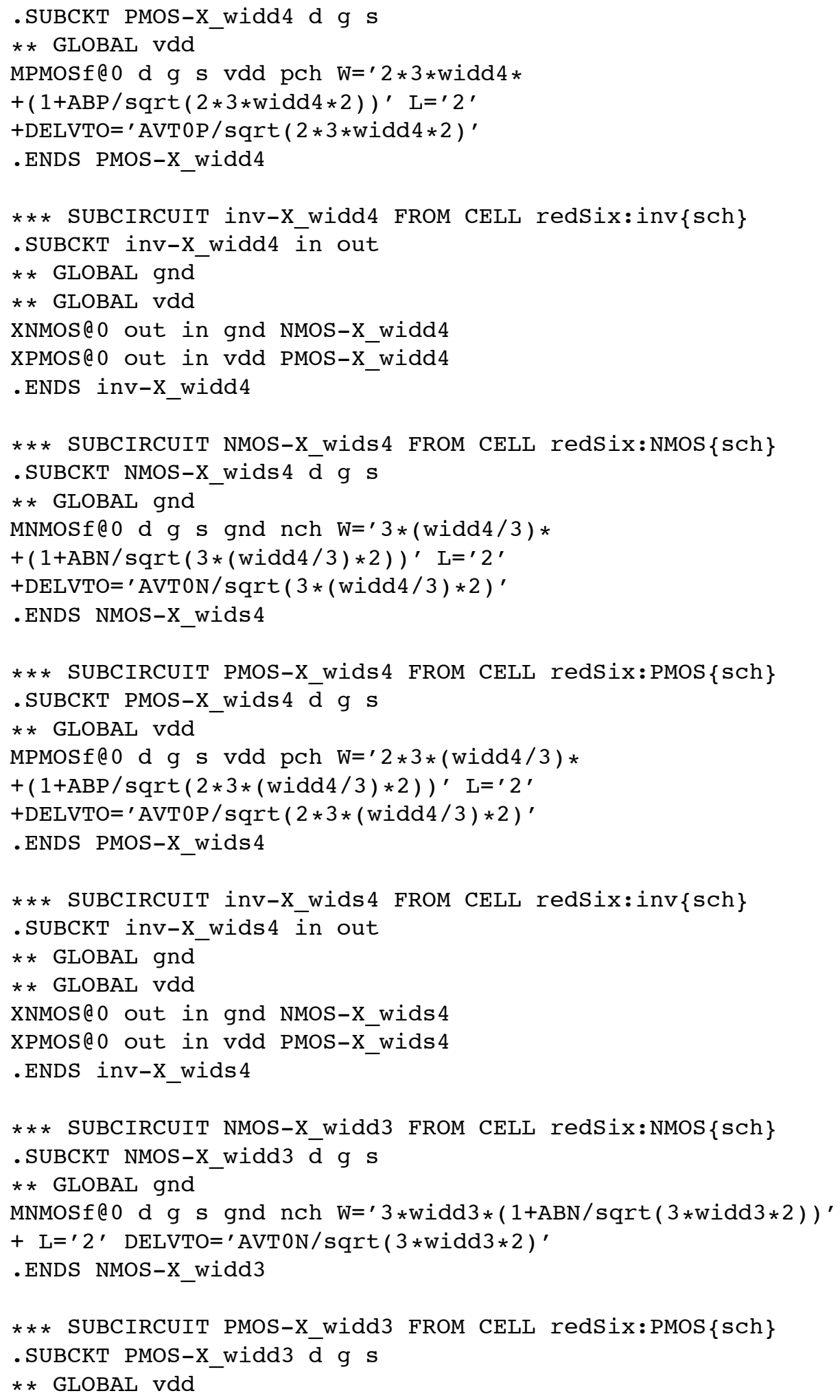


MPMOSf@0 d g $\mathrm{s}$ vdd pch $\mathrm{W}=' 2 * 3 *$ Widd $3 *(1+\mathrm{ABP} / \operatorname{sqrt}(2 * 3 * \mathrm{Widd} 3 * 2))^{\prime}$

$+\mathrm{L}={ }^{\prime} 2^{\prime} \mathrm{DELVTO}={ }^{\prime} \mathrm{AVTOP} / \operatorname{sqrt}(2 * 3 * \mathrm{Widd} 3 * 2)^{\prime}$

.ENDS PMOS-X_widd3

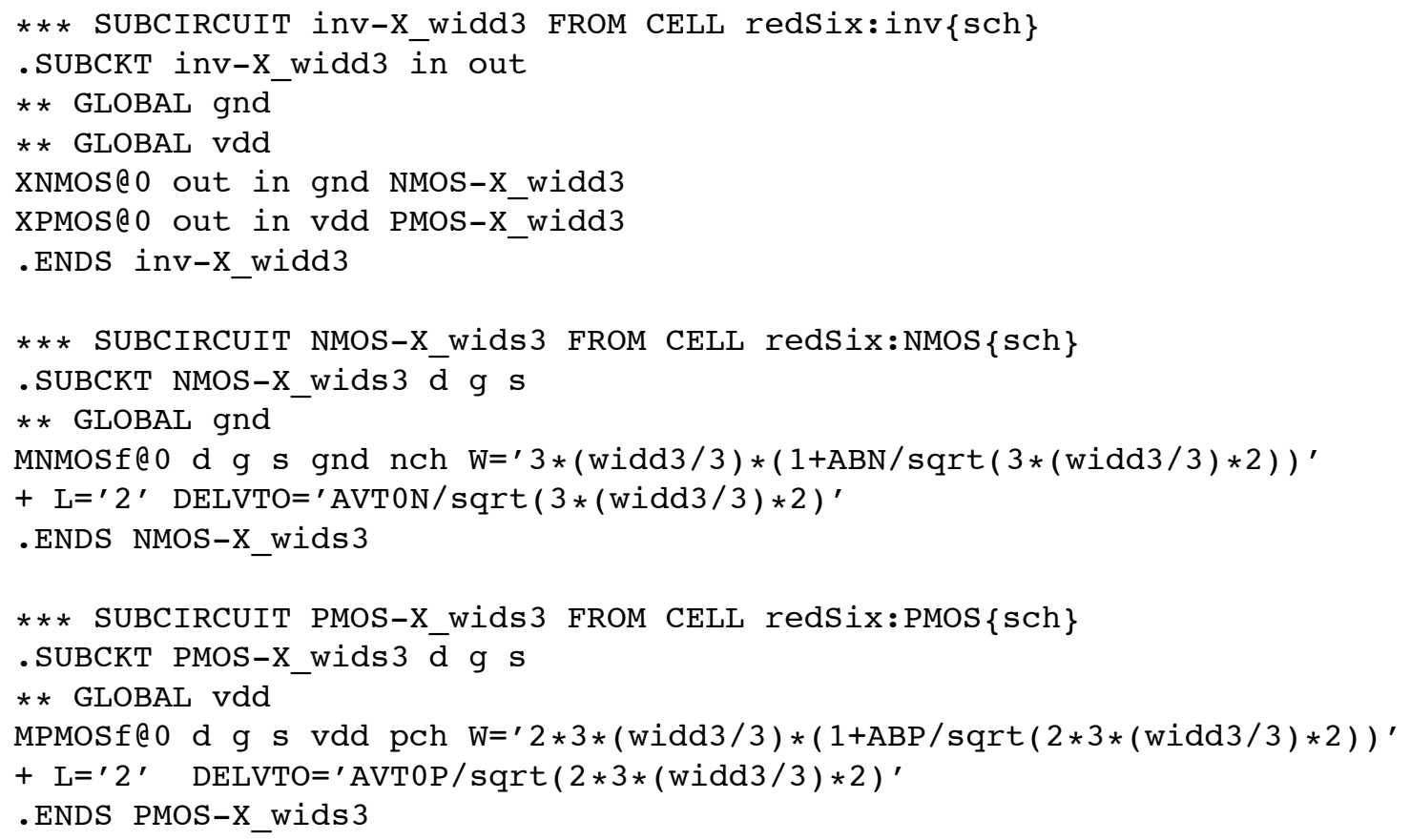




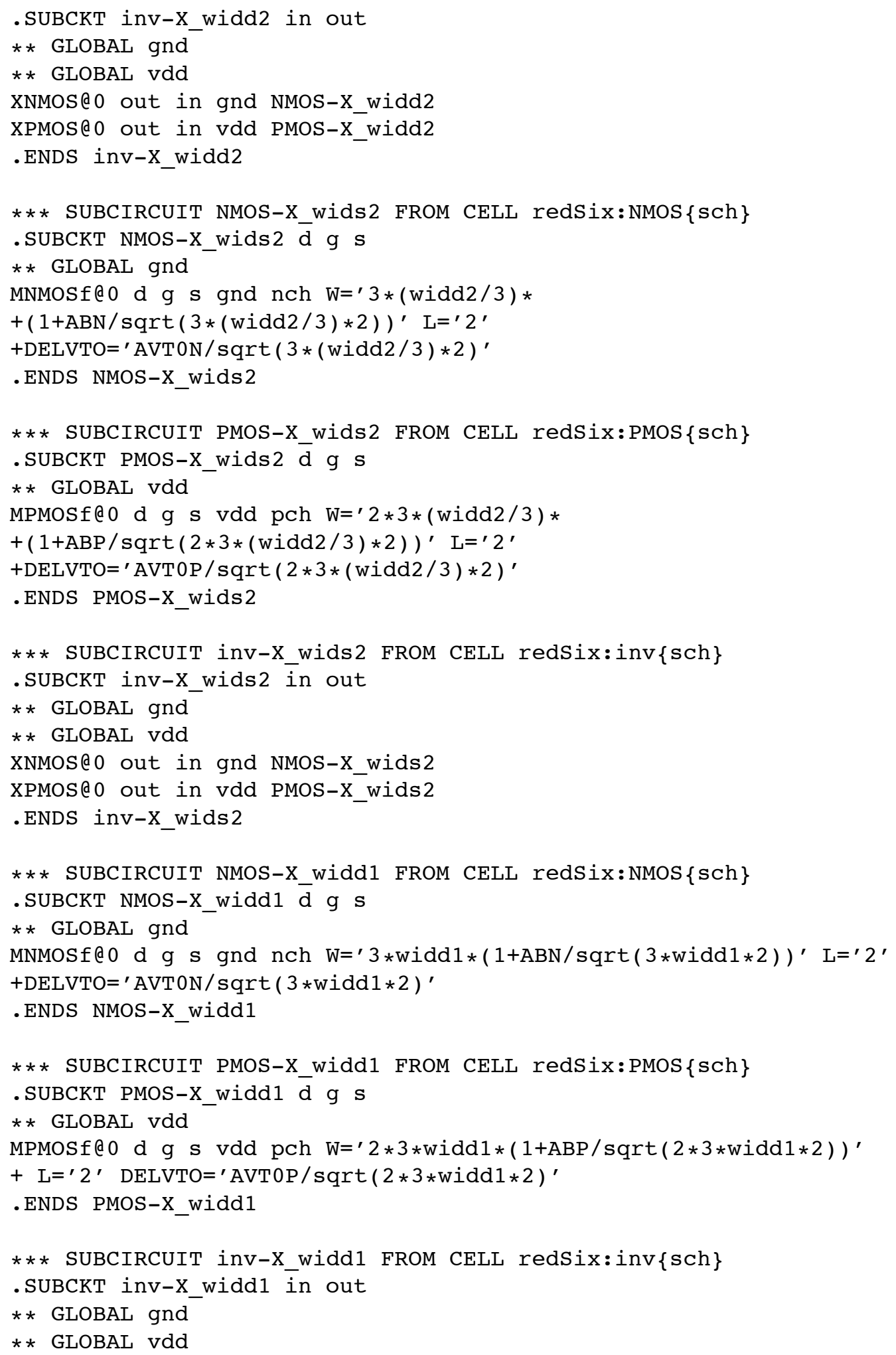


XNMOS@ 0 out in gnd NMOS-X_widd 1

XPMOS@ 0 out in vdd PMOS-X_widd 1

.ENDS inv-X_widd 1

*** SUBCIRCUIT NMOS-X_wids 1 FROM CELL redSix:NMOS $\{\mathrm{sch}\}$

.SUBCKT NMOS-X_wids 1 d g $s$

** GLOBAL gnd

MNMOSf@0 d g s gnd nch $\mathrm{W}={ }^{\prime} 3 *(\operatorname{widd} 1 / 3) *(1+\mathrm{ABN} / \operatorname{sqrt}(3 *(\operatorname{widd} 1 / 3) * 2))^{\prime}$

$+\mathrm{L}={ }^{\prime} 2^{\prime}$ DELVTO='AVTON $/ \operatorname{sqrt}(3 *(\operatorname{widd} 1 / 3) * 2)^{\prime}$

.ENDS NMOS-X_wids 1

*** SUBCIRCUIT PMOS-X_wids1 FROM CELL redSix:PMOS $\{\mathrm{sch}\}$

.SUBCKT PMOS-X_wids 1 d g s

** GLOBAL vdd

MPMOSf@0 d g s vdd pch $W=\prime 2 * 3 *(\operatorname{widd} 1 / 3) *(1+\mathrm{ABP} / \operatorname{sqrt}(2 * 3 *(\operatorname{widd} 1 / 3) * 2))^{\prime}$

$+\mathrm{L}={ }^{\prime} 2^{\prime}$ DELVTO='AVTOP $/ \operatorname{sqrt}(2 * 3 *(\text { widd } 1 / 3) * 2)^{\prime}$

.ENDS PMOS-X_wids 1

*** SUBCIRCUIT inv-X_wids1 FROM CELL redSix:inv\{sch\}

.SUBCKT inv-X_wids 1 in out

** GLOBAL gnd

** GLOBAL vdd

XNMOS@ 0 out in gnd NMOS-X_wids 1

XPMOS@ 0 out in vdd PMOS-X_wids 1

.ENDS inv-X_wids 1

.global gnd vdd

*** TOP LEVEL CELL: LUT_Noload_figure22 $\{\mathrm{sch}\}$

XLUTblue_@0 out1 in1 LUTblue_RT1_path

XLUTblue_@1 out2 in2 LUTblue_RT2_path

XLUTblue_@4 in3 out3 LUTblue_RT3_path

XLUTblue_@ 5 in 4 out4 LUTblue_RT5_path

VPulse 1 net 140 gnd pulse 0 ' $1 \mathrm{v}^{\prime}$ ' $30.5 \mathrm{~ns}^{\prime}$ '50ps' '50ps' '2ns' '4ns'

VPulsed 2 net 141 gnd pulse 0 ' $1 \mathrm{v}$ ' '30.5ns' '50ps' '50ps' '2ns' '4ns'

VPulse@ 3 net@142 gnd pulse 0 '1v' '0ns' '50ps' '50ps' '2ns' '4ns'

VPulse@ 4 net@143 gnd pulse 0 '1v' '0ns' '50ps' '50ps' '2ns' '4ns'

Xinv@ 5 Din4 in4 inv-X_widd4

Xinve6 net@140 Din4 inv-X_wids4

Xinve9 Din3 in3 inv-X_widd3

Xinve10 net@141 Din3 inv-X_wids3

Xinve13 Din2 in2 inv-X_widd2

Xinv@14 net@142 Din2 inv-X_wids2

Xinve17 Din1 in1 inv-X_widd1

Xinv@18 net@143 Din1 inv-X_wids1

* Trailer cards are described in this file:

.include '/u/mettalag/models/Models_90nm/NOLOAD/MOSIS_trailer_nl.hsp' .END 


\section{H.2 SPICE Simulation Setup File: HEADER}

Here is the basic file with default simulation parameters for SPICE. Its extension is '.hsp'. It contains a pointer to the 90nm CMOS process models for our GasP designs. We use the library with typical (TT) Process, Voltage and Temperature (PVT) settings.

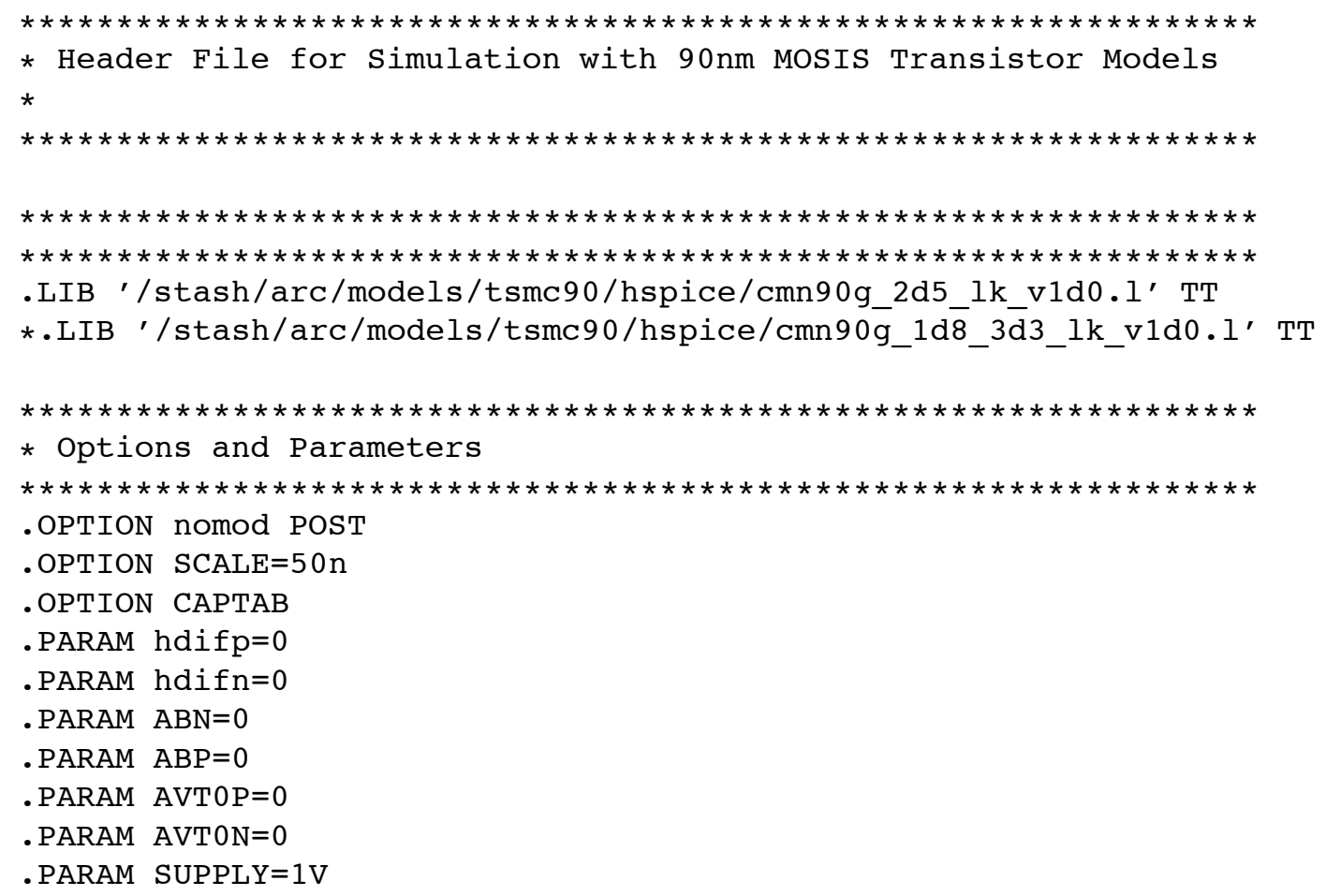




\section{H.3 SPICE Simulation Setup File: TRAILER}

Her is the SPICE trailer file with the .DATA sweep, transient analysis, and measurement details for the simulation environment in Figure 4.22 on page 84. It generates look up tables for four relative timing paths, by sweeping the driver sizes widd1, widd2, widd 3 and widd4. It times and measures the voltage levels on in 1 to in 4 and out 1 to out 4.

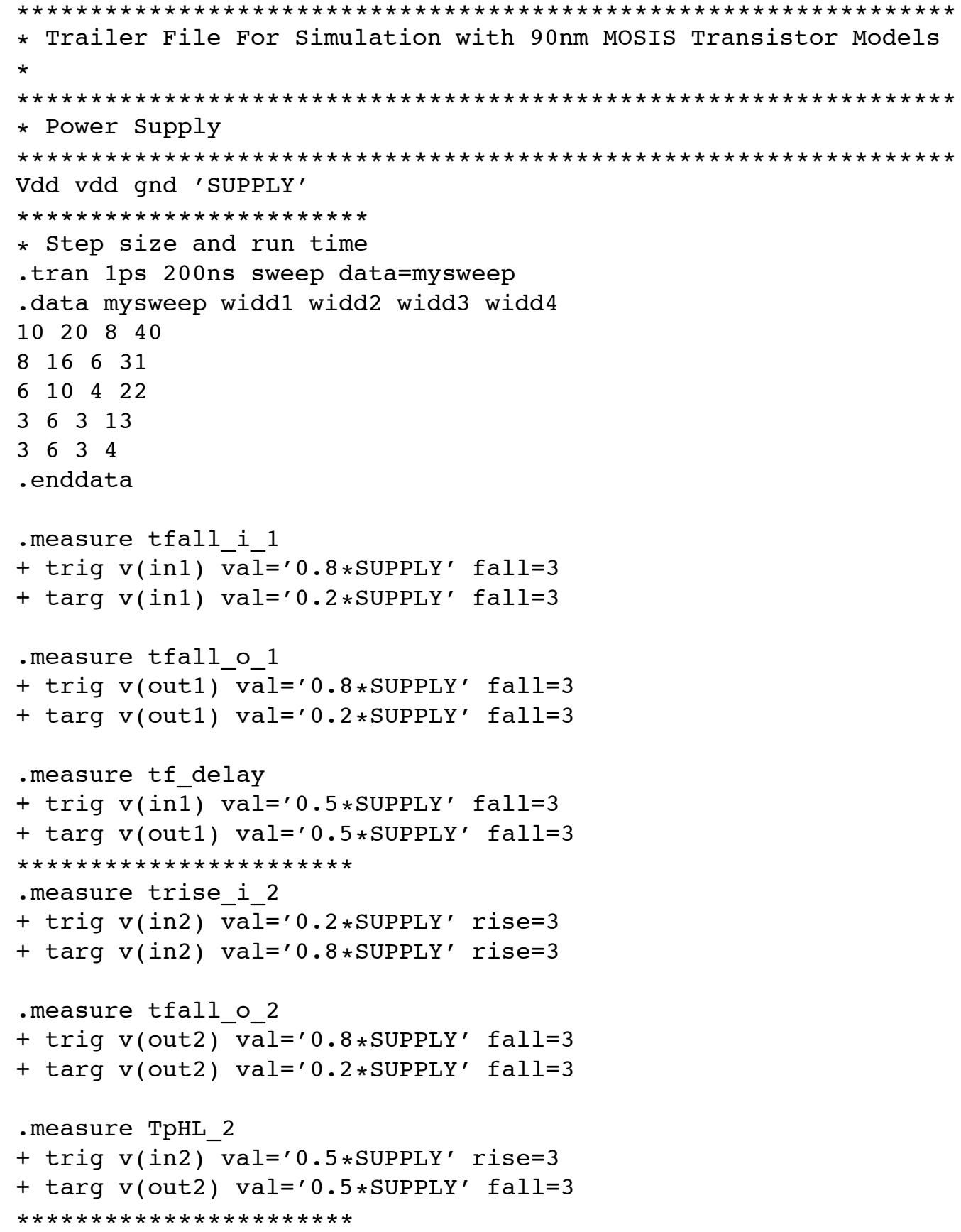




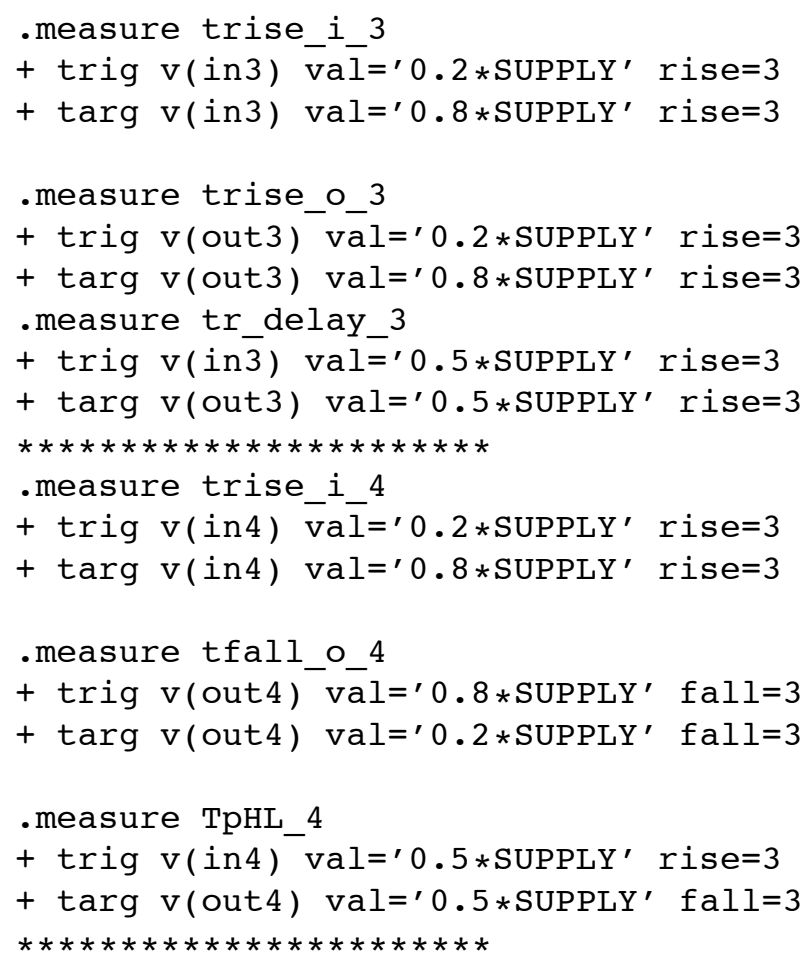




\section{H.4 SPICE Output File}

Below follows the SPICE output file with the look up table results for the four simple relative timing paths LUTblue:PRED ${ }_{i}-\mathrm{FIRE}_{i}-$, LUTblue:SUCC $i+\mathrm{FIRE}_{i}-$, LUTred/blue:FIRE ${ }_{i}+\mathrm{SUCC}_{i}+$, and LUTblue/green:FIRE $i+\mathrm{PRED}_{i}-$, simulated with zero external output load. The results were generated with the simulation environment in Figure 4.22 on page 84. The output file extension is '. $m t 0$ '. Per sweep, the file shows the sizes of sweep variable widd1, widd2, widd 3 and widd 4 , and the measured timing information in seconds. We measure:

For LUTblue:PRED ${ }_{i}-\mathrm{FIRE}_{i}-$ :

- tfall_i_1, the transition fall time, a.k.a. slope or slew time, on input in1

- tfall_o_1, the transition fall time, a.k.a. slope or slew time, on output out1

- tf_delay, the input-to-output delay for the falling transition from in 1 to out 1

For LUTblue:SUCC ${ }_{i}+\mathrm{FIRE}_{i}-$ :

- trise_i_2, the transition rise time, a.k.a. slope or slew time, on input in2

- tfall_o_2, the transition fall time, a.k.a. slope or slew time, on output out2

- tphl_2, input-to-output delay for the high to low transition from in2 to out2

For LUTred/blue:FIRE $i+\mathrm{SUCC}_{i}+$ :

- trise_i_3, the transition rise time, a.k.a. slope or slew time, on input in3

- trise_o_3, the transition rise time, a.k.a. slope or slew time, on output out3

- tr_delay_3, input-to-output delay for the rising transition from in 3 to out3

For LUTblue/green:FIRE ${ }_{i}+\mathrm{PRED}_{i}-$ :

- trise_i_4, the transition rise time, a.k.a. slope or slew time, on input in4

- tfall_o_4, the transition fall time, a.k.a. slope or slew time, on output out4

- tphl_4, the input-to-output delay for the high to low transition from in4 to out4

Note: the additional process parameters temper and alter\# can be ignored.

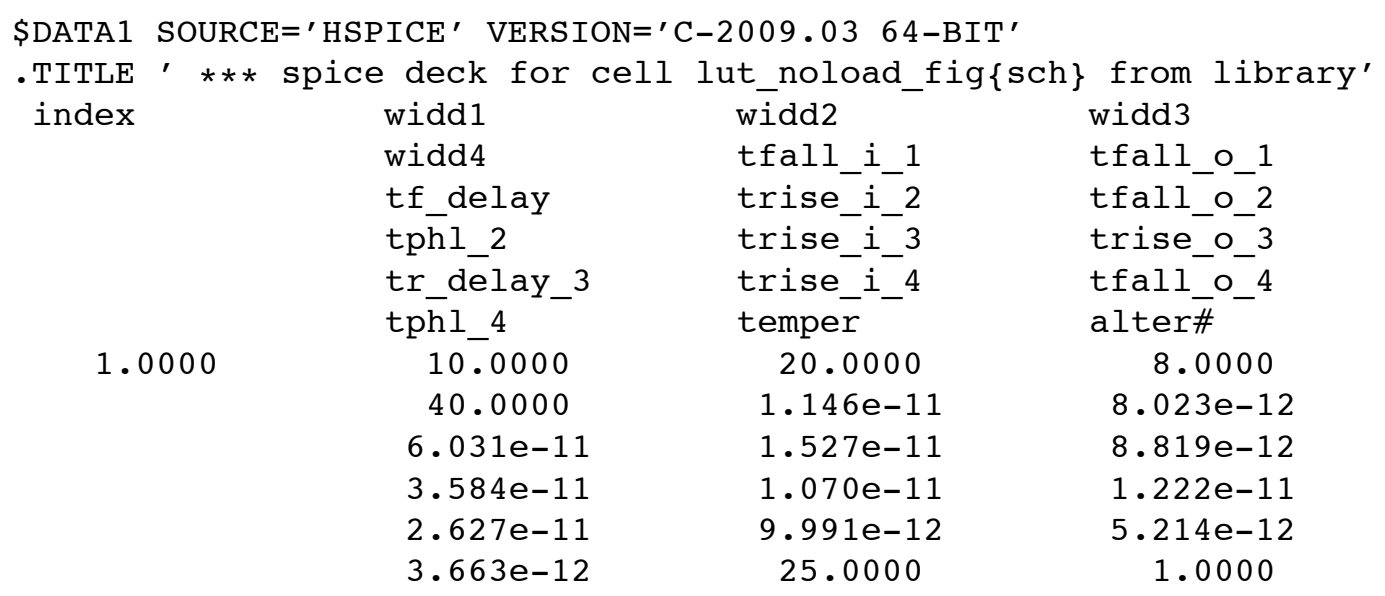




$\begin{array}{cccc}2.0000 & 8.0000 & 16.0000 & 6.0000 \\ 31.0000 & 1.236 \mathrm{e}-11 & 8.491 \mathrm{e}-12 \\ 6.081 \mathrm{e}-11 & 1.766 \mathrm{e}-11 & 8.597 \mathrm{e}-12 \\ 3.627 \mathrm{e}-11 & 1.223 \mathrm{e}-11 & 1.245 \mathrm{e}-11 \\ 2.673 \mathrm{e}-11 & 1.159 \mathrm{e}-11 & 5.448 \mathrm{e}-12 \\ 3.634 \mathrm{e}-12 & 25.0000 & 1.0000 \\ 3.0000 & 6.0000 & 10.0000 & 4.0000 \\ 22.0000 & 1.313 \mathrm{e}-11 & 8.300 \mathrm{e}-12 \\ & 6.113 \mathrm{e}-11 & 1.585 \mathrm{e}-11 & 8.464 \mathrm{e}-12 \\ & 3.739 \mathrm{e}-11 & 1.444 \mathrm{e}-11 & 1.271 \mathrm{e}-11 \\ & 2.761 \mathrm{e}-11 & 1.233 \mathrm{e}-11 & 5.582 \mathrm{e}-12 \\ & 3.696 \mathrm{e}-12 & 25.0000 & 1.0000 \\ 4.0000 & 3.0000 & 6.0000 & 3.0000 \\ & 13.0000 & 1.785 \mathrm{e}-11 & 8.381 \mathrm{e}-12 \\ & 6.319 \mathrm{e}-11 & 2.167 \mathrm{e}-11 & 8.275 \mathrm{e}-12 \\ & 3.918 \mathrm{e}-11 & 1.661 \mathrm{e}-11 & 1.294 \mathrm{e}-11 \\ & 2.852 \mathrm{e}-11 & 1.222 \mathrm{e}-11 & 5.982 \mathrm{e}-12 \\ & 3.380 \mathrm{e}-12 & 25.0000 & 1.0000 \\ 5.0000 & 3.0000 & 6.0000 & 3.0000 \\ & 4.0000 & 1.785 \mathrm{e}-11 & 8.381 \mathrm{e}-12 \\ & 6.319 \mathrm{e}-11 & 2.167 \mathrm{e}-11 & 8.275 \mathrm{e}-12 \\ & 3.918 \mathrm{e}-11 & 1.692 \mathrm{e}-11 & 1.251 \mathrm{e}-11 \\ & 2.846 \mathrm{e}-11 & 2.167 \mathrm{e}-11 & 8.482 \mathrm{e}-12 \\ & 3.760 \mathrm{e}-12 & 25.0000 & 1.0000\end{array}$




\section{Appendix I}

\section{TRANSLATING LOAD SIZE INTO LOAD CAPACITANCE}

The four appendices below contain the SPICE input and output files for translating the size of a load inverter into the capacitive load of that inverter. This translation is part of STEP 1, Section 5.2. The dual or nested sweep optimization process that we need for this translation differs from the sweeps used in Chapters 3-4. The differences show up in the SPICE simulation files. The organization of Appendix I is as follows:

- Appendix I.1 contains the SPICE netlist.

- Appendix I.2 contains the SPICE simulation HEADER file.

- Appendix I.2 contains the SPICE simulation TRAILER file.

- Appendix I.4 contains the SPICE output file with the translation information.

\section{I.1 SPICE Netlist}

Below follows the SPICE netlist for the simulation setup in Figure 5.1, page 95.

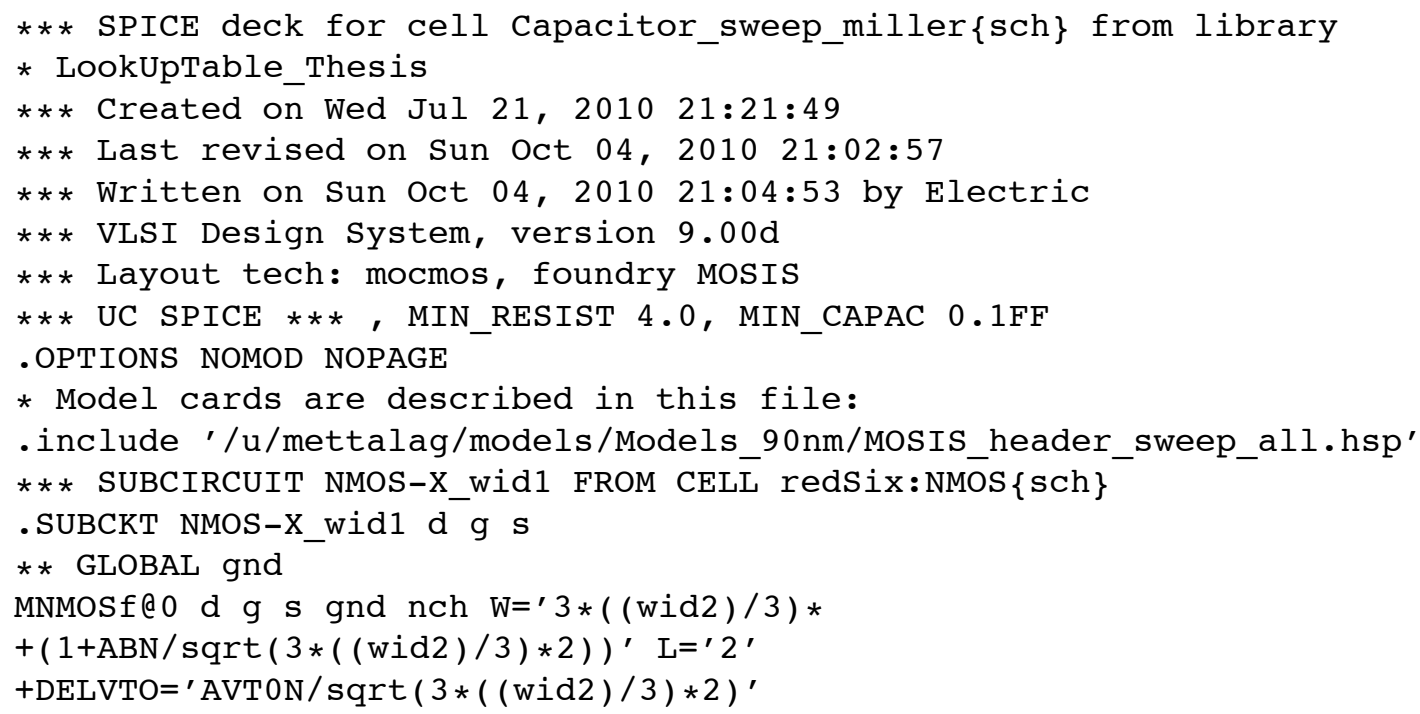


.ENDS NMOS-X_wid1

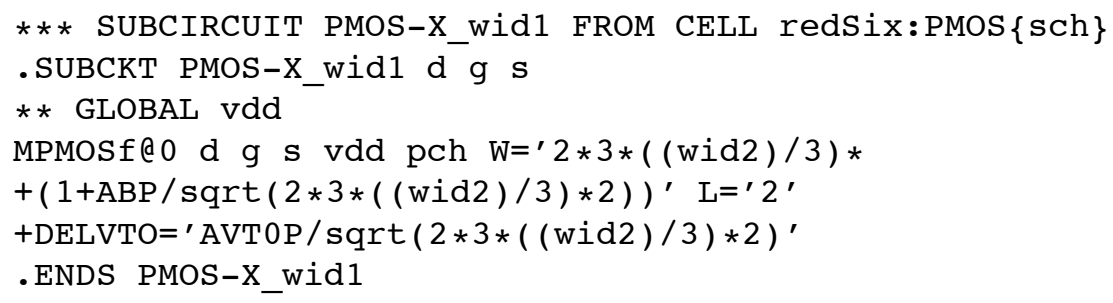




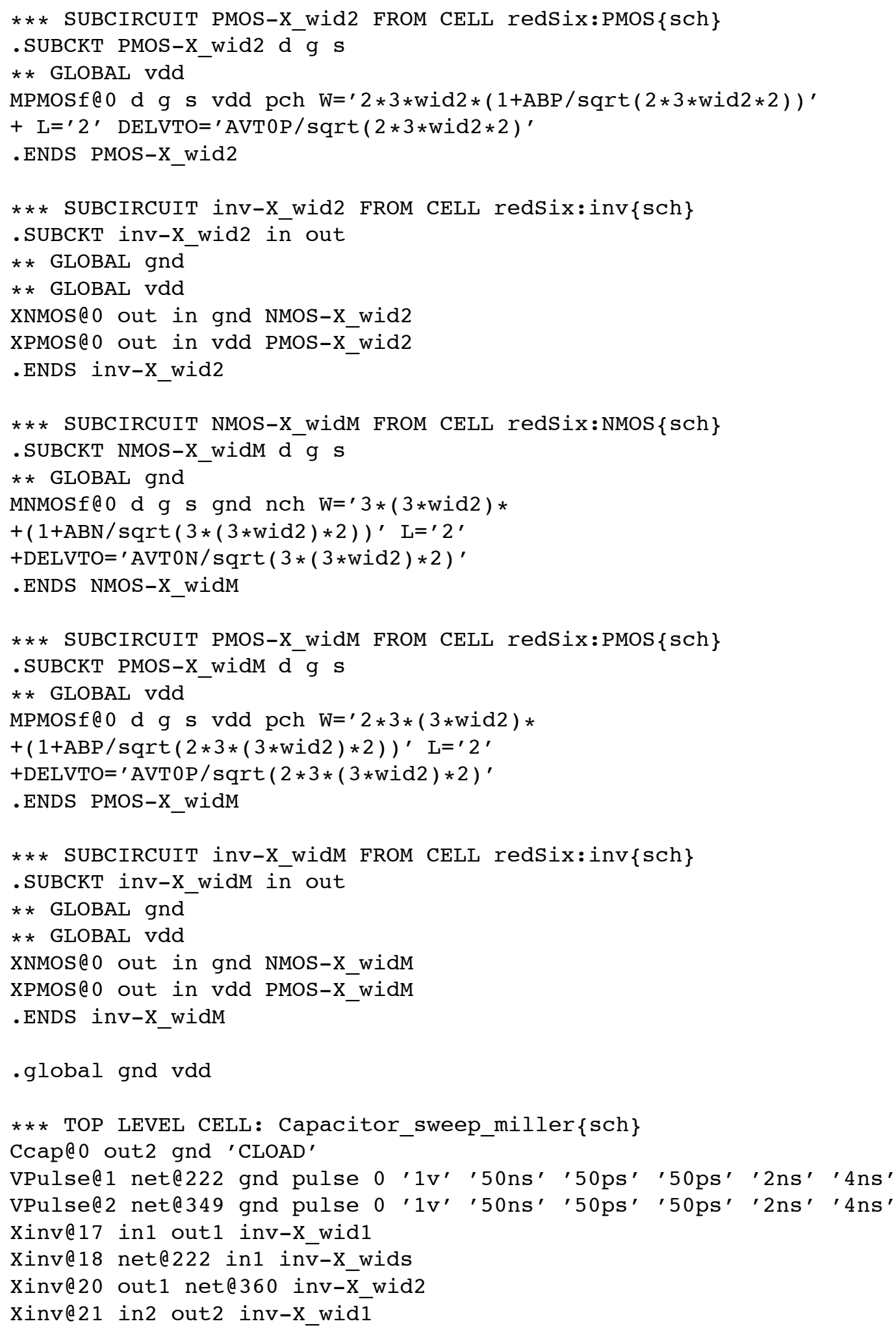


Xinve22 nete349 in2 inv-X wids

Xinv@23 net@ 360 outM inv- $\bar{x}$ widM

* Trailer cards are described in this file:

. include '/u/mettalag/models/Models_90nm/MosIS_trailer_Varcap.hsp'

. END 


\section{I.2 SPICE Simulation File: HEADER}

Here is the basic file with default simulation parameters for SPICE. Its extension is '.hsp'. It contains a pointer to the $90 \mathrm{~nm}$ CMOS process models for our GasP designs. We use the library with typical (TT) Process, Voltage and Temperature (PVT) settings. The key difference between this header and the one in Chapters 3-4 lies in the lines:

.PARAM CLOAD=OPTC $(1 \mathrm{fF}, 1 \mathrm{fF}, 1000 \mathrm{fF})$

-model OPT1 opt

The .PARAM and .MODEL commands here indicate a search for an optimal value for CLOAD, from $1 \mathrm{fF}$ to $1000 \mathrm{fF}$, starting at $1 \mathrm{fF}$. The default step size delta, which has been left out here, is $1 \mathrm{fF}$. The optimization goal is specified in the simulation trailer file in Appendix I.3. The .PARAM and .MODEL commands are explained in the HSPICE reference manual in [34], Chapter 2: HSPICE and HSPICE RF Netlist Commands, pages 218-221 respectively 198-204.

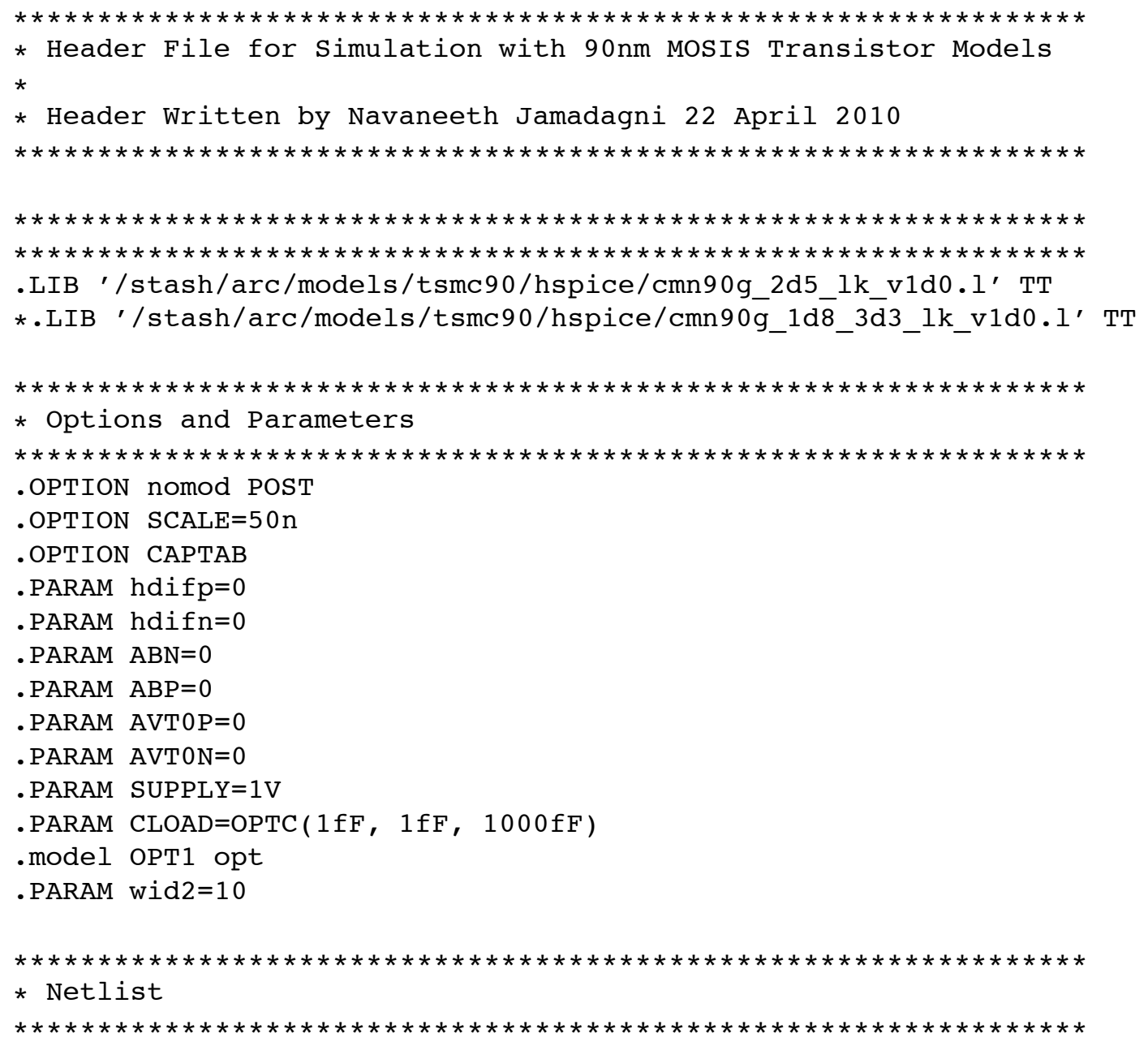




\section{I.3 SPICE Simulation File: TRAILER}

Here is the trailer file with a goal-directed optimization for load capacitance CLOAD in Figure 5.1, page 95. The value for CLOAD increases from $1 \mathrm{fF}$ up to $1000 \mathrm{fF}$ until the average delay tdavgc $=(\mathrm{tdrc}+\mathrm{tdfc}) / 2$ over the driver inverter driving CLOAD matches the average delay tdavg $=(\mathrm{tdr}+\mathrm{tdf}) / 2$ over the driver driving a wid2-size Load inverter.

The sweeps in this case are not encoded as .DATA sweep commands as in Chapters 3-4 but they are encoded as a series of separate .ALTER statements. Each .ALTER statement starts with a new wid2 sweep value, as indicated, and it performs a new goal-oriented transient analysis with fresh measurements for the given wid2 value. It repeats the transient analysis and measurements by sweeping the value of CLOAD until tdavgc=tdavg or until the difference between the two average delays is at its smallest.

For more details on .ALTER, .TRAN, and goal-oriented .MEASURE commands, see [34], Chapter 2: HSPICE and HSPICE RF Netlist Commands, pages 29-30, and 288-294, and 162-165 respectively.

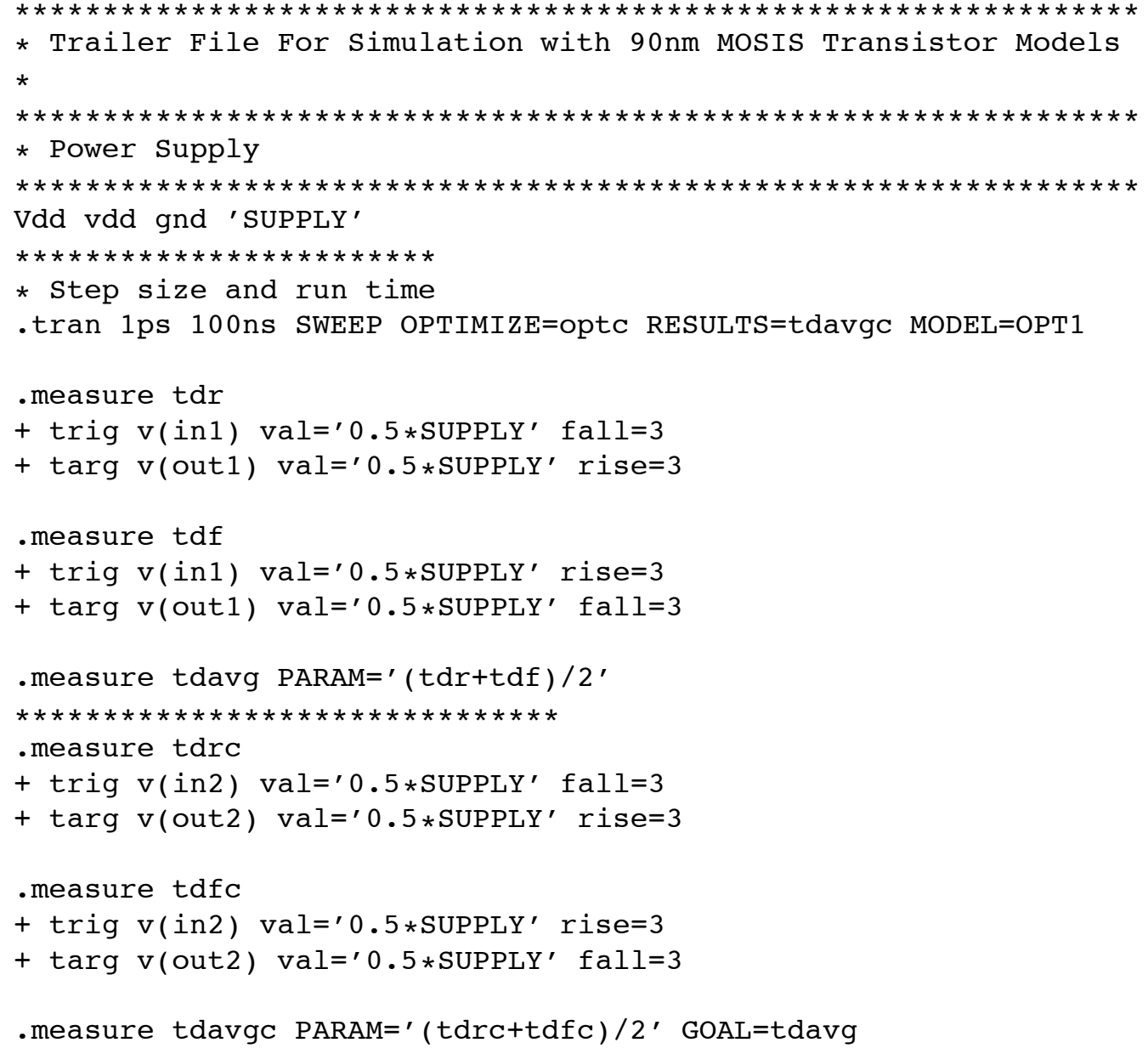




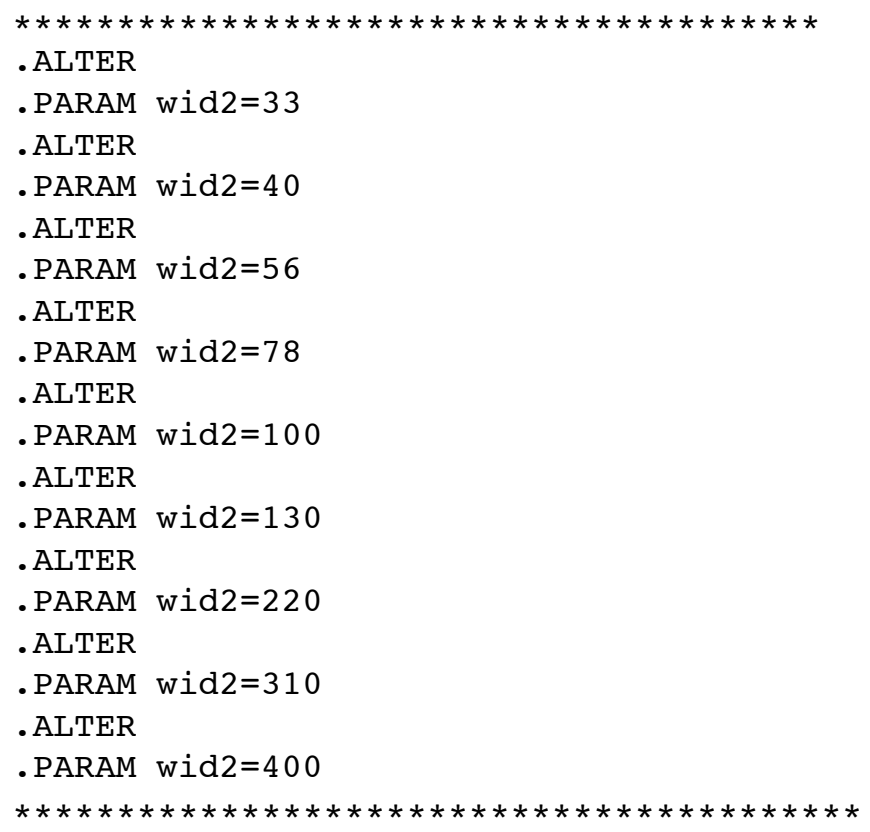




\section{I.4 SPICE Output File}

Here is the output file with the translation from a wid2 load size to a CLOAD load capacitance, as measured for the simulation setup in Figure 5.1, page 95. SPICE reports:

- wid2, the size of the LOAD inverter in the top schematics of Figure 5.1.

- cload, the capacitive load for CLOAD in the bottom schematics of Figure 5.1.

- tdr, the delay from in1 to out1 in Figure 5.1(top) for a rising transition on out1.

- tdf, the delay from in1 to out1 in Figure 5.1(top) for a falling transition on out1.

- tdavg, the average of tdr and tdf, i.e. (tdr $+\operatorname{tdf}) / 2$.

- tdrc, the delay from in2 to out2 in Figure 5.1(bottom) for a rising out2 transition.

- tdfc, the delay from in2 to out2 in Figure 5.1(bottom) for a falling out2 transition.

- tdavgc, the average of tdrc and tdfc, i.e. $(\operatorname{tdrc}+\operatorname{tdfc}) / 2$.

Note: the additional process parameters temper and alter\# can be ignored.

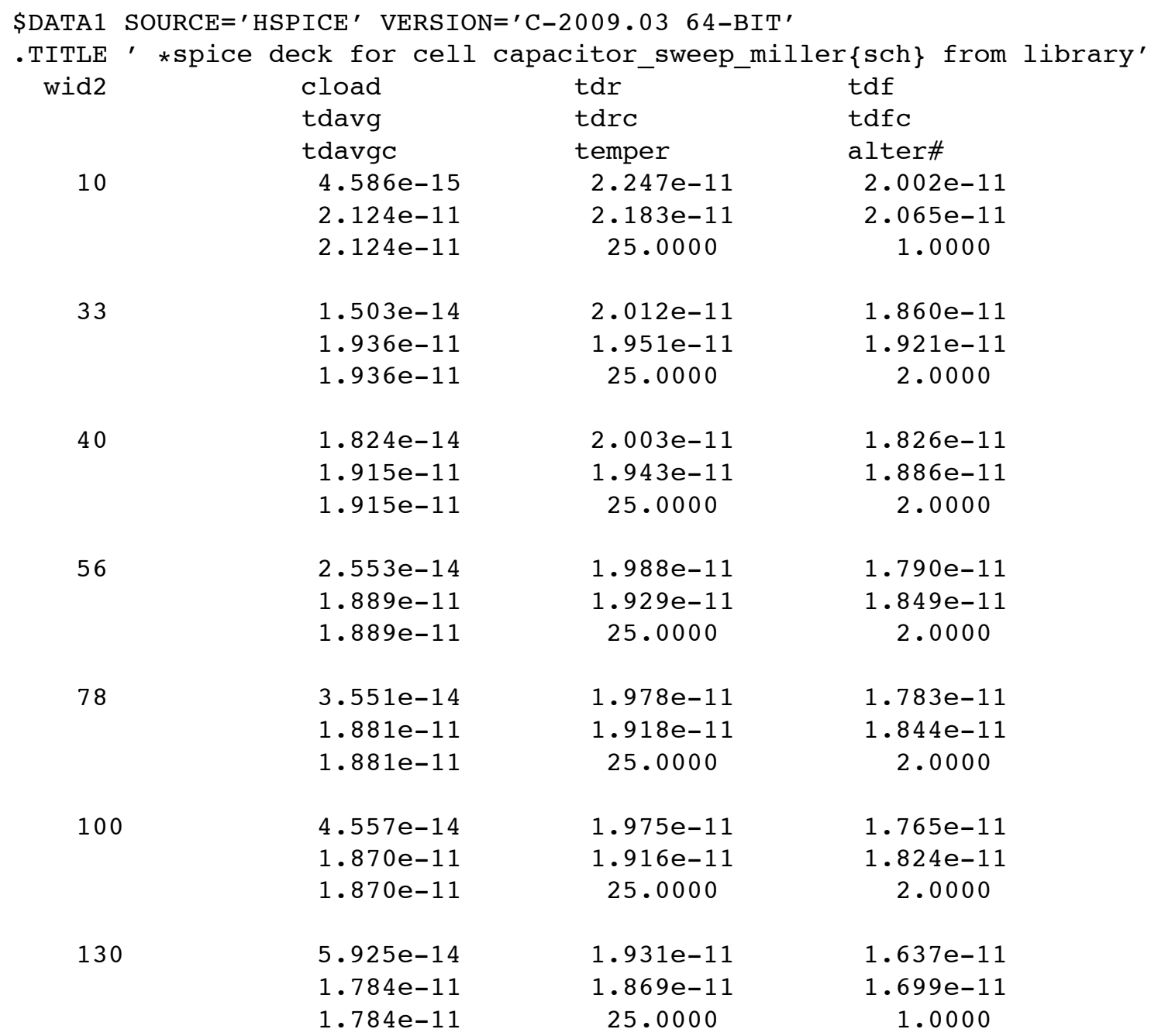


220

310

400
$1.014 \mathrm{e}-13$

$1.834 \mathrm{e}-11$

$1.834 \mathrm{e}-11$

$1.428 \mathrm{e}-13$

$1.772 \mathrm{e}-11$

$1.772 \mathrm{e}-11$

1. $851 e-13$

$1.795 \mathrm{e}-11$

$1.795 \mathrm{e}-11$
$1.918 e-11$

$1.853 \mathrm{e}-11$

25.0000

$1.865 \mathrm{e}-11$

$1.798 \mathrm{e}-11$

25.0000

$1.915 \mathrm{e}-11$

$1.848 \mathrm{e}-11$

25.0000
$1.751 \mathrm{e}-11$

$1.815 \mathrm{e}-11$

1.0000

$1.679 \mathrm{e}-11$

$1.746 \mathrm{e}-11$

1.0000

1. $675 e-11$

$1.741 \mathrm{e}-11$

1.0000 


\section{Appendix J}

\section{PRIMETIME NETLIST FOR THE TWO-STAGE GASP FIFO}

Verilog netlist file for the two-stage FIFO in Figure 5.11, page 106, where each 6-4 GasP module is replaced by the black box model shown in Figure 5.10, page 105. Only the pins of the black box are visible, the internal circuitry is invisible. File extension is '. $v$ '. File name is GASP_FIFO2.v.

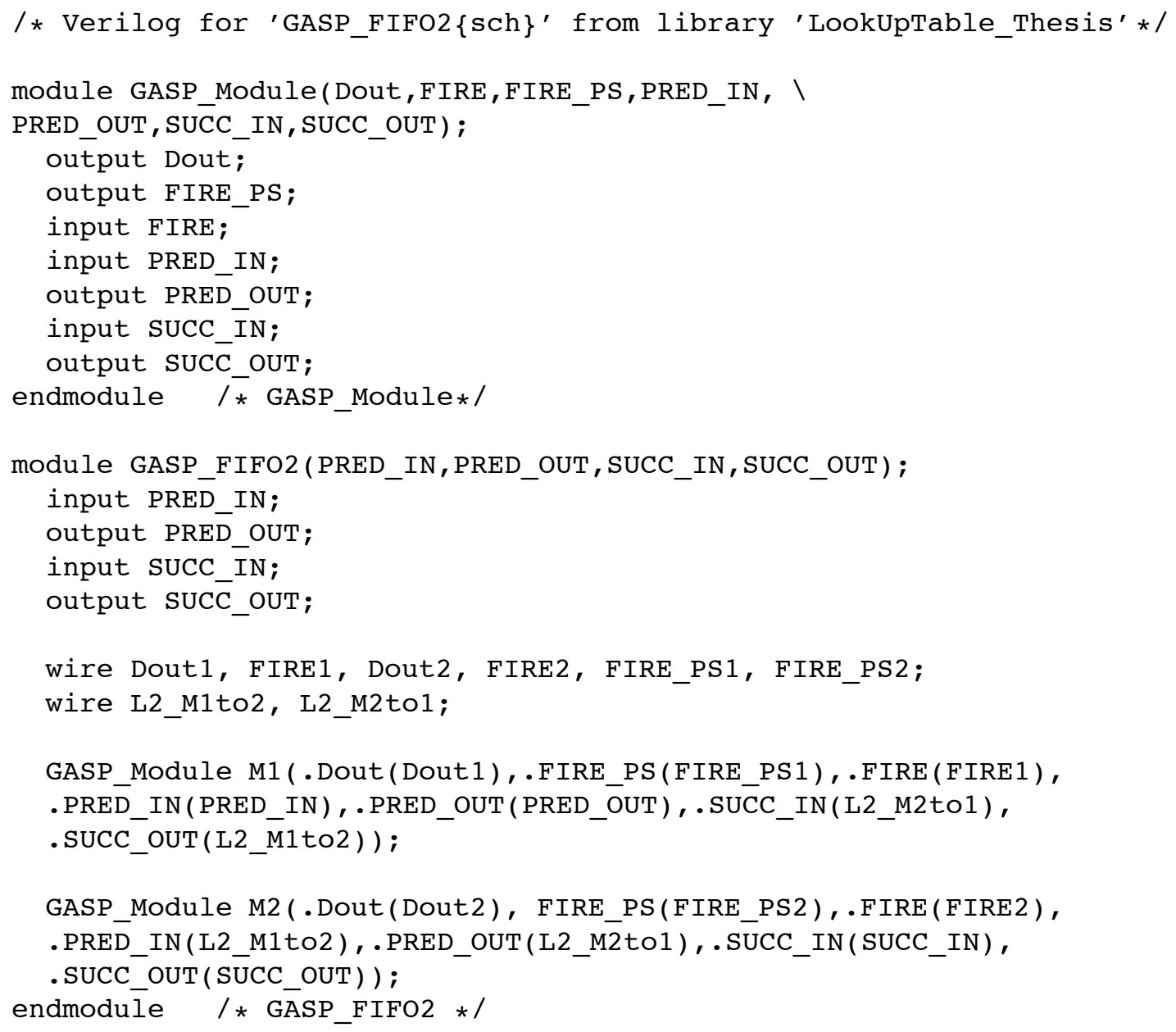




\section{Appendix K}

\section{PRIMETIME LIBRARY WITH SPICED LOOK UP TABLES}

The library file below is named ARC_typical_GasP.lib. It follows the Liberty file format that PrimeTime uses for its library definitions [33].

The file starts with definitions of the measure points for rise, fall and input-to-output times. It also indicates the typical Process, Voltage, and Temperature (PVT) conditions that we're using for the static timing analysis. These match the operating conditions that we used in Chapter 4 for generating our look up tables. Except for the $1 \mathrm{fF}$ load, $1 \mathrm{ps}$ time, and $1 \mathrm{~V}$ voltage units, we are not specifically using any of this initial setup information for the PrimeTime run of the two-stage FIFO in Figure 5.11 on page 106. All the timing information needed for the FIFO is stored in look up tables.

In STEP 3, Section 5.4, we already explained how our look up tables are embedded in this library file. Table 3.5, page 230 in [33], Liberty Reference Manual Version 2007.12, explains the timing types and timing _sense commands used with each look up table instance. For more details on the syntax and semantics of Liberty commands, see [33].

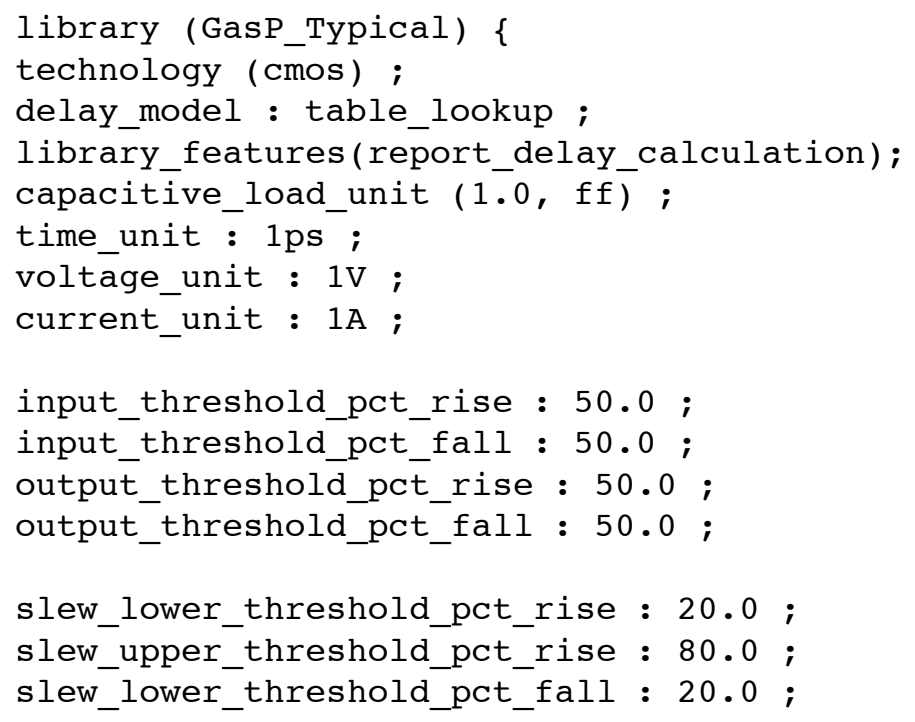




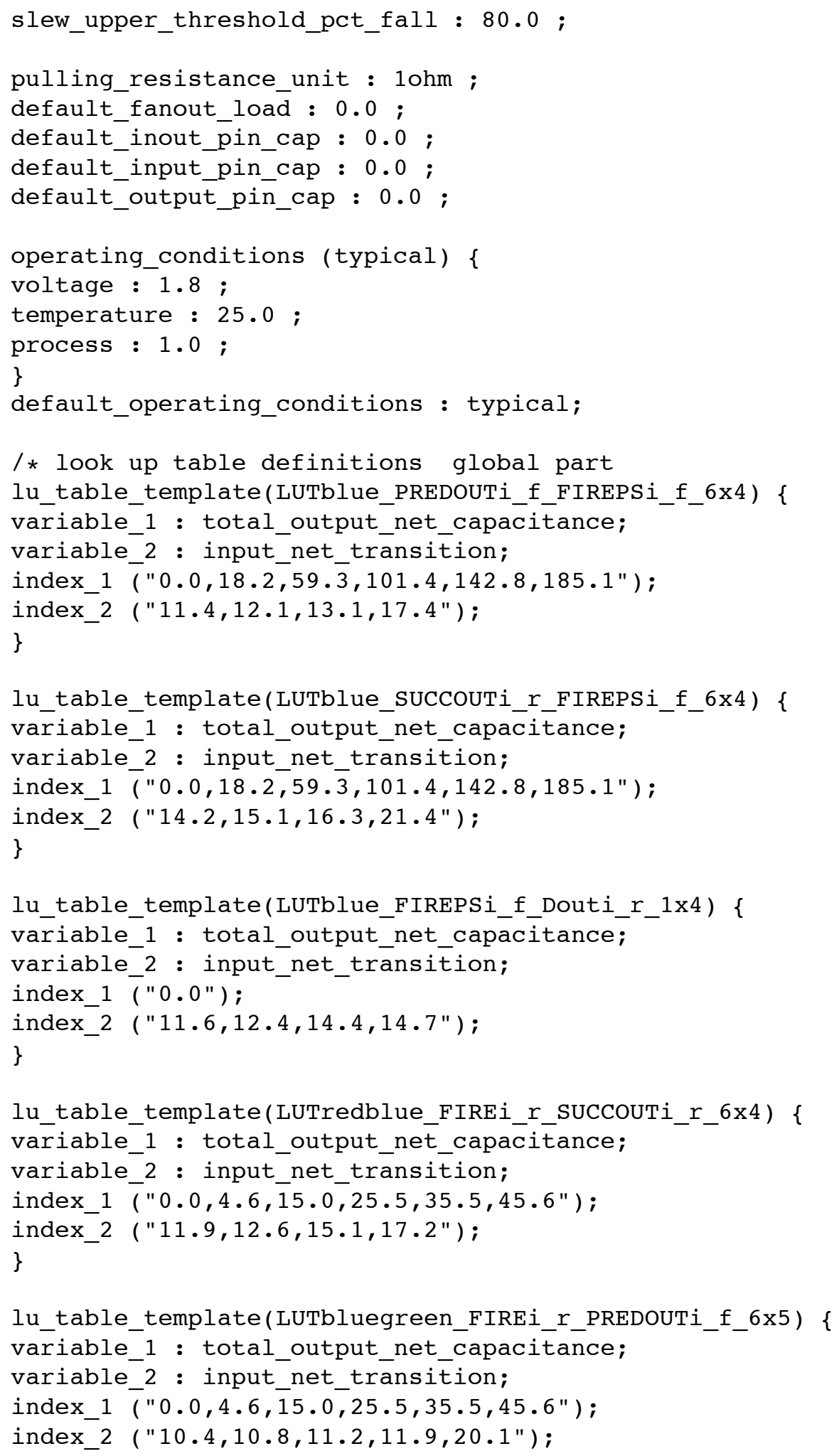




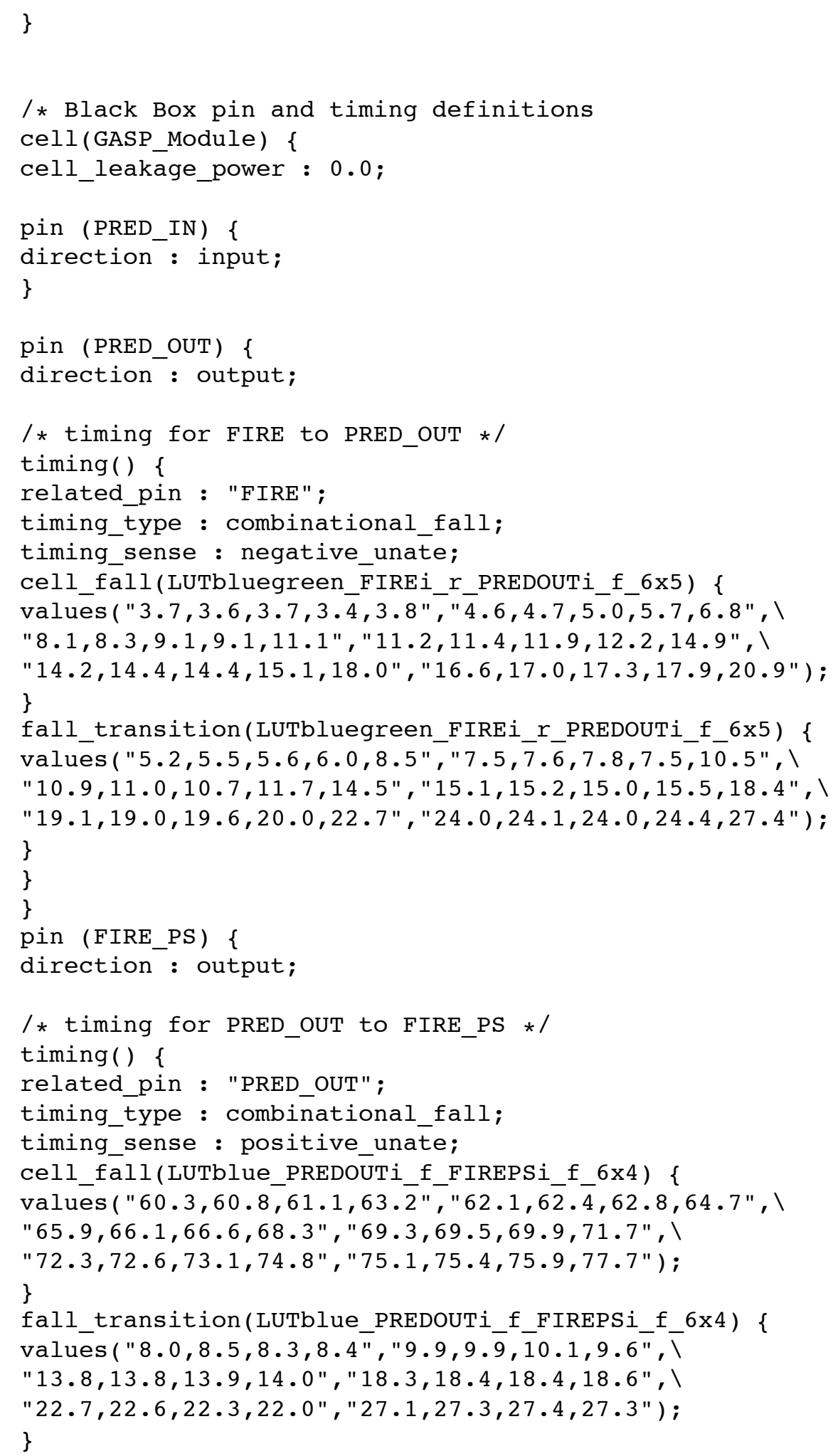




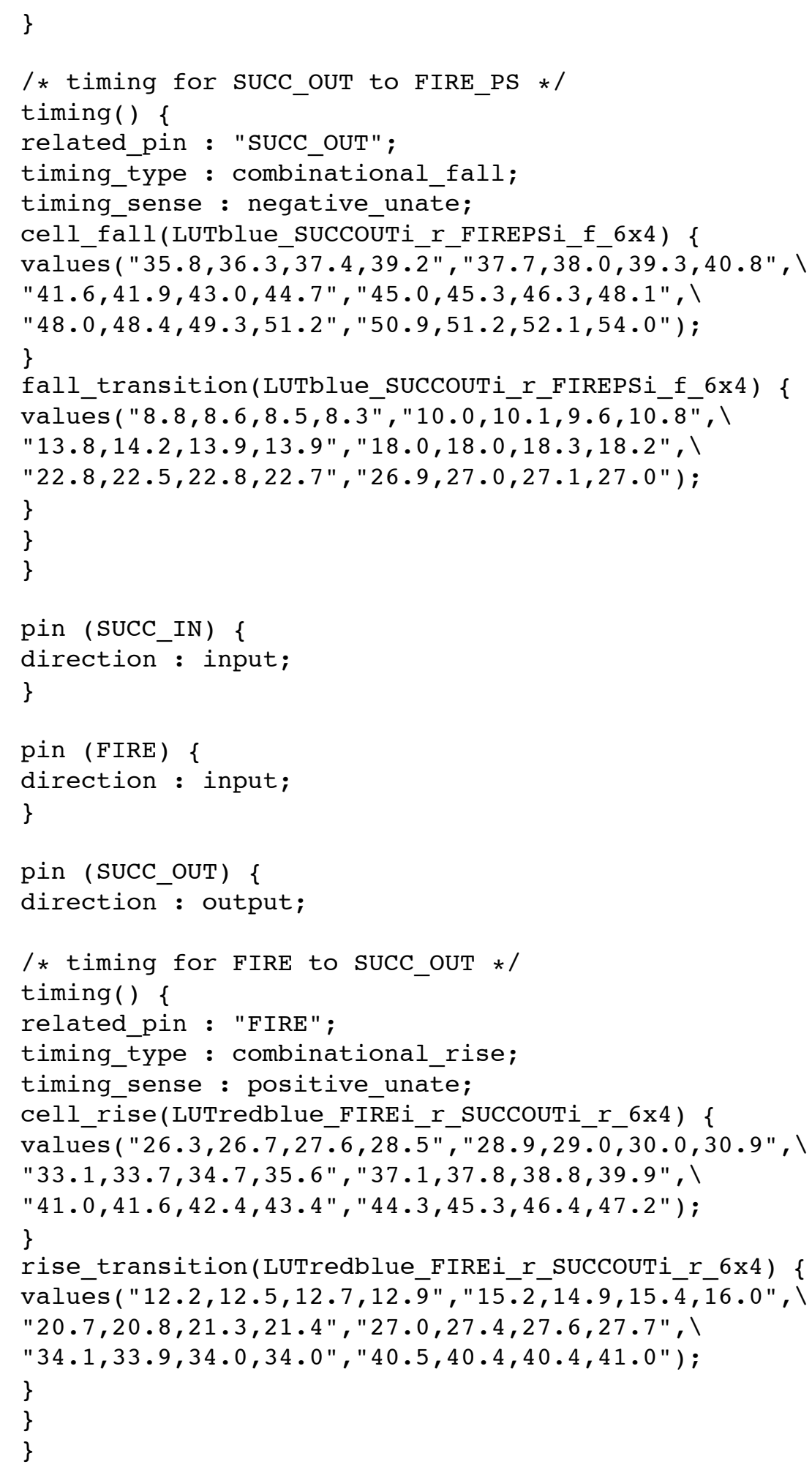




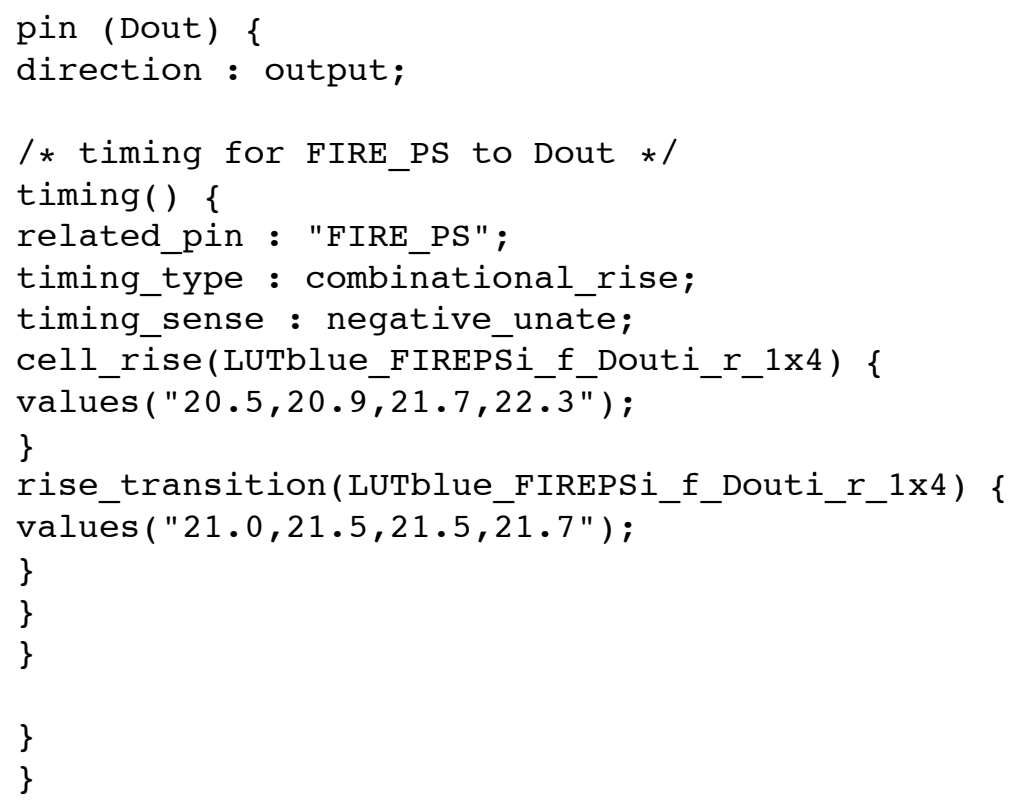




\section{Appendix L}

\section{PRIMETIME COMMANDS FOR VALIDATING RT1 TO RT4}

Below is the command file for validating the four relative timing constraints, RT1 to RT4 of Figure 5.12 on page 108. It contains a header with links to the netlist and library files, and it indicates which netlist module we're targeting: GASP_FIFO2 - the twostage FIFO. The file extension is '.tcl', and the full name of the file is GasP_STA_RT.tcl. Remember that the relative timing constraints in Figure 5.12 are the black box versions of the original relative timings specified in Figure 2.2 on page 13. We already explained the syntax and semantics of the validation commands in STEP 4, Section 5.5. Details on the usage, syntax, and semantics of PrimeTime can be found in [35].

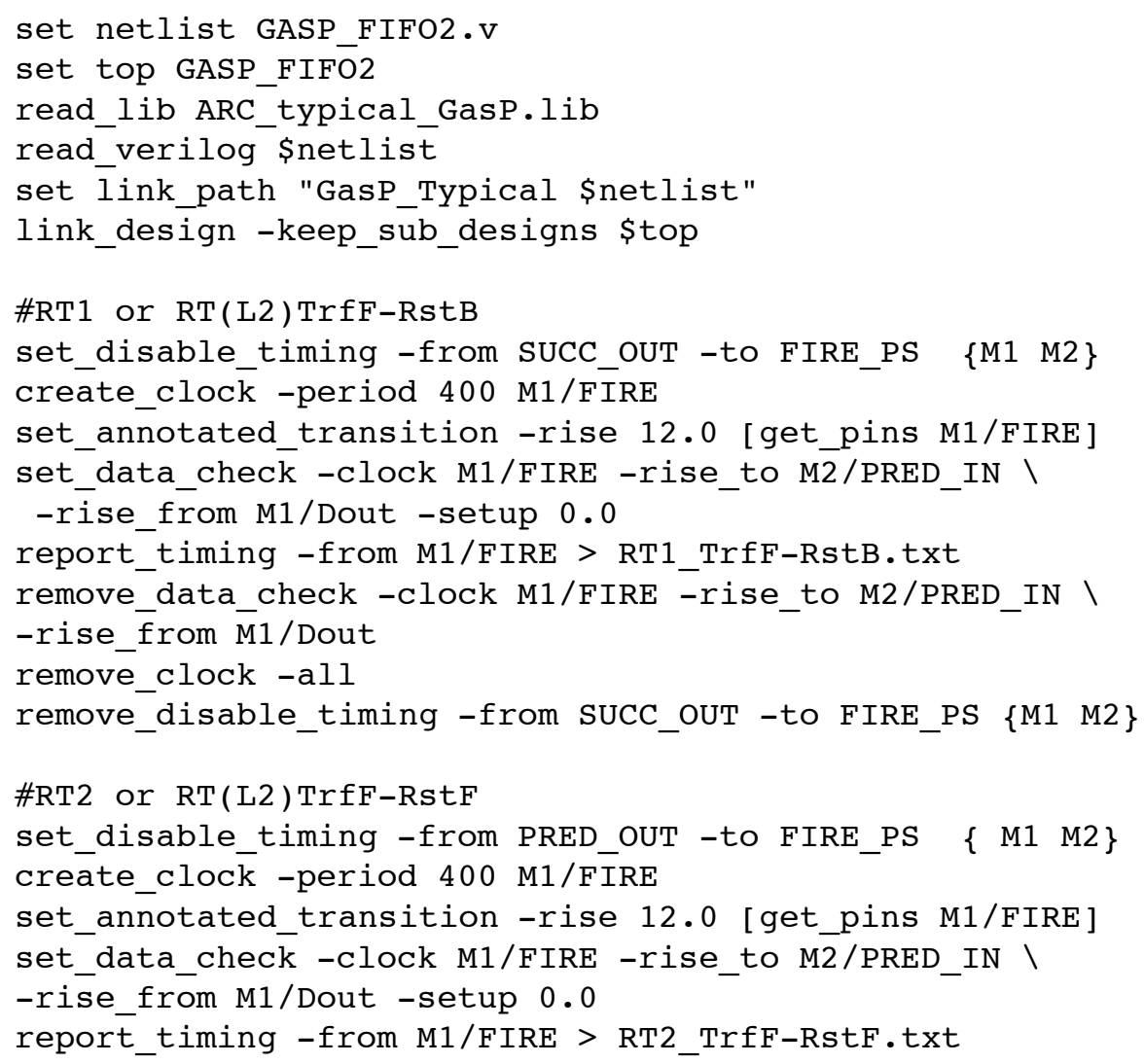




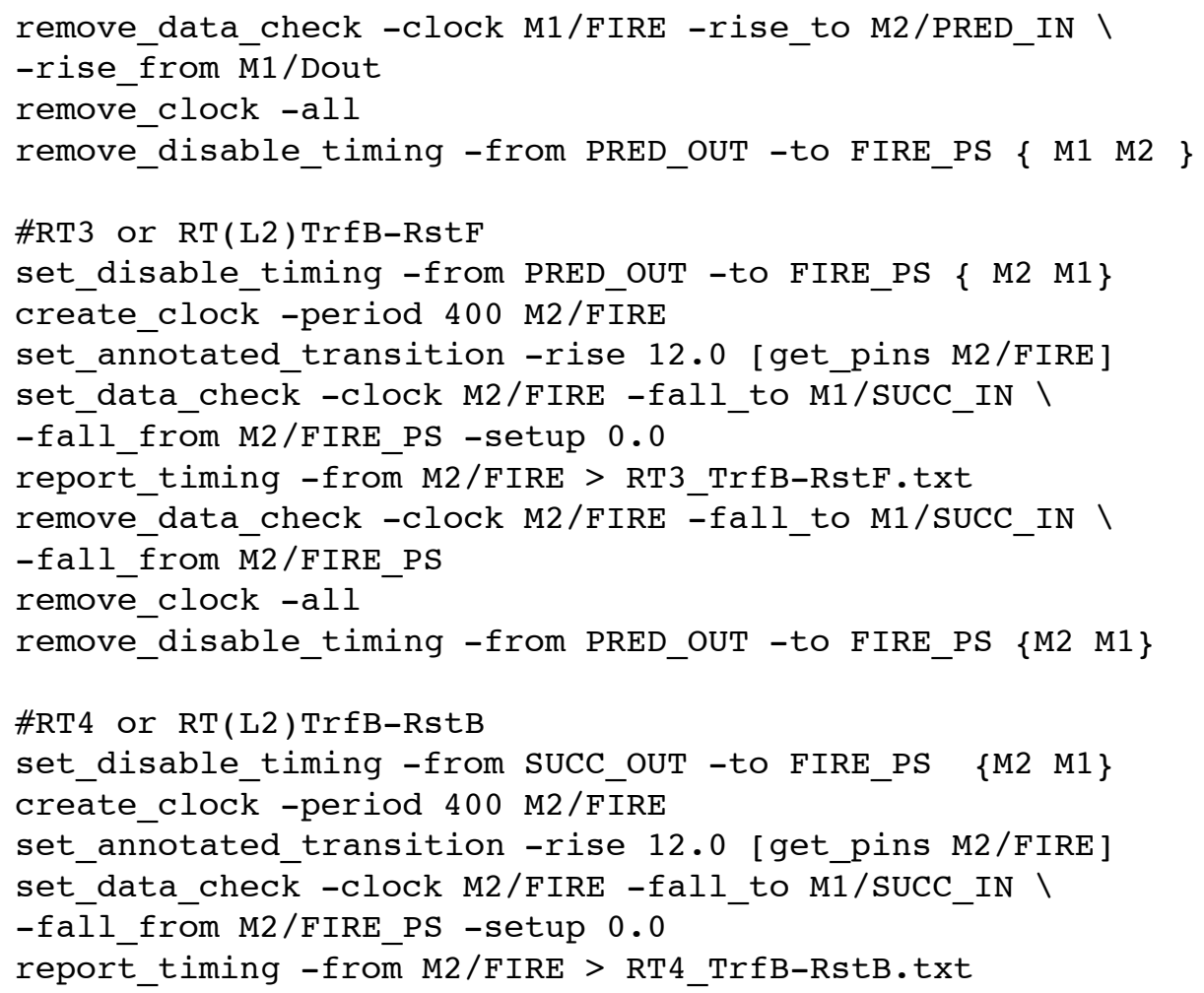




\section{Appendix M}

\section{PRIMETIME TIMING REPORTS}

To start the timing validation, using the Primetime netlist, library and command files in Appendix J, K, and L, we execute the following command lines [35]:

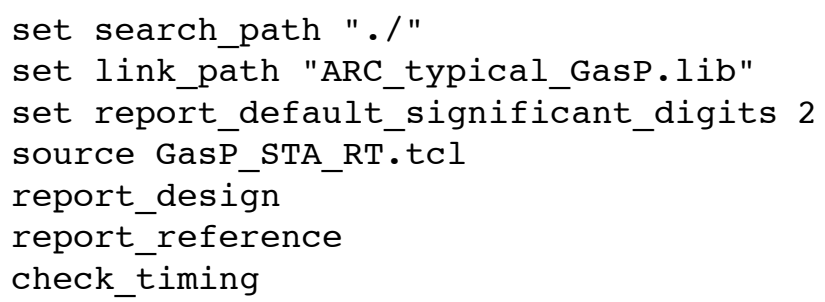

This yields four output files with timing reports, one for each relative timing constraint. The timing reports are listed in the following four Appendices M.1-M.4. They are discussed in STEP 5, Section 5.6. 
M.1 Timing Report for RT1 or RT(L2) ${ }^{\operatorname{TrfF}-R s t B}$

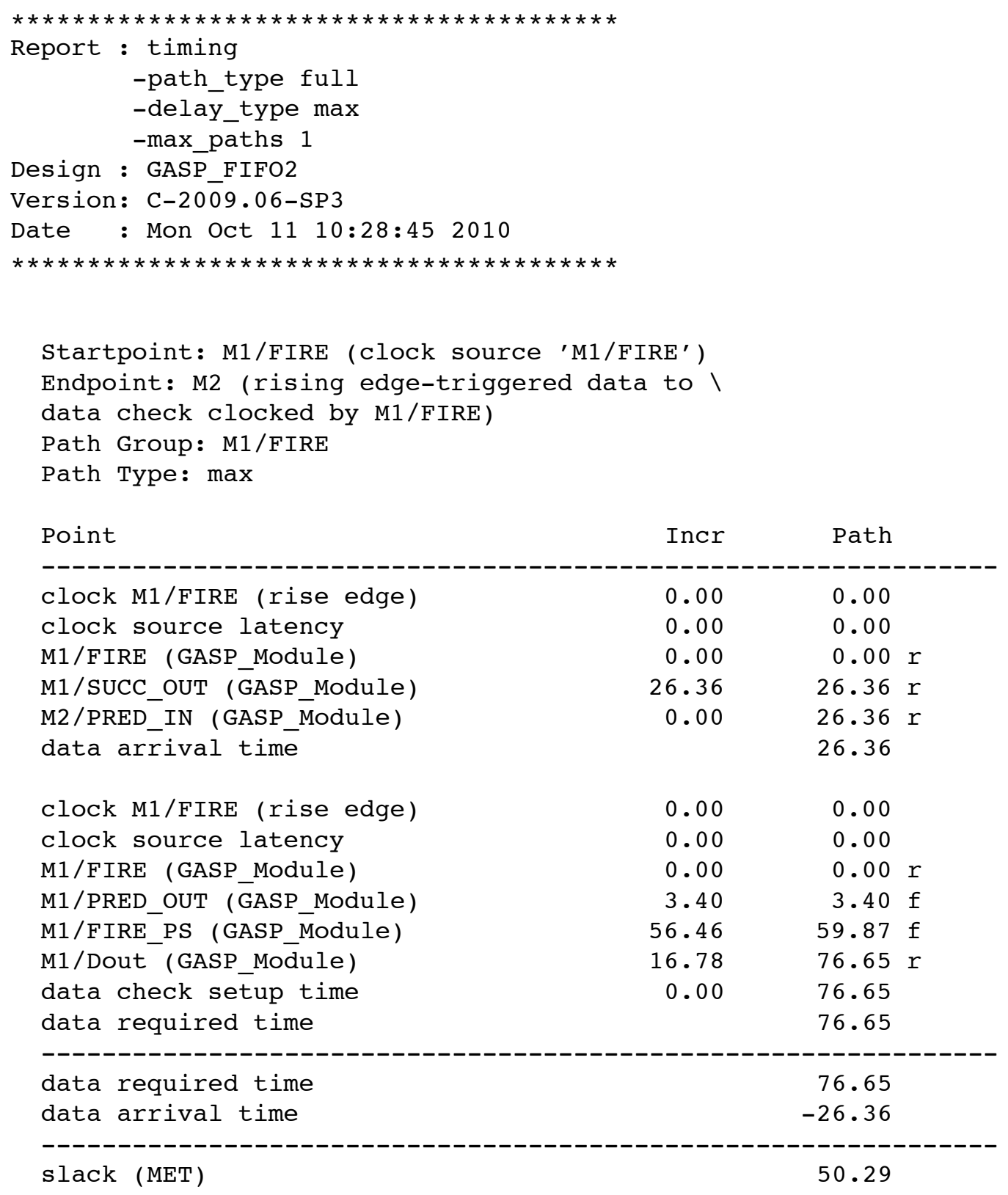


M.2 Timing Report for RT2 or RT(L2) ${ }^{\operatorname{TrfF}-\mathrm{RstF}}$

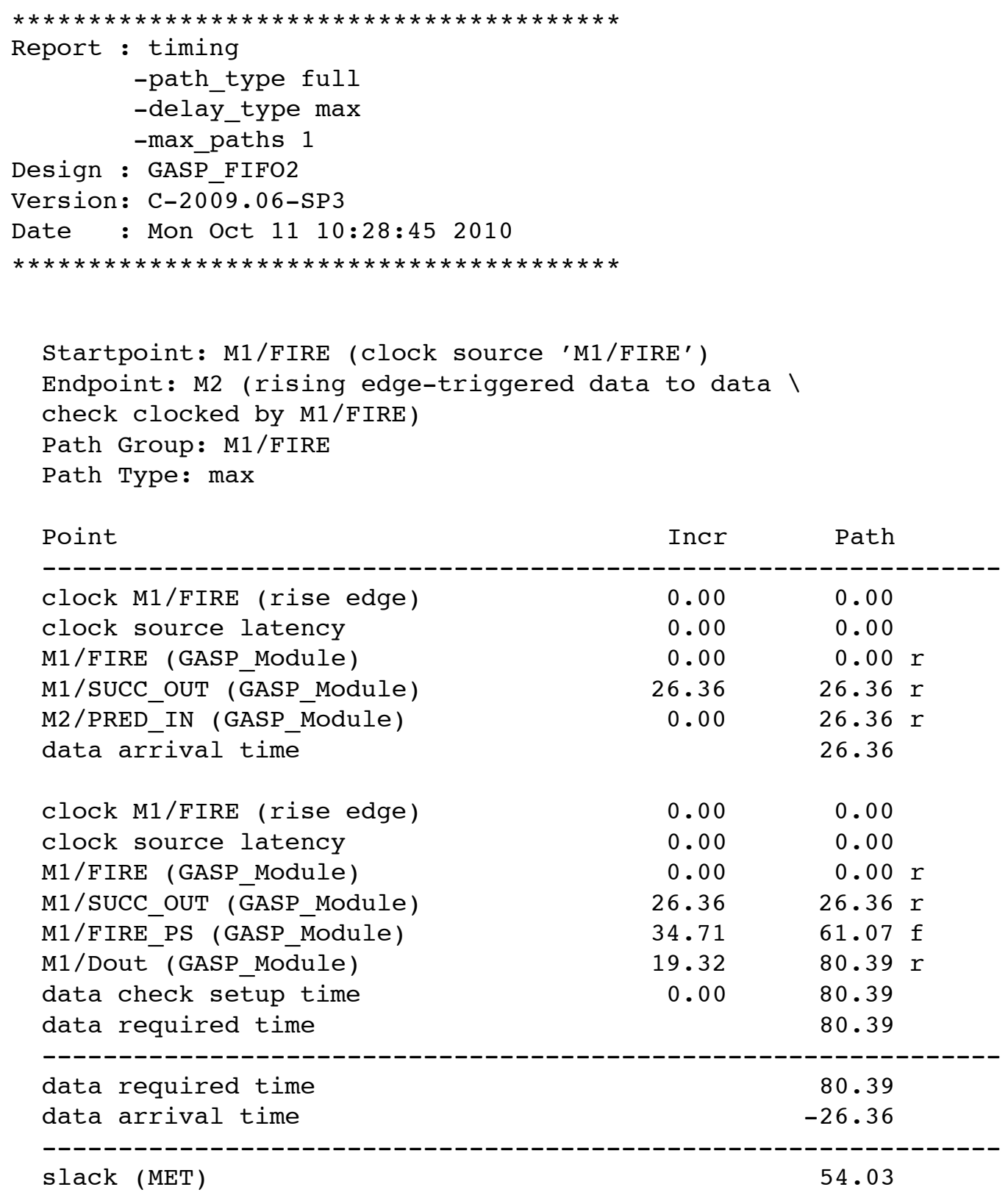


M.3 Timing Report for RT3 or RT(L2) ${ }^{\operatorname{TrfB}-R s t F}$

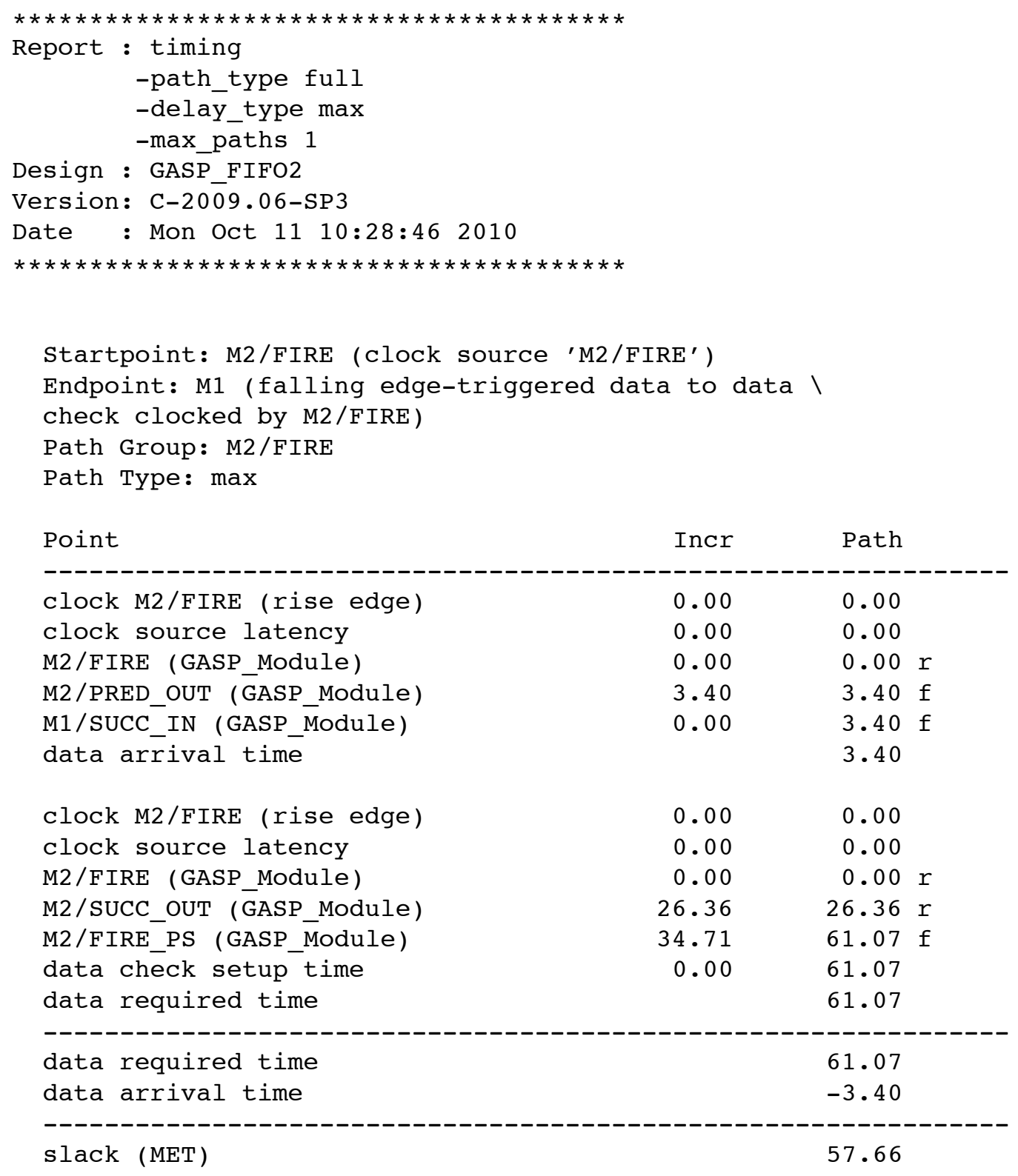


M.4 Timing Report for RT4 or RT(L2) ${ }^{\operatorname{TrfB}-R s t B}$

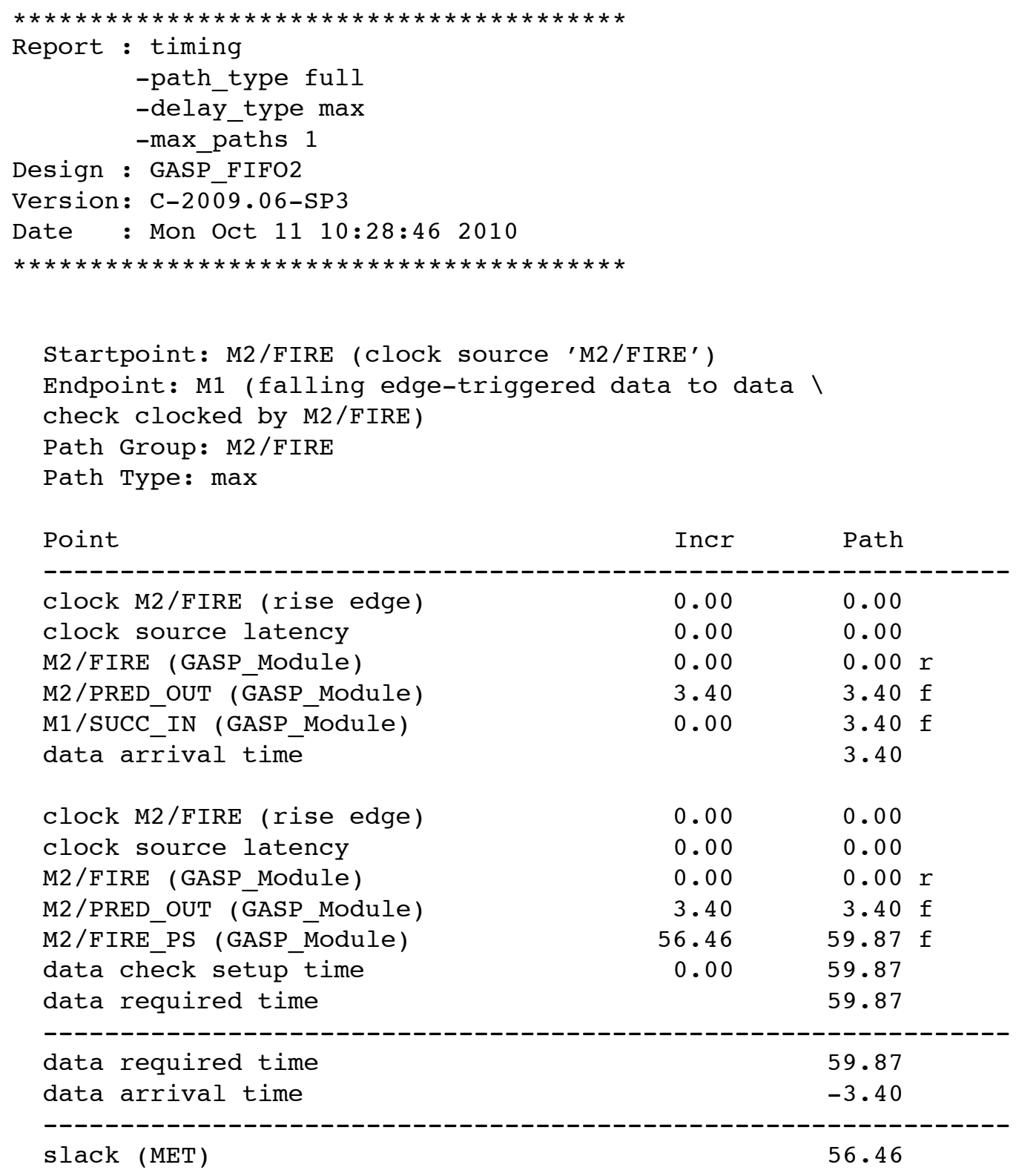




\section{Appendix N}

\section{BONUS MATERIAL: DISTANCE CONSTRAINT GRAPH}

Reprinted with permission from the Asynchronous Research Center (ARC) at Portland State University, this internal ARC report can be found in the thesis reference list as reference [15]. The full citation is as follows:

- Swetha Mettala Gilla. Distance Constraint Graph: A Graphical Representation for 6-4 GasP showing how Relative Timings constrain the Module Distances. Technical Report, ARC2009-smg01, Asynchronous Research Center, Portland State University, September, 2009. 


\title{
Asynchronous Research Center Portland State University
}

\author{
Subject: Distance Constraint Graph: A Graphical Representation for 6-4 GasP \\ showing how Relative Timings constrain the Module Distances \\ Date: $\quad$ September 1, 2009 \\ From: $\quad$ Swetha Mettala Gilla \\ ARC\#: 2009-smg01
}

\section{References:}

[1] Prasad Joshi, Peter A; Beerel, Marly Roncken and Ivan Sutherland Timing Verification of GasP Asynchronous Circuits: Predicted Delay Variations Observed by Experiment, 2008 Festschrift Series of Springer LNCS for the Festschrift of Willem-Paul de Roever, Kiel, Germany, 4 July 2008.

[2] Ivan Sutherland, Bob Sproull and David Harris, Logical Effort: Designing Fast CMOS Circuits, Morgan Kaufmann, San Francisco, 1999

\begin{abstract}
This paper follows up on the Festschrift publication in [1]. In [1], Joshi et al. analyze the relative timing constraints on which the correct operation of a 6-4 GasP circuit depends. They predict correct operation over a wide range of module distances provided the difference in the distances to predecessor and successor modules is limited. They predict failure if the distances differ by too much. Their analytical prediction is supported by experiments on a 90 nanometer test chip called "Infinity" which was built by Sun Microsystems and fabricated at TSMC.

In the present paper, I continue the analysis by Joshi et al. and calculate by how much the distances may differ and still maintain the two key relative timing constraints. I present this as a two-dimensional graph, which I call the Distance Constraint Graph.
\end{abstract}

The Distance Constraint Graph covers a range of wire distances from a given module to its predecessor and successor modules. It marks the minimum and maximum allowable difference from the equidistance line where the distances to the predecessor and successor modules are the same. It does this for the two key relative timing constraints and it overlays the results to show the pass-fail regions. Specifically, the graph indicates correct operation for all plausible wire lengths that the 90 nanometer Infinity chip would use without inserting 6-4 GasP repeater modules. For a quick preview of the Distance Constraint Graph, see Figure 3 on page 9 and Figure 4 on page 11.

This document contains information developed at the Asynchronous Research Center at Portland State University. Disclose this information to whomever you choose. Distribution is permitted with adequate reference to the source of the ideas and information. You may reproduce this material for any educational use. Copies of the material must contain this notice.

ARC\# 2009-smg01 Asynchronous Research Center

page 1 of 11 


\section{Background}

Before I present my analysis results, I will give some background on 6-4 GasP circuits, their basic operation, and the two key relative timing assumptions for correct operation.

\subsection{6-4 GasP Circuits}

Figure 1 shows two control stages, each with a 6-4 GasP circuit. The stages are connected in series by a bidirectional state wire L2 ${ }^{1}$ via connection ports SUCC and PRED, as shown in Figure 1. The basic operation of each 6-4 GasP circuit is as follows. When PRED is high and SUCC is low, signal FIRE rises. FIRE high does three things: (1) it clocks the latches to copy the present data (2) it raises SUCC to indicate that the data values are on their way, and (3) it lowers PRED to indicate that the data have been accepted and there is space again for new data.

Actions (2) and (3) have the additional side-effect of resetting the FIRE signal to low. Either (2) or (3) can do this: gate A synchronizes PRED high and SUCC low as PMOSAND function, but either PRED low or SUCC high will trigger the complementary NMOS-OR function of gate A. Thus, FIRE high self-resets to FIRE low, and there are two self-resetting loops to choose from: one through gates DEABC related to action (2) and another through gates XFABC related to action (3). Each one takes 5 gate delays.

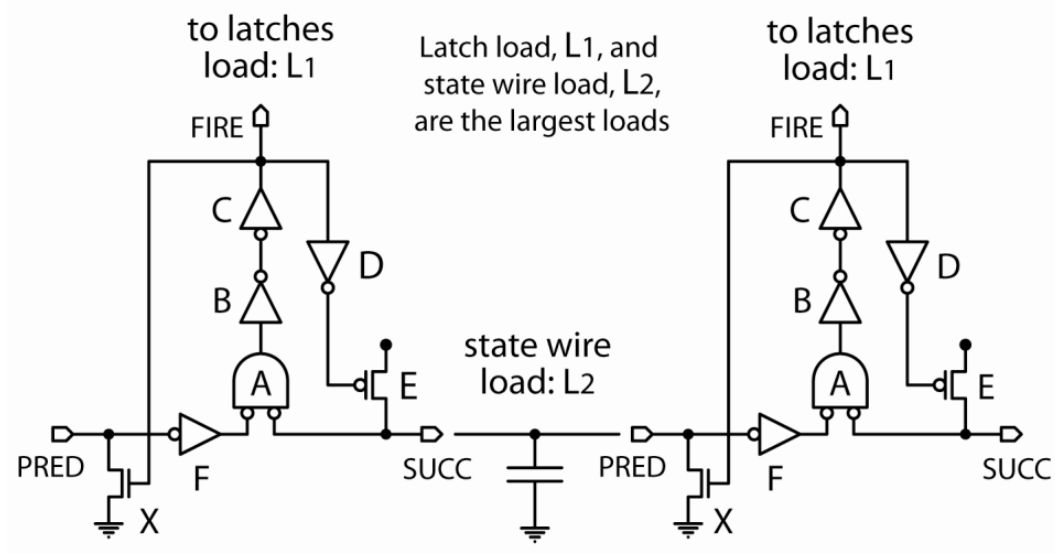

Figure 1 (Picture copied from Fig 1 in [1]) The picture shows two stages of 6-4 GasP circuit modules connected in series. The forward latency from gate $A$ in the first stage to gate $A$ in the second stage is 6 gate delays, and is covered by the path ABCDEF. The backward latency from gate $A$ in the second stage to gate $A$ in the first stage is 4 gate delays, and is covered by the path ABCX. This gives a cycle time of 10 gate delays. In addition to this global cycle time, each GasP module has two local self-resetting cycles of 5 gate delays each: in [1] these are called the successor loop, for ABCDE, and the predecessor loop, for ABCXF. It is these two self-resetting loops that incur the two key relative timing assumptions that cause the pass-fail circuit behavior that I analyze in this paper. The two relative timings are specified in Section 1.2, Figure 2.

\footnotetext{
${ }^{1}$ In the text of this paper, I loosely use L2 to denote either the state wire itself or its length or its load. I will be more precise in the delay calculations and presentation of the distance constraint graph in Section 2.
}

ARC\# 2009-smg01 Asynchronous Research Center

page 2 of 11 
In 6-4 GasP, one can count delay in terms of logic gate delays, because the modules are custom-designed using Logical Effort [2]. This means that the transistors in each logic gate are sized such that all logic gates have the same delay. Gate sizing takes into account the transistor sizes of the gates that it drives and the connecting wire lengths.

This works as long as one can adequately predict the wire lengths. Gates A, B, D, F in Figure 1 each drive a single local gate, and so one may assume that the connecting wire lengths are known in advance. The situation for gate $C$ is more complicated: $C$ drives two local gates, $D$ and $X$, and a number of latches with a total latch load of $L 1$, as well as a not so local wire between the gates and the latches. Fortunately, it is possible to place the latches near the 6-4 GasP controller, and design a macro module that contains both the data and the control and connects these with a fixed wire length.

The exceptions are gates $E$ and $X$ that drive state wire L2. The length of $L 2$ is dependent on the distance between the modules, which is not known until the final system layout. If $E$ and $X$ are sized using the wire lengths for $A, B, D$ and $F$, as was done in the Infinity chip, the gate delays for $E$ and $X$ will vary with different module distances and the delays of the self-resetting loops through DEABC and XFABC will vary correspondingly. If the delays vary by too much then the FIRE signal may selfreset before action (2) or (3) has completed, and cancel the action prematurely.

Premature cancellation of action (2) may result in loss of data because the successor stage never received the SUCC high indication that new data have arrived. Cancellation of action (3) may result in duplicate data because the predecessor stage never received the PRED low indicator to go ahead and replace the old data. To avoid this, Joshi et al. in [1] limit the delay variations due to module distance by enforcing so-called relative timing constraints. This is the topic of the next Section 1.2.

\subsection{Relative Timing (RT) Constraints}

Joshi et al. identified two key relative timing (RT) constraints to limit the delay variation between the two self-resetting loops of a GasP module. The two constraints state that one loop cannot shut off the other loop prematurely and cancel the associated action to the predecessor or successor module. The design and layout of 6-4 GasP circuits must maintain these constraints.

The two RT constraints, RT1 and RT2, are illustrated in Figure 2. Each constraint compares two path delays through a two-stage 6-4 GasP design, like Figure 1, and demands that one does not exceed the other. The lesser path delay is marked by a dotted line and corresponds to a forward or backward action through state wire L2. The larger path delay is marked by a solid line and corresponds to the self-resetting loop in the reverse direction. The paths are expressed as a sequence of up-going $(+)$ and down-going (-) gate output transitions. Figure 2 shows only the transitions for the key signals to the latches and neighboring GasP stages: FIRE, PRED and SUCC.

\section{ARC\# 2009-smg01 Asynchronous Research Center page 3 of 11}




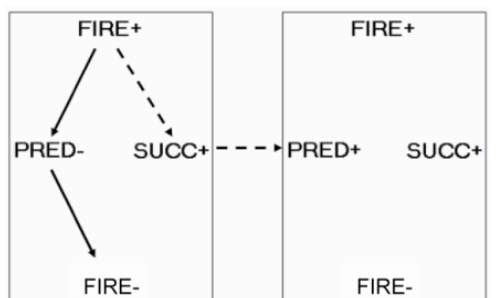

(a1)

(a2)

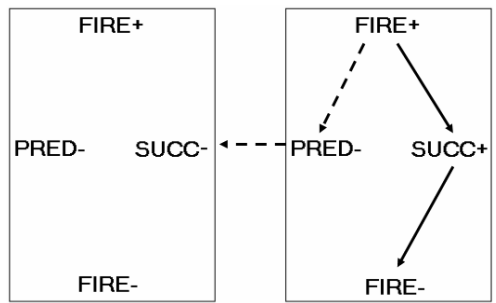

(b1)

(b2)

Figure 2 (Adapted from Fig 2 in [1]) The left and right sides show the two key relative timing constraints RT1 and RT2 for the two-stage 6-4 GasP design in Figure 1:

- $\quad$ RT1 is illustrated on the left for successive stages (a1)-(a2): the delay of the dotted forward path from FIRE+ to SUCC+ to PRED+ through gates DE in (a1) and up to (a2) must equal or be below the solid path delay for the self-resetting predecessor loop from FIRE+ to PRED- to FIRE- through gates XFABC in (a1).

- $\quad$ RT2 is illustrated on the right for successive stages (b1)-(b2): the delay of the dotted backward path from FIRE+ to PRED- to SUCC- through gate $X$ in (b2) and up to (b1) must equal or be below the solid path delay for the self-resetting successor loop from FIRE+ to SUCC+ to FIRE- through gates DEABC in (b2). Note that RT1 and RT2 use a different starting point for the self-resetting loops than the definitions in Figure 1 they starts from FIRE instead of from an input to gate $A$, but cover the same 5 gates - which is what matters.

The first timing constraint RT1 is illustrated for the left stage pair (a1)-(a2) of Figure 2. It states that PRED of the successor stage goes high before FIRE self-resets to low. In terms of the circuit diagram of Figure 1 this more-or-less translates to: gates DE drive PRED of the successor stage high through state wire L2 before the self-resetting path through gates XFABCD shuts off gate $E$ and stops it from driving PRED.

I emphasized that this translation "more-or-less" expresses what follows. This is because the RT1 expression in terms of FIRE, PRED and SUCC is slightly coarser: it stops at the FIRE input to gate D. The RT1 expression in Figure $\mathbf{2}$ factually translates to: the path delay through DE does not exceed that of XFABC. This makes RT1 more conservative than is intended. I will come back to this in Section 2.1, when I present the pass-fail regions for both the conservative and the intended RT1 versions.

The second timing constraint RT2 is illustrated on the right, for stage pair (b1)-(b2) of Figure 2. It states that SUCC of the previous stage goes low before FIRE self-resets to low. In terms of the circuit diagram of Figure 1, this exactly translates to: gate $X$ drives SUCC of the predecessor stage low through state wire L2 before the self-resetting path through gates DEABC shuts off gate $X$ and stops it from driving SUCC.

Note that timing constraint RT2 can be expressed as intended in terms of transitions ending on FIRE, PRED, and SUCC.

In the following Section 2, I will compute the values for L2 for which RT1 and RT2 hold. More precisely, I will compute the range of values for L2 PRED and L2 $2_{\text {SucC }}$ so that the gate delays for $X_{P R E D}$ driving L2PRED $_{\text {and for }} E_{\text {SUCC }}$ driving L2succ satisfy the RT1 path delay equation $D E_{S U C C} \leq X_{P R E D} F A B C$. And likewise, I will compute the range of values for $L_{2}$ PRED and L2succ that satisfy $R T 2$ path delay equation $X_{P R E D} \leq D E_{\text {SUCC }} A B C$.

ARC\# 2009-smg01 Asynchronous Research Center page 4 of 11 


\section{Computing Allowable Module Distances}

I use the term allowable module distances to indicate the range of wire lengths between 6-4-GasP modules that satisfy relative timing constraints RT1 and RT2. RT1 and RT2 are path-delay constraints; they are computed from the delays of the gates and the wires on the corresponding paths. This paper adopts the wire model used by Joshi et al in [1]. This means that a wire is modeled as a lumped capacitance, and its delay is incorporated into the delay of each gate that drives this lumped capacitance. Delays due to wire resistance are ignored. ${ }^{2}$

Joshi et al. built a spreadsheet that calculates the gate delays in a 6-4 GasP module using wire loads that are typical for the 90 nanometer chip design of Infinity. ${ }^{3}$ The delay calculations are based on the theory of Logical Effort [2]. Their spreadsheet calculations are repeated in Table 1 below.

With the help of Table 1 I compute the range of allowable module distances. I will first compute the path delays for RT1 and from these the allowable module distances for RT1. This is done Section 2.1. In Section 2.2, I do the same for RT2. I combine the two results in Section 2.3, where I give the full picture and relate my results back to the 90 nanometer results of the Infinity test chip presented by Joshi et al. in [1].

Table 1 (Adapted from Table 2 in [1]). Below follow the Logical Effort calculations of each gate delay in the 6-4 GasP module of Figure 1.The three columns with bold numbers for S, P and WL are fixed by design: gate size $S$ and its self-delay $P$ follow from the gate implementation and the gate complexity, and wire load WL follows from the wire dimensions. Short wires are indicated as 'wire' (values taken from column WL); the long wires L1 and L2 are indicated explicitly. Note that the calculation of the self delay P for gates $E$ and $X$ counts the diffusion output capacitance of both $E$ and $X$, because both their outputs connect to the state wire L2. Also note that the D2 values for $E$ and $X$ are different for different wire loads of $L 2$. The Table uses the logical effort

values of Table 1 in [1] (not included) to calculate the load per gate input: IL = ( $S$ * logical effort of the gate).

The formulas for gate delay, wire load delay, and total delay are: $\mathrm{D} 1=\mathrm{GL} / \mathrm{S}, \mathrm{D} 2=\mathrm{WL} / \mathrm{S}$, Total $=\mathrm{D} 1+\mathrm{P}+\mathrm{D} 2$.

\begin{tabular}{|c|c|c|c|c|c|c|c|c|c|}
\hline $\begin{array}{l}\text { Gate } \\
\text { Name }\end{array}$ & $\begin{array}{l}\text { Size } \\
\text { (S) }\end{array}$ & $\begin{array}{l}\text { Load } \\
\text { per } \\
\text { input } \\
\text { (IL) }\end{array}$ & $\begin{array}{l}\text { Drives next gates } \\
\text { and wire }\end{array}$ & $\begin{array}{c}\text { Next } \\
\text { gate load } \\
\text { (GL) }\end{array}$ & $\begin{array}{c}\text { Delay } \\
\text { from } \\
\text { GL } \\
\text { (D1) }\end{array}$ & $\begin{array}{l}\text { Self } \\
\text { delay } \\
\text { (P) }\end{array}$ & $\begin{array}{l}\text { Next } \\
\text { wire } \\
\text { load } \\
\text { (WL) }\end{array}$ & $\begin{array}{l}\text { Delay } \\
\text { from } \\
\text { WL } \\
\text { (D2) }\end{array}$ & $\begin{array}{c}\text { Total } \\
\text { delay } \\
{[\mathrm{T}]^{3}}\end{array}$ \\
\hline A & 18 & 30 & B+wire & 40 & 2.2 & 2 & 9 & 0.5 & 4.7 \\
\hline $\mathrm{B}$ & 40 & 40 & C+wire & 100 & 2.5 & 1 & 20 & 0.5 & 4 \\
\hline $\mathrm{C}$ & 100 & 100 & $\mathrm{D}+\mathrm{X}+\mathrm{L} 1_{\text {latches }}+\mathrm{L} 1_{\text {wire }}$ & $20+20+200$ & 2.4 & 1 & 100 & 1 & 4.4 \\
\hline $\mathrm{D}$ & 20 & 20 & E+wire & 40 & 2 & 1 & 20 & 1 & 4 \\
\hline $\mathrm{F}$ & 10 & 10 & A+wire & 30 & 3 & 1 & 5 & 0.5 & 4.5 \\
\hline $\begin{array}{c}E \\
\mathrm{~L} 2=15\end{array}$ & 60 & 40 & $A+F+L 2$ & $30+10$ & 0.67 & $\begin{array}{c}\text { with } X \\
=1\end{array}$ & 15 & 0.25 & 1.9 \\
\hline $\begin{array}{c}E \\
L 2=150\end{array}$ & 60 & 40 & $A+F+L 2$ & $30+10$ & 0.67 & $\begin{array}{c}\text { with } X \\
=1\end{array}$ & 150 & 2.5 & 4.2 \\
\hline $\begin{array}{c}X \\
L 2=15\end{array}$ & 60 & 20 & $A+F+L 2$ & $30+10$ & 0.67 & $\begin{array}{c}\text { with } E \\
=1\end{array}$ & 15 & 0.25 & 1.9 \\
\hline $\begin{array}{c}X \\
L 2=150\end{array}$ & 60 & 20 & $A+F+L 2$ & $30+10$ & 0.67 & $\begin{array}{c}\text { with } E \\
=1\end{array}$ & 150 & 2.5 & 4.2 \\
\hline
\end{tabular}

${ }_{3}^{2}$ Delays due to wire resistance and other long wires effects will be included in a follow-up paper.

${ }^{3}$ The delay unit here and in [1] is ' $T$ ' (tau): the characteristic delay of an inverter in the given process.

ARC\# 2009-smg01 Asynchronous Research Center

page 5 of 11 


\subsection{Path Delays and Allowable Module Distances for RT1}

From Figure 2(a1)-(a2) one can see that relative timing constraint RT1 involves both state wires at both ends of the 6-4 GasP module: the predecessor state wire participates in the solid backward path that self-resets FIRE from FIRE+ to FIRE-; the successor state wire participates in the dotted forward path from FIRE+ to PRED+.

To analyze RT1 it makes sense to distinguish these two state wires. I will use L2PRED to denote the state wire that connects the predecessor port PRED of a 6-4 GasP module to the previous stage. I will use L2succ to denote the state wire that connects the successor port SUCC of a 6-4 GasP module to the next stage. This matches with the terminology in Figure 1. For convenience, I wish to identify the gates that drive L2PRED respectively $L 2$ succ, so that the name of the gate indicates that the gate delay depends on the load value of L2PRED respectively L2 succ. To this end, I rename gate $X$ as $\mathrm{X}_{\mathrm{PRED}}$ and gate $\mathrm{E}$ as $\mathrm{E}_{\text {SUCC. }}$.

In Section 1.2, I distinguished two RT1 constraints: a conservative version and the intended version. The conservative version, which I will call RT1 $1_{\text {conservative, states that the }}$ forward path delay from FIRE+ to SUCC+ to PRED+ through D and $E_{\text {Succ }}$ must be less than or equal to the backward self-resetting path from FIRE+ to FIRE- through the series of gates $X_{P R E D}, F, A, B$ and $C$. The intended RT1 constraint version, which I will call RT1 $1_{\text {intended, }}$, is similar, but has one more gate in the backward path: $\mathrm{X}_{\text {PRED }} F A B C D$.

I use the gate delay calculation procedure in Table 1 to compute the backward selfresetting path delays for $X_{P R E D} F A B C$. For each fixed value of $L 2_{P R E D}$, this gives me the maximum forward path delay for $\mathrm{DE}_{\text {succ }}$ for which $\mathrm{RT} 1_{\text {conservative }}$ holds. Given this, I reverse-engineer the calculation procedure in Table 1 to obtain the corresponding maximum load value of L2succ that satisfies $R T 1_{\text {conservative. }}{ }^{4}$

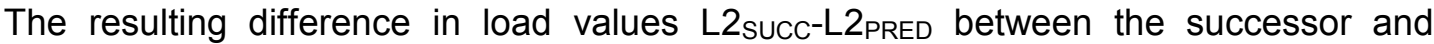
predecessor state wires represents the maximum allowable module distance in terms of load capacity. I call this the margin value. L2 SUCC-L2 $_{\text {PRED }}$ values below the margin value pass the relative timing constraint, those above fail the constraint.

The computations for the RT1 $1_{\text {conservative }}$ margin values are outlined in Table 2. It turns out that the margin value is approximately constant, 816 units of load, over the given range of $L 2$ PRED state wire loads.

\footnotetext{
${ }^{4}$ The load capacitance unit here and in [1] is ' $X$ ': the gate load, or capacitance, of the smallest inverter one can build in the given process.

${ }^{5}$ Reminder: I have used a lumped wire load. I ignored long wire delay effects, which are outside the scope of this study and which will be included in a follow-up paper.
}

ARC\# 2009-smg01 Asynchronous Research Center page 6 of 11 
Table 2 (RT1 $1_{\text {conservative }}$ margin) Calculations of the maximum allowable difference between the wire load L2succ of a given module to its successor module and the wire load L2PRED to its predecessor module. The calculations show that differences up to and including 816 units of load satisfy relative timing constraint RT1 of Figure 2(a1)-(a2), because $D_{S U C C} \leq X_{P R E D} F A B C$. The calculation procedure is as follows. Given the range of L2PRED values, I compute the path delays for $X_{P R E D} F A B C$. The computations are based on spreadsheet calculations using gate delays from Table 1 and its extension to a wide range of $L 2$ wire loads. The path delay

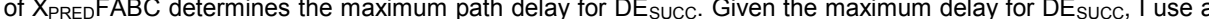
logarithmic search and additional spreadsheet calculations to get the corresponding maximum load for L2succ. This gives a maximum allowable difference of 816 units of load from L2succ to L2PRED.

\begin{tabular}{|c|c|c|c|c|}
\hline $\begin{array}{c}\text { L2PRED } \\
{[\mathrm{X}]^{4}} \\
\text { (fixed) }\end{array}$ & $\begin{array}{c}\text { XPRED FABC }{ }_{[\mathrm{T}]}{ }^{3} \\
\text { (computed from Table 1) }\end{array}$ & $\begin{array}{c}\mathrm{DE}_{\text {succ }} \\
{[\mathrm{T}]} \\
\text { (set to } \mathrm{X}_{\text {PRED }} \mathrm{FABC} \text { value) }\end{array}$ & $\begin{array}{c}\text { L2succ } \\
{[X]} \\
\text { (computed from } D_{\text {succ }} \text { ) }\end{array}$ & $\begin{array}{c}\text { L2succ - L2PRED } \\
{[\mathrm{X}]}\end{array}$ \\
\hline 10 & 19.4 & 19.4 & 826 & 816 \\
\hline 200 & 22.6 & 22.6 & 1016 & 816 \\
\hline 500 & 27.6 & 27.6 & 1316 & 816 \\
\hline 1000 & 35.9 & 35.9 & 1816 & 816 \\
\hline 2000 & 52.6 & 52.6 & 2816 & 816 \\
\hline 10000 & 185.9 & 185.9 & 10816 & 816 \\
\hline 12000 & 219.3 & 219.3 & 12816 & 816 \\
\hline 15000 & 269.3 & 269.3 & 15816 & 816 \\
\hline
\end{tabular}

The computations for the margin values of $\mathrm{RT} 1_{\text {intended }}$ are similar. The only difference is that the backward self-resetting path delay for $X_{P R E D} F A B C D$ is larger than the previous self-resetting path delay for $X_{P R E D} F A B C$ in Table 2. Specifically, it is larger by a fixed amount, namely by the gate delay value of $D$, which is independent of L2PRED.

From the previous calculations I know that the margin value for $\mathrm{RT} 1_{\text {conservative }}$ is constant over the given range of L2 PRED load values. This implies that the margin value for $\mathrm{RT} 1_{\text {intended }}$ is also constant, and moreover the constant value is larger than 816 . Table 3 gives the computations for the margin value of $\mathrm{RT} 1_{\text {intended. It suffices to do these }}$ computations for one fixed value of $L 2$ PRED because I know by now that the margin values for $L 2_{\text {SUCC }}$ - $2_{\text {PRED }}$ are the same for the entire $L 2_{\text {PRED }}$ range. ${ }^{5}$

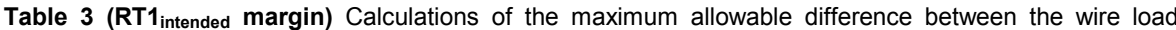
L2succ of a given module to its successor module and the wire load L2PRED to its predecessor module. The calculations show that differences up to and including 1056 units of load satisfy the intended relative timing constraint RT1 $1_{\text {intended }}$ for Figure 2(a1)-(a2), discussed in Section 1.2, because $D E_{S U C C} \leq X_{P R E D} F A B C D$. The calculation procedure is as in Table 2, but adds a fixed gate delay for gate $D$. The result is a larger maximum allowable difference of 1056 units of load from L2succ to L2PRED.

\begin{tabular}{|c|c|c|c|c|}
\hline $\begin{array}{l}\text { L2PRED } \\
{[\mathrm{XX}]^{4}} \\
\text { (fixed) }\end{array}$ & $\begin{array}{c}\text { X }_{\text {PRED FABCD }} \\
{[]^{3}{ }^{3}} \\
\text { (computed from Table 1) }\end{array}$ & $\begin{array}{c}\mathrm{DE}_{\text {succ }} \\
{[\mathrm{T}]} \\
\text { (set to } \mathrm{X}_{\mathrm{PRED}} \mathrm{FABCD} \text { value) }\end{array}$ & $\begin{array}{c}\text { L2succ } \\
{[X]} \\
\text { (computed from DE } E_{\text {succ }} \text { ) }\end{array}$ & $\begin{array}{c}\text { L2 } \text { SUCC }^{- \text {L2 }} \text { PRED } \\
{[\mathrm{X}]}\end{array}$ \\
\hline 10 & 23.4 & 23.4 & 1066 & 1056 \\
\hline
\end{tabular}

ARC\# 2009-smg01 Asynchronous Research Center

page 7 of 11 


\subsection{Path Delays and Allowable Module Distances for RT2}

The calculations for the margin values for relative timing constraint RT2 follow the same procedure as those for $\mathrm{RT} 1_{\text {conservative }}$ and $\mathrm{RT} 1_{\text {intended }}$ in Section 2.1.

I use the gate delay calculation procedure in Table 1 to compute the forward selfresetting path delays for $D E_{\text {succ }} A B C$. For each fixed value of $L 2_{s u c c}$, this gives me the maximum backward path delay for $X_{\text {PRED }}$ for which RT2 holds. Given this, I reverseengineer the calculation procedure in Table 1 to obtain the corresponding maximum load value of $L 2$ PRED. The resulting difference in load values $L 2_{\text {SUCC-L2PRED }}$ between the successor and predecessor state wires represents the maximum allowable module distance in terms of load capacity for RT2. This is the margin value for RT2, and it is

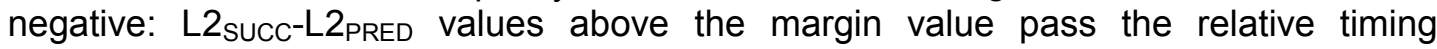
constraint, those below fail the constraint.

The computations for the RT2 margin values are outlined in Table 4. Also here, the margin value is approximately constant, -1026 load units, over the given range of L2succ state wire loads. ${ }^{6}$

Table 4 (RT2 margin) Calculations of the maximum allowable difference between the wire load L2succ of a given module to its successor module and the wire load L2PRED to its predecessor module. The calculations show that L2SUCC - L2PRED differences down to and including -1026 units of load satisfy relative timing constraint RT2 of Figure 2(b1)-(b2), because $X_{P R E D} \leq D_{S U C C} A B C$. The calculation procedure is similar to that of Table 2. Given the range of $L 2$ succ values, I compute the path delays for $D E_{\text {succ }} A B C$. This determines the maximum path delay for $X_{P R E D}$. From here, a logarithmic search gives the corresponding maximum load value for L2PRED, and a maximum allowable difference down to -1026 units of load from L2succ to L2PRED.

\begin{tabular}{|c|c|c|c|c|}
\hline $\begin{array}{c}\text { L2succ } \\
{[X]^{4}} \\
\text { (fixed) }\end{array}$ & $\begin{array}{c}\mathrm{DE}_{\text {succ }} \mathrm{ABC} \\
{[\mathrm{T}]^{3}} \\
\text { (computed from Table 1) }\end{array}$ & $\begin{array}{c}\text { X }_{\text {PRED }} \\
{[\mathrm{T}]} \\
\text { (set to } E_{\text {succ DABC value) }}\end{array}$ & $\begin{array}{c}\text { L2PRED } \\
{[X]} \\
\text { (computed from } X_{\text {PRED }} \text { ) } \\
\end{array}$ & $\begin{array}{c}\text { L2 } \text { SUCC }- \text { L2PRED } \\
{[\mathrm{X}]}\end{array}$ \\
\hline 10 & 18.9 & 18.9 & 1036 & -1026 \\
\hline 200 & 22.1 & 22.1 & 1226 & -1026 \\
\hline 500 & 27.1 & 27.1 & 1526 & -1026 \\
\hline 1000 & 35.4 & 35.4 & 2026 & -1026 \\
\hline 1500 & 43.8 & 43.8 & 2526 & -1026 \\
\hline 10000 & 185.4 & 185.4 & 11026 & -1026 \\
\hline 12000 & 218.8 & 218.8 & 13026 & -1026 \\
\hline 15000 & 268.8 & 268.8 & 16026 & -1026 \\
\hline
\end{tabular}

\footnotetext{
${ }^{6}$ Reminder: also in the delay calculations for RT2 I have used a lumped wire load. I ignored long wire delay effects, which fall outside the scope of this study and will be included in a follow-up paper.
}

ARC\# 2009-smg01 Asynchronous Research Center

page 8 of 11 


\subsection{The Full Picture: Distance Constraint Graph}

The graph in Figure 3 below helps visualize the result of the previous two Sections. This graph - which I call the Distance Constraint Graph - shows the allowable distances from a 6-4 GasP module to its predecessor and successor modules. I calculated the distance in units of length ${ }^{7}$ as well as in units of load. The solid and dashed blue lines are the margin lines for RT $1_{\text {conservative }}$ and $R T 1_{\text {intended. }}$. The green line is the margin line for RT2. Predecessor-successor pairs (L2PRED, L2succ) in the top-left

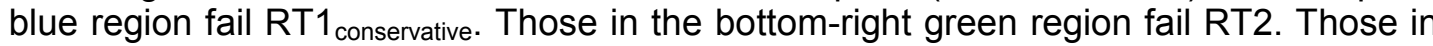
the white region in-between satisfy $R T 1_{\text {conservative }}$ (and hence also RT $1_{\text {intended }}$ ) and RT2.

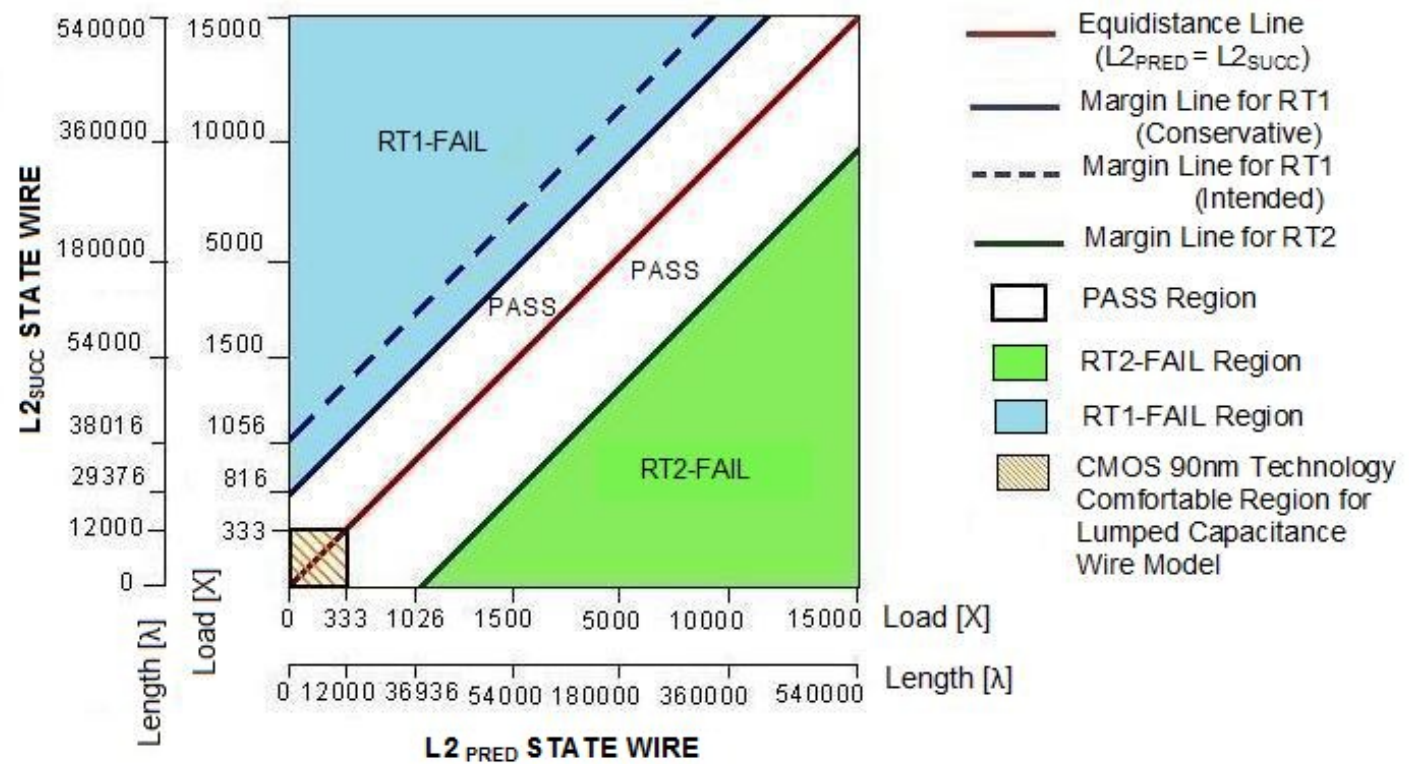

Figure 3 (Distance Constraint Graph) The graph shows the allowable distances from a 6-4 GasP module to its predecessor and successor modules. The X-axis shows the distance to the predecessor module, L2PRED. The Y-axis shows the distance to the successor module, L2succ. Both L2PRED and L2succ are expressed in units of length as well as in units of load. The conversion is based on the 90 nanometer CMOS technology used for Infinity, in which 36 units of length equals 1 unit of load - see also footnotes 4 and 7 . The solid and dashed blue lines are the margin lines for RT1 $1_{\text {conservative }}$ and RT1 $1_{\text {intended }}$ from Table 2 and Table 3 . The Predecessor-successor pairs (L2PRED, L2 2 SUCC) in the blue region above the solid line fail relative timing

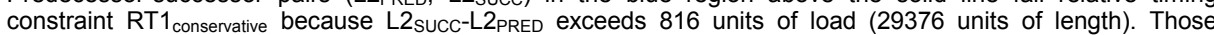

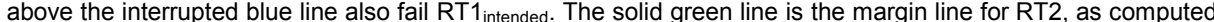
in Table 4. The predecessor-successor pairs (L2PRED, L2sucC) in the green region below it fail RT2 because L2 succ-L2PRED is below -1026 units of load (-36936 units of length). The (L2PRED, L2succ)-pairs in the white region between the blue and green lines satisfy both relative timing constraints RT $1_{\text {conservative }}$ and RT2.

\footnotetext{
${ }^{7}$ The length unit here and in [1] is ' $\lambda$ ' (lambda): 1/20-th of a micrometer in the TSMC 90 nanometer process used for Infinity.
}

ARC\# 2009-smg01 Asynchronous Research Center

page 9 of 11 
In addition to the allowable module distances and their relation to the key relative timing constraints, Figure 3 shows two other interesting facts.

1. The red equidistance line where L2PRED and L2 succ have the same load and length falls in the PASS region, approximately midway between the margin lines for RT1 $1_{\text {intended }}$ and RT2. This is good news: it makes designing GasP systems easier because the designer can assume equidistant module distances and still leave adequate margins to place and route the modules.

2. The orange-striped square box of about $333 \times 333$ units of load in the bottom-left corner of the graph represents the short-to-medium length wire region for the TSMC 90 nanometer CMOS manufacturing process that was used for Infinity. In this process, 333 units of wire load correspond to approximately 12000 units of wire length. This is the comfort region for the lumped capacitance wire model.

The anticipation is that for wires longer than 12000 units, the wire resistance is no longer negligible, and neither are the rise and fall times of the wire transitions. To mitigate these long wire effects, one can insert extra 6-4 GasP modules, called repeater modules, at state wire intervals of 12000 units of length.

Note that the orange-striped box is completely within the white PASS region of Figure 3. So, apparently, the repeater constraints to avoid long wires are much stricter than the 6-4 GasP relative timing constraints for functional correctness. ${ }^{8}$

\section{Conclusion}

The 6-4 GasP modules discussed in this paper are used to route data. The operation of each module is limited by relative timing constraints. In [1] Joshi et al. identified two key constraints, called RT1 and RT2, that limit the module distance between successive GasP modules in the router network. They also set up a framework to validate whether the distances from a 6-4 GasP module to its predecessor and successor are allowed.

In this paper, I introduce a new representation, the Distance Constraint Graph, to visualize the relation between the allowable module distances and the relative timing constraints RT1 and RT2. By looking at the graph one can immediately locate the pass and fail regions for each constraint for up to 15000 load units, i.e. 540000 length units.

There is one caveat: my current calculations ignore long wire effects - these will be taken into account in a follow-up paper. ${ }^{9}$

${ }^{8}$ I surely will investigate this further in my long-wire analysis.
${ }^{9}$ See also footnotes 5,6 and 8 .

ARC\# 2009-smg01 Asynchronous Research Center page 10 of 11 
Using the Distance Constraint Graph, it is immediately clear that the short to medium range module distances used in the 90 nanometer Infinity test chip are safe. Infinity uses up to 150 units of load, or 5400 units of length. Distances of length 5400 are safe regardless of the difference in distances to predecessor and successor modules.

Figure 4 offers an enlarged view of the orange-striped 6-4 GasP operating region from Figure 3 with short-to-medium range module distances.

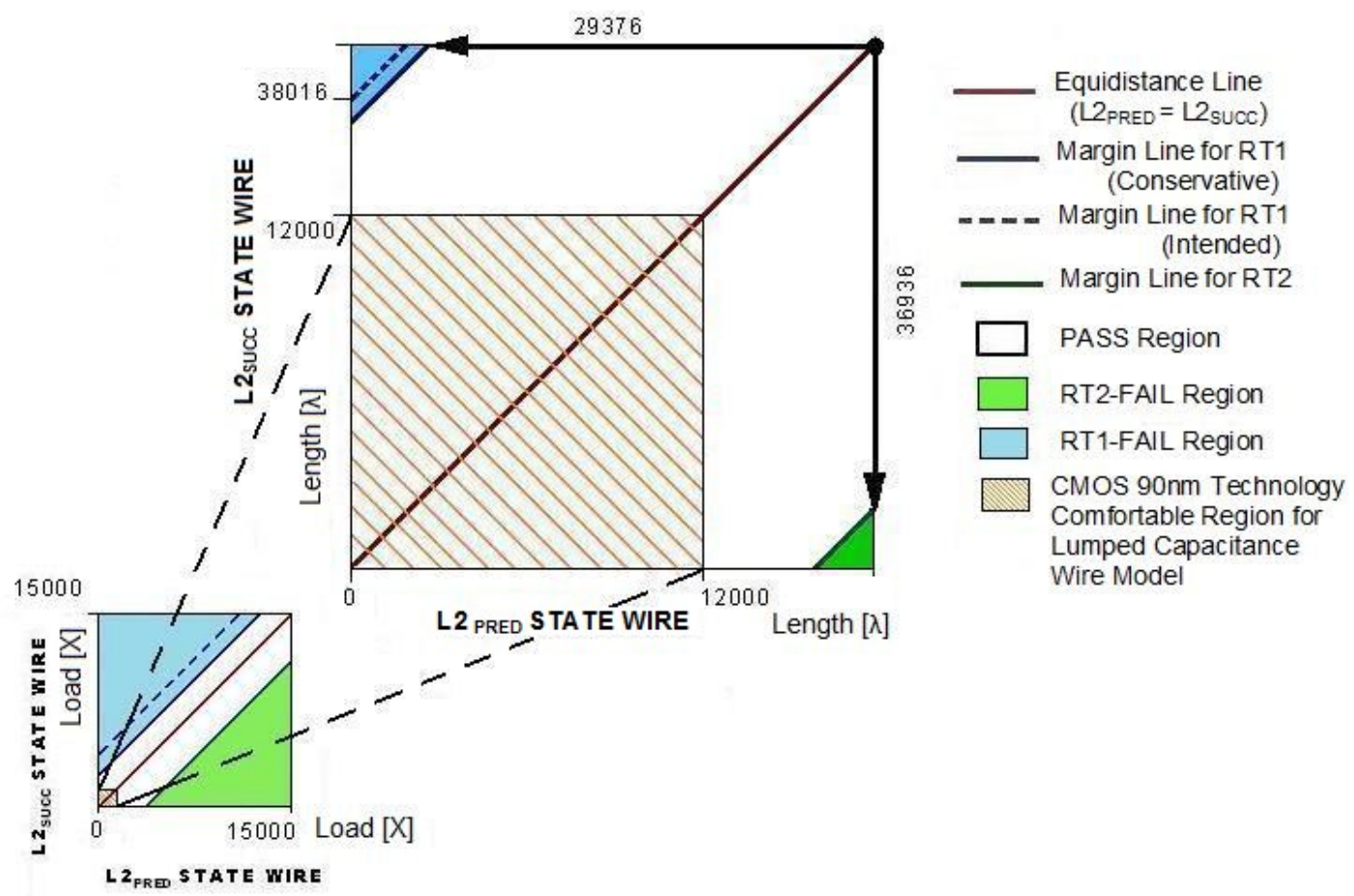

Figure 4 (Detail of Figure 3) Detailed segment of the Distance Constraint Graph for short-to-medium length module distances for 6-4 GasP in TSMC's 90 nanometer CMOS manufacturing process. The short-to-medium length distances are marked by the orange -striped region of $12000 \times 12000$ units of wire length. Note that the orange-striped region falls completely inside the white (PASS) region.

I anticipate that the long wire effect will kick in somewhere near the top and right boundaries of the orange-striped region. From there on, the margin lines for RT1 and RT2 will likely no longer be constant. My next research topic is to investigate how exactly the margin lines will change in relation to the individual module distances and in relation to the relative distance between predecessor and successor modules. 


\section{Appendix 0}

\section{BONUS MATERIAL: SHORT-TO-MEDIUM-TO-LONG WIRE STUDY}

(C) 2010 IEEE. Reprinted with permission, from the following publication source:

- Swetha Mettala Gilla, Marly Roncken and Ivan Sutherland. Long-Range GasP with Charge Relaxation. In Proc. IEEE International Symposium on Asynchronous Circuits and Systems (ASYNC), pages 185-195, 2010.

This IEEE publication can also be found in the thesis reference list as reference [18]. 


\title{
Long-Range GasP with Charge Relaxation
}

\author{
Swetha Mettala Gilla, Marly Roncken, and Ivan Sutherland \\ mettalag@pdx.edu,mroncken@cecs.pdx.edu, ivans@cecs.pdx.edu \\ Asynchronous Research Center \\ Maseeh College of Engineering and Computer Science \\ Portland State University, Portland, Oregon, USA
}

\begin{abstract}
GasP circuit modules communicate handshake signals in two directions over a single state wire. The 2008 Infinity test chip demonstrated GasP in $90 \mathrm{~nm}$ CMOS operating at four giga data items per second, but revealed that state wires about 5000 lambda long retard operation by about $10 \%$.

Simulations reported in this paper show that GasP modules will tolerate surprisingly long state wires, albeit at reduced throughput. The modules appear to operate correctly with state wires whose delay exceeds the drive time. With such long wires, the receiving module waits until passive distribution of charge brings the wire within range of the receiver's switching threshold. Having put enough charge into the wire, or vice-versa removed enough charge from it, the sending module may proceed with its next task. This result applies equally to other single-track signaling methods.

This behavior calls for a new kind of relative timing constraint to address when the wire charging or discharging process may cease rather than when the signal reaches the far end of the wire.
\end{abstract}

Keywords: GasP, Infinity chip, long wires, single-track, on-chip communication, self-timed, asynchronous circuits.

\section{Introduction}

Since publication of the first GasP family of circuits in 2001 [15] we have built and tested a variety of GasP chips. One chip experiment, called Infinity, consists of two rings of 100 GasP modules each that share a common section of 50 modules. The GasP modules in the Infinity test chip, which includes the Infinity experiment, form the basis of a wide variety of network-on-chip topologies. A sketch of the layout of the Infinity experiment appears in Figure 1(a).

Figure 1(b) offers a canopy diagram for separate operation of the two rings in Infinity. For this experiment data circulate in a single ring, avoiding contention for use of the
50 -stage shared section. The work reported in this paper began with the observation of the flat tops in this (measured) canopy diagram. The maximum throughput indicated by the two flat tops is about $10 \%$ below the peak throughput in Figure 1(c) as measured for other GasP rings on the chip. ${ }^{1}$

Additional experiments traced the $10 \%$ loss in throughput observed in Infinity to the longer state wires that connect the common ring section in the center column of Figure 1(a) to the individual ring sections in the side columns. The state wires from column to column are about 5000 lambda long, whereas the state wires within each column are only about 500 lambda long. This phenomenon prompted us to study the effect of wire delays in GasP.

Our first study, published by Joshi et al. in [5], uses a lumped capacitance model to examine the impact of a long state wire. It presents a logical effort model to compute the gate and path delays in GasP operations and to analyze the relative timing constraints on which the correct operation of a GasP module depends $[16,12]$. We observed that if the main effect of the long wire is to retard its driver, GasP modules should operate correctly over a large range of distances. Our analysis predicts correct operation provided the difference in the distances to predecessor and successor modules is limited. It predicts failure if these distances differ by too much. Moreover, the extra delay computed for the lumped capacitance model of a 5000 lambda long wire explains the $10 \%$ loss in throughput observed in Infinity.

However, the predicted maximum difference in module distances in the study by Joshi et al. is large enough to cast doubt on the adequacy of the lumped capacitance wire model, in particular for wires longer than 5000 lambda. Therefore, we started a second study that uses better models

\footnotetext{
${ }^{1}$ Silicon experiments and simulations in this paper all use a $90 \mathrm{~nm}$ CMOS process by TSMC in which lambda $(\lambda)$ is $50 \mathrm{~nm}$ and tau $(\tau)$ is about 8 psec. We prefer to use the process-normalized metrics lambda and tau rather than absolute metrics so as to make our results more readily applicable to other manufacturing processes.
} 


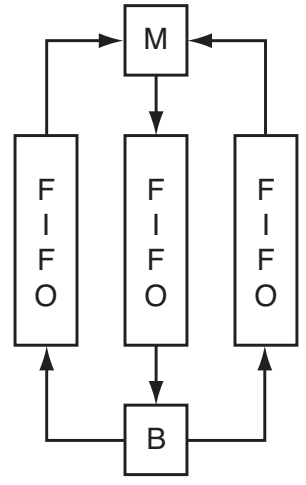

(a)

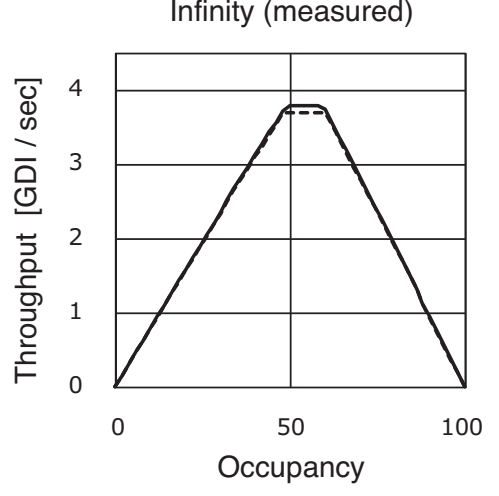

(b)
FIFO ring (measured)

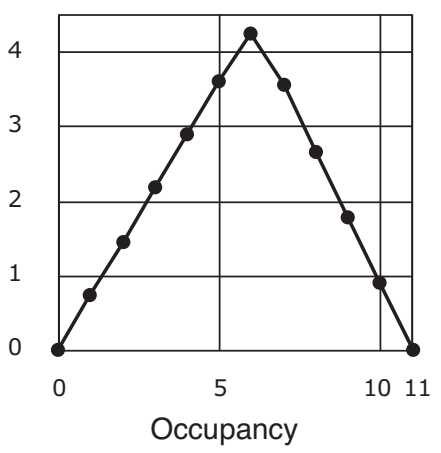

(c)

Figure 1 (a) Layout sketch of Infinity, and canopy diagrams for (b) Infinity and (c) an 11-stage FIFO ring on the same test chip. About (a)

The Infinity experiment has two rings of $100 \mathrm{GasP}$ modules each, with a common section of 50 modules. The experiment is approximately 17500 lambda tall by 15000 lambda wide, an area of about $0.6 \mathrm{~mm}^{2}$. Infinity got its name because its layout resembles the infinity symbol $\infty$. The chip was named after the Infinity experiment, which is the largest experiment on it. Infinity is laid out as three columns about 17500 lambda tall of 50 modules each, as shown. Most modules are about 4608 lambda wide and 288 lambda tall, although some are slightly taller. Each module carries 37 data bits plus 15 address bits. An addressable branch module $\mathrm{B}$ at the bottom end of the center column uses an address bit to steer arriving data to the left or right. A demand merge module $\mathrm{M}$ at the top of the center column accepts data from the side columns on a first-come-first-served basis. Additional test circuitry, omitted here, enables us to load and unload the rings with data and to count the number of data elements passing through each ring. A typical experiment involves loading the rings with a chosen number of data elements, running the circuit for a known time, and then reading the counts and unloading the data for inspection. Every experiment in the chip has observed flawless retention of data counts, sequence, and content.

About (b)-(c)

The canopy diagrams in (b) and (c) show the throughput of GasP rings in relation to their occupancy i.e. the number of data elements in the ring. The throughput is the ratio of the count of data elements that passed through the ring to the run time of the experiment. We have observed maximum throughputs over four giga data items per second $(4 \mathrm{GDI} / \mathrm{sec})$. The canopy diagram for the FIFO ring in (c) shows a peak throughput of $4.2 \mathrm{GDI} / \mathrm{sec}$. The solid- and broken-line canopy diagrams for the individual ring operations in Infinity in (b) have a flat top at about $3.8 \mathrm{GDI} / \mathrm{sec}$. A canopy diagram with a flat top indicates the presence of a slower than normal stage. The culprits for the $10 \%$ loss of throughput are the relatively long state wires between the side columns and the center column. The column-to-column state wires are about 5000 lambda long, which is ten times longer than the 500 lambda long state wires within each column. All state wires are slightly longer than the center-to-center distance between modules in order to reach multiple connections inside each module. The measurements that identify the long wires as the culprits can be found in [5], together with a theoretical foundation based on logical effort and a lumped capacitance wire model.

to describe the properties of longer wires. Our second study uses a distributed RC model [11, 17], capable of distinguishing voltage levels at the near and far ends of the state wire. For GasP, this matters, because the two ends play different roles in the handshake signaling over the state wire.

We were particularly eager to understand not only the impact of different near and far end delays in the state wires of GasP, but also the implications for the relative timing constraints that we must impose on the GasP circuit modules to ensure their correct operation.

This paper reports the results of our second study. Section 2 introduces the 6-4 GasP family used in Infinity and in our studies, and explains its communication mechanism. Given that neither Infinity nor the 6-4 GasP control circuits have been published before, Section 2 acts more as a tutorial on GasP than as a description of previously published work. ${ }^{2}$ Section 3 discusses the key relative timing constraints for long wire communication in GasP. Section 4 describes our simulation setup to analyze the potential and limitations for long-range GasP, and presents the results. Section 5 summarizes the results of both studies. We use a twodimensional graph, the Distance Constraint Graph [6], to help visualize how the results differ. Conclusion and work in progress follow in Section 6.

\footnotetext{
${ }^{2}$ Our previous publication [5] gives a partial description of 6-4 GasP, focusing only on key facets of its data transfer mechanism and ignoring keepers and other GasP design aspects presented here.
} 


\section{GasP Modules}

Infinity uses 6-4 GasP [14]. The 6-4 GasP family gets its name from the six logic gates on the path going forward from one module to the next, path $\mathrm{ABCDEF}$ in Figure 2, and from the four logic gates on the path going backwards, path $\mathrm{ABCX}$. The longer delay is in the forward direction because that is the direction in which the data elements are transferred. Copying data requires action on the part of the data latches, whereas moving an empty space or "bubble" backwards to declare the latches empty needs no action.

Figure 2 shows a 2-stage FIFO, with one 6-4 GasP module per stage. The two stages, $\mathrm{M}_{1}$ and $\mathrm{M}_{2}$, are connected by a bidirectional state wire L2 via ports $\mathrm{SUCC}_{1}$ and $\mathrm{PRED}_{2}$. When state wire $\mathrm{L} 2$ is high it indicates that $\mathrm{M}_{1}$ has new data for $\mathrm{M}_{2}$. We will refer to this as L2 being full. When state wire $\mathrm{L} 2$ is low it indicates that $\mathrm{M}_{2}$ has latched the proffered data and it is safe for $\mathrm{M}_{1}$ to change its data. We will refer to this as L2 being empty. ${ }^{3}$

When $\mathrm{PRED}_{2}$ is high and $\mathrm{SUCC}_{2}$ low, meaning $M_{1}$ has new data for $M_{2}$ and $M_{2}$ has space for it, $M_{2}$ raises its signal FIRE 2 . A high FIRE 2 signal starts three parallel actions: (1) the data latches of stage $\mathrm{M}_{2}$ are enabled to copy the proffered data, and (2) $\mathrm{SUCC}_{2}$ is raised via gates D and $\mathrm{E}$ to indicate to $\mathrm{M}_{2}$ 's successor that new data are available, and (3) $\mathrm{PRED}_{2}$ is lowered via gate $\mathrm{X}$ to declare $\mathrm{L} 2$ empty.

Note that for $\mathrm{FIRE}_{2}$ to rise, gate A must synchronize a high $\mathrm{PRED}_{2}$ signal with a low $\mathrm{SUCC}_{2}$ signal. But to get $\mathrm{FIRE}_{2}$ to fall, either a low $\mathrm{PRED}_{2}$ signal or a high $\mathrm{SUCC}_{2}$ signal suffices. Hence, actions (2) and (3) each have the additional side effect of resetting FIRE $_{2}$.

So, setting FIRE $_{2}$ high automatically leads to resetting FIRE $_{2}$ low, and there is a choice of two self-resetting loops that automate this: the forward loop DEABC, and the backward loop XFABC. Note that each loop has five gates.

The gate count matters. GasP modules are custom-designed using logical effort [16]. The transistors in each logic gate are sized such that all logic gates have approximately the same delay. Gate sizing takes into account both the transistor sizes of the driven gates and the loads of the connecting wires. As a result, we can count delay in terms of logic gate delays and we can compare path delays simply by counting and comparing the number of gates on each path.

This works as long as we can adequately predict the wire lengths. Gates A, B, D and F in Figure 2 each drive a single local gate, and so we may assume that the connecting wire lengths are known in advance.

\footnotetext{
${ }^{3}$ This paper addresses primarily the control portion of 6-4 GasP that generates the handshake operations between GasP modules. Other than giving an intuition of the data bundling relation between the handshake signaling on state wire L2 and the data being transferred, we omit any further discussion of data validity.
}

The situation for gate $\mathrm{C}$ is more complicated: $\mathrm{C}$ drives two local gates, $\mathrm{D}$ and $\mathrm{X}$. In addition, $\mathrm{C}$ drives a number of latches and the not so local wire L1 between gates D and $\mathrm{X}$ and the latches. In Infinity, the data latches are placed near the 6-4 GasP module that controls them. GasP module and latches together form a macro module with a fixed wire length for L1. This solves the gate sizing problem for C.

The exceptions are gates $\mathrm{E}$ and $\mathrm{X}$ that drive the $\mathrm{L} 2$ state wire. The length of $\mathrm{L} 2$ depends on the distance between the modules, and this is unknown until the system layout has been finalized. If $E$ and $X$ are sized using the wire lengths for A, B, D and F, as was done in Infinity, the gate delays for $\mathrm{E}$ and $\mathrm{X}$ will vary with different module distances and the throughput will vary accordingly, as demonstrated by the canopy diagrams in Figure 1(b)-(c).

So, without further investigation into the effects of long state wires, all we can say is that, for short state wires, the maximum throughput of 6-4 GasP is the throughput that corresponds to a cycle time of 10 gate delays: 6 to forward the data and 4 to move the empty space backward. The investigation with long state wires follows in Section 4, and is easier to understand after reading the design details on state wires and keepers coming up next.

\subsection{State Wires}

GasP state wires use a form of forward and back handshake protocol, called "single-track" signaling [1, 15, 9, 2]. In a single-track protocol, a single wire carries both the forward and the backward handshake signals. This is attractive, not only because a single wire occupies less space, but also because the handshake consumes minimum energy per cycle. To handshake, a transition must pass in each direction. The single wire does exactly that, with automatic return to the initial state after each pair of handshake signals.

The GasP modules in this paper use single-track handshake signaling to communicate control information only. They use single-rail signaling to communicate data, as do the handshake circuits discussed by van Berkel and Bink in [1]. The single-track designs discussed by Nyström et al. in [9] combine both the data and the control into a single-track communication protocol, as do Ferretti and Beerel in [2].

There are various ways to implement single-track signaling; van Berkel and Bink differentiate between "dynamic" and "overlapping" protocols [1]. Each participant in a singletrack signaling protocol must cease to drive the state wire soon enough to make room for the action of the other participant. Were one participant to drive the wire for too long, then both might end up driving it concurrently in opposite directions, consuming unnecessary energy and producing an indeterminate logic signal. How is this to be avoided? 


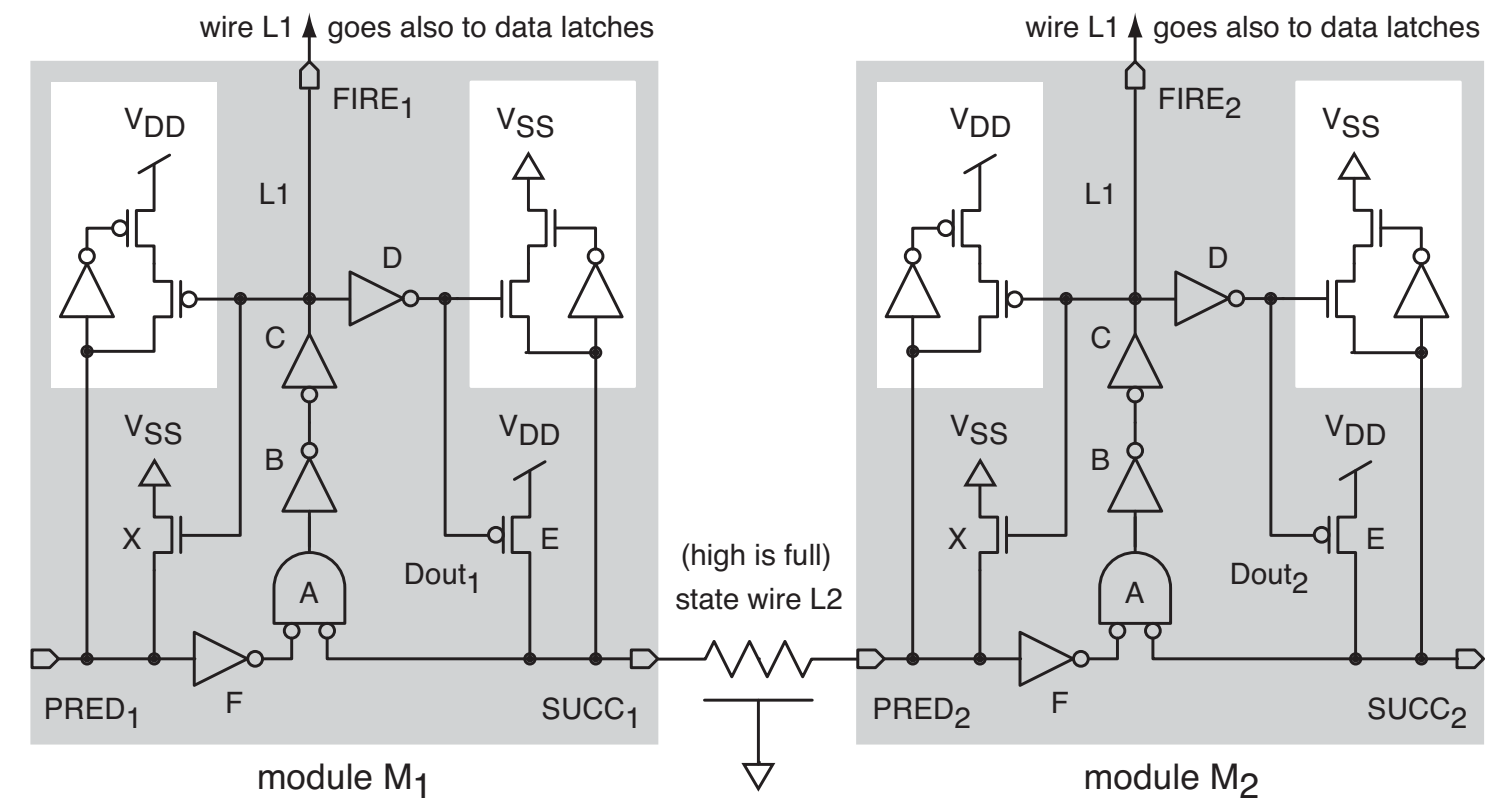

Figure 2 A two-stage FIFO with 6-4 GasP modules $\mathrm{M}_{1}$ and $\mathrm{M}_{2}$ connected by a state wire L2. The picture omits the additional state wires to the left and right of the modules shown. It also omits the data wires and latches. The transistors in each logic gate are sized such that all gates have approximately the same delay. The designation "6-4" refers to the forward-backward latencies. The forward latency from gate $\mathrm{A}$ in $\mathrm{M}_{1}$ to gate $\mathrm{A}$ in $\mathrm{M}_{2}$ is 6 gate delays, and is covered by the path $\mathrm{ABCDEF}$. The backward latency from gate $A$ in $M_{2}$ to gate $A$ in $M_{1}$ is 4 gate delays, and is covered by the path $A B C X$. This gives a cycle time of 10 gate delays. In addition to this global cycle time, each 6-4 GasP module has two local self-resetting cycles of 5 gate delays: the forward loop goes through ABCDE and the backward loop through ABCXF. The AND function A acts when (a) the predecessor state wire is high, indicating a full predecessor state with data, and (b) the successor state wire is low, indicating an empty successor state with space. The action produces a high pulse on the module's FIRE signal. The FIRE pulse renders the data latches transparent for a sufficiently long duration to capture the data. It also renders the predecessor state wire empty via transistor X, and it renders the successor state wire full via Dout and transistor E. By the latter two actions the FIRE signal shuts itself off, cutting its pulse time down to 5 gate delays. Half keepers, shown in the white insets, use small transistors to retain the voltage on the state wire when drivers $\mathrm{X}$ and $\mathrm{E}$ are off. The distributed RC symbol in the L2 state wire refers to the wire model that we use in this paper [11].

In the overlapping protocol, implemented by van Berkel and Bink, this is avoided because each participant drives the wire "long enough" for it to pass some threshold voltage that will alert the other participant who then takes over the driving role. The overlapping protocol fits in the quasi delay-insensitive design style deployed by Philips and Handshake Solutions [10].

GasP uses a dynamic implementation, which is simpler but relies on the relative timing of logic gates to avoid drive conflicts at the state wire. In GasP, the sender "briefly" drives the wire to one logic level, signaling the presence of data on adjacent data wires. The receiver, noticing the change in the wire's state, copies the corresponding data and then briefly drives the wire to the other logic level to indicate that it has absorbed the data values.
The single-track advantages in GasP are offset by a timing requirement inherent in the word "briefly". Because sender and receiver drive the shared state wire in opposite directions, each must take care to cease its drive promptly so that the other has free use of the wire. For a 6-4 GasP module, "briefly" means "about 5 gate delays" or half the minimum cycle time. This is where the local self-resetting loops in Figure 2 come into play, as will become clear by following one single-track handshake over state wire L2:

- Sending module $\mathrm{M}_{1}$ drives L2 high via transistor E. After $\mathrm{M}_{1}$ starts driving, it also promptly ceases driving via its 5 gate-delay forward self-resetting loop $\mathrm{ABCDE}$. On the receiving end, it takes at least 5 gate delays for $\mathrm{M}_{2}$ to sense a high voltage level on L2 and invert it through FABCX to drive L2 low. 
- After $\mathrm{M}_{2}$ starts driving L2 low via transistor X, it also promptly ceases driving via its 5 gate-delay backward self-resetting loop FABCX. On the reverse end, it takes at least 5 gate delays for $\mathrm{M}_{1}$ to sense a low voltage level on $\mathrm{L} 2$ and invert it via $\mathrm{ABCDE}$ to drive $\mathrm{L} 2$ high.

- Except for their separation in space, the P and $\mathrm{N}$ type driving transistors $\mathrm{E}$ and $\mathrm{X}$ act as the two halves of an inverter. However, the crossover current that exists when one transistor's drive turns on and the other's turns off is at least as low as that of an inverter with a shared drive. The crossover current diminishes with longer state wires, as we will explain from the waveforms in Figure 5, later in this paper.

The state wires in GasP carry state: they record the full or empty state of the communication link. To retain the state for arbitrary periods of time, e.g. when there is a shortage of data or of bubbles, there are so-called keepers on the state wire. These are discussed in Section 2.2.

\subsection{Keepers}

GasP modules include small keepers to retain the charge on state wires in the face of noise or leakage. Each module in Figure 2 includes a half-keeper on each state wire that it can drive. Each half-keeper will keep its state wire either only high or only low. It takes two half-keepers working together to retain the state on the wire. The two half-keepers involved are always on opposite ends of the state wire. For instance, the half-keeper that is responsible for keeping L2 low resides in module $\mathrm{M}_{1}$ which drives $\mathrm{L} 2 \mathrm{high}$. And vice versa, the half-keeper that is responsible for keeping L2 high resides in module $\mathrm{M}_{2}$ which drives $\mathrm{L} 2$ low.

The choice of "drive one level, keep the other" makes it easy to shut off the half-keeper right at the start of the drive. Module $\mathrm{M}_{1}$ uses internal control signal Dout ${ }_{1}$ to shut off its half-keeper for L2 while driving the state wire in the opposite direction, namely high. At the other end of the state wire, module $\mathrm{M}_{2}$ uses control signal $\mathrm{FIRE}_{2}$ to shut off its local half-keeper while driving L2 in the opposite direction, namely low. Shutting off the half-keeper right at the start of the drive avoids the energy loss that would occur were state wire L2 driven high while its half-keeper attempts to hold it low, or vice versa.

It is a happy result of the "drive high, keep low" and "drive low, keep high" arrangements in $\mathrm{M}_{1}$ and $\mathrm{M}_{2}$ that the halfkeepers themselves help drive state wire L2 in a useful way. When module $\mathrm{M}_{1}$ starts to drive L2 high, it immediately shuts off the half-keeper at its end of the state wire. The half-keeper in $\mathrm{M}_{2}$ at the other end is also shut off, because that end of L2 is still low.
Only when the far end of L2 reaches the switching voltage of the receiving gate does the half-keeper at the far end turn on, and in doing so assist in driving and ultimately keeping state wire L2 high. This effect, although weak, is observable in the waveforms of Figure 5 shown later in this paper.

\section{Relative Timing Constraints}

The 6-4 GasP circuits in the Infinity test chip were validated using the SPICE electrical simulation tool. We simulated the behavior of each and every transistor, wire capacitance, and logic function. This was a fairly laborious and computeintensive process. The results of these simulations showed proper operation over the range of state wire lengths actually needed in the test chip. Now that we know that the test chip works, we want to use the GasP modules in more complex designs. In particular, we want to use standard commercial static timing analysis tools and automatic placement and routing software to inspect timing margins and to drive the layout. This requires a deeper understanding of the conditions that make GasP work.

Our new approach to timing validation in GasP is based on static timing analysis of relative timing constraints $[4,13]$. Intuitively, relative timing constraints describe the order in which two signals must arrive at a point of convergence.

The key relative timing constraints for short to medium to long wire communication in GasP come from the presence of the two local self-resetting loops. These loops turn off the communication drive signal to the state wire and we must avoid letting them turn the drive off prematurely.

Take for instance module $\mathrm{M}_{1}$ in Figure 2. When gate $\mathrm{C}$ rises to start the FIRE 1 pulse, both loops DEABC and XFABC act to end the FIRE 1 pulse and this results in ending the drive on both $\mathrm{M}_{1}$ 's state wires. To guarantee that each state wire is driven long enough to complete its communication, we compare the communication delay to the self-resetting loop delays and require the former to beat the latter. Delays are counted from the start of the module's FIRE pulse. This produces four relative timing constraints per state wire.

For example, state wire L2 in Figure 2 produces the following four relative timing constraints:

- RT(L2) TransferForward: The forward transfer delay to drive both ends of L2 high through DE in module $\mathrm{M}_{1}$ is shorter than the forward self-resetting loop delay through DEABCDE in $\mathrm{M}_{1}$ to cease the drive.

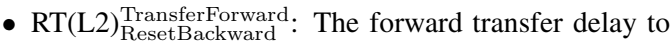
drive both ends of L2 high through DE in module $\mathrm{M}_{1}$ is shorter than the backward self-resetting loop delay through XFABCDE in $\mathrm{M}_{1}$ to cease the drive. 


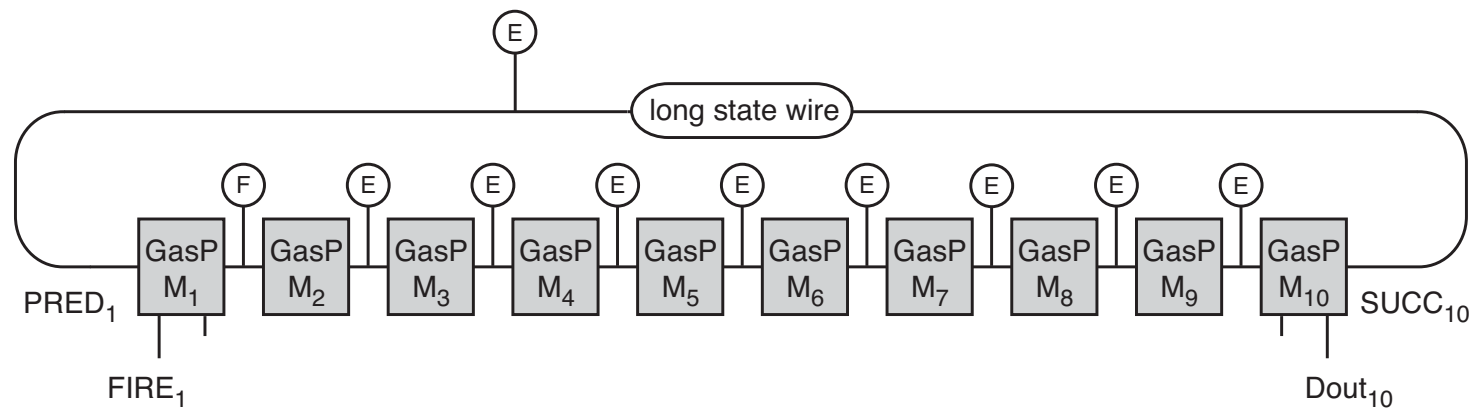

Figure 3 Our long-range GasP simulation set up includes a ring of ten 6-4 GasP modules, $\mathrm{M}_{1}$ to $\mathrm{M}_{10}$, connected by nine short state wires and one long state wire. The circles with designation $\mathrm{E}$ or F indicate the initial states of the state wires: E for empty, i.e. low, and $\mathrm{F}$ for full, i.e. high. The ring in this picture starts with one full state wire. The picture explicitly indicates the GasP signals PRED 1 and FIRE $_{1}$ of module $\mathrm{M}_{1}$ and $\mathrm{SUCC}_{10}$ and Dout 10 of module $\mathrm{M}_{10}$. These are the signals that play a direct role in the handshake communication over the long wire. The picture omits the corresponding signals for the other 6-4 GasP modules.

- RT(L2) TransferBackward: The backward transfer delay to drive both ends of L2 low through X in module $\mathrm{M}_{2}$ is shorter than the forward self-resetting loop delay through DEABCX in $\mathrm{M}_{2}$ to cease the drive.

- RT(L2) TransferBackward: The backward transfer delay to drive both ends of L2 low through $\mathrm{X}$ in module $\mathrm{M}_{2}$ is shorter than the backward self-resetting loop delay through XFABCX in $\mathrm{M}_{2}$ to cease the drive.

The M.Sc. thesis by Prasad Joshi [4] shows how to validate relative timing constraints for a given GasP design using PrimeTime, the Synopsys static timing analysis tool.

\section{Long-Range GasP Simulations}

To develop our understanding of long state wires in GasP we undertook a series of simulations.

First, we simulated the behavior of isolated long wires using a distributed RC model. We used four R-C-R sections for every 100 lambda of wire, matching the corresponding $90 \mathrm{~nm}$ TSMC wire resistance and capacitance numbers for state wires. We simulated the wire delays for wire lengths ranging from 100 to 60000 lambda. We observed that the delay in the wire is, as expected, roughly quadratic with its length. However, for longer wires, the far end of the wire exhibits very slow rise times which renders the precise meaning of "wire delay" suspect. We observed reasonable delays for wires up to 30000 lambda, or $1.5 \mathrm{~mm}$, long.

Armed with these observations, we set up simulations of 6-4 GasP modules separated by long state wires. Each simulation involves a ring of ten 6-4 GasP modules with nine short and exactly one long state wire. Different initializations of the state wires permit us to generate a canopy diagram to exhibit the impact of the long wire on throughput. The two extreme initializations for data-limited rings, with exactly one full state wire, and for bubble-limited rings, with exactly one empty state wire, will give us a larger time window to observe the dynamic aspects of the long wire and its keepers. Figure 3 shows the simulation set up for the data-limited case with exactly one full state wire.

We simulated each initial configuration with the long state wire set at a length of 1000, 4000, 10000, 20000, 24000, and 30000 lambda. To get reasonable simulation times, we used three R-C-R sections for every 1000 lambda of wire. This still produces a sufficiently fine-grained wire model. All simulated configurations with long state wire lengths up to and including 24000 lambda run correctly.

For the 30000 lambda long state wire we observed failures in the two extreme initialization scenarios with (a) exactly one full state wire and (b) exactly one empty state wire:

(a) When we start with one full state wire, initialized as in Figure 3, we lose the full state during the first handshake over the 30000 lambda long state wire, because module $\mathrm{M}_{10}$ shuts off its high drive before the wire has accumulated enough charge to rise from ground level to the switching voltage level of $\mathrm{M}_{1}$. The ring, now completely empty with nothing to work on, deadlocks.

(b) When we start with one empty state wire, or bubble, we see not a loss of the one and only bubble but the creation of an extra bubble. This is due to the fact that we initialized the ring with the empty state at the long state wire. During the first handshake over the 30000 lambda long state wire, module $\mathrm{M}_{10}$ behaves exactly like it did in the previous scenario: it shuts off its high drive before the wire has accumulated enough charge to rise from ground level to the switching level of $\mathrm{M}_{1}$. And so, we end up with two bubbles instead of one. With two bubbles, the round trip delay becomes just short enough to keep the ring going. 

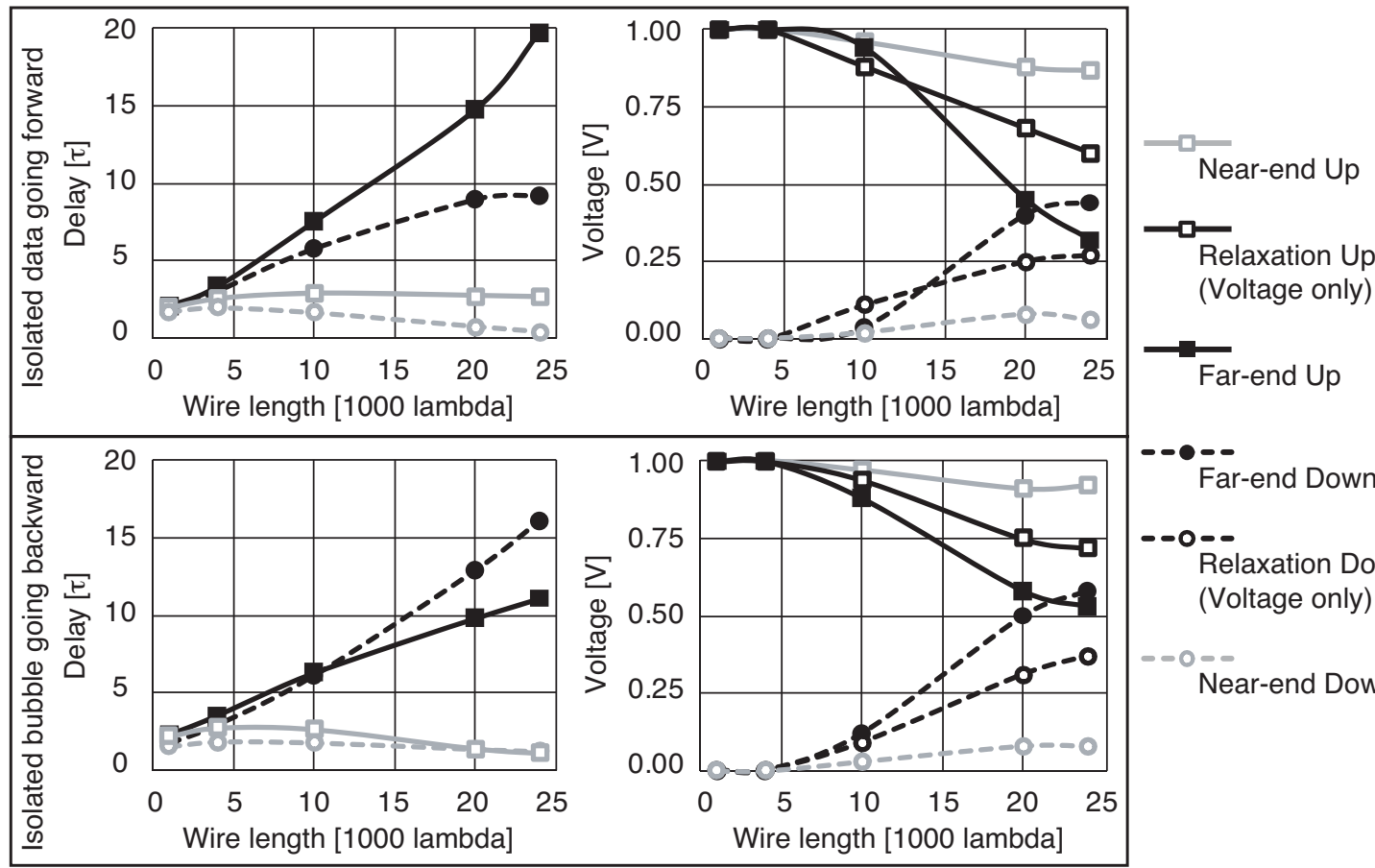

- - -

Far-end Down

$-0--$

Relaxation Down (Voltage only)

Near-end Down

Figure 4 Simulated delays for rising and falling transitions and their voltage levels at the near and far end of the long state wire. The top window shows the results for the simulation set up in Figure 3 with one full state wire. We measured the delays and voltages during a full-to-empty single-track handshake consisting of a rising transition followed by a falling transition in the reverse direction. The bottom window contains similar measurements for the opposite scenario with one empty state wire. The bottom measurements track an empty-to-full single-track handshake, consisting of a falling transition followed by a rising transition in the reverse direction. Notice how the delays and voltage levels at the near and far ends of the wire start to deviate for state wires longer than 5000 lambda. We measured the voltage levels at the end of the drive pulse, and again after charge relaxation when both ends have the same voltage. Notice how the 20000 and 24000 long state wires depend on charge relaxation to drive the far end above (top) or below (bottom) the 50\% voltage level and thus complete the first part of the handshake. Longer state wires have an easier task in completing the second part of the handshake, because there is less charge to take away (top) or add (bottom).

The rest of Section 4 focuses on the correctly functioning designs with long state wires up to 24000 lambda.

\subsection{Simulated Wire Delays and Voltage Levels}

We measured delays for rising and falling transitions in the long state wire at both the near end and the far end of the wire. The near and far end delays are measured from the time that the drive signal at Dout 10 or FIRE 1 reaches $50 \%$ of the supply voltage level to the times that the near and far ends of the wire reach $50 \%$ of the supply voltage level.

In dealing with GasP circuits, we have become accustomed to measuring time in gate delays. With that in mind, we calibrated all delays, including the wire delays, in terms of $\tau$, the normalized inverter delay with equal rise and fall times.
Figure 4 summarizes the measurement results that we obtained for the extreme simulation scenarios with one full state wire (top) and one empty state wire (bottom). We believe that these are the simulation scenarios that stress the GasP ring operations the most. The left-hand graphs show the normalized delays for transitions at the near and far ends of the long state wire. The right-hand graphs show the voltage levels at both ends, measured at the end of the drive pulse and again after charge relaxation.

For state wires up to 5000 lambda, the delays for rising and falling transitions take $2-4 \tau$, which is about one FO3 or FO4 gate delay, and about one gate delay in 6-4 GasP. The voltage levels change more or less simultaneously at both wire ends, with rail-to-rail voltage swings. The delay is almost entirely in driving the wire. Beyond 5000 lambda, delays and voltage levels start to deviate at the two wire ends. 


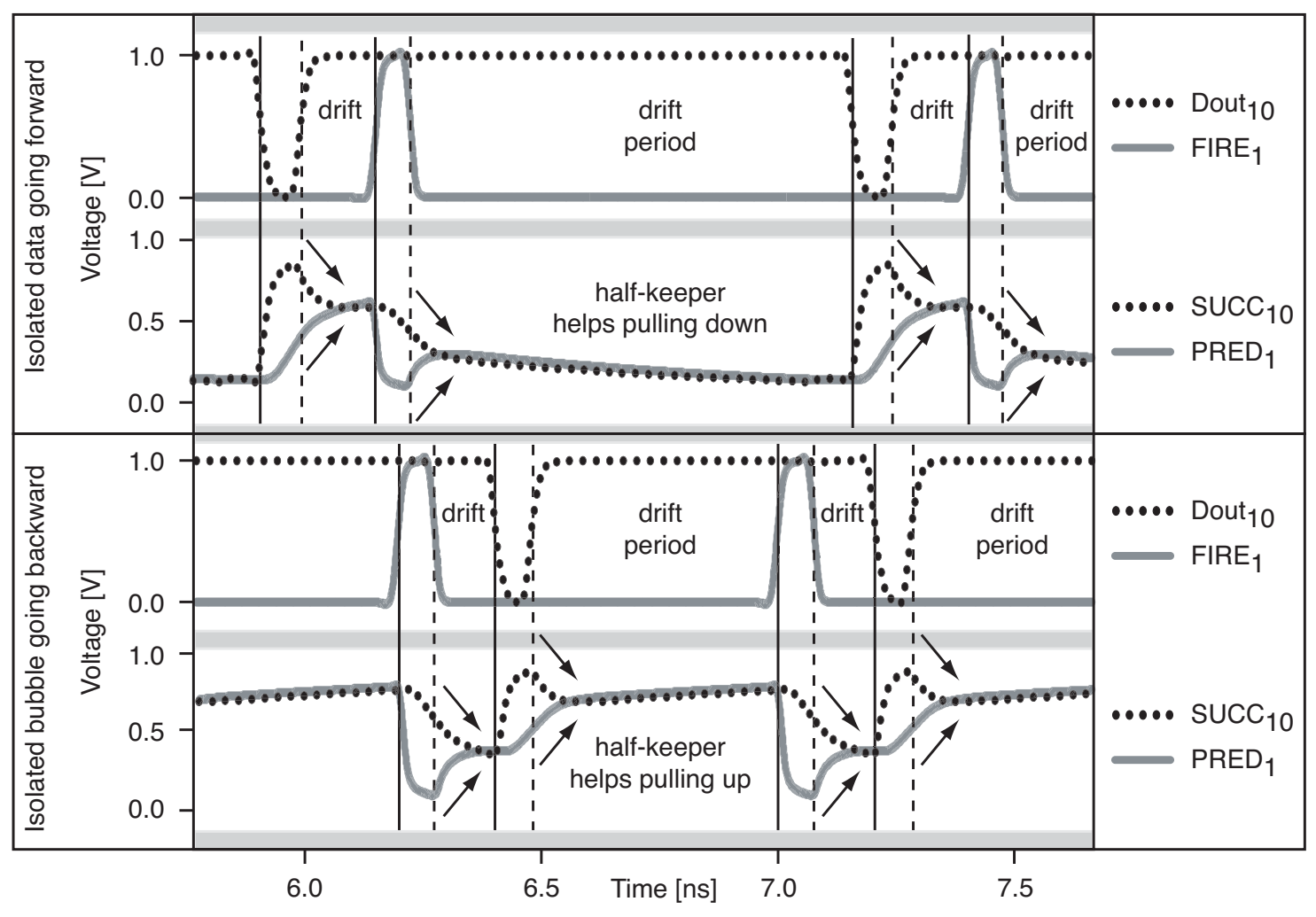

Figure 5 Voltage waveforms for the 10-stage GasP ring of Figure 3, with a long state wire of length 24000 lambda, and initialized with one full state wire (top) and with one empty state wire (bottom). The upper frames of each pair show the voltages to the $\mathrm{P}$ and $\mathrm{N}$ type drive transistors of the long state wire, viz. Dout ${ }_{10}$ and Fire ${ }_{1}$. The lower frames of each pair show the voltages at

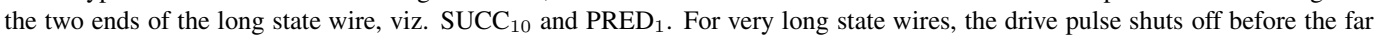
end of the wire reaches $50 \%$ of the supply voltage level. Thereafter, the near and far ends drift closer to the same voltage as the charge distributes along the length of the wire and relaxes into equilibrium. The half-keepers at the far-end turn on when the far end reaches its switching voltage, and in doing so assist in keeping the state wire at or closer to the chosen voltage level.

The delays and voltage levels in Figure 4 characterize the critical handshake communications over the long wire. The handshake communication in the top window starts with a low pulse on Dout ${ }_{10}$ that drives a rising transition over the wire, and is followed by a high pulse on $\mathrm{FIRE}_{1}$ that drives a falling transition back over the wire. The longest delay belongs to a rising transition over the 24000 lambda long state wire, and is about $20 \tau$. This delay is so long because the transition does not quite make it to the far end of the wire before the drive pulse turns off. In fact, when the drive pulse turns off, the far end has risen only to about $30 \%$ of the supply voltage level. After the drive ceases, the charge in the state wire distributes itself along the length of the wire and relaxes to reach an equilibrium at about $60 \%$ of the supply voltage level. It is the charge relaxation that completes the rising transition at the far end of the wire and in doing so enables the reverse handshake. Because the wire is only partly charged after the rising transition, the delay for the following falling transition is significantly shorter: it takes about $9 \tau$ to pull the far end back down to the $50 \%$ level.

The simulation results in the bottom simulation window of Figure 4 are similar. We expected the results in the top and bottom windows to be more symmetric. Upon inspection, we noticed that the gate delays in our 6-4 GasP modules are not as well matched as is possible with logical effort. The implications of both simulation results are similar, though.

Figure 5 shows the waveform details for the 24000 lambda simulations of Figure 4. As before, the top window gives the details for the simulation scenario with one full state wire, and the bottom window covers the simulation scenario with one empty state wire. 
The upper frames in both the top and bottom windows of Figure 5 show the waveforms for the drive pulses Dout 10 and FIRE $_{1}$. A low pulse on Dout 10 briefly drives P type transistor $\mathrm{E}$ in $\mathrm{M}_{10}$ and pulls the long state wire high at $\mathrm{SUCC}_{10}$. A high pulse on FIRE 1 briefly drives $\mathrm{N}$ type transistor $\mathrm{X}$ in $\mathrm{M}_{1}$ and pulls the long state wire low at $\mathrm{PRED}_{1}$. Each drive pulse lasts about 5 gate delays. The waveforms at the two wire ends follow in the lower frames.

Note that Dout ${ }_{10}$ is always higher in absolute voltage than FIRE $_{1}$. This indicates that transistors $\mathrm{E}$ and $\mathrm{X}$ are never both on at the same time. This behavior is characteristic of GasP circuits, even for short state wires. The result is that transistors $\mathrm{E}$ and $\mathrm{X}$ that act as the two halves of an inverter exhibit a lower crossover current than an inverter with shared drive. With longer state wires, the crossover current diminishes further and finally disappears, due to the extra wire delay that separates the drive pulses. There is no crossover current between $\mathrm{E}$ and $\mathrm{X}$ in the long state wire simulations of Figure 5.

The important message in Figure 5 is that the charge on the wire continues to distribute throughout the length of the wire even while the wire is undriven, i.e. even during the periods marked "drift". Thus, it suffices, during the available 5 gate delay period of drive, to insert or remove enough charge in the wire to ensure that its voltage will be clearly above or below its chosen threshold after the distribution finishes. The amount of charge that a long wire can accept in 5 gate delays is limited by the resistance of the wire rather than by the size of the driver. This is what limits the wire lengths of the uniform, minimum-width metal-2 or metal-3 state wires that we used in Infinity and in the simulation studies reported in this paper. As we pointed out earlier: this limit is somewhere between 24000 and 30000 lambda.

Charge distribution without drive, or "charge relaxation" as we call it in the title of this paper, permits GasP modules to use a single long wire for bi-directional handshake signals.

\subsection{Simulated Throughput}

The canopy diagrams in Figure 6 show how latency and throughput are affected by the long state wire.

Latency increases by the delay of the wire. With a longer wire, data and bubbles take longer for each trip around the ring because they must pass once through the long state wire. The increased latency lowers the left and right sides of the canopy diagram. This effect appears small because the long wire delay is a relatively small fraction of the total latency around the ring.

The long wire also limits throughput. The impact on throughput is pronounced because each and every element passing through the long wire must use it twice to execute

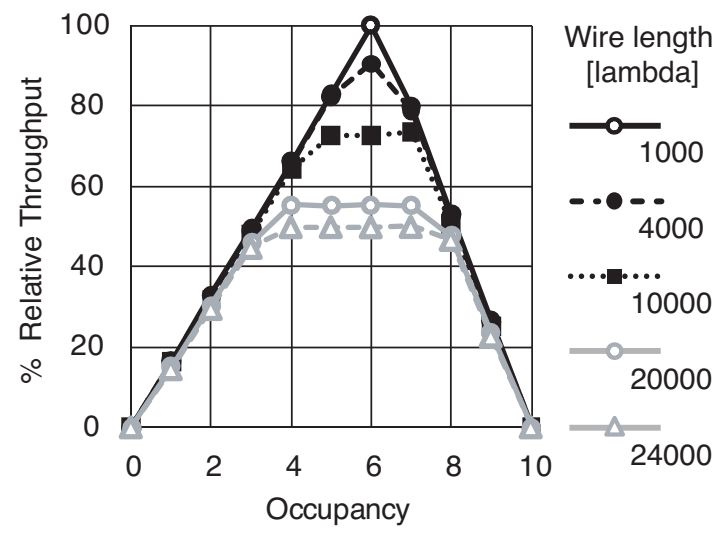

Figure 6 Simulated canopy diagrams for the 10-stage GasP ring of Figure 3 for various lengths of the long state wire. The lower sides show how longer wires slightly increase latency. The flat and lower tops show how longer wires dramatically decrease throughput.

a single-track handshake. Not only is the impact of the long state wire felt twice, but its effect accumulates because queued-up data elements and bubbles must wait for earlier handshakes to complete. This effect shows up dramatically as the flat and lower tops in the canopy diagrams for the longer state wires in Figure 6.

Note that the maximum throughput with the long state wire set to 24000 lambda is about half that of the maximum throughput with the long state wire set to 1000 lambda.

\section{Summary: Distance Constraint Graph}

Qualitatively, our simulations show that for sufficiently short state wires, the main impact of the wire is to retard the action of its driver. The capacitance of short wires dominates their resistance and so the driver "sees all of the wire". For such state wires, a larger driver can succeed in driving the wire faster. We can use logical effort to size the driver to provide an acceptable communication delay.

At some medium length of the state wire, however, increasing the wire length fails to retard further the action of its driver. In this regime, the driver saturates, pinning the near end of the wire to the power or ground rail. But the resistance of the wire itself limits the speed with which the far end of the wire can respond. In the $90 \mathrm{~nm}$ TSMC CMOS technology that we use, this regime sets in at 5000 lambda. In effect, the driver can no longer see the total capacitance of the wire, but only the capacitance of its near end. 


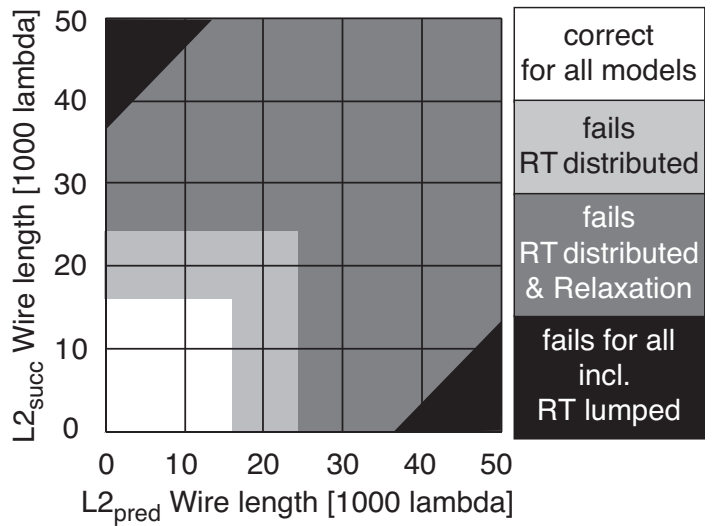

Figure 7 Distance Constraint Graph (90 nm CMOS), showing how the distance L2pred from a given 6-4 GasP module to its predecessor, and likewise $\mathrm{L} 2_{\text {succ }}$, from the module to its successor, are constrained according to: - (white area)

Relative timing under the distributed RC model, which predicts functionality for distances below 16000 lambda. - (light-grey area)

Charge relaxation, which predicts functionality for distances up to 24000 lambda.

- (everywhere except black area)

Relative timing under the lumped capacitance model, which predicts functionality, i.e. correct operation, when $\mathrm{L} 2_{\text {pred }}$ and L $2_{\text {succ }}$ differ by less than 37000 lambda.

At about 16000 lambda, the delays for rising and falling transitions to the far end of the wire begin to exceed the 5 gate delay self-resetting loops in the 6-4 GasP module. This is the point where the relative timing constraints of Section 3 begin to fail. The wire length is now long enough that the driver shuts off before the far end of the wire has risen or fallen to the $50 \%$ voltage level. We now enter the domain of long-range GasP and charge relaxation.

For long state wires, it is the charge on the wire that represents its state, not the voltage. Though the wire is a passive component, it acts through its charge, even when undriven. The charge in the wire distributes itself and relaxes to occupy the full length of the wire. The driver must put enough charge into it so that the wire relaxes within the switching voltage range of the receiver. Our simulations show that the driver can do this for state wires up to about 24000 lambda.

The above summary captures the results of our second study of wire delays in GasP. The distributed RC model that we use for the state wires in our second study significantly reduces the maximum module distance suggested by the simple lumped capacitance model used in our first study [5]. ${ }^{4}$

${ }^{4}$ The goal of our first study in [5] was to understand the effects of the
In the lumped capacitance model, the delay of a selfresetting loop depends on the delay of the state wire attached to it. With a lumped capacitance model, the forward self-resetting loop delay always exceeds the forward transfer delay and the backward self-resetting loop delay always exceeds the backward transfer delay. Thus, in this model, a self-resetting loop can never prematurely terminate the drive of "its own" state wire, but the self-resetting loop's partner still can. Therefore, the worst that can happen is that the delays of the two self-resetting loops in a GasP module differ enough so that the faster loop terminates prematurely the drive of the slower state wire. The lumped capacitance model predicts correct operation provided the difference in the lengths of the two state wires is below some maximum, which is 37000 lambda in our technology.

The Distance Constraint Graph [6] in Figure 7 gives a graphical overview of the results of both studies.

\section{Conclusion and Future Work}

The need to drive a state wire for "long enough" to deliver adequate charge presents a new kind of timing constraint. Unlike most constraints that consider the time of arrival of two signals, this one considers the shut off time of a drive signal. Moreover, it is couched not in terms of something that has happened, but in terms of something that will happen after the signal ceases.

Knowing the importance of charge gives us a better understanding of single-track communication. It is comforting to know that GasP modules can function, albeit with reduced throughput, with state wires so long that their internal delay is nearly a full GasP cycle. Such long wires deliver signals after their drive has ceased.

One might accommodate longer state wires by extending the drive period for a longer time. However, because GasP circuits obtain logical simplicity by representing a send or receive transaction as a pulse of standard duration, we are reluctant to make some GasP modules slower than others.

Exploring wire lengths beyond what might be considered reasonable, and still observing correct GasP functionality, has taught us that GasP designs offer quite a bit of freedom to trade off distance and cycle time.

These results apply equally to other single-track handshake protocols, including $[1,15,9,2]$. All single-track circuits, even those that are based on the conservative overlapping protocol by van Berkel and Bink in [1], cease to charge or

500 versus 5000 lambda long state wires in Infinity. According to the wire classification in Section 5 these are short wires. The lumped capacitance model was O.K. to use in the context of our first study. But it is not the correct model to understand the delay effects of medium and long wires in GasP, which is why we use a distributed RC model in our second study. 
discharge the wire, or wires in case of $[9,2]$, based only on information available at the near end. The present study extends the operational analysis available for single-track signaling beyond the lumped capacitance model.

We are currently investigating how to add long wires into the logical effort models for logic with interconnect [7], how to support them in our timing analysis tools [4], and how to engineer them to minimize the loss in latency and throughput [8].

Perhaps the most important implication of this study is that one can engineer the wires! Indeed, in the current era of circuit technology where the properties of wires dominate delay as well as power and area, one should engineer the wires as well as the transistors. For example, a $50 \%$ increase in the width and spacing allocated for a 6-4 GasP state wire can improve its delay by a factor of two at an area cost of less than $1 \%$ for a single-rail 64-bit datapath. It is wise to allocate more space to a single-track state wire that makes multiple transitions per transaction than to a bundled data wire that makes only one. Improvement of selected state wires may further optimize the design at locations where the bi-directional single-track signaling would otherwise fail to keep up with the uni-directional data, as for example shown in [3]. Understanding how to use wire engineering to improve distance and cycle time permits us to reason about and optimize the insertion and placement of GasP repeaters.

We hope soon to build a next test chip to confirm that real GasP circuits can indeed operate with long state wires. This chip will also explore how engineering the wires can improve performance. We hope that measurements from such a chip will shed light on the speed, the power demand, and the reliability of long-range GasP circuits.

Acknowledgments We gratefully acknowledge Jon Lexau for (running)* our $90 \mathrm{~nm}$ simulations. Sun Microsystems paid for the design, fabrication and testing of Infinity, and also sponsored the present work through a generous grant to the Portland State University Foundation.

\section{References}

[1] K. v. Berkel and A. Bink. Single-Track Handshake Signaling with Application to Micropipelines and Handshake Circuits. In Proc. Advanced Research in Asynchronous Circuits and Systems (ASYNC), pages 122-133, 1996.

[2] M. Ferretti and P. Beerel. High Performance Asynchronous Design Using Single-Track Full-Buffer Standard Cells. IEEE Journal of Solid-State Circuits, 41(6):1444$1454,2006$.

[3] R. Ho, J. Gainsley, and R. Drost. Long Wires and Asynchronous Control. In Proc. IEEE International Symposium on Asynchronous Circuits and Systems (ASYNC), pages 240-249, 2004.
[4] P. Joshi. Static Timing Analysis of GasP. Master of Science thesis, Electrical Engineering, Faculty of the USC Viterbi School of Engineering, University of Southern California, December 2008.

[5] P. Joshi, P. Beerel, M. Roncken, and I. Sutherland. Timing Verification of GasP Asynchronous Circuits: Predicted Delay Variations Observed by Experiment. In D. Dams, U. Hannemann, M. Steffen (Eds.): Willem-Paul de Roever Festschrift, LNCS 5930, pages 260-276. Springer-Verlag Berlin Heidelberg, 2010.

[6] S. Mettala Gilla. Distance Constraint Graph: A Graphical Representation for 6-4 GasP showing how Relative Timings constrain the Module Distances. Technical Report, ARC2009-smg01, Asynchronous Research Center, Portland State University, September 2009.

[7] A. Morgenshtein, E. Friedman, R. Ginosar, and A. Kolodny. Timing Optimization in Logic with Interconnect. In Proc. International Workshop on System Level Interconnect Prediction (SLIP), pages 19-26, 2008.

[8] F. Mu and C. Svensson. Analysis and Optimization of a Uniform Long Wire and Driver. IEEE Transactions on Circuits and Systems I: Fundamental Theory and Applications, 46(9):1086-1100, 1999.

[9] M. Nyström, E. Ou, and A. Martin. An Eight-Bit Divider Implemented with Asynchronous Pulse Logic. In Proc. IEEE International Symposium on Asynchronous Circuits and Systems (ASYNC), pages 229-239, 2004.

[10] A. Peeters and K. van Berkel. Single-rail Handshake Circuits. In Proc. Asynchronous Design Methodologies, pages 53-62, 1995.

[11] J. Rabaey, A. Chandrakasan, and B. Nikolić. Digital Integrated Circuits: A Design Perspective. Prentice-Hall, 2003.

[12] K. Stevens, R. Ginosar, and S. Rotem. Relative Timing. In Proc. IEEE International Symposium on Advanced Research in Asynchronous Circuits and Systems (ASYNC), pages 208218, 1999.

[13] K. Stevens, R. Ginosar, and S. Rotem. Relative Timing. IEEE Transactions on Very Large Scale Integration (VLSI) Systems, 11(1):129-140, 2003.

[14] I. Sutherland. A Six-Four GasP Tutorial. Technical Report, UCIES2007-is49 at http://fleet.cs.berkeley.edu/docs, 2007.

[15] I. Sutherland and S. Fairbanks. GasP: A Minimal FIFO Control. In Proc. IEEE International Symposium on Asynchronous Circuits and Systems (ASYNC), pages 46-53, 2001.

[16] I. Sutherland, B. Sproull, and D. Harris. Logical Effort: Designing Fast CMOS Circuits. Morgan Kaufmann, San Francisco, 1999.

[17] N. Weste and D. Harris. CMOS VLSI Design: A Circuits and Systems Perspective. Addison-Wesley, 2005. 
Appendix P

BONUS MATERIAL: THESIS POWERPOINT PRESENTATION 

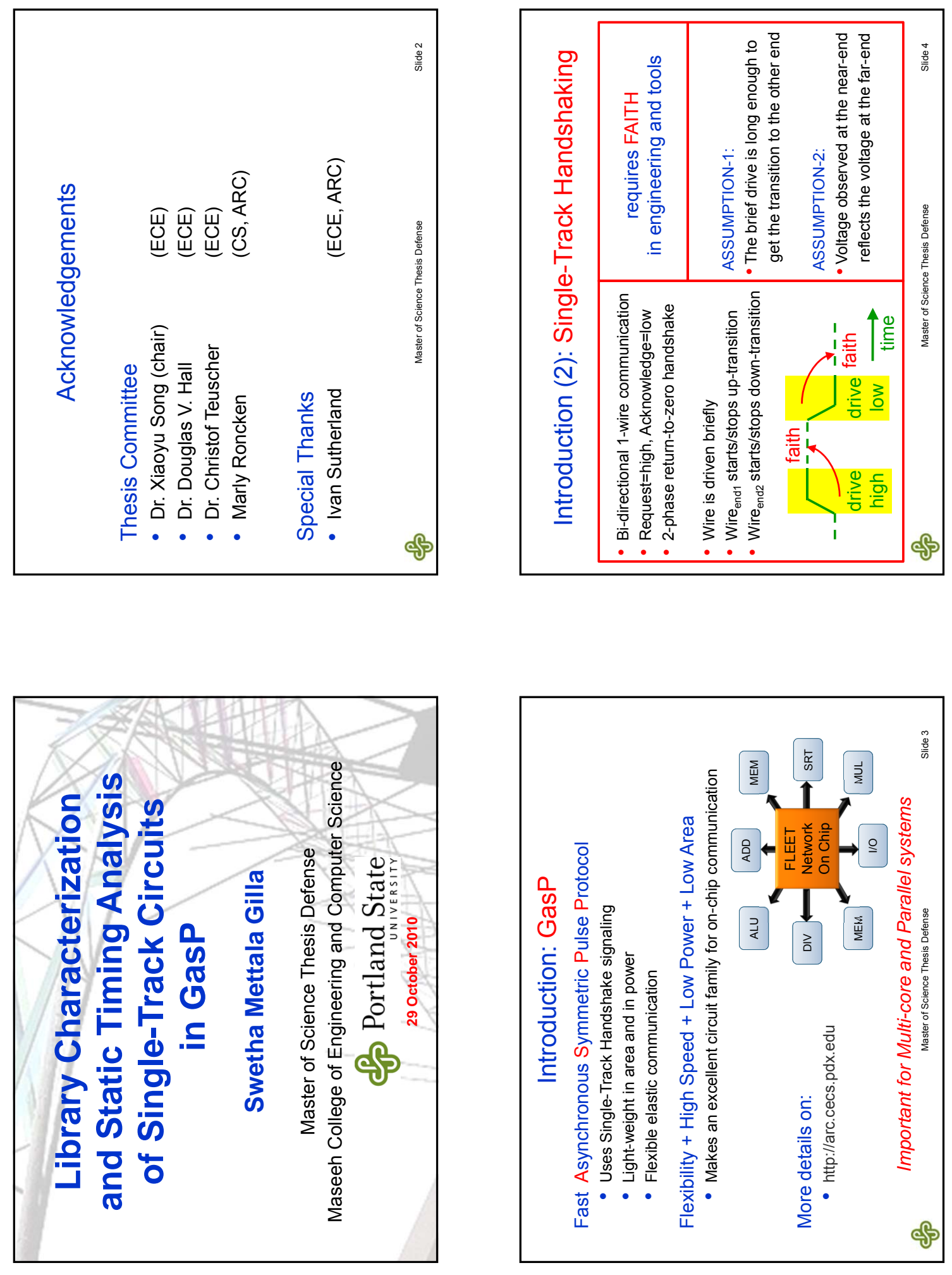

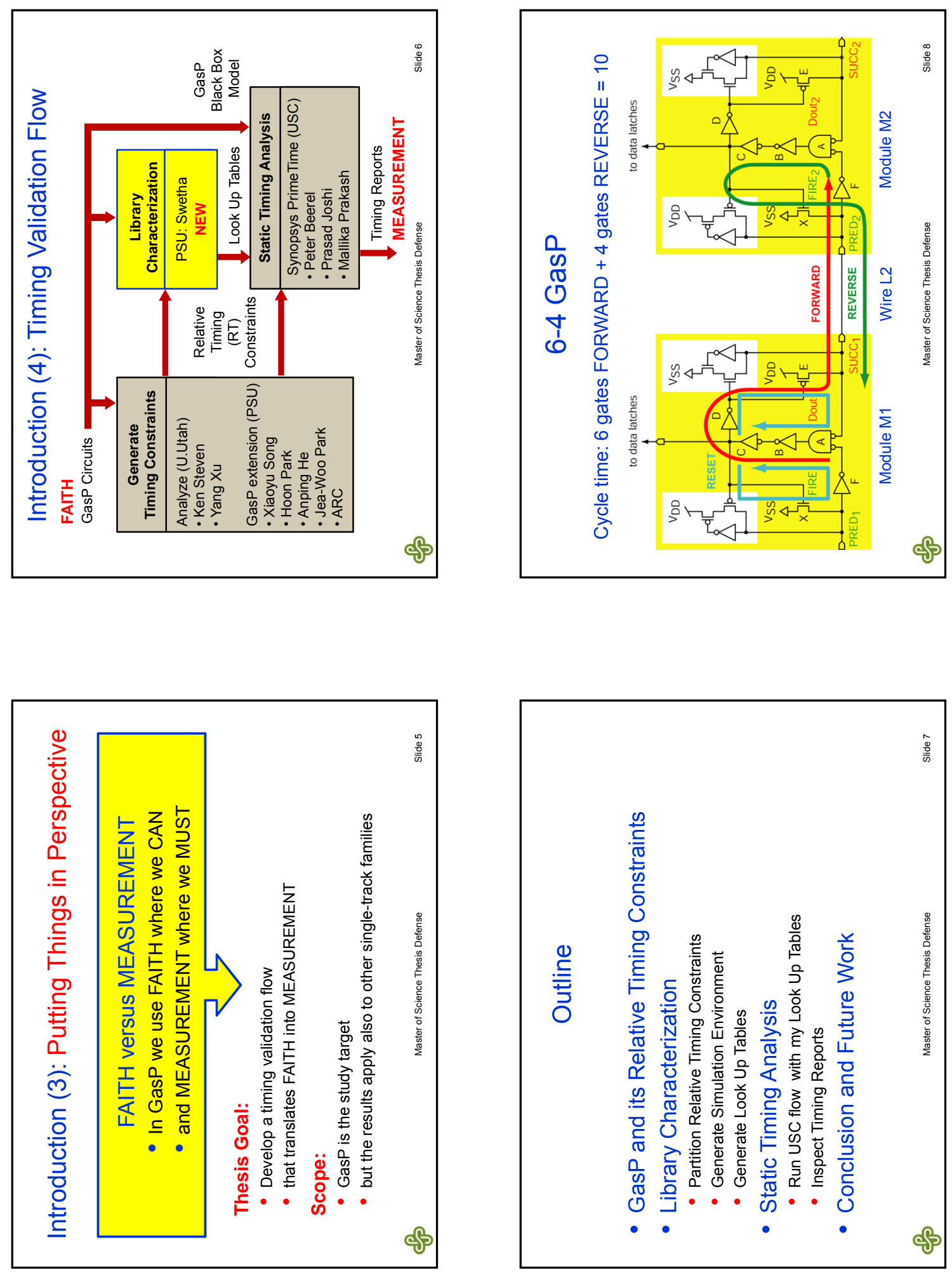

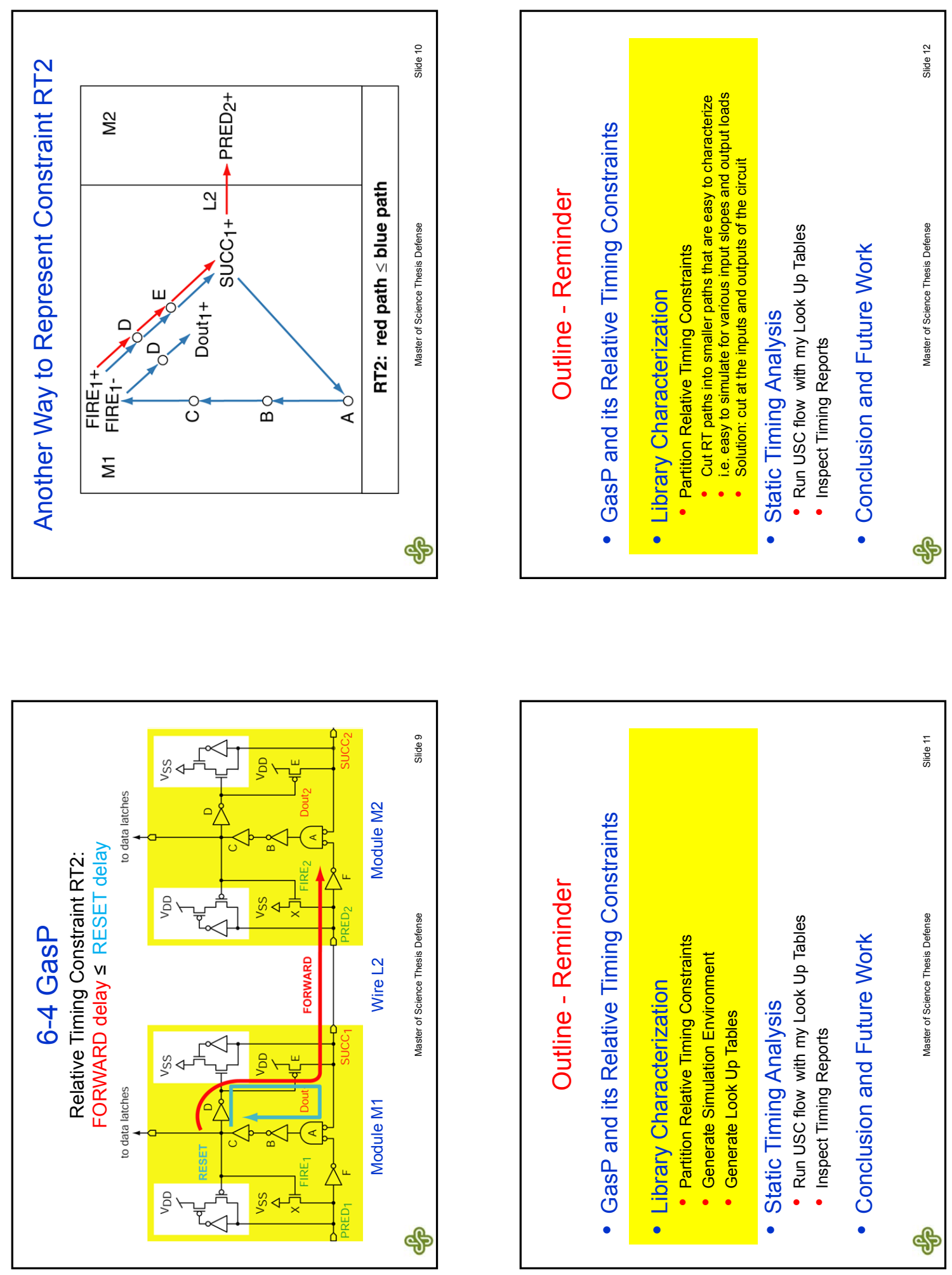

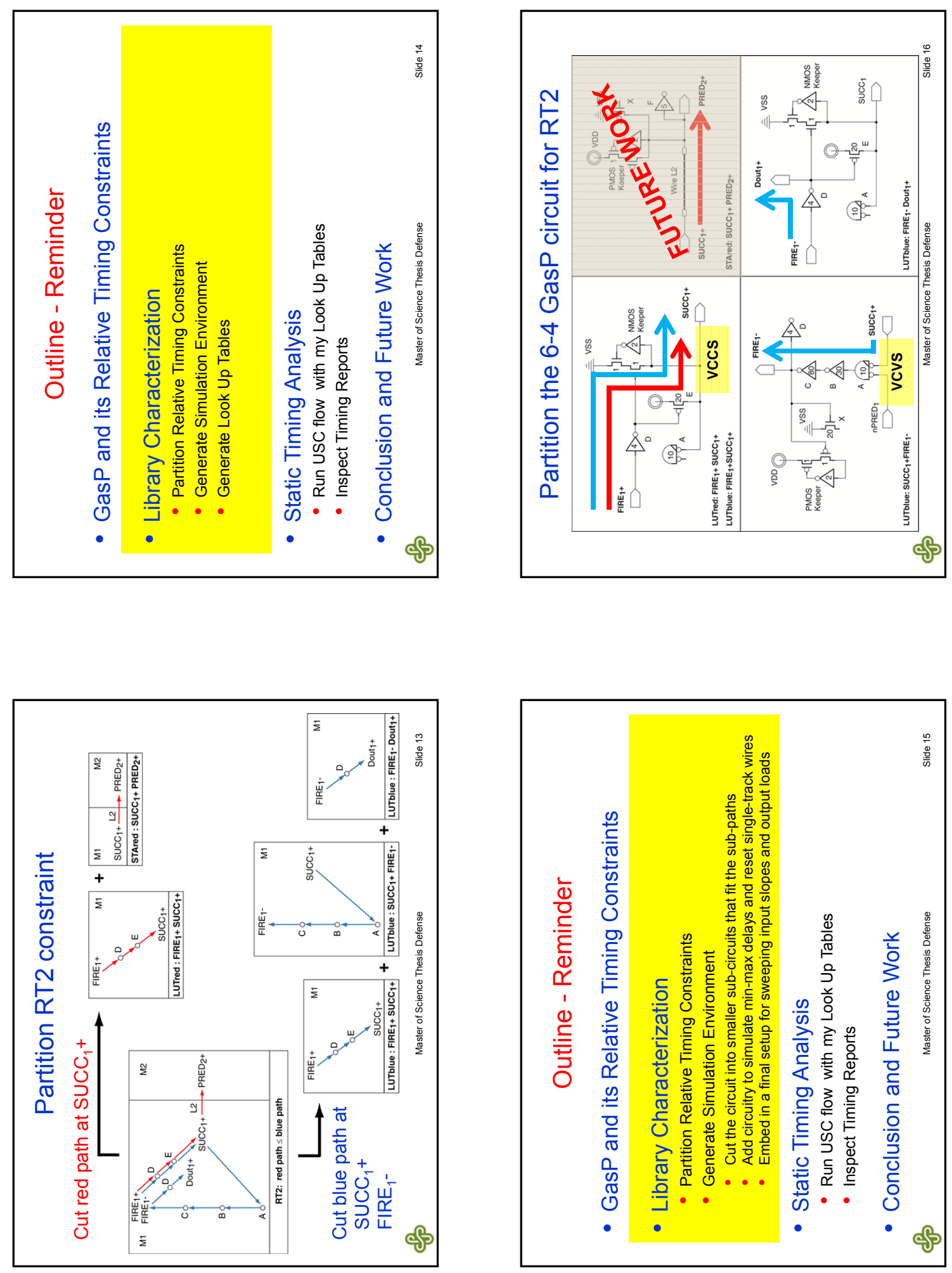

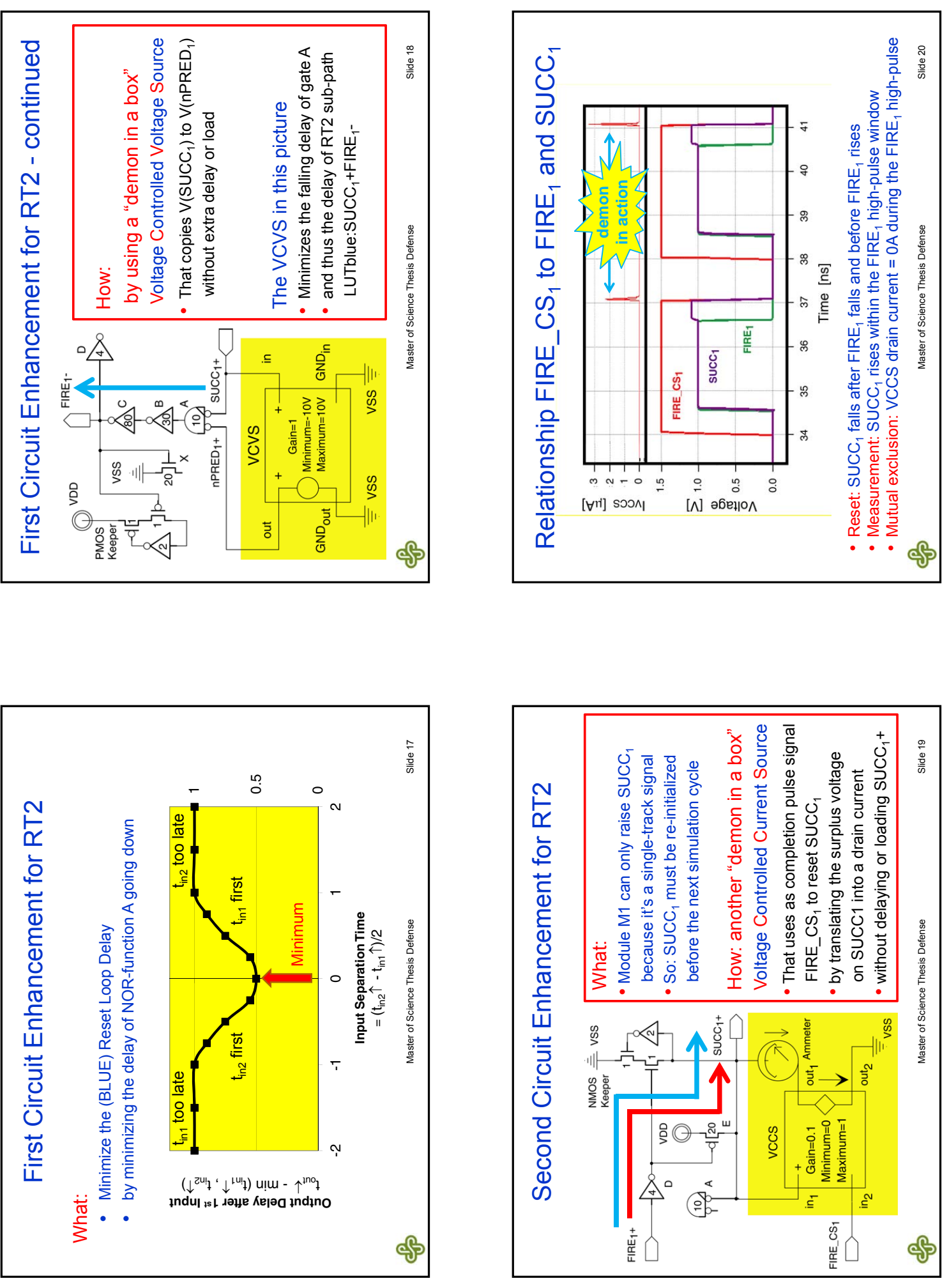

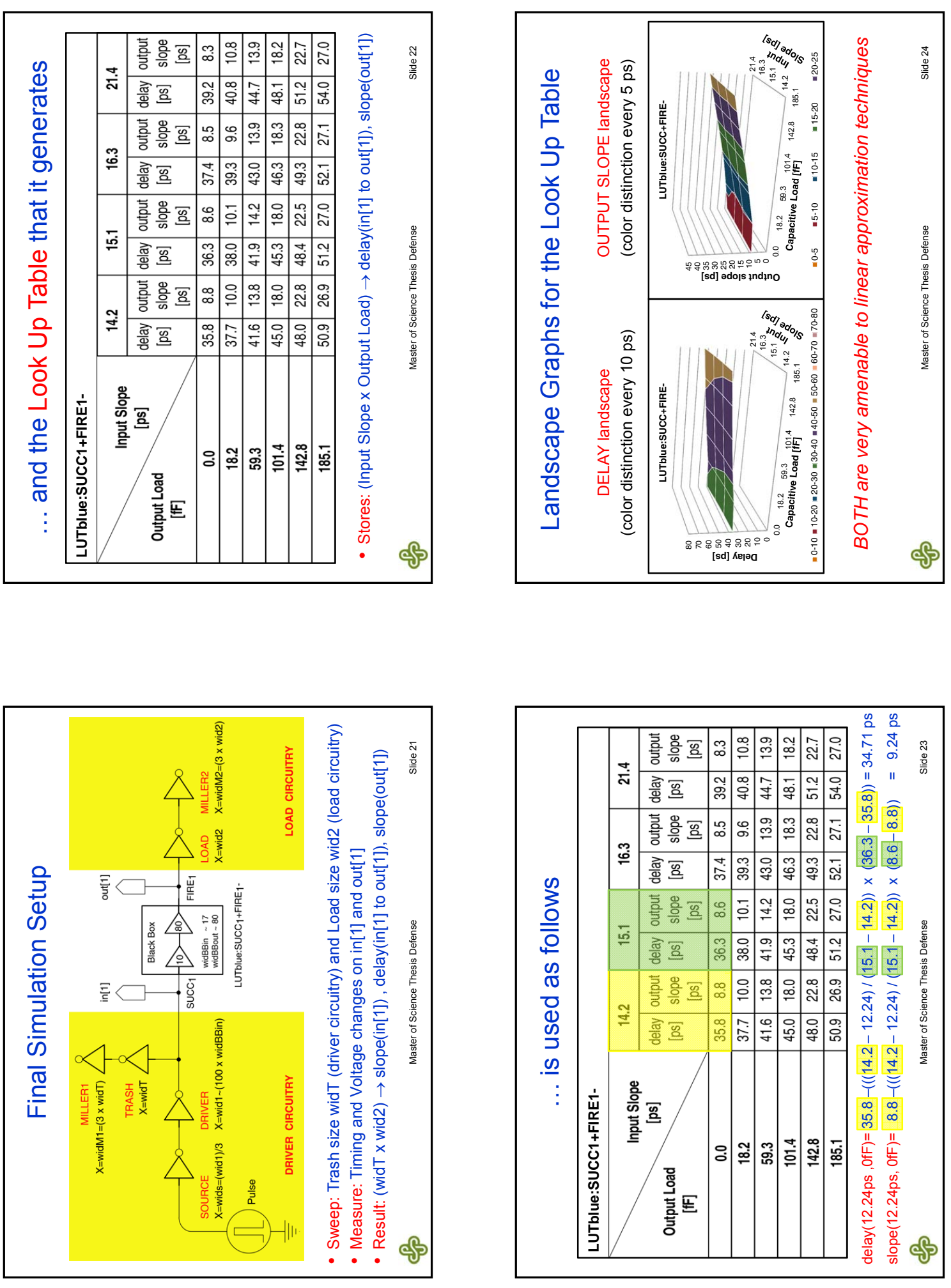

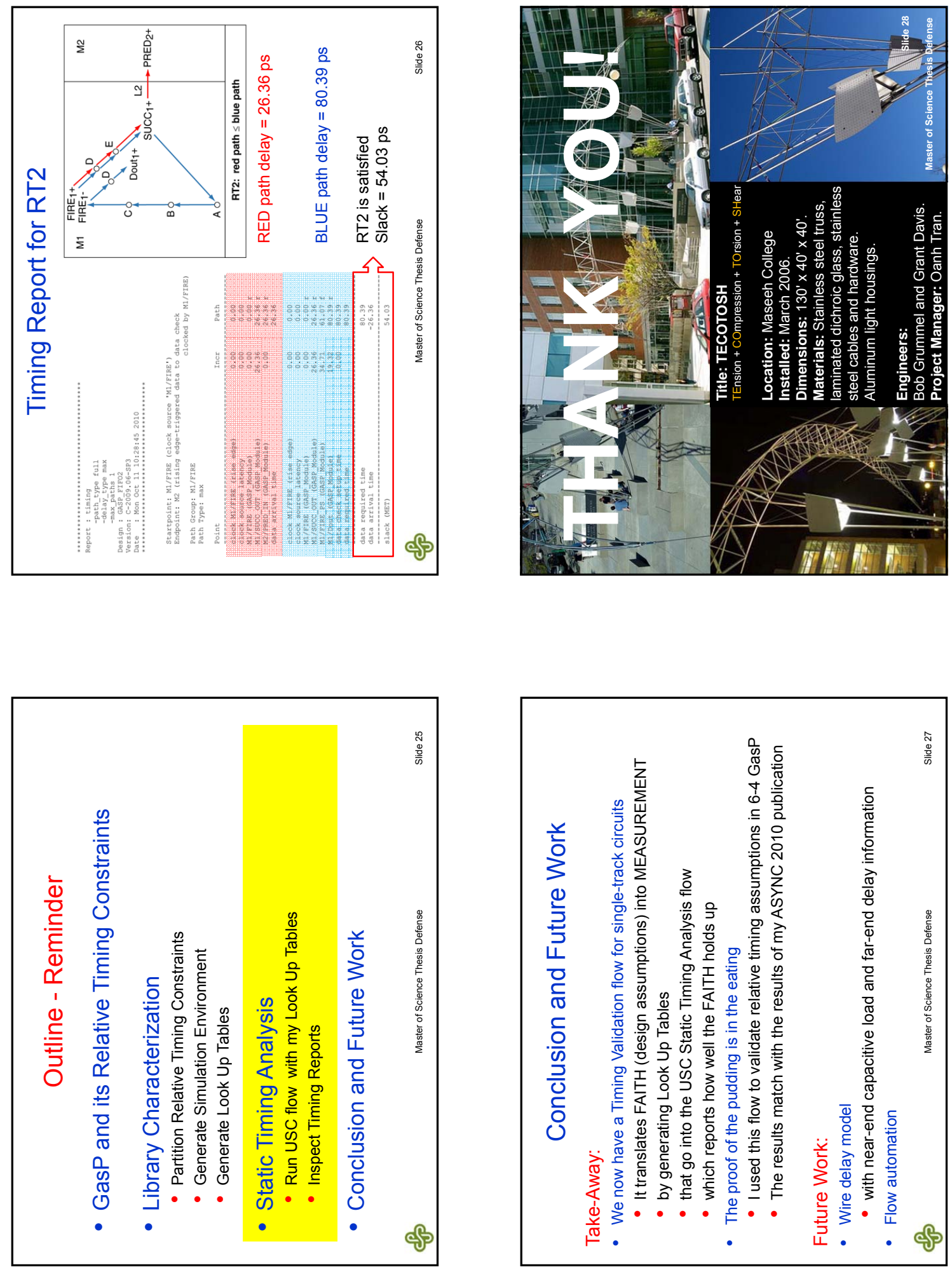